RATIONAL DESIGN OF NANOSTRUCTURED POLYMER ELECTROLYTES AND SOLID - LIQUID INTERPHASES FOR LITHIUM BATTERIES

\author{
A DISSERTATION \\ PRESENTED TO THE FACULTY OF THE GRADUATE SCHOOL \\ OF CORNELL UNIVERSITY
}

IN PARTIAL FULFILLMENT OF THE REQUIREMENTS FOR THE DEGREE OF DOCTOR OF PHILOSOPHY

BY

SNEHASHIS CHOUDHURY

AUGUST 2018 
(C) 2018 SNEHASHIS CHOUDHURY 


\title{
RATIONAL DESIGN OF NANOSTRUCTURED POLYMER ELECTROLYTES AND SOLID - LIQUID INTERPHASES FOR LITHIUM BATTERIES
}

\author{
Snehashis Choudhury, Ph. D.
}

Cornell University 2018

Advances in understanding of the basic science and engineering principles the underpin performance of electrochemical storage technologies is imperative for significant progress in portable electrical storage. In this regard, metal based batteries comprising of a reactive metal (like $\mathrm{Li}, \mathrm{Na}, \mathrm{Al}$ ) as anode have attracted significant attention because of their promise of improving the anode-specific capacity by as much 10-fold, compared to the current state-of-art Li-ion battery using graphitic anode. Perhaps their greatest advantage lies in the possibility of using of a Li-free high-capacity cathode like oxygen that can improve the gravimetric energy density of batteries from $\sim 0.3 \mathrm{kWh} / \mathrm{kg}$ to $\sim 12 \mathrm{kWh} / \mathrm{kg}$ (i.e. comparable to the useful energy available from combustion of hydrocarbons). A persistent challenge with batteries based on metallic anodes, concerns their propensity to fail by short-circuits produced by dendrite growth during battery recharge, as well as by runaway of the cell resistance due to internal side reactions with liquid electrolytes. The work reported in this thesis utilizes multiscale transport modeling and experiments to fundamentally understand and to thereby develop rational designs for polymer electrolytes and electrode - electrolyte interphases that overcome these challenges . On the basis of a linear stability analysis of dendrite growth during 
metal electrodeposition, it is shown that the length - scale on which transport occurs near the electrodes can be as important as electrolyte modulus in stabilizing metals against dendrite formation. To evaluate this proposal, cross-linked polymer electrolytes were designed with tunable pore size and the stability of metal electrodeposition was quantified in these systems. Direct visualization of electrodeposition using these electrolytes showed remarkable agreement with the theoretical predictions. Furthermore, when operated in a battery, the crosslinked membrane demonstrated stable galvanostatic cycling of lithium metal anodes for several hundreds of hours. Importantly, these studies showed that while the tendency for battery failure by dendrite-induced short-circuits can be reduced, the issue of capacity-fading as a result of continuous reactions of the metal with liquid electrolyte persists. Through multiscale modeling of ion transport, artificial solid electrolyte interphase designs are proposed for lithium-oxygen batteries to enable stable recharge and low overpotentials even with chemically reactive liquid electrolytes. 


\section{BIOGRAPHICAL SKETCH}

Snehashis Choudhury was born in a small town of Brajrajnagar in the state of Odisha in India. He went to high-school in Kolkata, India. As a child, his favorite topics were Mathematics, Chemistry and Economics. Following his high-school, he decided to pursue Engineering over his second choice of Economics. He went to National Institute of Technology Calicut to pursue Bachelor of Technology in Chemical Engineering in the year 2009. The four years of experience in NIT Calicut was one of the best time of his life, where he made several great friends and also learnt about independent research. He joined Cornell University for his Masters of Engineering in the School of Chemical and Biomolecular Engineering in the year 2013. He worked in the group of Professor Lynden Archer, thereafter continued as a PhD student in the following year. In this $\mathrm{PhD}$, he started his work on designing covalently grafted hairy nanoparticles to understand their structure and dynamics. In his second year Cornell, he developed an interest in understanding the instabilities and thereafter stabilizing of metal-based batteries. He found that the prior-designed hairy nanoparticles are excellent candidates in serving as solid or gel electrolytes to inhibit battery short-circuits in lithium metal batteries. However, a glaring failure mechanism he discovered in these metal batteries, was the unwanted side reactions at the battery interfaces causing slow fading in the capacity. He took this challenge head-on by designing artificial interfaces to inhibit these side reactions, thereby enabling stable cycling of a solid polymer electrolyte in a high energy metal battery. He will always be grateful to all the collaborators and group members for their constant support not only in research but also in other endeavors. 


\section{DEDICATED TO MY FAMILY, MY STUDENTS AND TO ALL THOSE WHO}

LIVE WITH A PASSION 


\section{ACKNOWLEDGMENTS}

I have to apologize as my words will not do justice to all the help and support I have received from everyone in my $\mathrm{PhD}$ journey and in my life. I would like to firstly thank Prof. Lynden Archer for his help not only in my $\mathrm{PhD}$ work but also in my career counseling. His knowledge in a wide spectrum of subjects and his passion for science has always driven me to improve every single day I spent at Cornell. I greatly appreciate the help from my committee members Prof. Yong Joo and Prof. Geoffrey Coates. I greatly enjoyed the discussions with them on my research.

Perhaps one of the most satisfying and inspiring experience at Cornell was my Teaching Assistant responsibilities. Overall, I served as a TA for four different times in the Chemical Engineering and Physics department. I want to thank every student in these classes, who have inspired me and helped me in discovering my love for teaching. I am grateful to Prof. Julius Lucks, Prof. William Olbricht and Prof. Chris Alabi for providing me guidance and as well as independence in conducting lectures and recitations in my TA classes.

Thanks to all the former and current members of Archer group. I am greatly thankful to Rajesh for being my mentor in first year at Cornell as a Master of Engineering student. I have to say, I wouldn't have been in the $\mathrm{PhD}$ program without Rajesh's guidance. I am sincerely grateful to Akanksha who have been a great friend, mentor and collaborator. I will always miss our long conversations about research, people and life 
with her while simultaneously working long hours in lab. Also, in course of my $\mathrm{PhD}, \mathrm{I}$ met a great human being and researcher, Zhengyuan, whose dedication towards research was extraordinary. I thank him for making my life so easy in handling different projects and serving as a great partner in everything I worked on in my $\mathrm{PhD}$. Over the five years at Cornell, I have worked with several undergraduate and masters students who have been more of my mentor than vice-versa. I am thankful to Charles, Dylan and Sanjuna for their constant support in research and making my experience in the group so memorable.

A big thanks to Himanshu, Prayag, Samanvaya, Sanjuna, Dylan, Ritesh, Pooja, Zhengyuan, Anubhav, Yue, Alex, Kaihang, Nijam, Rohit, Prajwal, Rahul, Mun Sek, Sampson for their strong support in my in research and life. I am greatly obliged to all my collaborators outside Archer group, Prof. Tomas Arias, Prof. Mendoza Cortes, Prof. Ravishankar Sundaraman, Prof. Donald Koch and Dr. A. Nijamudheen. I am specially grateful to Professor Lena Kourkoutis and Dr. Michael Zachman for their constant support in cryo-electron microscopy. Also, I am thankful to all the staff scientists for their help at Argonne National Lab and Cornell High Energy Synchrotron Facicility.

Lastly, and most importantly, I want to thank my parents and brother for their love and sacrifice all through my life. 


\section{TABLE OF CONTENTS}

ABSTRACT

BIOGRAPHICAL SKETCH V V V V V V V v

DEDICATION Vi

ACKNOWLEDGEMENT vii

CHAPTER 1: INTRODUCTION ........................................... 1

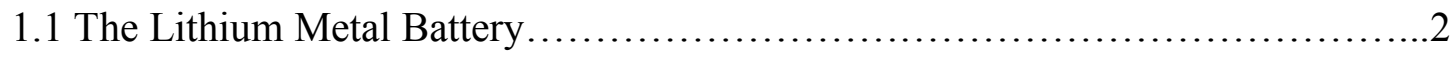

1.2 Rational Design Principles.............................................. 5

1.2.1 Nanostructured Electrolytes...........................................

1.2.2 Solid-Liquid Interphases......................................... 10

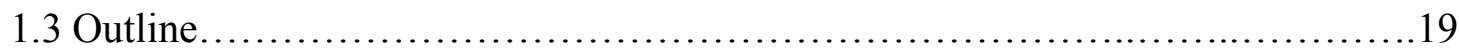

CHAPTER 2: SELF-SUSPENDED SUSPENSIONS OF COVALENTLY GRAFTED

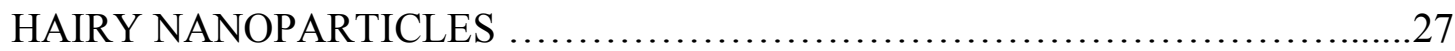

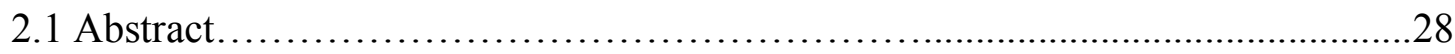

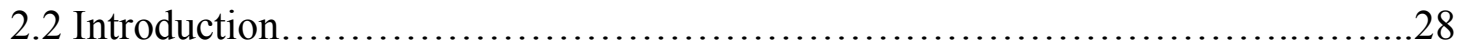

2.3 Experimental Section...................................................... 31

2.3.1 Synthesis of Self-Suspended Covalently Grafted Nanoparticles................31

2.3.2 Characterization..................................................... 33

2.3.3 Small Angle X-Ray Scattering Measurements........................... 33

2.3.4 Rheology Measurements............................................. 34 
2.4 Results and Discussion...................................................... 34

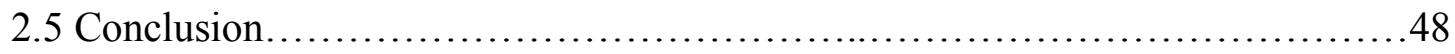

Acknowledgement................................................................ 49

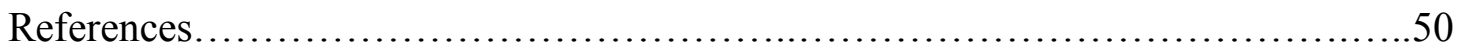

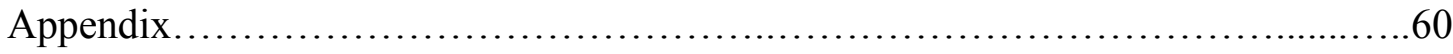

CHAPTER 3: A HIGHLY CONDUCTIVE, NON-FLAMMABLE POLYMERNANOPARTICLE HYBRID ELECTROLYTE _..................................69

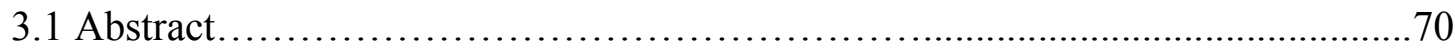

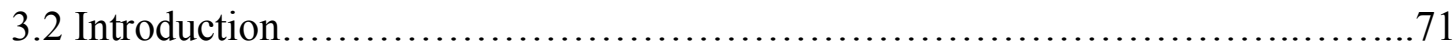

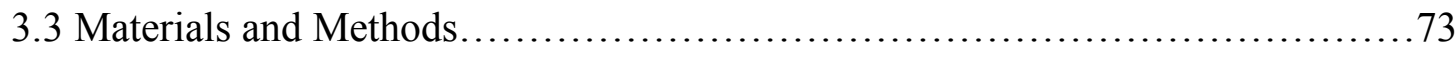

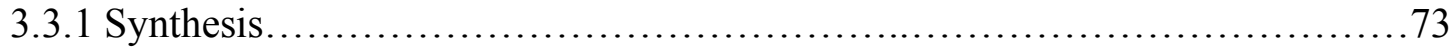

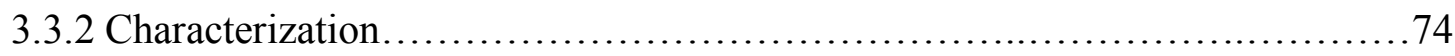

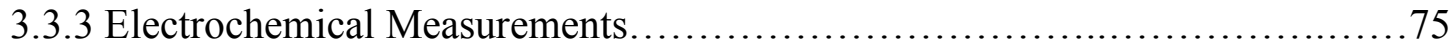

3.3.4 Characterizing Flammability ........................................... 75

3.4 Results and Discussion................................................. 77

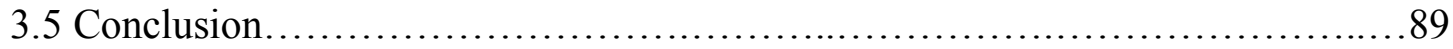

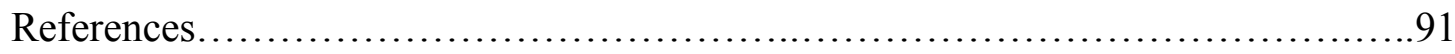

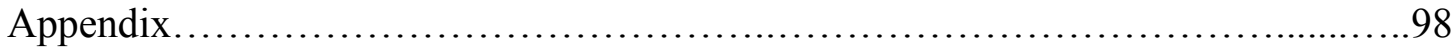

CHAPTER 4: HYBRID HAIRY NANOPARTICLE ELECTROLYTES STABILIZE

LITHIUM METAL BATTERIES............................................ 102 


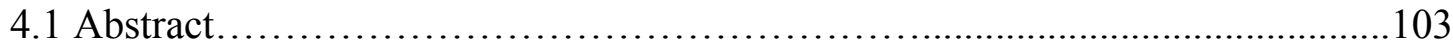

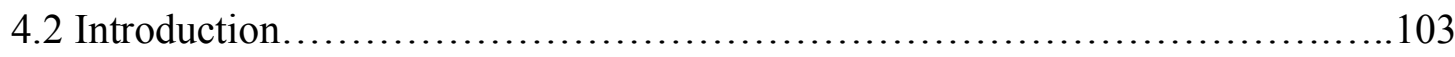

4.3 Materials and Methods..................................................... 107

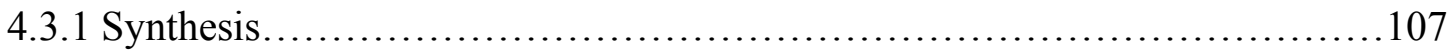

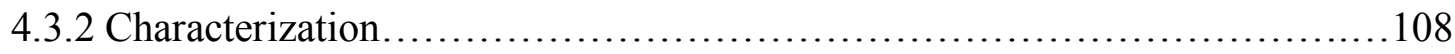

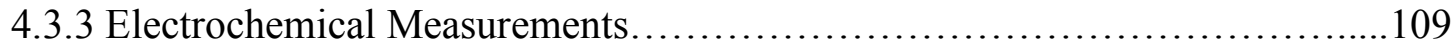

4.3.4 Analyzing the Columbic Efficiency .....................................110

4.3.5 Cell Lifetime Study .....................................................111

4.4 Results and Discussion.................................................111

4.4.1 Physical Characterization and Ion Transport................................73

4.4.2 Structural Factor Analysis..............................................114

4.4.3 Variation of Interfacial Resistance ...................................... 118

4.4.4 Surface Characterization of Li Anode..................................... 120

4.4.5 Enhanced Electrochemical Stability of Nanocomposites......................123

4.4.6 Analyzing Galvanostatic Performance......................................124

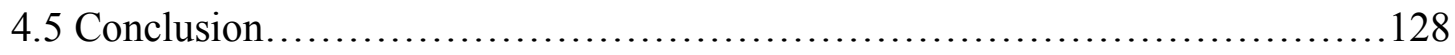

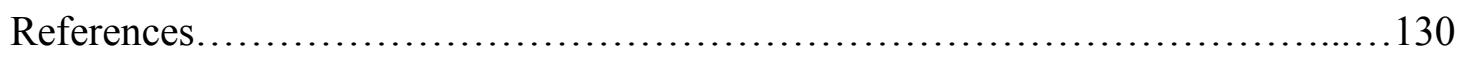

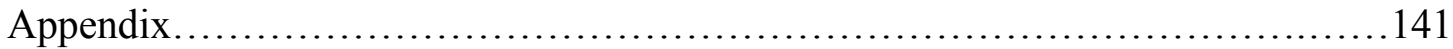

CHAPTER 5: A HIGHLY REVERSIBLE ROOM TEMPERATURE LITHIUM

METAL BATTERY BASED ON CROSS-LINKED HAIRY NANOPARTICLES.146

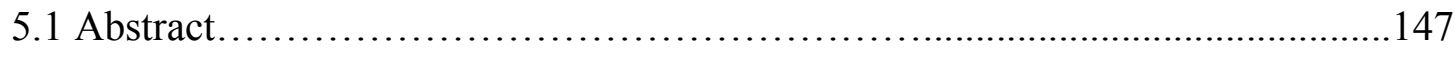

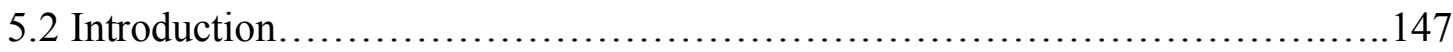


5.3 Methods

150

5.3.1 Materials

5.3.2 Nanoparticle-Polymer Crosslink Synthesis and Composite Electrolyte

Preparation

5.3.3 TEM And Small Angle X-Ray Scattering.

5.3.4 Mechanical Properties

152

5.3.5 Electrochemical Characterization.

5.3.6 Cell Lifetime and Failure Studies 153

5.3.7 Measuring the Coulombic Efficiency. 153

5.3.8 Half-Cell Testing. 154

5.4 Results 155

5.4.1 Synthesis and Physical Characterization of Crosslinked Membrane. .155

5.4.2 Mechanical and Electrochemical Properties of Crosslinked Membrane 159

5.4.3 Analyzing Stability of Lithium Electrodeposition Using Crosslinked

Membranes. 162

5.5 Discussion. 169 
Acknowledgments

170

References............................................................ 171

Appendix.............................................................. 179

CHAPTER 6: CONFINING ELECTRODEPOSITION OF METALS IN

STRUCTURED ELECTROLYTES......................................... 193

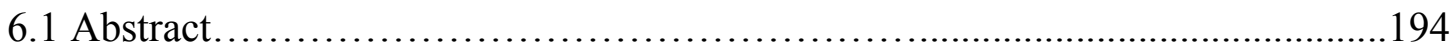

6.2 Significance........................................................ 195

6.3 Introduction........................................................ 195

6.4 Materials and Methods.................................................... 198

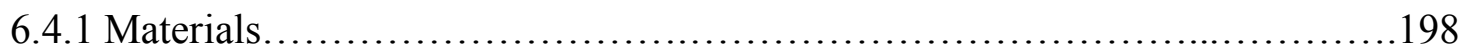

6.4.2 Linear Stability Analysis........................................... 198

6.4.3 Crosslinked Hairy Nanoparticles Synthesis.................................. 198

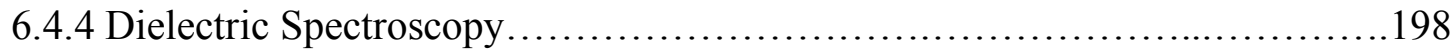

6.4.5 Transmission Electron Microscopy...................................... 199

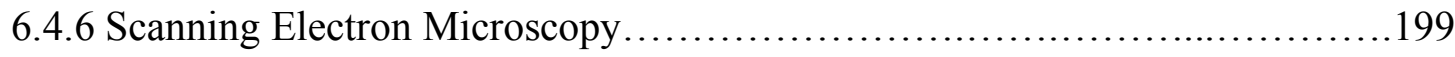

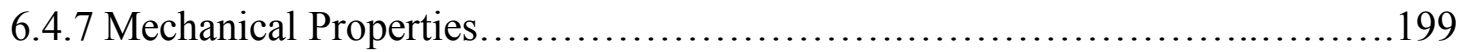

6.4.8 Direct Visualization Experiments...................................200

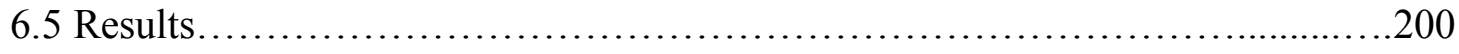

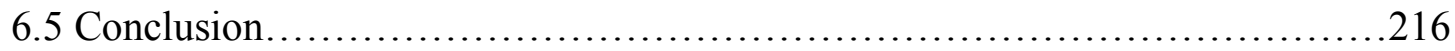

Acknowledgements...................................................217

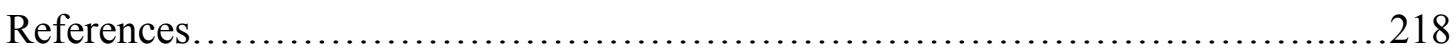




\section{CHAPTER 7: SOFT COLLOIDAL GLASSES AS SOLID-STATE}

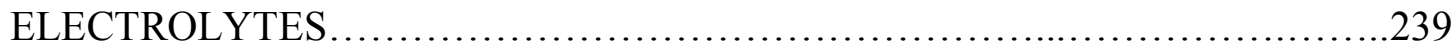

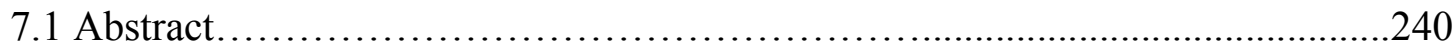

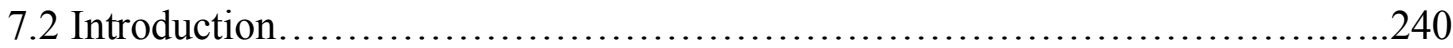

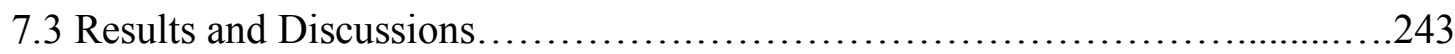

7.3.1 Synthesis and Chemical Analysis......................................243

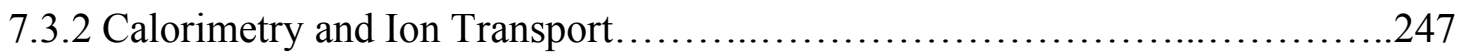

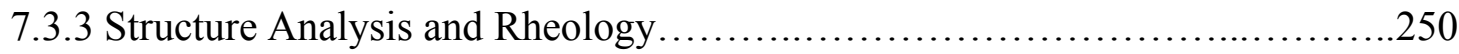

7.3.4 Analysis of Electrochemical Performance...............................257

7.5 Conclusion........................................................261

Acknowledgements..................................................... 261

References.......................................................... 263

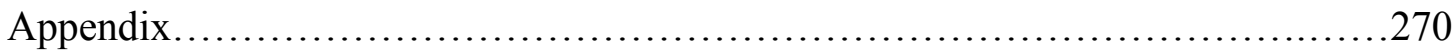

CHAPTER 8: SOLID POLYMER INTERPHASES FOR LITHIUM METAL

BATTERIES ......................................................285

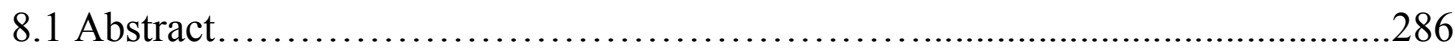

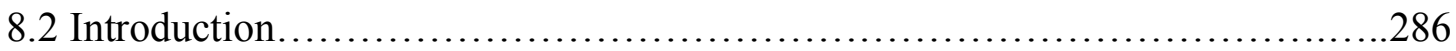

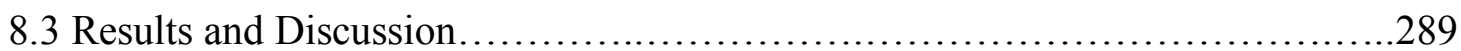

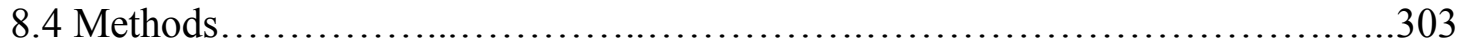

8.4.1 Fabrication of crosslinked polymer network and coated lithium................303 
8.4.2 Material Characterization......................................................

8.4.3 Electrochemical Characterization..............................................304

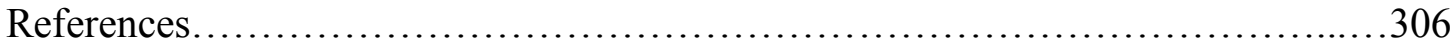

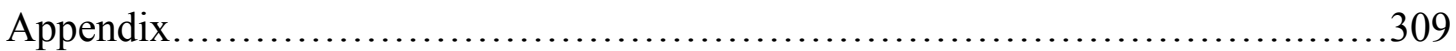

CHAPTER 9: STABILIZING POLYMER ELECTROLYTES IN HIGH-VOLTAGE

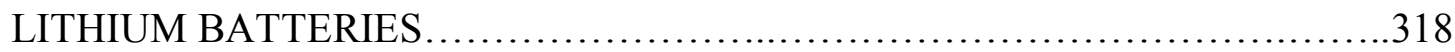

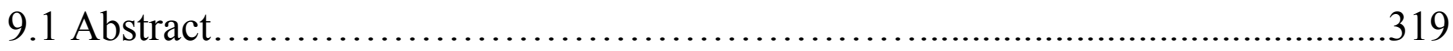

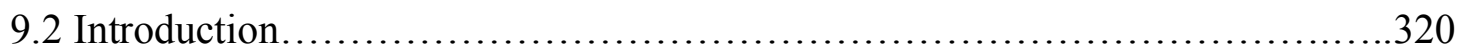

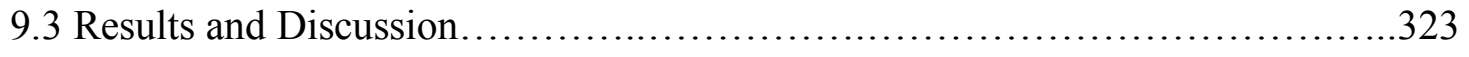

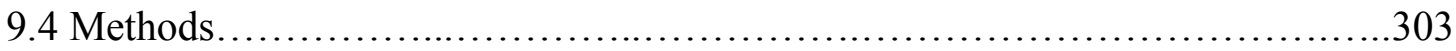

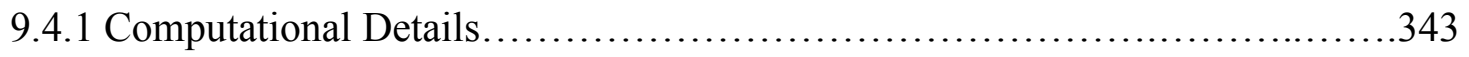

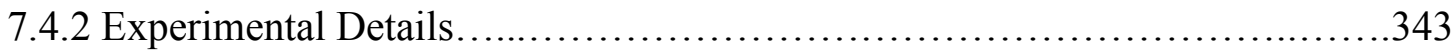

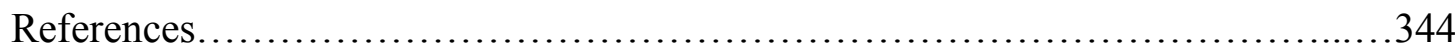

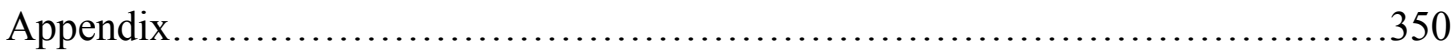

CHAPTER 10: LITHIUM FLUORIDE ADDITIVES FOR STABLE CYCLING OF

LITHIUM BATTERIES AT HIGH CURRENT DENSITIES..........................377

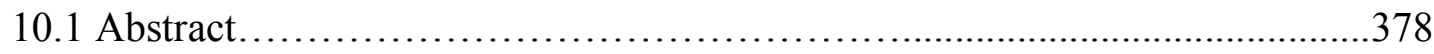

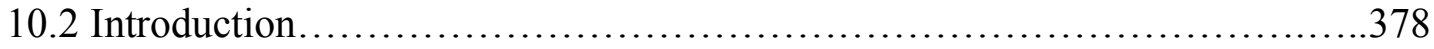

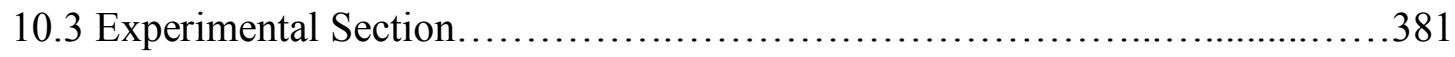

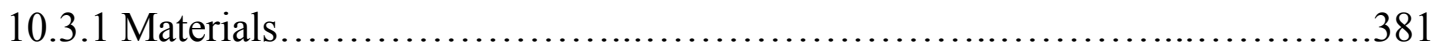

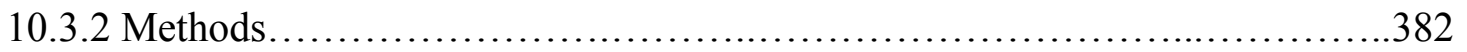




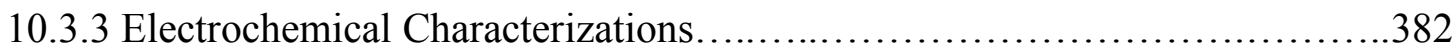

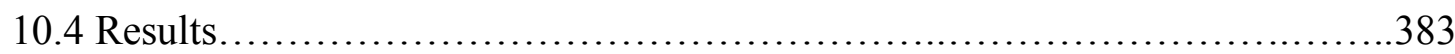

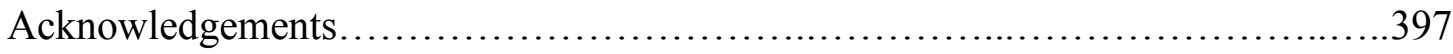

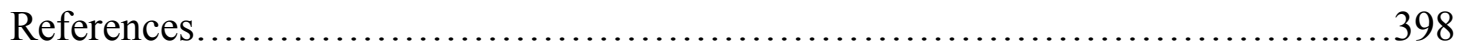

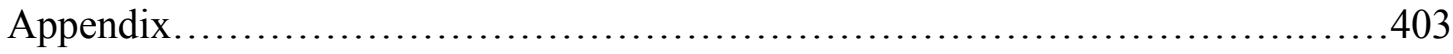

CHAPTER 11: DESIGNING SOLID-LIQUID INTERPHASES FOR SODIUM

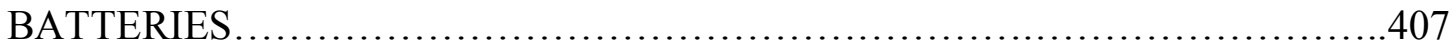

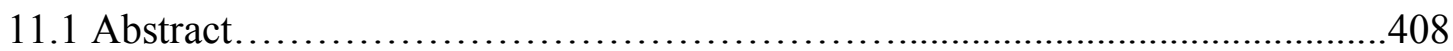

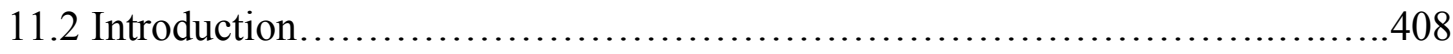

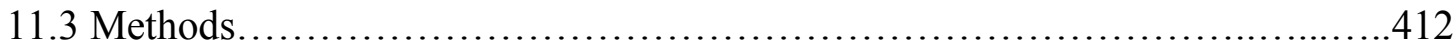

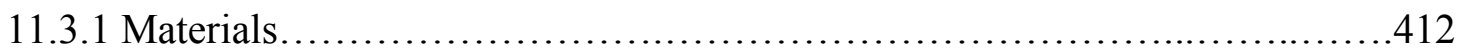

11.3.2 Sodium Bromide and Other Halide Coating Formation ......................412

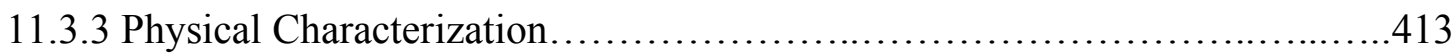

11.3.4 Electrochemical Characterization.............................................. 414

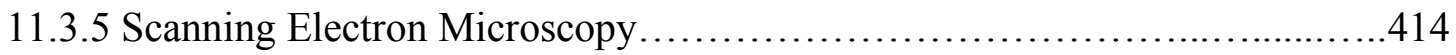

11.3.6 Focused Ion Beam/Scanning Electron Microscopy............................415

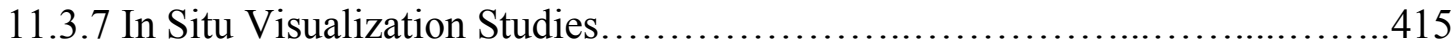

11.3.8 Cell Lifetime and Failure Studies..........................................416

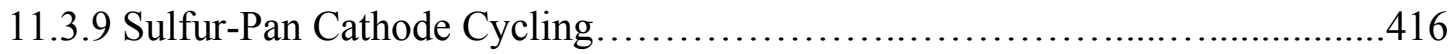

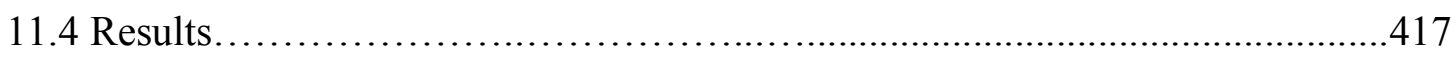

11.4.1 Joint Density-Functional Theory (JDFT) Study of SEI......................417

11.4.2 Formulation and Stability a NaBr-Based SEI Layer on Sodium Metal.........420 
11.4.4 Electrodeposition of Sodium Metal with $\mathrm{NaBr}$ Coated Anode................428

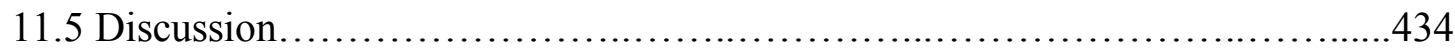

Acknowledgements....................................................435

References............................................................... 436

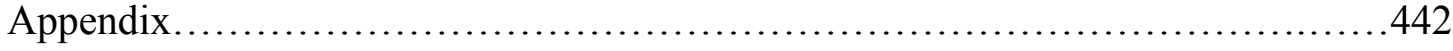

CHAPTER 12: ELECTROLESS FORMATION OF HYBRID LITHIUM ANODES

FOR HIGH INTERFACIAL ION TRANSPORT ..............................450

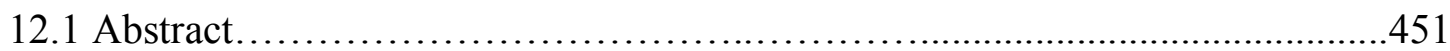

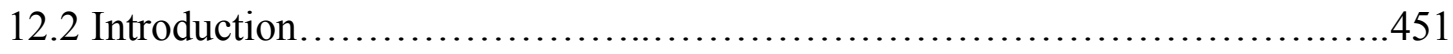

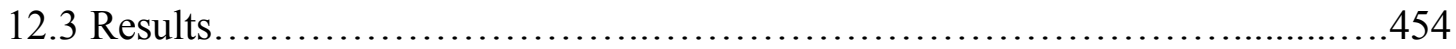

12.3 Conclusion......................................................468

Acknowledgements....................................................469

References.......................................................4 470

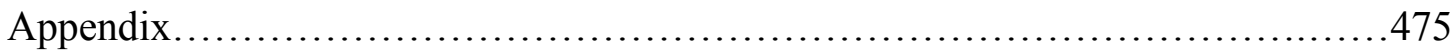

CHAPTER 13: DESIGNER INTERPHASES FOR THE LITHIUM-OXYGEN

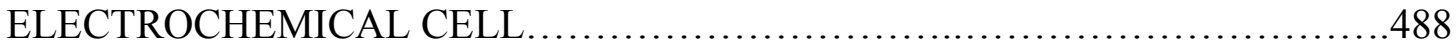

13.1 Abstract........................................................ 489

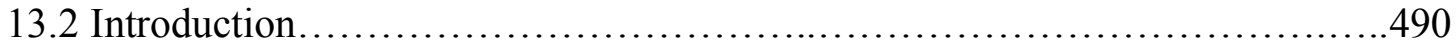

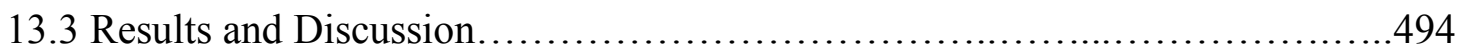

13.3.1 Understanding the Anode Protection Mechanism.........................494

13.3.1.1 Characterization of the Anode..........................................494 
13.3.1.2 Lithium-Electrolyte Stability .......................................50

13.3.1.3 Anode Protection Mechanism..........................................504

13.3.2 Understanding the Cathode Stabilization Mechanism......................506

13.3.2.1 Characterizing Cathode Products...........................................506

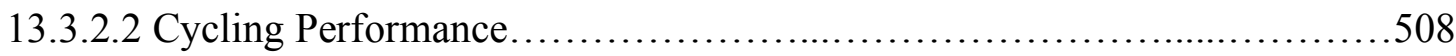

13.3.2.3 Cathode Stabilization Mechanism..........................................511

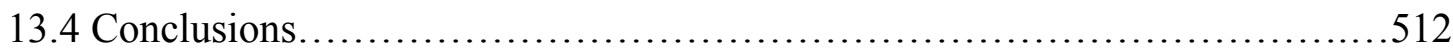

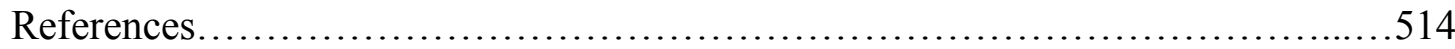

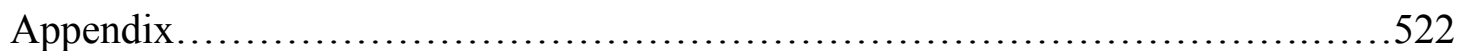

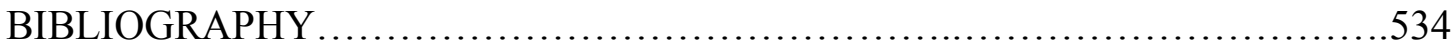


CHAPTER 1

INTRODUCTION 


\subsection{The Lithium Metal Battery}

Upsurge in consumer electronics and electrical appliances have necessitated the need for high power, lightweight, long lasting batteries at low cost. In this regard, lithium ion batteries have found their place in the commercial world because of their robustness and reversibility ${ }^{1-3}$. Moving forward in the line of energy storage advancement, the next generation batteries are predicted to also base on lithium metal, however, instead of using a graphitic anode for lithium intercalation, an absolute lithium film can serve as anode. Although, this replacement may seem trivial, use of lithium metal anode can mean a paradigm shift in battery technology. Some of the advantages of a lithium metal full cell battery over lithium ion batteries are as follows: 1) High power density, because rate of intercalation of Li ions into graphite anode is much slower than Li ion plating onto lithium anode; 2) Low cost, as use of Li anode avoids the cost of graphite in the anode; 3) Lightweight, for not using graphitic anode; 4) High energy density, use of Li anode provides the liberty of using many cathode materials like sulfur and oxygen that have the higher specific capacity than any currently used cathode ${ }^{4}$; 5) Batteries can be made in variety of form factors.

However, the main bottleneck in the practicality of Lithium metal batteries is the safety issue and low reversibility. During the alternate charge and discharge cycles, lithium may get unevenly deposited onto the anode, resulting in growth of dendritic structures having the potential of puncturing the separator and causing internal short circuit and at times explosion ${ }^{1}$. The low reversibility is related with the slow degradation of electrolyte due to parasitic reactions with the anode ${ }^{5}$. Although, these problems also persistent in Lithium ion batteries, they exacerbated in a Lithium metal 
battery, where the reactive surface of lithium is exposed to the organic electrolyte. Broadly, these issues can be divided in three categories-; 1) morphological instability inevitable dendritic deposition, especially at high current densities; and 2) chemical instability - unregulated reactions at electrode-electrolyte interface and 3) hydrodynamic instability - unstable ion transport that create space charge near the electrode surface. We believe that the origin of these instabilities is a three-stage process $^{6}$, as shown in Figure 1.1. In stage-1, the electrolyte passivates the Li metal electrode by side reactions owing to its high reactivity, however this passive layer so called solid-electrolyte interface (SEI) is non-uniform, creating gradients of surface conductivity. In stage-2, the Li ions selectively electrodeposit on regions having higher conductivity, leading to formation of uneven and sharp nucleates (deposits). These rough deposits increase the surface area of contact between the electrode and electrolyte, thus amplifying the unwanted parasitic reactions resulting in batterycapacity fade over time. Finally, in stage-3, by repeated battery cycling, the lithium ions continue to deposit on the tips of these nucleates due to higher local electric field causing the formation of needle like dendrites that grow bigger in size to ultimately short-circuiting the cell, which often accompanied with fire and explosions. My PhD thesis is bifurcated in two major topics- 1) designing theory-inspired nanostructured electrolytes for preventing the proliferation of dendrite. 2) understanding the role of interfacial chemistry in the nucleation step of electrodeposition. There has been extensive scientific research in this field for past four decades to understand the origin of such instabilities in a battery and techniques to mitigate them. 


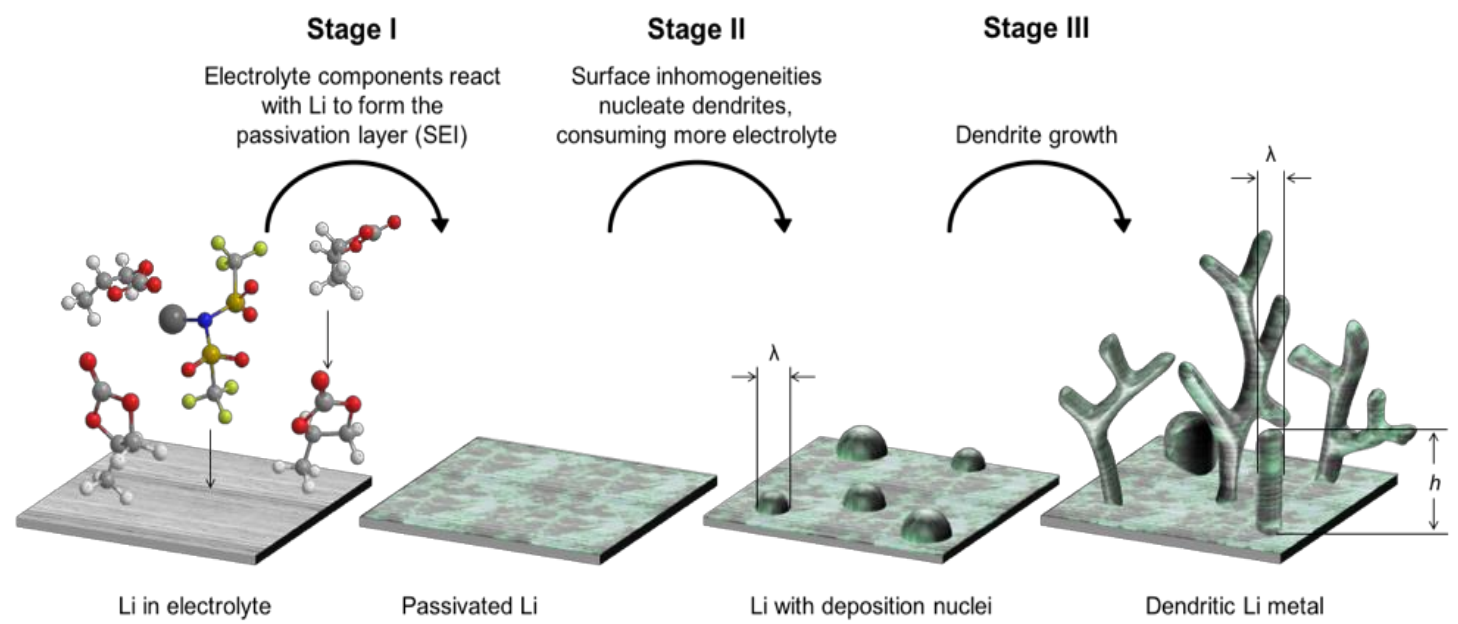

Figure 1.1 Schematic showing different stages of instabilities during electrodeposition on a lithium metal anode 


\subsection{Rational Design Principles}

The three frameworks discussed in the last section all lead to different recommendations for enabling $\mathrm{Li}$ anodes. Some common methods include use of additives for stabilizing electrode-electrolyte interface ${ }^{7-12}$, high mechanical strength separator for providing compression force on the surface ${ }^{13-16}$, nanostructured electrolytes for controlling the length-scale of electrodeposition ${ }^{17-19}$ and ion transport modification using single ion conducting electrolytes ${ }^{20,21}$. Overall, thee strategies have led to significant attention among the researcher to fundamentally understand the transport and thermodynamic aspects of nanostructured electrolytes and solid-liquid interphases. The on-going efforts in these two broad areas are discussed here:

\subsubsection{Nanostructured Electrolytes}

Solid-state electrolytes have recently gained significant attention because of the general notion in battery literature that although chemical modifications in liquid electrolyte recipe can extend the lifetime of battery to a significantly large timescale, but it cannot explicitly ensure safety. Previous work by Monroe and Newman using solid mechanics showed that dendrite growth can be prevented using a solid electrolyte with modulus twice that of the electrode. ${ }^{22,23}$ In this regard, ceramics have been of primary focus of investigation as candidates for SSE's. Previously, Dudney et al. ${ }^{24}$ designed a SSE with LiPON chemistry that demonstrated over 10,000 cycles of galvanostatic charge and discharge in Lithium vs. NMO configuration, with minimal capacity fading and close to $100 \%$ coulombic efficiency as shown in Figure 1.2. The long cycle-life performance sets a benchmark for rechargeable battery operation; 


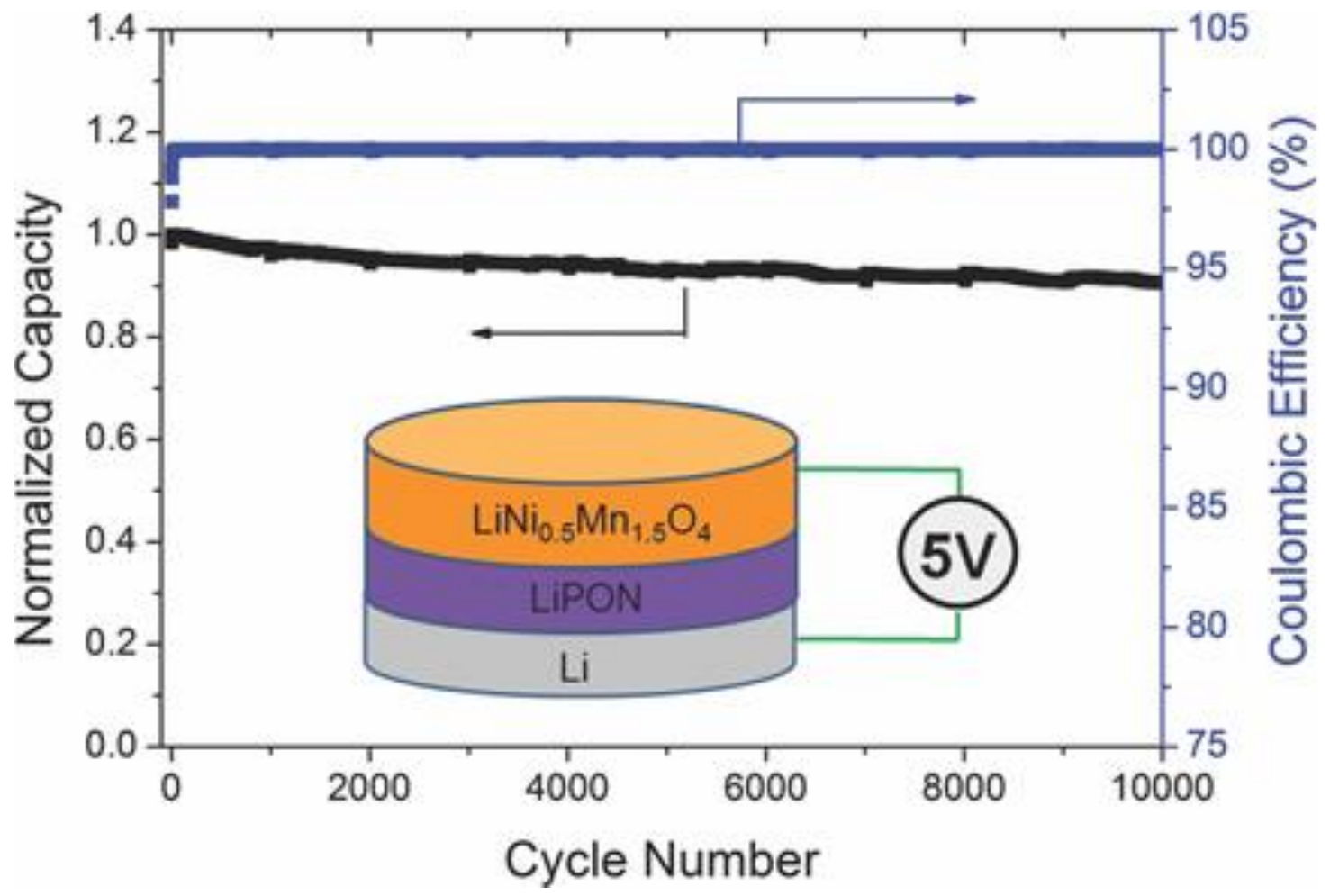

Figure 1.2: LiPON based solid - state electrolyte used in Li\|NMO micro battery showing stable performance for over 10,000 cycles with high capacity retention and coulombic efficiency. (Adapted from: Li, J., Ma, C., Chi, M., Liang, C. \& Dudney, N. J. Solid Electrolyte: the Key for High- Voltage Lithium Batteries. Adv. Energy Mater. $5,1401408(2015))$ 
which to best of our knowledge hasn't been reported in batteries based on liquid electrolytes. However, there are major shortcomings associated with the LiPON solid electrolyte and the most notable is the low ionic conductivity $\left(<10^{-5} \mathrm{~S} \mathrm{~cm}^{-1}\right)$ at room temperature. It is for this reason, the cell reported in the mentioned work is microscale and similar performance hasn't been replicated in pouch level or even coin-type cell configurations. There has been intensive research in this area for the chemical modification of the ceramic electrolytes for improving the conductivity, however the electrochemical stability is seen to deteriorate. Another major disadvantage with the ceramic-based solid electrolytes is the poor solid-solid contact between electrode and electrolyte that causes major setback in the interfacial conductivity. Further, the brittleness and high cost of raw material imparts a huge challenge in terms of commercialization of the metal batteries with inorganic solid electrolytes.

Recent on nanostructured electrolytes based on alumina membrane ${ }^{18,25}$ and crosslinked hairy nanoparticles ${ }^{17}$ that has gained significant attention owing to the unprecedented stable cycling and simplicity of implementation. Tikekar et al. ${ }^{16,26}$ using linear stability analysis of dendrite growth analyzed different properties of electrolyte components required for 'dendrite-free' battery operation. In a nutshell, the electrode surface was modeled a perturbation equation: $H_{c}=L+H_{c} e^{\sigma t} e^{i k x}$ where, $\sigma$ represent the dendrite growth rate and $k$ as the inverse deposition length-scale. The growth rate was further shown to depend on multiple factors like surface tension, modulus and anion transference number of the electrolyte. On analyzing dendrite growth with during small electrode - perturbations (that represent the initial nucleation size during deposition), it was seen that under typical operating conditions, the surface tension of 
electrolyte-electrode interphase plays a major role in limiting the growth rate of dendrites at low-order nucleate sizes, in fact below a critical size absolute stability can be attained as shown in the state diagram in Figure 1.3. Further it is evident that the critical nucleate size can be manipulated by varying anion transference number and the electrolyte modulus. In light of these revelations, we systematically designed nanoporous alumina separator with varying pore-size. It was seen that pore-size correlated with the deposition length-scale, such that the high-modulus separator framework restricted the higher-than-pore dendrite growth. Thus, it was possible to eliminate battery failure by short circuits even in liquid electrolytes. Building on this diagnostic experimentation, we designed polymer composite membrane using crosslinked hairy nanoparticles for a scalable and low-cost solution. The membrane was designed by interlinking polyethylene oxide grafted silica nanoparticles with polypropylene glycol based cross-linker. In contrast to most previously reported polymer electrolytes, the crosslinked membrane simultaneously showed good mechanical strength $(\sim 1 \mathrm{MPa})$ and high ionic conductivity at room temperature $(\sim 5 \mathrm{mS} / \mathrm{cm})$, which is a consequence of the high crosslinking node points in these membranes. Direct visualization experiments were performed to understand the effect of pore-size on dendrite growth, which showed remarkable agreement with the theoretical predictions. Furthermore, when operated in a battery, the crosslinked membrane showed one of the highest short-circuit time compared to similar electrolytes reported in the literature. Unlike previous studies of nanocomposite electrolytes where nanoparticles were used as fillers in polymer solutions, this was one of the first studies, where nanoparticles were linked with polymer matrix using 


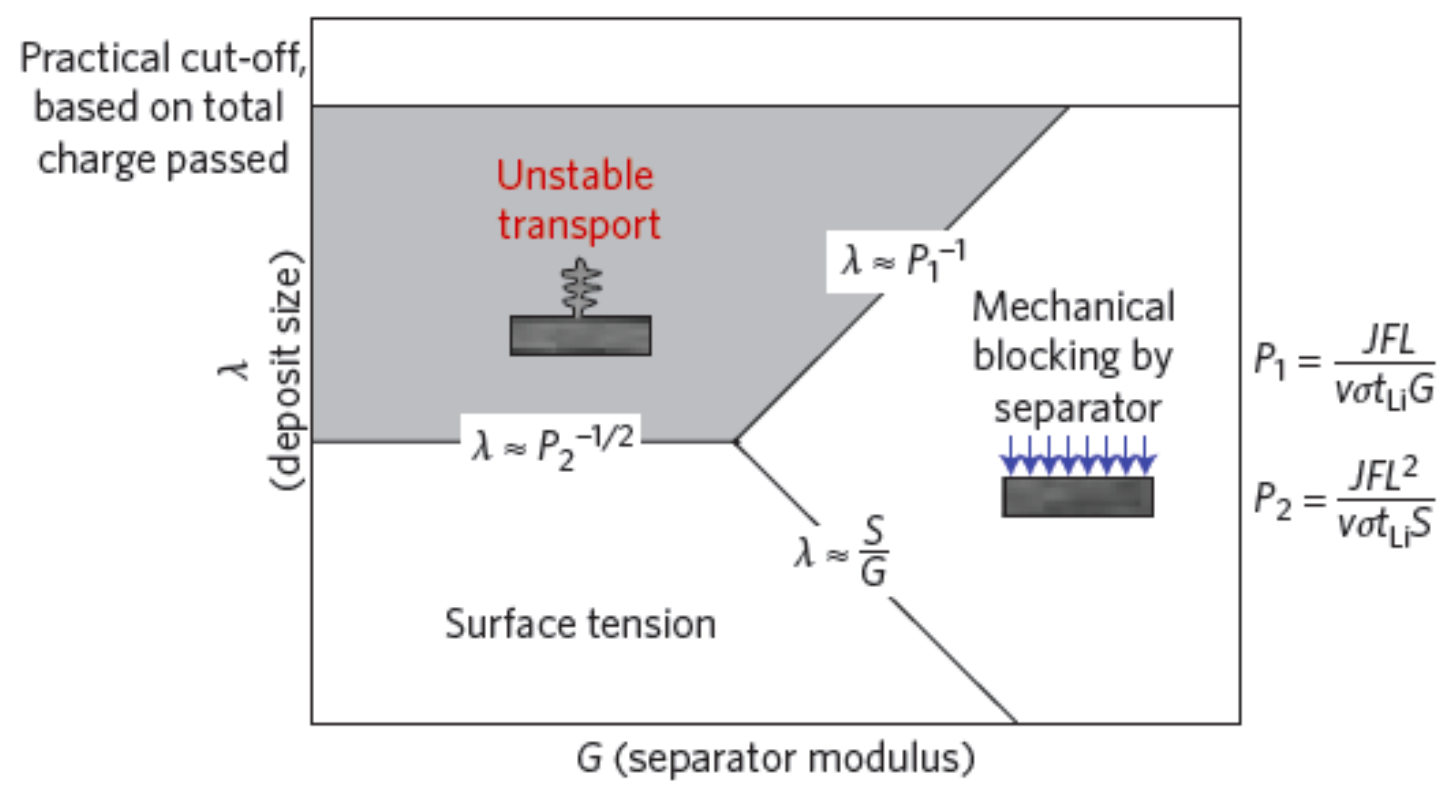

Figure 1.3: Theoretical state diagram showing how different parameters like surface tension, deposit size, transference number or modulus affects the deposition stability 
covalent bonding. Consequently, there have several follow-up studies involving synthesis of advanced materials with similar architecture for energy applications. ${ }^{27-31}$

Other nanostructure designs for inhibiting dendrite growth include on single ion conducting electrolytes with silica nanoparticle fillers with sulfonate groups tethered on their surface ${ }^{21}$ as well as UV-crosslinked pegylated sulfonic groups ${ }^{32}$ (see Figure 1.4). These electrolytes have been reported to have transference number higher than 0.8 , while having high ionic conductivity at room temperature. As previously mentioned, the high transference number or fixed anionic species lowers the threshold of instability during electrodeposition, thus it was observed that stable surface could be obtained with traditional separators. In another set of studies, it was reported that ionic liquids (eg. Imazolium, piperidium) when tethered to the silica nanoparticle surface $^{33,34}$, or crosslinked by electric field demonstrated stable cycling in lithium metal batteries ${ }^{35}$. These observations throw light on two different stabilizing mechanisms; ionic liquids act as supporting electrolytes in battery electrolyte, such that these species tend to crowd on specific unstable electrode regions thereby normalizing the overall electric field. Also, the silica nanoparticles as well as ionic liquids serve as stabilization agents for the SEI layer for preventing side reactions. ${ }^{36}$ Thus, it is clear that once can achieve significant gain in both scientific knowhow and battery performance on combining and unifying these stabilizing agents.

\subsubsection{Solid-Liquid Interphases}

Chemical modification of the solid-electrolyte interphase (SEI) is an elegant way of eliminating the dendrite forming nucleates. Conventional electrolytes form a relatively thick and insulating SEI layer on the Li metal, which cracks during Li ion insertion 


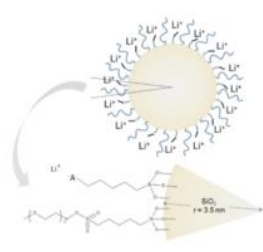

d

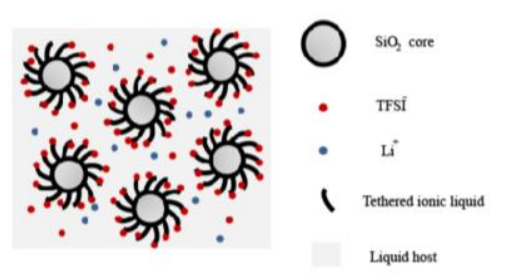

b

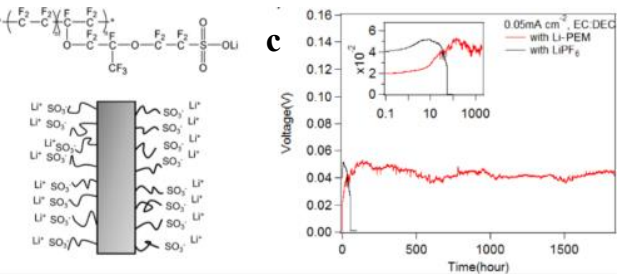

e

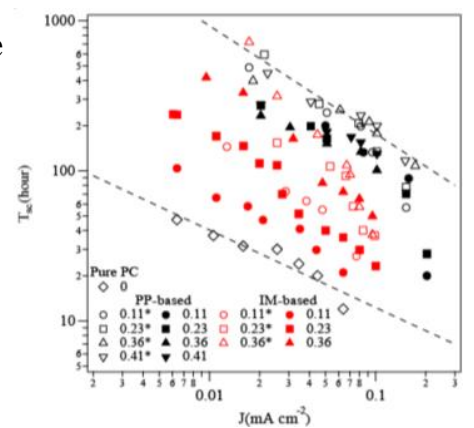

Figure 1.4: Ionic polymers and functionalized nanoparticle salts: (a) nanoparticle grafted with sulfonic acid-lithium salts; (b) polymerized ionic membrane; (c) polarization showing $>1500$ stable cycling with ionic membrane; (d) ionic liquid functionalized silica nanoparticles; (e) comparison of short circuit time of IL based nanoparticles with control liquid electrolytes 
and desertion, exposing fresh metal to the electrolyte leading to repeated formation of interfacial species, which can consume the electrolyte. The chemical modifications in this context apply to an artificial SEI layer, which often comprises of a different chemistry from the bulk electrolyte by use of additives or thin film technology. While the behavior of the interface appears in the aforementioned theories in form of the surface energy, the interface and the interfacial layers are not explicitly modeled, rendering this approach a somewhat trial-and-error effort.

Recently, a Stanford group showed that $\sim 99 \%$ coulombic efficiency can be achieved for a LMB by a two-fold anode protection technique ${ }^{33}$ comprised of: 1) stable SEI forming electrolytes with additives, and 2) a thin-film protective coating of interconnected hollow carbon nanospheres that shield the anode preventing direct contact with electrolyte ${ }^{33}$. This result provides a futuristic perspective toward stable lithium metal battery by combining the benefits of a stable electrolyte and a thin-film protector.

Aurbach et al. $(2002)^{5}$ realized that no electrolytes are stable when in contact with lithium metal causing the formation of a passivation layer that worsens at high charge and discharge rates due to the large volume changes. However, some electrolytes have indeed shown to form a more stable SEI layer than others. For example, 1,3 Dioxalane (DOL) undergoes ring opening reaction at the Li surface to form an elastic surface layer of polydioxalane oligomers that expand and contract with lithium insertion and desertion ${ }^{34}$. Similarly, glyme based electrolytes like Dimethoxyethane (monoglyme), diglyme, tetraglyme are also good for formation of alkoxy based SEI (ROLi), which stabilizes electrodeposition in both $\mathrm{Li}$ and $\mathrm{Na}$ metal batteries ${ }^{35,36}$. In contrast, 
Propylene Carbonate (PC) and other carbonate-based electrolytes form a very unstable, thick and insulating passivation layer mostly comprising of Lithium Carbonate $\left(\mathrm{Li}_{2} \mathrm{CO}_{3}\right)$ that can yield a maximum efficiency of $\sim 77 \%$ in absence of any additives. ${ }^{37}$ Thus, it is evident that the chemical composition of the interfacial layer is of utmost importance in determining the degree of coulombic stability.

Recently, Joint Density Functional Theory (JDFT) calculations ${ }^{38}$ revealed that surface diffusion barriers for $\mathrm{Li}$ are much lower in halide salts ( $\mathrm{LiBr}, \mathrm{LiI}, \mathrm{LiF}$ ) compared to regular SEI salts like $\mathrm{LiOH}$ and $\mathrm{Li}_{2} \mathrm{CO}_{3}$ (see Figure 1.5). This means that $\mathrm{Li}$ ions can move laterally and rearrange on the interfacial layer before getting deposited as Li atoms, thus having a lesser chance of forming dendritic nucleates. Based on this concept, Lu et al. ${ }^{39}$ used LiF additive in the electrolyte that showed remarkable results in terms of dendrite suppression and capacity retention (see Figure 1.6). An LiF rich SEI layer can also act as a thin film barrier between the anode and electrolyte, which is recently confirmed by coulombic efficiency measurements in presence of carbonatebased electrolytes resulting in over $10 \%$ improvement ${ }^{40}$. In other studies, an interfacial layer of $\mathrm{LiF}$ is formed by using fluoride-based additives in the electrolyte. These include Hydrogen Fluoride $(\mathrm{HF})^{41}$ and Fluoro-ethylene Carbonate (FEC), which stabilizes both Li metal ${ }^{42}$ and Silicon based anodes ${ }^{43}$ giving very high capacity for several cycles. Use of dual salt of LiTFSI and LiFSI is also one such technique, where the side reaction between these two salts in presence of lithium metal forms a thin layer of LiF that improves the efficiency of the battery ${ }^{34}$. Excess use of salt (near saturation point), LiFSI in DME electrolyte leads to the same outcome as it is known 


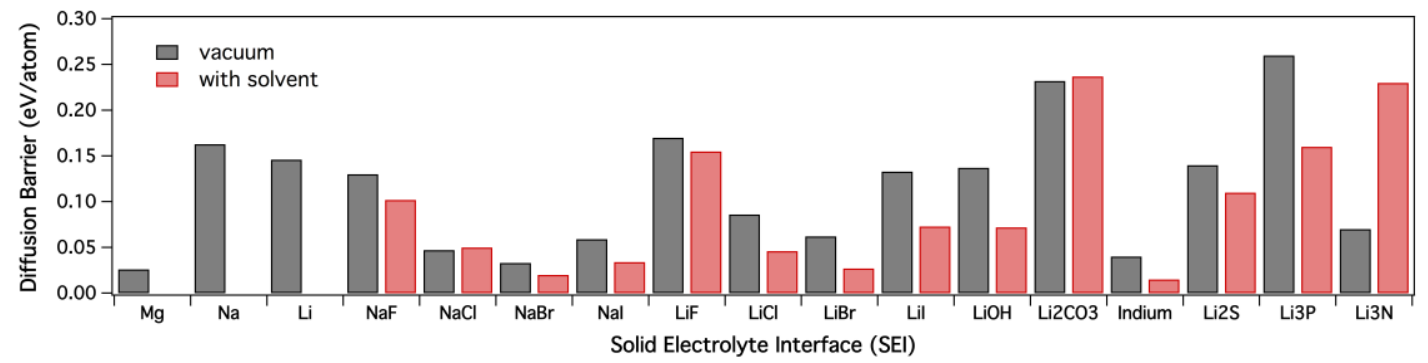

Figure 1.5: Bar Chart showing the Surface Diffusion Barrier for various compounds typically exist in the interfacial layer of lithium or sodium metal battery cycling using liquid as well as solid superionic electrolytes 
from computational chemistry methods that, LiFSI decomposes to $\mathrm{LiF}$, which ultimately protects both the anode and cathode ${ }^{35}$.

There have been several other additives used in LMBs for improving the battery performance. Of these, Lithium Nitrate $\left(\mathrm{LiNO}_{3}\right)$ is a prominent candidate, because its presence in the electrolyte can significantly improve the efficiency of a Lithium sulfur battery to nearly $100 \%$, whereas a neat electrolyte has below $60 \%$ efficiency due to polysulfide shuttling that attacks the lithium metal continuously causing corrosion and failure ${ }^{44,45}$. While the mechanism behind this phenomenon is still debatable, Aurbach et al (2009) ${ }^{46}$ shed some light on the abundance of oxy-sulfur and oxy-nitrogen species in the SEI layer of lithium in presence of $\mathrm{LiNO}_{3}$ and polysulfides. It can be inferred that these species prevent the access of polysulfides to fresh lithium; even the smooth and compact morphology of the SEI layer, points toward the same direction ${ }^{44}$. A similar behavior is also seen when the lithium anode is protected by a layer of $\mathrm{Li}_{3} \mathrm{~N}$, which possess the additional advantage of high Li conductivity ${ }^{61}$. Other additives include Vinylene Carbonate ${ }^{47-49}$, Sultones ${ }^{50,51}, \mathrm{LiBOB}^{52,53}$, and many others. Some of these additives have additional advantages. For example, alkyl phosphates and fluorine-based additives act as flame-retardants by reducing the self-heating rates significantly ${ }^{54}$.

As the investigation towards finding the right additive for LMB intensifies, current trends indicate that previously used additives for graphite-based lithium ion batteries work efficiently with appropriate compositions. It is however, clear, that additivesbased lithium battery stabilization is a rather empirical science that depends upon the battery configuration as well as component combinatorics. A recent study shows that 


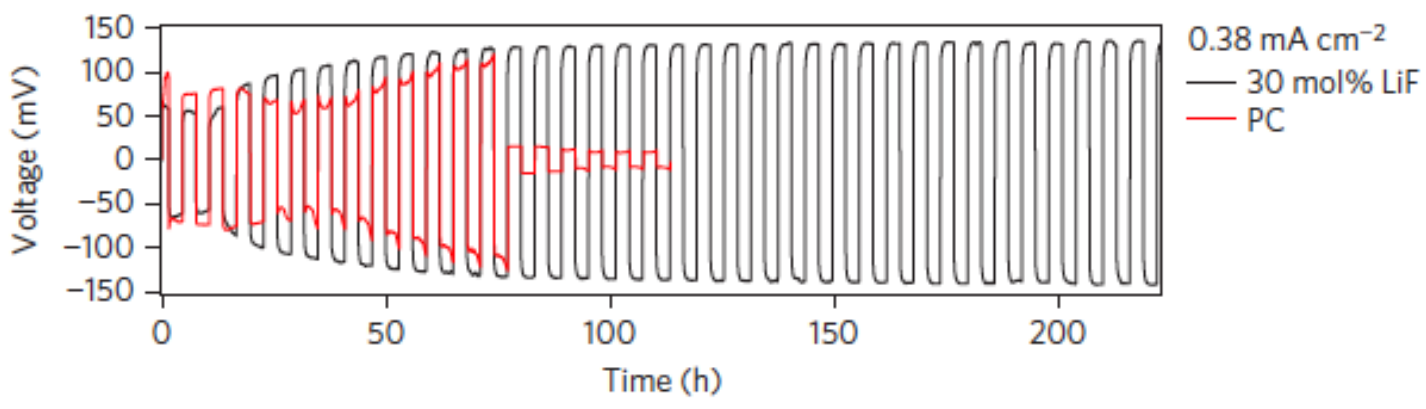

Figure 1.6: Strip Plate measurement using symmetric lithium cell showing stable cycling with LiF added electrolyte in black in contrast to sudden short circuit with a liquid electrolyte without any additive. 
rationalistic selection of additives can dramatically improve the specific capacity of a $\mathrm{Li}-\mathrm{O}_{2}$ battery with a high energy efficiency of $\sim 93.2 \%$ for several hundred cycles even in presence of water ${ }^{55}$. Using relatively high concentration of LiI additives promotes the formation of $\mathrm{LiOH}$ in presence of water on cathode surface. This gives lower insulating properties and $\mathrm{LiOH}$ easily reduced in presence of iodide anion that serves as a redox mediator ${ }^{55}$.

The second approach in lithium metal protection is ex-situ formation of a thin-film that serves as artificial SEI layer protecting the anode from parasitic reactions with electrolytes and dendrite formation. This is a multidisciplinary field where a broad range of techniques can be utilized to manufacture thin-films on lithium. This approach can also benefit from the two previous practice methods viz. modification of transport and enhancement of elasticity, providing a rationale for design. The work of Song et al. (2015) ${ }^{18}$ employing Nafion as surface protection layer for Li anodes demonstrates this confluence of methods. Another recent example is the use of Atomic Layer Deposition (ALD) technique for depositing a monolayer of alumina on lithium surface ${ }^{56}$. An emerging ex-situ coating of lithium metal anode include usage of a secondary metal coating based on tin (shown in Figure 1.7) or indium that can act as a host for the lithium ions to alloy or intercalate before electrodeposition Such a monolayer works excellently for preventing corrosion of lithium metal from not only the electrolyte but also ambient air. ${ }^{42,43}$ In another study, Polyacrylonitrile (PAN) was oxidized to enhance ion transport and electrospun to form PAN nanofibers, which was then placed on the electrode surface ${ }^{57}$. This nanostructured network allowed smooth electrodeposition by confining the lithium ion plating inside the network. The other 


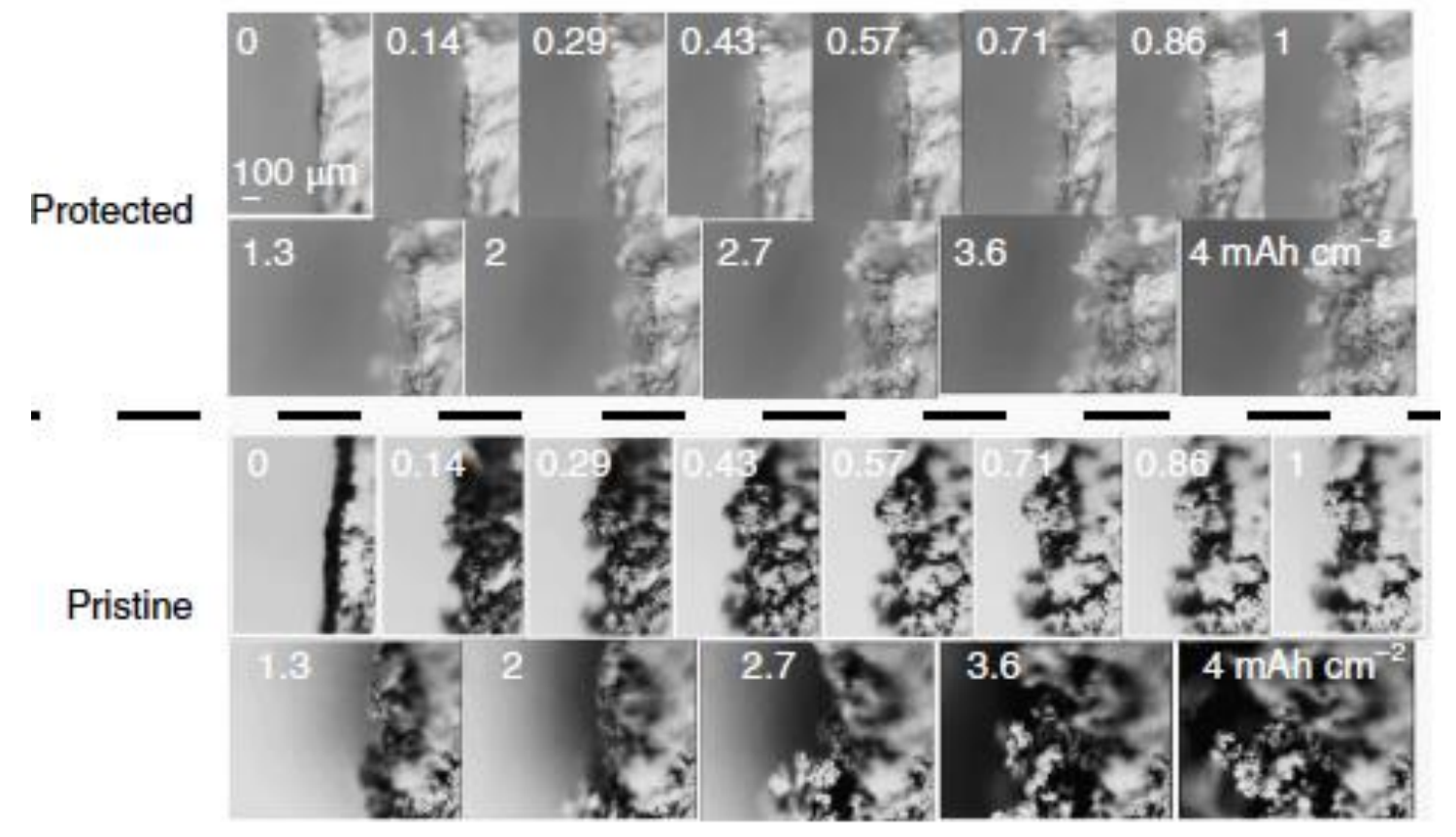

Figure 1.7: Direct Visualization experiment with and without protection with a Tin coating. The control lithium shows dendritic rough deposits, while the tin protection slows down the growth rate. 
artificial SEI formers include Boron Nitride nanosheets ${ }^{58}$, PEG tethered silica nanoparticles ${ }^{59}$, etc.

In the future, polymer based artificial SEI layer would be popular because of several reasons, including ease of industrial scale manufacturing, low cost, minimal reduction in ion conductivity if the polymer has its intrinsic ion transport pathway. Recent work on crosslinked polymer networks of different chemistries has shown good ion

conduction ability as well as dendrite suppression capability ${ }^{23,27}$. In situ crosslinking or network formation with rational choice of polymer chemistry on the lithium metal can be an effective means of lithium metal protection.

\subsection{Outline}

In the present work, we utilize multiscale transport modeling and experiments to fundamentally understand and to thereby develop rational designs for polymer electrolytes and electrode-electrolyte interphases that overcome the rampant instabilities in metal-based batteries. In Chapter $2-4$, we show novel architectures of polymer grafted nanoparticles where poly (ethylene oxide) is grafted covalently on the surface of silica nanoparticles. Even in absence of any suspending media these hybrid nanoparticles show good phase stability, confirmed using Transmission Electron Microscopy and Small Angle X-ray Scattering. Furthermore, they show unusual properties like temperature induced jamming and shoot-up in startup of shear flow. On suspending in a liquid electrolyte, they show simultaneous good conductivity and high mechanical modulus. Furthermore, the silica nanoparticles act as good flame retardant even when suspended in flammable electrolyte solvents. 
In Chapter $5-6$, we discuss on crosslinked polymer electrolytes and their role in preventing dendritic growth in metal batteries. These membranes re synthesized by linked hairy nanoparticles with poly(propylene oxide) polymers. Their texture is rubbery, and they can soak significant amount of electrolyte to successfully address the dilemma between conductivity and modulus. There membranes serve as mode nanoporous media because the silica nanoparticles act as barriers to impede dendrite growth while the interparticle spacing hosts oligomer chains that enable metal ion transport. Also, one can explicitly control the pore size of these membranes by tuning the volume fraction of nanoparticles in the membranes. We performed direct visualization electrodeposition to understand the effect of pore size on dendritic growth. The results were validated using a linear stability analysis calculation. In a battery, the membranes showed excellent performance in symmetric cell cycling as well as when paired with a commercial cathode.

Importantly, these studies showed that while the tendency for battery failure by dendrite-induced short-circuits can be reduced in polymer electrolytes, the issue of capacity-fading as a result of continuous reactions of the metal with liquid electrolyte persists. An additional striking fact in the electrodeposition literature not addressed by the linear stability analysis is that certain metals, including Magnesium, do not form dendrites. In Chapter $7-9$ we show how multiscale analysis of transport at electrochemical interfaces enables design of stable solid-liquid interphases for reactive metal batteries. Specifically, we used Density Functional Theory (DFT) calculations to quantify the diffusion energy barrier of ions on $\mathrm{Mg}$, Li, $\mathrm{Na}$ surfaces and interestingly it seen that the diffusion barrier of $\mathrm{Mg}(0.02 \mathrm{eV} / \mathrm{atom})$ is several folds lower than $\mathrm{Li}$ 
(0.14eV/atom) or $\mathrm{Na}(0.16 \mathrm{eV} /$ atom $)$ metals. In fact, the diffusion barrier of $\mathrm{Li}_{2} \mathrm{CO}_{3}$, $\mathrm{Li}_{2} \mathrm{O}$ (the commonly found compounds in lithium interface) is even higher, which is consistent with the dendritic electrodeposition in such batteries. However, in quest for finding stable interfaces, we observed that most metal halides ( $\mathrm{LiF}, \mathrm{LiBr}, \mathrm{NaF}$ etc.) as well as Indium metal have much lower diffusion barrier. In other words, halide-rich or Indium coated interfaces on lithium or sodium can lead to stable electrodeposition similar to Mg deposition. The predictions from the DFT model were validated using ex-situ scanning electron microscopy as well as in-situ optical microscopy. The nucleation pattern, indeed, showed a strike difference between usual (carbonate-rich) and halide-rich lithium interfaces. Based on these fundamental understanding, in Chapter 10, a solid electrolyte interphase in lithium metal batteries were artificially designed using organo-metallic reactions to enable enhanced reversibility in high energy density Lithium-Oxygen battery that demonstrated extended capacity retention and longer cycle life. 


\section{REFERENCES}

1. Tarascon, J. M. \& Armand, M. Issues and challenges facing rechargeable lithium batteries. Nature 414, 359-67 (2001).

2. Dresselhaus, M. S. \& Thomas, I. L. Alternative energy technologies. Nature 414, 332-7 (2001).

3. Armand, M. \& Tarascon, J.-M. Building better batteries. Nature 451, 652-7 (2008).

4. Bruce, P. G., Freunberger, S. a, Hardwick, L. J. \& Tarascon, J.-M. Li-O2 and Li-S batteries with high energy storage. Nat. Mater. 11, 19-29 (2012).

5. Aurbach, D., Zinigrad, E., Cohen, Y. \& Teller, H. A short review of failure mechanisms of lithium metal and lithiated graphite anodes in liquid electrolyte solutions. Solid State Ionics 148, 405-416 (2002).

6. Tikekar, M. D., Choudhury, S., Tu, Z. \& Archer, L. A. Design principles for electrolytes and interfaces for stable lithium-metal batteries. Nat. Energy 1, $16114(2016)$.

7. Zhang, S. S. A review on electrolyte additives for lithium-ion batteries. $J$. Power Sources 162, 1379-1394 (2006).

8. Pieczonka, N. P. W. et al. Impact of lithium bis(oxalate)borate electrolyte additive on the performance of high-voltage spinel/graphite Li-ion batteries. $J$. Phys. Chem. C 117, 22603-22612 (2013).

9. Choudhury, S. \& Archer, L. A. Lithium Fluoride Additives for Stable Cycling of Lithium Batteries at High Current Densities. Adv. Electron. Mater. 1-6 (2015). doi:10.1002/aelm.201500246 
10. Guo, J., Wen, Z., Wu, M., Jin, J. \& Liu, Y. Vinylene carbonate-LiNO3: A hybrid additive in carbonic ester electrolytes for SEI modification on Li metal anode. Electrochem. commun. 51, 59-63 (2015).

11. Aurbach, D. et al. On the use of vinylene carbonate (VC) as an additive to electrolyte solutions for Li-ion batteries. Electrochim. Acta 47, 1423-1439 (2002).

12. Li, B., Xu, M., Li, T., Li, W. \& Hu, S. Prop-1-ene-1,3-sultone as SEI formation additive in propylene carbonate-based electrolyte for lithium ion batteries. Electrochem. commun. 17, 92-95 (2012).

13. Khurana, R., Schaefer, J. L., Archer, L. A. \& Coates, G. W. Suppression of lithium dendrite growth using cross-linked polyethylene/poly(ethylene oxide) electrolytes: a new approach for practical lithium-metal polymer batteries. $J$. Am. Chem. Soc. 136, 7395-7402 (2014).

14. Bouchet, R. et al. Single-ion BAB triblock copolymers as efficient electrolytes for lithium-metal batteries. Nat. Mater. 12, $452-457$ (2013).

15. Srivastava, S., Schaefer, J. L., Yang, Z., Tu, Z. \& Archer, L. A. 25Th Anniversary Article: Polymer-Particle Composites: Phase Stability and Applications in Electrochemical Energy Storage. Adv. Mater. 26, 201-34 (2014).

16. Tikekar, M. D., Archer, L. A. \& Koch, D. L. Stability Analysis of Electrodeposition across a Structured Electrolyte with Immobilized Anions. $J$. Electrochem. Soc. 161, A847-A855 (2014).

17. Choudhury, S., Mangal, R., Agrawal, A. \& Archer, L. A. A highly reversible 
room-temperature lithium metal battery based on crosslinked hairy nanoparticles. Nat. Commun. 6, 10101 (2015).

18. Tu, Z., Kambe, Y., Lu, Y. \& Archer, L. A. Nanoporous Polymer-Ceramic Composite Electrolytes for Lithium Metal Batteries. Adv. Energy Mater. 4, 1300654 (2014).

19. Tu, Z., Nath, P., Lu, Y., Tikekar, M. D. \& Archer, L. A. Nanostructured Electrolytes for Stable Lithium Electrodeposition in Secondary Batteries. Acc. Chem. Res. 48, 2947-2956 (2015).

20. Lu, Y. et al. Stable Cycling of Lithium Metal Batteries Using High Transference Number Electrolytes. Adv. Energy Mater. 5, 1402073 (2015).

21. Schaefer, J. L., Yanga, D. A. \& Archer, L. A. High Lithium Transference Number Electrolytes via Creation of 3-Dimensional, Charged, Nanoporous Networks from Dense Functionalized Nanoparticle Composites. Chem. Mater. 25, 834-839 (2013).

22. Monroe, C. \& Newman, J. The Impact of Elastic Deformation on Deposition Kinetics at Lithium/Polymer Interfaces. J. Electrochem. Soc. 152, A396 (2005).

23. Monroe, C. \& Newman, J. The Effect of Interfacial Deformation on Electrodeposition Kinetics. J. Electrochem. Soc. 151, A880 (2004).

24. Li, J., Ma, C., Chi, M., Liang, C. \& Dudney, N. J. Solid Electrolyte: the Key for High-Voltage Lithium Batteries. Adv. Energy Mater. 5, 1401408 (2015).

25. Tu, Z. et al. Nanoporous Hybrid Electrolytes for High-Energy Batteries Based on Reactive Metal Anodes. Adv. Energy Mater. 7, 1602367 (2017).

26. Tikekar, M. D., Archer, L. A. \& Koch, D. L. Stabilizing electrodeposition in 
elastic solid electrolytes containing immobilized anions. Sci. Adv. 2, (2016).

27. Hu, J. et al. Flexible Organic-Inorganic Hybrid Solid Electrolytes Formed via Thiol-Acrylate Photopolymerization. Macromolecules 50, 1970-1980 (2017).

28. Zhang, J. et al. Flexible and ion-conducting membrane electrolytes for solidstate lithium batteries: Dispersion of garnet nanoparticles in insulating polyethylene oxide. Nano Energy 28, 447-454 (2016).

29. Li, Y., Wong, K. W., Dou, Q. \& Ng, K. M. A single-ion conducting and shearthinning polymer electrolyte based on ionic liquid-decorated PMMA nanoparticles for lithium-metal batteries. J. Mater. Chem. A 4, 18543-18550 (2016).

30. Lu, Q. et al. Dendrite-Free, High-Rate, Long-Life Lithium Metal Batteries with a 3D Cross-Linked Network Polymer Electrolyte. Adv. Mater. 29, 1604460 (2017).

31. Lee, Y.-G. et al. Dendrite-Free Lithium Deposition for Lithium Metal Anodes with Interconnected Microsphere Protection. Chem. Mater. 29, 5906-5914 (2017).

32. Ma, L., Nath, P., Tu, Z., Tikekar, M. \& Archer, L. A. Highly Conductive, Sulfonated, UV-Cross-Linked Separators for Li-S Batteries. Chem. Mater. 28, 5147-5154 (2016).

33. Lu, Y., Das, S. K., Moganty, S. S. \& Archer, L. a. Ionic liquid-nanoparticle hybrid electrolytes and their application in secondary lithium-metal batteries. Adv. Mater. 24, 4430-5 (2012).

34. Wei, S. et al. A stable room-temperature sodium-sulfur battery. Nat. Commun. 
7, $11722(2016)$.

35. Wei, S. et al. Highly Stable Sodium Batteries Enabled by Functional Ionic Polymer Membranes. Adv. Mater. 29, 1605512 (2017).

36. Choudhury, S., Agrawal, A., Wei, S., Jeng, E. \& Archer, L. A. Hybrid Hairy Nanoparticle Electrolytes Stabilizing Lithium Metal Batteries. Chem. Mater. 28, 2147-2157 (2016).

37. Barghamadi, M. et al. Lithium-sulfur batteries - the solution is in the electrolyte, but is the electrolyte a solution? Energy Environ. Sci. 7, 3902-3920 (2014).

38. Li, W. et al. The synergetic effect of lithium polysulfide and lithium nitrate to prevent lithium dendrite growth. Nat. Commun. 6, 7436 (2015).

39. Chen, L., Wang, K., Xie, X. \& Xie, J. Effect of vinylene carbonate (VC) as electrolyte additive on electrochemical performance of Si film anode for lithium ion batteries. J. Power Sources 174, 538-543 (2007).

40. Pires, J. et al. Role of propane sultone as additive to improve the performance of lithium-rich cathode material at high potential. $R S C A d v$. (2015). doi:10.1039/C5RA05650K

41. Xu, K., Zhang, S. \& Jow, T. R. LiBOB as Additive in LiPF[sub 6]-Based Lithium Ion Electrolytes. Electrochem. Solid-State Lett. 8, A365 (2005).

42. Tu, Z. et al. Fast ion transport at solid-solid interfaces in hybrid battery anodes. Nat. Energy (2018). doi:10.1038/s41560-018-0096-1

43. Choudhury, S. et al. Electroless Formation of Hybrid Lithium Anodes for Fast Interfacial Ion Transport. Angew. Chemie Int. Ed. 56, 13070-13077 (2017). 
CHAPTER 2

SELF-SUSPENDED SUSPENSIONS OF COVALENTLY GRAFTED HAIRY

NANOPARTICLES 


\subsection{Abstract}

Dispersions of small particles in liquids have been studied continuously for almost two centuries for their ability to simultaneously advance understanding of physical properties of fluids and their widespread use in applications. In both settings, the suspending (liquid) and suspended (particle) phases are normally distinct and uncoupled on long length and time-scales. In this study, we report on the synthesis and physical properties of a novel family of covalently grafted nanoparticles that exist as self-suspended suspensions with high particle loadings. In such suspensions, we find that the grafted polymer chains exhibit unusual, multiscale structural transitions and enhanced conformational stability at sub-nanometer and nanometer length scales. On mesoscopic length-scales, the suspensions exhibit exceptional homogeneity and colloidal stability, which we attribute to steric repulsions between grafted chains, which prevent close contact, and a space filling constraint on the tethered chains, which inhibits phase segregation. On macroscopic length scales, the suspensions exist as neat fluids, which exhibit soft glassy rheology and, counter-intuitively, display enhanced elasticity upon increasing temperature. This feature is discussed in terms of increased interpenetration of the grafted chains and jamming of the nanoparticles.

\subsection{Introduction}

Dispersions of small particles in simple liquids have been studied for at least a century to understand their interaction forces and dynamics ${ }^{1-4}$. In recent years interest in suspensions of particles with nanometer-sized dimensions has grown in response to their exceptional promise for applications in multiple fields of technology. In 
medicine, they are receiving increasing attention as therepeutics ${ }^{5,6}$ and for biomedical imaging $^{7-10}$. In energy harvesting and storage, nanosize particles have been reported to provide attractive attributes when used as tunable components in the anode, cathode, or electrolyte ${ }^{11-24}$. Because of the small size of the particles, surface forces dominate and the difficulty in preparing dispersions of un-aggregated nanoparticles is well known and extensively studied. This challenge has nevertheless hindered fundamental studies of the materials and delayed progress in understanding their colloidal science $^{25-27}$. A variety of approaches have been reported in the literature for controlling phase stability of large and small particles. Only two are regarded as sufficiently versatile to be employed in practice: electrostatic stabilization ${ }^{28,29}$ using charges physically adsorbed to the particle surface in solution; and steric stabilization using physically/chemically attached polymers ${ }^{30-35}$.

Recently, the concept of solvent-less nanoparticle fluids has been proposed, which structurally resemble block copolymers micelles and multi-arm star polymers ${ }^{36-42}$. These nanoparticle fluids are comprised of polymer chains grafted to nanoparticles at such high coverage that the particles exhibit remarkable phase stability and fluidity in the absence of a solvent ${ }^{25,43,44}$. Theoretical studies show that the exceptional colloidal stability of such self-suspended suspensions arise from two sources, steric forces between the tethered polymer chains and by the space filling constraints these chains experience in the absence of any suspending medium ${ }^{45,46}$. Density functional theoretical and molecular simulation studies further show that each nanoparticle in a self-suspended material carries its own share of the suspending fluid (the tethered 
polymer) on its back such that exactly one neighboring core is excluded by each hairy nanoparticle. This feature of the materials simultaneously make them analogous to incompressible, single-component fluids comprised of molecular units and leads to a vanishing structure factor $S(q->0)=0{ }^{45,46}$ and good model systems for understanding interactions, structure, and dynamics of soft colloids ${ }^{45-49}$.

A fundamental question that arises in the context of using self-suspended materials as model systems for soft colloids arises from the fidelity of the ligand coupling ${ }^{34-35}$ possible with the ionic sulfonic acid - amine bond most commonly used for creating the most widely studied materials ${ }^{25,43,50}$. Additionally, recent work by Fernandez et al. show that electrostatic interactions between ionically linked core and corona can lead to leading to complex layering of the charged core and corona ${ }^{51}$. In particular, these authors found that the diffusivity of the grafted polymeric chains do not correlate with the hard sphere like diffusivity of the core $e^{51,52}$ and contended that exchange of polymers between a bilayer of chains tethered to the particles creates a dynamic interface between the core and polymer ${ }^{51,52}$. In a model self-suspended system of nanoparticles, this exchange is undesirable. Herein we report a synthesis strategy for creating truly self-suspended suspensions of nanoparticles in which polymeric ligands are covalently grafted to nanoparticles at coverage where the system spontaneously exhibits a homogeneous fluid state in the absence of any solvent. The materials open new opportunities for both fundamental studies and for applications where the particles must be exposed to high-dielectric constant, polar solvents that may dissociate the polymer-particle linkages in their ionic counterparts. We show by means 
of scattering experiments and rheology that the materials are self-suspended, exhibit hierarchical structure, and soft glassy fluid rheology.

\subsection{Experimental Section}

\subsubsection{Synthesis of self-suspended covalently grafted nanoparticles}

Figure 2.1(a) shows the reaction scheme used for the synthesis. Briefly these covalently grafted hairy nanoparticles are synthetized in a two-step process in which the polymer is first functionalized with a silane group, after which it is grafted to the silica nanoparticle surface. In the first step of reaction, the Polymer (in this case Polyethylene Oxide,) was attached to a Silane group, by the reacting the isocyanate group in 3-(Triethoxysilyl) propyl isocyanate (purchased from Sigma Aldrich) to the amine group present in Amino-Polyethylene Oxide (MW 5000Da, purchased from Polymer Source) in stoichiometric ratio, creating a stable urethane bond between the

core and corona. In the next step, the Silanized PEO is reacted to the silanol groups on the surface of colloidal silica. The excess polymer chains were removed from the system by repeated centrifugation in a chloroform-hexane mixture. This is an important step, in order to make sure that there are no extra polymeric chains other than what is carried by the nanoparticles. The inorganic content of these hairy nanoparticles was analyzed after each centrifuge cycle using Thermo-gravimetric Analysis (TGA) on TGA Q1000 (TA Instruments). The inorganic content was found to reduce with successive cycles, finally reaching a constant value. Systems with grafting densities of 1.18 chains $/ \mathrm{nm}^{2}, 1.03$ chains $/ \mathrm{nm}^{2}, 0.703$ chains $/ \mathrm{nm}^{2}$ and 0.576 chains $/ \mathrm{nm}^{2}$ were synthesized and characterized. 
a)
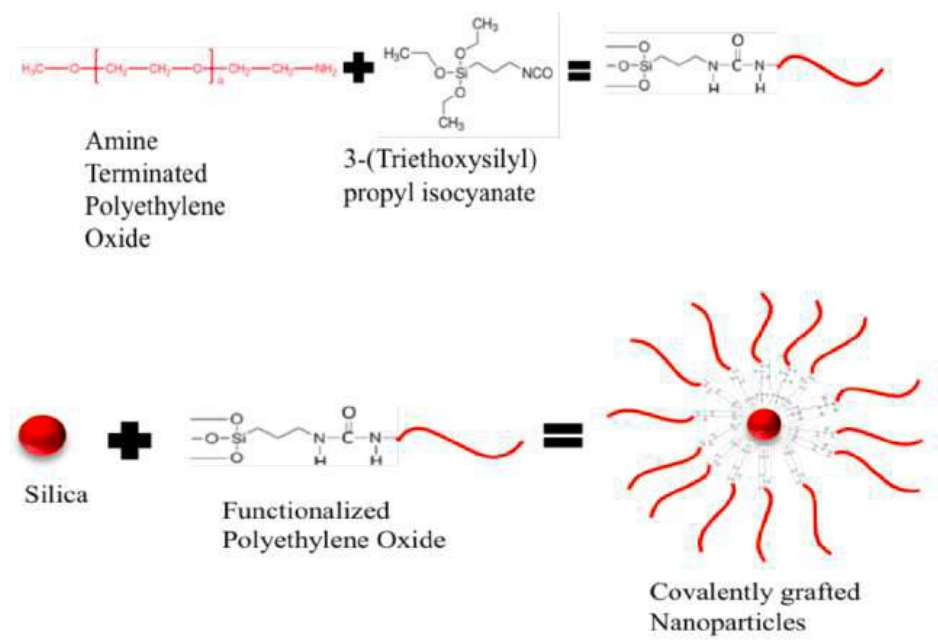

b)
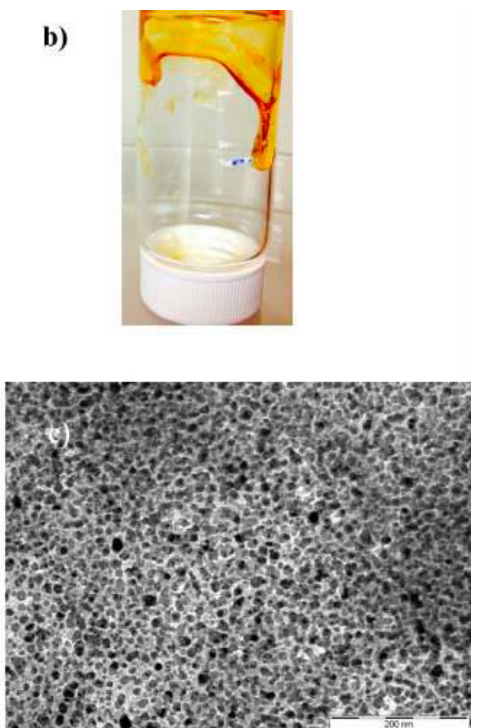

Figure 2.1 a) Reaction schematic for synthesizing the functionalized PEO and then tethering it to silica. b) Upturned vial showing liquid-like behavior of solventless covalently grafted nanoparticles. c) A typical transmission electron microscopy (TEM) image of the covalently grafted hairy nanoparticles. The scale for all the images is $200 \mathrm{~nm}$. 


\subsubsection{Characterization}

${ }^{1} \mathrm{H}$ NMR (Nuclear Magnetic Resonance) spectra were collected on NOVA $600 \mathrm{MHz}$ NMR operating at $599.50 \mathrm{MHz}$ at $25^{\circ} \mathrm{C}$ to confirm the formation of covalent bond. The chemical shifts were referenced to $\mathrm{CDCl}_{3}$ as standards. $2 \mathrm{D}{ }^{1} \mathrm{H}-{ }^{13} \mathrm{C}$ short-range correlation spectra were recorded through edited HSQC (Heteronuclear Single Quantum Correlation) using HSQCAD sequence in $\mathrm{CDCl}_{3}$. $\mathrm{HMBC}$ (Heteronulear Multiple Bond Correlation) experiment was performed with gradient HMBCAD sequence in $\mathrm{CDCl}_{3}$ for long-range correlation. Melting temperature of tethered and free PEO chains were studied using Differential Scanning Calorimetry on a DSC Q2000 (TA Instruments).

Further analysis of chain conformations was done using Attenuated Total ReflectanceFourier Transform Infrared Spectroscopy (ATR FT-IR) on a Nicolet iS10 FTIR spectrometer (Thermo Fisher Scientific) equipped with deuterated triglycine sulfate (DTGS) detector and SMART iTR diamond ATR accessory.

\subsubsection{Small Angle X-ray Scattering measurements}

Small Angle X-ray Scattering (SAXS) measurements were performed at Station D1 of Cornell High Energy Synchrotron Source (CHESS) using a point collimated X-ray beam. All the samples were smeared on a thermal sample cell and the measurements were performed at different temperatures above melting temperature of PEO. The measured scattering intensity, $I(q)$ depends on wave vector $q$ and particle volume fraction $\varphi$ as:

$$
I(q, \varphi)=\mathrm{P}(q) \mathrm{S}(q, \varphi)
$$


Where, $\mathrm{P}(q)$, and $\mathrm{S}(q, \varphi)$ represent the particle form factor and the inter-particle structure factor. Since in the limit of infinite dilution $S(q, \varphi \rightarrow 0) \sim 1$, the particle form factor can thus be obtained from the scattering intensities of dilute aqueous suspensions of particle. The structure factor can then be obtained by normalizing the scattered intensity with the form factor.

\subsubsection{Rheology measurements}

Oscillatory Shear Measurements were performed on an MCR501 (Anton Paar) Rheometer using a $10 \mathrm{~mm}$ cone and plate fixture at temperatures ranging from $70^{\circ} \mathrm{C}$ to $150^{\circ} \mathrm{C}$. All the suspensions were presheared to erase any strain history. Variable amplitude oscillatory measurements were performed at a fixed angular frequency of $\omega=10 \mathrm{rad} / \mathrm{s}$.

\subsection{Results and Discussion}

On macroscopic length scales, these materials exhibit liquid-like behavior, even in the absence of a solvent as evident from Figure 1(b), while at nano-scale, as observed from the Transmission Electron Micrograph (TEM) for these systems, shown in Figure 2.1(c), each nanoparticle is uniformly dispersed and well segregated from each

other. It is remarkable that there is no aggregation or phase separation in the sea of nanoparticles.

The formation of covalent bond is mapped using FTIR and NMR techniques. Figure 2.2(a) shows the Infra-red Spectra for Monoamine terminated Polyethylene oxide, Silane Propyl Isocyanate and the Silane terminated PEO. It is clearly observed that the 

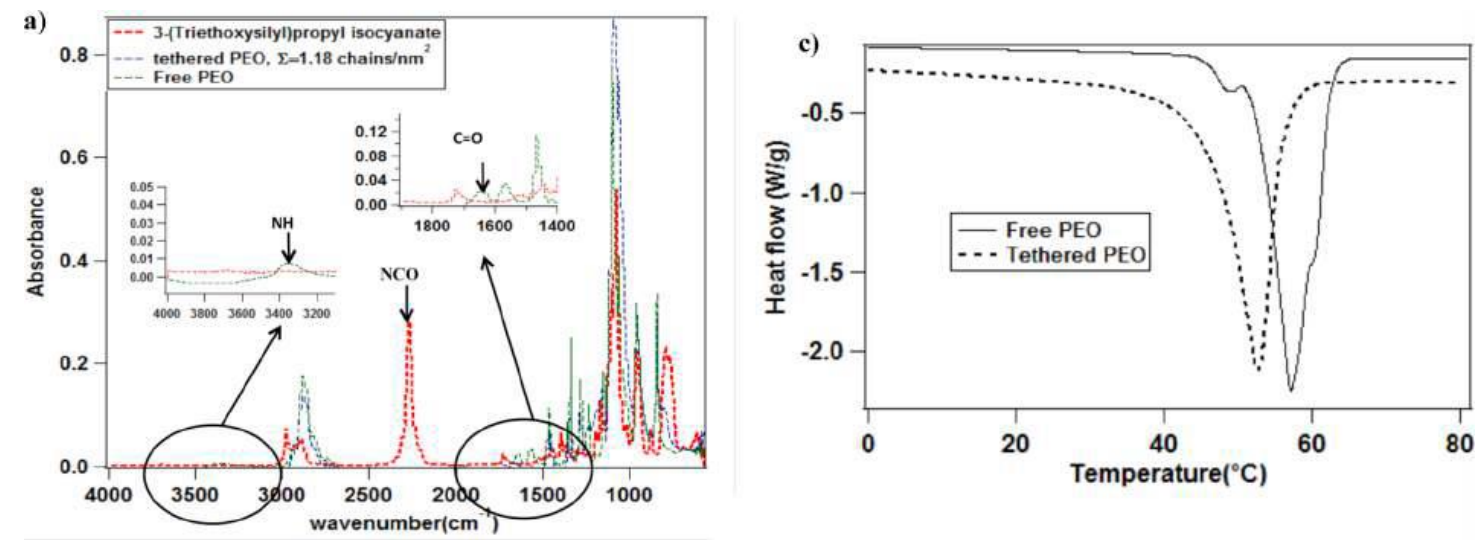

b)
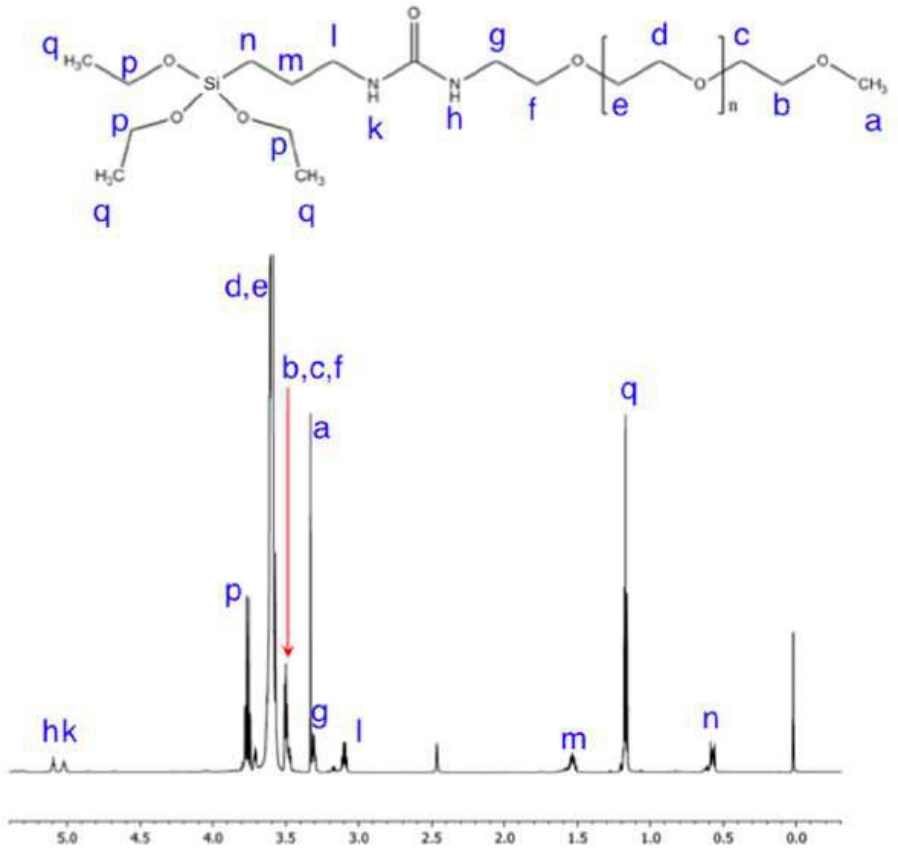

Figure 2.2 (a) FTIR spectra of a tethered PEO chain, free PEO, and 3(triethoxysilyl)propyl isocyanate. (b) 1H NMR spectra with structural assignments of functionalized PEO. (c) DSC thermograms of free PEO (solid line) and tethered PEO chains (dashed lines). Free PEO chains have three types of crystallite structuresextended, once folded, and twice folded-as seen on going from high to low temperature corresponding to the three peaks while the tethered chains have just the extended-type crystallite structure and thus only one peak in the DSC. 
-NCO peak $\left(2270 \mathrm{~cm}^{-1}\right)$ present in the Silane Propyl Isocyanate is consumed, while in the Silane terminated PEO, there is evidence of the formation of the urethane bond indicated by the $-\mathrm{NH}$ bond $\left(3350 \mathrm{~cm}^{-1}\right)$ and $-\mathrm{C}=\mathrm{O}$ bond $\left(1640 \mathrm{~cm}^{-1}\right)^{53}$. Also, to further confirm the reaction step, two-dimensional HSQC and HMBC NMR experiments were performed on Silanized PEO (shown in Supplementary Figure 2.2). Figure 2.2(b), shows the ${ }^{1} \mathrm{H}$ NMR spectrum with structural assignments of the functionalized PEO. All the chemical species and bonds in the expected structure of the Silanized PEO are observed in the NMR spectrum. This confirms the formation of a covalent bond between silane isocyanate and the PEO chain.

The stability of these covalently grafted particles is contrasted with their ionic counterparts using ultracentrifuge at $10000 \mathrm{rpm}$ for one hour in water. Supplementary Figure $2.3(\mathrm{a}, \mathrm{b})$ shows the solid content of the ionic and covalently grafted hairy nanoparticles before and after ultracentrifugation in water. Owing to the ionic linkage and dissociation of ions in a high-dielectric constant medium, there is a noticeable loss of polymer chains in the ionically grafted materials under a high centrifugal force in the presence of water. This can be contrasted with the covalently grafted materials where the net polymer content is essentially completely preserved after ultracentrifugation under the same conditions! Further, it is shown in Supplementary Figure 2.3 that the amplitude sweep curves obtained from rheological measurements overlap for samples before and after ultracentrifugation. It has been previously reported that an untethered PEO has three melting peaks owing to three types of crystallites while there is just one melting peak (single crystallite) present in tethered 
a)

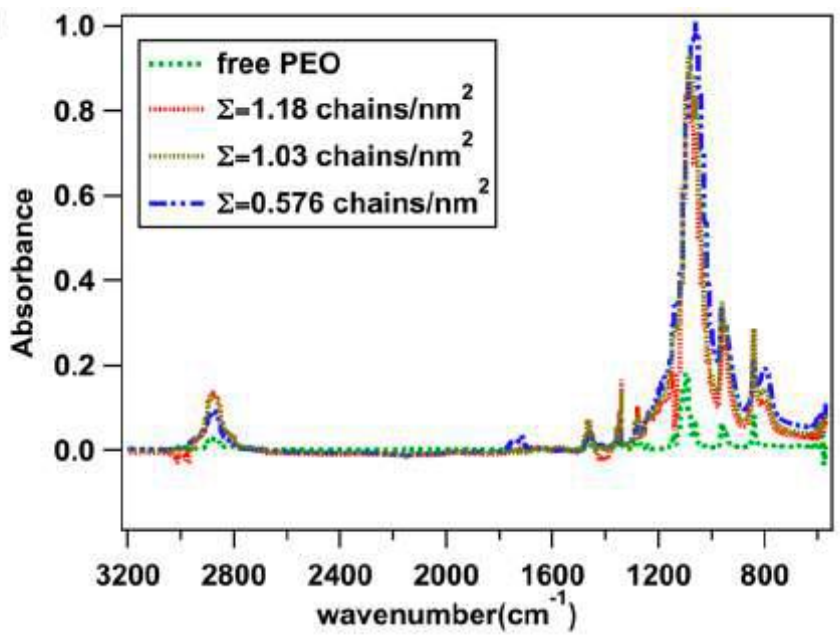

b)

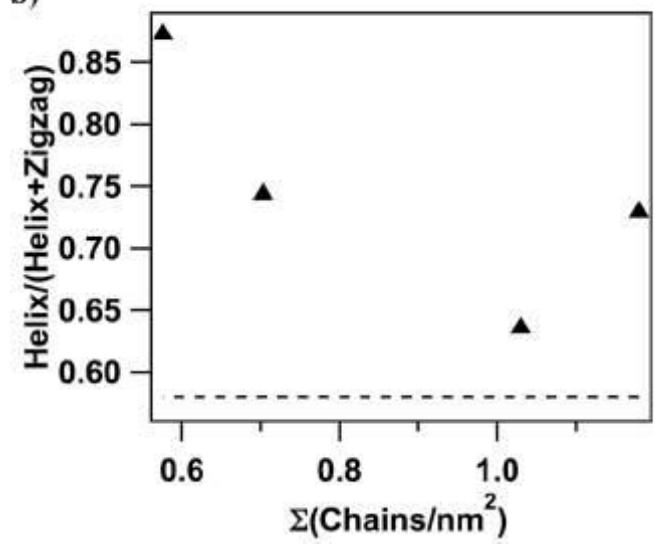

c)

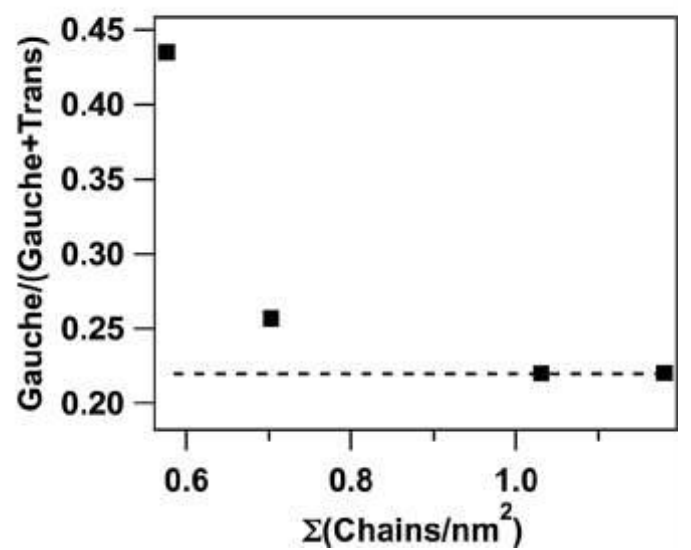

Figure 2.3 (a) FTIR spectra for tethered PEO chains with different grafting densities and for free PEO chains. (b) Intensity ratios as obtained from the Gaussian fitting between helix and zigzag structures of tethered (squares) and free (dashed line) PEO chains. (c) Intensity ratio between gauche (wavenumber $\approx 1357 \mathrm{~cm}^{-1}$ ) and trans (wavenumber $\approx 1342 \mathrm{~cm}^{-1}$ ) conformations of $\mathrm{C}-\mathrm{C}$ bonds of tethered (triangles) and free (dashed line) PEO chains. The decrease in grafting density leads to increased helix and gauche conformations for the tethered chains, indicating enhanced stability. 
PEO polymer ${ }^{54}$. Figure 2.2(c) indeed shows the reduction of melting modes from three to one in the DSC thermogram of PEO after the above steps, again confirming the absence of free chains and covalent linkage of the PEO onto silica particles.

The reduction of the degrees of freedom in polymer chains by surface confinement has been previously reported to lead to stable conformations ${ }^{54,55}$. Figure 2.3(a) reports results from FTIR measurements on covalent $\mathrm{SiO}_{2}$-PEO systems with different grafting densities. For a PEO polymer, the chain conformations can be determined using the relative intensities of the FTIR peaks ${ }^{18,54,56,57}$. It has been previously reported that the most stable conformation in a PEO strand is trans-trans-gauche, followed by trans-trans-trans in $\left(-\mathrm{O}-\mathrm{CH}_{2}-\mathrm{CH}_{2}\right)$ which, ultimately form the building blocks for helix-like and zigzag unit cells, respectively ${ }^{18,54,56,57}$. These hairy nanoparticles can be characterized using only $\mathrm{C}-\mathrm{C}$ trans and gauche conformation modes of the $\mathrm{CH}_{2}$ (1342$1360 \mathrm{~cm}^{-1}$ ) (shown in Table STI of Supplementary Information). The relative FTIR intensities were measured exactly by de-convolution the peak using Gaussian function (shown in Supplementary Figure 2.4). Figure 2.3(b) and 2.3(c) show relative conformational abundance in angstrom scale and nanometer scale respectively. The tethered PEO chains are shown to have higher abundance of gauche conformations at lower grafting density (shown in Figure 2.3(b)). It is known that the gauche state has lower energy compared to the trans state in a C-C bond ${ }^{18,54}$. Thus, it can be concluded that at the molecular level the PEO chains are more thermodynamically stable when tethered at a lower grafting density. At the mesoscale, the helix and zigzag type unit cells were counted by adding up the intensities at each assigned peak, as given in 

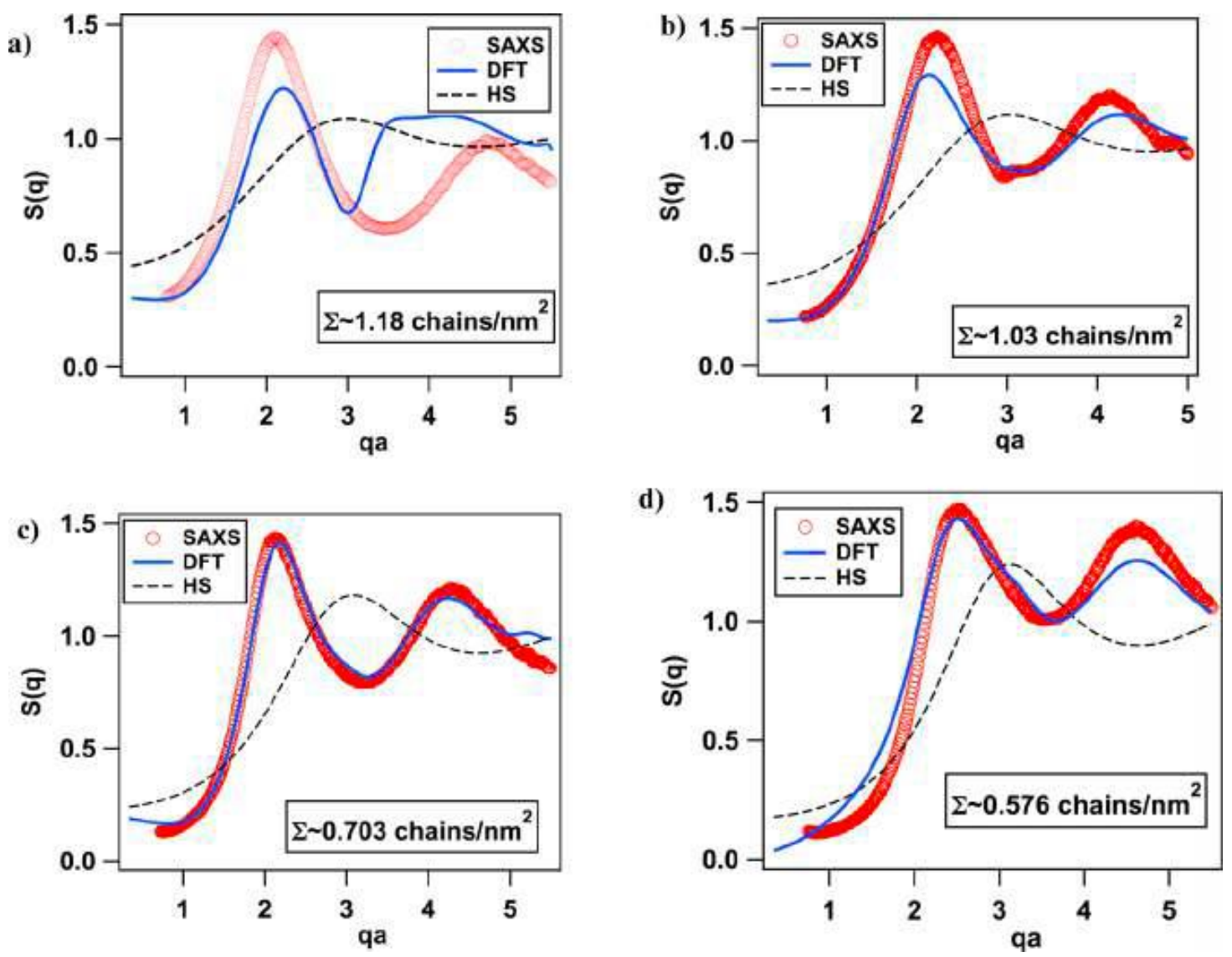

Figure 2.4 Structure factor, $\mathrm{S}(\mathrm{q})$, varies with the wave vector nondimensionalized with the core radius, qa, for (a) $\Sigma \approx 1.18$ chains $/ \mathrm{nm}^{2}$, (b) $\Sigma \approx 1.03$ chains $/ \mathrm{nm}^{2}$, (c) $\Sigma \approx$ 0.703 chains $/ \mathrm{nm}^{2}$, and (d) $\Sigma \approx 0.576$ chains $/ \mathrm{nm}^{2}$. The red circles are for experimental values, the solid blue lines represent the DFT fit, and the dotted black lines are for hard-sphere calculations. Symbols in (d) are for hard-sphere calculations. 
Table STI of Supplementary Information. Figure 2.3(c) shows the proportion of these two types of structures at various grafting densities $(\Sigma)$. Again, the PEO chains in lower grafting densities are seen to have higher stability owing to the fact that the helix unit cell is more stable than the zigzag type ${ }^{18,54}$.

To further understanding of the structural changes in these materials with variation in grafting density were obtained from Small Angle X-ray Scattering (SAXS) measurements. The scattering intensities shown in Supplementary Figure 2.5, show the absence of an upturn in low q region ${ }^{58}$, which indicates that the particles are welldispersed with no aggregation or phase separation. Figure 4 reports the structure factor $S(q)$ for different grafting densities $(\Sigma)$ as a function of the wave vector $q$ nondimensionalised with the particle core radius $a$. The structure factors determined from experiment are compared with the predictions from DFT theory for self-suspended $\mathrm{NOHMs}^{45,59}$, and with reference hard-sphere systems. It is evident from Figure 2.4 that both the experiment and DFT theory show stronger peaks in $S(q)$ than the corresponding hard-sphere suspensions, which indicate an enhanced particle-particle correlation. Also, the first peak is shifted to a smaller $q$ value, which implies a larger inter-particle separation due to steric repulsion from the chains, and lower $S(q)$ values in the low $q$ region is a direct manifestation of the entropic penalty imposed on the tethered chains to uniformly fill the spaces between the cores. ${ }^{45}$ A notable feature of the self-suspended covalently grafted nanoparticles is the presence of a stronger first peak than the second peak in $S(q)$. The first peak is now understood to be an indication of the steric repulsion of the chains while the second peak is a reflection of entropic 

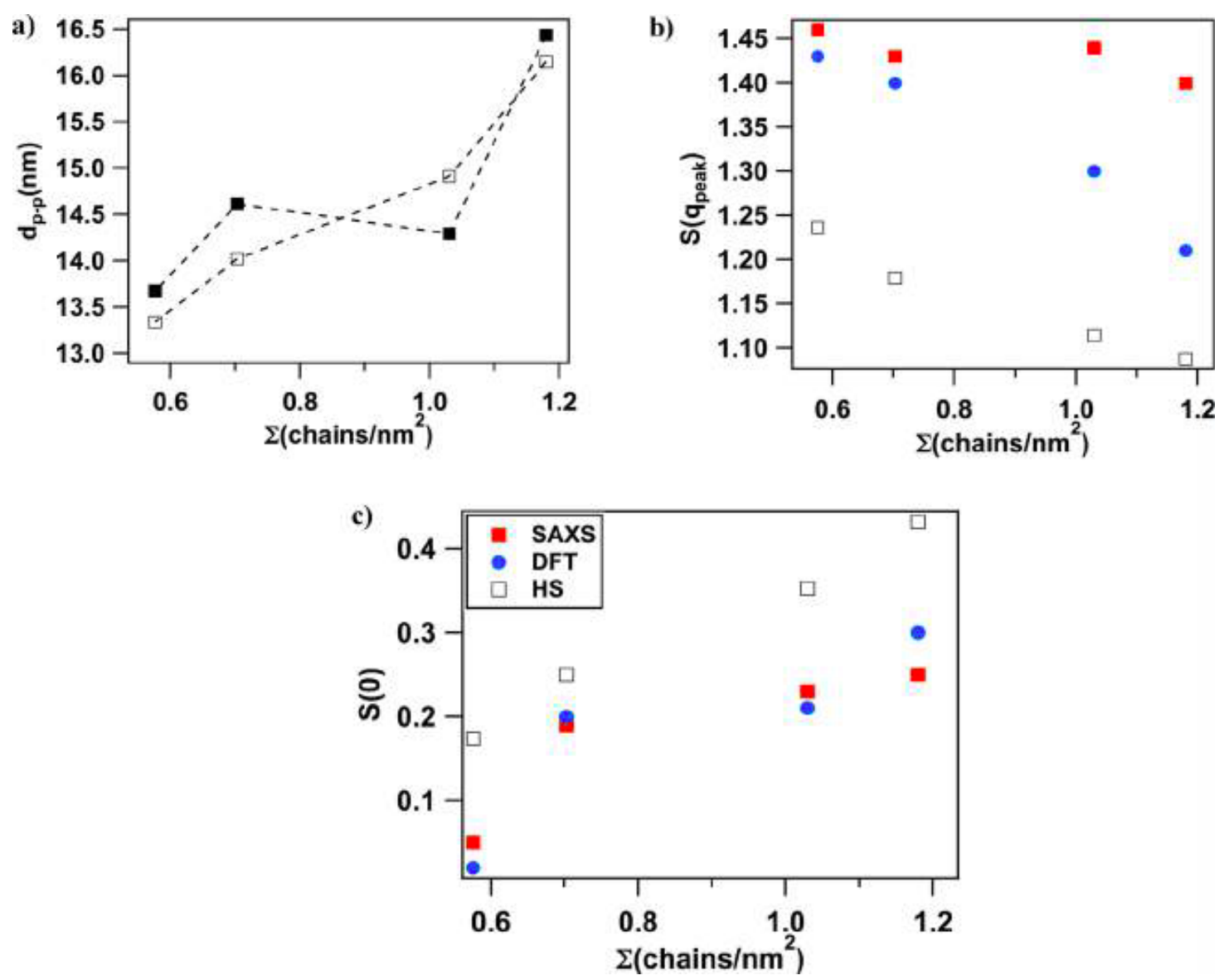

Figure 2.5 (a) Interparticle distance, $d p-p$, extracted from the experimental $S(q)$ (closed symbols) increases with grafting density. A similar trend is observed for theoretical dp-p (open symbols). The dashed lines are a guide to the eye. (b) The height of first peak of $\mathrm{S}(\mathrm{q})$ decreases while the (c) $\mathrm{S}(0)$ values increases with increasing grafting density. The experimental values agree well with DFT, and the HS $\mathrm{S}(0)$ values are seen to be higher. 
attraction between the chains. In contrast to previous observations in ionically grafted particles ${ }^{59}$ where the first peak was found to be weaker for densely grafted systems, the stronger first peak observed for the present systems even at higher grafting densities is a consequence of the permanent bond between the chains and the particle surface. Remarkably, this observation of a weaker second peak has also been observed in computer simulations of self-suspended particles ${ }^{47}$. It was postulated that this trend reflects the fact that chains in covalently grafted hairy nanoparticles are directly tethered to the surface of the core as opposed to previous studies where electrostatic interactions between the positively charged core with negatively charged corona ${ }^{52,59}$ produced stronger $S(q)$ peaks at higher $q$. The recently developed DFT theory ${ }^{59}$ predicts polydispersities in core size and grafting densities to fit the experimental data. Supplementary Figure 2.1 shows the polydispersity in core size, as extracted from Gaussian fitting of a dilute suspension of the silica nanoparticles. It shows an average size of $10 \pm 2 \mathrm{~nm}$, which corresponds to a polydispersity of $20 \%$ in core size. On assuming a polydispersity of $20 \%$ in core size, as extracted from Gaussian fitting of the dilute suspension of silica nanoparticles (Supplementary Figure 2.1), we obtain the polydispersities in grafting density for different systems from DFT, as reported in Table 2.1. It can be noted that with increasing $\Sigma$, or decreasing particle volume fraction, the polydispersity in grafting density increases. This suggests that the stronger entropic constraints on the chains at lower volume fraction can be released more efficiently by introducing more polydispersity in the grafting density. 
Figure 2.5a) compares the inter-particle distance, $d_{p-p}$ extracted from the first peak position of $S(q)$ with $\Sigma$. It can be seen that the inter-particle distance increases with $\Sigma$ which is not surprising as higher grafting density means that the tethered chains are more effective in keeping the cores apart, and thus exhibit an effectively higher steric repulsion as compared to systems with lower grafting density. The experimental $d_{p-p}$ is found to be roughly consistent with the theoretical estimate of $\mathrm{d}_{\mathrm{p}-\mathrm{p}}=2 a(0.63 / \varphi)^{1 / 3}$, where $\varphi$ is particle volume fraction. A similar trend is manifested in the decrease of peak height of first peak $S\left(q_{\text {peak }}\right)$ (Figure 2.5(b)) with increasing grafting density. Since densely tethered cores are able to push each other more due to stronger steric repulsion by the tethered chains, this results in a decrease of correlation amongst the nearest neighbors as opposed to the sparsely tethered cores where the chains are not able to stretch out as much and thus the particles are much closer, and the correlation is hence much enhanced. A potentially even more interesting feature is the increasing $S(q)$ value at low $q \rightarrow 0$ with the increase in $\Sigma$, as shown in Figure 2.5(c). A lower $S(0)$ value at lower grafting densities indicates a more uniform distribution for particles than at higher grafting densities. The $S(0)$ value for experimental systems was extracted by performing a quadratic fit for $S(q)$ in the low q region $(q a<1.5)$ and was then extrapolated to $q=0$. It is noteworthy, that the $S(0)$ values for experiment are comparable to theory and are much lower than the hard sphere values, which is strong evidence of a more uniform distribution for self-suspended particles as opposed to hard spheres. 

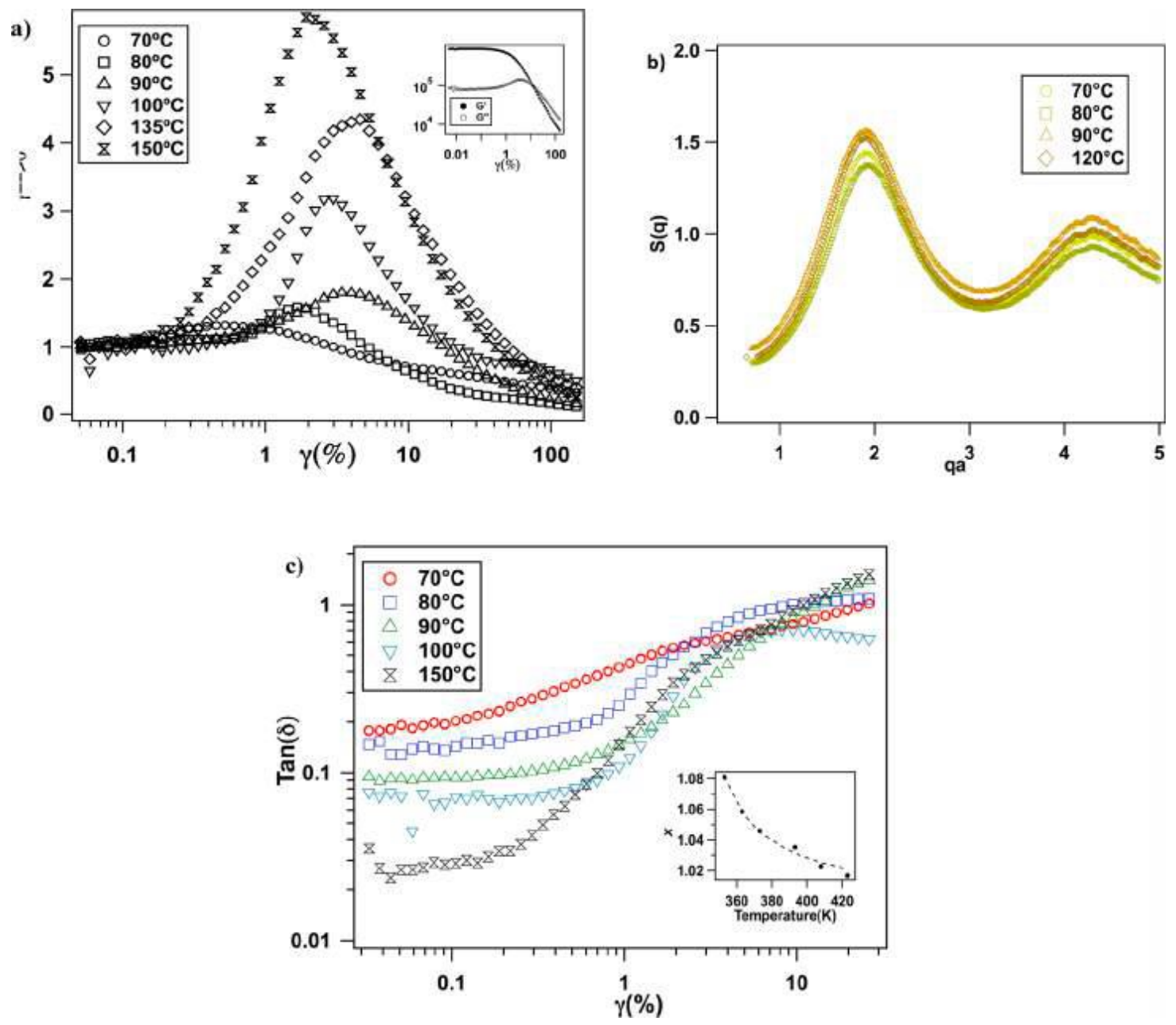

Figure 2.6 (a) The height of the normalized loss maximum, $G^{\prime \prime} / G^{\prime \prime} \gamma \rightarrow 0$, increases with temperature. The inset shows a typical soft glassy response of the material. All measurements are performed at $\omega=10 \mathrm{rad} / \mathrm{s}$. (b) The interparticle distance does not change with temperature, as observed from the variation of the first peak of structure factor $\mathrm{S}(\mathrm{q})$ with wave vector $\mathrm{q}$ at different temperatures for the system. (c) The loss tangent, $\tan \delta$, decreases with temperature. All results are for $\Sigma \approx 1.18$ chains $/ \mathrm{nm} 2$. The inset shows a decrease in noise temperature, $X$, with temperature. The dashed line is the VFT fit to the data. 
Next, we performed oscillatory shear experiments at variable shear strain to investigate the properties of these self-suspended nanoparticles on macroscopic length scale. The observed material response (inset of Figure 2.6(a)) is typical of a soft glass $^{43,60,61}$ wherein the storage modulus $G^{\prime}$ dominates the loss modulus $G^{\prime \prime}$ at low strain values and on further increasing the strain a prominent maximum is observed in $G^{\prime \prime}$ which is now understood to be associated with breaking of the cages neighboring particles exert on each other.

On investigating the effects of temperature variation (shown in Figure 2.6(a)) we observe that the loss maximum increases with temperature indicating enhanced jamming as the height of $G^{\prime \prime}$ is a reflection of the degree of jamming in the system. As previously seen in ionically grafted silica nanoparticles ${ }^{50}$, variation of temperature does not change the location of the first peak in the structure factor $S(q)$ (Figure 2.6b)) of these covalently grafted systems as well, which implies that change in temperature does not alter the inter-particle spacing of the cores. This justifies that the increase in temperature leads to effectively stiffer corona chains resulting in an enhanced corona inter-penetration, and thus leads to tighter and more jammed cages with increase in temperature. This trend is further confirmed by decrease in loss tangent, $\tan \delta$ $\left(=\mathrm{G}^{\prime \prime} / \mathrm{G}^{\prime}\right)$ with increase in temperature (Figure 2.6(c)).

The temperature induced jamming of the system can be quantified using a parameter referred to as noise temperature, $X$ which has been described in the soft glassy rheology (SGR) model ${ }^{61,62}$ as an indication of the amount of energy available for each 
particle to hop out of its potential energy well in the energy landscape. The noise temperature can be related to loss tangent as: $X=1+\frac{2}{\pi} \delta^{42,52}$. The inset of Figure 2.6c) shows a decreasing trend for $X$ with increase in temperature for $\Sigma \sim 1.18$ chains $/ \mathrm{nm}^{2}$, which implies that the particles have lesser energy available for hopping at higher temperatures, thus leading to jamming of the system with increase in temperature. Similar behavior with temperature is seen at lower grafting densities of 0.703 and 0.576 chains $/ \mathrm{nm}^{2}$ as shown in the supporting information. It is also striking that the dependence of $X$ on $\mathrm{T}$ follows the Vogel-Fulcher-Tammann (VFT) fit $^{63}$ : $X=A \exp \left(\frac{B}{T-T^{*}}\right)$, where $\mathrm{A}$ is the high temperature value of $X, \mathrm{~B}$ is the activation energy and $\mathrm{T}^{*}$ is the Vogel temperature. All the values are listed in TableI. It is remarkable that the $\mathrm{T}^{*}$ values are close to the melting point of PEG for all the systems, indicating that the tethered chains play a crucial role in determine the dependence of $X$ on $\mathrm{T}$. It is also notable that the value of A is always close to unity, indicating that the colloidal glass transition occurs at high temperature. The thermal jamming observed here is reminiscent of thermal vitrification that is observed in star polymers. ${ }^{64,65} \mathrm{In}$ those systems, the star polymers form clusters due to improved solvent quality on heating. It is rather different from our systems, as the space filling constraint imposed on the tethered chains prevents any formation of clusters or any inhomogeneties. Also, the solvent is attached to the core and thus the suspending medium and the suspension are the same, which excludes any possibility of an improvement in solvent quality. 

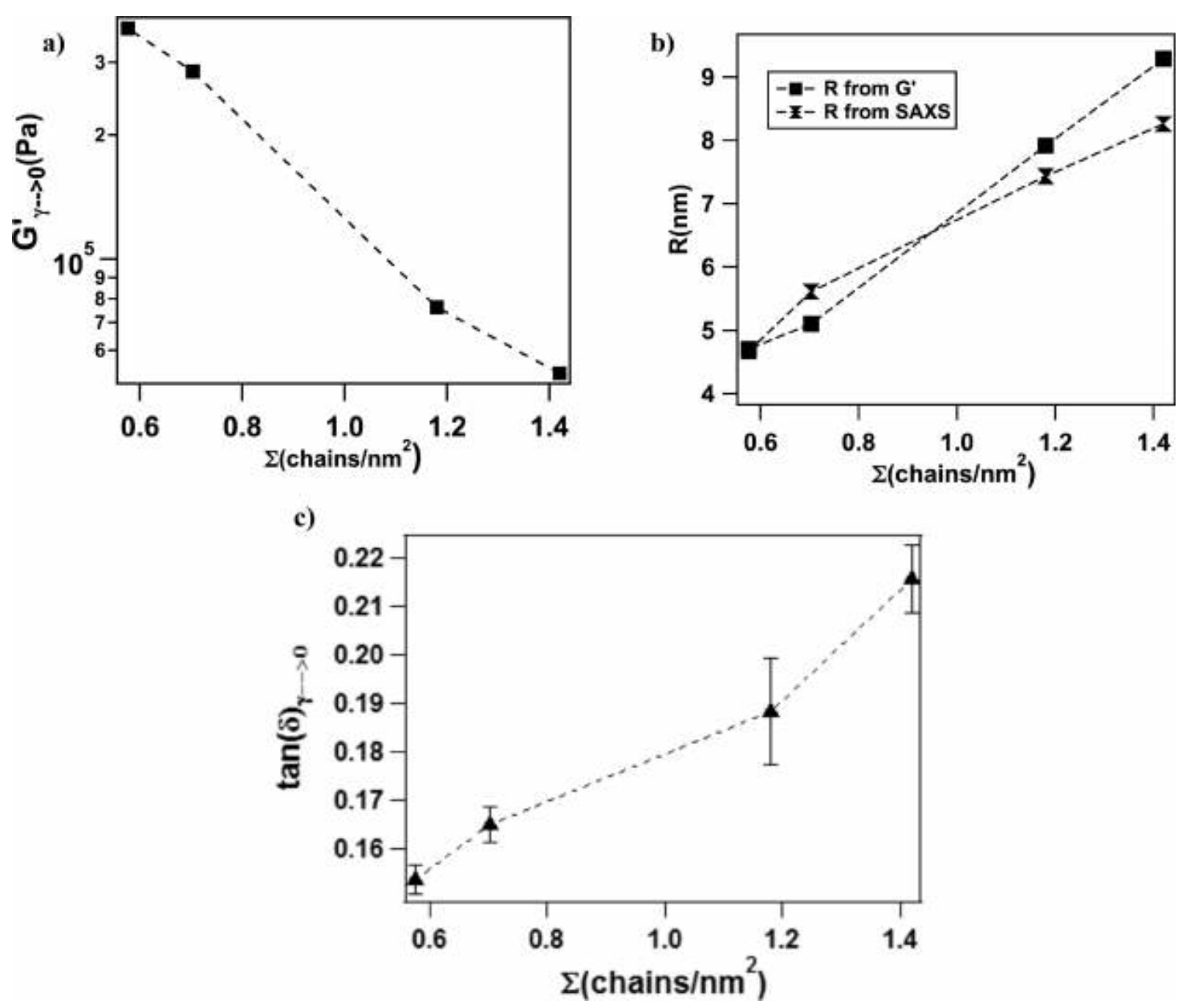

Figure 2.7 (a) $\mathrm{G}^{\prime}$ in the linear viscoelastic regime decreases with increasing grafting density while (b) the cage size, $R$, estimated from $\mathrm{G}^{\prime}$ and SAXS, and (c) the linear viscoelastic loss tangent, $\tan \delta_{\gamma 0}$, increases with increasing grafting density. The lines are guides for the eye. 
The effect of variation of grafting density $(\Sigma)$ also has a strong effect on the variation of cage strength. From Figure 2.7a) it is apparent that the value of $G^{\prime}$ in the linear viscoelastic regime decreases with increase in $\Sigma$. Previous studies have shown that $G^{\prime}$ varies as $\sim \mathrm{kT} / \mathrm{r}^{2}$ loc $\mathrm{D}$, where $\mathrm{r}_{\text {loc }}$ is the particle localization length within the cage and $\mathrm{D}$ is the particle diamtere, maximum distance which can be moved by the particle inside the cage ${ }^{66,67}$ However, since in our systems, the cage is determined by the interactions of the tethered chains while the particles are fixed relative to the chains, the cage size is determined by the extent of inter-penetration of the chains. So, we assume the localization length of the cage similar to its maximum size, and thus use $G^{\prime} \sim k T / R^{3}$, where $2 R$ is the cage size. The cage size estimated from $G^{\prime}$ is found to be in close agreement with the cage size obtained from SAXS data as $2 \mathrm{R}=\mathrm{d}_{\mathrm{p}-\mathrm{p}}-2 a$ (Figure 2.6b)). The cage size is found to increase with an increase in $\Sigma$, which implies that the system becomes less jammed on increasing the grafting density. This behavior is further confirmed by plotting tan $\delta$ as a function of $\Sigma$ (Figure 2.6c)). The increase in $\tan \delta$ with $\Sigma$ indicates un-jamming of the system on adding more chains to the particles, which further suggests that the cage strength is lowered by an increase in grafting density.

\subsection{Conclusion}

In summary, we report a facile synthesis route for creating self-suspended nanoparticles in which each particle permanently carries its own share of liquid in the form of covalently tethered polymer chains. The materials are found to exhibit outstanding phase stability in absence of any solvent; they are among the first example 
of a nanoparticle-polymer composite in which each and every building block is itself a nanocomposite. On nanometer length scales, the materials show the dominance of thermodynamically stable conformational modes in the polymer strands compared to free polymer. At mesoscopic length-scales, we observe that these systems exhibit less heterogeneity as opposed to their ionic counterparts and have stronger steric repulsions due to the absence of any electrostatic interactions between the core and corona conforming to the observations from DFT results. On macroscopic length scales, dynamic rheology measurements firmly place the materials in the universality class of soft glasses, but we find that temperature can enhance jamming in the systems, an unexpected result for a true soft glass where the cage energy is considered substantially higher than $k T$.

\section{Acknowledgement}

This work was supported by the National Science Foundation, Award No. DMR1006323 and by Award No. KUS-C1-018-02, made by King Abdullah University of Science and Technology (KAUST). Use of the Cornell High Energy Synchrotron Source was supported by the U.S. DOE under Contract No. DE-AC02-06CH11357. This work made use of the Cornell Center for Materials Research Shared Facilities, which are supported through the NSF MRSEC program (DMR-1120296). We thank Dr. Rajesh Mallavajula for his insights and ideas. We also acknowledge Dr. Ivan Keresztes for the help with the NMR experiment. We would also like to thank Adithya Sagar Gurram for his help with DFT calculations. 


\section{REFERENCES}

(1) Einstein, A. Investigations on the Theory of the Brownian Movement. Ann. $d$. Phys 1906, 19, 371-381.

(2) Batchelor, G. K. Sedimentation in a Dilute Dispersion of Spheres. J. Fluid Mech. 1972, 52, 245-268.

(3) Batchelor, G. K. Brownian Diffusion of Particles with Hydrodynamic Interaction. J. Fluid Mech. 1976, 74, 1-29.

(4) Batchelor, G. K. The Effect of Brownian Motion on the Bulk Stress in a Suspension of Spherical Particles. J. Fluid Mech. 1977, 83, 97-117.

(5) Davis, M. E.; Chen, Z. G.; Shin, D. M. Nanoparticle Therapeutics: An Emerging Treatment Modality for Cancer. Nat. Rev. Drug Discov. 2008, 7, $771-782$.

(6) Salata, O. V. Applications of Nanoparticles in Biology and Medicine. 2004, 6, $1-6$.

(7) Phillips, E.; Penate-Medina, O.; Zanzonico, P. B.; Carvajal, R. D.; Mohan, P.; Ye, Y.; Humm, J.; Gonen, M.; Kalaigian, H.; Schoder, H.; et al. Clinical Translation of an Ultrasmall Inorganic Optical-PET Imaging Nanoparticle Probe. Sci. Transl. Med. 2014, 6, 260ra149-ra260ra149. 
(8) Kim, Y.; Lobatto, M. E.; Kawahara, T.; Lee Chung, B.; Mieszawska, A. J.;

Sanchez-Gaytan, B. L.; Fay, F.; Senders, M. L.; Calcagno, C.; Becraft, J.; et al.

Probing Nanoparticle Translocation across the Permeable Endothelium in

Experimental Atherosclerosis. Proc. Natl. Acad. Sci. U. S. A. 2014, 111, $1078-$ 1083.

(9) Rocco, M. a; Kim, J.-Y.; Burns, A.; Kostecki, J.; Doody, A.; Wiesner, U.;

DeLisa, M. P. Site-Specific Labeling of Surface Proteins on Living Cells Using Genetically Encoded Peptides That Bind Fluorescent Nanoparticle Probes.

Bioconjug. Chem. 2009, 20, 1482-1489.

(10) Bradbury, M. S.; Phillips, E.; Montero, P. H.; Cheal, S. M.; Stambuk, H.;

Durack, J. C.; Sofocleous, C. T.; Meester, R. J. C.; Wiesner, U.; Patel, S.

Clinically-Translated Silica Nanoparticles as Dual-Modality Cancer-Targeted

Probes for Image-Guided Surgery and Interventions. Integr. Biol. (Camb).

2013, 5, 74-86.

(11) Liu, S.; Wang, H.; Imanishi, N.; Zhang, T.; Hirano, a.; Takeda, Y.; Yamamoto, O.; Yang, J. Effect of Co-Doping Nano-Silica Filler and N-Methyl-NPropylpiperidinium Bis(trifluoromethanesulfonyl)imide into Polymer Electrolyte on Li Dendrite Formation in Li/poly(ethylene Oxide)Li(CF3SO2)2N/Li. J. Power Sources 2011, 196, 7681-7686. 
(12) Lu, Y.; Korf, K.; Kambe, Y.; Tu, Z.; Archer, L. a. Ionic-Liquid-Nanoparticle Hybrid Electrolytes: Applications in Lithium Metal Batteries. Angew. Chemie 2014, 126, 498-502.

(13) Schaefer, J. L.; Moganty, S. S.; Yanga, D. a.; Archer, L. a. Nanoporous Hybrid Electrolytes. J. Mater. Chem. 2011, 21, 10094.

(14) Croce, F.; Appetecchi, G. B.; Persi, L.; Scrosati, B. Nanocomposite Polymer Electrolytes for Lithium Batteries. Nature 1998, 394, 456-458.

(15) Bruce, P. G.; Scrosati, B.; Tarascon, J.-M. Nanomaterials for Rechargeable Lithium Batteries. Angew. Chem. Int. Ed. Engl. 2008, 47, 2930-2946.

(16) Gurevitch, I.; Buonsanti, R.; Teran, a. a.; Gludovatz, B.; Ritchie, R. O.; Cabana, J.; Balsara, N. P. Nanocomposites of Titanium Dioxide and PolystyrenePoly(ethylene Oxide) Block Copolymer as Solid-State Electrolytes for Lithium Metal Batteries. J. Electrochem. Soc. 2013, 160, A1611-A1617.

(17) Moganty, S. S.; Srivastava, S.; Lu, Y.; Schaefer, J. L.; Rizvi, S. a.; Archer, L. a. Ionic Liquid-Tethered Nanoparticle Suspensions: A Novel Class of Ionogels. Chem. Mater. 2012, 24, 1386-1392.

(18) Chrissopoulou, K.; Andrikopoulos, K. S.; Fotiadou, S.; Bollas, S.; Karageorgaki, C.; Los, D. C.; Voyiatzis, G. A.; Anastasiadis, S. H. Crystallinity and Chain Conformation in PEO / Layered Silicate Nanocomposites. 2011, $9710-9722$. 
(19)

Zheng, G.; Lee, S. W.; Liang, Z.; Lee, H.-W.; Yan, K.; Yao, H.; Wang, H.; Li, W.; Chu, S.; Cui, Y. Interconnected Hollow Carbon Nanospheres for Stable Lithium Metal Anodes. Nat. Nanotechnol. 2014, 9, 618-623.

(20) Liu, S.; Imanishi, N.; Zhang, T.; Hirano, a.; Takeda, Y.; Yamamoto, O.; Yang, J. Effect of Nano-Silica Filler in Polymer Electrolyte on Li Dendrite Formation in Li/poly(ethylene oxide)-Li(CF3SO2)2N/Li. J. Power Sources 2010, 195, $6847-6853$.

(21) Lu, Y.; Das, S. K.; Moganty, S. S.; Archer, L. a. Ionic Liquid-Nanoparticle Hybrid Electrolytes and Their Application in Secondary Lithium-Metal Batteries. Adv. Mater. 2012, 24, 4430-4435.

(22) Baker, G. L.; Colsons, S. Composite Polymer Electrolytes Using Fumed Silica Fillers : Rheology and Ionic Conductivity. 1994, 2359-2363.

(23) Srivastava, S.; Schaefer, J. L.; Yang, Z.; Tu, Z.; Archer, L. a. 25Th Anniversary Article: Polymer-Particle Composites: Phase Stability and Applications in Electrochemical Energy Storage. Adv. Mater. 2014, 26, 201-234.

(24) Agrawal, A.; Choudhury, S.; Archer, L. A. A Highly Conductive, NonFlammable Polymer-Nanoparticle Hybrid Electrolyte. RSC Adv. 2015. DOI: $10.1039 / \mathrm{C} 5 \mathrm{RA} 01031 \mathrm{D}$

(25) Srivastava, S.; Agarwal, P.; Archer, L. a. Tethered Nanoparticle-Polymer Composites: Phase Stability and Curvature. Langmuir 2012, 28, 6276-6281. 
(26) Balazs, A. C.; Emrick, T.; Russell, T. P. Nanoparticle Polymer Composites: Where Two Small Worlds Meet. Science 2006, 314, 1107-1110.

(27) Krishnamoorti, R. Strategies for Dispersing Nanoparticles in Polymers. MRS Bull. 2007, 32 .

(28) Park, B. J.; Vermant, J.; Furst, E. M. Heterogeneity of the Electrostatic Repulsion between Colloids at the Oil-water Interface. Soft Matter 2010, 6, 5327.

(29) Fritz, G.; Scha, V.; Willenbacher, N.; Wagner, N. J. Electrosteric Stabilization of Colloidal Dispersions. 2002, 6381-6390.

(30) Zhulina, E. B.; Borisov, O. V; Priamitsyn, V. a. Theory of Steric Stabilization of Colloid Dispersions by Grafted Polymers. J. Colloid Interface Sci. 1990, $137,495-511$.

(31) Smith, G. D.; Bedrov, D. Dispersing Nanoparticles in a Polymer Matrix: Are Long, Dense Polymer Tethers Really Necessary? Langmuir 2009, 25, 11239 11243.

(32) Kalb, J.; Dukes, D.; Kumar, S. K.; Hoy, R. S.; Grest, G. S. End Grafted Polymer Nanoparticles in a Polymeric Matrix: Effect of Coverage and Curvature. Soft Matter 2011, 7, 1418. 
(33) Chevigny, C.; Jestin, J.; Gigmes, D.; Schweins, R.; Di-Cola, E.; Dalmas, F.; Bertin, D.; Boué, F. “Wet-to-Dry” Conformational Transition of Polymer Layers Grafted to Nanoparticles in Nanocomposite. Macromolecules 2010, 43, 4833-4837.

(34) Green, D. L.; Mewis, J.; Engineering, C.; Uni, V.; Way, E.; Charlottes, V. Connecting the Wetting and Rheological Behaviors of Poly ( Dimethylsiloxane ) -Grafted Silica Spheres in Poly ( Dimethylsiloxane ) Melts. 2006, 9546-9553.

(35) Hasegawa, R.; Aoki, Y.; Doi, M. Optimum Graft Density for Dispersing Particles in Polymer Melts. 1996, 9297, 6656-6662.

(36) Roovers, J.; Paul, L. Z.; Zwan, M. Van Der; Iatrou, H.; Hadjichristidisi, N. Regular Star Polymers with 64 and 128 Arms. Models for Polymeric Micelles? Macromolecules 1993, 4324-4331.

(37) Watanabe, H.; Yao, M.; Sato, T.; Osaki, K. Non-Newtonian Flow Behavior of Diblock Copolymer Micelles : Shear-Thinning in a Nonentangling Matrix. Macromolecules 1997, 9297, 5905-5912.

(38) Watanabe, H. Nonlinear Rheology of Diblock Copolymer Micellar Dispersion: A Review of Recent Findings. J. Nonnewton. Fluid Mech. 1999, 82, 315-329.

(39) Sato, T.; Watanabe, H.; Osaki, K. Relaxation of Spherical Micellar Systems of Styrene - Isoprene Diblock Copolymers . 1 . Linear Viscoelastic and Dielectric Behavior. Macromolecules 1996, 9297, 3881-3889. 
(40) Willner, L.; Richter, J. J. D.; Roovers, J.; Z, L.; Festkorperforschung, I.; Gmbh, F. J. Structural Investigation of Star Polymers in Solution by Small Angle Neutron Scattering. Macromolecules 1994, 3821-3829.

(41) Adams, J. L.; Graessley, W. W. Rheology and the Microphase Separation Transition in Styrene-Isoprene. Macromolecules 1994, 6026-6032.

(42) Fetters, L. J.; Andrea, D. K.; J, D. S. P.; Quack, G. F.; Vitus, F. J. Rheological Behavior of Star-Shaped Polymers. Macromolecules 1993, 647-654.

(43) Agarwal, P.; Qi, H.; Archer, L. a. The Ages in a Self-Suspended Nanoparticle Liquid. Nano Lett. 2010, 10, 111-115.

(44) Fernandes, N. J.; Akbarzadeh, J.; Peterlik, H.; Giannelis, E. P. Terms of Use Synthesis and Properties of Highly Dispersed Ionic Silica À Poly ( Ethylene Oxide ) Nanohybrids. 2013, 1265-1271.

(45) Yu, H.-Y.; Koch, D. L. Structure of Solvent-Free Nanoparticle-Organic Hybrid Materials. Langmuir 2010, 26, 16801-16811.

(46) Chremos, A.; Panagiotopoulos, A. Z.; Yu, H.-Y.; Koch, D. L. Structure of Solvent-Free Grafted Nanoparticles: Molecular Dynamics and DensityFunctional Theory. J. Chem. Phys. 2011, 135, 114901. 
(47) Hong, B.; Chremos, A.; Panagiotopoulos, A. Z. Simulations of the Structure and Dynamics of Nanoparticle-Based Ionic Liquids. Faraday Discuss. 2012, $154,29$.

(48) Chremos, A.; Panagiotopoulos, A. Z.; Koch, D. L. Dynamics of Solvent-Free Grafted Nanoparticles. J. Chem. Phys. 2012, 136, 044902.

(49) Goyal, S.; Escobedo, F. a. Structure and Transport Properties of Polymer Grafted Nanoparticles. J. Chem. Phys. 2011, 135, 184902.

(50) Agarwal, P.; Srivastava, S.; Archer, L. a. Thermal Jamming of a Colloidal Glass. Phys. Rev. Lett. 2011, 107, 268302.

(51) Jespersen, M. L.; Mirau, P. A.; Meerwall, E. Von; Vaia, R. A.; Rodriguez, R.; Ḱ, E. P. G. Canopy Dynamics in Nanoscale Ionic Materials. 2010, 4, 37353742.

(52) Fernandes, N. J.; Wallin, T. J.; Vaia, R. a.; Koerner, H.; Giannelis, E. P. Nanoscale Ionic Materials. Chem. Mater. 2014, 26, 84-96.

(53) Coleman, M. M.; Skrovanek, D. J.; Hu, J.; Painter, P. C. Hydrogen Bonding in Polymer Blends. 1. FTIR Studies of Urethane-Ether Blends. Macromolecules $1988,21,59-65$.

(54) Kim, S. a; Archer, L. a. Hierarchical Structure in Semicrystalline Polymers Tethered to Nanospheres. Macromolecules 2014, 47, 687-694. 
(55) Agarwal, P.; Kim, S. a.; Archer, L. a. Crowded, Confined, and Frustrated: Dynamics of Molecules Tethered to Nanoparticles. Phys. Rev. Lett. 2012, 109, 258301.

(56) Matsuura, H.; Fukuhara, K. Vibrational Spectroscopic Studies of Conformation of Poly ( oxyethylene ). 11. Conformation- Spectrum Correlations. 1986, 24, $1383-1400$.

(57) Deng, Y.; Dixon, J. B.; White, G. N. Bonding Mechanisms and Conformation of Poly(ethylene Oxide)-Based Surfactants in Interlayer of Smectite. Colloid Polym. Sci. 2005, 284, 347-356.

(58) Glatter, O.; Kratky, O. Small Angle X-Ray Scattering; United Sta.; Academic Press: New York, 1982.

(59) Yu, H.-Y.; Srivastava, S.; Archer, L. a; Koch, D. L. Structure Factor of Blends of Solvent-Free Nanoparticle-Organic Hybrid Materials: Density-Functional Theory and Small Angle X-Ray Scattering. Soft Matter 2014, 10, 9120-9135.

(60) Mason, T. G.; Weitz, D. A. Linear Viscoelasticity of Colloidal Hard SPhere Suspensions near the Glass Transition. 1995, 75, 2770-2773.

(61) Sollich, P.; Lequeux, F.; Hébraud, P.; Cates, M. Rheology of Soft Glassy Materials. Phys. Rev. Lett. 1997, 78, 2020-2023. 
(62) Sollich, P. Rheological Constitutive Equation for a Model of Soft Glassy Materials. Phys. Rev. E 1998, 58, 738-759.

(63) Angell, C. a.; Ngai, K. L.; McKenna, G. B.; McMillan, P. F.; Martin, S. W. Relaxation in Glassforming Liquids and Amorphous Solids. J. Appl. Phys. 2000, $88,3113$.

(64) Kapnistos, M.; Vlassopoulos, D.; Fytas, G.; Mortensen, K.; Fleischer, G.; Roovers, J. Reversible Thermal Gelation in Soft Spheres. Phys. Rev. Lett. 2000, $85,4072-4075$.

(65) Loppinet, B.; Stiakakis, E.; Vlassopoulos, D.; Fytas, G.; Roovers, J. Reversible Thermal Gelation in Star Polymers : An Alternative Route to Jamming of Soft Matter. 2001, 8216-8223.

(66) Mason, T. G. Estimating the Viscoelastic Moduli of Complex Fluids Using the Generalized Stokes-Einstein Equation. Rheol. Acta 2000, 39, 371-378.

(67) Shah, S. a.; Chen, Y.-L.; Schweizer, K. S.; Zukoski, C. F. Viscoelasticity and Rheology of Depletion Flocculated Gels and Fluids. J. Chem. Phys. 2003, 119, 8747. 


\section{APPENDIX}

Supplementary Information for Chapter 2

Figures-

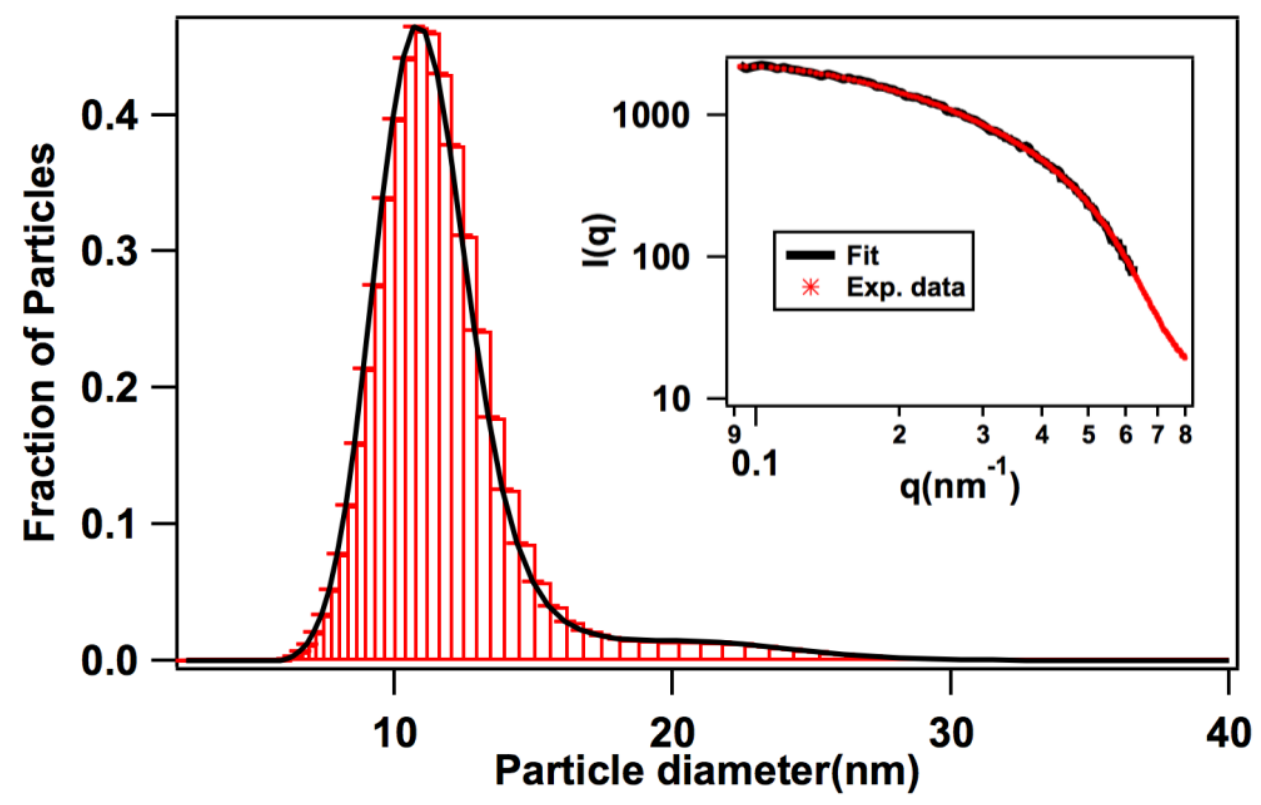

Supplementary Figure 2.2. a) $\mathrm{HSQC}{ }^{1} \mathrm{H}^{13}{ }^{13} \mathrm{C}$ in $\mathrm{CDCl}_{3}$ at $25^{\circ} \mathrm{C}$. b) $\mathrm{HMBC}{ }^{1} \mathrm{H}^{-13} \mathrm{C}$ in $\mathrm{CDCl}_{3}$ at $25^{\circ} \mathrm{C}$ 


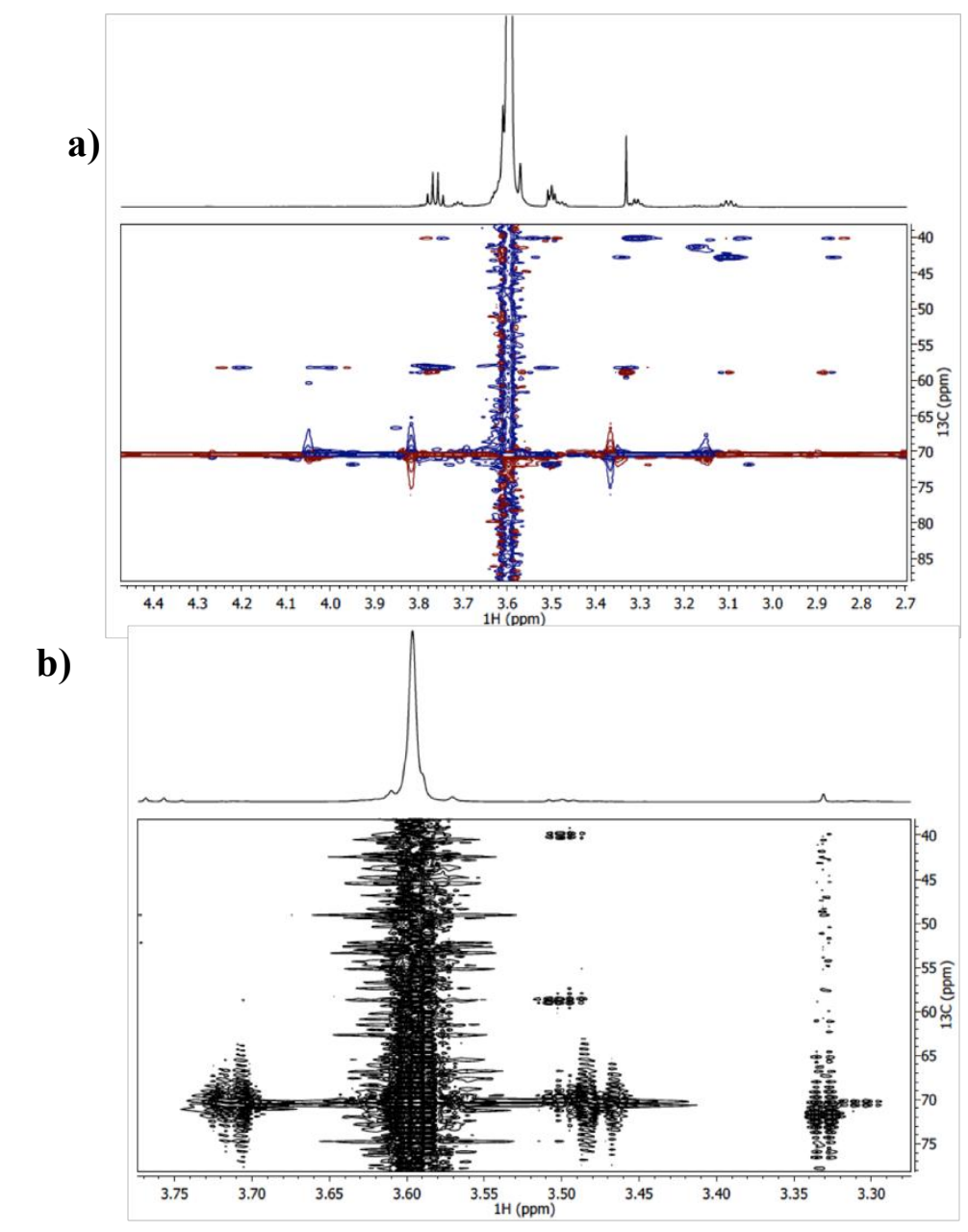

Supplementary Figure 2.1. Size distribution of Silica nanoparticles as determined from SAXS analysis. The solid line denoted Gaussian fits to the data. Inset: Experimental scattering intensity for Silica nanoparticles(red dots) and the 

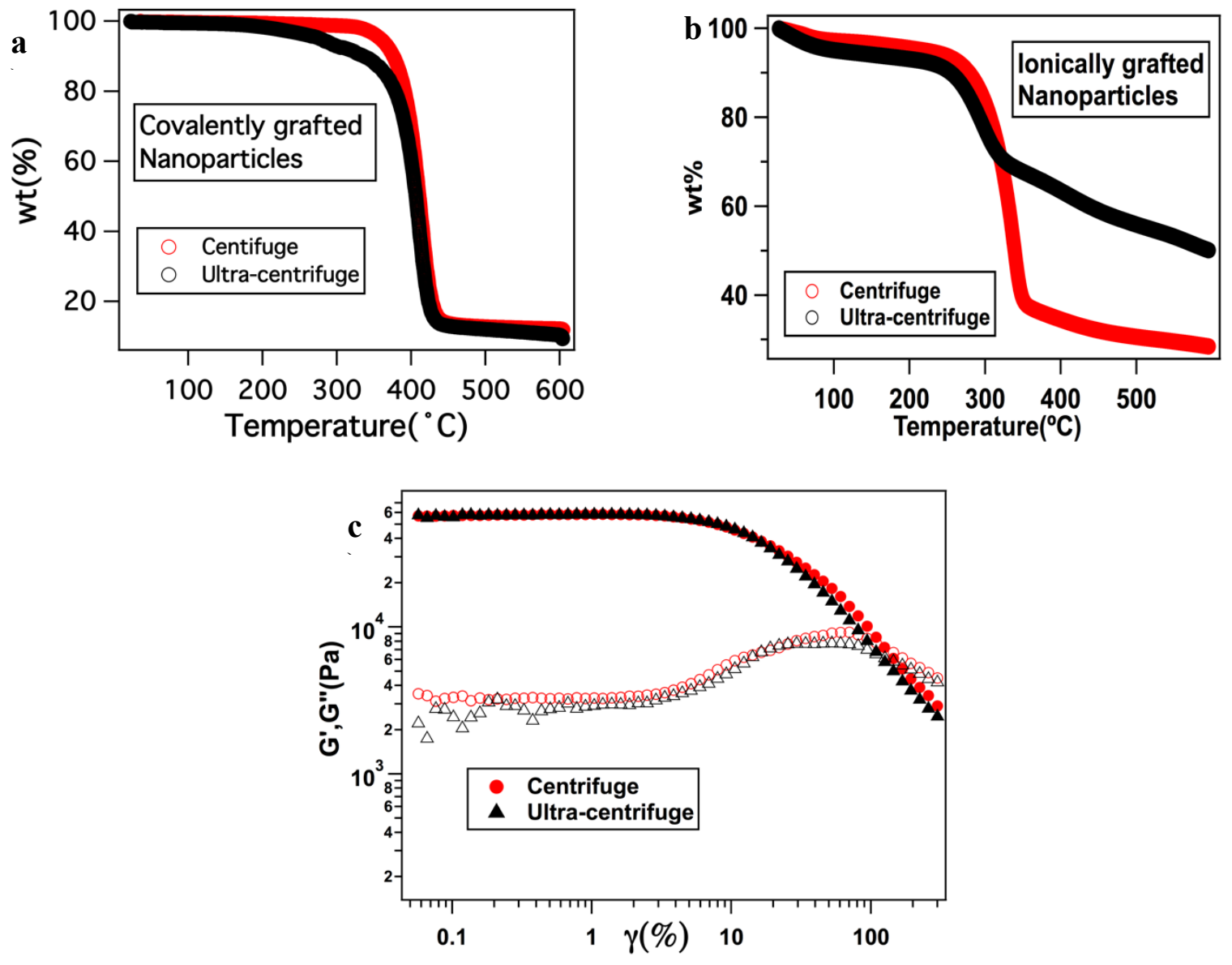

Supplementary Figure 2.3. Comparison of centrifuge and ultra-centrifuge for a) covalently grafted nanoparticles and b) Ionically grafted nanoparticles. It can be observed that the resultant weight \% for the covalent system is the same from both the methods while for the ionic system the weight fraction of silica goes on decreasing when ultracentrifuged. c) Amplitude sweep measurement of the covalently grafted sample for normal centrifuge and after ultra-centrifuge. The two measurements can be seen to overlap. 


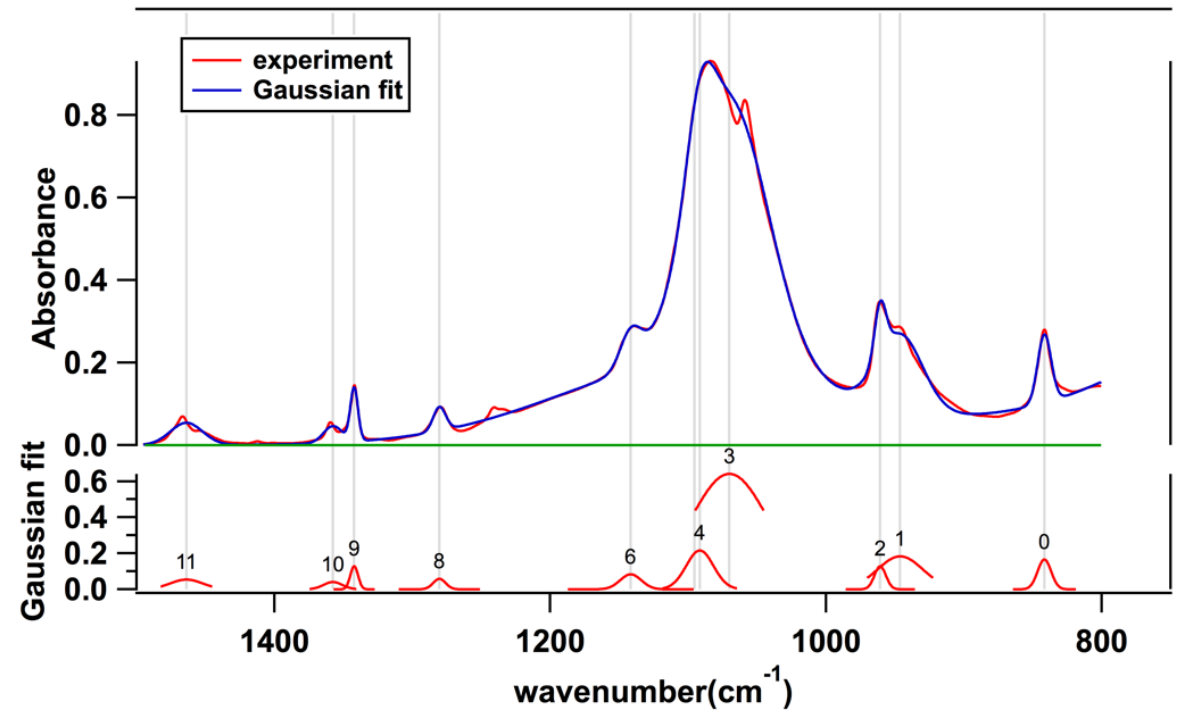

Supplementary Figure 2.4 Gaussian fitting of the FT-IR peaks for tethered PEO chains of grafting density, $\Sigma \sim 1.03$ chains $/ \mathrm{nm}^{2}$. 


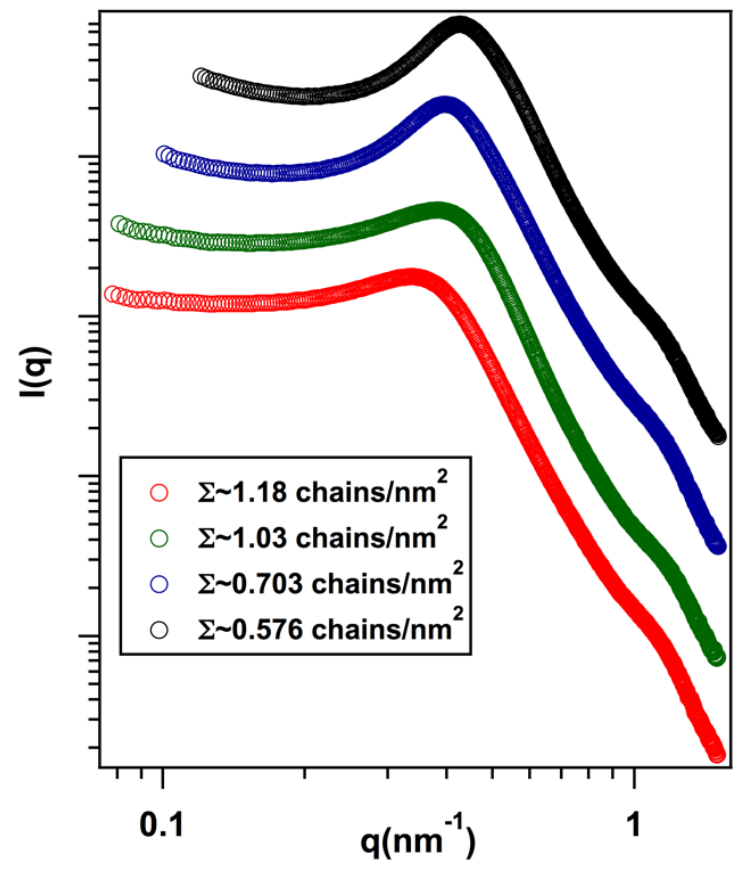

Supplementary Figure 2.5. Variation of intensity(I(q)) as measured from SAXS experiments with $\mathrm{q}$ at different grafting densities. 

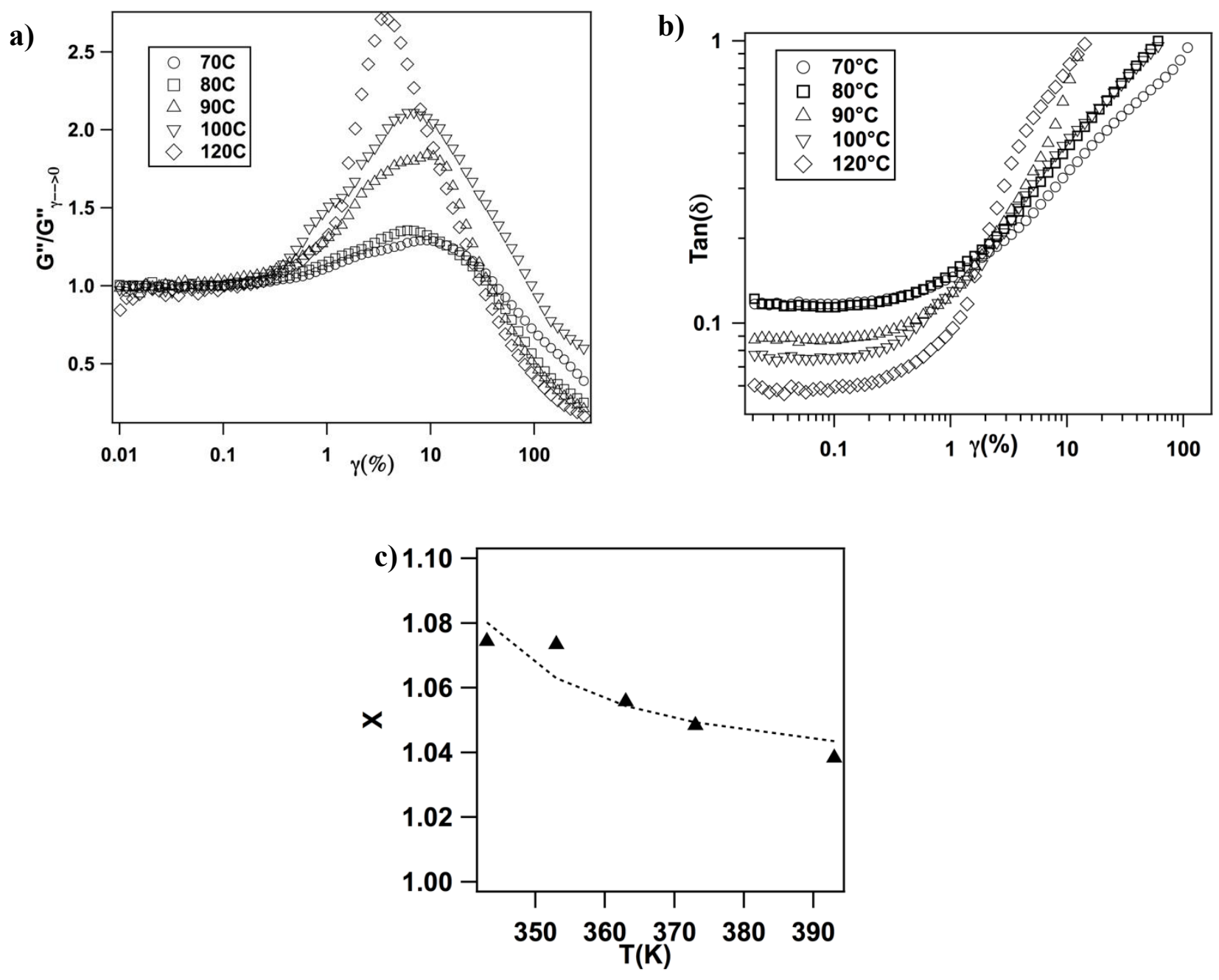

Supplementary Figure 2.6 a) Variation of normalised loss modulus G"/G" ${ }_{\gamma \rightarrow 0}$ and b) $\tan (\delta)$ with strain amplitude at different temperatures. All the measurements are performed at $\omega=10 \mathrm{rad} / \mathrm{s} \mathrm{c}$ ) Similar trends are seen in noise temperature $X$ variation with temperature. The results are for system with $\Sigma \sim 0.703$ chains $/ \mathrm{nm}^{2}$ 

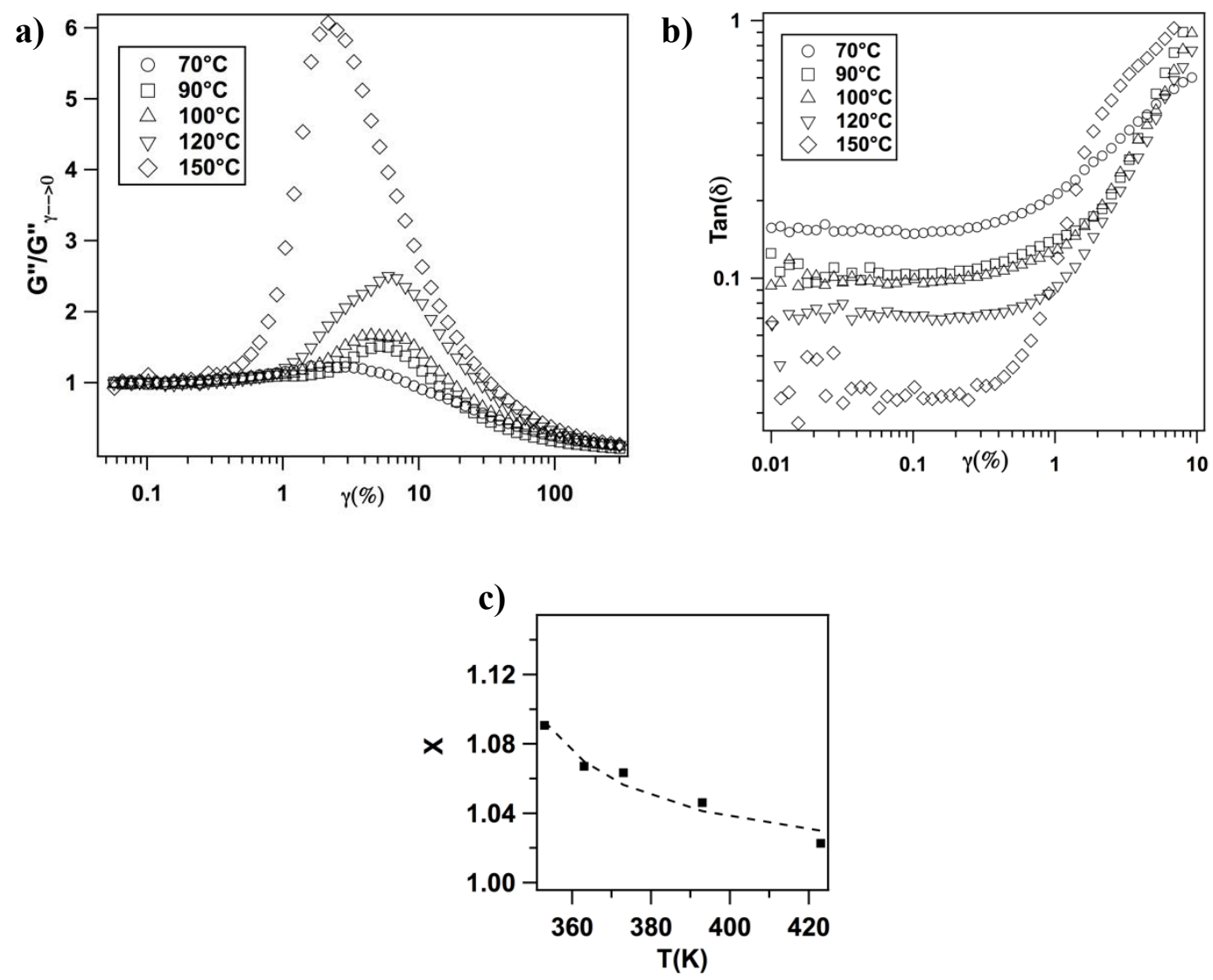

Supplementary Figure 2.7 a) Variation of normalised loss modulus G"/G" ${ }_{\gamma \rightarrow 0}$ and b) $\tan (\delta)$ with strain amplitude at different temperatures and at $\omega=10 \mathrm{rad} / \mathrm{s} \mathrm{c}$ ) Similar trends are seen in noise temperature $X$ variation with temperature. The results are for system with $\Sigma \sim 0.576$ chains $/ \mathrm{nm}^{2}$ 

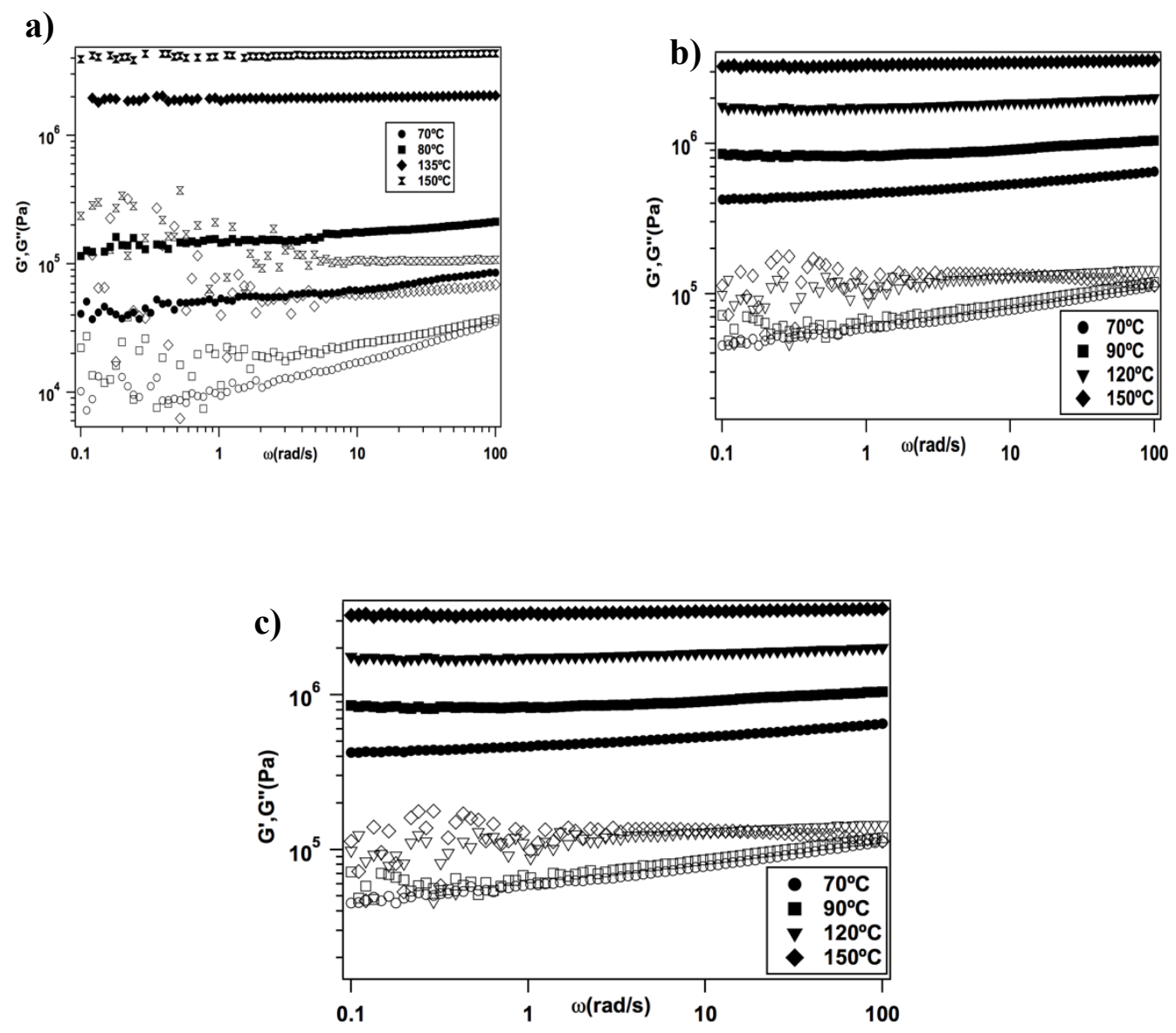

Supplementary Figure 2.8 Frequency sweep measurements at $\gamma=0.1 \%$ at different temperatures for a) $\Sigma \sim 1.18$ chains $/ \mathrm{nm}^{2}$ b) $\Sigma \sim 0.703$ chains $/ \mathrm{nm}^{2}$ and c) $\Sigma \sim 0.576$ chains $/ \mathrm{nm}^{2}$. Storage Modulus, $G^{\prime}$ (closed symbols) is found to be always greater than the loss modulus, G" (open symbols) 
Supplementary Table 2.1. Assignment of FTIR peaks for free and tethered chains to the corresponding conformations and structure of crystal unit cell

\begin{tabular}{|c|c|c|c|c|}
\hline $\begin{array}{l}\text { Free PEO peak } \\
\text { position }\left(\mathrm{cm}^{-1}\right)\end{array}$ & $\begin{array}{l}\text { Tethered } \\
\text { PEO(1.18chains/ } \\
\left.\mathrm{nm}^{2}\right) \text { peak } \\
\text { position }\left(\mathrm{cm}^{-1}\right)\end{array}$ & $\begin{array}{l}\text { Vibrational } \\
\text { assignment }\end{array}$ & ormations & unit cell \\
\hline 842 & 841 & C-O stretch, $\mathrm{CH}_{2}$ rock & mostly C-C gauche & helix \\
\hline 949 & 950 & C-O stretch, $\mathrm{CH}_{2}$ rock & $\mathrm{C}-\mathrm{C}$ gauche, $\mathrm{C}-\mathrm{O}$ trans & helix \\
\hline 961 & 961 & C-O stretch, $\mathrm{CH}_{2}$ rock & & zigzag \\
\hline 1060 & 1064 & $\begin{array}{l}\mathrm{C}-\mathrm{O} \text { stretch, C-C stretch } \\
\mathrm{CH}_{2} \text { rock }\end{array}$ & & helix \\
\hline 1098 & 1094 & C-O stretch, C-C stretch & & \\
\hline 1146 & 1150 & C-O stretch, C-C stretch & & \\
\hline 1238 & 1238 & $\mathrm{CH}_{2}$ twist (anti-symmetric) & C-O trans & helix \\
\hline 1279 & 1280 & $\mathrm{CH}_{2}$ twist (symmetric) & $\mathrm{C}-\mathrm{O}$ gauche & helix \\
\hline 1341 & 1342 & $\mathrm{CH}_{2}$ wag (anti-symmetric) & C-C trans & zigzag \\
\hline 1358 & 1357 & $\mathrm{CH}_{3}$ wag (anti-symmetric) & C-C gauche & helix \\
\hline
\end{tabular}


CHAPTER 3

\section{A HIGHLY CONDUCTIVE, NON-FLAMMABLE POLYMER- NANOPARTICLE HYBRID ELECTROLYTE}




\subsection{Abstract}

We report on physical properties of lithium-ion conducting nanoparticle-polymer hybrid electrolytes created by dispersing bidisperse mixtures of polyethylene glycol (PEG)-functionalized silica nanoparticles in an aprotic liquid host. At high particle contents, we find that the ionic conductivity is a non-monotonic function of the fraction of larger particles $x_{L}$ in the mixtures, and that for the nearly symmetric case $x_{L} \approx 0.5$ (i.e. equal volume fraction of small and large particles), the room temperature ionic conductivity is nearly ten-times larger than in similar nanoparticle hybrid electrolytes comprised of the pure small or large particle components used in the mixtures. Complementary behaviors are seen in the activation energy for ion migration and effective tortuosity of the electrolytes, which both exhibit minima near $x_{L} \approx 0.5$. Characterization of the electrolytes by dynamic rheology reveals that the maximum conductivity coincides with a distinct transition in soft glassy properties from a jammed to partially jammed and back to jammed state, as the fraction of large particles is increased from 0 to 1 ; indicating that the conductivity enhancement arises from purely entropic loss of correlation between nanoparticle centers arising from particle size dispersity. As a consequence of these features, we show that it is possible to create hybrid electrolytes with $1 \mathrm{MPa}$ elastic moduli and $1 \mathrm{mS} / \mathrm{cm}$ ionic conductivities at room temperature using common aprotic liquid media as the electrolyte solvent. Remarkably, we also find that even in highly flammable liquid media, the bidisperse nanoparticle hybrid electrolytes can be formulated to exhibit low or no flammability without compromising their favorable ionic conductivity and mechanical properties. 


\subsection{Introduction}

Significant amount of research has been devoted towards improving the portability, power, lifetime and safety of secondary rechargeable batteries with exclusive focus on battery electrolytes and ion conducting membranes ${ }^{1-6}$. Polymer nanocomposite is a special class of such an electrolyte that has created new platforms to mitigate the issues associated with conventional flammable liquid electrolytes ${ }^{4-8}$. These nanocomposite electrolytes have improved the safety as well as portability of batteries and have prevented electrolyte leakage, thus eliminating the need of a physical separator $^{9-15}$. The inorganic particles in polymer electrolytes affect the ion transport mainly as passive filler and sometimes as active filler ${ }^{9}$. As a passive filler, they act as plasticizers for the polymers preventing crystallization, thus speeding up the segmental dynamics of polymer host and enhancing the ion transport ${ }^{9,12,14-16}$. However, an optimum nanoparticle loading is required to ensure a well-dispersed state of the particles, such that the ion transport pathway is not disrupted due to high particle concentration. Active fillers directly participate in the ion transfer process either by providing additional cations/anions or by surface reaction with mobile ions. They are shown to improve the cation transference number that ultimately results in significant increment in the coulombic stability of the batteries ${ }^{9,11,12,15,17,18}$.

Perhaps, the greatest benefit of these nanocomposite electrolytes is their integration towards higher mechanical stability. It has been previously reported that a high modulus electrolyte can be effective in preventing dendrite-induced short circuit in a Lithium metal battery ${ }^{19-25}$. Nanocomposite based batteries have shown encouraging 
results in this regard ${ }^{26-28,16}$. Although all the above properties are of relevant interest for application in rechargeable battery industry, low ambient conductivity and high interfacial resistance hinder the practicality of the nanocomposite electrolytes ${ }^{9,26,29,30}$. Weston and Steele ${ }^{31}$ were the first to make improvements towards developing a decently ion conducting and highly mechanical stable electrolyte by adding ceramic fillers in low volume fractions to polyethylene oxide polymer. However, achieving practical conductivity at high particle loadings, where the mechanical strength is maximized, has been a tough task for over a decade.

Recent studies on hybrid electrolytes based on polymer-tethered nanoparticles have shown a significant promise towards this step ${ }^{32-34}$. These hybrid electrolytes based on hairy nanoparticles have good mechanical and electrochemical properties, and at the same time they provide enough room for nano-engineering to improve the current state of art even further. The polymer-grafted nanoparticles have been shown to exhibit interesting physical properties like viscoelasticity ${ }^{35}$, thermal jamming ${ }^{36}$, and star polymer like relaxation ${ }^{37}$. Previously, studies on binary mixture of star polymers have gained significant attention by showing that addition of smaller star polymers to bigger ones leads to a transition from glassy state to liquid state ${ }^{38,39}$. Similar studies on the self-suspended binary mixtures of these hairy nanoparticles have demonstrated that addition of either small or bigger particles leads to un-jamming of the system ${ }^{40}$. Currently, we focus on this jamming transition observed in binary hairy nanoparticles in the context of an electrolyte and try to utilize it to build a better battery. 
In this article we report on ionic conductivity, mechanical properties, and structure of hybrid electrolytes comprised of a bidisperse blend of SiO2-PEG hairy nanoparticles dispersed in propylene carbonate (PC). The study focuses on silica particles with diameters of $10 \mathrm{~nm}$ and $25 \mathrm{~nm}$ covalently functionalized with PEG oligomers. Our specific interest is in understanding the effect of both the total particle volume fraction, $\Phi$, and relative volume fraction of the bigger particles $\mathrm{xL}-$ at a fixed $\Phi-$ on suspension structure and physical properties. We find that the additional degree of freedom provided by $\mathrm{xL}$ allows the structure and transport properties of polymernanoparticle hybrid electrolytes to be tuned in novel ways to achieve both high ionic conductivity and good mechanical performance. To our knowledge, this is the first study to systematically investigate the effect of particle size dispersity on conductivity of nanoparticle-polymer hybrid electrolytes.

\subsection{Materials and Methods}

\subsubsection{Synthesis}

Silica nanoparticles (Ludox, SM-30 and TM-50; Sigma Aldrich) with diameters of $10 \mathrm{~nm}$ and $25 \mathrm{~nm}$, respectively, were grafted by covalent attachment of a trimethoxysilane functionalized polyethylene glycol methyl ether (PEG, MW $\sim 500 \mathrm{~g} / \mathrm{mol}$, Gelest chemicals) in aqueous solution using a previously reported silane chemistry ${ }^{41,42}$ (see schematic in Figure 3.1(a)). The grafting densities were computed from analysis of the residual inorganic content using thermal gravimetric analysis (TGA) to be $\Sigma \sim 1.3$ chains $/ \mathrm{nm} 2$ and $\Sigma \sim 1.5$ chains $/ \mathrm{nm} 2$ for $10 \mathrm{~nm}$ and $25 \mathrm{~nm}$ particles, respectively. Following synthesis, the hairy particles were purified using a 
two-step process, first by dialysis using a snake skin membrane to remove any dissolved salts along with unattached free PEG chains; and second by repeated centrifugation at $8500 \mathrm{rpm}$ for 10 minutes using a chloroform(solvent)-hexane(nonsolvent) mixture to completely remove any remaining unattached PEG oligomers. The particles were then subsequently dried in convection oven at $55^{\circ} \mathrm{C}$ overnight and at least for $12 \mathrm{hrs}$ under high vacuum. The dried sample was quickly transferred to Argon filled glove box for storage and subsequent modification. Electrolytes were prepared inside the glove box by suspending the hairy nanoparticles in the electrolyte solvent propylene carbonate (PC, Sigma Aldrich) at various core volume fractions, $\Phi$ ranging from 0.1 to 0.5 . For each $\Phi$, the relative fraction of bigger particles with respect to the overall particle volume fraction, i.e. $\Phi \mathrm{L} / \Phi=\mathrm{xL}$, in the $\mathrm{SiO} 2-\mathrm{PEG} / \mathrm{PC}$ suspensions was varied. The resulting solution of hybrid nanoparticles in PC was doped with bis(trifluoromethanesulfone imide) (LiTFSi, Sigma Aldrich) salt to create SiO2PEG/PC hybrid electrolytes containing 1M LiTFSI based on the total organic content (i.e. PEG corona and PC electrolyte solvent).

\subsubsection{Characterization}

The particle weight fraction in the SiO2-PEG/PC-LiTFSi hybrid electrolytes was determined from thermal gravimetric analysis (TGA) by heating the sample at 10 ${ }^{\circ} \mathrm{C} / \mathrm{min}$ to $600{ }^{\circ} \mathrm{C}$. The structure and dispersion state of the nanoparticles was characterized by angle-resolved Small Angle X-ray Scattering (SAXS) measurements at Station D1 of Cornell High Energy Synchrotron Source (CHESS) using a point 
collimated X-ray beam. All studied materials were smeared on a sample cell and the measurements were performed at $30^{\circ} \mathrm{C}$.

Dynamic rheological properties were studied using frequency- and strain-dependent oscillatory shear measurements at $30^{\circ} \mathrm{C}$ on a MCR 301 rheometer outfitted with $10 \mathrm{~mm}$ diameter, $2^{\circ}$ cone and plate fixtures. The frequency sweep measurements were performed at a low strain, $\gamma=0.05 \%$, which is within the linear viscoelastic regime for the materials. The strain-dependent oscillatory shear measurements were performed at a fixed angular frequency of $\omega=10 \mathrm{rad} / \mathrm{s}$.

\subsubsection{Electrochemical measurements}

The ionic conductivity of the SiO2-PEG/PC-LiTFSI hybrid electrolytes was measured as a function of temperature, ranging from $0^{\circ} \mathrm{C}$ to $105^{\circ} \mathrm{C}$, using a Novocontrol Broadband Dielectric spectrometer. For each temperature, the frequency was varied from $0.1-3 \times 10^{6} \mathrm{~Hz}$. The DC conductivity at each temperature was obtained from the plot of real part of the conductivity with frequency using the procedure described by Jonscher43. The $\operatorname{Re}\left[\right.$ conductivity] can be expressed as $\sigma^{\prime}(\omega)=\sigma_{\mathrm{DC}}+\mathrm{A} \omega \mathrm{s}$; where $\mathrm{A}$ is a constant. The DC conductivity can thus be estimated from the plateau value of the plot between $\operatorname{Re}[$ conductivity] and $\omega$.

\subsubsection{Characterizing Flammability}


The flammability of electrolytes containing the hairy SiO2-PEG nanoparticles was

studied by suspending the particles in a more flammable electrolyte mixture, ethylene

a)

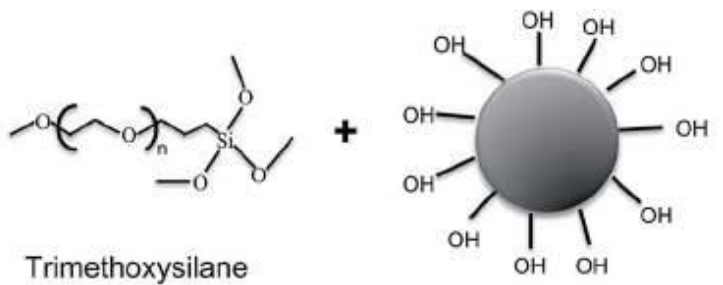

Silica Nanoparticle terminated PEG

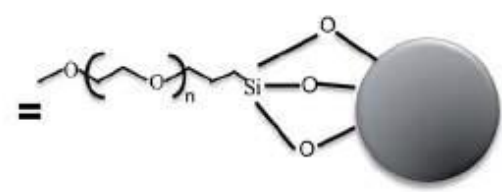

PEG attached to Silica

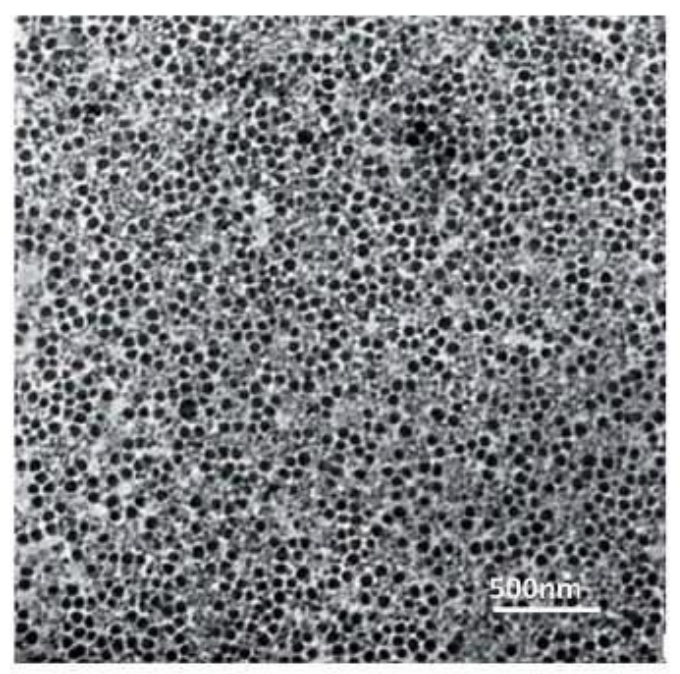

c)

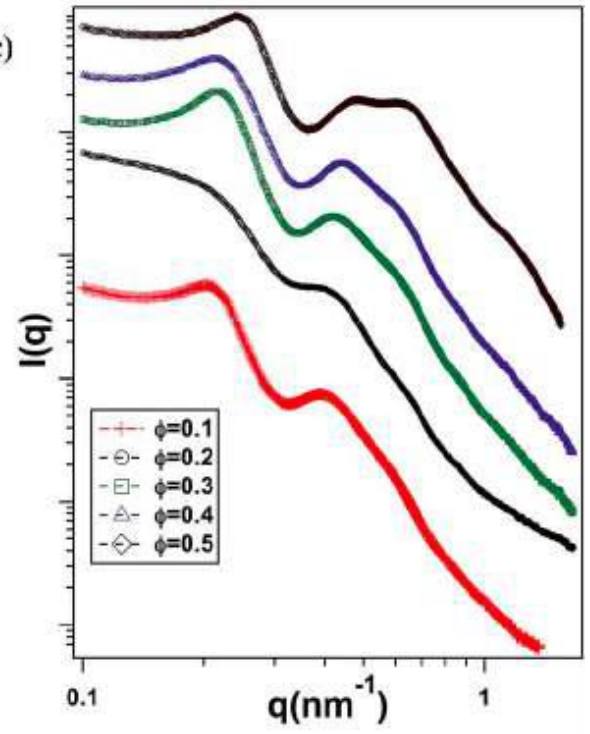

d)

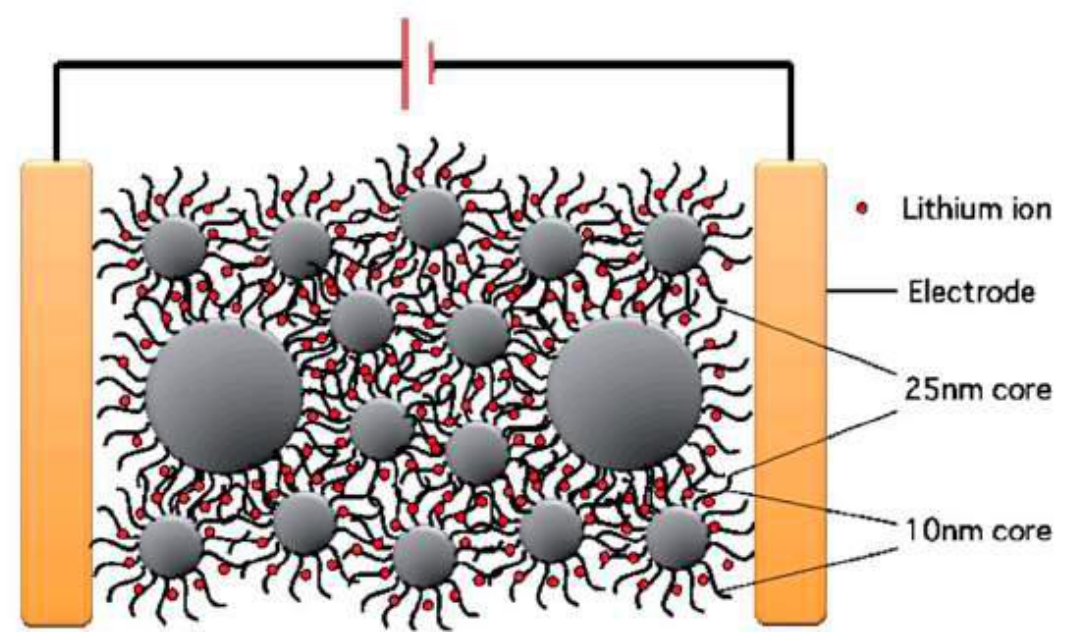

carbonate/diethyl carbonate (EC: DEC, Sigma Aldrich). This approach was necessary 
Figure 3.1 Physical Characterization - (a) schematic of hairy nanoparticle synthesis. (b) Transmission Electron Micrograph (TEM) image of binary hairy nanoparticle composite with $\mathrm{xL}$. 0.5. (c) Intensity (I(q)) as a function of wave vector (q) for $\mathrm{xL}$. 0.5 at different F, obtained from SAXS measurements. (d) Pictorial representation of a battery with binary hairy nanoparticle composite electrolyte. because the PC solvent used for the other studies is flammable only at very high temperatures. The samples used for this component of the study were doped with $1 \mathrm{M}$ of Lithium hexafluorophosphate (LiPF6, Sigma Aldrich). 0.2g of SiO2PEG/EC:DEC/LiPF 6 hybrid electrolytes at $\mathrm{xL}=0.5$ and various $\Phi$ were transferred to Aluminum pans. Material flammability was studied by igniting each electrolyte specimen with butane torch lighter and recording images at the time of ignition, $4 \mathrm{~s}$ after ignition, and at the time the flame self-extinguished.

\subsection{Results and Discussion}

Figure 3.1(b) is a Transmission electron micrograph (TEM) of a SiO2-PEG/PC hybrid electrolyte with $\Phi=0.5$ and $\mathrm{xL}=0.5$. The particles are observed to be quite well dispersed in the PC host. Figure 3.1(c) reports the wavenumber (q)-dependent scattering intensity (I(q)) for SiO2-PEG/PC hybrid electrolyte obtained using SAXS measurements. The results are reported for a fixed value of $x L=0.5$ at different $\Phi$. It is evident that at any $\Phi$, in the low q region, $I(q)$ is at best a very weak function of $q$, whereas in the high q region, $\mathrm{I}(\mathrm{q})$ varies as q-4. Both observations are characteristic of un-aggregated well-dispersed spherical nanoparticles confirming the good dispersion state of the materials inferred from the small-area TEM measurements 44,45 . 
Figure 3.2 shows the temperature dependent DC conductivity of SiO2-PEG/PC-

LiTFSi hybrid electrolytes at various particle volume fractions $(\Phi)$ and for a range of $x L$ values $(0 \leq x L \leq 1)$. The data is well described by the Vogel-Fulcher-Thamann (VFT) temperature dependent conductivity, $\mathrm{S}(\mathrm{T})=\mathrm{A} \exp (-\mathrm{Ea} / \mathrm{k}(\mathrm{T}-\mathrm{T} 0))$, over much of

a)

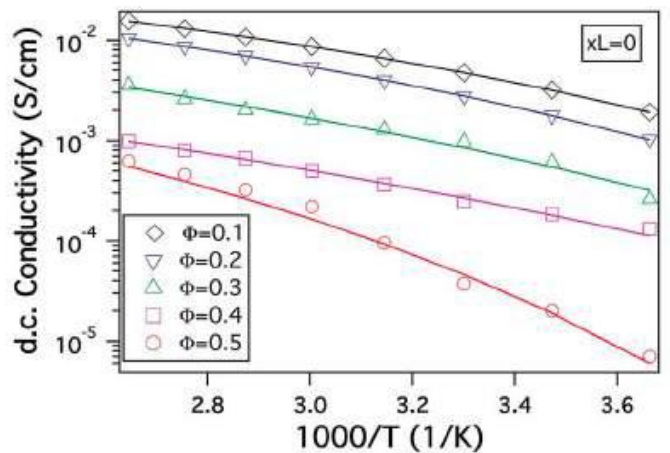

c)

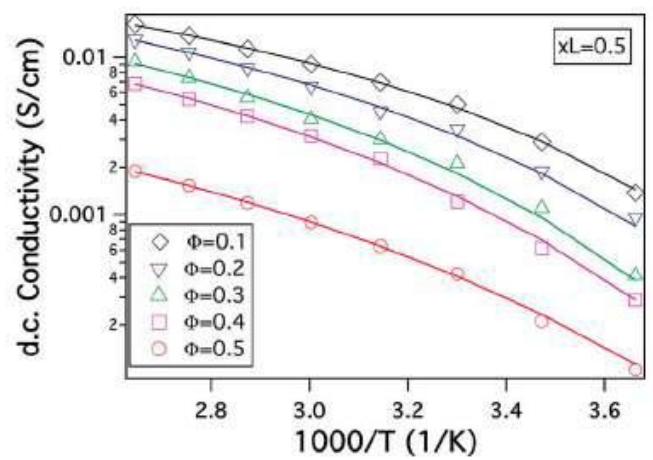

b)

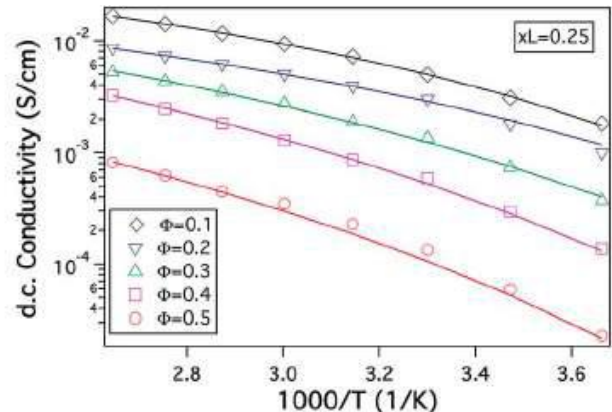

d)

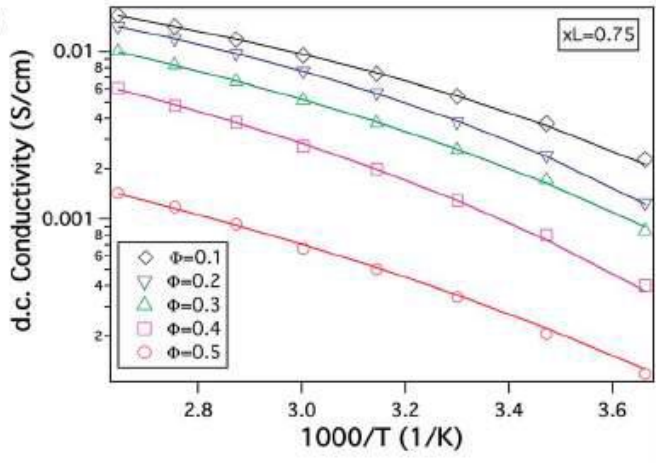

e)

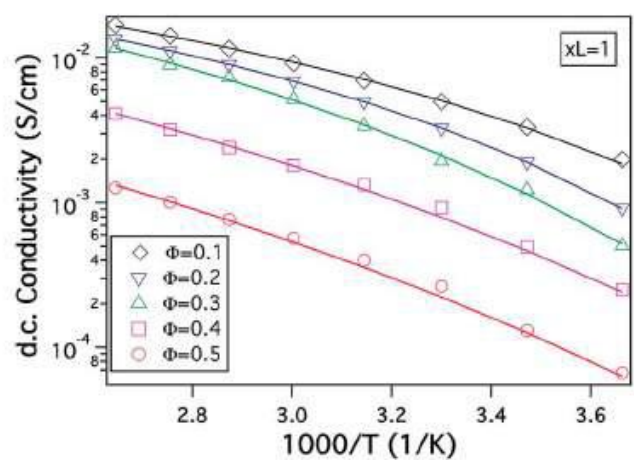

Figure 3.2 Electrochemical properties - conductivity as a function of inverse of temperature for different core volume fraction $(\mathrm{F})$ at various fractions of bigger nanoparticles in the binary composite electrolyte indicated by $\mathrm{xL}$, as, (a) $\mathrm{xL}=0$; (b) $\mathrm{xL}=0.25 ;$ (c) $\mathrm{xL}=0.5 ;$ (d) $\mathrm{xL}=0.75 ;$ (e) $\mathrm{xL}=1$. 
the temperature range and for all the electrolytes studied. Here the prefactor A, with units $\mathrm{S} / \mathrm{cm}$, is proportional to the number of mobile ions present in the electrolyte, Ea is the activation energy for ion mobility in $\mathrm{kJ} / \mathrm{mole}$, $\mathrm{k}$ is the gas constant in $\mathrm{kJ} /$ mole- $\mathrm{K}$, and To is the empirical reference Temperature in K.46 The fact that conductivity for all electrolyte compositions studied are well described by the VFT relation over the entire range of temperature implies that there is no melting or crystallization transition, and that there is no phase separation.

In order to understand the effect of particle size dispersity on ionic conductivity, the DC conductivity measured at a fixed temperature $\mathrm{T}=30 \mathrm{C}$ is plotted as a function of $\mathrm{xL}$ at different $\Phi$, in Figure 3.3(a). It can be observed that at $\Phi=0.1$ and 0.2 , the conductivity does not vary much with changes in xL. However, for $\Phi \geq 0.3$ conductivity for the bidisperse $\mathrm{SiO} 2-\mathrm{PEG} / \mathrm{PC}-\mathrm{LiTFSi}$ hybrid electrolytes is noticeably higher than the conductivity of hybrid electrolytes comprised of the pure small $(\mathrm{xL}=0)$ or big ( $\mathrm{xL}=1) \mathrm{SiO} 2-\mathrm{PEG}$ hairy nanoparticles. This effect is most pronounced at $\Phi=0.5$, where a clear maximum is seen near $\mathrm{xL}=0.5$. The conductivity is also plotted as a function of overall particle volume fraction for different xL values in Supplementary Figure 3.1. There is a greater drop in the conductivity on increasing the particle volume fraction for pure small $(\mathrm{xL}=0)$ and big $(\mathrm{xL}=1) \mathrm{SiO} 2-\mathrm{PEG} / \mathrm{PC}-\mathrm{LiTFSi}$ hybrid 
electrolytes than for the bidsiperse hybrid electrolytes; the effect being particularly visible in electrolytes with $\mathrm{xL}=0.5$ and 0.75 .

a)

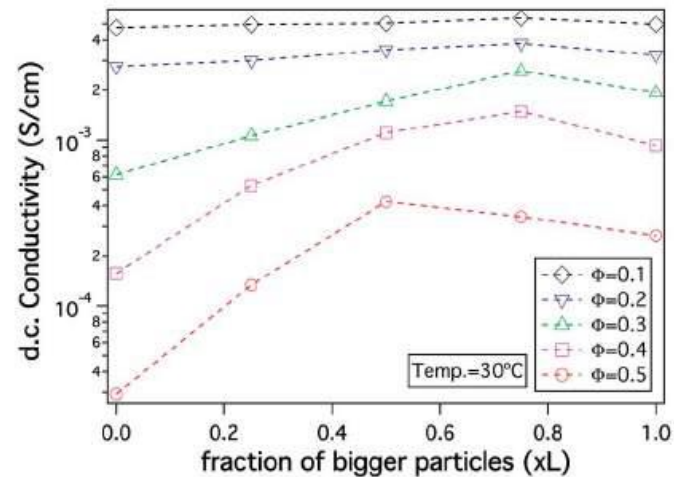

c)

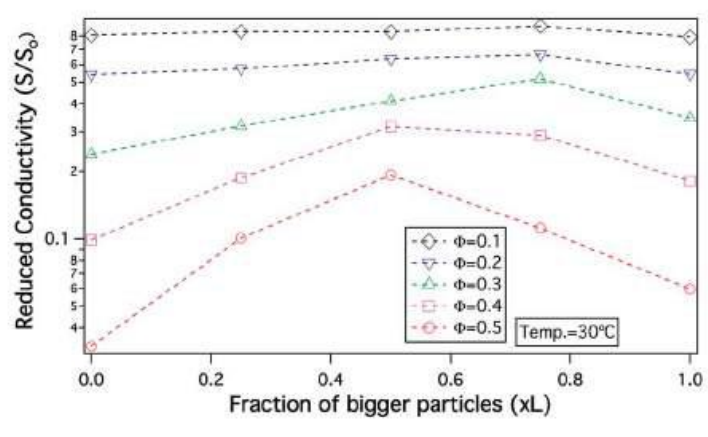

b)

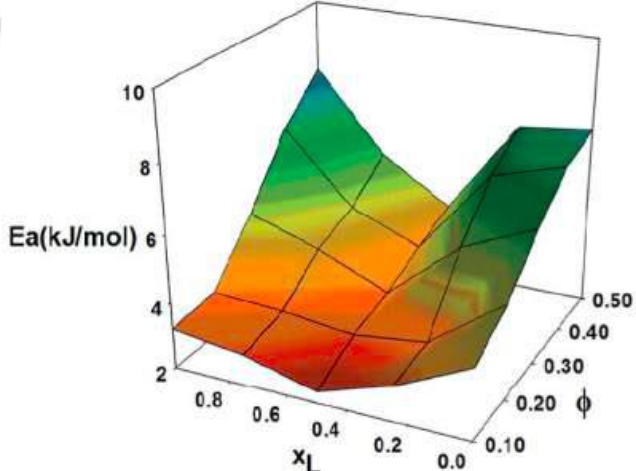

d)

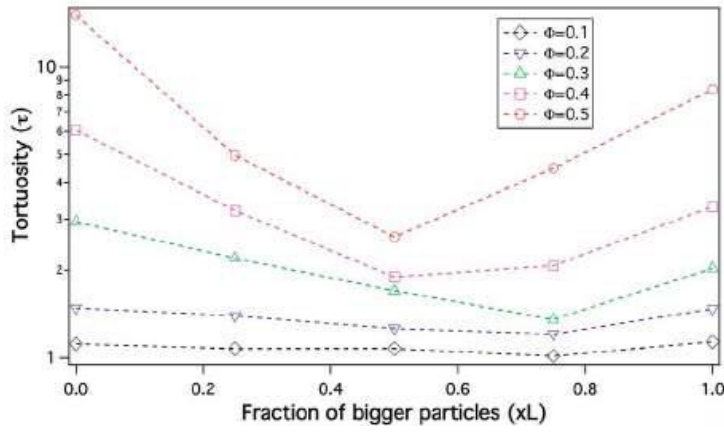

Figure 3.3 Analysis of ion transport - (a) conductivity as a function of $x L$ at $30^{\circ} \mathrm{C}$ at different F; (b) activation energy landscape at different $\mathrm{xL}$ values and corresponding F; (c) reduced conductivity given by the ratio of actual conductivity to that of the organic content, shown as a function of $x \mathrm{~L}$ at $30^{\circ} \mathrm{C}$ (d) tortuosity versus $\mathrm{xL}$. 
Activation energies computed from the VFT fits of temperature-dependent conductivity provides additional insights about the nature of ion transfer processes in electrolytes. It is known that for electrolytes with higher activation energy there is a significant change in conductivity associated with temperature, which is correlated with more practical risks, such as thermal runaway and fire in cells in which there are exothermic side reactions ${ }^{47}$. Figure 3.3(b) reports the activation energy landscape obtained from VFT fits at different $\Phi$ and xL. It is seen that for high $\Phi$ the activation energy is greatest for the pure small $(x L=0)$ or pure big $(x L=1)$ hybrid electrolyte and that it is minimum near $\mathrm{xL} \approx 0.5$ (see also, Table ST1 in Supplementary Information). Consistent with the conductivity data, the effect becomes weaker as the overall particle volume fraction, $\Phi$, in the electrolytes is reduced. Thus, the bidisperse system of nanocomposite electrolytes can find wide applications as electrolytes that can be used at ambient temperatures, while sustaining sudden thermal shocks.

We hypothesize that the observed enhancement in conductivity in bidisperse $\mathrm{SiO}_{2}$ PEG/PC-LiTFSI hybrid electrolytes at high $\Phi$ and $\mathrm{xL} \approx 0.5$ may arise due to reduced correlation between particle-centers in the bidisperse materials. In particular at the high particle volume fractions, where bidispersity has the most noticeable effect on conductivity and activation energy, the positions of $\mathrm{SiO}_{2}-\mathrm{PEG}$ nanospheres in a monodisperse suspension are more correlated than in the bidisperse case. The tethered PEG corona chains are hence more crowed and confined in electrolytes containing either pure small or pure large $\mathrm{SiO}_{2}$-PEG particles. Such crowding of surface grafted chains has already been reported to lead to dramatically slower chain reorientation 
dynamics for cis-1,4-polyisoprene tethered to $\mathrm{SiO}_{2} \cdot{ }^{35}$ The motion of ions mediated by the confined PEG chains would therefore be expected to be correspondingly impaired and sluggish, which is consistent with the higher activation energy. In contrast, for a bidisperse suspension of $\mathrm{SiO}_{2}$-PEG nanospheres, neighboring particles exert weaker constraints on each other because of the size dispersity. In other words, at high particle loadings, the system is less jammed due to the heterogeneities introduced by addition of a different sized species ${ }^{40,48,49}$, thus loosening the packing of particles and reducing the confinement on tethered PEG chains, increasing their mobility and lowering the activation energy. This freedom would in turn increase the rate at which ions migrate in the electrolyte, reflected in a higher ionic conductivity. This hypothesis can also account for the weaker of particle size dispersity at lower $\Phi$ where the tethered PEG chains are not confined and ion motion is controlled more in coordination with the mobile PC phase.

Since, the organic phase in the $\mathrm{SiO}_{2}$-PEG/PC-LiTFSi hybrid electrolytes is comprised of both PC and tethered PEG chains, it is useful to resolve the relative content of PEG and PC in different electrolytes to better understand the effect of bidispersity of the nanocores on electrolyte properties. The conductivity of PC/PEG-LITFSi liquid electrolyte mixtures was measured as a function of composition and the results are reported in Supplementary Figure 3.2. A straight-line fit of the data allows us to determine how the content of PC/PEG influences ionic conductivity in the absence of particles. Using this knowledge, the conductivity contribution (So) attributable to the organic content for each electrolyte shown in Figure 3.3(a) can be recovered. The 
relative PC content in each sample is tabulated in Supplementary Table 1. In order to isolate the contribution of the nanocores, we plot the reduced conductivity as S/So versus the fraction of bigger particles $(\mathrm{xL})$ in Figure 3.3(c). The effect of particle size dispersity is now more profound, but again the maximum is most readily apparent for hybrids with the highest $\Phi$ and the maximum is clear. Hence, we conclude that the idea of unjamming a high-volume fraction nanoparticle hybrid electrolyte by introducing bidispersity is not a function of the chemistry of the electrolyte solvent, making the concept a potentially powerful tool in other fields, such as ion exchange chromatography, desalination, and electroplating, where ion transport through complex media is relevant.

To understand the nature of the inter-particle spaces in the $\mathrm{SiO}_{2}-\mathrm{PEG} / \mathrm{PC}$ hybrid electrolytes, we look at the trends in 'tortuosity' of these materials, as previously proposed by Carman ${ }^{50}$. The conductivity of an electrolyte is a direct reflection of the tortuous nature of the conducting phase, where, tortuosity is defined as the ratio between the actual distance covered by the ions to travel across the bulk and the shortest distance between the two points. In the context of electrolytes, the conductivity of a neat electrolyte is suppressed by the addition of nanoparticles or other non-conducting entities depending upon the resulting porosity, which is equivalent to the volume fraction of the conductive phase in a suspension electrolyte. The experimental conductivity, S, is lower than that obtained empirically, $\operatorname{So}(1-\Phi)$, this discrepancy is quantified using the term effective tortuosity ${ }^{51}$. Figure 3.3(d) reports tortuosity as a function of $\mathrm{xL}$ for different volume fractions for the binary hairy 
nanoparticle electrolytes. It is seen that for low volume fractions, there is little change across the range of $x \mathrm{~L}$, and the tortuosity values remain close to unity. This is consistent with intuition that, at low particle volume fraction, the nature of the conducting pathway across the electrolyte is almost same as for the particle-free electrolyte and size dispersity of the particulate phase has no obvious effect on conductivity. On increasing the particle volume fraction, the tortuosity of the pure small or pure big $\mathrm{SiO}_{2}$-PEG/PC-LiTFSI electrolyte becomes greater than 10, however the bidisperse hybrid electrolytes are seen to have substantially lower effective tortuosity. This behavior can be attributed to the unjamming of densely packed $\mathrm{SiO}_{2}-$ PEG nanoparticles, facilitating nearly unimpeded transport of ions in the electrolyte. Although empirical models for tortuosity for different shaped randomly spaced particles exist,${ }^{51-55}$ such models do not yet exist for the surface-functionalized particles used in this study, making it difficult at the present time to understand the significance of the experimentally determined values for tortuosity.

The changes in ionic conductivity are associated with important changes in mechanical properties of the electrolytes. It can be seen from Figure 3.4(a) that for a given $x L$ value, on changing $\Phi$ from 0.2 to 0.5 , the system undergoes a well-studied transition from a viscoelastic liquid in which the loss modulus, G", is larger than the storage modulus G', to a soft glass, characterized by G' $>$ G” at low shear strains, and the tell tale maximum in G" at higher strains associated with yielding and flow $\left(G^{\prime}>G^{\prime}\right)$ of the material ${ }^{56,57}$. In particular, at low $\Phi$ and for $x L=0$ or $1, G{ }^{\prime}>G^{\prime}$, indicating the electrolytes exhibit liquid-like behavior; remarkably, even under these 

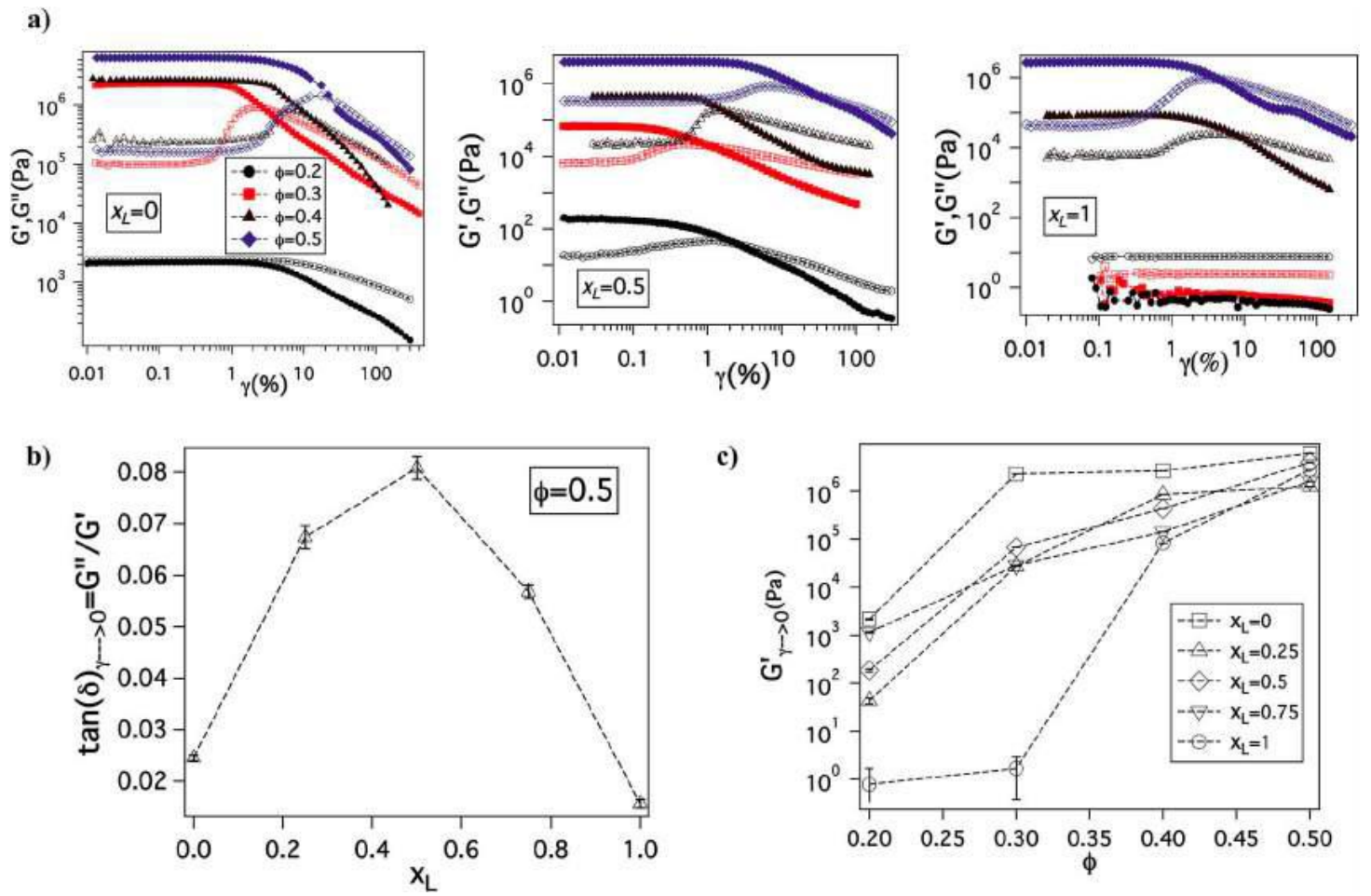

Figure 3.4: Rheological properties- a) Evolution of storage modulus, G' (closed symbol) and loss modulus, G" (open symbol) as a function of amplitude strain, $\gamma$ with particle volume fraction, $\Phi$ at $x_{L}=0,0.5$ and 1 on going from left to right. b) Variation of Loss Tangent, $\tan (\delta)=G^{\prime \prime} / G^{\prime}$ obtained at $\gamma \rightarrow 0$ as a function of $x_{L}$ at $\Phi=0.5$. Since $\tan (\delta)$ is the ratio of loss modulus to storage modulus, the higher the loss tangent the more fluid-like or un-jammed the system is. It can be observed that addition of either small or big particles leads to increase in un-jamming of the particles as compared to the pure species. c) Storage modulus at $\gamma \rightarrow 0$ varying as a function of $\Phi$ at different $x_{L}$ values. It can be seen that at high $\Phi$, the modulus values are extremely high, more than $10^{6}$. 
conditions the bidisperse mixture with $\mathrm{xL}=0.5$ shows soft glassy behavior. In concentrated particulate suspensions, soft glassy rheology is understood to result from crowding and trapping of particles in cages formed due to the presence of neighboring particles. Upon increasing the shear strain, these cages break producing the burst of dissipated energy as particles move relative to each other, which manifests as a maximum in G".58,59 This implies that in such suspensions high shear strains can transform a jammed material to a less solid-like, processable form; removal of the strain causes the cage structure to reform and the solid-like jammed state is restored.

The degree of jamming in a soft glassy material can be quantified using the value of its loss tangent $\left(\tan \delta=G^{\prime} / G^{\prime}\right)$, measured at low stains in the linear viscoelastic regime of oscillatory shear measurements. Figure 3.4(b) shows tan $\delta$ value as a function of $x \mathrm{~L}$, it is seen that addition of either the bigger or smaller particles to their pure cohorts leads to an increase in $\tan \delta$; implying that introduction of heterogeneity leads to less jamming of the system. Remarkably, at $\Phi=0.5$, where the electrolyte exhibits soft glassy rheology over the entire range of $x L, \tan \delta$ exhibits a clear maximum near $\mathrm{xL}=0.5$, i.e. exactly where the maximum in ionic conductivity and minimum in activation energy for the electrolytes is observed. This finding succinctly shows that the enhanced conductivity for the bidisperse $\mathrm{SiO}_{2}-\mathrm{PEG} / \mathrm{PC}$ hybrid electrolytes is a result of reduced jamming. Figure 3.4(c) shows the viscoelastic storage modulus (G') of these electrolytes as a function of volume fraction for various xL. At high particle loading, these electrolytes display G' values around 5MPa (Supplementary Table 3.1), 
which is only modestly lower than the modulus of hybrid electrolytes created using the pure small or pure large particles at $\Phi=0.5$.

The favorable ionic conductivity and mechanical properties of these bidisperse $\mathrm{SiO}_{2}$ PEG/PC-LiTFSI hybrid electrolytes at relatively high nanoparticle concentrations is already attractive for the application related investigations outlined in the introduction. Since silica and PEG are both non- or poorly-flammable materials, it is possible that at the high particle loadings where size dispersity has the greatest effect on conductivity and rheology of the electrolytes, it may also have a positive effect on their safety. To explore this aspect of the materials, we briefly studied their flammability using a previously disclosed protocol. ${ }^{13}$ The experiments are complicated by the fact that propylene carbonate, the electrolyte solvent used in the study so far, is only flammable at very high temperatures. In order to correctly assess the role of particles on flammability, we have created similar nanoparticle hybrid electrolytes in a more flammable electrolyte solvent (EC: DEC with $\left.1 \mathrm{M} \mathrm{LiPF}_{6}\right)$. Figure 3.5 reports the results from these experiments in the form of snapshots of an electrolyte with $\mathrm{xL}=0.5$, with varying nanoparticle contents at different times following ignition. Figure 3.5(a) shows the physical appearance of the electrolyte specimen at each volume fraction before ignition. Figure 3.5(b) shows the same materials at the time of ignition, i.e. at $\mathrm{t}=0$. It can be seen that the electrolyte specimen with $\Phi=0.4$ initially catches fire, it is extinguished within one second of ignition; whereas the specimen with $\Phi=0.5$ is highly fire resistant. At the same time, the neat electrolyte without any particle or the samples with lower core volume fractions burn when ignited, as also seen in Figure 
(a)
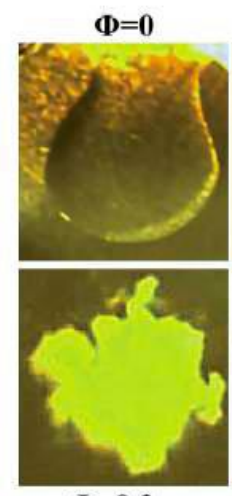

$\Phi=0.3$

(c)
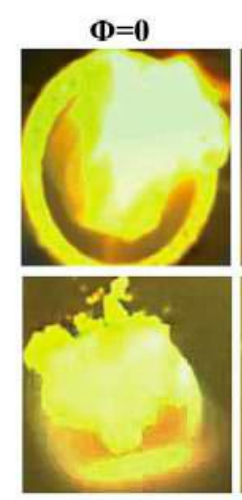

$\Phi=0.3$ $\mathbf{t}<\mathbf{0}$
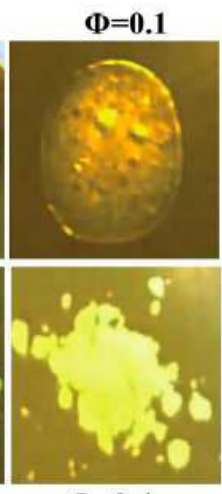

$\Phi=0.4$

$$
\mathrm{t}=\mathbf{4 s}
$$
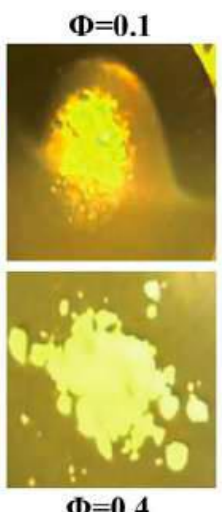

$\Phi=0.2$
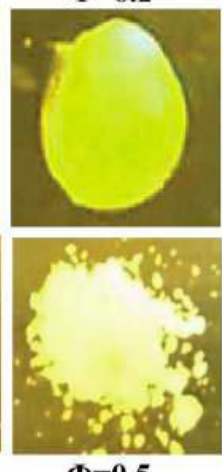

$\Phi=0.5$
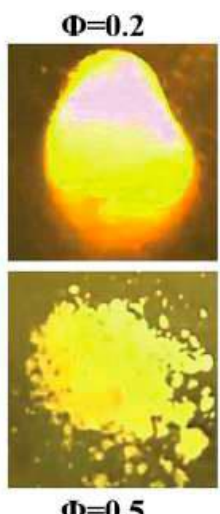

(b)
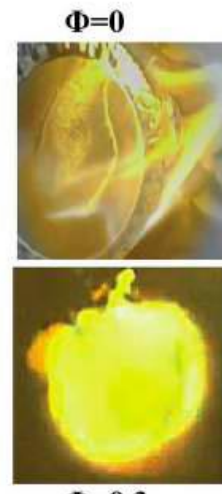

$\Phi=0.3$

(d)

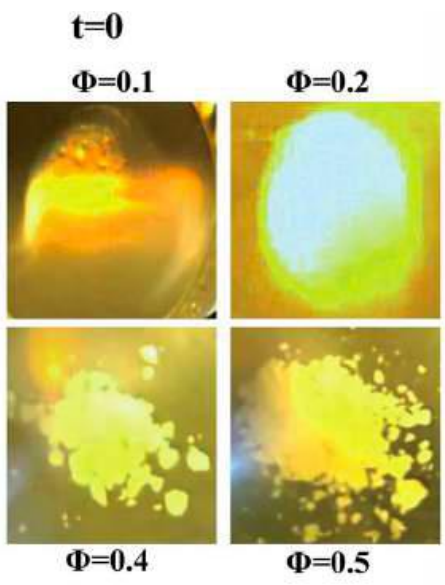

\section{Extinguishing time}

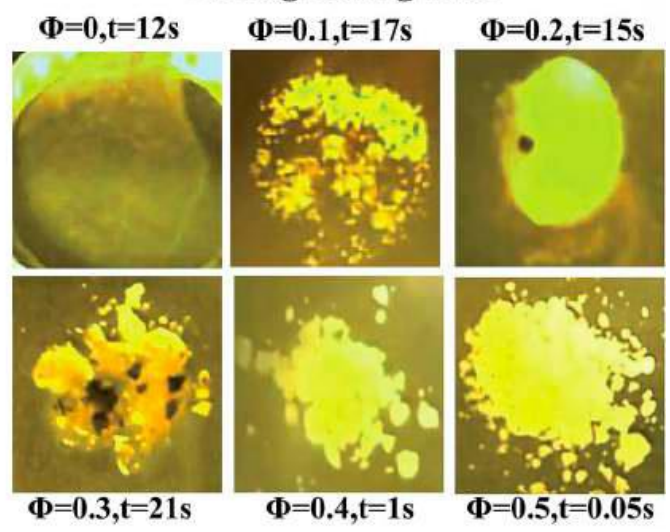

Figure 3.5: Flammability Test- Electrolyte samples with different particle volume fraction a) before ignition i.e. $t<0$. b) At ignition time i.e. $t=0$ c) after ignition i.e. at $\mathrm{t}=4 \mathrm{~s}$ d) At the extinguishing time. The binary electrolyte sample used here corresponds to $x_{L}=0.5$. 
3.5(c). Figure 3.5(d) marks the time required for the fire to completely extinguish. Among the flammable samples, the particle-free electrolyte burns out the quickest, while the electrolytes with $\Phi=0.3$ take the longest to completely extinguish; implying that the degree of flammability decreases for higher volume fractions. The bidisperse hybrid electrolytes with $\Phi=0.4$ and 0.5 therefore appear to be good candidates for future applications-oriented studies.

\subsection{Conclusion}

In summary, we have synthesized nanoparticle hybrid electrolytes based on bidisperse mixtures of PEG-functionalized silica nanoparticles in aprotic electrolyte solvents. The nanoparticles are found to be well dispersed and un-aggregated in their liquid hosts, even at high volume fractions. The conductivity of electrolytes containing nanoparticles with a bimodal size distribution is found to exhibit a pronounced maximum when the volume fraction of small and large particles is the same. This observation is attributed to the ability of large/small particles in a concentrated suspension of the opposite counterpart to produce disordering of the suspension by lowering correlation among the polydisperse particles. The tortuosity as well as activation energy values obtained from the measured conductivity show pronounced minima that correlate with the maximum conductivity, lending support to the idea that loose packing of the cores in a binary suspension is responsible for the observed enhancement in conductivity. Oscillatory shear rheology measurements show that the hybrid electrolytes transits from a jammed state to relatively unjammed and back to a jammed state when the fraction of bigger particles in the mixture is increased from 0 
to 1 . At high nanoparticle volume contents, where the effect of particle size dispersity on conductivity is greatest, little changes are seen in the elastic modulus of the materials. In particular, it is possible to create nanoparticle hybrid electrolytes with 5 MPa mechanical modulus and $1 \mathrm{mS} / \mathrm{cm}$ level ionic conductivity at room temperature. Preliminary studies of flammability show that even if the electrolyte solvent used in such bidisperse nanoparticle hybrid electrolytes is a high-flammability aprotic liquid, the electrolytes can be formulated to exhibit low flammability. Thus, we conclude that nanoparticle hybrid electrolytes created using bidisperse mixtures of PEGfunctionalized particles in conventional aprotic liquids provide an attractive platform for tuning multiple properties of contemporary interest for applications. 


\section{REFERENCES}

(1) Dunn, B.; Kamath, H.; Tarascon, J.-M. Electrical Energy Storage for the Grid: A Battery of Choices. Science 2011, 334, 928-935.

(2) Yang, P.; Tarascon, J.-M. Towards Systems Materials Engineering. Nat. Mater. 2012, 11, 560-563.

(3) Bouchet, R.; Maria, S.; Meziane, R.; Aboulaich, A.; Lienafa, L.; Bonnet, J.; Phan, T. N. T.; Bertin, D.; Gigmes, D.; Devaux, D.; et al. Efficient Electrolytes for Lithium-Metal Batteries. Nat. Mater. 2013, 12, 452-457.

(4) Tarascon, J. M.; Armand, M. Issues and Challenges Facing Rechargeable Lithium Batteries. Nature 2001, 414, 359-367.

(5) Dresselhaus, M. S.; Thomas, I. L. Alternative Energy Technologies. Nature $2001,414,332-337$.

(6) Armand, M.; Tarascon, J.-M. Building Better Batteries. Nature 2008, 451, $652-657$.

(7) Zhou, M.; Cai, T.; Pu, F.; Chen, H.; Wang, Z.; Zhang, H.; Guan, S. Graphene/carbon-Coated Si Nanoparticle Hybrids as High-Performance Anode Materials for Li-Ion Batteries. ACS Appl. Mater. Interfaces 2013, 5, 3449-3455.

(8) Goodenough, J. B.; Kim, Y. Challenges for Rechargeable Li Batteries $\dagger$. Chem. Mater. 2010, 22, 587-603.

(9) Srivastava, S.; Schaefer, J. L.; Yang, Z.; Tu, Z.; Archer, L. a. 25Th Anniversary Article: Polymer-Particle Composites: Phase Stability and Applications in Electrochemical Energy Storage. Adv. Mater. 2014, 26, 201-234. 
(10) Croce, F.; Sacchetti, S.; Scrosati, B. Advanced, Lithium Batteries Based on High-Performance Composite Polymer Electrolytes. J. Power Sources 2006, 162, 685689.

(11) Bruce, P. G.; Scrosati, B.; Tarascon, J.-M. Nanomaterials for Rechargeable Lithium Batteries. Angew. Chem. Int. Ed. Engl. 2008, 47, 2930-2946.

(12) Croce, F.; Appetecchi, G. B.; Persi, L.; Scrosati, B. Nanocomposite Polymer Electrolytes for Lithium Batteries. Nature 1998, 394, 456-458.

(13) Kashiwagi, T.; Du, F.; Douglas, J. F.; Winey, K. I.; Harris, R. H.; Shields, J. R. Nanoparticle Networks Reduce the Flammability of Polymer Nanocomposites. Nat. Mater. 2005, 4, 928-933.

(14) Tang, C.; Hackenberg, K.; Fu, Q.; Ajayan, P. M.; Ardebili, H. High Ion Conducting Polymer Nanocomposite Electrolytes Using Hybrid Nanofillers. 2012, $1152-1156$.

(15) Croce, F.; Scrosati, B. Nanocomposite Lithium Ion Conducting Membranes. Ann. N.Y. Acad Sci. 984 2003, 207, 194-207.

(16) Liu, S.; Imanishi, N.; Zhang, T.; Hirano, a.; Takeda, Y.; Yamamoto, O.; Yang, J. Effect of Nano-Silica Filler in Polymer Electrolyte on Li Dendrite Formation in Li/poly(ethylene oxide)-Li(CF3SO2)2N/Li. J. Power Sources 2010, 195, 6847-6853. (17) Schaefer, J. L.; Yanga, D. a.; Archer, L. a. High Lithium Transference Number Electrolytes via Creation of 3-Dimensional, Charged, Nanoporous Networks from Dense Functionalized Nanoparticle Composites. Chem. Mater. 2013, 25, 834-839.

(18) Jung, S.; Kim, D. W.; Lee, S. D.; Cheong, M.; Nguyen, D. Q. Fillers for SolidState Polymer Electrolytes : Highlight. 2009, 30. 
Electrodeposition Kinetics. J. Electrochem. Soc. 2004, 151, A880.

(20) Monroe, C.; Newman, J. The Impact of Elastic Deformation on Deposition Kinetics at Lithium/Polymer Interfaces. J. Electrochem. Soc. 2005, 152, A396.

(21) Hallinan, D. T.; Mullin, S. a.; Stone, G. M.; Balsara, N. P. Lithium Metal

Stability in Batteries with Block Copolymer Electrolytes. J. Electrochem. Soc. 2013, 160, A464-A470.

(22) Tikekar, M. D.; Archer, L. a.; Koch, D. L. Stability Analysis of

Electrodeposition across a Structured Electrolyte with Immobilized Anions. J.

Electrochem. Soc. 2014, 161, A847-A855.

(23) Tu, Z.; Kambe, Y.; Lu, Y.; Archer, L. a. Nanoporous Polymer-Ceramic

Composite Electrolytes for Lithium Metal Batteries. Adv. Energy Mater. 2014, 4, n/a $-\mathrm{n} / \mathrm{a}$.

(24) Khurana, R.; Schaefer, J. L.; Archer, L. a; Coates, G. W. Suppression of Lithium Dendrite Growth Using Cross-Linked Polyethylene/poly(ethylene Oxide) Electrolytes: A New Approach for Practical Lithium-Metal Polymer Batteries. J. Am. Chem. Soc. 2014, 136, 7395-7402.

(25) Lu, Y.; Tu, Z.; Archer, L. a. Stable Lithium Electrodeposition in Liquid and Nanoporous Solid Electrolytes. Nat. Mater. 2014, 13, 961-969.

(26) Stone, G. M.; Mullin, S. a.; Teran, a. a.; Hallinan, D. T.; Minor, a. M.; Hexemer, a.; Balsara, N. P. Resolution of the Modulus versus Adhesion Dilemma in Solid Polymer Electrolytes for Rechargeable Lithium Metal Batteries. J. Electrochem. Soc. 2012, 159, A222-A227. 
(27) Gurevitch, I.; Buonsanti, R.; Teran, a. a.; Gludovatz, B.; Ritchie, R. O.;

Cabana, J.; Balsara, N. P. Nanocomposites of Titanium Dioxide and Polystyrene-

Poly(ethylene Oxide) Block Copolymer as Solid-State Electrolytes for Lithium Metal Batteries. J. Electrochem. Soc. 2013, 160, A1611-A1617.

(28) Liu, S.; Wang, H.; Imanishi, N.; Zhang, T.; Hirano, a.; Takeda, Y.; Yamamoto, O.; Yang, J. Effect of Co-Doping Nano-Silica Filler and N-Methyl-N-

Propylpiperidinium Bis(trifluoromethanesulfonyl)imide into Polymer Electrolyte on Li Dendrite Formation in Li/poly(ethylene Oxide)-Li(CF3SO2)2N/Li. J. Power Sources 2011, 196, 7681-7686.

(29) Baker, G. L.; Colsons, S. Composite Polymer Electrolytes Using Fumed Silica Fillers : Rheology and Ionic Conductivity. 1994, 2359-2363.

(30) Singh, M.; Odusanya, O.; Wilmes, G. M.; Eitouni, H. B.; Gomez, E. D.; Patel, A. J.; Chen, V. L.; Park, M. J.; Fragouli, P.; Iatrou, H.; et al. Effect of Molecular Weight on the Mechanical and Electrical Properties of Block Copolymer Electrolytes. 2007, 4578-4585.

(31) Weston, J. E.; Steele, B. C. H. Effects Of Inert Fillers On The Mechanical And Electrochemical Properties Of Lithium Salt-Poly ( Ethylene Oxide ) Polymer Electrolytes _ I B. 1982, 7, 75-79.

(32) Nugent, J. L.; Moganty, S. S.; Archer, L. a. Nanoscale Organic Hybrid Electrolytes. Adv. Mater. 2010, 22, 3677-3680.

(33) Schaefer, J. L.; Moganty, S. S.; Yanga, D. a.; Archer, L. a. Nanoporous Hybrid Electrolytes. J. Mater. Chem. 2011, 21, 10094. 
(34) Gowneni, S.; Ramanjaneyulu, K.; Basak, P.; Division, P. C.; Technology, C.; Institutes, N.; Energy, S.; Pradesh, A. Polymer-Nanocomposite Brush-like Architectures as an All-Solid Electrolyte Matrix. ACS Nano 2014, 8, 11409-11424. Agarwal, P.; Qi, H.; Archer, L. a. The Ages in a Self-Suspended Nanoparticle Liquid. Nano Lett. 2010, 10, 111-115.

(36) Agarwal, P.; Srivastava, S.; Archer, L. a. Thermal Jamming of a Colloidal Glass. Phys. Rev. Lett. 2011, 107, 268302.

(37) Agarwal, P.; Kim, S. a.; Archer, L. a. Crowded, Confined, and Frustrated: Dynamics of Molecules Tethered to Nanoparticles. Phys. Rev. Lett. 2012, 109, 258301. Mayer, C.; Zaccarelli, E.; Stiakakis, E.; Likos, C. N.; Sciortino, F.; Munam, a; Gauthier, M.; Hadjichristidis, N.; Iatrou, H.; Tartaglia, P.; et al. Asymmetric Caging in Soft Colloidal Mixtures. Nat. Mater. 2008, 7, 780-784. Zaccarelli, E.; Mayer, C.; Asteriadi, a.; Likos, C.; Sciortino, F.; Roovers, J.; Iatrou, H.; Hadjichristidis, N.; Tartaglia, P.; Löwen, H.; et al. Tailoring the Flow of Soft Glasses by Soft Additives. Phys. Rev. Lett. 2005, 95, 268301.

$$
\text { Agrawal, A.; Yu, H.; Srivastava, S.; Narayanan, S.; Lynden, A. Jamming and }
$$
Un-Jamming in Binary Soft Colloids.

(41) Zhang, Q.; Archer, L. a. Poly(ethylene oxide)/Silica Nanocomposites: Structure and Rheology. Langmuir 2002, 18, 10435-10442.

(42) Moganty, S. S.; Jayaprakash, N.; Nugent, J. L.; Shen, J.; Archer, L. a. IonicLiquid-Tethered Nanoparticles: Hybrid Electrolytes. Angew. Chemie 2010, 122, 9344-9347. 
(43) Jonscher, A. K. The "Universal” Dielectric Response. Nature 1977, 267.

(44) Glatter, O.; Kratky, O. Small Angle X-Ray Scattering; United Sta.; Academic Press: New York, 1982.

(45) Srivastava, S.; Agarwal, P.; Archer, L. a. Tethered Nanoparticle-Polymer Composites: Phase Stability and Curvature. Langmuir 2012, 28, 6276-6281.

(46) Duclot, M.; Levy, M. Salt-Polymer Complexes: Strong or Weak Electrolytes? $1996,85,149-157$.

(47) Wong, D. H. C.; Thelen, J. L.; Fu, Y.; Devaux, D.; Pandya, A. a; Battaglia, V. S.; Balsara, N. P.; DeSimone, J. M. Nonflammable Perfluoropolyether-Based Electrolytes for Lithium Batteries. Proc. Natl. Acad. Sci. U. S. A. 2014, 111, 33273331.

(48) Sentjabrskaja, T.; Babaliari, E.; Hendricks, J.; Laurati, M.; Petekidis, G.; Egelhaaf, S. U. Yielding of Binary Colloidal Glasses. Soft Matter 2013, 9, 4524. (49) Sentjabrskaja, T.; Hermes, M.; Poon, W. C. K.; Estrada, C. D.; CastanedaPriego, R.; Egelhaaf, S. U.; Laurati, M. Transient Dynamics during Stress Overshoots in Binary Colloidal Glasses. Soft Matter 2014, 10.

(50) Carman, P. C. Flow of Gases through Porous Media. New York Acad. Press 1956.

(51) Bouchet, R.; Phan, T. N. T.; Beaudoin, E.; Devaux, D.; Davidson, P.; Bertin, D.; Denoyel, R. Charge Transport in Nanostructured PS-PEO-PS Triblock Copolymer Electrolytes. 2014.

(52) Then, E. The Tortuosity Concept in Fixed and Fluidized Bed. 1993, 48, $2173-$ 2175. 
(53) Khirevich, S.; Höltzel, A.; Daneyko, A.; Seidel-Morgenstern, A.; Tallarek, U. Structure-Transport Correlation for the Diffusive Tortuosity of Bulk, Monodisperse, Random Sphere Packings. J. Chromatogr. A 2011, 1218, 6489-6497.

(54) Bouchet, R.; Denoyel, R. Influence of Molecule Size on Its Transport. 2010, $82,2668-2679$.

(55) Thorat, I. V.; Stephenson, D. E.; Zacharias, N. a.; Zaghib, K.; Harb, J. N.;

Wheeler, D. R. Quantifying Tortuosity in Porous Li-Ion Battery Materials. J. Power Sources 2009, 188, 592-600.

(56) Frith, W. J.; Strivens, T. A.; Mewis, J. Dynamic Mechanical Properties of Polymerically Stabilized Dispersions. J. Colloids Interface Sci. 1990, 139.

(57) Larson, R. G. The Structure and Rheology of Complex Fluids; 1999.

(58) Sollich, P.; Lequeux, F.; Hébraud, P.; Cates, M. Rheology of Soft Glassy

Materials. Phys. Rev. Lett. 1997, 78, 2020-2023.

(59) Sollich, P. Rheological Constitutive Equation for a Model of Soft Glassy Materials. Phys. Rev. E 1998, 58, 738-759. 


\section{APPENDIX}

Supplementary Information for Chapter 3

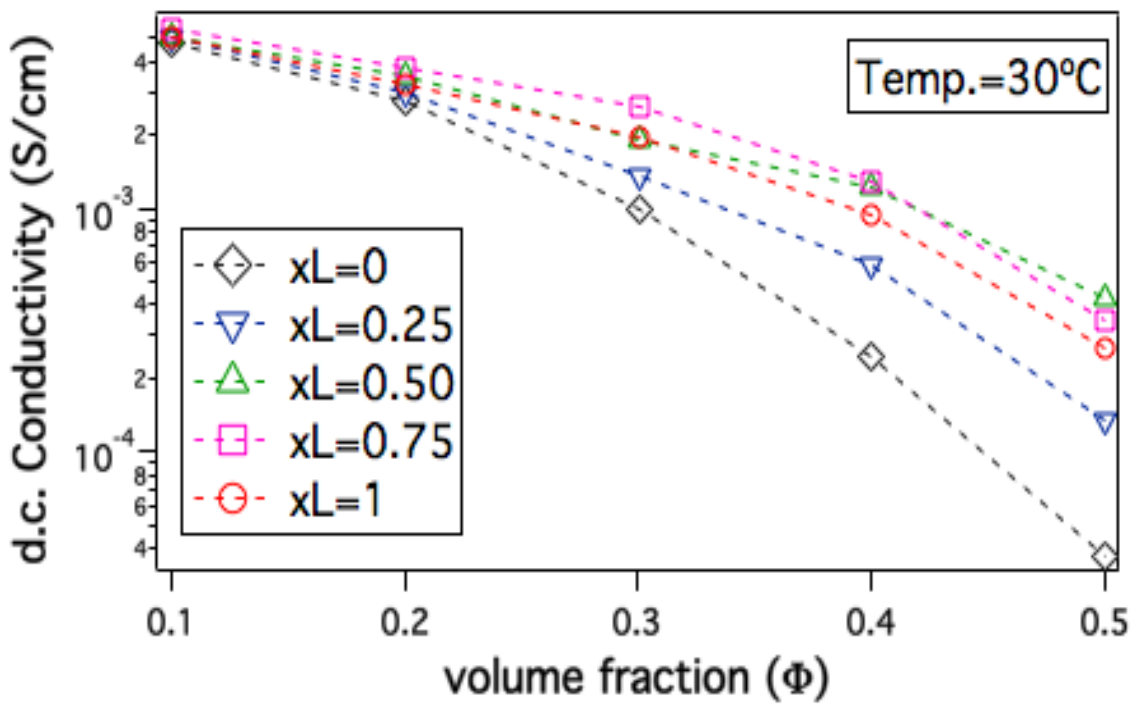

Supplementary Figure 3.2: Conductivity as a function of volume fraction of PC in a mixture of PC and Peg. It is fitted to a linear regression. The conductivity values obtained from this line at different organic content are used to normalize the actual conductivity for the resnective hvhrid samnles 


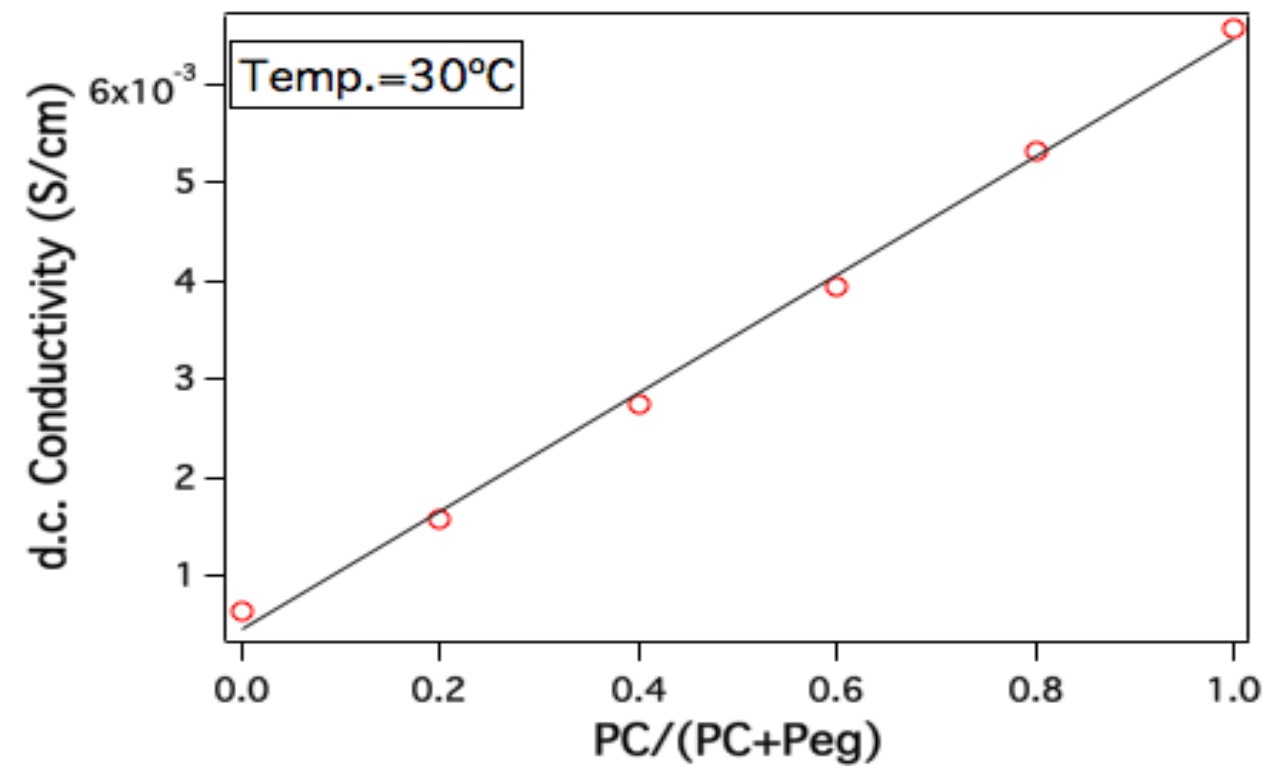

Supplementary Figure 3.1: Conductivity as a function of volume fraction for different binary ratios, while the conductivity for pure samples decrease significantly, that of binary mixtures are not as low at particle loading 

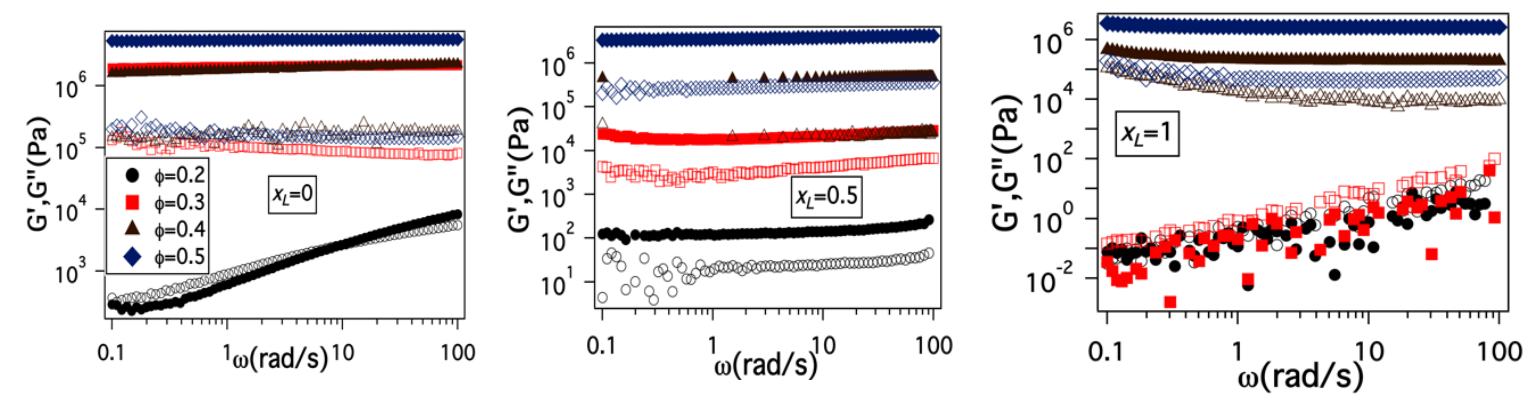

Supplementary Figure 3.3: Variation of storage modulus, $G^{\prime}$ (closed symbols) and loss modulus, G"(open symbols) with angular frequency, $\omega(\mathrm{rad} / \mathrm{s})$ at different particle volume fraction, $\Phi$ for varying $x_{L}$ values.

Supplementary Table 3.1. Relative volume fraction of $\mathrm{PC}$ with respect to $\mathrm{PEG}$, storage modulus in the limit of zero stain, $\mathrm{G}_{\gamma \rightarrow 0}^{\prime}$; DC ionic conductivity at $30^{\circ} \mathrm{C}$, $\sigma_{\mathrm{DC}}$; normalized ionic conductivity with neat blend of PC-PEG, $\mathrm{S} / \mathrm{S}_{0}$; and pseudoactivation energy, $\mathrm{E}_{\mathrm{a}}$ at different values of $\Phi$ with variation in $x_{L}$. 


\begin{tabular}{|c|c|c|c|c|c|}
\hline \multicolumn{6}{|c|}{$\Phi=0.2$} \\
\hline$x_{L}$ & $\begin{array}{l}\text { Volume fraction of } \\
\mathrm{PC} /(\mathrm{PC}+\mathrm{PEG})\end{array}$ & $\mathrm{G}_{\gamma_{\rightarrow 0}}^{\prime}(\mathrm{Pa})$ & $\sigma_{D C}(\mathrm{~S} / \mathrm{cm})$ & $\mathrm{S} / \mathrm{S}_{0}(\mathrm{~S} / \mathrm{cm})$ & $\mathrm{E}_{\mathrm{a}}(\mathrm{kJ} / \mathrm{mol})$ \\
\hline 0 & 0.77 & 2170 & $2.77 \mathrm{E}-03$ & $5.41 \mathrm{E}-01$ & 4.83 \\
\hline 0.25 & 0.78 & 43.4 & $3.01 \mathrm{E}-03$ & $5.74 \mathrm{E}-01$ & 3.23 \\
\hline 0.50 & 0.83 & 187.14 & $3.49 \mathrm{E}-03$ & $6.36 \mathrm{E}-01$ & 2.95 \\
\hline 0.75 & 0.87 & 1160.83 & $3.81 \mathrm{E}-03$ & $6.64 \mathrm{E}-01$ & 3.20 \\
\hline 1.0 & 0.91 & 0.44 & $3.25 \mathrm{E}-03$ & $5.43 \mathrm{E}-01$ & 3.32 \\
\hline \multicolumn{6}{|c|}{$\Phi=0.3$} \\
\hline 0 & 0.61 & $2.3 \mathrm{E}+06$ & $6.81 \mathrm{E}-04$ & $2.38 \mathrm{E}-01$ & 6.04 \\
\hline 0.25 & 0.63 & $2.7 \mathrm{E}+04$ & $1.06 \mathrm{E}-03$ & $3.18 \mathrm{E}-01$ & 5.07 \\
\hline 0.50 & 0.69 & $6.7 \mathrm{E}+04$ & $1.72 \mathrm{E}-03$ & $4.12 \mathrm{E}-01$ & 3.25 \\
\hline 0.75 & 0.75 & $2.88 \mathrm{E}+04$ & $2.60 \mathrm{E}-03$ & $5.17 \mathrm{E}-01$ & 4.17 \\
\hline 1.0 & 0.85 & 0.65 & $1.94 \mathrm{E}-03$ & $3.45 \mathrm{E}-01$ & 4.87 \\
\hline \multicolumn{6}{|c|}{$\Phi=0.4$} \\
\hline 0 & 0.33 & $2.6 \mathrm{E}+06$ & $1.57 \mathrm{E}-04$ & $9.89 \mathrm{E}-02$ & 7.0 \\
\hline 0.25 & 0.44 & $8.5 \mathrm{E}+05$ & $5.31 \mathrm{E}-04$ & $1.87 \mathrm{E}-01$ & 6.37 \\
\hline 0.50 & 0.55 & $4.3 \mathrm{E}+05$ & $1.11 \mathrm{E}-03$ & $3.16 \mathrm{E}-01$ & 3.75 \\
\hline 0.75 & 0.66 & $1.4 \mathrm{E}+05$ & $1.49 \mathrm{E}-03$ & $2.89 \mathrm{E}-01$ & 4.61 \\
\hline 1.0 & 0.77 & $8.3 E+04$ & $9.29 \mathrm{E}-04$ & $1.81 \mathrm{E}-01$ & 6.73 \\
\hline \multicolumn{6}{|c|}{$\Phi=0.5$} \\
\hline 0 & 0.10 & $6.1 \mathrm{E}+06$ & $2.93 \mathrm{E}-05$ & $3.32 \mathrm{E}-02$ & 7.33 \\
\hline 0.25 & 0.14 & $1.2 \mathrm{E}+06$ & $1.34 \mathrm{E}-04$ & $1.01 \mathrm{E}-02$ & 7.11 \\
\hline 0.50 & 0.28 & $4.5 \mathrm{E}+06$ & $4.24 \mathrm{E}-04$ & $1.92 \mathrm{E}-01$ & 4.18 \\
\hline 0.75 & 0.43 & $1.6 \mathrm{E}+06$ & $3.44 \mathrm{E}-04$ & $1.12 \mathrm{E}-01$ & 5.51 \\
\hline 1.0 & 0.65 & $2.8 \mathrm{E}+06$ & $2.65 \mathrm{E}-04$ & $5.97 \mathrm{E}-02$ & 7.93 \\
\hline
\end{tabular}


CHAPTER 4

HYBRID HAIRY NANOPARTICLE ELECTROLYTES STABILIZE

LITHIUM METAL BATTERIES 


\subsection{Abstract}

Rechargeable batteries comprising an energetic metal (e.g. Li, Na, Al) at the anode provide unparalleled opportunities for increasing the energy stored in batteries either on a per unit mass or volume basis. A major problem that has hindered development of such batteries for the last three decades concerns the electrochemical and mechanical instability of the interface between an energetic metal and an ion conducting organic liquid electrolyte. This study reports that hybrid electrolytes created by blending low volatility liquids with a bi-disperse mixture of hairy nanoparticles provide multiple attractive attributes for engineering electrolytes that are stable in the presence of reactive metals and at high charge potentials. Specifically, we report that such hybrid electrolytes exhibit exceptionally high voltage stability $(>7 \mathrm{~V})$ over extended times; protect the Li metal anode by forming a particle-rich coating on the electrode that allows stable-long term cycling of the anode at high columbic efficiency; and manifest low bulk and interfacial resistance at room temperature that enables stable cycling of $\mathrm{Li} / \mathrm{LiFePO}_{4}$ half cells at a $\mathrm{C} / 3$ rate. We also investigate connections between particle curvature and ion transport in the bulk and at interfaces in such bi-disperse hybrid electrolytes.

\subsection{Introduction}

A rechargeable battery that uses metallic lithium as the anode is among the most sought-after technologies for portable storage of electrical energy. Such batteries are attractive for multiple reasons. First, lithium has the lowest redox potential (-3.04V vs. Standard Hydrogen Electrode (SHE)); Second, lithium has a low gravimetric density 
$\left(0.534 \mathrm{gm} / \mathrm{cm}^{3}\right)$ and high theoretical capacity $(3860 \mathrm{mAh} / \mathrm{gm}) \cdot{ }^{1-3}$ Third, because the anode is lithium, the cathode in such lithium metal batteries (LMBs) can be an unlithiated material, such as sulfur, oxygen, or carbon dioxide/oxygen mixtures, which opens up opportunities for batteries with very high specific energies (SE), relative to today's Li-ion technology (e.g. $\mathrm{SE}_{\mathrm{Li}-\mathrm{ion}}=0.15 \mathrm{kWh} / \mathrm{kg} ; \mathrm{SE}_{\mathrm{Li}-\mathrm{S}}=2.5 \mathrm{kWh} / \mathrm{kg} ; \mathrm{SE}_{\mathrm{Li}-\mathrm{O} 2 / \mathrm{CO} 2}$ $=10.5 \mathrm{kWh} / \mathrm{kg}$ ). Four decades of focused research aimed at creating LMBs that live up to the promise of this technology has revealed multiple major problems associated with the reactivity of the metal with liquid electrolytes and the stability of $\mathrm{Li}$ electrodeposition at the anode during cell recharge.

During normal battery operation exposed surfaces of a Li metal electrode react with the electrolyte to form a passivation layer loosely termed the solid electrolyte interface (SEI), which ideally would prevent further side reactions between the Li electrode and electrolyte. In practice, the SEI formed on a Li metal surface breaks and reforms due to the expansion and compression of lithium, during plating (charge) and stripping (discharge), resulting in continuous consumption of electrolyte. ${ }^{4}$ The lifetime and cyclability of a lithium metal battery is therefore now understood to depend on the ability to create a mechanically and electrochemically robust SEI layer, which also allows for relatively fast transport of $\mathrm{Li}$-ions across the electrolyte/electrode interface. After extensive studies, Aurbach et al. ${ }^{5}$ in 2002 concluded that none of the aprotic organic electrolyte solvents in current use is compatible with a lithium metal anode because all react with $\mathrm{Li}$ to form unstable SEI layers at the high current densities required for practical battery operation. Researchers have pursued several approaches 
for stabilizing the SEI on a Li metal anode. Chemical electrolyte additives, such as vinylene carbonate ( $\mathrm{VC})$, that react preferentially with Li metal to form cross-linked polymers have been used as sacrificial agents to create mechanically robust passivating layers on $\mathrm{Li}$ that also offer interfacial ionic conductivities comparable to a bulk liquid electrolyte. ${ }^{6-8}$ More recently, a larger number of electrolyte additives, including $\mathrm{LiBOB}^{9}, \mathrm{LiNO}_{3}{ }^{10}, \mathrm{LiF}^{11,12}$, and sultones ${ }^{13,14}$ have been shown to produce SEI compositions that not only limits contact between the electrolyte solvent and Limetal, but which allow the SEI layer to retain its mechanical integrity during charge and discharge cycles. Methods of introducing such stabilizing components indirectly in the SEI using specific solvents, e.g. Fluoroethylene Carbonate ${ }^{15}$, or salts (e.g. binary LiTFSI-LiFSI ${ }^{16}$ or excess LiFSI $^{1}$ ) in the electrolyte have also been reported. In a recent departure from these approaches, Cui et al. ${ }^{4}$ showed that a thin film comprising of hollow carbon nanospheres on the lithium surface provides a robust artificial SEI layer that improved columbic efficiency of a Li metal anode to over $99 \%$. Similar results have been observed with coatings of Boron Nitride ${ }^{17}$ and Polyacrylonitile ${ }^{18}$ on Li metal. Problems involving parasitic reaction between $\mathrm{Li}$ anodes and liquid electrolytes are exacerbated under conditions of uneven/dendritic electrodeposition of the metal during cell recharge because such deposition increases the contact surface area between the electrolyte and anode, promoting formation of new SEI after every charge cycle. Modifying ion transport in the electrolyte via single ion conducting species $^{19,20}$ or at the electrolyte/electrode interface by means of halide salts ${ }^{12,21}$ have been shown to prevent growth of dendritic structures during charging. Nanostructured 
separators with high modulus components have also been reported to yield flatter, more compact deposition. ${ }^{7,12,22-25}$

Weston and Steele ${ }^{26}$ in 1982 were to our knowledge among the first to propose using nanocomposites, comprised of nanoparticle fillers in a liquid or polymer based electrolyte, as electrolytes in rechargeable batteries. Since then there have been myriad studies that have established the benefits of such nanocomposite electrolytes for making portable, leakage-free, non-flammable batteries. ${ }^{24,27,28}$ High modulus inorganic fillers have also been successfully utilized in the past to suppress rough electrodeposition. ${ }^{24}$ In this respect, surface modification of such fillers by special ionically active species like $\mathrm{PEO}^{29}$, anionic groups ${ }^{20}$, ionic liquids (IL) ${ }^{30,31}$ have been illustrated as particularly effective in stabilizing electrodeposition of $\mathrm{Li}$ as a result of ion transport modifications. Korf et al. ${ }^{32}$, for example, recently used silica nano-fillers covalently grafted with the ionic liquid 1-methyl-1-propylpiperidinium bis(trifluoromethanesulfone) imide in a liquid propylene carbonate (PC) electrolyte in symmetric Li/Li cells and, interestingly, on postmortem analysis of the Li electrodes following several cycles of plating and stripping found that the regions where nanoparticles adsorb to the Li electrodes exhibited significantly smoother morphology compared to those where nanoparticles are absent. This finding is important because it is counter to what one would expect if the insulating $\mathrm{SiO}_{2}$ particles are assumed to retard ion transport to the electrode it also opens up opportunities for more targeted use of nanocomposites as interfacial stabilizers and as building blocks for creating artificial SEI layers on battery electrodes. 
Unfortunately, previous reports on nanocomposite electrolytes suggest that their ionic conductivity are too low at high particle volume fractions to realize these beneficial effects under ambient conditions. ${ }^{33,34}$ Very recently we showed that this difficulty can be overcome by deliberately introducing size polydispersity to suspensions of hairy nanoparticles in liquid electrolyte hosts. ${ }^{35}$ Herein, we build on these findings to create nanocomposite SEI layers on Li metal electrodes and to carry out an in-depth exploration of their transport and interfacial properties. We find that a SEI layer enriched with a polydisperse, nanoparticle-rich electrolyte imparts novel interfacial and transport behaviors at the electrode/electrolyte interface of a LMB and may also be used to enhance the high-voltage stability of aprotic liquid electrolytes.

\subsection{Materials and Methods}

\subsubsection{Synthesis}

Trimethoxysilane functionalized polyethylene glycol methyl ether (PEG, MW 500g/mol, Gelest chemicals) were grafted to silica nanoparticles with size $10 \mathrm{~nm}$ (Ludox, SM-30, Sigma Aldrich) and 25nm (Ludox, TM-50, Sigma Aldrich) using a previously described method. ${ }^{57}$ The particles were purified by performing dialysis using a snake skin membrane to remove an unattached PEG chains, followed by repeated centrifugation using ethanol-hexane as solvent-non-solvent to remove any residual PEG from the material. The particles were then dried at $60^{\circ} \mathrm{C}$ for 24 hours in a convection oven, followed by vacuum drying for 48 hours. Thermo Gravimetric Analysis (TGA) was used to estimate the residual inorganic content to compute the 
grafting densities (number of chains per unit surface area) which were evaluated to be $\Sigma \sim 1.3$ chains $/ \mathrm{nm}^{2}$ and $\Sigma \sim 1.5$ chains $/ \mathrm{nm}^{2}$ for $10 \mathrm{~nm}$ and $25 \mathrm{~nm}$ particles respectively. Hybrid electrolytes were prepared in Argon filled glove box by dispersing the hair nanoparticles in propylene carbonate (PC, Sigma Aldrich) at various core volume fractions $\phi$ ranging from 0.2 to 0.4 . At each $\phi$, the relative fraction of larger particles added was varied which is given by the parameter $x_{L}=\phi_{L} / \phi .1 \mathrm{M}$ of bis(trifluoromethanesulfoneimide) (LiTFSI, Sigma Aldrich) salt was added to the resultant solution of hybrid silica nanoparticles in $\mathrm{PC}$ to create $\mathrm{SiO}_{2}-\mathrm{PEG} / \mathrm{PC}$ hybrid electrolytes.

\subsubsection{Characterization}

The dispersion and structure of hybrid particles was determined from Transmission Electron Microscopy (TEM) and Small Angle X-ray Scattering (SAXS) measurements. For TEM measurements, dilute suspensions of the hybrid electrolyte dissolved in chloroform were dropped on copper grids, with subsequent solvent evaporation and annealing at $70^{\circ} \mathrm{C}$ for 48 hours.

SAXS measurements were performed at D-1 beamline of Cornell High Energy Synchrotron Source (CHESS) using a point-collimated X-ray beam source. The scattered x-ray intensity from the hybrid electrolyte is measured with the variation in the wave-vector $q$ and can be given as a function of particle form factor $\mathrm{P}(q, D)$ and structure factor $\mathrm{S}(q \phi, D)$

$$
\mathrm{I}(q, \phi, D)=\Phi \Delta \rho_{\mathrm{e}} \mathrm{VP}(q, D) \mathrm{S}(q, \phi, D)
$$


Where, $\mathrm{V}$ and $\Delta \rho_{\mathrm{e}}$ is the volume of a single particle core, and electron density contrast between the particle and the surrounding medium respectively. The form factor $\mathrm{P}(q, D)$ gives the information about the size and shape of a single particle.

The glass transition temperature of the electrolytes was determined from Differential Scanning Calorimetric (DSC, Q2000 TA instruments) measurements.

\subsubsection{Electrochemical Measurements}

The ionic conductivities of the electrolytes were measured at room temperature using a Novocontrol Broadband Dielectric spectrometer with a frequency range of $0.1-3 \times 10^{6}$ Hz. The DC conductivities were obtained from the plateau of real part of the conductivity versus frequency curve. ${ }^{74}$ The molar diffusivities were obtained using the Nernst-Einstein Equation:

$$
D=\frac{\sigma k_{B} T}{C_{0} q^{2}}
$$

where, $\sigma\left(\mathrm{S}-\mathrm{m}^{2} / \mathrm{mol}\right)$ is the D.C. molar ionic conductivity, $\mathrm{k}_{\mathrm{B}}(\mathrm{J} / \mathrm{K})$ is the Boltzmann constant, $\mathrm{T}(\mathrm{K})$ is the temperature, $\mathrm{C}_{0}\left(\# / \mathrm{m}^{3}\right)$ is the ion concentration and $\mathrm{q}(\mathrm{C})$ is the charge of the diffusing entity.

The AC impedance measurements were performed on a Solortron Electrochemical Impedance Spectrometer using a symmetric coin cell with lithium metal as the electrodes and $\mathrm{SiO}_{2}-\mathrm{PEG} / \mathrm{PC}$ as the electrolyte at different $\phi$ and $x_{L}$ at ambient temperature. The frequency was varied from $1 \mathrm{MHz}$ to $0.1 \mathrm{~Hz}$ at amplitude of $50 \mathrm{mV}$. 
Error bars in the final impedances were evaluated as deviations from running each measurement for 12 symmetric cells.

Electrochemical stability voltage window was determined by linear scan voltammetry measurements performed at a scan rate $1 \mathrm{mV} / \mathrm{s}$ between $-0.2 \mathrm{~V}$ and $6.5 \mathrm{~V}$. The stability was further confirmed by performing floating-point test measurements where the voltage was stepped up to $7 \mathrm{~V}$, with each voltage step of $0.5 \mathrm{~V}$ maintained for 5 hours.

\subsubsection{Analyzing the Columbic Efficiency}

Li| electrolyte |Stainless steel 2032 type coin cells were assembled in an Argon-filled Glove Box. Control liquid electrolyte comprising 1M LiTFSi-PC with 1wt\% Lithium Nitrate $\left(\mathrm{LiNO}_{3}\right)$ and $2 \mathrm{vol} \%$ vinylene carbonate and a standard Celgrad separator were compared against hybrid nanoparticles dispersed in the same electrolyte with $\phi=0.3$ and $x_{L}=0.5$. In both cases, prior to the measurements, cells were conditioned by cycling them between 0 to $0.5 \mathrm{~V}$ for 10 cycles, to ensure the formation of a stable SEI layer on the electrodes as shown previously. ${ }^{7}$ To characterize the columbic efficiency, the conditioned cells were first discharged at a constant current density of $0.25 \mathrm{~mA} / \mathrm{cm}^{2}$ for 2 hours to transfer an amount of lithium corresponding to $0.50 \mathrm{mAh} / \mathrm{cm}^{2}$ of charge from the Li electrode to the stainless steel electrode. The amount of charge recovered in the reverse cycle was then recorded where the cell was charged back to $0.5 \mathrm{~V}$ at the same current density, and the fractional recovery between the two cycled was used to determine the columbic efficiency. 
The lithium electrode surface was characterized using a similar procedure for hybrid electrolytes with $\phi=0.2$ and $x_{L}=0.5$ without any additives. The coin cells were disassembled inside the glove box and the lithium foil was repeatedly washed with pure PC to dissolve any excess of particles on the surface. Scanning Electron Microscopy (SEM) measurements were performed on the lithium foil to image the electrode surface.

\subsubsection{Cell lifetime study}

$\mathrm{LiFePO}_{4}$ slurry was prepared by mixing the active material, super-P carbon and PVDF binder in 8:1:1 ratio in a ball-mill in presence of NMP solvent. The slurry was casted on an aluminium sheet using a doctor blade to prepared cathode sheets of $0.5 \mathrm{mAh} / \mathrm{cm}^{2}$ surface capacity. Half-cells were made in glovebox with Lithium foil as anode, Lithium Iron Phosphate as cathode and the hybrid binary nanocomposite as electrolyte. The batteries were cycled at constant current between the voltage limits of $2.5 \mathrm{~V}$ and $3.8 \mathrm{~V}$.

\subsection{Results and Discussion}

\subsubsection{Physical Characterization and Ion Transport}

Aggregation and phase separation are among the most important hurdles that have limited application of nanoparticle-polymer composites in many fields of technology. ${ }^{33,36-40}$ Surface functionalization of particles with short polymer chains is a widely practiced technique for creating uniform dispersion of particles in polymers and liquid hosts. ${ }^{41-49}$ Here we utilize previous chemistry to densely graft short 
polyethylene glycol methyl ether (PEGME, MW 500g/mol) chains to $\mathrm{SiO}_{2}$ nanoparticles $\left(d_{\mathrm{p}}=10 \mathrm{~nm}\right.$ and $\left.25 \mathrm{~nm}\right)$ and dispersed these particles and their binary mixtures in Propylene Carbonate (PC)-based liquid electrolytes. The dispersion state of hairy nanoparticles in the resultant hybrid electrolytes was analyzed by observing their transmission electron micrographs (TEM). Figure 4.1(a) shows that irrespective of the volume ratio $\left(x_{L}\right)$ of larger $\left(d_{\mathrm{p}}=25 \mathrm{~nm}\right)$ to smaller $\left(d_{\mathrm{p}}=10 \mathrm{~nm}\right)$ particles, the particles appear as well-dispersed objects in the hybrid electrolytes. This finding is confirmed by analyzing the variation in the scattered intensity $\mathrm{I}(q)$ of X-rays from the bulk hybrid electrolytes using small-angle x-ray scattering (SAXS). Figure 1(b) reports typical $\mathrm{I}(q)$ vs $q$ data from SAXS measurements performed on a hybrid electrolyte with fixed volume fraction of nanoparticles $(f=0.3)$, but with varying fractions $x_{L}$ of the larger particles. $\mathrm{I}(q)$ is seen to exhibit a plateau for all $x_{L}$ values in the low $q$ region and a $q^{-4}$ scaling in the high $q$ region. Both of these features are known characteristics of uniformly dispersed, un-aggregated spheres. ${ }^{41,50}$

Previous studies on bi-disperse suspensions of star polymers and hairy nanoparticles have shown that introduction of a larger species to a mono-disperse suspension comprised of smaller ones results in a transition from a highly jammed glass to a weakly jammed suspension, and, for large enough size ratios, ultimately to a liquid state. $^{51-53}$ This transition has also been reported in self-suspended suspensions of bidisperse hairy particles and is thought to reflect differences in the degree of interpenetration of tethered oligomer chains produced by differences in curvature of the two different nanocore populations. ${ }^{53}$ As a consequence, tethered oligomer chains in a 

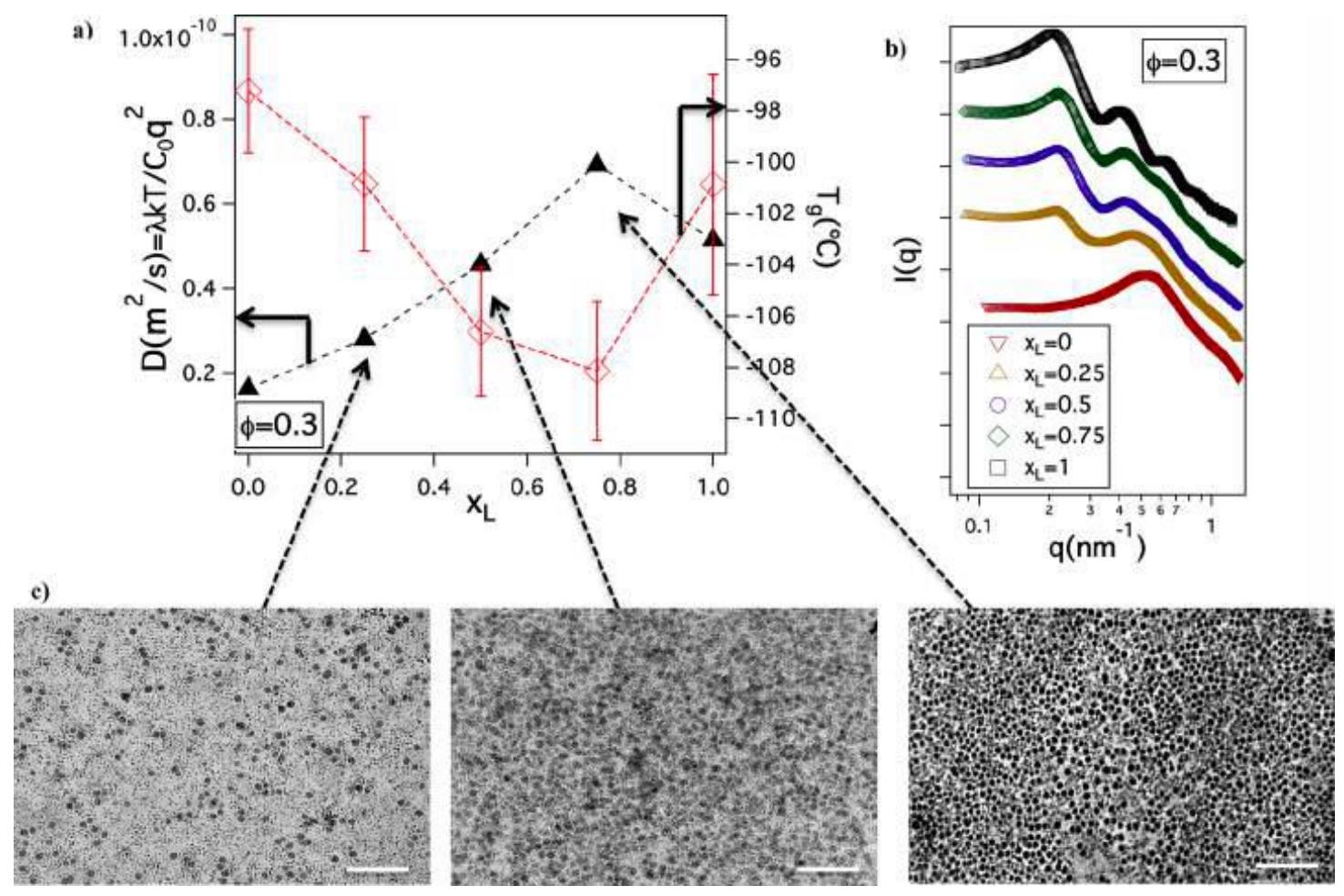

Figure 4.1 Physical Characterization: a) Variation in ionic diffusivity, D (triangles) as calculated from Nernst- Einstein equation, and glass transition temperature, $\operatorname{Tg}$ (diamonds) with fraction of larger particles, $\mathrm{xL}$. b) Intensity, I(q) as a function of wave vector $\mathrm{q}$ at $\Phi=0.3$, as obtained from SAXS measurements for different values of $\mathrm{xL}$, increasing from bottom to top. c) Transmission Electron Micrographs (TEM) for bi-disperse hybrid electrolytes at $\Phi=0.3$ for $\mathrm{xL}=0.25,0.5$ and 0.75 . The scale bar for all the images is $500 \mathrm{~nm}$. 
bi-disperse blend of hairy particles may acquire higher configurational entropy even at the same overall particle volume fraction. It has been observed previously that lithiumion transport in electrolytes containing PEO occur predominantly by local diffusion of polymer chain segments and hopping of Li-ions between ether groups on PEO chains. ${ }^{33,34}$ Figure 4.1 (a) reports the diffusivity of lithium ions obtained, via the Nernst-Einstein equation, from ionic conductivity data for a $\mathrm{SiO}_{2}-\mathrm{PEGME} / \mathrm{PC}$ hybrid electrolyte with $\phi=0.3$. The figure shows that the diffusivity is a maximum near $x_{L}=$ 0.75. This higher diffusivity can be explained in terms of the lower interpenetration and hence higher mobility of PEGME chains in the binary hairy particle mixtures; the higher mobility of PEGME chains allows Li-ions in an electrolyte to migrate at a faster rate, which results in an increase in ionic conductivity and higher ionic diffusivity in the bi-disperse hybrid electrolytes. Figure 4.1(a) also reports the glass transition temperature, $\mathrm{T}_{\mathrm{g}}$, for $\mathrm{SiO}_{2}-\mathrm{PEGME} / \mathrm{PC}$ hybrid electrolytes as a function of $x_{L}$. Consistent with the explanation of the enhanced diffusivity at $x_{L} \approx 0.75$, it is seen that $\mathrm{T}_{\mathrm{g}}$ exhibits a minimum at $x_{L}$ close to where the maximum in diffusivity is observed. A reduced $\mathrm{T}_{\mathrm{g}}$ for the bi-disperse suspension implies an increased free volume, which is consistent with our hypothesis that the tethered PEGME chains are less constrained in the bi-disperse hybrid electrolytes. Supplementary Figure 4.1 presents results for electrolytes with $\phi=0.2$ and 0.4 and shows that the observation of a maximum diffusivity at $x_{L} \approx 0.75$ and $\mathrm{T}_{\mathrm{g}}$ minimum near this value is a generic feature of these materials.

\subsubsection{Structural Factor Analysis}


Further insight about the structure of the $\mathrm{SiO}_{2}$-PEGME/PC electrolytes and about the confinement of PEGME chains can be obtained by analyzing the SAXS structure factor $\mathrm{S}(q)$ of the materials. Figure 4.2(a) compares the experimentally obtained $\mathrm{S}(q)$ with the calculated structure factor for a binary hard sphere (HS) suspension using the Percus-Yevick (PY) approximation. ${ }^{54}$ To account for the fact that a few PEGME chain segments nearest the anchor points on the particles are likely to be completely correlated with their particle substrate ${ }^{42}$ the theoretical $\mathrm{S}(q)$ approximate the particle size in terms of an effective radius, $\mathrm{a}_{\mathrm{eff}, \mathrm{i}}=\mathrm{a}_{\mathrm{i}}+\mathrm{n}_{\mathrm{m}, \mathrm{i}} \mathrm{l}_{\mathrm{m}} \cos (\theta / 2)$, such that a certain number of monomers, $\mathrm{n}_{\mathrm{m}, \mathrm{i}}$, are assumed to be part of the core. Here $\mathrm{i}=\mathrm{s}, 1$ for smaller particles and larger particles respectively, $a_{i}$ is the original radius of the particle, $l_{\mathrm{m}}=0.35 \mathrm{~nm}$ which is the monomer length for PEO, and $\theta=68^{\circ}$ which is the bond tetrahedral angle. In analyzing the experimental data using the PY approximation, $\mathrm{n}_{\mathrm{m}, \mathrm{i}}$ was treated as an unknown variable and its value adjusted so that the peak positions of $\mathrm{S}(q)$ computed for a binary HS suspension model coincide with those seen in the experimental $\mathrm{S}(q)$. It can be observed from Figure 4.2(a) that whereas the $\mathrm{S}(q)$ peak amplitudes and positions estimated from PY-HS analysis are in good agreement with the experimental values for electrolytes with low $f$, the first $\mathrm{S}(q)$ maxima are much higher than the predicted ones in electrolytes with high $f$. A higher first-peak height for $\mathrm{S}(q)$ implies a stronger inter-particle correlation, which results in the hybrid particles from enhanced interaction of tethered corona chains. ${ }^{55-57}$ Supplementary Figure 4.2 reports the corresponding results for electrolytes with a range of particle size polydispersities, $x_{L}=0,0.25,0.75$ and 1 . The discrepancy in the calculated and measured peak heights, especially the first peak, is clearly greatest in systems with the largest asymmetry in 

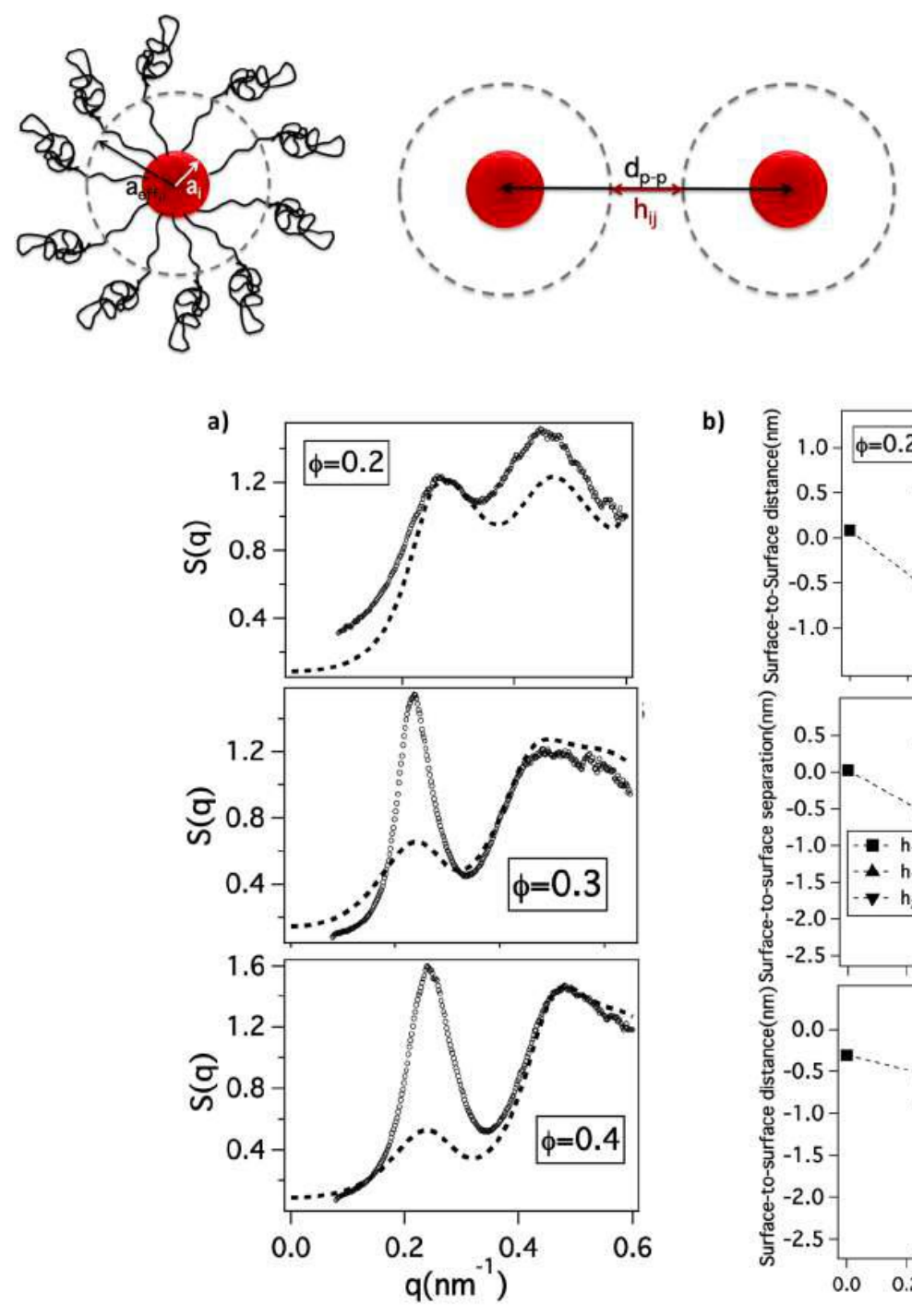

b)

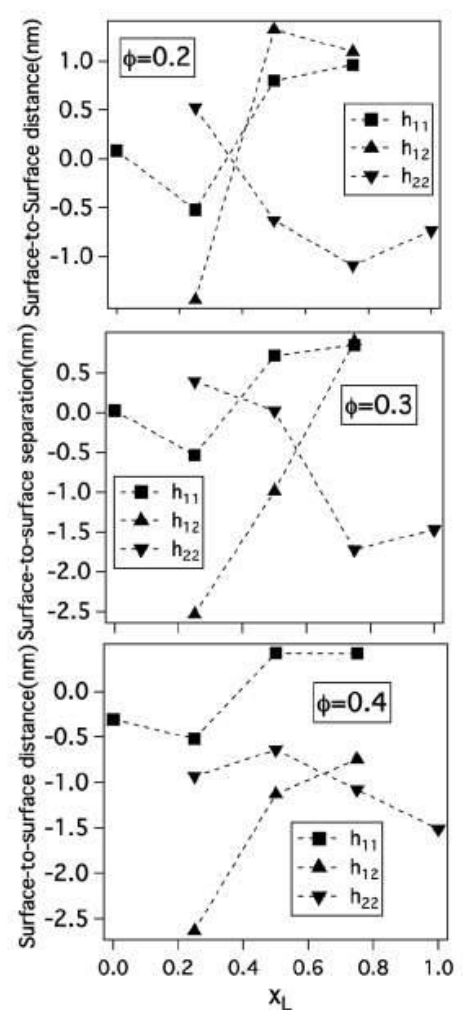

Figure 4.2 Structure Factor: a) Evolution of Structure factor, S(q) with the wave vector $\mathrm{q}$ for different volume fractions, $\Phi=0.2,0.3$ and 0.4 at $\mathrm{xL}=0.5$. The open symbols are the experimentally obtained $\mathrm{S}(\mathrm{q})$ values and the dashed lines are predictions from binary HS model for the same systems. The schematic above illustrates the effective particle radius, which includes some fraction of monomers as part of the core. b) Surface-to-surface distances, h11, h12 and h22 for the same volume fractions as a function of $\mathrm{xL}$. The graphics illustrates the two scenarios for surface-surface distances which will be positive when there is no overlap and negative when the polymer chains have significant overlap. 
nanoparticle size. The discrepancy is thought to reflect enhanced interaction between tethered oligomer chains in the hybrid particle electrolytes, which are not included in the PY-HS analysis.

Additional insights about the structure of the electrolytes can be obtained from the PYHS predictions by resolving the structure factor into its three components- $S_{11}, S_{12}$ and $\mathrm{S}_{22}$ where 1 and 2 denote smaller and larger particles respectively (see Supplementary Figure 4.3). From the first peak positions of each of these components, three different inter-particle distances can be obtained viz., $d_{p-p, 11}, d_{p-p, 12}$ and $d_{p-p, 22}$. Figure $4.2(b)$ show the variation in surface-to-surface distance for $\phi=0.2,0.3$ and 0.4 , as a function of fraction of larger particles $x_{L}$. The surface-to-surface distance is calculated by subtracting the effective core diameter from the inter-particle distance i.e. $h_{\mathrm{ij}}=\mathrm{d}_{\mathrm{p}-\mathrm{p}, \mathrm{ij}}$ $2 a_{\text {eff }, \mathrm{ij}}$, where $\mathrm{i}, \mathrm{j}=1$ and 2 and $\mathrm{i}=\mathrm{j}$ when analyzing the distance between the same species. It can be observed that for certain values of $f$ and $x_{L}, \mathrm{~h}_{\mathrm{ij}}$ is negative which implies that the corona chains overlap or inter-penetrate for those regions. It can be seen that as $f$ increases, as expected $h_{\mathrm{ij}}$ assumes higher negative values, implying higher degree of interpenetration and thereby confinement for tethered chains at higher volume fractions. For any core volume fraction $f$, it can also be observed that initial addition of larger particles to a pure mono-disperse suspension of smaller ones, first decreases the distance between the smaller particles after which the $h_{11}$ value increases with further addition of larger particles. For the same $x_{L}$ values, however, $\mathrm{h}_{22}$ is larger for a smaller fraction of large particles and decreases with further increase in $x_{L}$. This implies that for smaller $x_{L}$ values, the larger particles confine the smaller particles and 
increases the distance between themselves, and subsequent increase in $x_{L}$ values leads to an increase in the average distance between smaller particles. This interpretation is confirmed from the $\mathrm{h}_{12}$ values, which always increase with increase in $x_{L}$, meaning that the distance between a smaller and a larger particle always increases. Also, for the pure mono-disperse suspensions of smaller and larger particles the $h_{i j}$ values are either negative or close to zero, which implies that the tethered corona chains inter-penetrate much more in these suspensions, while $h_{\mathrm{ij}}$ values for bi-disperse suspensions are either positive or less negative than their mono-disperse counterparts, which indicates less interpenetration and hence reduced confinement of corona chain motions in bidisperse suspension. This observation is in agreement with the trends seen in the diffusivity and $\mathrm{T}_{\mathrm{g}}$ values, which confirms the hypothesis that in a bi-disperse suspension the tethered PEO chains are less confined or have higher mobility which subsequently leads to a higher ionic conductivity values for these hybrid electrolytes as observed previously. ${ }^{35}$

\subsubsection{Variation of interfacial resistance}

Figure 4.3(a)-(c) report Nyquist plots obtained using electrochemical impedance spectroscopy measurements to evaluate the impedance in bulk electrolyte and at the electrode-electrolyte interface in symmetric $\mathrm{Li} / \mathrm{Li}$ cells. The experimental plot is fitted using the circuit model shown schematically in Figure 4.3, where $\mathrm{R}_{\mathrm{b}}$ corresponds to the resistance in bulk electrolyte, $\mathrm{R}_{\text {int }}$ represents interfacial resistance to ion migration at the electrode-electrolyte interface, $\mathrm{CPE}$ is the constant phase element capacitance near the electrode surface and $\mathrm{W}_{\mathrm{d}}$ is the Warburg diffusion element. ${ }^{23}$ Figure $4.3(\mathrm{~d})$ 

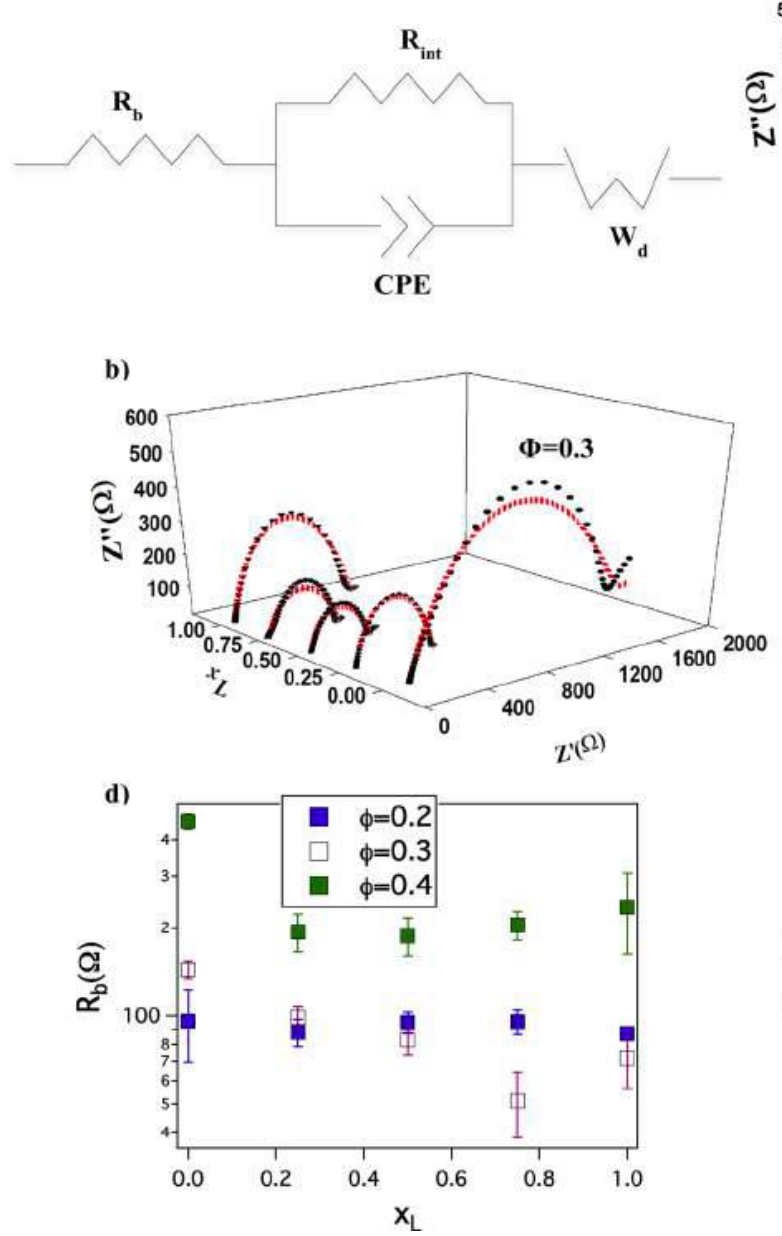
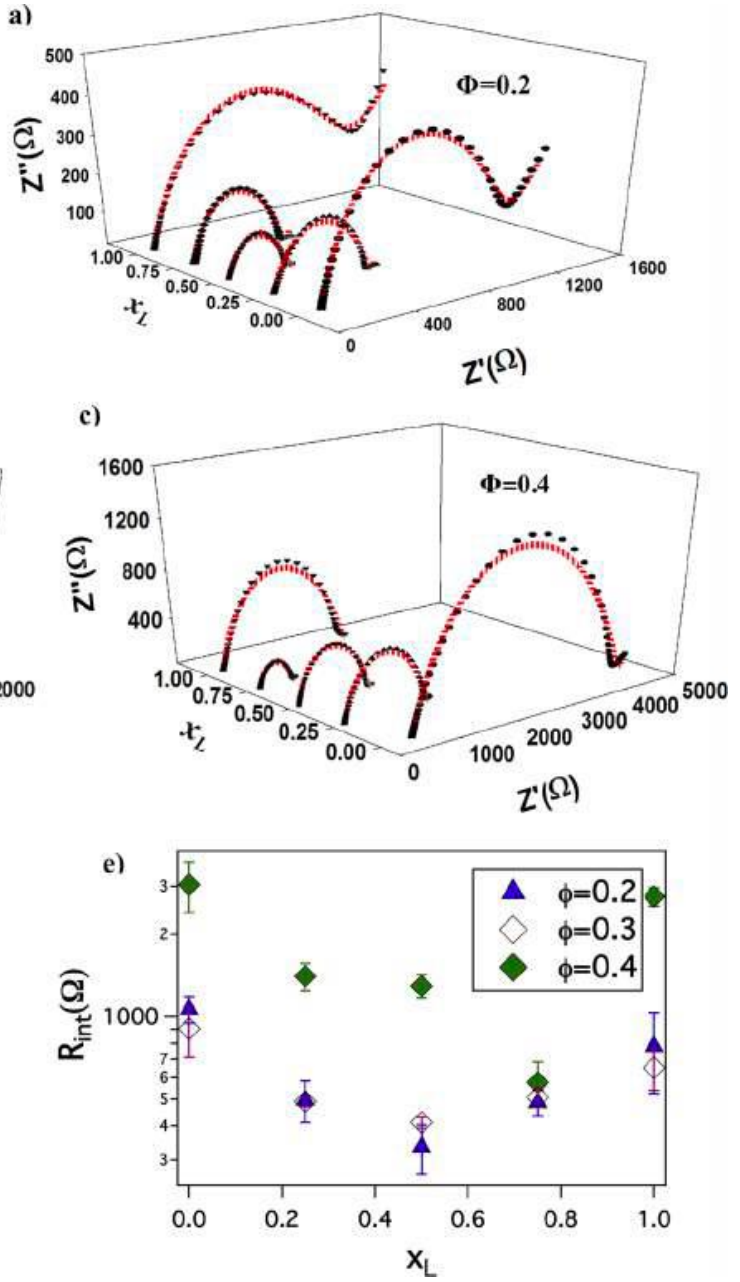

Figure 4.3 Electrochemical Characterization: Impedance spectra for electrolytes with core volume fractions a) $\Phi=0.2$, b) 0.3 , and c) 0.4 measured at different fractions of larger particles added, xL. The black symbols are experimental data and the red lines are fit to the equivalent circuit model as shown. d) Bulk resistance, $\mathrm{Rb}$ and e) Interfacial resistance, Ri measured at different core volume fractions as a function of $x L$. 
and (e) summarize the magnitude of $\mathrm{R}_{\mathrm{b}}$ and $\mathrm{R}_{\mathrm{int}}$ as a function of $x_{L}$ at different core volume fractions $f$, respectively. While $\mathrm{R}_{\mathrm{b}}$ does not show significant variation with $x_{L}$, $\mathrm{R}_{\text {int }}$ decreases significantly and exhibits similar trends to those discussed earlier for the ionic diffusivity and $T_{g}$. We postulate that the relative invariance in $R_{b}$ occurs because of adsorption of significant fraction of particles from the bulk electrolyte to the electrode surface, which results in bulk resistance values similar to those of liquid electrolytes with low nanoparticle loadings where ion transport is dominated by diffusion of electrolyte solvent-ion associates. This would then also lead to variations in interfacial resistance similar to trends seen in diffusivity due to the presence of particles.

\subsubsection{Surface Characterization of $\mathrm{Li}$ anode}

In order to evaluate the hypothesis that surface adsorption of $\mathrm{SiO}_{2}-\mathrm{PEGME}$ particles is the source of the weaker dependence of $\mathrm{R}_{\mathrm{b}}$ and $\mathrm{R}_{\text {int }}$ on $x_{L}$, we performed Scanning Electron Microscopy (SEM) analysis of the lithium electrodes harvested from symmetric $\mathrm{Li} / \mathrm{Li}$ cells following galvanostatic cycling at a low current density of $0.03 \mathrm{~mA} / \mathrm{cm}^{2}$ for 10 cycles. Prior to SEM analysis, the electrodes were vigorously washed with pure PC to remove any loosely bound material. Figure 4.4(a) shows a representative SEM image of the $\mathrm{Li}$ metal electrode for small and higher magnifications. It is apparent that a dense layer of bi-disperse particles of size $10 \mathrm{~nm}$ and $25 \mathrm{~nm}$ are adsorbed to the surface of the electrode. The hairy particles appear to form a continuous, protective film on the electrode surface. 
It has been observed in a typical Lithium ion battery (LIB) that during charging or discharging, oppositely charged ions accumulate near the surface of respective electrodes. ${ }^{58-60}$ The electrochemical capacitance (EC) near the electrode surface can be determined from the relaxation frequency $f_{0}$ deduced from the Nyquist plot $^{61-63}$ and its relationship, $2 \pi f_{0} \mathrm{R}_{\mathrm{int}} \mathrm{C}_{\mathrm{d}}=1$, to the interfacial resistance and capacitance. ${ }^{64}$ The calculated capacitance can be written in terms of the dielectric constant at the interface as, $\mathrm{C}_{\mathrm{d}}=\varepsilon \varepsilon_{0} \mathrm{~A} / l$ where, $\varepsilon$ is the dielectric permittivity of the material at the interface, $\varepsilon_{0}$ is the vacuum permittivity, $\mathrm{A}$ is the surface area of the electrode and $l$ is the thickness of the capacitive layer. Figure 4.4(b) shows the variation in the electrochemical capacitance $\mathrm{C}_{\mathrm{d}}$ as a function of $x_{L}$ for $f=0.3$. It is apparent that hybrid electrolytes based on bi-disperse $\mathrm{SiO}_{2}$-PEGME nanoparticles display higher interfacial capacitance than the mono-disperse particle suspension. This observation can be explained either in terms of an increase in the effective dielectric constant or a reduction in the SEI layer thickness as $x_{L}$ increases. Based on the earlier observation that the particles form a stable, dense film on the Li anode, we conclude that $l$ is likely to be a fixed number of the order of average particle diameter in the electrolytes. Inserting this value in the above formula, allows us to estimate $\varepsilon$ and, from it, an apparent Debye screening length $\left(I=\sqrt{\frac{e e_{o} R T}{2 F^{2} C_{0}}}\right)$, which is also shown as a function of $x_{L}$ in Fig. $4.4 \mathrm{~b}$. Here $\mathrm{F}$ is Faraday's constant and $\mathrm{C}_{0}$ is the molar concentration of salt in the electrolyte. For a pure $\mathrm{PC}$ electrolyte containing $1 \mathrm{M}$ salt, $\lambda \approx 0.26 \mathrm{~nm}$, which is comparable to the highest values obtained in Fig. 4b, but somewhat larger than the values estimated for the electrolytes with $x_{L}=0$ or $x_{L}=1$, which is consistent with expectations for the 
a)

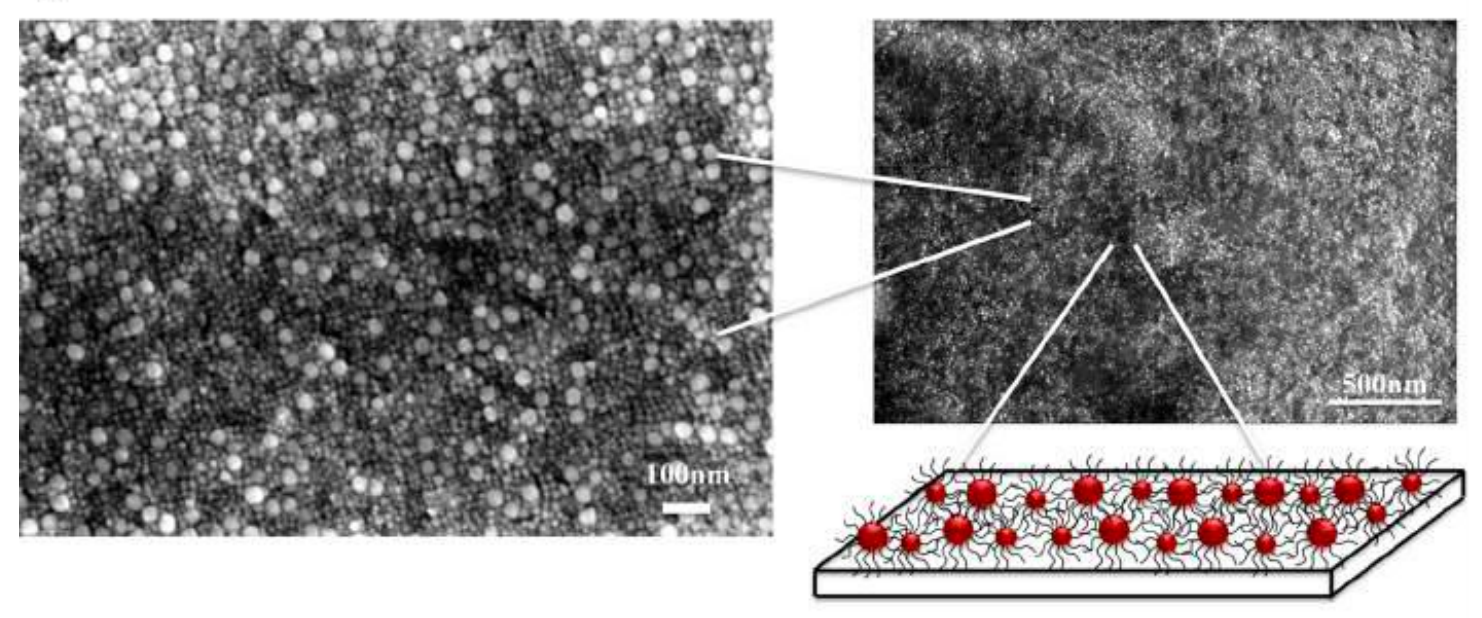

b)

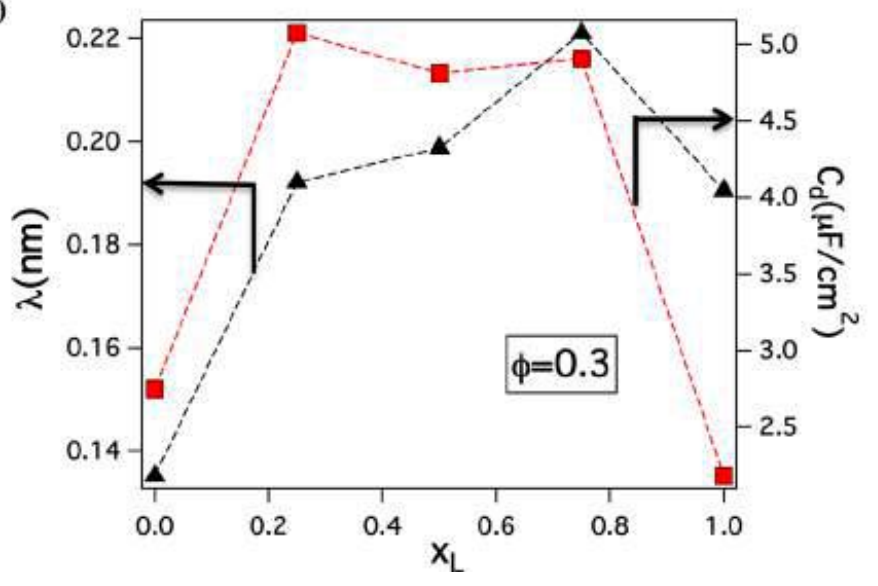

Figure 4.4 Surface Characterization: a) Scanning Electron Micrographs (SEM) for lithium electrode surface after the symmetric cell was cycled for 10 cycles at $0.03 \mathrm{~mA} / \mathrm{cm} 2$ with the bi-disperse hybrid electrolyte. The schematic illustrates the electrode surface as observed in SEM, where the particles form protective layer on the electrode surface. b) Electrical double layer, $\lambda$ (triangles) and electrochemical capacitance per unit area, $\mathrm{Cd}$ (squares) for $\phi=0.3$ as a function of $\mathrm{xL}$. 
factor of 5 to 10 difference in the reported dielectric constants for PEG and $\mathrm{PC}^{36,65-68}$. One explanation of the high $\mathrm{C}_{\mathrm{d}}$ and / values apparent at intermediate $x_{L}$ is that they reflect greater access of PC to the SEI, which would increase $\varepsilon$. It is important to note however, that even the largest / values are more than an order of magnitude lower than 1, meaning that the electric field gradients in the SEI layer formed by a hairy particle coating of Li are substantially smaller than in a pure electrolyte, which would lower both the diffusive and electro-convective flux of anions to the Li metal surface. The $\mathrm{Li}^{+}$transference number $\left(\mathrm{t}_{\mathrm{Li}}{ }^{+}\right)$was determined by the Bruce-Scrosati method ${ }^{69,70}$ (see Supplementary Information) which was found to be approximately 0.32 for this electrolyte, in agreement with previously reported transference numbers for PEG based electrolytes. ${ }^{34}$ The presence of particles in the SEI therefore does not influence the lithium ion transference number, which is consistent with expectations based on the theoretical predictions of Jacob ${ }^{71}$, where it was found that the transference number has a strong dependence of the ratio of pore size to double layer thickness.

\subsubsection{Enhanced electrochemical stability of nanocomposites}

If the Li metal in a LMB is unprotected, the electrolyte continuously reacts with the anode to form insulating products, which increase the interfacial resistance of the battery with time. The interface stability in symmetric cells containing $\mathrm{SiO}_{2}-$ PEGME/PC hybrid electrolyte with $f=0.3$ and $x_{L}=0.5$ were evaluated using impedance measurements as a function of time for a period of 2 months. It can be seen from Figure 4.5(a) that the time-resolved impedance plots overlay well onto each other, indicating that the particle-rich SEI layer imparts long-term enhanced chemical 
stability to Li-metal/electrolyte interface. This enhanced stability is consistent with the existence of a passivating particle film on the $\mathrm{Li}$ anode as deduced from the earlier SEM experiments. Figure 4.5(b) reports results from linear-sweep voltammetry and electrochemical floating-point measurements on lithium cells containing the hybrid electrolytes. The linear sweep voltagram reveals that at higher particle volume fractions (e.g. $f=0.3$ and $f=0.4$ ) and for $x_{L}=0.5$ the hybrid electrolytes are stable up to $6.5 \mathrm{~V}$ vs. $\mathrm{Li}^{2} / \mathrm{Li}^{+}$. In contrast, electrolytes containing lower fractions $f=0.2$ of particles (inset of Figure 4.5(b)) become unstable at around 4V. The initial peak at $0.2 \mathrm{~V}$ in all the measurements is the cathodic peak associated with the $\mathrm{Li} / \mathrm{Li}^{+}$ reaction. ${ }^{7,12}$ Its presence for all of the materials studied means that this process is not compromised in the hybrid electrolytes. Similar trends were also observed in the floating-point tests in Figure 4.5(c), where hybrid electrolytes containing higher nanoparticle volume fractions are seen to manifest negligible leakage currents even after exposure to voltages as high as $7.5 \mathrm{~V}$ for 5 hours. In contrast, the particle-free PC electrolyte or a blend of un-tethered PEG in PC already exhibits high leakage currents at $5 \mathrm{~V}$. Together, these results clearly show that $\mathrm{SiO}_{2}-\mathrm{PEGME} / \mathrm{PC}$ hybrid electrolytes with high $\mathrm{SiO}_{2}$ are exceptionally stable, in agreement with previous reports. ${ }^{72}$

\subsubsection{Analyzing galvanostatic performance}

As a final assessment of the ability of a nanoparticle-rich SEI to stabilize Li metal anodes against reaction with liquid electrolytes, we analyzed the columbic efficiency obtained in galvanostatic measurements employing Li/stainless steel electrodes. In these experiments, a fixed amount of $\mathrm{Li}^{+}$ions is stripped from lithium metal and 

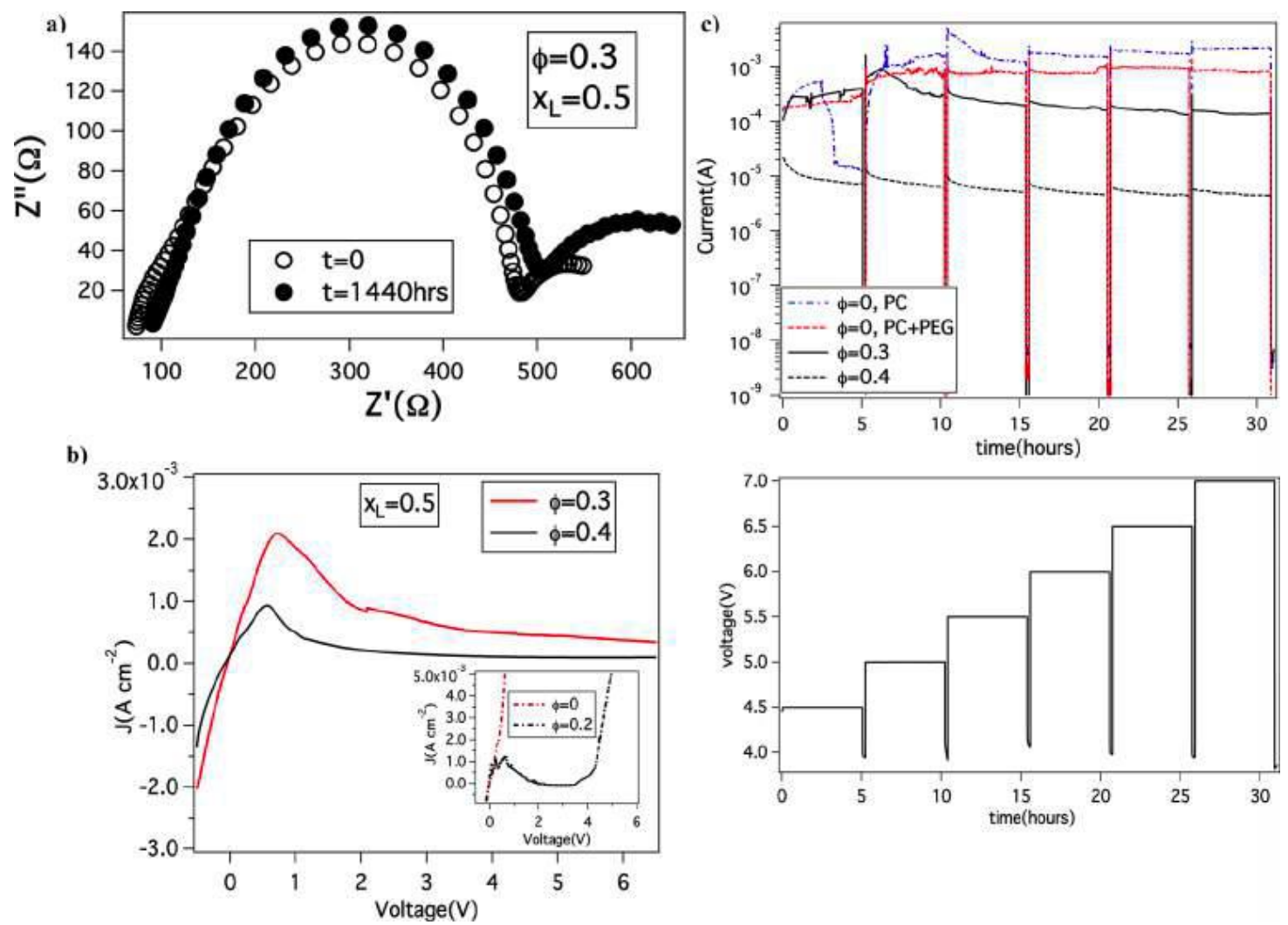

Figure 5 Electrochemical Stability: a) Initial impedance spectra (open symbols) for a symmetric cell with hybrid particle electrolyte at $\Phi=0.3$ and $x L=0.5$ compared with the spectra at $\mathrm{t}=1440$ hours (closed symbols). b) Variation in faradic current density, $\mathrm{J}$ as a function of voltage as measured from Linear sweep voltammetry measurements for $\mathrm{xL}=0.5$ compared for $\Phi=0.3$ (red line) and $\Phi=0.4$ (black line). Inset shows the measurements for neat PC (red line) and $\Phi=0.2$ (black line). c) Leak current measured as a function of time for different steps of voltage as measured in a floating-point test. Profiles for symmetric cell with hybrid electrolyte at $\Phi=0.3$ (black solid line) and $\Phi=0.4$ (black dashed line) are compare against neat electrolytes with PC (blue line) and with PEG and PC (red line) blended in the same ratio as that for hybrid particle electrolyte with $\Phi=0.3$. 
deposited to the stainless-steel substrate and plated back in successive runs. By measuring the capacity ratio for the strip and plate segments of the cycle, the columbic efficiency (CE) of the cell can be quantified. It has been reported that PC-based electrolytes spontaneously form an insulating and unstable SEI layer on $\mathrm{Li}$, yielding a low $\mathrm{CE}$ of $\sim 70 \%{ }^{73}$ It has also been shown that the $\mathrm{CE}$ for PC can be improved by employing $\mathrm{VC}$ and $\mathrm{LiNO}_{3}$ as electrolyte additives. ${ }^{6,7}$ To study the effect of particles on CE of Li metal electrodes, we utilized a PC-based electrolyte reinforced with $1 \%$ $\mathrm{LiNO}_{3}$ and $2 \% \mathrm{VC}$ as a control. It can be seen from Figure 4.6(a) that although both the control and hybrid PC electrolytes exhibit high $\mathrm{CE}$, the control electrolyte is unable to maintain the improvements beyond $\sim 25$ cycles, implying that the $\mathrm{VC}$ and $\mathrm{LiNO}_{3}$ are consumed. In contrast, the $\mathrm{SiO}_{2}-\mathrm{PEGME} / \mathrm{PC}$ hybrid electrolyte displays high $\mathrm{CE}$ for at least 80 cycles. In the control experiment, despite use of additives, the PC electrolyte decomposes over cycles due to its low stability window and uneven electrodeposition which exposes higher effective surface area of the electrode for the electrolyte to react, thus the additive fails to maintain high columbic efficiency over $\sim 25$ cycles. On the other hand, as previously inferred from electrochemical stability tests and electron microscopy, the $\mathrm{SiO}_{2}$-PEGDME form a protective monolayer on the electrode surface that prevents constant exposure of liquid electrolyte with lithium surface and breakdown of the additive rich SEI. This synergistic effect of the additives and $\mathrm{SiO}_{2}$-PEGDME helps in maintaining high columbic efficiency large number of cycles compared to the control without particles. Figure 4.6(b) shows the roomtemperature performance of the binary hybrid nanocomposite electrolytes in a $\mathrm{Li} / \mathrm{LiFePO}_{4}$ half-cell in which a $\mathrm{SiO}_{2}-\mathrm{PEGME} / \mathrm{PC}$ hybrid with $f=0.3$ and $x_{L}=0.5$ is 
a)
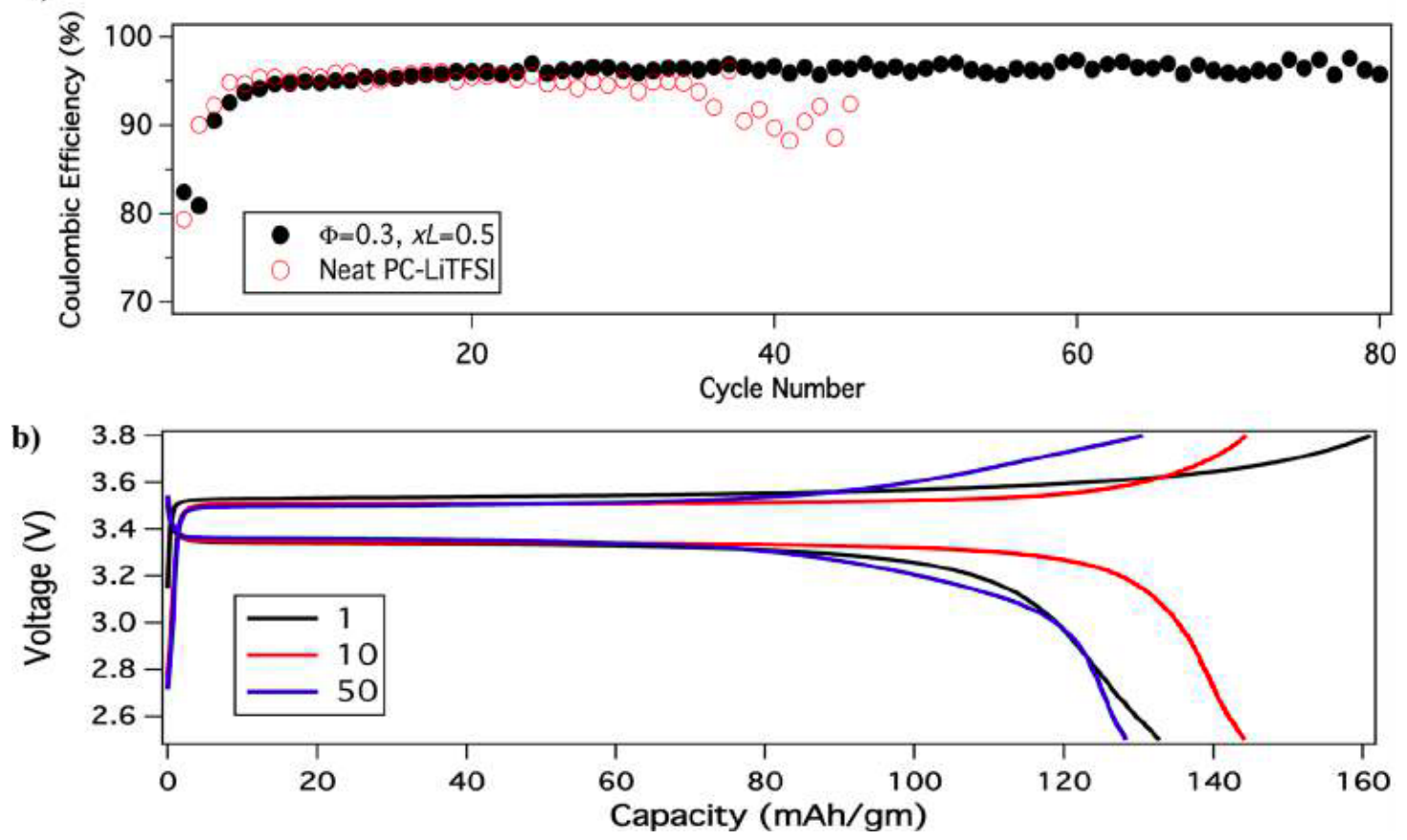

Figure 6 Galvanostatic performance: a) Comparison of columbic efficiency as a function of cycle number for neat PC electrolyte and hybrid particle electrolyte with $\Phi=0.3$ and $\mathrm{xL}=0.5$ for $\mathrm{Li} \mid$ electrolyte|Stainless Steel configuration. Measurements were performed at a current density of $0.25 \mathrm{~mA} / \mathrm{cm} 2$. b) Charge-discharge profiles for Li/LFP half-cell containing hybrid particle electrolyte with $\Phi=0.3$ and $x L=0.5$ at a fixed rate of $\mathrm{C} / 3$ in the initial, 10th and 50th cycle. 
employed. The figure reports the voltage profiles (voltage versus capacity) for the $1^{\text {st }}$, $10^{\text {th }}$ and $50^{\text {th }}$ cycles of charge and discharge. The low efficiency in the first cycle is associated with irreversible reactions that help in forming a stable passivation layer on the $\mathrm{Li}$ anode. The results in Figure 4.6(b) shows that even after 50 cycles the cells maintain a high discharge capacity of $\sim 130 \mathrm{mAh} / \mathrm{gm}$ at $\mathrm{C} / 3$ rate.

\subsection{Conclusion}

In conclusion, we have studied hybrid electrolytes created by blending a low-volatility carbonate (PC) with a bi-disperse mixture of $\mathrm{SiO}_{2}$-PEGDME nanoparticles. We show that hybrid electrolytes based on bi-disperse hairy nanoparticles can be designed to provide multiple attractive features, including exceptional high voltage stability ( $>$ $7 \mathrm{~V})$ over extended times; protection of a Li metal anode that allows stable-long term cycling of the anode at high columbic efficiency; and low bulk and interfacial resistance at room temperature that enables stable cycling of $\mathrm{Li} / \mathrm{LiFePO}_{4}$ half cells at a $\mathrm{C} / 3$ rate.

The origin of the enhanced ion mobility in bi-disperse hybrid electrolytes is shown by means of DSC measurements, to be the reduction in $\mathrm{T}_{\mathrm{g}}$ of the tethered PEG chains and more fundamentally from SAXS analysis, to arise from an increase in mobility of tethered PEGME chains due to lower levels of chain interpenetration caused by differences in curvature of the larger and smaller particles in the bi-disperse electrolytes. By means of interfacial impedance measurements, it was further shown that the interfacial mobility of hybrid electrolytes at a Li metal electrode is a much 
stronger function of polydispersity than the bulk resistance. We hypothesize that this difference arises from the spontaneous adsorption of particles to the high energy Li metal electrode to form a dense protective film. Post-mortem analysis of the $\mathrm{Li}$ electrode surface following galvanostatic cycling shows that a dense particle-rich film accumulates at the Li metal surface, which increases the interfacial capacitance and appears to be very effective in limiting access of liquid electrolyte to the Li metal surface without compromising interfacial transport of $\mathrm{Li}$-ions. In contrast to control, particle-free electrolytes, hybrid electrolytes based on bi-disperse hairy nanoparticles are shown to enable Li/stainless steel cells with high columbic efficiency for at least 80 cycles and $\mathrm{Li} / \mathrm{LiFePO}_{4}$ cells with high discharge capacity in extended cycling at room-temperature. 


\section{REFERENCES}

(1) Qian, J.; Henderson, W. a; Xu, W.; Bhattacharya, P.; Engelhard, M.; Borodin, O.; Zhang, J.-G. High Rate and Stable Cycling of Lithium Metal Anode. Nat. Commun. 2015, 6, 6362.

(2) Xu, W.; Wang, J.; Ding, F.; Chen, X.; Nasybulin, E.; Zhang, Y.; Zhang, J.-G. Lithium Metal Anodes for Rechargeable Batteries. Energy Environ. Sci. 2014, $7,513$.

(3) Tu, Z.; Nath, P.; Lu, Y.; Tikekar, M. D.; Archer, L. A. Nanostructured Electrolytes for Stable Lithium Electrodeposition in Secondary Batteries. Acc. Chem. Res. 2015, acs.accounts.5b00427.

(4) Zheng, G.; Lee, S. W.; Liang, Z.; Lee, H.-W.; Yan, K.; Yao, H.; Wang, H.; Li, W.; Chu, S.; Cui, Y. Interconnected Hollow Carbon Nanospheres for Stable Lithium Metal Anodes. Nat. Nanotechnol. 2014, 9, 618-623.

(5) Aurbach, D.; Zinigrad, E.; Cohen, Y.; Teller, H. A Short Review of Failure Mechanisms of Lithium Metal and Lithiated Graphite Anodes in Liquid Electrolyte Solutions. Solid State Ionics 2002, 148, 405-416.

(6) Guo, J.; Wen, Z.; Wu, M.; Jin, J.; Liu, Y. Vinylene carbonate-LiNO3: A Hybrid Additive in Carbonic Ester Electrolytes for SEI Modification on Li Metal Anode. Electrochem. commun. 2015, 51, 59-63. 
(7) Choudhury, S.; Mangal, R.; Agrawal, A.; Archer, L. A. A Highly Reversible Room-Temperature Lithium Metal Battery Based on Crosslinked Hairy Nanoparticles. Nat. Commun. 2015, 1-9.

(8) Chen, L.; Wang, K.; Xie, X.; Xie, J. Effect of Vinylene Carbonate (VC) as Electrolyte Additive on Electrochemical Performance of Si Film Anode for Lithium Ion Batteries. J. Power Sources 2007, 174, 538-543.

(9) Xu, K.; Zhang, S.; Jow, T. R. LiBOB as Additive in LiPF[sub 6]-Based Lithium Ion Electrolytes. Electrochem. Solid-State Lett. 2005, 8, A365.

Li, W.; Yao, H.; Yan, K.; Zheng, G.; Liang, Z.; Chiang, Y.-M.; Cui, Y. The Synergetic Effect of Lithium Polysulfide and Lithium Nitrate to Prevent Lithium Dendrite Growth. Nat. Commun. 2015, 6, 7436.

(11) Choudhury, S.; Archer, L. A. Lithium Fluoride Additives for Stable Cycling of Lithium Batteries at High Current Densities. Adv. Electron. Mater. 2015, 1-6.

(12) Lu, Y.; Tu, Z.; Archer, L. A. Stable Lithium Electrodeposition in Liquid and Nanoporous Solid Electrolytes. Nat. Mater. 2014, 13, 961-969.

(13) Pires, J.; Timperman, L.; Castets, A.; Santos-pena, J.; Dumont, E.; Levasseur, S.; Dedryvere, R.; Tessier, C.; Anouti, M. Role of Propane Sultone as Additive to Improve the Performance of Lithium-Rich Cathode Material at High Potential. RSC Adv. 2015. 
(14) Li, B.; Xu, M.; Li, T.; Li, W.; Hu, S. Prop-1-Ene-1,3-Sultone as SEI Formation Additive in Propylene Carbonate-Based Electrolyte for Lithium Ion Batteries. Electrochem. commun. 2012, 17, 92-95.

(15) Etacheri, V.; Haik, O.; Goffer, Y.; Roberts, G. A.; Stefan, I. C.; Fasching, R.; Aurbach, D. Effect of Fluoroethylene Carbonate (FEC) on the Performance and Surface Chemistry of Si-Nanowire Li-Ion Battery Anodes. Langmuir 2012, 28, 965-976.

(16) Miao, R.; Yang, J.; Feng, X.; Jia, H.; Wang, J.; Nuli, Y. Novel Dual-Salts Electrolyte Solution for Dendrite-Free Lithium-Metal Based Rechargeable Batteries with High Cycle Reversibility. J. Power Sources 2014, 271, 291-297.

(17) Luo, W.; Zhou, L.; Fu, K.; Yang, Z.; Wan, J.; Manno, M.; Yao, Y.; Zhu, H.; Yang, B.; Hu, L. A Thermally Conductive Separator for Stable Li Metal Anodes. Nano Lett. 2015, 150810091733009.

(18) Liang, Z.; Zheng, G.; Liu, C.; Liu, N.; Li, W.; Yan, K.; Yao, H.; Hsu, P.-C.; Chu, S.; Cui, Y. Polymer Nanofiber-Guided Uniform Lithium Deposition for Battery Electrodes. Nano Lett. 2015, 150330172314009.

Lu, Y.; Tikekar, M.; Mohanty, R.; Hendrickson, K.; Ma, L.; Archer, L. A. Stable Cycling of Lithium Metal Batteries Using High Transference Number Electrolytes. Adv. Energy Mater. 2015, 5, n/a - n/a. 
(20) Schaefer, J. L.; Yanga, D. A.; Archer, L. A. High Lithium Transference Number Electrolytes via Creation of 3-Dimensional, Charged, Nanoporous Networks from Dense Functionalized Nanoparticle Composites. Chem. Mater. 2013, 25, 834-839.

(21) Ozhabes, Y.; Gunceler, D.; Arias, T. A. Stability and Surface Diffusion at Lithium-Electrolyte Interphases with Connections to Dendrite Suppression. arXiv 2015, 1504.05799, 1-7.

(22) Khurana, R.; Schaefer, J. L.; Archer, L. A; Coates, G. W. Suppression of Lithium Dendrite Growth Using Cross-Linked Polyethylene/poly(ethylene Oxide) Electrolytes: A New Approach for Practical Lithium-Metal Polymer Batteries. J. Am. Chem. Soc. 2014, 136, 7395-7402.

(23) Tu, Z.; Kambe, Y.; Lu, Y.; Archer, L. A. Nanoporous Polymer-Ceramic Composite Electrolytes for Lithium Metal Batteries. Adv. Energy Mater. 2014, $4, \mathrm{n} / \mathrm{a}-\mathrm{n} / \mathrm{a}$.

(24) Gurevitch, I.; Buonsanti, R.; Teran, a. a.; Gludovatz, B.; Ritchie, R. O.; Cabana, J.; Balsara, N. P. Nanocomposites of Titanium Dioxide and PolystyrenePoly(ethylene Oxide) Block Copolymer as Solid-State Electrolytes for Lithium Metal Batteries. J. Electrochem. Soc. 2013, 160, A1611-A1617. 
(25) Bouchet, R.; Maria, S.; Meziane, R.; Aboulaich, A.; Lienafa, L.; Bonnet, J.;

Phan, T. N. T.; Bertin, D.; Gigmes, D.; Devaux, D.; et al. Efficient Electrolytes for Lithium-Metal Batteries. Nat. Mater. 2013, 12, 452-457.

(26) Weston, J. E.; Steele, B. C. H. Effects of inert fillers on the mechanical and electrochemical properties of lithium salt-poly ( ethylene oxide ) polymer electrolytes_i b. Solid State Ionics 1982, 7, 75-79.

(27) Croce, F.; Scrosati, B. Nanocomposite Lithium Ion Conducting Membranes. Ann. N.Y. Acad Sci. 984 2003, 207, 194-207.

(28) Agrawal, A.; Choudhury, S.; Archer, L. A. A Highly Conductive, NonFlammable Polymer-Nanoparticle Hybrid Electrolyte. RSC Adv. 2015.

(29) Srivastava, S.; Schaefer, J. L.; Yang, Z.; Tu, Z.; Archer, L. A. 25Th Anniversary Article: Polymer-Particle Composites: Phase Stability and Applications in Electrochemical Energy Storage. Adv. Mater. 2014, 26, 201234.

(30) Lu, Y.; Korf, K.; Kambe, Y.; Tu, Z.; Archer, L. A. Ionic-Liquid-Nanoparticle Hybrid Electrolytes: Applications in Lithium Metal Batteries. Angew. Chemie 2014, 126, 498-502.

(31) Lu, Y.; Das, S. K.; Moganty, S. S.; Archer, L. A. Ionic Liquid-Nanoparticle Hybrid Electrolytes and Their Application in Secondary Lithium-Metal Batteries. Adv. Mater. 2012, 24, 4430-4435. 
(32) Korf, K. S.; Lu, Y.; Kambe, Y.; Archer, L. A. Piperidinium Tethered Nanoparticle-Hybrid Electrolyte for Lithium Metal Batteries. J. Mater. Chem. A 2014, 2, 11866-11873.

(33) Nugent, J. L.; Moganty, S. S.; Archer, L. a. Nanoscale Organic Hybrid Electrolytes. Adv. Mater. 2010, 22, 3677-3680.

(34) Schaefer, J. L.; Moganty, S. S.; Yanga, D. A.; Archer, L. A. Nanoporous Hybrid Electrolytes. J. Mater. Chem. 2011, 21, 10094.

(35) Agrawal, A.; Choudhury, S.; Archer, L. A. A Highly Conductive, NonFlammable Polymer-nanoparticle Hybrid Electrolyte. $R S C A d v .2015,5$, 20800-20809.

(36) Zhang, Q.; Archer, L. A. Poly(ethylene oxide)/Silica Nanocomposites: Structure and Rheology. Langmuir 2002, 18, 10435-10442.

(37) Moganty, S. S.; Jayaprakash, N.; Nugent, J. L.; Shen, J.; Archer, L. A. IonicLiquid-Tethered Nanoparticles: Hybrid Electrolytes. Angew. Chemie 2010, 122, 9344-9347.

(38) Hussain, F. Review Article: Polymer-Matrix Nanocomposites, Processing, Manufacturing, and Application: An Overview. J. Compos. Mater. 2006, 40, 1511-1575. 
(39) Palmqvist, A. E. C. Synthesis of Ordered Mesoporous Materials Using Surfactant Liquid Crystals or Micellar Solutions. Curr. Opin. Colloid Interface Sci. 2003, 8, 145-155.

(40) Balazs, A. C.; Emrick, T.; Russell, T. P. Nanoparticle Polymer Composites : Meet Two Small Worlds. Science (80-. ). 2013, 314, 1107-1110.

(41) Mangal, R.; Srivastava, S.; Archer, L. A. Phase Stability and Dynamics of Entangled Polymer-nanoparticle Composites. Nat. Commun. 2015, 6, 1-9.

(42) Green, D. L.; Mewis, J.; Engineering, C.; Uni, V.; Way, E.; Charlottes, V. Connecting the Wetting and Rheological Behaviors of Poly ( Dimethylsiloxane ) -Grafted Silica Spheres in Poly ( Dimethylsiloxane ) Melts. Langmuir 2006, 9546-9553.

(43) Srivastava, S.; Agarwal, P.; Archer, L. A. Tethered Nanoparticle-Polymer Composites: Phase Stability and Curvature. Langmuir 2012, 28, 6276-6281.

(44) McEwan, M.; Green, D. Rheological Impacts of Particle Softness on Wetted Polymer-Grafted Silica Nanoparticles in Polymer Melts. Soft Matter 2009, 5, 1705.

(45) Dutta, N.; Green, D. Nanoparticle Stability in Semidilute and Concentrated Polymer Solutions. Langmuir 2008, 24, 5260-5269. 
(46) Lindenblatt, G.; Schärtl, W.; Pakula, T.; Schmidt, M. Structure and Dynamics of Hairy Spherical Colloids in a Matrix of Nonentangled Linear Chains. Macromolecules 2001, 34, 1730-1736.

(47) Oh, H.; Green, P. F. Polymer Chain Dynamics and Glass Transition in Athermal Polymer/nanoparticle Mixtures. Nat. Mater. 2009, 8, 139-143.

(48) Meng, D.; Kumar, S. K.; D. Lane, J. M.; Grest, G. S. Effective Interactions between Grafted Nanoparticles in a Polymer Matrix. Soft Matter 2012, 8, 5002.

(49) Ohno, K.; Morinaga, T.; Takeno, S.; Tsujii, Y.; Fukuda, T. Suspensions of Silica Particles Grafted with Concentrated Polymer Brush: Effects of Graft Chain Length on Brush Layer Thickness and Colloidal Crystallization. Macromolecules 2007, 40, 9143-9150.

Glatter, O.; Kratky, O. Small Angle X-Ray Scattering; United Sta.; Academic Press: New York, 1982.

(51) Zaccarelli, E.; Mayer, C.; Asteriadi, A.; Likos, C.; Sciortino, F.; Roovers, J.; Iatrou, H.; Hadjichristidis, N.; Tartaglia, P.; Löwen, H.; et al. Tailoring the Flow of Soft Glasses by Soft Additives. Phys. Rev. Lett. 2005, 95, 268301.

(52) Mayer, C.; Zaccarelli, E.; Stiakakis, E.; Likos, C. N.; Sciortino, F.; Munam, A; Gauthier, M.; Hadjichristidis, N.; Iatrou, H.; Tartaglia, P.; et al. Asymmetric Caging in Soft Colloidal Mixtures. Nat. Mater. 2008, 7, 780-784. 
(53) Agrawal, A.; Yu, H.-Y.; Srivastava, S.; Choudhury, S.; Narayanan, S.; Archer, L. Dynamics and Yielding of Binary Self-Suspended Nanoparticle Fluids. Soft Matter 2015, 11, 5224-5234.

(54) Ashcroft, N. W.; Langreth, D. C. Structure of Binary Liquid Mixtures. I. Phys. Rev. 1967, 16, 685-692.

(55) Srivastava, S.; Shin, J. H.; Archer, L. a. Structure and Rheology of Nanoparticle-polymer Suspensions. Soft Matter 2012, 8, 4097.

(56) Yu, H.-Y.; Srivastava, S.; Archer, L. A; Koch, D. L. Structure Factor of Blends of Solvent-Free Nanoparticle-Organic Hybrid Materials: Density-Functional Theory and Small Angle X-Ray Scattering. Soft Matter 2014, 10, 9120-9135.

(57) Choudhury, S.; Agrawal, A.; Kim, S. A; Archer, L. A. Self-Suspended Suspensions of Covalently Grafted Hairy Nanoparticles. Langmuir 2015, 31, $3222-3231$.

Qu, D.; Qu, D.; Shi, H.; Shi, H. Studies of Activated Carbons Used in DoubleLayer Capacitors. J. Power Sources 1998, 74, 99-107.

(59) Shi, H. Activated Carbons and Double Layer Capacitance. Electrochim. Acta 1996, 41, 1633-1639.

(60) Choi, N. S.; Chen, Z.; Freunberger, S. a.; Ji, X.; Sun, Y. K.; Amine, K.; Yushin, G.; Nazar, L. F.; Cho, J.; Bruce, P. G. Challenges Facing Lithium Batteries and 
Electrical Double-Layer Capacitors. Angew. Chemie - Int. Ed. 2012, 51, 999410024.

(61) Flandrois, S.; Simon, B. Carbon Materials for Lithium-Ion Rechargeable Batteries. Carbon N. Y. 1999, 37, 165-180.

(62) Shiraishi, S.; Kurihara, H.; Tsubota, H.; Oya, A.; Soneda, Y.; Yamada, Y. Electric Double Layer Capacitance of Highly Porous Carbon Derived from Lithium Metal and Polytetrafluoroethylene. Electrochem. Solid-State Lett. 2001, 4, A5-A8.

(63) Largeot, C.; Portet, C.; Chmiola, J.; Taberna, P. L.; Gogotsi, Y.; Simon, P. Relation between the Ion Size and Pore Size for an Electric Double-Layer Capacitor. J. Am. Chem. Soc. 2008, 130, 2730-2731.

(64) Lanfredi, S.; Rodrigues, a. C. M. Impedance Spectroscopy Study of the Electrical Conductivity and Dielectric Constant. J. Appl. Phys. 1999, 86, 2215.

(65) Payne, R.; Theodorou, I. E. Dielectric Properties and Relaxation in Ethylene Carbonate and Propylene Carbonate. J. Phys. Chem. 1972, 76, 2892-2900.

(66) Simeral, L.; Ameyib, R. L.; Amey, L. Dielectric Properties of Liquid Propylene Carbonate '". J. Phys. Chem. 1970, 74, 1968-1971.

(67) Sengwa, R. J.; Kaur, K.; Chaudhary, R. Dielectric Properties of Low Molecular Weight Poly ( Ethylene Glycol ) S. Polym Int. 2000, 608, 599-608. 
(68) Schneider, U.; Lunkenheimer, P.; Brand, R.; Loidl, A. Broadband Dielectric Spectroscopy on Glass-Forming Propylene Carbonate. Phys. Rev. E. Stat. Phys. Plasmas. Fluids. Relat. Interdiscip. Topics 1999, 59, 6924-6936.

(69) Bruce, P. Conductivity and Transference Number Measurements on Polymer Electrolytes. Solid State Ionics 1988, 28-30, 918-922.

(70) Appetecchi, G. B. A New Class of Advanced Polymer Electrolytes and Their Relevance in Plastic-Like, Rechargeable Lithium Batteries. J. Electrochem. Soc. 1996, 143, 6.

(71) Jorne, J. Transference Number Approaching Unity in Nanocomposite Electrolytes. Nano Lett. 2006, 6, 2973-2976.

(72) Capuano, F.; Croce, F.; Scrosati, B. Composite Polymer Electrolytes. J. Electrochem. Soc. 2003, 203, 197-203.

(73) Ding, F.; Xu, W.; Graff, G. L.; Zhang, J.; Sushko, M. L.; Chen, X.; Shao, Y.; Engelhard, M. H.; Nie, Z.; Xiao, J.; et al. Dendrite-Free Lithium Deposition via Self-Healing Electrostatic Shield Mechanism. J. Am. Chem. Soc. 2013, 135, $4450-4456$.

(74) Jonscher, A. K. The "Universal” Dielectric Response. Nature 1977, 267, 673679. 


\section{APPENDIX}

Supplementary Information for Chapter 4

1. Diffusivity and Glass Transition Variation:
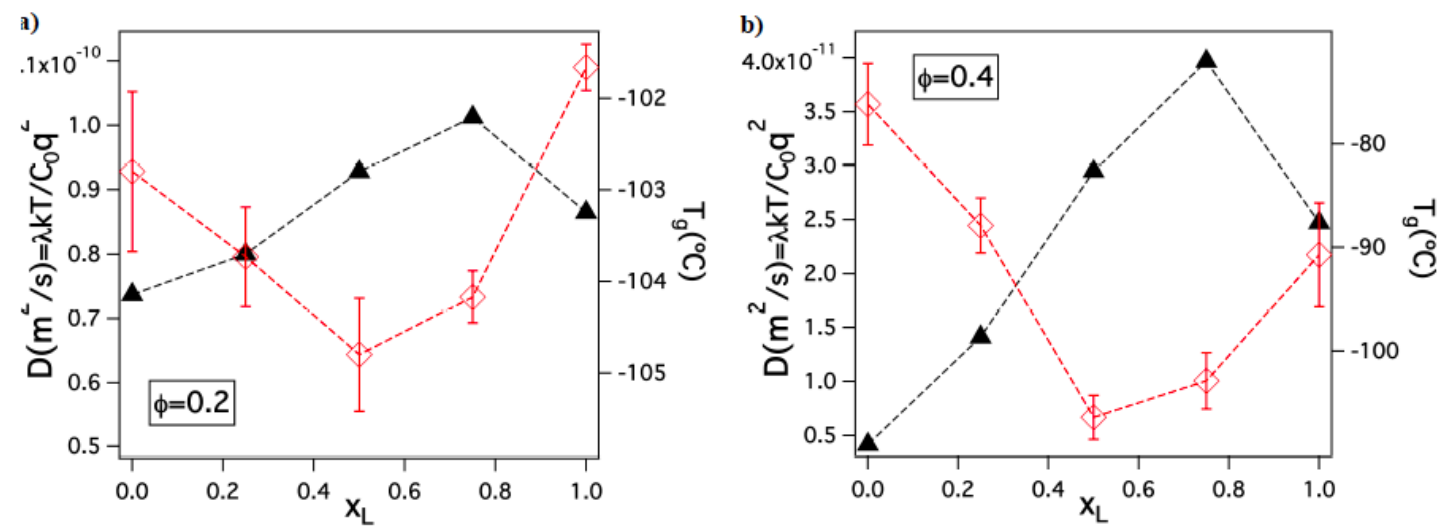

Supplementary Figure 4.1 Variation of glass transition temperature (open symbols) and diffusivity (close symbols) as a function of fraction of larger particles $\mathrm{xL}$, for core volume fraction a) $\phi=0.2$ and b) $\phi=0.4$. The dashed lines are guide to eye. 

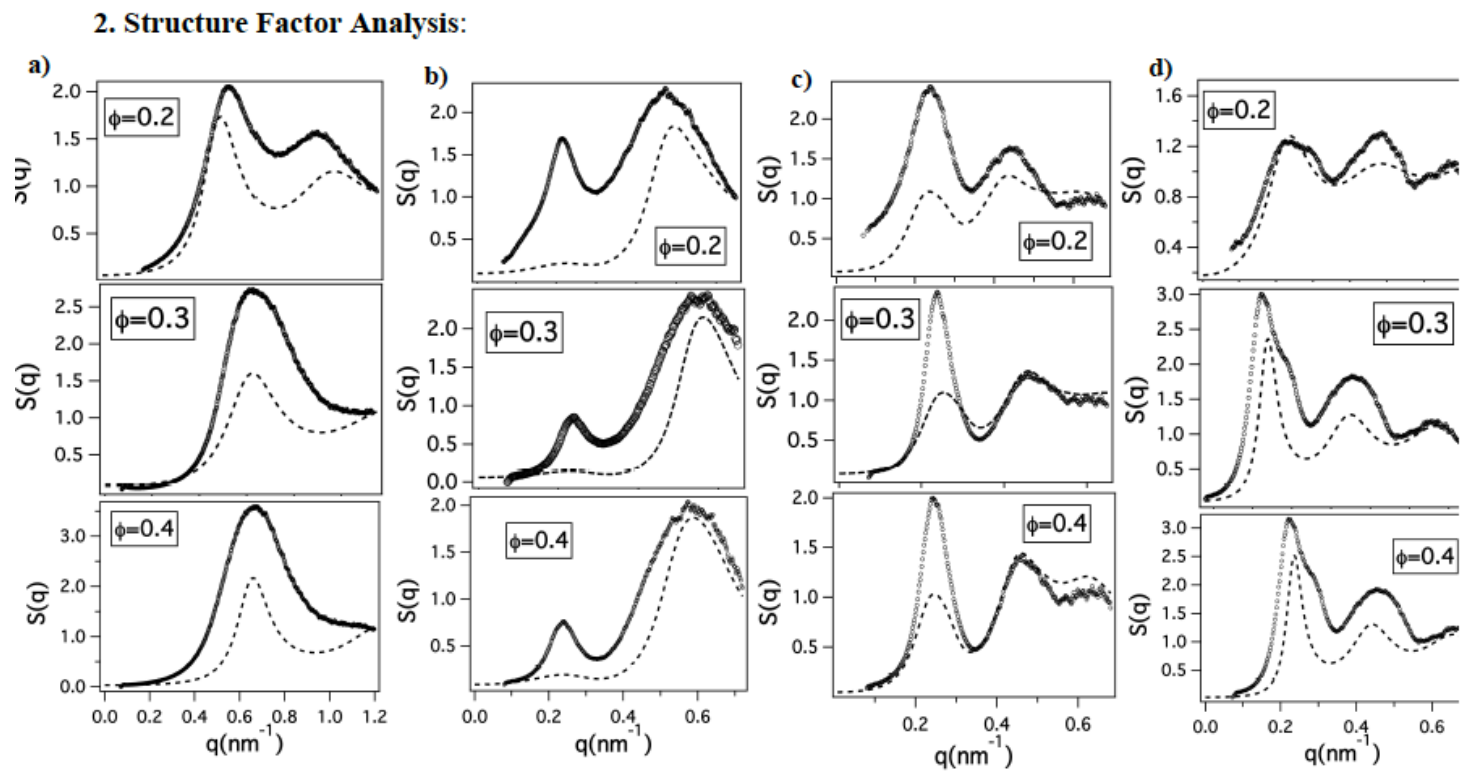

Supplementary Figure 4.2 Evolution of structure factor $\mathrm{S}(\mathrm{q})$ as a function of the wave vector $\mathrm{q}$ at volume fractions $\Phi=0.2,0.3$ and 0.4 for a) $\mathrm{xL}=0, \mathbf{b}) \mathrm{xL}=0.25$, c) $x L=0.75$, and d) $x L=1$. The open symbols are experimental values and the dashed lines are fit to experimental S(q) using binary Hard Sphere model. 

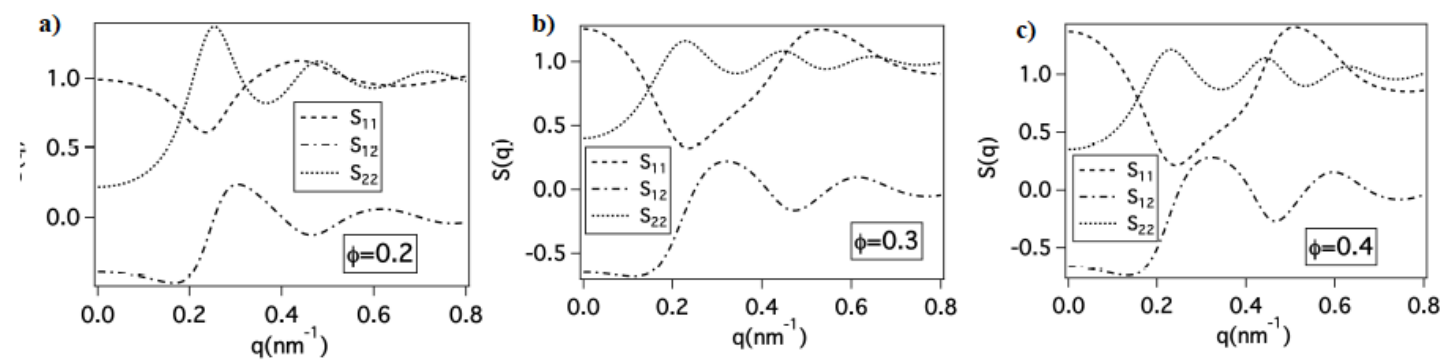

Supplementary Figure 4.3 Variation in three components of structure factor viz., S11, S12, and S22 as estimated from Binary Hard Sphere model predictions. Here, 1 and 2 indicate smaller and larger particles respectively. The structure factors are shown for $\mathrm{xL}=0.5$ for different volume fraction a) $\Phi=0.2$, b) $\Phi=0.3$, and c) $\Phi=0.4$. 

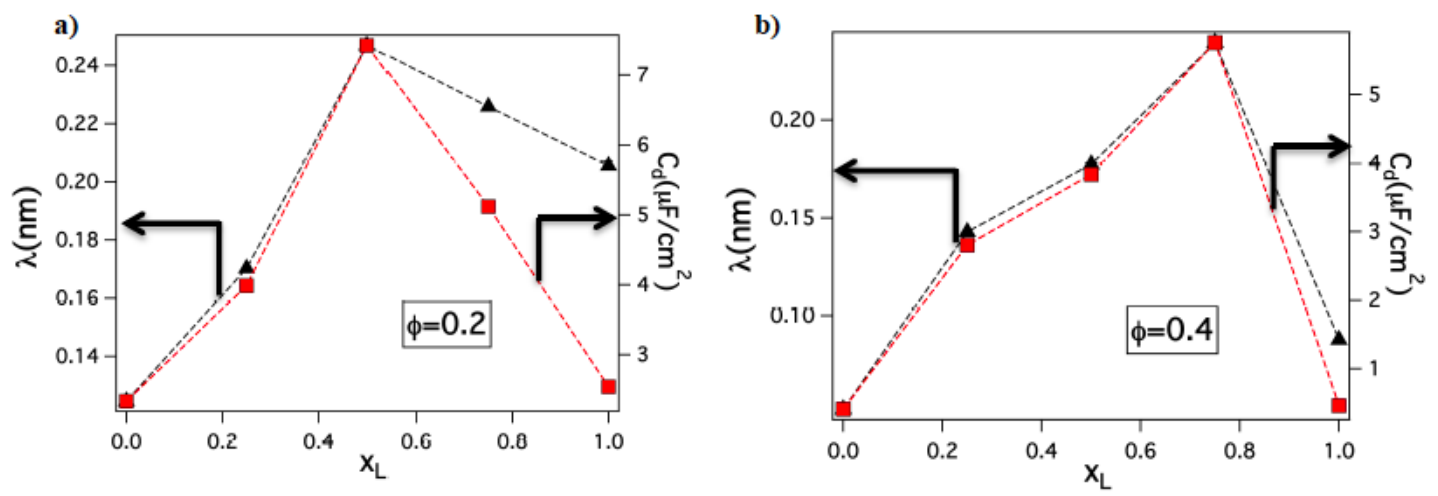

Supplementary Figure 4.4 Electrical double layer (square symbols) and electrochemical capacitance (triangle symbols) for different values of $\mathrm{xL}$ at a) $\Phi=0.2$ and b) $\Phi=0.4$ 

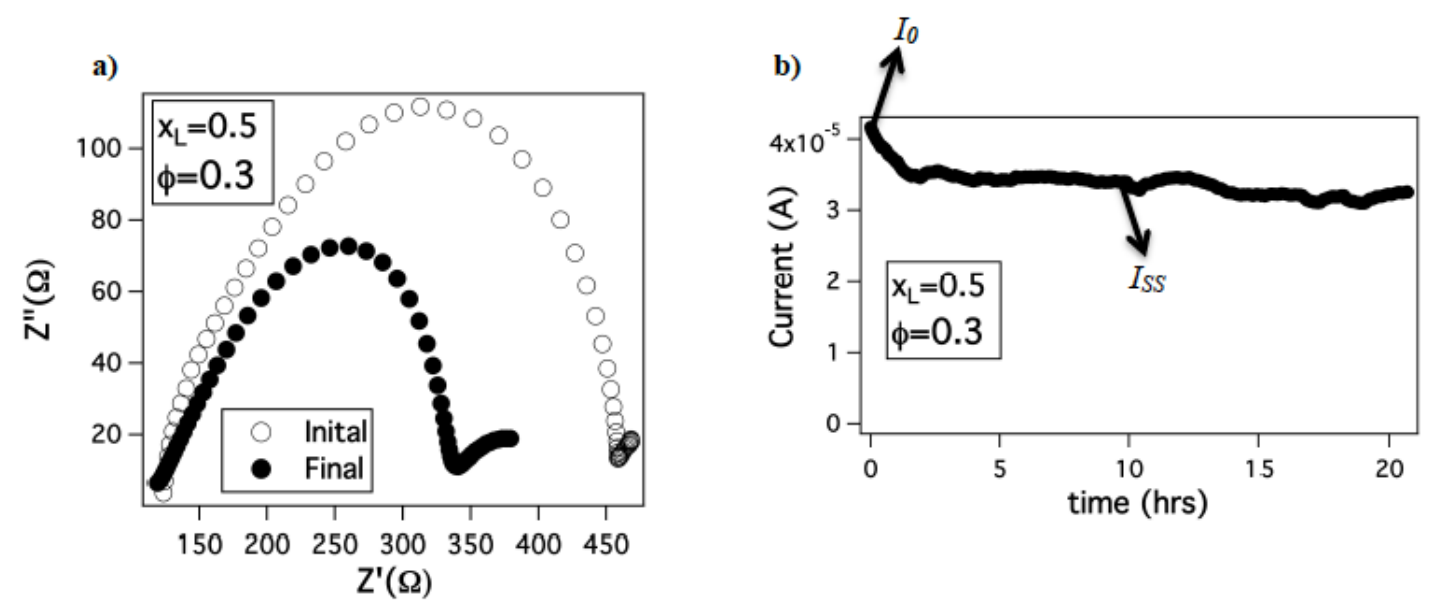

Supplementary Figure 4.5 a) Electrochemical impedance measurements for a symmetric lithium cell before polarization (open symbols) and after steady state is reached (closed symbols). $\mathrm{R}_{0}$ and $\mathrm{R}_{\mathrm{ss}}$ were determined by fitting these impedances to the circuit model mentioned in the main text. b) Current decay for the cell undergoing polarization at $20 \mathrm{mV}$ potential. $\mathrm{I}_{0}$ and $\mathrm{I}_{\mathrm{SS}}$ were used in the calculations as observed in the plot. 
CHAPTER 5

\begin{abstract}
A HIGHLY REVERSIBLE ROOM TEMPERATURE LITHIUM METAL BATTERY BASED ON CROSS-LINKED HAIRY NANOPARTICLES
\end{abstract}




\subsection{Abstract}

Rough electrodeposition, uncontrolled parasitic side-reactions with electrolytes, and dendrite induced short-circuits have hindered development of advanced energy storage technologies based on metallic $\mathrm{Li}, \mathrm{Na}$ and $\mathrm{Al}$ electrodes. Solid polymer electrolytes and nanoparticle-polymer composites have shown promise as candidates to suppress Li dendrite growth, but the challenge of simultaneously maintaining high mechanical strength and high ionic conductivity at room temperature has so far been unmet in these materials. Here, we report a facile and scalable method of fabricating tough, freestanding membranes that combine the best attributes of solid polymers, nanocomposites, and gel-polymer electrolytes. Hairy nanoparticles are employed as multifunctional nodes for polymer cross-linking, which produces mechanically robust membranes that are exceptionally effective in inhibiting dendrite growth in a lithium metal battery. The membranes are also reported to enable stable cycling of Lithium batteries paired with conventional intercalating cathodes. Our findings appear to provide an important step towards room temperature dendrite-free batteries.

\subsection{Introduction}

The search for portable, high capacity and safe electrical energy storage technologies remains one of the paramount motivators for materials research. High voltage cathodes, high energy anodes, and highly conductive, but stable electrolytes for lithium-ion batteries (LIBs) have received a lop-sided share of the attention by researchers because of their multiple attractive features, including high energy density,

light weight, high operating voltage and minimal memory effects ${ }^{1-3}$. Secondary 
lithium metal batteries (LMBs), wherein metallic lithium serves as the anode, are an attractive alternative to LIBs, but are known to have a serious problem associated with dendrite-induced short circuits ${ }^{4}$. During repeated cycles of charge and discharge, the uneven deposition of Li-ion on this metal lead to the formation of ramified structures, which grow unstably, puncture the separator, and ultimately causes cell failure by short circuiting the anode and cathode. Over the years, a growing number of studies have explored electrolyte and separator platforms to suppress the dendrite growth in an effort to enable $\mathrm{LMBs}^{5,6}$. Recent efforts have focused on stabilizing the surface of the $\mathrm{Li}$ anode using electrolyte additives ${ }^{7-9}$, hybrid ionic liquid nanostructures ${ }^{10,11}$, or by using a high modulus separator ${ }^{12-14}$, which can also provide a means of applying compression forces to stabilize the anode during deposition. Infusion of a nanoporous ceramic or polymer membrane separator with a liquid electrolyte that can facilitate $\mathrm{Li}$ ions transfer, without compromising the mechanical properties of the nanoporous membrane, provides a more straightforward route towards mechanically strong, roomtemperature electrolytes/separators that prevent dendrite growth ${ }^{15,16}$.

Solid or gel-polymer electrolyte have been researched extensively for their ability to enable batteries in various form factors that are leakage free, flexible, yet safer ${ }^{12-14,17-}$ ${ }^{21}$. However, these gel electrolyte systems have consistently underperformed in terms of the ionic conductivity requirements for room-temperature operation of advanced batteries $^{22}$. In a block copolymer solid electrolyte system, the ratio of the hard nonconducting phase to the soft conducting phase determines the mechanical strength of the system. It has been shown for instance that in poly(styrene)-poly(ethylene oxide) 
(PS-PEO) electrolytes, a PS/PEO molar ratio of around unity provides a good balance between mechanical strength and ionic conductivity ${ }^{23}$. However, the abundance of the non-conducting, reinforcing PS phase still results in low bulk conductivity relative to liquid electrolytes, necessitating elevated temperature battery operation, which is a limitation for many consumer-based applications. Nanocomposite electrolytes comprised of liquid or polymeric electrolytes reinforced with nanoparticle fillers can achieve higher modulus at lower reinforcing material content, which potentially offers multiple straightforward paths towards electrolytes with high modulus and acceptable room-temperature ionic conductivity ${ }^{24-28}$. Uniform dispersion of the fillers in the polymer host is understood to be a prerequisite to prevent particle agglomeration and local inhomogeneity in the electrolyte medium. Unfortunately, strong attractive Van der Waals and depletion forces on the particles result in particle aggregation and phase separation $^{29,30}$. Several recent studies have shown that various physical and chemical modifications of nanoparticle-polymer interactions can lead to dramatic improvements in phase stability and electrolyte properties of such systems ${ }^{29,31-34}$.

A strategy towards a hybrid electrolyte platform, which can provide high ionic conductivity and attractive mechanical properties, is designing a cross-linked polymer web in which hairy nanoparticles serve as cross-linkers. A perhaps obvious benefit of this design is that chemistry introduced on the surface of the precursor particles can be presented in the pores of the cross-linked material to selectively pass Li ions while hindering the unstable dendrite growth. Here, we report the first realization of this concept and show that an electrolyte with the proposed design combining the 
advantages of a nanocomposite and solid polymer electrolyte. In the systems reported herein, a hydrophobic polymer provides a porous conductive pathway for Li ions while a short hydrophilic oligomer tethered to the nanoparticle surface constrain the network providing structure and mechanical strength. The nanoparticle-induced crosslinking of the polymer prevents particle aggregation, which is known to compromise flexibility and elasticity of nanocomposites.

\subsection{Methods}

\subsubsection{Materials}

Ludox SM30 colloidal silica $(d=10 \pm 2 \mathrm{~nm})$, poly(propylene glycol)-toluene-2,4diisocyante, propylene carbonate, Bis(trifluoromethane) sulfonamide lithium salt, ethylene carbonate/ diethylene carbonate (1v:1v) with lithium hexafluorophosphate were all purchased from Sigma Aldrich. Hydoxy terminated poly (ethylene oxide)silane was obtained from Gelest. All the chemicals were used as received in appropriate conditions.

\subsubsection{Nanoparticle-Polymer Crosslink Synthesis and Composite Electrolyte Preparation}

Crosslinked-Nanoparticle-Polymer-Composites (CNPC) were synthesized using a two-step process. In the first step, colloidal silica was grafted with hydroxypoly(ethylene oxide)-silane [500Da] in water by the reaction of silanol groups of the PEO chain and $\mathrm{OH}$ - groups on the silica particle. A predetermined amount of the 
Ludox SM30 and a large excess of the hydroxy-PEO-Silane was first heated at $80^{\circ} \mathrm{C}$ for a period of two days, before centrifuging the thus obtained mixture in a chloroform-hexane mixed solvent five times at a speed of $8500 \mathrm{rpm}$ for 10 minutes in order to remove all unlinked PEO from the hairy nanoparticles. The recovered $\mathrm{OH}-$ $\mathrm{PEO}-\mathrm{SiO}_{2}$ hairy particle suspension was rigorously dried and saved for the next reaction step. In the second step, poly(propylene glycol)-toluene-2,4-diisocyante and the $\mathrm{OH}-\mathrm{PEO}-\mathrm{SiO}_{2}$ nanoparticles were combined in a 4:1 ratio by weight and dissolved anhydrous chloroform using a vortex mixer. The obtained solution was casted onto a rectangular Teflon mould of desired size. The mixture was allowed to crosslink overnight under ambient conditions to produce CNPC films/membranes of desired thicknesses. The resultant membranes were characterized using TGA, DSC and FTIR. In order to use the synthesized CNPC membranes as battery electrolytes, they were soaked for a period of two days in conventional liquid electrolytes in an Argon-filled Glove Box.

\subsubsection{TEM and Small Angle X-ray Scattering}

The nanoscale structure of the as prepared CNPC membranes was characterized using a FEI T12 Spirit TEM. For these measurements, a thin layer of the precursor material was casted onto the TEM grid, allowing it to crosslink on the grid. Before TEM imaging, coated grids were dried overnight using the same procedure used to prepare the freestanding CNPC membranes. Small Angle X-ray Scattering (SAXS) measurements were performed at the Cornell High Energy Synchotron Source 
(CHESS) on a strip of the CNPC film using a point collimated X-ray source at room temperature.

\subsubsection{Mechanical Properties}

Mechanical properties of CNPC membranes and CNPC electrolytes were analyzed using dynamic mechanical analysis (DMA Q800) at room temperature. A fixed strain of $0.1 \%$ was applied on the strip over a range of frequency of $1 \mathrm{~Hz}$ to $100 \mathrm{~Hz}$, in order to obtain the storage modulus of the samples. A similar experiment was done on the Silica-PPO suspension using an Anton Paar MCR501 shear Rheometer in order to characterize the effect of cross-linking on mechanical properties of the materials.

\subsubsection{Electrochemical Characterization}

The ionic conductivity of CNPC electrolytes was measured as a function of temperature using a Novocontrol N40 Broadband Dielectric instrument. The d.c. conductivity was obtained from the plateau, high frequency region of the a.c. conductivity versus frequency data. Impedance Spectroscopy measurements were performed using a Solatron frequency analyzer. A two-electrode, symmetric Li|Li cell in which the CNPC electrolyte was sandwiched between lithium foils was studied in a frequency range from $10^{-3} \mathrm{~Hz}$ to $10^{7} \mathrm{~Hz}$. Linear sweep and cyclic voltammetry were performed at a sweep rate of $1 \mathrm{mV} / \mathrm{s}$ between $-0.2 \mathrm{~V}$ to $6.5 \mathrm{~V}$ using $\mathrm{Li} / \mathrm{CNPC}$ electrolyte/Stainless Steel cells to quantify the voltage stability window of the electrolytes. 


\subsubsection{Cell Lifetime and Failure Studies}

Symmetric 2032 type Li|Li coin cells containing either liquid of CNPC electrolytes were prepared inside an Argon-filled Glove Box. To facilitate comparisons, all cells used in this component of the study also included a standard Celgard separator, with a diameter of $6.4 \mathrm{~mm}$. The cells were evaluated using both galvanostatic (strip-plate) cycling and in step current (polarization) modes over a range of current density using a Neware CT-3008 battery tester. In the 'strip-plate' experiments, the cells were charged and discharged at a predetermined current density for a period of three hours each. Failure was deduced either from a sudden drop of the resultant voltage waveform or when the waveform exhibited new harmonics and displayed irregular time dependence. In the polarization experiments, the symmetric cells were constantly charged at a particular current density until the cell failed. In this case failure was determined either by a sudden drop in the resultant voltage profile or by the appearance of irregular transients in the voltage profile. Postmortem characterization of the lithium surface after cycling was used to corroborate our conclusions about failure from the electrical response of the cells and for assessing the surface structure of $\mathrm{Li}$ in cells that showed no electrical signatures of failure. For this purpose, cells were dissembled in the glove box and the Li foil removed and was washed repeatedly in pure PC to remove the electrolyte, before performing SEM (LEO155FESEM) analysis.

\subsubsection{Measuring the Coulombic Efficiency}


Li| electrolyte |Stainless steel 2032 type coin cells were assembled in an Argon-filled Glove Box. Control experiments using liquid electrolytes comprised of 1M LiTFSIPC with $1 \%$ (w.t.) $\mathrm{LiNO}_{3}$ and $2 \%$ (vol.) vinylene carbonate additives and a standard Celgard separator were compared to measurements using CNPC electrolytes created by soaking CNPC membranes in these same liquid electrolytes. In both cases, prior to the measurements, cells were conditioned by cycling them between 0 to $0.5 \mathrm{~V}$ for 10 cycles, following a procedure reported in literature ${ }^{44}$ thought to enable formation of a stable SEI layer on the electrodes. To characterize the columbic efficiency, the conditioned cells were first discharged at a constant current density of $0.25 \mathrm{~mA} / \mathrm{cm}^{2}$ for 2 hours to transfer an amount of lithium corresponding to $0.50 \mathrm{mAh} / \mathrm{cm}^{2}$ of charge from the Li electrode to the stainless steel electrode. The amount of charge recovered in the reverse cycle when the cell is charged back to $0.5 \mathrm{~V}$ at the same current density was recorded, and the fractional recovery used to determine the Columbic efficiency.

\subsubsection{Half-cell testing}

Both LTO and LFP cathodes were prepared by mixing the active materials with Super P carbon and PVDF binder in the ratio of 8:1:1, by weight, in the presence of NMP, and casting a layer of the resultant slurry onto aluminum foil. Next, the cathode was dried first at room temperature and then at $70^{\circ} \mathrm{C}$ overnight under vacuum. The surface capacity of both the films was maintained constant as $0.5 \mathrm{mAh} / \mathrm{cm}^{2}$. Li|CNPC electrolyte|LTO and Li|CNPC electrolyte|LFP 2032 type coin cells were assembled under argon environment in a glove box. 


\subsection{Results}

\subsubsection{Synthesis and Physical characterization of crosslinked membrane}

Figure 5.1(a) is a schematic of the reaction steps involved in the synthesis of our cross-linked hybrid electrolyte material. First, short-chained oligomers, poly(ethylene oxide)-500Da, are grafted onto silica particles to form nanoparticle organic hybrids with reactive end groups. The PEO-tethered silica particles are next linked with difunctional poly(propylene oxide)-2000Da (PPO) to form the cross-linked nanoparticle-polymer composite (CNPC) in a desired macroscopic shape. The materials used in the present study achieve high levels of cross-linking by tworeaction mechanisms: dimerization between isocyanate end groups in PPO and reaction between isocyanate group in PPO and end-functional hydroxyl groups on the hairy nanoparticles to form stable urethane linkages. The CNPC membrane (Figure 5.1(c)) is mechanically tough and has a 'rubbery' texture. In order to use it as a composite electrolyte, the membrane was soaked in a liquid electrolyte comprised of propylene carbonate (PC) containing 1M lithium bis(trifluoromethanesulfonyl)imide (LiTFSI) salt for a period of two days. This step yields a material with a swollen, gellike appearance (shown in Figure 5.1(d)), but with little loss of the solid nanocomposite material's mechanical strength. In contrast, it is seen that on soaking with electrolyte the un-crosslinked components separate out in the liquid, leaving behind a transparent film with no observable particle aggregates. For brevity, the CNPC membranes infiltrated with liquid electrolytes are hereafter referred to as CNPC electrolytes. 
The reaction progress can be facilely mapped using Fourier transform infrared spectroscopy (FTIR) as illustrated in Supplementary Figure 5.2. It is evident that the $\mathrm{NCO}$ bond at $\left(2270 \mathrm{~cm}^{-1}\right)$ present in the neat PPO-diisocyanate polymer is consumed in the reaction and at same time generates a urethane linkage, evident by the $-\mathrm{NH}$ vibration $\left(3288 \mathrm{~cm}^{-1}\right)$ and $-\mathrm{C}=\mathrm{O}$ bond peak $\left(1680 \mathrm{~cm}^{-1}\right)$. TGA analysis of these materials (Supplementary Figure 5.2) shows that the freestanding crosslinked film contains $6 \%$ silica by weight and in the final CNPC electrolyte material the silica content is $2 \%$ (Supplementary Table 5.2), thus there is a preponderance of ionconducting entity in the material. Significantly, because the silica particles are nano sized ( $\sim 10 \mathrm{~nm})$ and have multiple chains attached to their surface ( $\sim 55$ chains/particle), each particle provides a large number of node points for cross-linking the PPO polymer. Thus, even a low particle content in the precursor material is expected to enable extensive cross-linking via particle nodes, which should lead to dramatic improvements in mechanical properties without compromising room temperature ionic conductivity of the liquid electrolyte hosted by a CNPC membrane.

The synthesized CNPC membranes were characterized using differential scanning calorimetry (DSC) measurements (Supplementary Figure 5.3), which reveal a low glass transition temperature $\left(T_{\mathrm{g}}\right)$ of $-63^{0} \mathrm{C}$ for neat PPO polymer, after crosslinking, $T_{\mathrm{g}}$ increases to $-42^{\circ} \mathrm{C}$. The dispersion and arrangement of nanoparticles in the crosslinked polymer matrix is important for the targeted application as nanostructured electrolytes. Figure 5.1(b) shows a 2D image of a thin layer of the material obtained from Transmission Electron Microscopy (TEM). The image shows that the particles 
organize in disordered inter-connected, string-like phases to produce tortuous pores with an average diameter of $24 \pm 2 \mathrm{~nm}$ estimated from the TEM micrograph (see Supplementary Figure 5.4). Small Angle X-ray Scattering (SAXS) has been used previously to study particle packing and polymer-nanoparticle interactions in bulk nanocomposite based electrolyte materials ${ }^{35-37}$. Figure 5.1(e) shows the experimental Intensity $\mathrm{I}(q)$ as a function of wave number $(q)$ obtained from SAXS measurements on CNPCs. The solid line through the experimental I $(q)$ data was obtained using the Beaucage Unified model (see Supporting Information). The power-law scattering regime $I(q) \square q^{-a}$, with exponent $a » 2$ observed at low q indicates that the particles in the materials are organized in the form of mass fractals with sizes in the range 50 $\mathrm{nm}$ to $70 \mathrm{~nm}$. The shoulder at $q=q_{s} \gg 0.6 \mathrm{~nm}-1$ implies that the scattering originates from particles with average diameter $d=2 p / q_{s} » 10.5 \mathrm{~nm}$, which is close to the average diameter of the $\mathrm{SiO}_{2}$ nanocores used for synthesizing the materials. Finally, the absence of any additional structure contribution (maxima) in I(q) in the intermediate and high $q$ regime means that the primary particles are reasonably far apart. The interparticle distance can be theoretically calculated assuming random packing of hard spheres in suspension, $\mathrm{d}_{\mathrm{p}-\mathrm{p}}=d(0.63 / \phi)^{1 / 3}$, where $d$ is the nanoparticle diameter and $\phi$ is the volume fraction of particles in the polymer network. This analysis yields an interparticle spacing of approximately $27 \mathrm{~nm}$, which is comparable to the particle separation of $24 \pm 2 \mathrm{~nm}$ deduced from analysis of the TEM image in Figure 5.1(b) (see Supplementary Figure 5.4). Thus, the TEM and SAXS results imply that in a freestanding CNPC membrane, randomly distributed particles organize in string-like phases to form tortuous path for ion and mass transport through the 


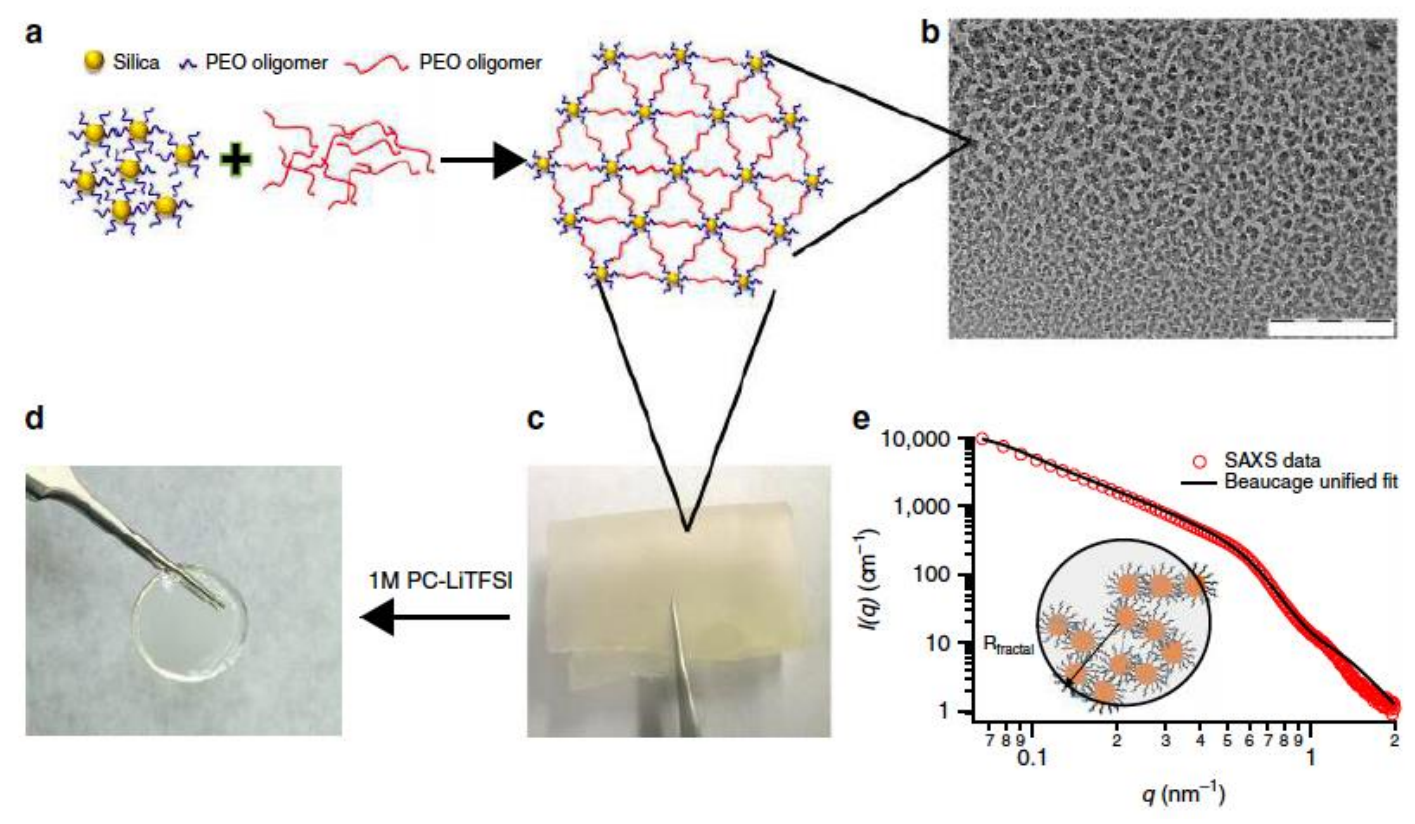

Figure 5.1: From hybrid nanostructure to macroscale membrane: Clockwise. a, The first row shows the scheme of reaction involved in the synthesis of the freestanding Crosslinked Nanoparticle Polymer Composite. Silica tethered with Hydroxyterminated Polyethylene Oxide is reacted with Polypropylene Oxide-Diisocyanate with a weight ratio of 1:4 at room temperature in a Teflon mould of appropriate shape. b, TEM image of the Crosslinked membrane. The black circles are silica particle dispersed in the polymer matrix. c, The photograph of a free-standing membrane is shown. Physically it has a rubbery texture and is transperent. d, Image of the wet polymer gel is shown which is obtained by long time soaking of the prepared membrane in unimolar electrolyte liquid. e, Scattered intensity $I(q)$ vs wave vector $q$ profile obtained from SAXS measurements. Solid line represents the fit for Beaucage Unified model to the data. The fit in the low $q$ regime shows a power law scattering $\left(I(q) \sim q^{-\alpha}\right)$ with an exponent $(\alpha)$ of around 2 , indicating the presence of mass fractals 
materials. Such nanostructured materials have been hypothesized, largely without proof, to be excellent candidates to hinder dendrite-induced short circuit in Lithium batteries $^{13,14,20}$.

\subsubsection{Mechanical and Electrochemical properties of crosslinked membrane}

Figure 5.2(a) reports the storage modulus as a function of frequency for a freestanding CNPC film before and after soaking in liquid electrolyte. The storage modulus of a suspension of PPO in silica is also shown in the figure. Clearly, cross-linking increments the modulus by several orders of magnitude. The mechanical strength of the material is about five orders of magnitude higher than a neat PEO based electrolyte, and is comparable to the elastic shear modulus reported for dry PS-PEO Block copolymer-based electrolytes ${ }^{23,38}$. Additionally, it is seen that even after soaking in a liquid electrolyte, the elastic modulus of the CNPC material remains high; it is higher than those recently reported for cross-linked PEO-PE-PEO solid polymer electrolytes. ${ }^{14}$ This latter feature we attribute to the large number of cross-links made to a single particle and the short length and stiffness of the polymer fragments used as cross-linkers. Figure 5.2(b) reports the dc conductivity in Arrhenius plot for CNPC electrolytes obtained by soaking the as synthesized CNPC membranes in two widely studied liquid electrolytes, PC-LiTFSI and EC/DEC-LiPF6. In comparison to the neat (no nanoparticles) liquid electrolytes, the conductivity is lower, by about one order of magnitude, but is still high enough at room temperature for most lithium battery applications. This high conductivity is attributed to the low glass transition temperature of PPO and the low fraction of non-conductive nanoparticles needed to 
produce materials with high mechanical strength. The conductivity versus temperature data is well described by the Vogel Tamman Fulcher (VFT) relationship $\sigma=A \exp (-$ $\left.\mathrm{B} /\left(T-T_{\mathrm{O}}\right)\right)$, where $\mathrm{A}$ is the pre-exponential factor corresponding to conductivity at infinite temperature, $\mathrm{B}$ is the Activation Energy and $T_{\mathrm{O}}$ is the reference temperature. The respective coefficients for the different systems are tabulated in Supplementary Table 5.1.

Figure 5.2(c) reports temperature-dependent impedance spectra for CNPC electrolytes obtained by soaking the membranes in PC-1MLiTFSI electrolyte. The measurements were performed in symmetric $(\mathrm{Li} \mid \mathrm{Li})$, two-electrode cells. The solid lines through the data were obtained using an equivalent circuit model previously reported for nanocomposite based electrolyte systems ${ }^{39-41}$. The bulk and interfacial resistance deduced from the Nyquist plots are plotted as a function of temperature in Figure S5. It is evident that both resistances decrease with temperature and the bulk resistance is always lower than the interfacial resistance. This implies that ion transport through the tortuous materials is in reality easier relative to ion transfer across the electrolyte/electrode interface. It is apparent nonetheless that the bulk and interfacial resistances (Supplementary Figure 5.5) exhibit similar dependence on temperature, indicating that both have similar activation energy. It can therefore be concluded that the CNPC electrolytes make good contact with the Li metal, as just the polymer itself appears to limit ion transport across the interface. The electrochemical stability for the CNPC electrolyte was analyzed at room temperature using cyclic voltammetry in a prototype cell with the following configuration, $\mathrm{Li}|\mathrm{CNPC}+\mathrm{PC}-1 \mathrm{MLiTFSI}|$ Stainless 

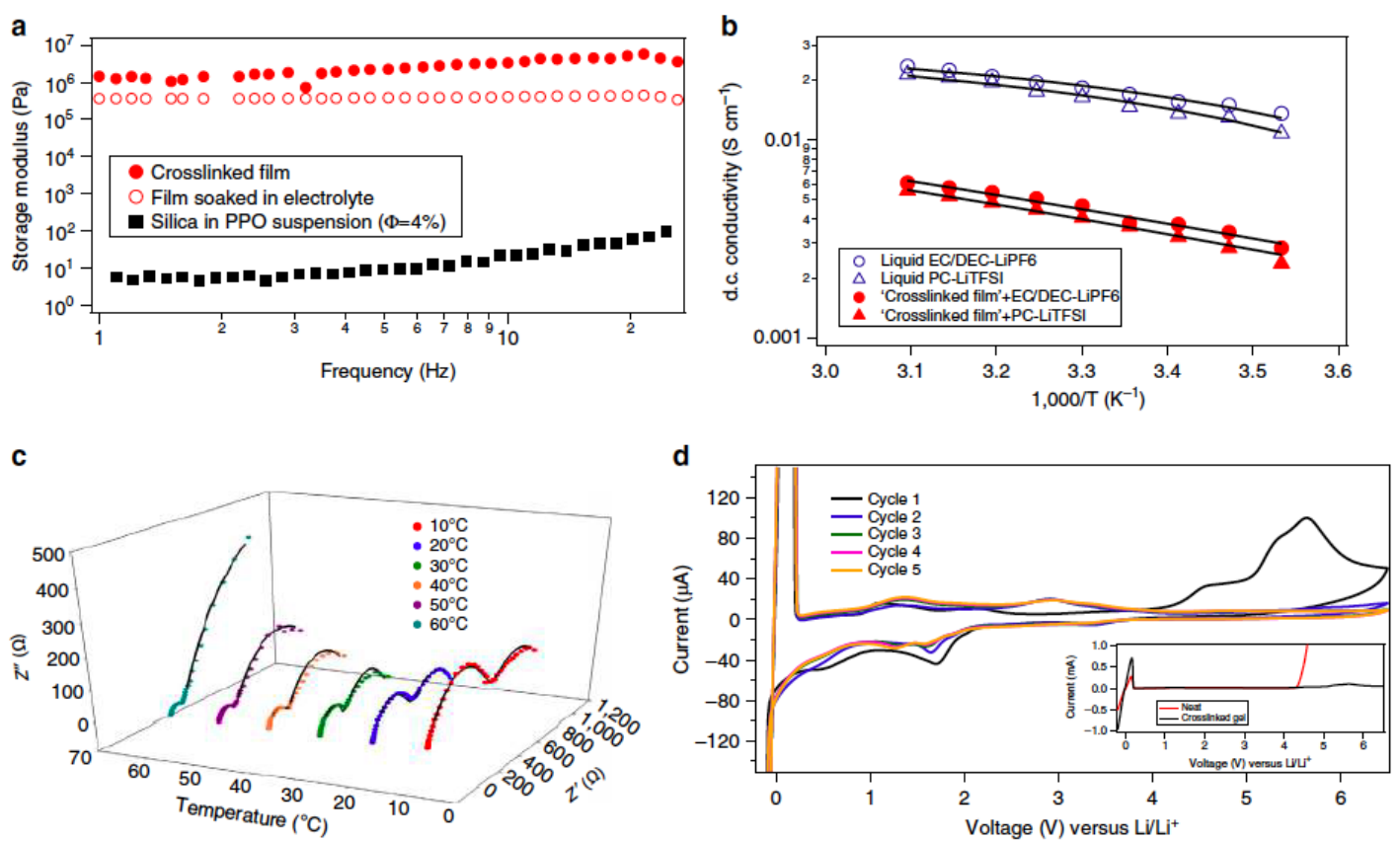

Figure 5.2: Good mechanical and electrochemical properties: a, Storage Modulus (Pa) as a function of frequency, comparing the dry polymer network and gel electrolyte with a suspension of Silica in PPO polymer with same volume fraction. The modulus of crosslinked gel is more than five orders of magnitude higher than the suspension. b, Conductivity as a function of inverse absolute temperature. The markers show measured values, while the continuous lines are fitted VFT curves. The crosslinked gel show conductivity of an order of magnitude less than the intrinsic value of eletrolyte. c, Impendence Spectroscopy results for a symmetric Lithium battery, with crosslinked gel electrolyte, are plotted against temperature; where the xaxis denote temperature, y-axis denote real Impedance (ohms) and z-axis denote imaginary Impedance (ohms). d, Cyclic Voltametry of a cell with configuration Li/Crosslinked gel/Stainless Steel is shown for 5 cycles, where $\mathrm{x}$-axis represent voltage and y-axis, current. The inset compares the stability of the crosslinked gel with neat electrolyte of 1M PC-LiTFSI 
Steel. Measurements were performed between $-0.2 \mathrm{~V}$ to $6.5 \mathrm{~V}$ and a scan rate of $1 \mathrm{mV} / \mathrm{s}$ was employed. Figure 5.2(d) shows the current as a function of Voltage for the CNPC electrolyte at different cycle numbers, while the inset shows the equivalent first cycle results obtained using a PC-1MLiTFSI liquid electrolyte. The significant current peaks at $\sim-0.2 \mathrm{~V}$ vs. $\mathrm{Li}^{+} / \mathrm{Li}$ and $+0.2 \mathrm{~V}$ vs. $\mathrm{Li}^{+} / \mathrm{Li}$ indicate that plating and stripping of $\mathrm{Li}$ ions onto/from stainless steel ${ }^{42}$. As previously reported ${ }^{43}$, a passivation layer is formed on the $\mathrm{Li}$ metal surface at $4.1 \mathrm{~V} \mathrm{vs.} \mathrm{Li}^{+} / \mathrm{Li}$, indicated by the current peak, which disappears in subsequent cycles. The CNPC electrolyte also shows a high stability window, with cathodic stability of at least $5 \mathrm{~V} \mathrm{vs.} \mathrm{Li}^{+} / \mathrm{Li}$, which is superior to what is observed for a PC-LiTFSI liquid electrolyte.

Figure 5.4.3 Analyzing stability of Lithium electrodeposition using crosslinked membranes

Figure 5.3 (a, d) report results from a so-called 'strip-plate test' in which symmetric $\mathrm{Li} \mid \mathrm{Li}$ cells are charged and discharged sequentially for a period of three hours. The voltage profiles for liquid electrolytes are shown in Supplementary Figure 5.6. It is clear that cells containing the CNPC electrolyte exhibit remarkably high cycling stability in comparison to standard cells with the same liquid electrolyte infused in the CNPC membrane. At a current density of $0.20 \mathrm{~mA} / \mathrm{cm}^{2}$, the cells show stable voltage profiles for more than 500 hours. In comparison, a control symmetric cell with the same electrolyte and without the cross-linked membranes fails within 60 hours (see Supplementary Figure 5.6). At a higher current density of $1 \mathrm{~mA} / \mathrm{cm}^{2}$, the cells run for more than 120 hours, whereas, the voltage profile for the control cell is already 
unstable from the first cycle of the test. Figure 5.3 reports the morphology of the Lithium anode surface using Scanning Electron Microscopy (SEM) after 100 hours of cycling for both types of cells discussed above. At a current density of $0.20 \mathrm{~mA} / \mathrm{cm}^{2}$, the anode surface is smooth with only sporadic patches of rough deposits (Figure 5.3(b)) for cells based on the CNPC electrolyte. In contrast, clear sharp dendrites are formed on the surface of the anode cycled in the control liquid electrolyte (Figure 5.3(c)). For cells cycled at the higher current density of $1 \mathrm{~mA} / \mathrm{cm}^{2}$ the SEM images show that after 100 hours, a dendritic structure is visibly present on the anode surface cycled in both the CNPC and control liquid electrolytes, however, the control cells display comparatively sharper and much larger dendrites.

In an LMB, electrolytes are also prone to degradation as a result of parasitic chemical and electrochemical reactions with the reactive lithium metal anode. Uneven electrodeposition exacerbates this failure mode by creating fresh surface for additional reactions. The coulombic efficiency is an important parameter that allows these effects to be quantified and tracked from cycle to cycle; it can therefore be considered a surrogate measure of the stability of the Solid Electrolyte Interface (SEI) on the lithium metal anode. Several recent studies have considered methods for improving the stability of the SEI layer using protective films on Li anodes. ${ }^{44,45}$ Procedures ranging from deployment of sacrificial, surface-reactive additives in liquid electrolytes, ${ }^{7,946-48}$ to use of electrolytes saturated with salt ${ }^{49}$ have been reported to be effective in stabilizing lithium metal. 
We performed cycling studies in which a fixed amount of $\mathrm{Li}$ is transferred from a lithium metal electrode onto a stainless-steel substrate and quantify the recovery in the reverse process when $\mathrm{Li}$ is stripped from the substrate to determine the coulombic efficiency. Understanding that surface protection of lithium is required for meaningful coulombic efficiencies in LMBs, the control measurements were performed using liquid electrolytes comprised of $\mathrm{PC}$ containing $1 \mathrm{wt} \% \mathrm{LiNO}_{3}$, and $2 \mathrm{wt} \%$ vinylene carbonate (VC) as an additive, which are reported to form a stable SEI on $\mathrm{Li}^{48}$ In contrast to the symmetric cell studies discussed in the previous section where reasonable cycling was possible in liquid electrolytes in cells using O-ring style separators, we discovered that the poor adhesion between deposited lithium and the stainless steel counterelectrode caused lithium to separate and become electrically disconnected from the stainless steel substrate, resulting in poor columbic efficiencies. Replacing the O-rings with a conventional Celgard separator eliminated this problem.

The coulombic efficiency obtained in this manner is plotted in Figure 5.3(g) as a function of cycle number at a current density of $0.25 \mathrm{~mA} / \mathrm{cm}^{2}$ for the control liquid electrolyte and for CNPC electrolytes (CNPC membranes soaked in the control liquid electrolyte). A typical voltage-capacity curve measured at the $40^{\text {th }}$ cycle of these measurements is provided in the Supplementary Figure 5.8. PC is known to form a very unstable SEI layer, yielding a low coulombic efficiency $(\sim 75 \%)^{50}$, however, it is evident that the additives improve the efficiency to over $90 \%$ both in liquid PC and in the CNPC electrolytes. Nevertheless, it is seen from Figure 5.3(g) that the neat electrolyte fails to sustain high coulombic efficiency beyond 45 cycles, while the 

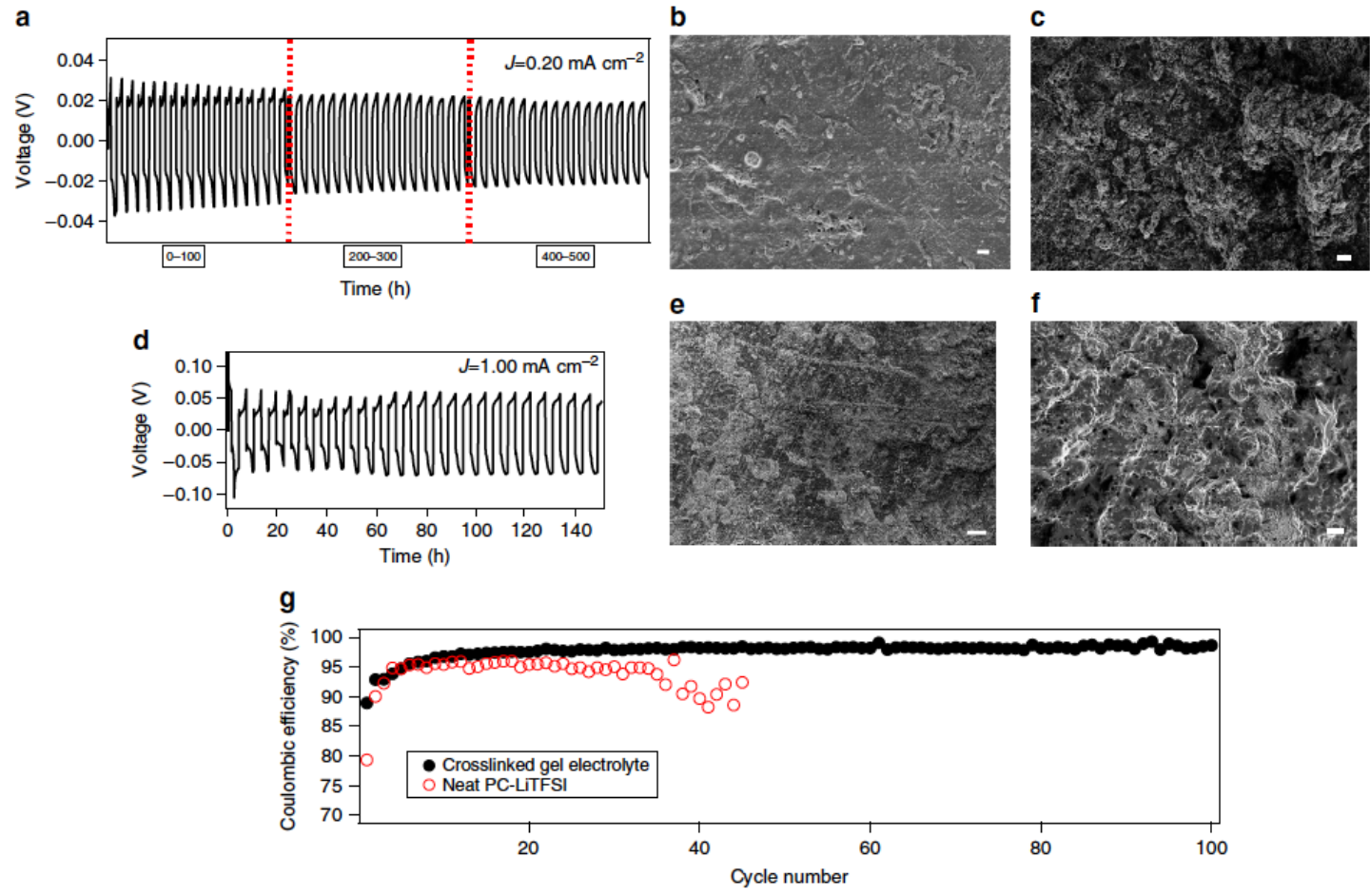

Figure 5.3: Inhibiting dendrites and enabling smooth electrodeposition: a, PlateStrip cycles for a symmetric cell comprising of crosslinked gel electrolyte is shown at a current density of $0.20 \mathrm{~mA} / \mathrm{cm}^{2}$, it shows stable performance for over 500 hours. $\mathbf{b}$, The morphology of Lithium metal anode after 100 hours of cycling of with the crosslinked gel electrolyte is shown, where very smooth surface is observed. c, Similarly, the morphology of Lithium metal anode neat electrolyte is shown, where dendritic structures of Lithium is seen to have formed. d, Volatge vs. time plots at 1.00 $\mathrm{mA} / \mathrm{cm}^{2}$ for crosslinked gel based cells are shown, the cells show no sign of short circuit for at least 120 hours. e, The post mortem analysis shows scatters of Lithium structures. f, The Lithium surface after 100 hours of cycling shows dense dendrites that are capable of shorting the cells. All white scale bars measure 20 microns. g, Electrochemical tests with Li| electrolyte| Stainless Steel configuration at a current density of $0.25 \mathrm{~mA} / \mathrm{cm}^{2}$ comparing coulombic efficiency as a function of cycle numbers for pristine and crosslinked gel electrolytes with $2 \%$ (vol.) V.C. and 1\%(wt.) $\mathrm{LiNO}_{3}$ additive. 
CNPC electrolytes maintain high coulombic efficiency for at least 100 cycles. This can be rationalized by our earlier observation of smoother electrodeposition in the latter electrolytes, which helps in preventing the rupture and reformation of SEI layer in successive charge and discharge cycles.

Polarization experiments at a fixed current density provide a more aggressive protocol for evaluating stability of lithium electrodeposition. Figure 5.4(a) compares the cell lifetimes obtained using strip-plate and polarization measurements. A typical voltage vs. time curve is shown in the Supplementary Figure 5.9. It is again apparent that the CNPC electrolytes enable cells that deliver over two orders of magnitude improvement in lifetime. The short circuit times $\left(T_{\mathrm{SC}}\right)$ deduced for both the polarization test and strip-plate cycling studies can be fitted with a power-law function of the form, $T_{\mathrm{SC}}=\mathrm{A} . J^{\mathrm{K}}$, where $J$ is the current density and A is a transport coefficient. The power law exponents for the strip-plate and polarization measurements are, respectively, 1.84 and 1.35 ; consistent with previous reports ${ }^{14,43}$. Figure 5.4(a) compares the short circuit time from our plate-strip and polarization experiments with results from a wide variety of literature studies. For example, Rosso et al. ${ }^{51}$ studied the stability of lithium electrodeposition in high-molecular-weight poly(ethylene oxide) containing lithium salt at $90^{\circ} \mathrm{C}$. The figure shows that at equivalent current densities, the cross-linked nanocomposite electrolyte based cells register substantially higher short circuit times in comparison to those obtained in the polymer electrolytes studied by Rosso et al. Figure 5.4(a) also reports results from the work of Liu et al. ${ }^{39,40,52}$ who performed electrochemical experiments in visualization 
cells in which surface modified silica-PEO based nanocomposite electrolytes, solid polymer electrolytes comprised of PEOxLiTFSI repeating units, and combinations of the two were used to stabilize deposition in lithium metal batteries at elevated temperature; from Sannier et. al. ${ }^{53}$ who performed symmetric cell studies using gel polymer membranes based on PEO and PVdF-HFP polymers; and from Khurana et al. ${ }^{14}$ using cross-linked PEO-PE-PEO polymers. It is important to note that all of these experiments were performed at elevated operating temperature between $60^{\circ} \mathrm{C}$ and $90^{\circ} \mathrm{C}$ to overcome the poor room temperature ionic conductivity of the electrolyte materials. It is apparent from the figure that the CNPC electrolytes deliver as good or better performance than all of these other studies with the important difference that the experiments the CNPC electrolytes were all performed at room temperature! Finally, Figure 5.4(a) reports results from simple liquid electrolytes (here termed Neat electrolytes) and other state-of-art nanocomposite and polymer based electrolytes reported previously to be effective inhibitors of dendrite growth in LMBs operated at room temperature. Comparison of these results with those obtained in the present study also show that cells that utilize CNPC electrolytes exhibit superior ability to stabilize electrodeposition of Li than any of the other room-temperature electrolyte/separator materials. ${ }^{11,54}$ On the basis of these results, we therefore conclude that the CNPC electrolytes reported in the present study are promising candidates towards the goal of dendrite-free room temperature LMBs.

To further characterize the galvanostatic performance of our electrolyte materials, LMBs were constructed in which metallic lithium was paired with Lithium Titanium 


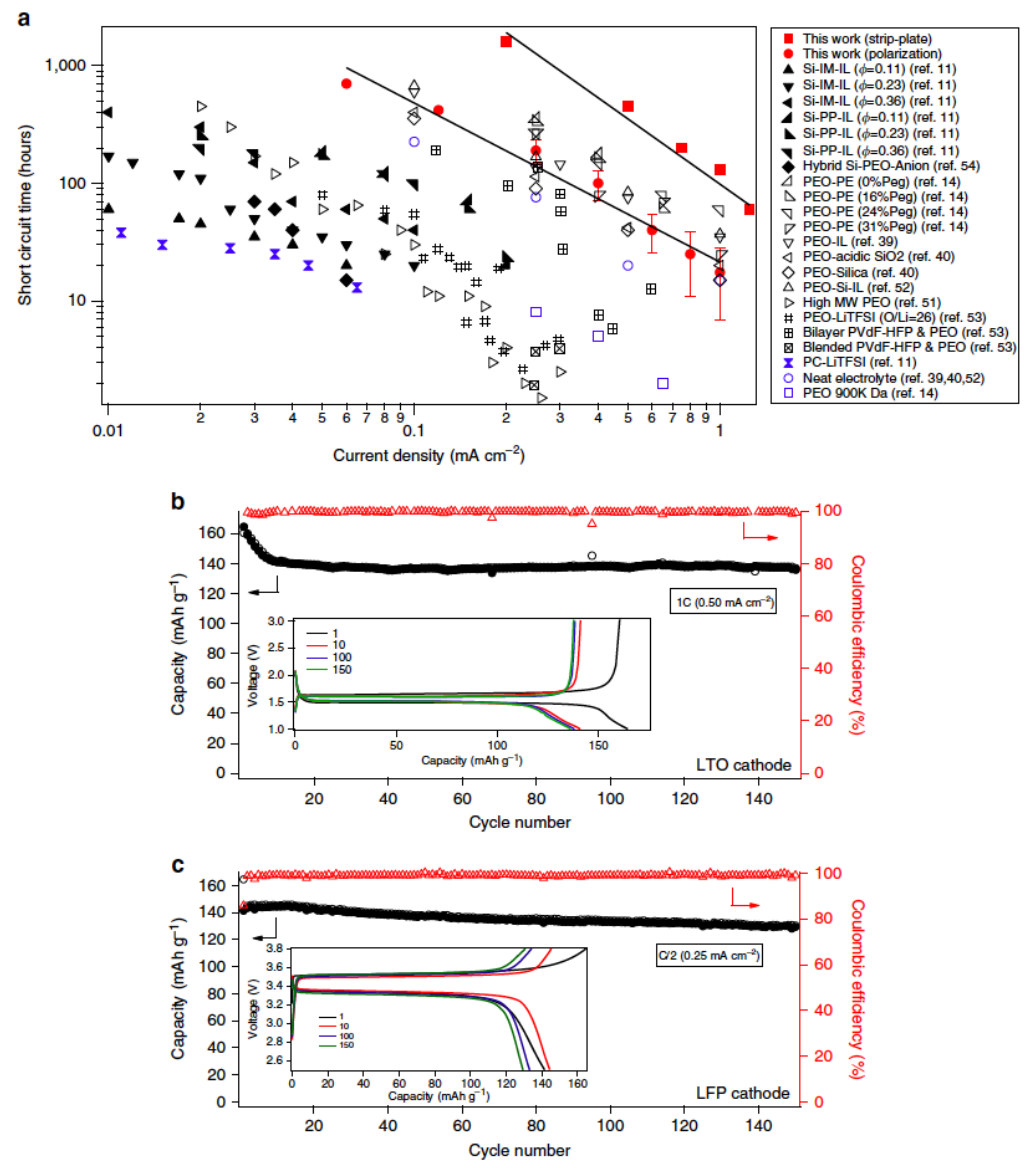

Figure 5.4: High short circuit time and good cyclic stability: a, Short circuit time of crosslinked gel electrolytes compared with other state of art battery performance. Red squares and red circles indicate the Tsc for strip-plate test and polarization test in this work respectively. The black filled symbols represent polarization tests done at Room Temperature, while the open symbols represent elevated temperature experiments. Black closed triangles represent Silica tethered with Imidazolium (SiIM-IL) and Piperidinium Ionic Liquid (Si-PP-IL) at various volume fractions of Silica, indicated in parenthesis ${ }^{11}$. Black closed diamonds indicate anion tethered hybrid silica nanocomposites ${ }^{54}$. The high temperature data include crosslinked PE-PEO solid polymer with different plasticizer content given in parenthesis ${ }^{14}$. Other data points are PVdF-HFP/PEO composite ${ }^{53}$, high molecular weight polymer $^{51}$, silica -polymer composite $^{40}$, polymer with ionic liquid ${ }^{39}$ as well as their combination ${ }^{52}$. The blue symbols indicate neat/pristine electrolyte systems. b, Cycling Performance for Li| crosslinked gel| LTO is shown at $1 \mathrm{C}\left(0.50 \mathrm{~mA} / \mathrm{cm}^{2}\right)$. The inset shows the voltage profiles of the same. c, Cyclic performance for Li| crosslinked gel| LFP at C-rate of $\mathrm{C} / 2\left(0.25 \mathrm{~mA} / \mathrm{cm}^{2}\right)$ is shown, with inset showing voltage profiles. In last two figures, closed black symbols indicate discharge capacity, open black indicate charge capacity. The red triangles denote coulombic efficiency. 
Oxide (LTO) and Lithium Iron Phosphate (LFP) as the cathode. For these studies, CNPC membranes were soaked in the commonly used EC: DEC (1v: 1v) electrolyte solvent mixture containing $1 \mathrm{M} \mathrm{LiPF}_{6}$ salt. Again the CNPC electrolytes exhibited $\mathrm{mS} / \mathrm{cm}$ level room-temperature ionic conductivities, allowing the batteries to be operated at room temperature. For practical applications, it is important for a battery to have high capacity for large number of cycles, even at high current densities. Figure 5.4(b) reports the cycling performance of $\mathrm{Li}\left|\mathrm{CNPC}+\mathrm{EC}: \mathrm{DEC} 1 \mathrm{MLiPF}_{6}\right| \mathrm{LTO}$ cells at a current density of $0.5 \mathrm{~mA} / \mathrm{cm}^{2}$. The batteries retain high capacity for at least 150 cycles. Figure 5.4(c) reports the performance of a battery where we paired metallic lithium with a LFP cathode in the CNPC electrolyte. At a current density of $0.25 \mathrm{~mA} / \mathrm{cm}^{2}$, the battery retains a capacity of over $120 \mathrm{mAh} / \mathrm{cm}^{2}$ upto 150 cycles. The inset of the figures shows the voltage profiles of the respective cells exhibit clear plateaus and low IR losses. These results clearly show that the CNPC electrolytes have good compatibility with lithium metal and with conventional high-performance intercalating cathodes and anodes. This makes them promising candidate materials for LMBs in which a metallic lithium anode is paired with conventional intercalating cathodes.

\subsection{Discussion}

Our findings provide a novel pathway towards nanostructured membranes in which chemistry introduced on the surface of nanoparticles can be internalized in the pores for regulating ion and mass transport. Here we illustrate the approach using hairy nanoparticles that can be cross-linked with rigid polymers to create ion-conducting 
membranes with good mechanical properties. Because each particle is functionalized with up to 55 reactive groups, this allows one to achieve highly cross-linked materials at very low particle contents. This makes it possible to create electrolyte membranes with both high mechanical modulus $\left(G_{N}=1 \mathrm{MPa}\right)$ and high, liquid-like ionic conductivity $\left(\sigma_{\mathrm{o}}=5 \mathrm{mS} / \mathrm{cm}\right)$ at ambient temperature, where traditional high modulus electrolyte systems fail to maintain high conductivity. The cross-linked polymer nanoparticle composite (CNPC) electrolytes are shown to be exceptionally effective in promoting smooth, dendrite-free electrodeposition of lithium metal at intermediate current densities. Comparison of the lithium metal anode lifetimes achievable in the new materials with those reported for other polymer, block-copolymer, cross-linked polymers and polymer-nanoparticle composites show that CNPC systems are the most promising for room-temperature LMB systems. Further, it is shown that these materials work efficiently in LMBs based on low-voltage LTO and intermediate voltage LFP cathodes, where they can be reversibly cycled with high discharge capacity for over 150 cycles, at high current densities.

\section{Acknowledgments}

This work was supported by the National Science Foundation, Award No. DMR1006323 and by Award No. KUS-C1018-02, made by King Abdullah University of Science and Technology (KAUST). Small-angle X-ray Scattering facilities available through the Cornell High Energy Synchotron Source (CHESS) were used in the study. CHESS is supported by the NSF \& NIH/NIGMS via NSF award DMR-1332208. 


\section{REFERENCES}

1. Armand, M. \& Tarascon, J.-M. Building better batteries. Nature 451, 652-7 (2008).

2. Dresselhaus, M. S. \& Thomas, I. L. Alternative energy technologies. Nature 414, 332-7 (2001).

3. Dunn, B., Kamath, H. \& Tarascon, J.-M. Electrical energy storage for the grid: a battery of choices. Science 334, 928-35 (2011).

4. Tarascon, J. M. \& Armand, M. Issues and challenges facing rechargeable lithium batteries. Nature 414, 359-67 (2001).

5. Kim, H., Jeong, G., Kim, Y.U., Kim, J.H., Parke, C.M., and Sohn, H.J., Metallic anodes for next generation secondary batteries. Chem. Soc. Rev. 42, 9011-34 (2013).

6. Xu, W. et al. Lithium metal anodes for rechargeable batteries. Energy Environ. Sci. 7, 513 (2014).

7. Pieczonka, N. P. W. et al. Impact of lithium bis(oxalate)borate electrolyte additive on the performance of high-voltage spinel/graphite Li-ion batteries. $J$. Phys. Chem. C 117, 22603-22612 (2013). 
8. Li, B., Xu, M., Li, T., Li, W. \& Hu, S. Prop-1-ene-1,3-sultone as SEI formation additive in propylene carbonate-based electrolyte for lithium ion batteries. Electrochem. commun. 17, 92-95 (2012).

9. Aurbach, D. et al. On the use of vinylene carbonate (VC) as an additive to electrolyte solutions for Li-ion batteries. Electrochim. Acta 47, 1423-1439 (2002).

10. Lu, Y., Das, S. K., Moganty, S. S. \& Archer, L. A. Ionic liquid-nanoparticle hybrid electrolytes and their application in secondary lithium-metal batteries. Adv. Mater. 24, 4430-5 (2012).

11. Lu, Y., Korf, K., Kambe, Y., Tu, Z. \& Archer, L. A. Ionic-Liquid-Nanoparticle Hybrid Electrolytes: Applications in Lithium Metal Batteries. Angew. Chemie 126, 498-502 (2014).

12. Bouchet, R. et al. efficient electrolytes for lithium-metal batteries. Nat. Mater. 12, 452-457 (2013).

13. Gurevitch, I. et al. Nanocomposites of Titanium Dioxide and PolystyrenePoly(ethylene oxide) Block Copolymer as Solid-State Electrolytes for Lithium Metal Batteries. J. Electrochem. Soc. 160, A1611-A1617 (2013).

14. Khurana, R., Schaefer, J. L., Archer, L. A \& Coates, G. W. Suppression of lithium dendrite growth using cross-linked polyethylene/poly(ethylene oxide) 
electrolytes: a new approach for practical lithium-metal polymer batteries. $J$. Am. Chem. Soc. 136, 7395-402 (2014).

15. Tu, Z., Kambe, Y., Lu, Y. \& Archer, L. A. Nanoporous Polymer-Ceramic Composite Electrolytes for Lithium Metal Batteries. Adv. Energy Mater. 4, n/a$\mathrm{n} / \mathrm{a}(2014)$.

16. Tikekar, M. D., Archer, L. A. \& Koch, D. L. Stability Analysis of Electrodeposition across a Structured Electrolyte with Immobilized Anions. $J$. Electrochem. Soc. 161, A847-A855 (2014).

17. Fuller, J., Breda, A. C. \& Carlin, R. T. Ionic Liquid-Polymer Gel Electrolytes. J. Electrochem. Soc. 144, 8-11 (1997).

18. Zhang, J., Sun, B., Huang, X., Chen, S. \& Wang, G. Honeycomb-like porous gel polymer electrolyte membrane for lithium ion batteries with enhanced safety. Sci. Rep. 4, 6007 (2014).

19. Stephan, A. M. Review on gel polymer electrolytes for lithium batteries. Eur. Polym. J. 42, 21-42 (2006).

20. Cheng, X.-B., Peng, H.-J., Huang, J.-Q., Wei, F. \& Zhang, Q. Dendrite-Free Nanostructured Anode: Entrapment of Lithium in a 3D Fibrous Matrix for Ultra-Stable Lithium-Sulfur Batteries. Small 1-7 (2014). 
21. Hallinan, D. T., Mullin, S. A., Stone, G. M. \& Balsara, N. P. Lithium Metal Stability in Batteries with Block Copolymer Electrolytes. J. Electrochem. Soc. 160, A464-A470 (2013).

22. Hallinan, D. T. \& Balsara, N. P. Polymer Electrolytes. Annu. Rev. Mater. Res. 43, 503-525 (2013).

23. Singh, M. et al. Effect of Molecular Weight on the Mechanical and Electrical Properties of Block Copolymer Electrolytes. Macromolecules 4578-4585 (2007).

24. Croce, F., Appetecchi, G. B., Persi, L. \& Scrosati, B. Nanocomposite polymer electrolytes for lithium batteries. Nature 394, 456-458 (1998).

25. Tang, C., Hackenberg, K., Fu, Q., Ajayan, P. M. \& Ardebili, H. High Ion Conducting Polymer Nanocomposite Electrolytes Using Hybrid Nanofillers. Nano Lett. 1152-1156 (2012).

26. Bertasi, F. et al. Single-Ion-Conducting Nanocomposite Polymer Electrolytes for Lithium Batteries Based on Lithiated-Fluorinated-Iron Oxide and Poly(ethylene glycol) 400. Electrochim. Acta (2015).

27. Agrawal, A., Choudhury, S. \& Archer, L. A. A highly conductive, nonflammable polymer-nanoparticle hybrid electrolyte. $R S C A d v, \mathbf{5 , 2 0 8 0 0 . ~ ( 2 0 1 5 ) . ~}$ 
28. Croce, F., Sacchetti, S. \& Scrosati, B. Advanced, lithium batteries based on high-performance composite polymer electrolytes. J. Power Sources 162, 685689 (2006).

29. Balazs, A. C., Emrick, T. \& Russell, T. P. Nanoparticle polymer composites: where two small worlds meet. Science 314, 1107-10 (2006).

30. Krishnamoorti, R. Strategies for Dispersing Nanoparticles in Polymers. MRS Bull. 32, (2007).

31. Smith, G. D. \& Bedrov, D. Dispersing nanoparticles in a polymer matrix: are long, dense polymer tethers really necessary? Langmuir 25, 11239-43 (2009).

32. Chandran, S., Begam, N., Padmanabhan, V. \& Basu, J. K. Confinement enhances dispersion in nanoparticle-polymer blend films. Nat. Commun. 5, 3697 (2014).

33. Patra, T. K. \& Singh, J. K. Polymer directed aggregation and dispersion of anisotropic nanoparticles. Soft Matter 10, 1823-30 (2014).

34. Srivastava, S., Agarwal, P. \& Archer, L. A. Tethered nanoparticle-polymer composites: phase stability and curvature. Langmuir 28, 6276-81 (2012).

35. Litschauer, M., Peterlik, H. \& Neouze, M.-A. Nanoparticles/Ionic Linkers of Different Lengths: Short-Range Order Evidenced by Small-Angle X-ray Scattering. J. Phys. Chem. C 113, 6547-6552 (2009). 
36. Litschauer, M., Puchberger, M., Peterlik, H. \& Neouze, M.-A. Anion metathesis in ionic silica nanoparticle networks. J. Mater. Chem. 20, 1269 (2010).

37. Moganty, S. S. et al. Ionic Liquid-Tethered Nanoparticle Suspensions: A Novel Class of Ionogels. Chem. Mater. 24, 1386-1392 (2012).

38. Stone, G. M. et al. Resolution of the Modulus versus Adhesion Dilemma in Solid Polymer Electrolytes for Rechargeable Lithium Metal Batteries. J. Electrochem. Soc. 159, A222-A227 (2012).

39. Liu, S. et al. Lithium Dendrite Formation in Li/Poly(ethylene oxide)-Lithium Bis(trifluoromethanesulfonyl)imide and N-Methyl-N-propylpiperidinium Bis(trifluoromethanesulfonyl)imide/Li Cells. J. Electrochem. Soc. 157, A1092 (2010).

40. Liu, S. et al. Effect of nano-silica filler in polymer electrolyte on Li dendrite formation in Li/poly(ethylene oxide)-Li(CF3SO2)2N/Li. J. Power Sources 195, 6847-6853 (2010).

41. Schaefer, J. L., Moganty, S. S., Yanga, D. A. \& Archer, L. A. Nanoporous hybrid electrolytes. J. Mater. Chem. 21, 10094 (2011).

42. Georén, P. \& Lindbergh, G. On the use of voltammetric methods to determine electrochemical stability limits for lithium battery electrolytes. J. Power Sources 124, 213-220 (2003). 
43. Lu, Y., Tu, Z. \& Archer, L. A. Stable lithium electrodeposition in liquid and nanoporous solid electrolytes. Nat. Mater. 13, 961-969 (2014).

44. Zheng, G. et al. Interconnected hollow carbon nanospheres for stable lithium metal anodes. Nat. Nanotechnol. 9, 618-623 (2014).

45. Luo, W. et al. A Thermally Conductive Separator for Stable Li Metal Anodes. Nano Lett. 15 (9), 6149-6154 (2015).

46. Li, W. et al. The synergetic effect of lithium polysulfide and lithium nitrate to prevent lithium dendrite growth. Nat. Commun. 6, 7436 (2015).

47. Pires, J. et al. Role of propane sultone as additive to improve the performance of lithium-rich cathode material at high potential. $R S C A d v$. 5, 42088-42094 (2015).

48. Guo, J., Wen, Z., Wu, M., Jin, J. \& Liu, Y. Vinylene carbonate-LiNO3: A hybrid additive in carbonic ester electrolytes for SEI modification on Li metal anode. Electrochem. commun. 51, 59-63 (2015).

49. Qian, J. et al. High rate and stable cycling of lithium metal anode. Nat. Commun. 6, 6362 (2015).

50. Ding, F. et al. Dendrite-free lithium deposition via self-healing electrostatic shield mechanism. J. Am. Chem. Soc. 135, 4450-6 (2013). 
51. Rosso, M., Gobron, T., Brissot, C., Chazalviel, J.-N. \& Lascaud, S. Onset of dendritic growth in lithium/polymer cells. J. Power Sources 97-98, 804-806 (2001).

52. Liu, S. et al. Effect of co-doping nano-silica filler and N-methyl-Npropylpiperidinium bis(trifluoromethanesulfonyl)imide into polymer electrolyte on $\mathrm{Li}$ dendrite formation in $\mathrm{Li} /$ poly(ethylene oxide)-Li(CF3SO2)2N/Li. J. Power Sources 196, 7681-7686 (2011).

53. Sannier, L., Bouchet, R., Rosso, M. \& Tarascon, J. M. Evaluation of GPE performances in lithium metal battery technology by means of simple polarization tests. J. Power Sources 158, 564-570 (2006).

54. Schaefer, J. L., Yanga, D. A. \& Archer, L. A. High Lithium Transference Number Electrolytes via Creation of 3-Dimensional, Charged, Nanoporous Networks from Dense Functionalized Nanoparticle Composites. Chem. Mater. 25, 834-839 (2013). 


\section{APPENDIX}

Supplementary Information for Chapter 5

\section{Supplementary Figures}

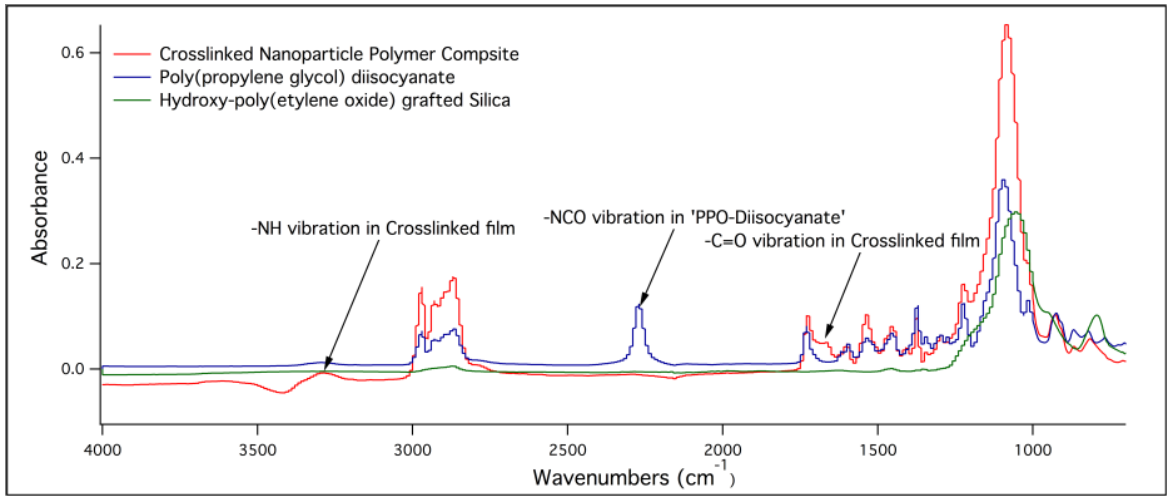

\section{Supplementary Fig. 5.1: Fourier Transform- Infrared Spectroscopy (FTIR)}

Characterization of the Reaction Scheme: The reaction process is confirmed by the IR-peak transition involved in urethane reaction. The $-\mathrm{NCO}$ peak of the PPO Diisocyanate disappears, while characteristic peaks of $-\mathrm{NH}$ vibration and shift in the $\mathrm{C}=\mathrm{O}$ peak appear because of the urethane bond formation in the final product. 


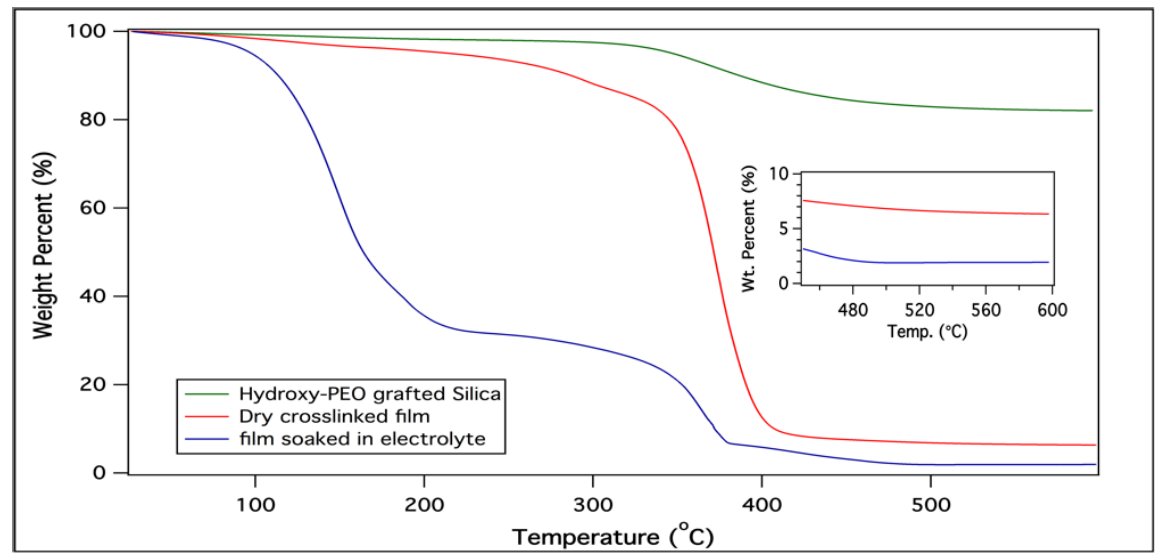

Supplementary Fig. 5.2: TGA Analysis: The initial silica content in the hairy nanoparticle (Si-PEO) was $83 \%$, which reduces to $6 \%$ silica in the final product of crosslinked film. Thus, the non-conducting entity in the entire material is remarkably low, in-spite of having decent mechanical strength. Further soaking the CNPC in 1M electrolytes comprising of PC-LiTFSI, reduces the silica content close to $2 \%$, as shown in the inset of the figure. Also, it is evident that the film is remarkably stable, such that there is degradation of the product up-to $120^{\circ} \mathrm{C}$, where PC starts to degrade. 


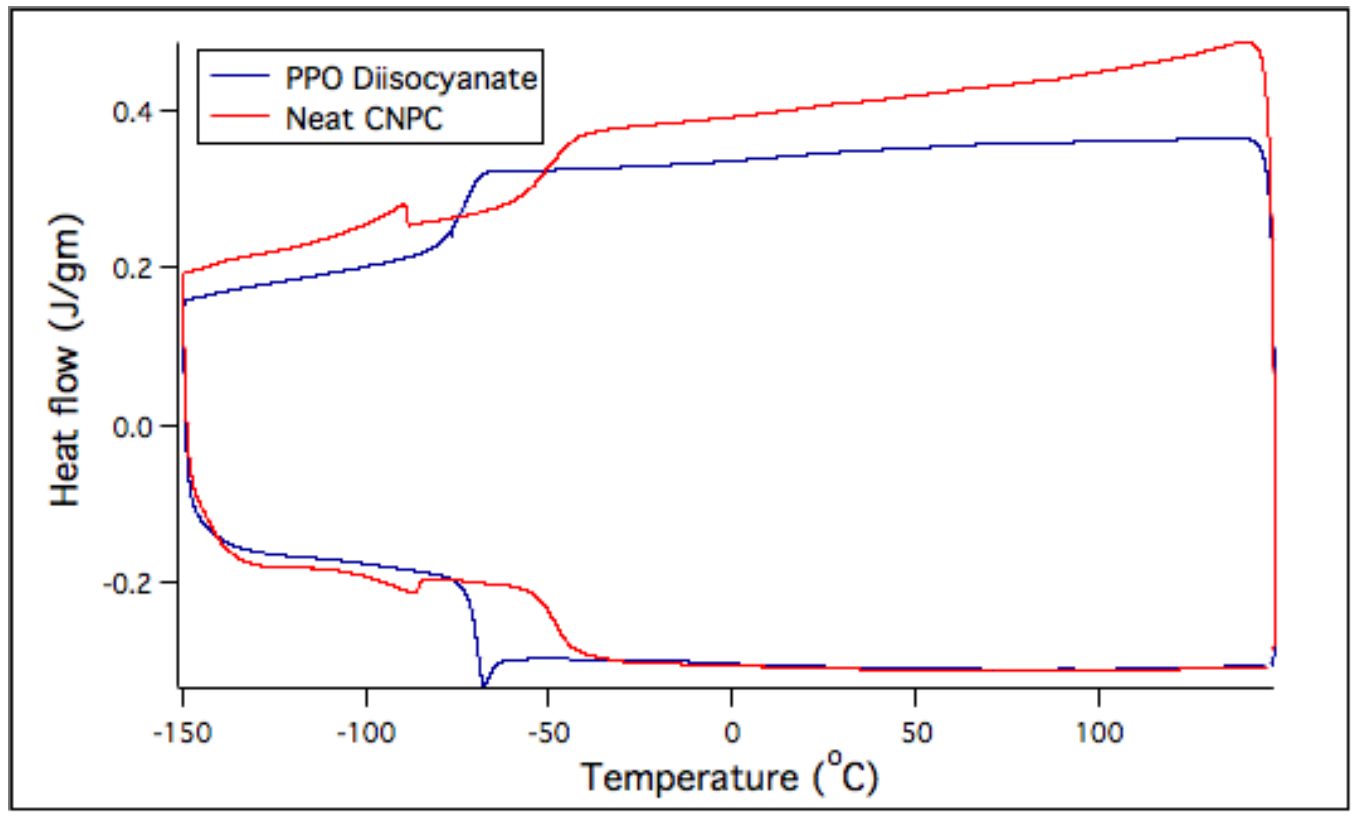

Supplementary Fig. 5.3: DSC Characterization of: The glass transition temperature of the PPO polymer increases from $-63^{\circ} \mathrm{C}$ to $-42^{\circ} \mathrm{C}$ due to the reduction of free volume as the chains are constricted due to the crosslinking. However, the crosslinked film is still in amorphous state at room temperature where it is used as electrolyte in battery systems. The amorphous nature of the polymer membrane enables higher ionic conductivity compared to other high MW PEO based electrolyte at room temperature. 


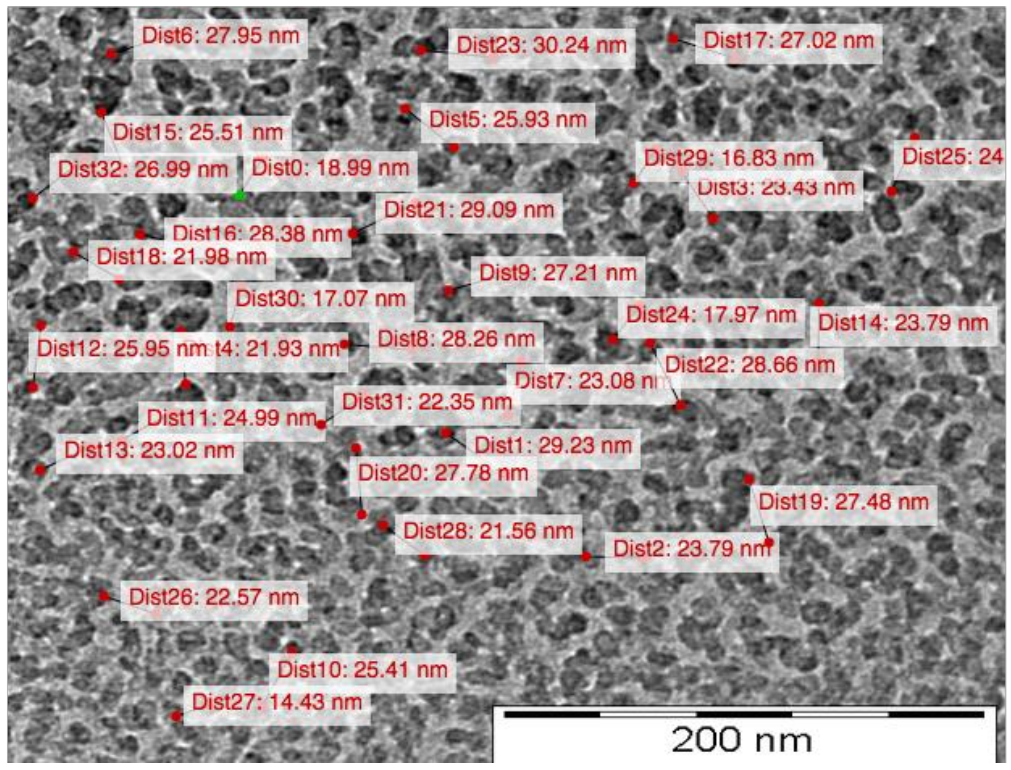

Supplementary Fig. 5.4: TEM Analysis: The inter-particle spacing in the crosslinked polymer is estimated by graphical analysis of TEM micrograph. 

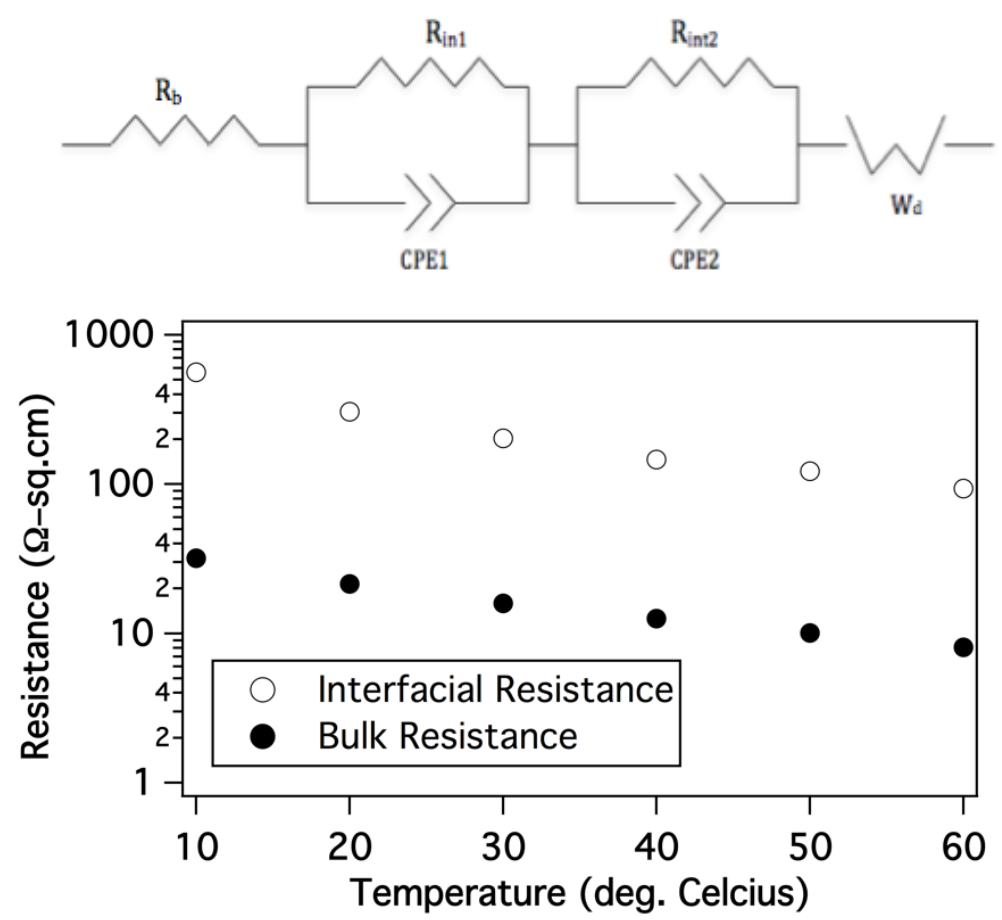

Supplementary Fig. 5.5: Equivalent Electric Circuit for the Impedance Spectroscopy results: The Nyquist plot obtained by the measurement of the Impedance at a wide range of frequency can be fitted by an equivalent circuit shown above. The bulk resistance and the interfacial resistance, thus obtained are plotted against temperature; and it is seen that the interfacial resistance is always higher than the bulk resistance, which indicates interface limited ion transfer. 
(a)

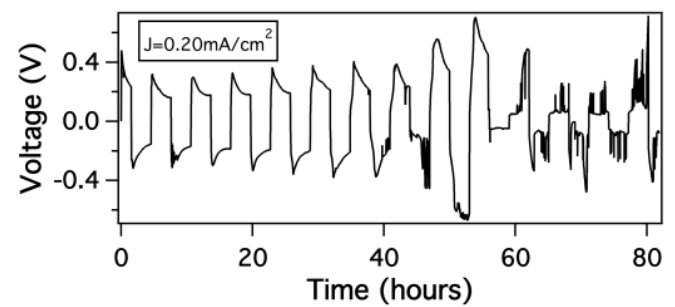

b

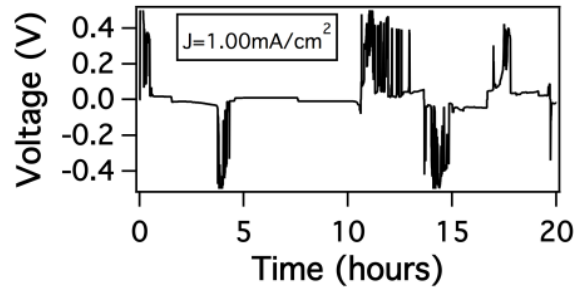

Supplementary Fig. 5.6: Strip-Plate measurement of a symmetric Lithium cell without CNPC separator: The voltage profile of a control cell $(\mathrm{Li} / \mathrm{PC}+\mathrm{LiTFSI} / \mathrm{Li})$ is plotted against time. a, it is seen that at current density of $0.20 \mathrm{~mA} / \mathrm{cm} 2$ about 55 hours of charging-discharging, the voltage profile gets distorted and the voltage range drops down which is a signature of dendrite induced short circuit. $\mathbf{b}$, at current density of $1.00 \mathrm{~mA} / \mathrm{cm} 2$, the voltage profile is unstable even at the start of cycle. 


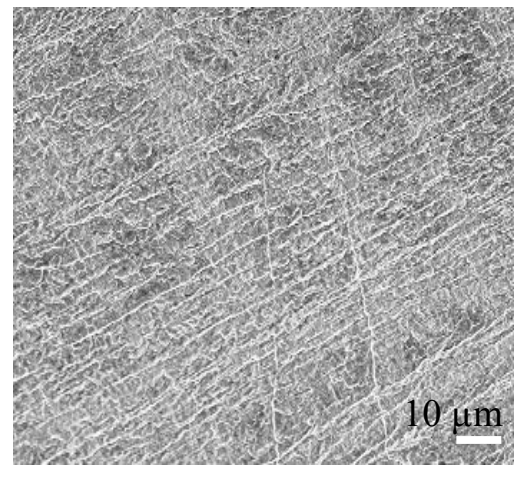

Supplementary Fig. 5.7: SEM image of Pristine Lithium: Surface of Lithium is presented in order to compare the changes in surface morphology after cycling using neat and crosslinked gel based electrolytes. (Scale bar is 10 microns) 


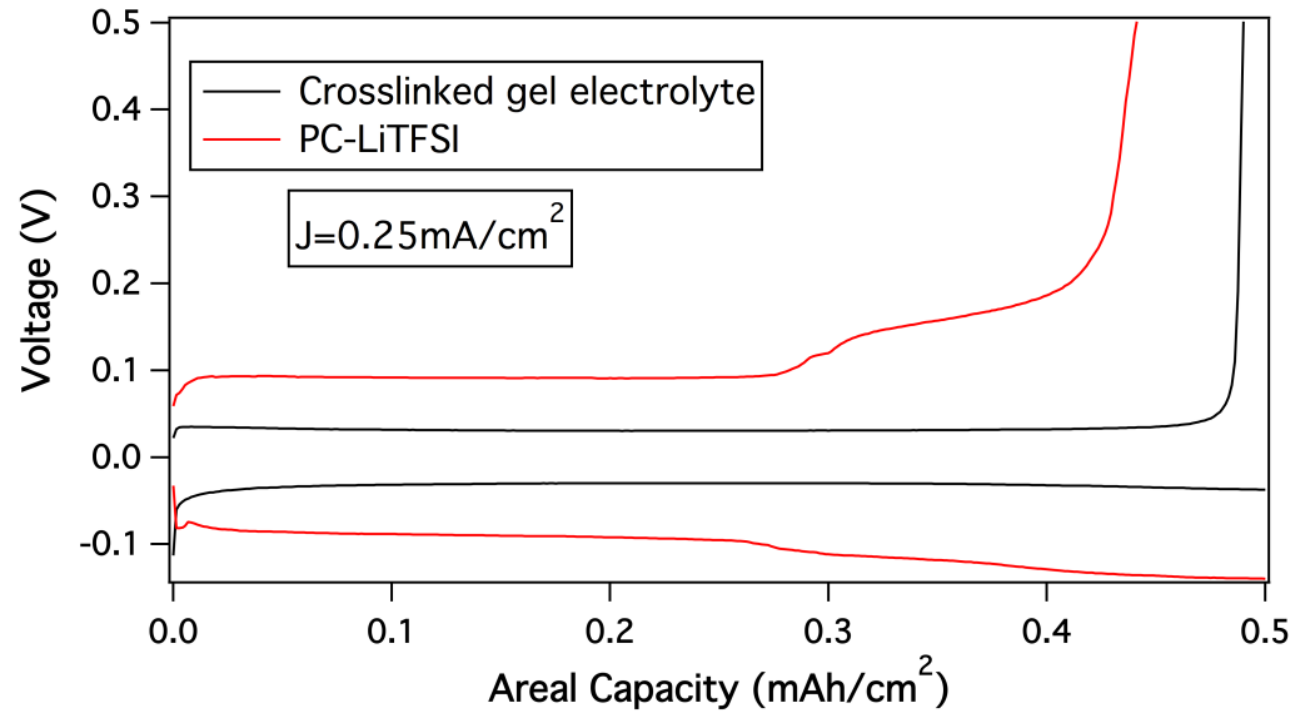

Supplementary Fig. 8: Coulombic Efficiency Test using Li| electrolyte| Stainless Steel configuration showing $40^{\text {th }}$ cycle: Batteries with crosslinked gel electrolyte and pristine PC-LiTFSI were cycled at $0.25 \mathrm{~mA} / \mathrm{cm}^{2}$. It is seen that at the $40^{\text {th }}$ cycle, the neat electrolyte exhibits deposition at a much lower voltage and also low coulombic efficiency compared to the crosslinked gel electrolyte. 


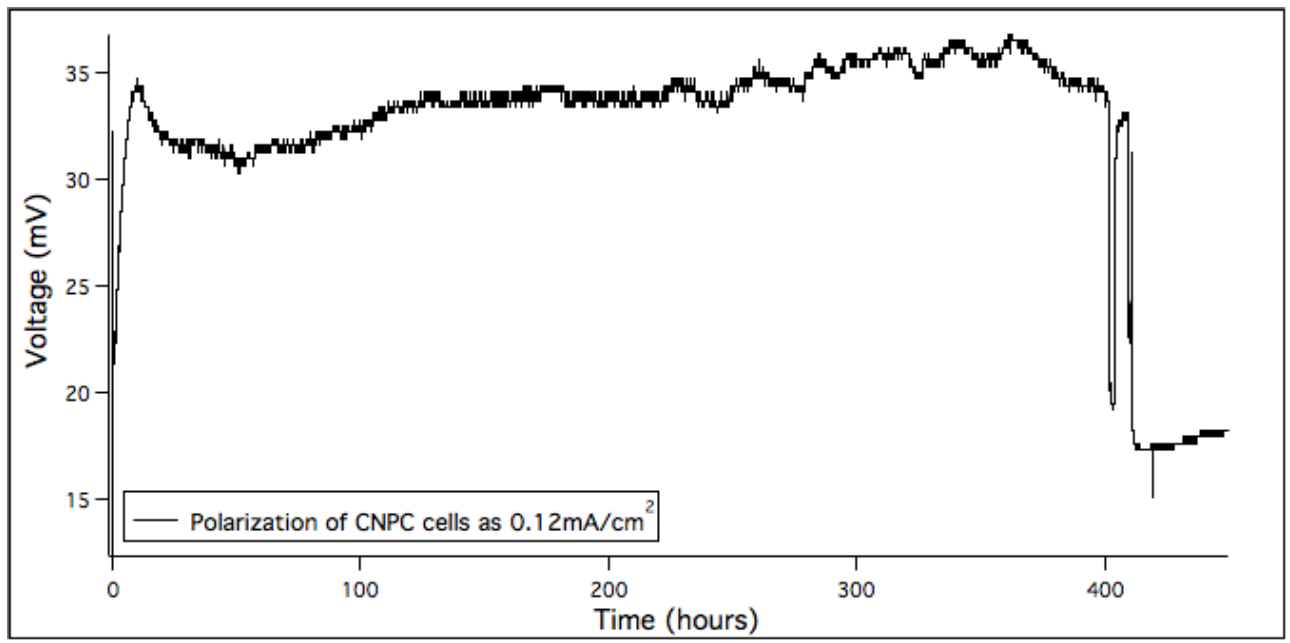

\section{Supplementary Fig. 5.9: Polarization curve of symmetric Lithium cell with} crosslinked gel electrolyte: A symmetric Lithium cell consisting of crosslinked gel electrolyte is charged constantly at a current density of $0.12 \mathrm{~mA} / \mathrm{cm}^{2}$. It is seen that the cell successfully depositing Li ion onto the anode surface for about 400 hours before failing, indicated by drop in the voltage profile. 


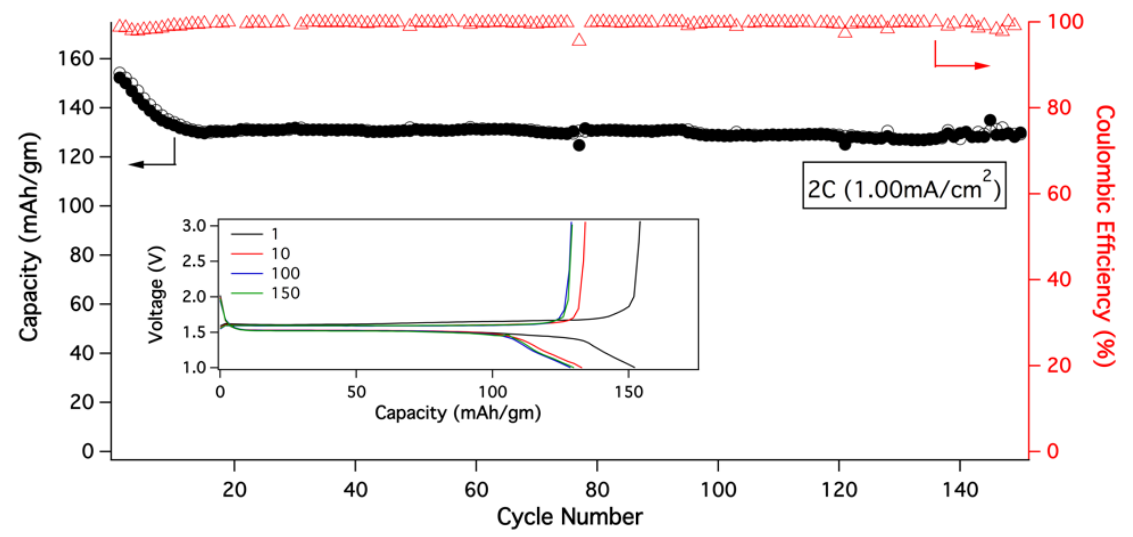

Supplementary Fig. 5.10: Cycling Performance using LTO cathode: It is seen that using the crosslinked gel electrolyte, a LTO based battery cycles well for over 150 cycles at a high current density of $1 \mathrm{~mA} / \mathrm{cm}^{2}$ 


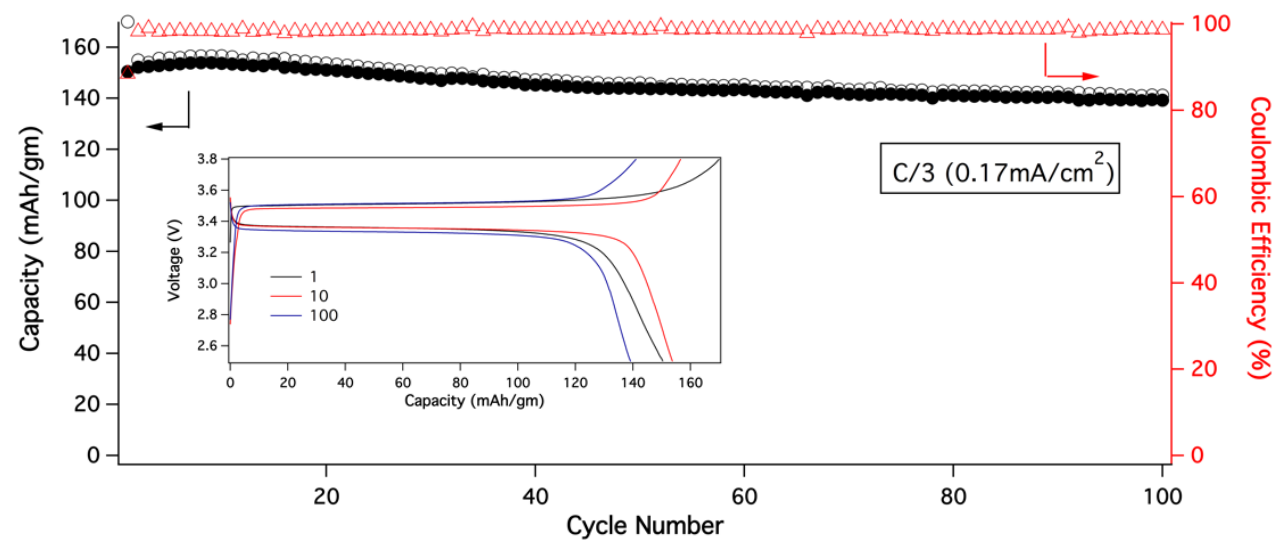

Supplementary Fig. 5.11: Cycling Performance of $\mathrm{LiFePO}_{4}$ battery using crosslinked gel electrolyte: It is seen that at a C-rate of $\mathrm{C} / 3$, the battery cycles with minimum fade for at least 100 cycles. 


\section{Supplementary Tables}

\section{Supplementary Table 5.1: Content of different components at successive}

synthesis stage: The weight percent of the Silica, PEO, PPO, electrolyte is given in table. It is seen that ultimately in the crosslinked gel electrolyte contains as low as $2 \%$ silica, still having a relatively high mechanical modulus.

\begin{tabular}{|c|c|c|c|}
\hline Type of product & Si-PEO & Si-PEO-PPO & Si-PEO-PPO-Electrolyte \\
\hline Component & Wt. \% & Wt. \% & Wt. \% \\
\hline Silica & 83 & 6 & 2 \\
\hline PEO & 17 & 1.3 & 0.4 \\
\hline PPO & - & 92.7 & 30.9 \\
\hline Electrolyte & - & - & 66.7 \\
\hline
\end{tabular}


Supplementary Table T2: VFT parameters of the different electrolyte configuration: $\sigma=A \exp \left(-\mathrm{B} /\left(\mathrm{T}-\mathrm{T}_{\mathrm{O}}\right)\right)$, where $A$ is the pre-exponential factor corresponding to conductivity at infinite temperature, B is the Activation Energy and $T_{0}$ is the reference temperature.

\begin{tabular}{|c|c|c|c|}
\hline Parameters & $\begin{array}{c}\mathbf{A} \\
(\mathbf{S} / \mathbf{c m})\end{array}$ & $\begin{array}{c}\mathbf{B} \\
(\mathbf{K})\end{array}$ & $\begin{array}{c}\text { To } \\
(\mathbf{K})\end{array}$ \\
\hline LiPF6-EC/DEC & 0.045 & 56.9 & 237 \\
\hline LiTFSI-PC & 0.044 & 61 & 239 \\
\hline Crosslinked film-LiPF6-EC-DEC & 0.239 & 870.5 & 84.3 \\
\hline Crosslinked film-LiTFSI-PC & 0.237 & 891.5 & 85 \\
\hline
\end{tabular}




\section{Supplementary Methods:}

The size of clusters was obtained by fitting the SAXS data with Beaucage unified equation [1] as shown below.

$$
I(q)=A \exp \left(-\frac{q^{2} R_{p}^{2}}{3}\right)+B \operatorname{erf}\left(\frac{\left(\frac{q R_{p}}{\sqrt{6}}\right)^{3}}{q}\right)^{p_{1}}+\sum_{i} C_{i} \exp \left(-\frac{q^{2} R_{p}^{2}}{3}\right) \operatorname{erf}\left(\frac{\left(\frac{q R_{c, i}}{\sqrt{6}}\right)^{3}}{q}\right)^{p_{2, i}}
$$

First two terms (Guinier and power law) contribute to the scattering for spheres in a dilute suspension with radius $a=\sqrt{\frac{5}{3}} R_{p} \sim 4-5 \mathrm{~nm}$ and power law exponent $p_{1}(\sim 4)$. A and $\mathrm{B}$ are the Guinier and Porod scaling factors. The last term contributes to the scattering from the fractal objects in the low $q$ regime with $R_{\text {fractal }}=\sqrt{\frac{5}{3}} R_{c} \sim 51-72 \mathrm{~nm}$ with a power exponent $p_{2} \sim 2$, indicating the fractals to be mass fractals. Since the low q regime has only the power law scattering no Guinier term has been included suggesting that the $R_{\text {fractal }}$ obtained from the fitting will be the lower bound as exact dimension cannot be determined. $\mathrm{C}$ is the pre-factor factor for the power law scattering in the low $q$. Absence of any additional structure contribution in intermediate to high $q$ suggests that the particles are reasonably far apart.

\section{Supplementary References}

1. Beaucage, G., Small-angle scattering from polymeric mass fractals of arbitrary massfractal dimension. Journal of Applied Crystallography 29 (2), 134-146 (1996) 


\section{CHAPTER 6}

\section{CONFINING ELECTRODEPOSITION OF METALS IN STRUCTURED}

\section{ELECTROLYTES}




\subsection{Abstract}

Electrochemical cells based on alkali metal ( $\mathrm{Li}, \mathrm{Na}$ ) anodes have attracted significant recent attention because of their promise for producing large increases in gravimetric energy density for energy storage in batteries. To facilitate stable, long-term operation of such cells a variety of structured electrolytes have been designed in different physical forms, ranging from soft polymer gels to hard ceramics, including porous ceramics that host a liquid or molten polymer in their pores. In almost every case, the electrolytes are reported to be substantially more effective than anticipated by early theories in improving uniformity of deposition and lifetime of the metal anode. These observations have been speculated to reflect the effect of electrolyte structure in regulating ion transport to the metal electrolyte interface, thereby stabilizing metal electrodeposition processes at the anode. Here, we create, and study model structured electrolytes composed of covalently linked polymer grafted nanoparticles that host a liquid electrolyte in the pores. The electrolytes exist as free-standing membranes with effective pore-size that can be systematically manipulated through straightforward control of the volume fraction of the nanoparticles. By means of physical analysis and direct visualization experiments we report that at current densities approaching the diffusion limit, there is a clear transition from unstable to stable electrodeposition at $\mathrm{Li}$ metal electrodes in membranes with average pore sizes below $500 \mathrm{~nm}$. We show that this transition is consistent with expectations from a recent theoretical analysis that takes into account local coupling between stress and ion transport at metal-electrolyte interfaces. 


\subsection{Significance}

Electrochemical cells based on lithium and sodium metal anodes are considered among the most versatile platforms for high-energy electrical energy storage. Unfortunately, unstable dendritic metal deposition at currents below the diffusion limit lead to premature cell failure by internal short-circuits. In a volatile electrolyte, the ohmic heat generated by these shorts pose significant safety risks. The manuscript reports that electrodeposition of metals can be stabilized by confining ion transport to length scales below a few hundred nanometers. It is also shown that dendrite growth can be arrested in electrolytes with mechanical moduli well below that of the metal. This finding contradicts current orthodoxy, which holds that solid-state electrolytes with moduli higher than the metal are required for preventing dendrite growth.

\subsection{Introduction}

Rechargeable electrochemical cells based on alkali metal anodes provide opportunities to substantially push the energy storage limits of batteries. Such cells achieve this feat both by increasing the amount of electrical energy that can be stored on a mass or volume basis at the anode and by enabling more flexible material choices for the cathode. ${ }^{1-6}$ Parasitic reactions between the chemically reactive anode ${ }^{7-11}$ and proliferation of rough, dendritic/mossy electrodeposition at the metal anode during battery recharge have been reported to reduce stability of the cells by increasing the likelihood of failure by internal short circuits and by increasing the surface area of reactive metals in contact with electrolytes. ${ }^{12,13}$ 
The stability analysis of electrodeposition reported by Monroe and Newman ${ }^{14,15}(\mathrm{MN})$ is to our knowledge the first to employ continuum modeling of ion transport processes and mechanics in bulk electrolytes to predict morphological transitions at a metal anode. This work showed that marginal stability is achieved at any current density in an electrolyte with mechanical modulus about twice that of the metal electrode. One study using block copolymer electrolytes based on hard polystyrene and ion conductive poly(ethylene oxide) segments to systematically adjust the electrolyte modulus provided early support for this prediction. ${ }^{16-19}$ This finding, however, stands in contrast to a large and growing body of recent experimental reports which show that rough deposition of metals can be inhibited in gel-like electrolytes ${ }^{20,21}$, cross-linked polymer electrolytes ${ }^{18,22,23}$ and in ion conducting polymer electrolytes, ${ }^{24-26}$ all with mechanical moduli three or more orders of magnitude below that of the metal electrode. Recently, Zhao et al. ${ }^{27}$ showed that a composite polymer electrolyte with immobilized ions and ceramic fillers can indeed inhibit dendrites at ambient conditions. Even more dramatic are reports which show that dendrite-induced shortcircuits in lithium and sodium batteries can be delayed or even eliminated at low and moderate current densities in liquid electrolytes in which a fixed fraction of anions is maintained at the interface, ${ }^{28-33}$ or in liquid electrolytes in which halide ions are present at the electrode-electrolyte interface. ${ }^{11,34-37}$ Essentially nothing is known about the working mechanisms by which such low-modulus materials prevent metal dendrite proliferation at either low or high current densities, relative to the diffusion limiting current $J^{*}$, and a variety of qualitative arguments have been proposed to explain their working mechanism. 
The present study is motivated by recent theoretical work by Tikekar et al., ${ }^{7,38}$ which extended the $\mathrm{MN}$ analysis to consider the effect of local stress-transport coupling with ion migration profiles and the impact such coupling has on stability of metal electrodeposition. This analysis shows that at current densities below $J^{*}$, stable electrodeposition of metals can be achieved under a broad range of conditions, including in electrolytes with shear moduli as low as $0.1 \mathrm{MPa}$ (i.e. more than four orders of magnitude lower than that of the metal electrode). Another conclusion from the analysis is that electrolytes created by hosting simple liquids in porous materials should be able to prevent rough electrodeposition of any metal when the average pore size of the host falls-below a certain critical electrolyte-dependent value. This prediction is important because it means that solid-state electrolytes are not required to stabilize electrodeposition at metal anodes in batteries; it is also testable. Here, we design structured electrolytes based on cross-linked nanoparticle-polymer hybrid membranes in which the effective pore size can be facilely manipulated by changing the volume fraction of particles in the precursor material. We also use these electrolytes to systematically investigate the effect of electrolyte network structure and mechanical properties on the stability of metal deposition at currents close to the critical current density, $J^{*}$. The study takes advantage of an optical visualization technique that allows time-dependent changes in morphology of the metal-electrolyte interface to be directly imaged. Used in combination with the analysis of Tikekar et al. ${ }^{38}$, these efforts are shown to lead to a comprehensive understanding of how and why structured electrolytes that do not meet the MN modulus criteria are able to prevent metal dendrite proliferation. 


\subsection{Materials and Methods:}

\subsubsection{Materials}

Lithium rods, Ethylene Carbonate, Dimethylene carbonate, Lithium

Hexafluorophosphate, Anydrous Copper Chloride, Dimethyl Sulfoxide, Glass fibers

Ludox SM30 colloidal silica ( $\mathrm{d}=10 \pm 2 \mathrm{~nm})$, poly(propylene glycol)-toluene-2,4-

diisocyante were all purchased from Sigma Aldrich. Hydoxy terminated poly

(ethylene oxide)-silane was obtained from Gelest. Copper foil was obtained from Alfa

Aesar. All the chemicals were used as received in after rigorous drying in a $\sim 0 \mathrm{ppm}$

water level and $<5$ ppm oxygen glove box.

\subsubsection{Linear Stability Analysis}

The details regarding the linear stability analysis is presented in a previous paper by

Tikekar et al. ${ }^{38}$

\subsubsection{Crosslinked Hairy Nanoparticles Synthesis}

The reaction procedure for crosslinking between the grafted silica nanoparticles and functionalized PPO polymer is given in a previous work. ${ }^{18}$ Different crosslinked nanoparticles with varying pore sizes was synthesized by adjusting the ratio between the silica nanoparticles and the PPO polymer as given in the Supplementary Table 1. The ratio between the liquid electrolyte and the crosslinked composite was kept constant at $2: 1$ by weight.

\subsubsection{Dielectric Spectroscopy}

The dielectric spectroscopy measurements were done using a Novocontrol N40 Broadband Dielectric instrument. The crosslinked hairy nanoparticles as well as neat PPO (without liquid electrolyte) were casted at the center of a teflon o-ring. The 
measurements were done in a frequency range from $10^{-3}$ to $10^{7} \mathrm{~Hz}$ at various temperatures

\subsubsection{Transmission Electron Microscopy}

The TEM measurements were performed to understand the nanostructure of the crosslinked hairy nanoparticles using a FEI T12 Spirit TEM. A thin layer of the materials was casted onto the TEM grid using chloroform as the solvent and it was allowed to crosslink in the TEM grid. Before the measurements, the samples were dried overnight at $60^{\circ} \mathrm{C}$.

\subsubsection{Scanning Electron Microscopy}

SEM analysis was done using the LEO155FESEM instrument. In this experiment, symmetric lithium coin cell was constructed using the crosslinked electrolyte (r.c.p. $=$ $20 \mathrm{~nm}$ ). 'Plate-strip' measurements were performed at a current density of 0.1 $\mathrm{mA} / \mathrm{cm} 2$ for 100 hours, with each cycle comprising of 3 hours; thereafter the lithium electrode was extracted and dried in the ante-chamber of glove-box overnight.

\subsubsection{Mechanical Properties}

The crosslinking process was studied mechanically by casting the precursor materials in the rheometer with parallel-plate geometry of diameter $25 \mathrm{~mm}$ using a ARES Rheometer. Specifically, for time-sweep measurements, a constant strain amplitude of $5 \%$ and fixed frequency of $10 \mathrm{~Hz}$ was applied until the storage modulus reached a fixed value. The frequency sweep measurements were performed at a fixed strain amplitude of $5 \%$ between $1 \mathrm{~Hz}$ to $100 \mathrm{~Hz}$ for all materials. All the measurements were done without any addition of liquid electrolytes and at the same temperature value of $60^{\circ} \mathrm{C}$. 


\subsubsection{Direct Visualization experiments}

The visualization experiment was carried out for understanding the electrodeposition process with various forms of electrolytes mentioned in the manuscript. In all the measurements the ratio between the weight of the composite material and liquid electrolyte (1M LiPF 6 EC/DMC) was kept to be constant at 1:2. The details of the visualization cell for lithium deposition measurements is presented in a previous paper. ${ }^{10}$ The dendrite growth was done using a semi-automatic analysis in MATLAB, where the sizes of the focused dendrites are measured over time.

\subsection{Results}

Figure 6.1(a) shows the synthetic route used to create cross-linked hairy nanoparticlebased electrolytes. Briefly, silica nanoparticles (diameter $\sim 10 \mathrm{~nm}$ ) are first grafted in aqueous solution with an alkoxy silane terminated oligomeric polyethylene glycol $\left(\mathrm{PEO}-\mathrm{OH}, M_{\mathrm{w}}=500 \mathrm{~g} / \mathrm{mol}\right)$. As shown in our previous work, ${ }^{18,39,40}$ the approach produces $\mathrm{SiO}_{2}$ nanoparticles densely grafted $\left(\sum \approx 1\right.$ chain $/ \mathrm{nm}^{2}$; from Thermal gravimetric analysis (TGA)) with PEO chains bearing a reactive terminal hydroxyl group. The resultant $\mathrm{SiO}_{2}-\mathrm{PEO}-\mathrm{OH}$ nanoparticles are subsequently used as multifunctional node points for cross-linking poly(propylene oxide) (PPO, $M_{\mathrm{w}}=2000$ $\mathrm{g} / \mathrm{mol}$ ) chains functionalized with isocyanate groups at both ends. A straightforward calculation shows that on average there are $\sim 300$ PEO-OH molecules anchored to a single $\mathrm{SiO}_{2}$ nanoparticle, meaning that materials with a high degree of cross-linking are achieved. Additionally, because the PEO chains are short and the PPO linkers are amorphous at ambient temperatures, the cross-linked materials exist as free-standing 
membranes with high flexibility and toughness for silica content of $6.5 \%$ (by weight) (see Figure 6.1(b)). The large number of grafting sites available on each $\mathrm{SiO}_{2}$ nanoparticle makes it possible to introduce different functionalities, including ionic species $^{41}$ or flame retardants such as phosphates ${ }^{42}$ in the materials. Cross-linking these nanostructures provides a facile route to nanoporous membranes in which the surface chemistry of the pores can be manipulated for regulating the ion transport characteristics or for preventing thermal runway in lithium batteries. Furthermore, by varying the volume fraction of particles it is possible to systematically change the configuration of the particle-tethered PEO and PPO chains in the cross-linked materials to create membranes with vastly different pore sizes and mechanical properties.

It is possible to monitor the progress of the cross-linking reaction by measuring the time-dependent development of elasticity in the materials by means of dynamic shear rheological measurements (Figure 6.1(c)). Specifically, we employed oscillatory shear to continuously measure the storage and loss modulus of the initial slurry of PEOgrafted nanoparticles and PPO-diisocyanate chains at $60^{\circ} \mathrm{C}$ using a small-amplitude shear strain of $1 \%$ and a fixed oscillatory shear frequency of $10 \mathrm{~Hz}$. It is observed that the storage modulus increases over time indicative of the cross-linking process, which reaches its maximum value in about 24 hours. It is important to note that although the chemical reaction between an isocyanate and hydroxyl group is very fast, the overall cross-linking reaction kinetics are under diffusion control as it becomes progressively harder for PPO chains tethered to one particle to bond to a neighboring site on another particle to increase network elasticity. It can also be seen from Fig. 6.1(c) that the loss 
a

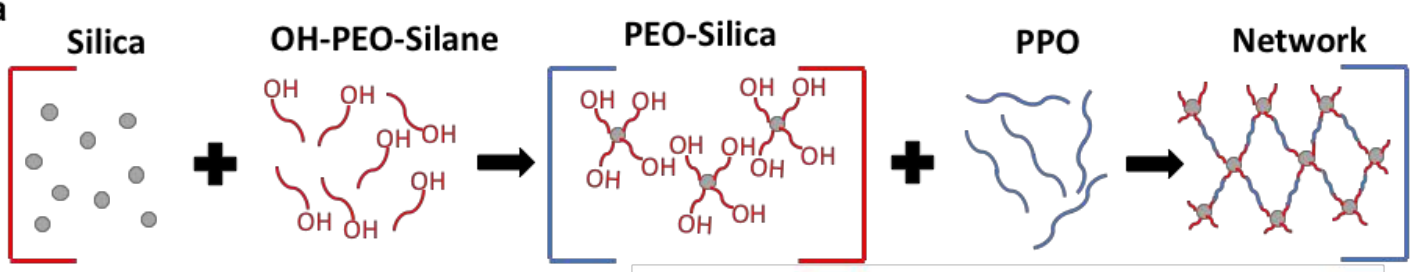

b

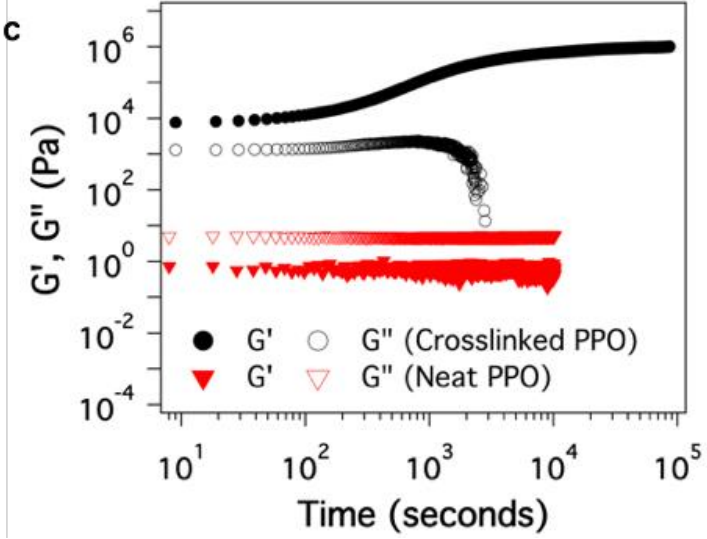

Figure 6.1: Synthesis Crosslinked Hairy Nanoparticles: (a) Schematic showing synthesis procedure of the crosslinked nanoparticles; (b) Photograph of the free-standing membrane with $6.8 \%$ (by weight) silica content; (c) Time sweep measurements under oscillatory shear at strain $1 \%$ and frequency of $10 \mathrm{~Hz}$ at $60^{\circ} \mathrm{C}$ 
modulus decreases and rolls over to very low values at long times, consistent with the gradual loss of dissipation processes as the network becomes more fully cross-linked. These effects are most dramatically demonstrated by comparison of the storage and loss moduli for the unlinked PPO polymer. It is seen that whereas the loss modulus dominates storage for the unlinked, liquid-like material, the storage modulus dominates as cross-linking nears completion. It is remarkable that at just $6.8 \mathrm{w} \%$ of $\mathrm{SiO}_{2}$ in the materials there is increment of over five orders of magnitude in modulus. More in-depth knowledge of the configuration and dynamics of the linker polymer chains is possible from dielectric relaxation measurements. It is known that polymers such as poly(propylene oxide) and cis-1,4-polyisoprene possess type-A dipole that facilitate alignment of the polymer chain end-to-end vector in the direction of an imposed electric field; thus one can quantify the end-to-end chain relaxation from the dielectric loss spectrum. ${ }^{43,44}$ Additionally, from the magnitude of the loss peak, one can calculate the dielectric strength, which for flexible, amorphous chains can be used to estimate the relative degree of chain stretch. ${ }^{45}$ Supplementary Figure 6.1(a), (b) show the loss permittivity of the neat PPO and cross-linked PPO at various frequencies and temperatures. The experimental data was fitted using Havriliak-Negami (H-N) equation: $\varepsilon^{*}(\omega)=\varepsilon_{\infty}+\Delta \varepsilon /\left[\left(1+(\mathrm{i} \omega \tau)^{\alpha}\right)^{\beta}\right]$, where $\alpha$ and $\beta$ are associated with broadening dielectric-peaks, $\tau$ represent the $\mathrm{H}-\mathrm{N}$ relaxation time, $\omega$ is frequency, $\varepsilon_{\infty}$ is dielectric constant $\left(\varepsilon^{\prime}\right)$ at high frequency limit, and $\Delta \varepsilon$ is the dielectric strength. The relaxation times at various temperature obtained from the $\mathrm{H}-\mathrm{N}$ fits are shown in Supplementary Figure 6.1(c). It can be seen that the relaxation time 
for all temperatures follow Vogel-Fulcher-Tammann (VFT) model, represented by the continuous lines in the figure, The VFT model is given as: $\tau=A$ $\exp \left(-\mathrm{B} / \mathrm{k}\left(\mathrm{T}-\mathrm{T}_{0}\right)\right)$, where, $A$ is the prefactor, $B$ is the activation energy and $T_{o}$ is reference temperature, shown in Supplementary Table 6.1. It can be seen that the activation energy of the relaxation dynamics of PPO polymer increases dramatically from $9.8 \mathrm{~kJ} /$ mole to $20 \mathrm{~kJ} /$ mole as a result of cross-linking, which is consistent with expectations based on the molecular weight of the polymers and high levels of crosslinking achieved. ${ }^{18}$ Comparison of the dielectric strength for the untethered and tethered PPO indicates that the linkers are also highly stretched (shown in Supplementary Figure 6.1(d)). For random coil type-A dielectric polymer chains, the dielectric strength $\Delta \varepsilon$ is proportional to the mean squared end-to-end vector of the polymer chain. The results in Supplementary Figure 6.1d) show that $\Delta \varepsilon$ measured using the cross-linked membranes is approximately 250 times larger than the corresponding value for the linear unlinked polymer. Considering that the molecular weight of the PPO chains is just $2 \mathrm{kDa}$, the large increases in $\Delta \varepsilon$ also imply that dipoles on the particle tethered chains are also highly correlated.

The cross-linked hairy nanoparticles provide an opportunity to understand the underlying physics of metal electrodeposition in heterogeneous environment, because these materials serve as model nanoporous structures such that the silica nanoparticles act as non-conductive physical barriers and the polymeric chains in the inter-particle gaps as pathways for ion migration. In this context, the volume fraction of silica nanoparticles in the entire composite was varied and the corresponding inter-particle distance was estimated by assuming random closed packing (r.c.p.) arrangement of 
spherical nanoparticles: $d_{p-p}=d\left[(0.63 / \Phi)^{1 / 3}-1\right.$, as reported in Supplementary Table 6.2 for different weight fraction of silica content. We utilized Transmission Electron Microscope (TEM) to analyze the spatial arrangement of the nanoparticles in the polymer composite as shown in Supplementary Figure 6.2(a) for r.c.p. pore-sizes of $20 \mathrm{~nm}, 100$ and $500 \mathrm{~nm}$. The interparticle distance of the silica nanoparticles was furthered measured from the TEM images and the corresponding variation was fitted to a Normal Distribution function obtained from the respective mean and standard deviations and plotted as inset of the images. The average interparticle distances measured from TEM is observed to be similar to r.c.p. pore sizes (Figure 6.2(a)). However, the variance of the distribution is seen to increase with decreasing silica volume fractions (Supplementary Figure 6.3). We furthered performed frequency sweep measurements at a constant amplitude of 5\% for different crosslinked PPO as well as neat PPO polymer. The storage and loss modulus of obtained from the measurements are plotted in Supplementary Figure 6.2(b) and (c), respectively. The results show that the composite materials at all crosslinking densities have higher G' than G”, indicating elastic behavior that is unseen at such low core volume fractions in colloidal suspensions. The elastic modulus is seen to progressively increase with increasing core volume fraction, the associated cage volume $(k T / G)$ is reported in Figure 6.2(a). Membranes with r.c.p. pore size $20 \mathrm{~nm}$ show the highest shear modulus of approximately $1 \mathrm{MPa}$, while in the uncross-linked state the materials are simple Newtonian liquid. The higher variance of spatial distribution of silica nanoparticles and reminiscence of elasticity at low core fractions indicate that the particles form hierarchical string-like mass fractals due to the fixed length of the crosslinker (PPO), 
a
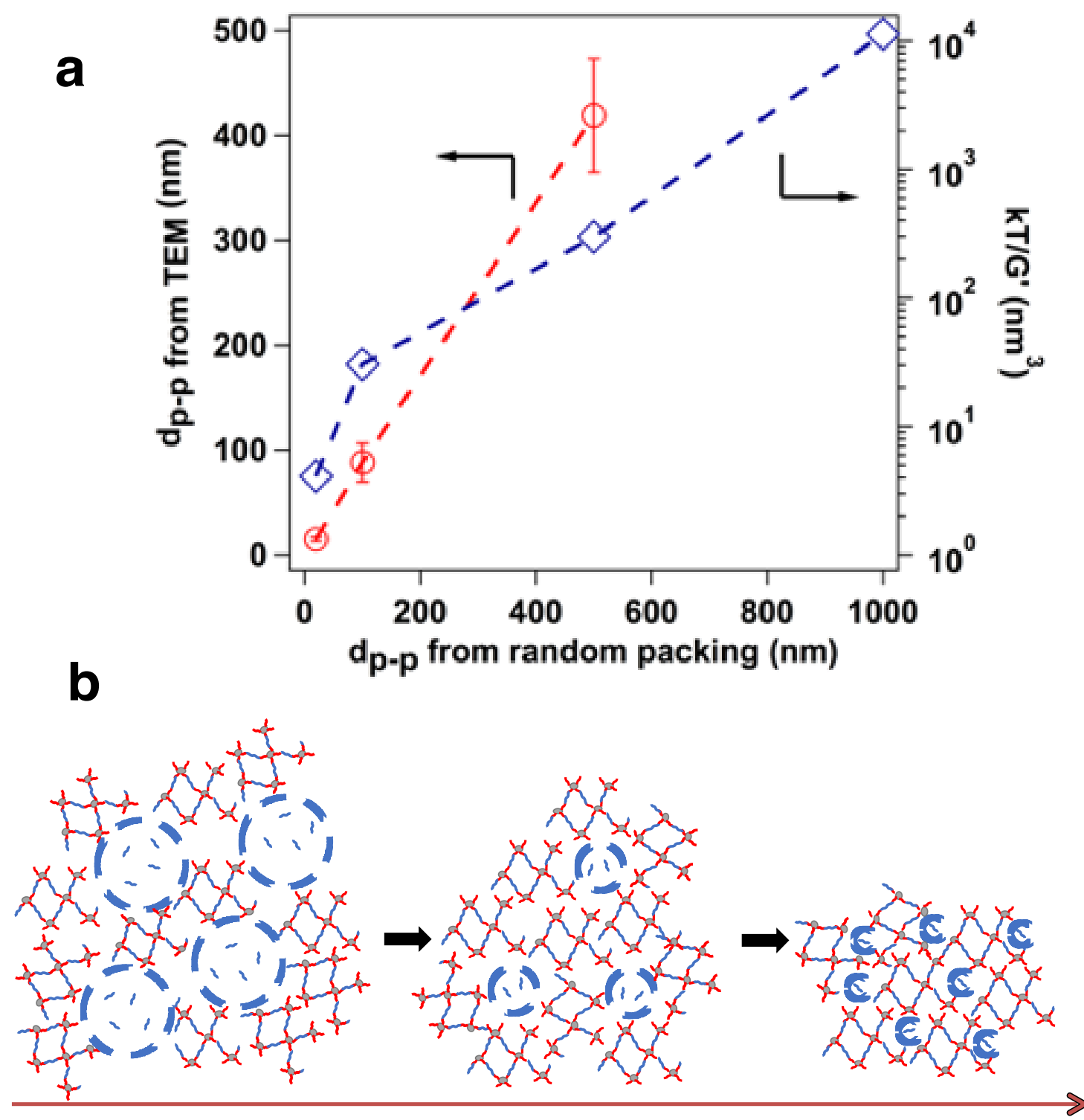

Increasing silica concentration in polymer network

Figure 6.2: Characterization of pore architecture in the crosslinked structure: (a)

Comparing the pore sizes obtained from TEM as well as cage sizes obtained from frequency sweep measurements with the random closed packing pore sizes; (b) Schematic showing the changes in pore structures with the variation of silica nanoparticle volume fractions. In all the sub-parts, the sample names are characterized by the random closed packing interparticle distance between silica nanoparticles in the composites 
as shown in in Figure 6.2(b), also confirmed using small-angle x-ray scattering in our previous work $^{46}$.

Next, we systematically study the morphology of the lithium-electrolyte interface in the cross-linked hairy nanoparticles soaked with liquid electrolyte using direct visualization of the lithium metal anode during electrodeposition in an optical cell. The cell comprises of a lithium metal and stainless-steel electrodes separated by a chamber containing electrolyte of $1 \mathrm{M} \mathrm{LiPF} 6$-EC/DMC. ${ }^{10}$ For the conditions used in the experiments, the diffusion limited current density for the different membranes were calculated from the measured conductivity and transference number: $J^{*}=$ $2 z c_{o} F D_{a p p}\left(t_{a} L\right)^{-1}$. Here, $z$ is the charge number of cation, $F$ is the Faraday constant, $c_{o}$ is salt concentration, $t_{a}$ is the anion transference number and $\mathrm{L}$ is the inter-electrode spacing $(1 \mathrm{~mm})$ and $D_{a p p}$ is the diffusion coefficient. Using the Bruce-Vincent method $^{33}$ the transference number (see Supplementary Figure 6.4), was determined to be 0.38 for the crosslinked nanoparticles (r.c.p. $=20 \mathrm{~nm}$ ). The variation of conductivity and critical current density $\left(\mathrm{J}^{*}\right)$ for the crosslinked nanoparticles at different pore sizes are reported in Supplementary Figure 6.5. It is seen that $J^{*}$ varies in the range $\sim 5$ to $9 \mathrm{~mA} / \mathrm{cm}^{2}$ for the materials studied. Using the previously reported values of $\mathrm{Li}^{+}$transference number $(0.36)$ and lithium ion diffusivity $\left(3^{*} 10^{-6} \mathrm{~cm}^{2} / \mathrm{sec}\right)$ for $1 \mathrm{M}$ EC:DMC-LiPF 6 electrolyte, the diffusion limited current density can be estimated to be $9 \mathrm{~mA} / \mathrm{cm}^{2}$ for the same inter-electrode spacing. ${ }^{47,48}$

Figure 6.3(a) reports snapshots of the negative electrode at different stages of electrodeposition for the cases without any separator, with a commercial glass fiber separator and crosslinked hairy nanoparticles (r.c.p. pore size $=20 \mathrm{~nm}$ ) at a constant 
current density of $8 \mathrm{~mA} / \mathrm{cm}^{2}$, which 0.9 times the $J^{*}$ calculated for liquid electrolyte. Through visual inspection, it is obvious that $\mathrm{Li}$ electrodeposition in the absence of a separator is uneven, mossy and blackened, which is indicative of the side reactions between electrode and electrolyte as well as porous nature of the deposited layers. At first appearance, the deposits are already quite large, at least $100 \mu \mathrm{m}$ in diameter, which is much larger than typical Li dendrite nucleate sizes assumed in the literature. ${ }^{6}$ Deposition studies based on a commercial glass fiber separator shows that deposition is relatively compact until a capacity of around $2 \mathrm{mAh} / \mathrm{cm}^{2}$ (i.e. approximately 10 $\mu \mathrm{m} / \mathrm{cm}^{2} \mathrm{Li}$ is transferred), where after dendritic structures develop and proliferate in time, ultimately piercing the separator and continue their growth through the separator. This sequence is associated with the short-circuiting phenomena described in the introduction section and can lead to catastrophic cell failure by fire, explosion, or both. In comparison to the cases with and without separator, the deposition pattern with the cross-linked hairy nanoparticles display uniform deposition for entire one hour of the charging process.

By tracking the advancing front of the Li electrode over time, it is possible to quantify the dendrite growth as a function of time (see Figure 6.3(b)). The apparent growth rate obtained from the initial time through the assumption of linearity is shown in the inset. The larger sized error bars for the neat electrolyte case is a reflection of the unevenness of electrodeposition. The apparent growth rate of dendrite in the commercial glass fiber separator is calculated to be $\sim 0.13 \mu \mathrm{m} / \mathrm{sec}$, while for the crosslinked hairy nanoparticles it is estimated to be $0.03 \mu \mathrm{m} / \mathrm{sec}$ which is modestly larger 

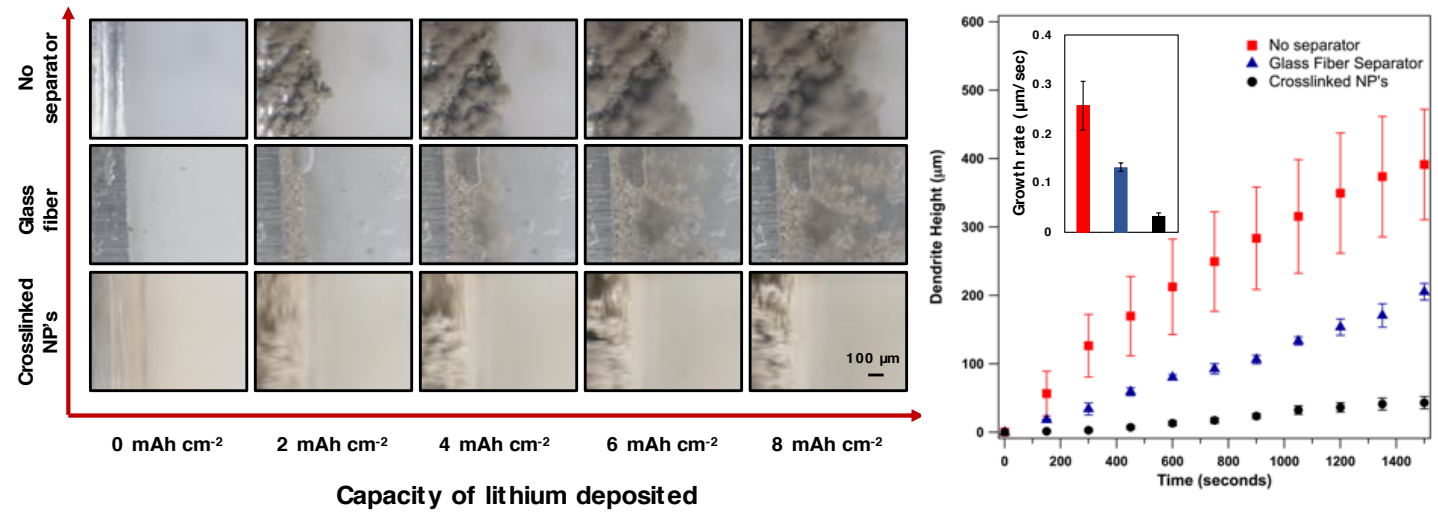

Figure 6.3: Stability of electrodeposition by direct visualization of electrode: (a) Snapshots of the electrode and electrolyte in every 15 minutes during charging at the rate of $8 \mathrm{~mA} \mathrm{~cm}^{-2}$; (b) Height of dendrite at various points of the electrode for the initial 1500 seconds, the inset compares the growth rate by assuming a linear growth for electrodeposition at $8 \mathrm{~mA} \mathrm{~cm}^{-2}$ 
than the theoretical rate $(0.011 \mu \mathrm{m} / \mathrm{sec})$ for perfectly smooth electrodeposition at 8 $\mathrm{mAh} / \mathrm{cm}^{2}$.

This idea that one could control the stability of electrodeposition by manipulating the nanostructure of an electrolyte (Supplementary Figure 6.6) was previously discussed in the theoretical analysis by Tikekar et al. ${ }^{7,38}$ and has also been adapted in an adhoc manner to explain previous experimental results. ${ }^{49,50}$ Deposition in membranes with pores larger than the critical nucleate diameter at which electrodeposition is unstable, is in this analysis thought to lead to unchecked growth of dendrites that penetrate through this pores, as in the case of the glass fiber separator. In contrast, in the crosslinked hairy nanoparticles the pore size of $20 \mathrm{~nm}$ is evidently much smaller than the nucleate size leading to a significant retardation of dendrite growth. To determine the effect of SEI formation and chemical instability on the topological features observed during the electrodeposition process, we performed post-mortem analysis of lithium electrode after repeated charge and discharge cycling in a coin cell. Specifically, we utilized a symmetric lithium battery with the crosslinked nanoparticle electrolyte $(20 \mathrm{~nm})$ and operated the battery at a current of $0.1 \mathrm{~mA} / \mathrm{cm}^{2}$ for 100 hours, with each half-cycle comprising of 3 hours, as shown in Supplementary Figure S7. We intentionally chose low current densities for the operation to isolate the effect of chemical and not morphological instabilities. The SEM image of the extracted lithium metal is seen to be smooth and unperturbed (Supplementary Figure 6.8). This provides evidence that the observed evolution of electrode surface in visualization experiment is dominant by dendritic growth of lithium and not due to parasitic reactions at the interface. 

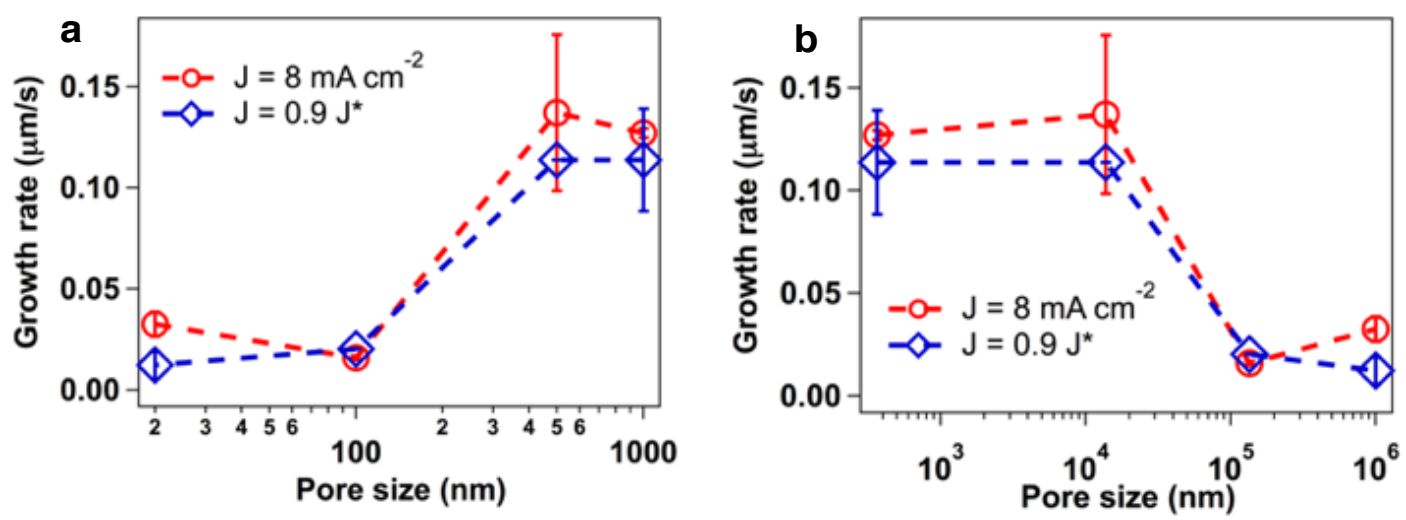

Figure 6.4: Analyzing pore size dependence on dendrite growth: (a), (b) Growth rate at different pore sizes and corresponding elastic modulus, respectively for the crosslinked hairy nanoparticles at current density of $8 \mathrm{~mA} / \mathrm{cm}^{2}$ as well as for variable current densities matching 0.9 times of the limiting current densities measured for respective samples 
One could make these tentative ideas more concrete by interrogating the evolution of the interface in cross-linked electrolytes in which the average pore size is systematically varied. Supplementary Figure 6.9 show the snapshots of deposition at same current density of $8 \mathrm{~mA} / \mathrm{cm}^{2}$, and Supplementary Figure 6.10 report the analytical results from such studies in which the morphology of the Li-electrolyte interface is characterized in cross-linked nanoparticles with varying pore size (or, weight fraction of $\mathrm{SiO}_{2}$ in the composite). The liquid electrolyte weight was maintained to be same in all cases reported in this work, which is twice as that of the composite weight. The results show that when the effective pore size of the membrane is $1000 \mathrm{~nm}$, dendrite growth is unimpeded and rapid. However, at high volume fractions of silica nanoparticles corresponding to interparticle distance of order 500 $\mathrm{nm}$, Supplementary Figure 6.10 shows two regimes of dendrite-growth, wherein, the growth rate at the initial time is low, followed by an accelerated rate of dendrite propagation at longer time. The results in Supplementary Figure 6.9 also shows that for the membranes with $500 \mathrm{~nm}$ pores, dendrites with 'needle-like' morphology are visible at $4 \mathrm{mAh} / \mathrm{cm}^{2}$, in contrast to the mossy-blackened deposited lithium apparent when the pores are larger and in neat electrolytes. Furthermore, it is seen that in membranes with effective pore size of around $100 \mathrm{~nm}$, the deposition is similarly restricted as for the $20 \mathrm{~nm}$ case, implying that there is a threshold pore size between $500 \mathrm{~nm}$ and $100 \mathrm{~nm}$ where the resistance to dendrite proliferation is large enough to prevent growth.

Figure 6.4(a) and (b) summarizes the growth rate deduced from experiments as a function of average membrane pore size and elastic modulus, respectively at 8 
$\mathrm{mA} / \mathrm{cm}^{2}$ as well as for varying current densities such that $J / J^{*}$ remains constant at 0.9 (see Supplementary Figure 6.5). Optical snapshots of the initial deposition and the corresponding current densities (at $\left.J / J^{*}=0.9\right)$ are reported in Supplementary Figure 6.11. The experimental results reveal a sudden transition from stable deposition to dendrite growth between 100 to $500 \mathrm{~nm}$ pore-size in agreement with our hypothesis that electrodeposition stability is function of electrolyte length-scale. Also, the shear modulus measured from rheology doesn't have a linear relation with growth rate can be rationalized by the fact that the crosslinked membrane is comprised of building blocks having different inherent modulus $\left(\mathrm{SiO}_{2}\right.$ and polymer). As the deposition takes place in the inter-particle gap, the growing 'dendrites' only experience the modulus of the liquid electrolyte and polymers, thus the length scale of the polymeric phase plays a greater role than the overall modulus of the material. We also analyze the growth rate of dendrites as a function of pore size obtained from TEM micrograph analysis as well as the pore volume calculated from plateau modulus as $k T / G^{\prime}$, As shown in Supplementary Figure 6.12, a similar non-linear behavior is observed.

Figure 6.5(a) plots the perturbation growth rate versus wavelength deduced from the analysis of Tikekar et al. ${ }^{38}$ It is seen that the growth rate is negative for small wave lengths and goes through a maximum at a particular wavelength that decreases as the current density approaches critical current density, $J^{*}$. The first of these results can be shown to arise from the greater influence of surface tension in suppression growth of small dendrite nucleates, while the presence of the maximum reflects the effect of the physical (macroscopic) dimensions of the simulation cell in restricting nucleate size below certain limits. As the current density increases, the growth rate of the fastest 

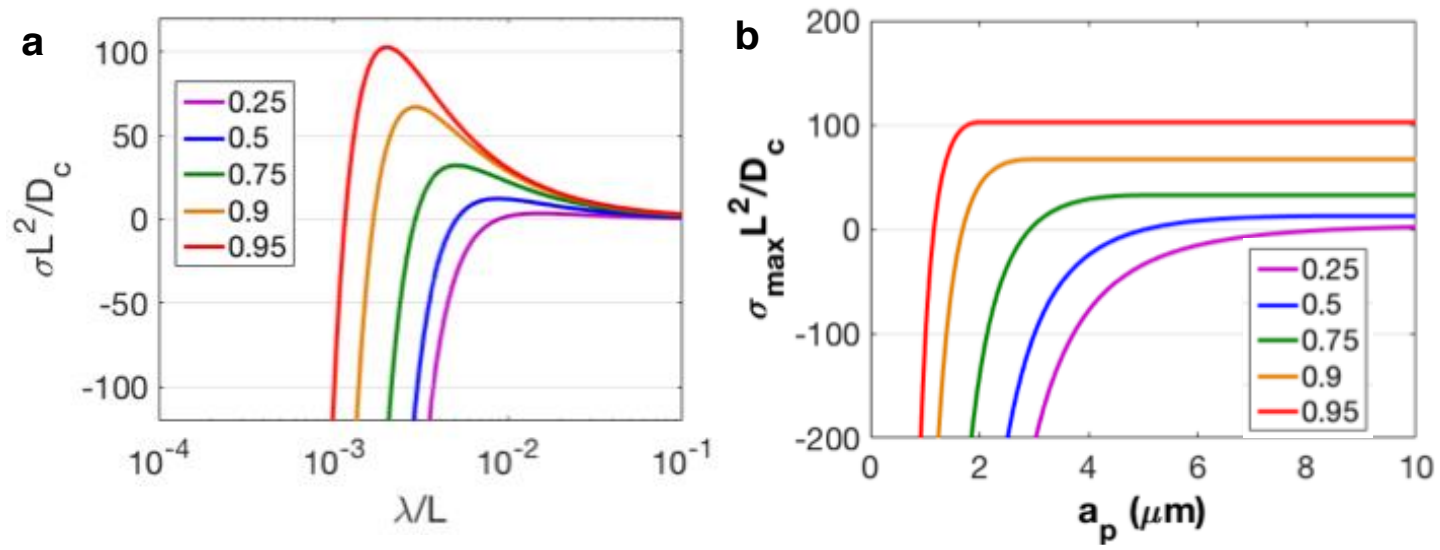

Figure 6.5: Linear Stability analysis of dendrite growth: (a) Normalized Growth rate of the deposited nucleate at different normalized wave numbers obtained by linear stability analysis; (b) Maximum growth at different theoretical pore sizes at various effective currents. The different lines in parts (a) and (b) represent different values of $\mathrm{J} / \mathrm{J}^{*}$ 
(most unstable) mode increases and its wavelength decreases because the interfacial tension has to compete with progressively more aggressive electroconvection to stop the roughening. The fastest growing mode can therefore be thought of as representative of the typical size of dendrites that one would observe in an experiment under similar conditions. A porous material would restrict the size of the growing dendrite, since the deposition can only happen on length scales smaller than the pore size. In Figure 6.5(b), we show the calculated growth rate for the fastest growing mode as a function of the critical wavelength. For very large pores, the pore size is predicted to have no effect on the dendrite growth, and the growth occurs more or less at the same rate as in the absence of the porous material. For smaller pores however, the pore diameter restricts the sizes of the dendrites that can form in them, causing the growth rate of the fastest mode to plummet. Below a certain size, there can be no unstable deposition, as surface tension adequately competes with current density driven dendritic growth at all possible length scales. This critical pore size decreases with current density due to the requirement of a higher contribution from surface tension as seen in Supplementary Figure 6.13. The inset in Figure 6.13 plots the analogous growth rate deduced from experiments as a function of membrane pore size for a fixed current density, $J / J^{*} \sim 0.9$. The experimental results show a similar trend as calculated from the linear stability analysis, wherein there is sudden transition from stable deposition to dendrite growth. The transition point from the theoretical prediction corresponding to non-zero growth is $\sim 1.6 \mu \mathrm{m}$, while the experiments indicate the crossover between 100-500 nm pore-size as previously mentioned in Figure 6.4(a). Considering the variety of sources of error in comparing the experiments and theory, 
including the tortuosity of the pores in the cross-linked materials, the polydispersity of the particle size, uncertainty in the value of the Li transference number under the conditions of the experiments ${ }^{48}$, and the deviation from linearity in calculation of initial growth rate, the agreement between the theoretical and observed transition is fair.

\subsection{Conclusion}

In conclusion, we utilize a crosslinked network by covalently linking polymer-grafted nanoparticles, where varying the volume fraction of the nanoparticles in the composite can conveniently change the effective pore size. It was shown that these crosslinked nanoparticles when used as gel electrolytes showed stable electrodeposition in contrast to mossy and dendritic morphology for cases without separator and with glass fibers respectively. We performed visualization experiments for different pore sizes determined by volume fractions to demonstrate that below $500 \mathrm{~nm}$, the deposition is smooth and compact, while dendritic for other cases. These experimental observations were rationalized using linear stability analysis of dendrite growth at different perturbation length scale representing pore diameter of the electrolytes. It was understood that below the limiting current density, the electrodeposition can be stabilized by implementing a separator with pore-size lower than the length scale of the most unstable nucleate. Overall, this work will provide guidelines for designing solid state electrolytes and separators for metal-based batteries. 


\section{Acknowledgements}

This work was supported by the National Science Foundation, Division of Materials

Research, through Award No. DMR-1609125. 


\section{REFERENCES}

1. Lin, D., Liu, Y. \& Cui, Y. Reviving the lithium metal anode for high-energy batteries. Nat. Nanotechnol. 12, 194-206 (2017).

2. Cheng, X. et al. A Review of Solid Electrolyte Interphases on Lithium Metal Anode. Adv. Sci. 3, 1-20 (2016).

3. Cheng, X.-B., Zhang, R., Zhao, C.-Z. \& Zhang, Q. Toward Safe Lithium Metal Anode in Rechargeable Batteries: A Review. Chem. Rev. 117, 10403-10473 (2017).

4. Ma, L., Hendrickson, K. E., Wei, S. \& Archer, L. A. Nanomaterials: Science and applications in the lithium-sulfur battery. Nano Today 10, 315-338 (2015).

5. Zhang, W. et al. Design Principles of Functional Polymer Separators for HighEnergy, Metal-Based Batteries. Small 1703001-n/a doi:10.1002/smll.201703001

6. Wei, S., Choudhury, S., Tu, Z., Zhang, K. \& Archer, L. A. Electrochemical Interphases for High-Energy Storage Using Reactive Metal Anodes. Acc. Chem. Res. (2017). doi:10.1021/acs.accounts.7b00484

7. Tikekar, M. D., Archer, L. A. \& Koch, D. L. Stabilizing electrodeposition in elastic solid electrolytes containing immobilized anions. Sci. Adv. 2, (2016).

8. Chazalviel, J.-N. Electrochemical aspects of the generation of rampified metallic electrodeposits. 42, 7355-7367 (1990).

9. Bai, P., Li, J., Brushett, F. R. \& Bazant, M. Z. Transition of lithium growth mechanisms in liquid electrolytes. Energy Environ. Sci. 9, 3221-3229 (2016). 
10. Choudhury, S. et al. Electroless Formation of Hybrid Lithium Anodes for Fast Interfacial Ion Transport. Angew. Chemie Int. Ed. 56, 13070-13077 (2017).

11. Choudhury, S., Wei, S., Ozhabes, Y., Gunceler, D. \& Nath, P. Designing Solidliquid Interphases for Sodium Batteries. Nat. Commun. 8, (2017).

12. Wood, K. N. et al. Dendrites and Pits: Untangling the Complex Behavior of Lithium Metal Anodes through Operando Video Microscopy. ACS Cent. Sci. 2, 790-801 (2016).

13. Zheng, G. et al. Interconnected hollow carbon nanospheres for stable lithium metal anodes. Nat. Nanotechnol. 9, 618-623 (2014).

14. Monroe, C. \& Newman, J. The Effect of Interfacial Deformation on Electrodeposition Kinetics. J. Electrochem. Soc. 151, A880 (2004).

15. Monroe, C. \& Newman, J. The Impact of Elastic Deformation on Deposition Kinetics at Lithium/Polymer Interfaces. J. Electrochem. Soc. 152, A396 (2005).

16. Stone, G. M. et al. Resolution of the Modulus versus Adhesion Dilemma in Solid Polymer Electrolytes for Rechargeable Lithium Metal Batteries. J. Electrochem. Soc. 159, A222-A227 (2012).

17. Agrawal, A., Choudhury, S. \& Archer, L. a. A highly conductive, nonflammable polymer-nanoparticle hybrid electrolyte. $R S C A d v$. 5, 20800-20809 (2015).

18. Choudhury, S., Mangal, R., Agrawal, A. \& Archer, L. A. A highly reversible room-temperature lithium metal battery based on crosslinked hairy nanoparticles. Nat. Commun. 6, 10101 (2015).

19. Lu, Y., Korf, K., Kambe, Y., Tu, Z. \& Archer, L. a. Ionic-Liquid-Nanoparticle 
Hybrid Electrolytes: Applications in Lithium Metal Batteries. Angew. Chemie 126, 498-502 (2014).

20. Zhang, J., Sun, B., Huang, X., Chen, S. \& Wang, G. Honeycomb-like porous gel polymer electrolyte membrane for lithium ion batteries with enhanced safety. Sci. Rep. 4, 6007 (2014).

21. Stephan, a. M. Review on gel polymer electrolytes for lithium batteries. Eur. Polym. J. 42, 21-42 (2006).

22. Wu, H. et al. Stable Li-ion battery anodes by in-situ polymerization of conducting hydrogel to conformally coat silicon nanoparticles. Nat. Commun. 4 , 1943 (2013).

23. Khurana, R., Schaefer, J. L., Archer, L. A. \& Coates, G. W. Suppression of lithium dendrite growth using cross-linked polyethylene/poly(ethylene oxide) electrolytes: a new approach for practical lithium-metal polymer batteries. $J$. Am. Chem. Soc. 136, 7395-7402 (2014).

24. Porcarelli, L., Gerbaldi, C., Bella, F. \& Nair, J. R. Super Soft All-Ethylene Oxide Polymer Electrolyte for Safe All-Solid Lithium Batteries. Sci. Rep. 6, 19892 (2016).

25. Long, L., Wang, S., Xiao, M. \& Meng, Y. Polymer electrolytes for lithium polymer batteries. J. Mater. Chem. A 4, 10038-10069 (2016).

26. Gurevitch, I. et al. Nanocomposites of Titanium Dioxide and PolystyrenePoly(ethylene oxide) Block Copolymer as Solid-State Electrolytes for Lithium Metal Batteries. J. Electrochem. Soc. 160, A1611-A1617 (2013).

27. Zhao, C.-Z. et al. An anion-immobilized composite electrolyte for dendrite-free 
lithium metal anodes. Proc. Natl. Acad. Sci. 114, 11069 LP-11074 (2017).

28. Tu, Z. et al. Designing Artificial Solid-Electrolyte Interphases for Single-Ion and High-Efficiency Transport in Batteries. Joule (2017). doi:https://doi.org/10.1016/j.joule.2017.06.002

29. Choudhury, S. et al. Designer interphases for the lithium-oxygen electrochemical cell. Sci. $A d v$. 3, (2017).

30. Ma, L., Nath, P., Tu, Z., Tikekar, M. \& Archer, L. A. Highly Conductive, Sulfonated, UV-Cross-Linked Separators for Li-S Batteries. Chem. Mater. 28, $5147-5154$ (2016).

31. Lu, Y. et al. Stable Cycling of Lithium Metal Batteries Using High Transference Number Electrolytes. Adv. Energy Mater. 5, 1402073 (2015).

32. Oh, H. et al. Poly(arylene ether)-Based Single-Ion Conductors for Lithium-Ion Batteries. Chem. Mater. 28, 188-196 (2016).

33. Bouchet, R. et al. Single-ion BAB triblock copolymers as efficient electrolytes for lithium-metal batteries. Nat. Mater. 12, 452-457 (2013).

34. Choudhury, S. \& Archer, L. A. Lithium Fluoride Additives for Stable Cycling of Lithium Batteries at High Current Densities. Adv. Electron. Mater. 1-6 (2015). doi:10.1002/aelm.201500246

35. Lu, Y., Tu, Z. \& Archer, L. A. Stable lithium electrodeposition in liquid and nanoporous solid electrolytes. Nat. Mater. 13, 961-969 (2014).

36. Seh, Z. W., Sun, J., Sun, Y. \& Cui, Y. A Highly Reversible Room-Temperature Sodium Metal Anode. ACS Cent. Sci. 1, 449-455 (2015).

37. Zhang, X., Cheng, X., Chen, X., Yan, C. \& Zhang, Q. Fluoroethylene 
Carbonate Additives to Render Uniform Li Deposits in Lithium Metal Batteries. Adv. Funct. Mater. 27, 1605989 (2017).

38. Tikekar, M. D., Archer, L. A. \& Koch, D. L. Stability Analysis of Electrodeposition across a Structured Electrolyte with Immobilized Anions. $J$. Electrochem. Soc. 161, A847-A855 (2014).

39. Choudhury, S., Agrawal, A., Kim, S. a \& Archer, L. a. Self-Suspended Suspensions of Covalently Grafted Hairy Nanoparticles. Langmuir 31, 3222$3231(2015)$.

40. Choudhury, S., Agrawal, A., Wei, S., Jeng, E. \& Archer, L. A. Hybrid Hairy Nanoparticle Electrolytes Stabilizing Lithium Metal Batteries. Chem. Mater. 28, 2147-2157 (2016).

41. Wei, S. et al. Highly Stable Sodium Batteries Enabled by Functional Ionic Polymer Membranes. Adv. Mater. 29, 1605512 (2017).

42. Stalin, S., Choudhury, S., Zhang, K. \& Archer, L. A. Multifunctional CrossLinked Polymeric Membranes for Safe, High-Performance Lithium Batteries. Chem. Mater. 30, 2058-2066 (2018).

43. Agarwal, P., Kim, S. A. \& Archer, L. A. Crowded, Confined, and Frustrated: Dynamics of Molecules Tethered to Nanoparticles. Phys. Rev. Lett. 109, $258301(2012)$.

44. Ding, Y. et al. Dielectric Spectroscopy Investigation of Relaxation in C 60 Polyisoprene Nanocomposites. 3201-3206 (2009). doi:10.1021/ma8024333

45. Adachi, K. \& Kotaka, T. Dielectric normal mode relaxation. Prog. Polym. Sci. 18, 585-622 (1993). 
46. Choudhury, S., Mangal, R., Agrawal, A. \& Archer, L. A. A highly reversible room-temperature lithium metal battery based on crosslinked hairy nanoparticles. Nat. Commun. 6, 10101 (2015).

47. Nyman, A., Behm, M. \& Lindbergh, G. Electrochemical characterisation and modelling of the mass transport phenomena in LiPF6-EC-EMC electrolyte. Electrochim. Acta 53, 6356-6365 (2008).

48. Valden, L. O. \& Reimers, J. N. Transport Properties of LiPF6-Based Li-Ion Battery Electrolytes. J. Electrochem. Soc. 152, A882 (2005).

49. Tu, Z., Nath, P., Lu, Y., Tikekar, M. D. \& Archer, L. A. Nanostructured Electrolytes for Stable Lithium Electrodeposition in Secondary Batteries. Acc. Chem. Res. 48, 2947-2956 (2015).

50. Tu, Z. et al. Nanoporous Hybrid Electrolytes for High-Energy Batteries Based on Reactive Metal Anodes. Adv. Energy Mater. 7, 1602367 (2017).

51. Rosso, M., Gobron, T., Brissot, C., Chazalviel, J.-N. \& Lascaud, S. Onset of dendritic growth in lithium/polymer cells. J. Power Sources 97-98, 804-806 (2001). 


\section{APPENDIX}

Supplementary Information for Chapter 6
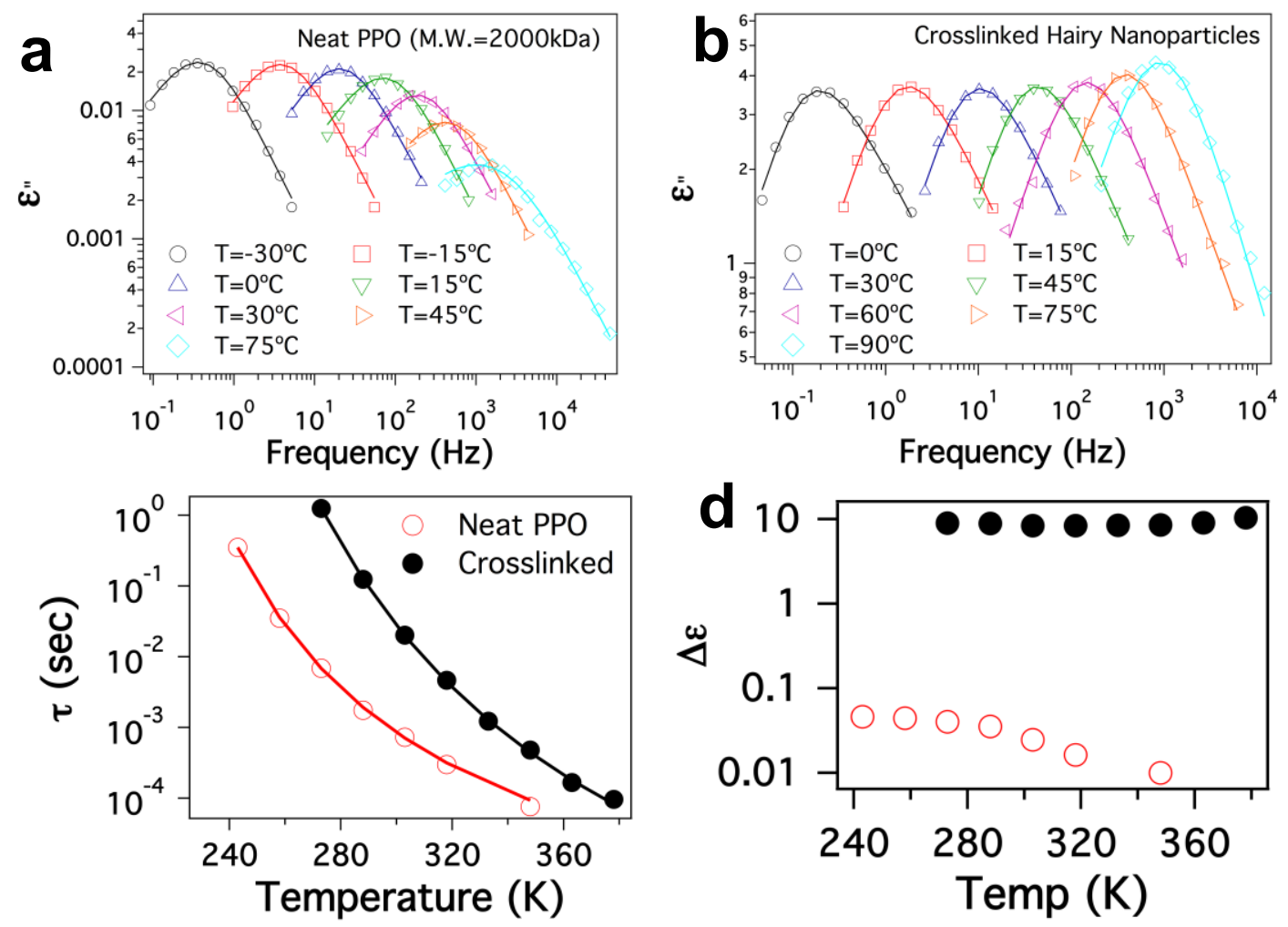

\section{Supplementary Figure 6.1: Dielectric Relaxation of Crosslinked Hairy}

Nanoparticles: (a), (b) Dielectric Permittivity at various temperatures fitted with the H-N model for Neat PPO and crosslinked hairy nanoparticles, respectively; (c) Polymer relaxation time as a function of temperatures fitted with a VFT model; (d) comparison between the dielectric strength between the neat PPO and crosslinked PPO, the symbols are same as in part c. 
a

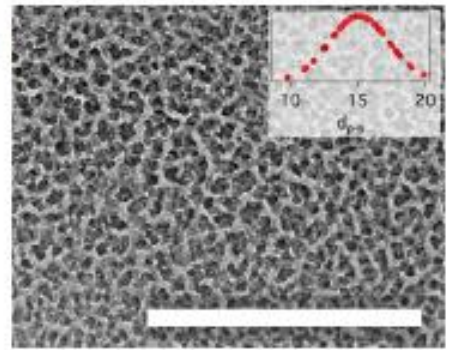

b

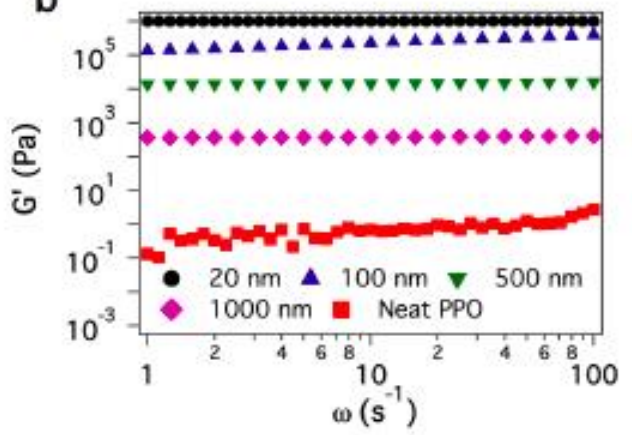

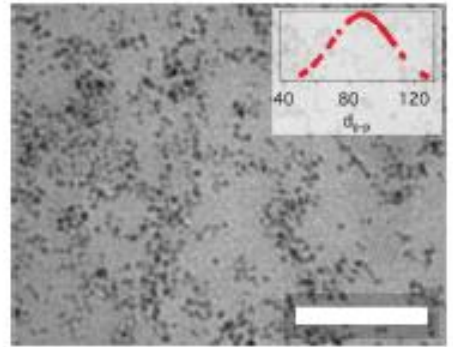

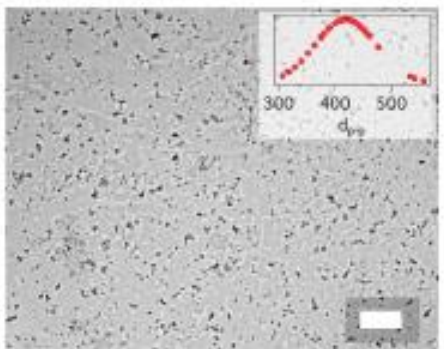

C

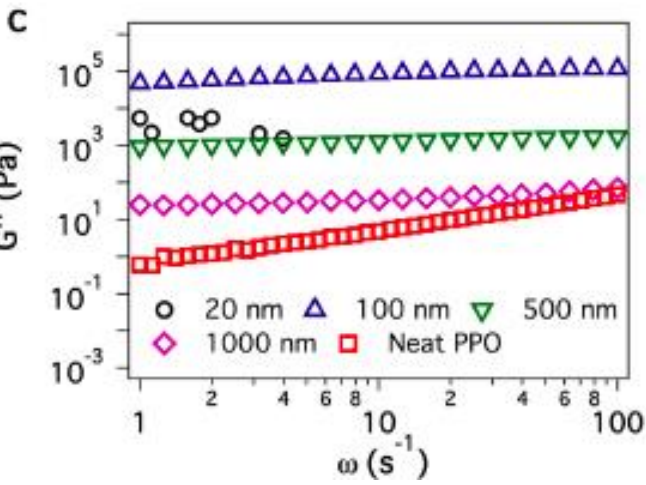

\section{Supplementary Figure 6.2: Characterization of pore architecture in the}

crosslinked structure: (a) TEM micrographs of crosslinked nanoparticles. The scale bar represents $200 \mathrm{~nm}$. From left to right, the samples are r.c.p. pore sizes $20 \mathrm{~nm}, 100 \mathrm{~nm}$ and $500 \mathrm{~nm}$. (b) Storage Modulus and (c) Loss Modulus obtained through frequency sweep measurements at strain of $5 \%$ for different crosslinked samples and neat $\mathrm{PPO}$ at $60^{\circ} \mathrm{C}$ 


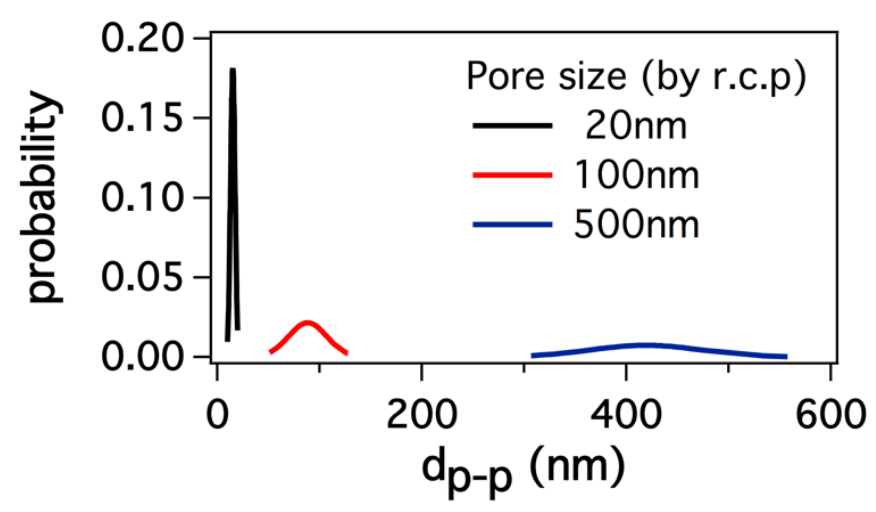

Supplementary Figure 6.3: Normal Distribution of interparticle distances obtained by analysis of TEM images for crosslinked hairy nanoparticles with different random closed packing pore sizes 


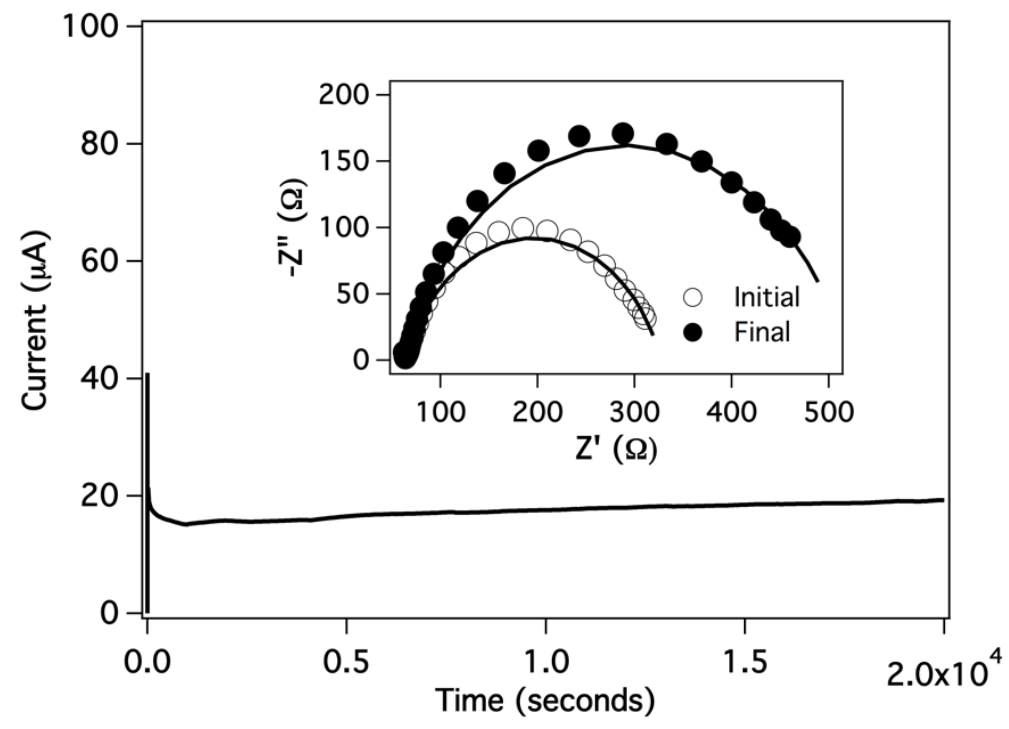

Supplementary Figure 6.4: Polarization of a symmetric lithium cell using the crosslinked hairy nanoparticle electrolyte $($ r.c.p. $=20 \mathrm{~nm})$ soaked with the electrolyte $1 \mathrm{M} \mathrm{EC/DMC} \mathrm{LiPF} 6$ at $20 \mathrm{mV}$. The inset shows the impedance results before and after polarization. 


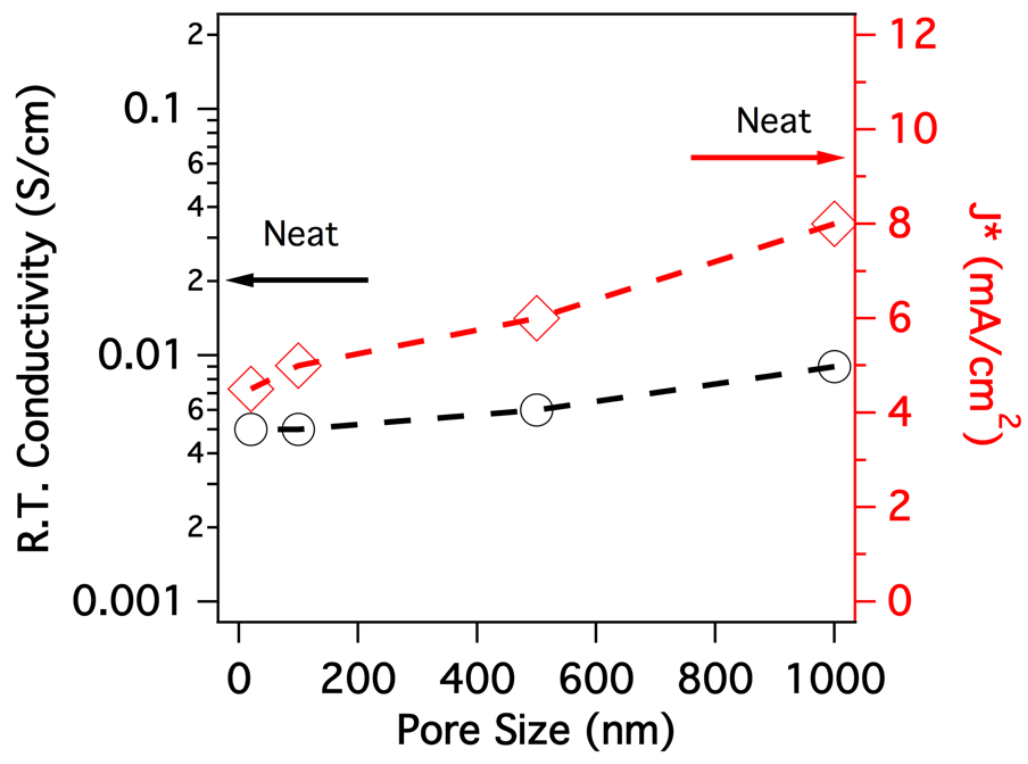

Supplementary Figure 6.5: Room temperature conductivity and limiting current density variation with different pore sized membranes. The arrows show the corresponding values for a neat electrolyte of $1 \mathrm{M} \mathrm{EC/MC} \mathrm{LiPF}_{6}$. 

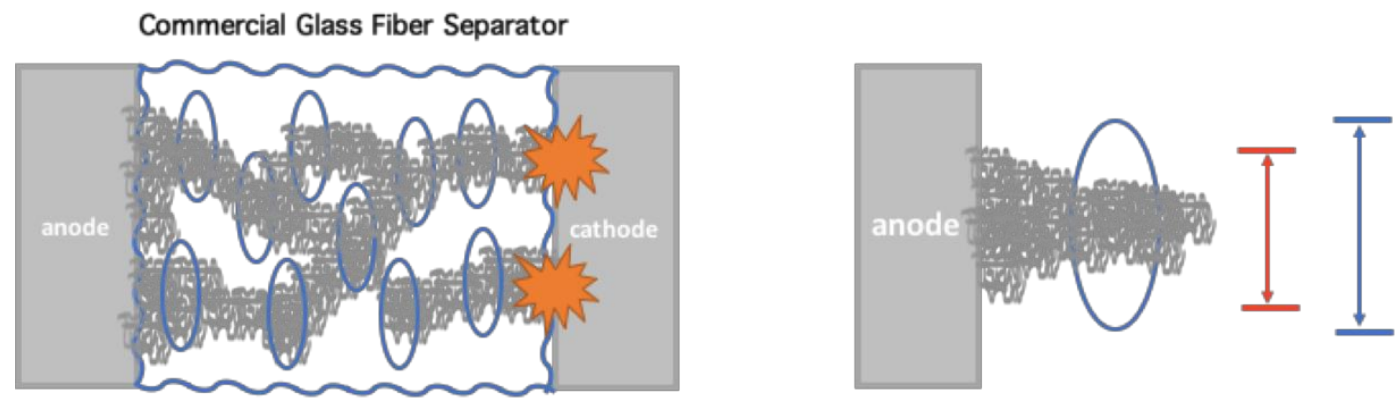

\section{Crosslinked Hairy Nanoparticles}
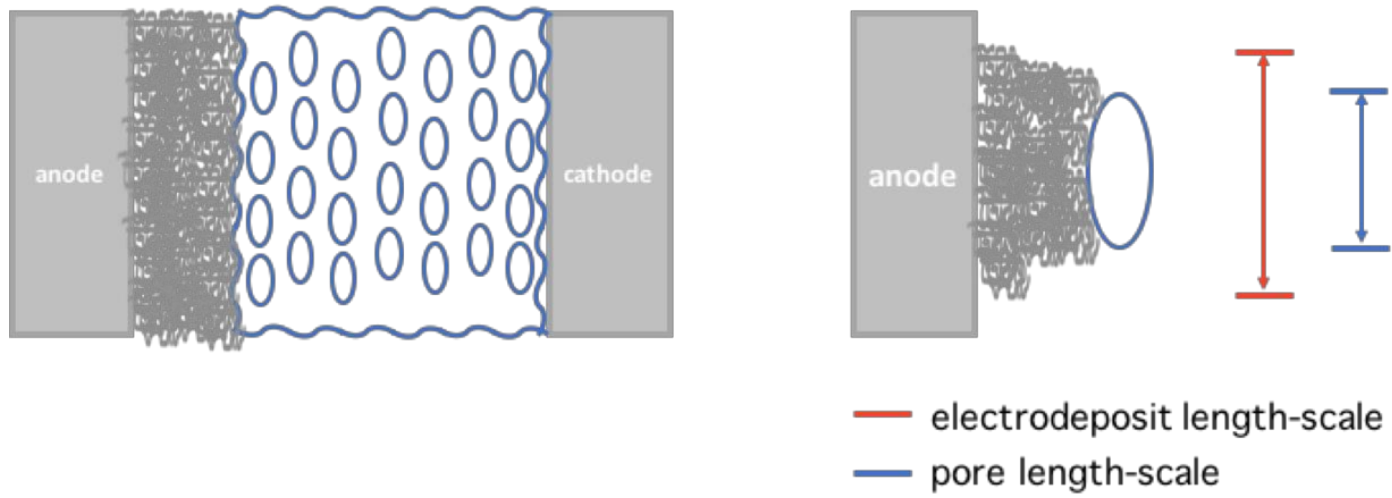

Supplementary Figure 6.6: Schematic representing the idea that the pore size of the electrolyte/separator is important and related to the stability of electrodeposition. In this figure, the crosslinked nanoparticles have random closed packing pore size of 20 $\mathrm{nm}$ 


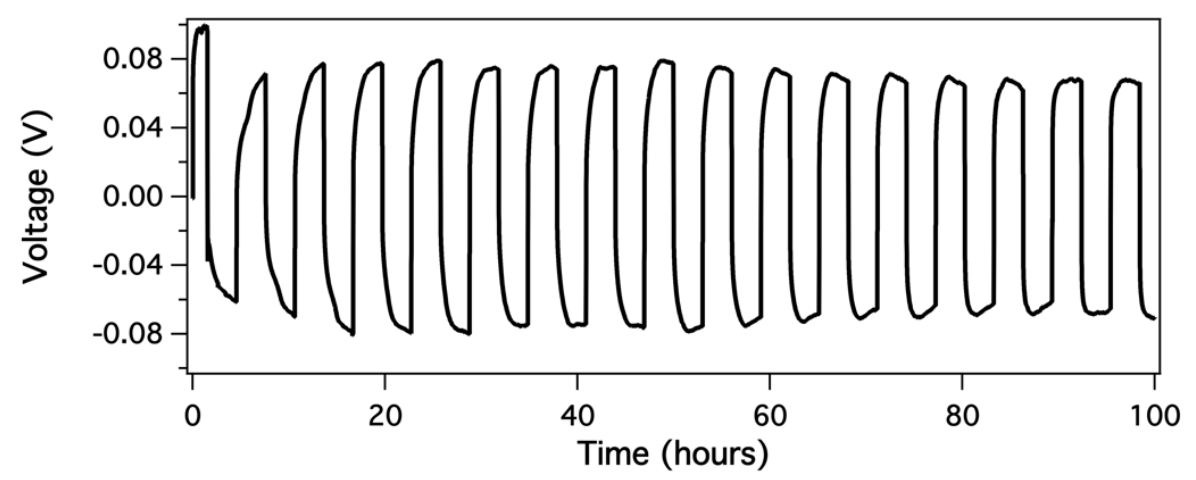

Supplementary Figure 6.7: Charge and discharge cycles in a symmetric lithium coin cell using the crosslinked hairy nanoparticles electrolyte with pore size of $20 \mathrm{~nm}$. The battery was operated at a current density of $0.1 \mathrm{~mA} / \mathrm{cm} 2$ with each half cycle is 3 hour long. 

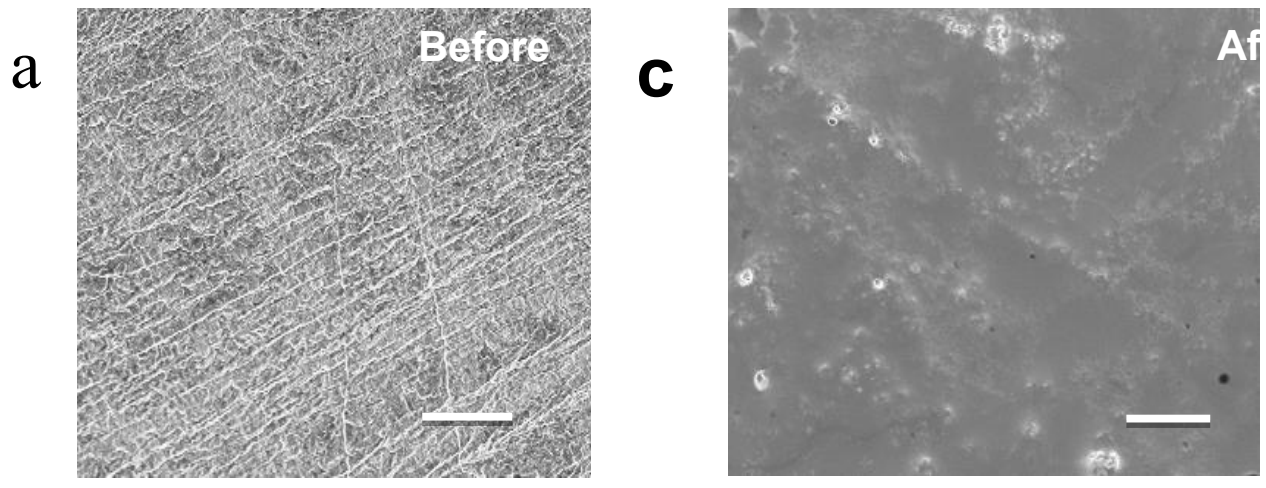

Supplementary Figure 6.8: (a), (b) SEM images of lithium electrode surface before and after cycling in a symmetric lithium cell for 100 hours at $0.1 \mathrm{~mA} / \mathrm{cm}^{2}$. 


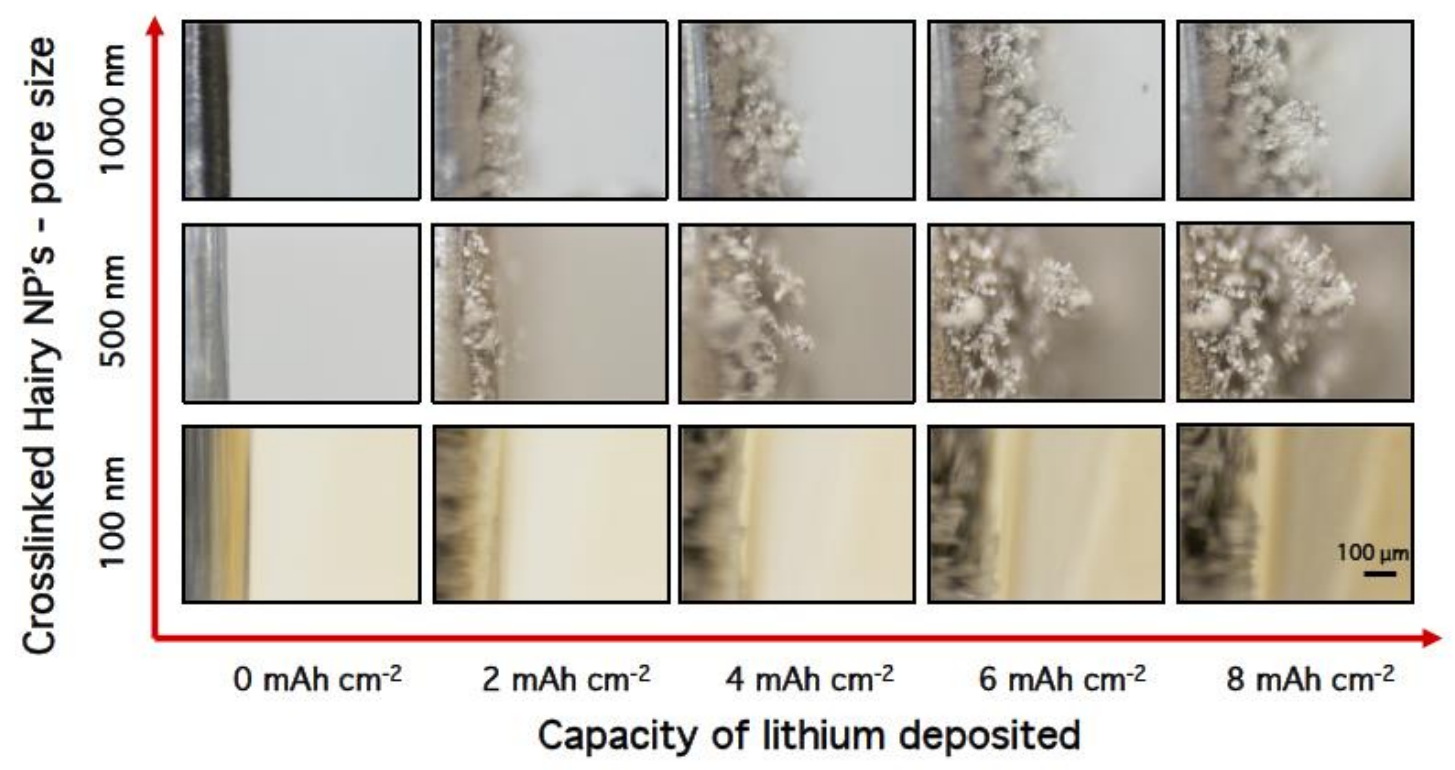

Supplementary Figure 6.9: Electrodeposition with different pore size of the crosslinked nanoparticles: Snapshots of the electrode and crosslinked electrolyte with pore sizes of 1000, 500 and $100 \mathrm{~nm}$ in every 15 minutes during charging at the rate of $8 \mathrm{~mA} \mathrm{~cm}-2$ 


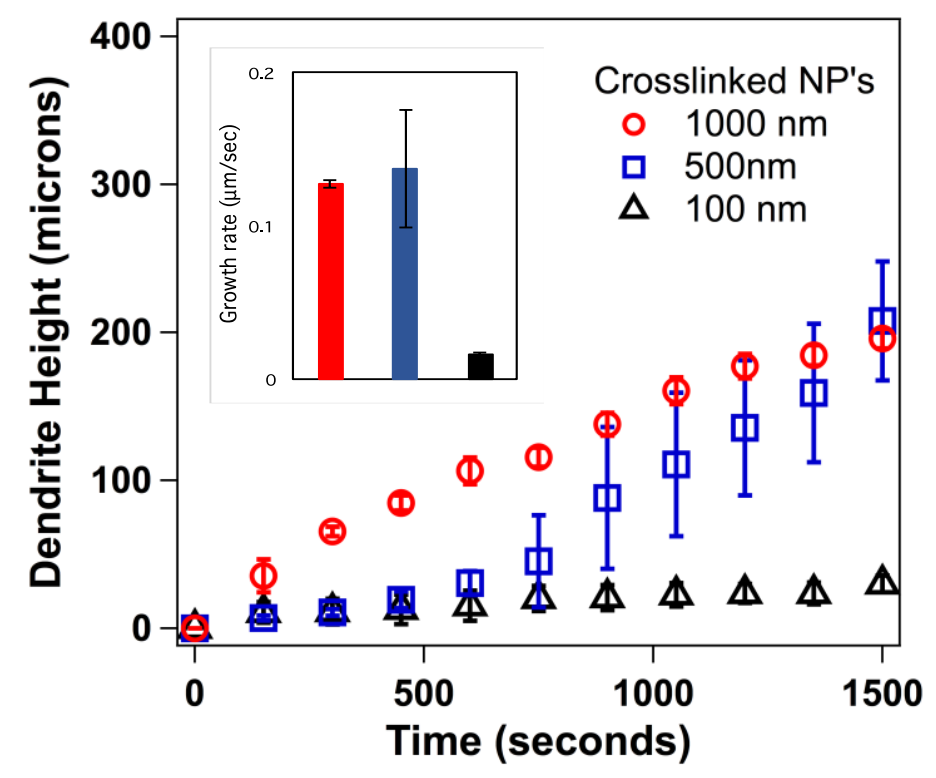

Supplementary Figure 6.10: Height of dendrite at various points of the electrode for the initial 1500 seconds, the inset compares the growth rate by assuming a linear growth for the visualization experiment in Figure $\mathrm{S} 9$ at a current density of $8 \mathrm{~mA} \mathrm{~cm}^{-2}$; 
a

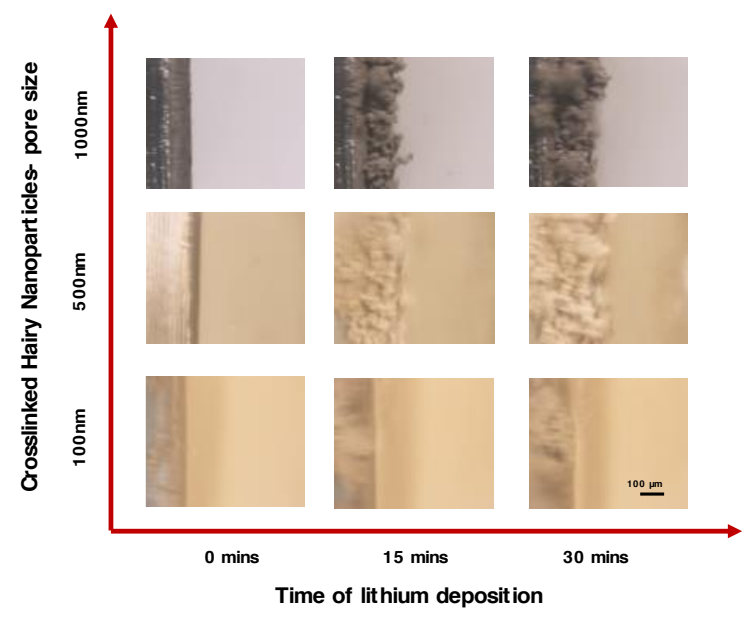

b

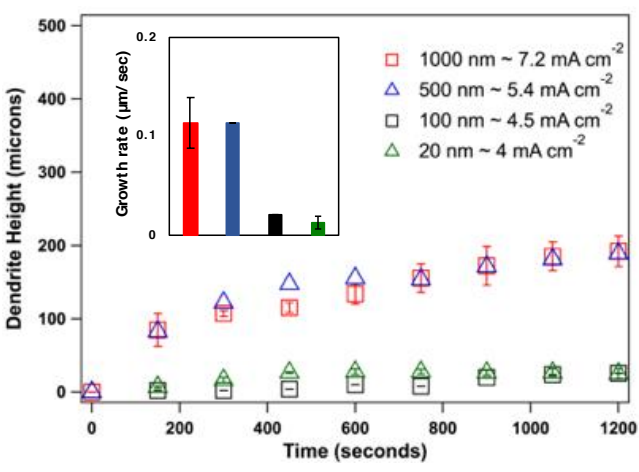

Supplementary Figure 6.11: (a) Snapshots of electrodeposition with different crosslinked membrane pore sizes at variable current density such that the $\mathrm{J} / \mathrm{J} *$ is maintained at 0.9 for each case; (b) Height of dendrite as a function of time for different samples. The absolute values of current densities are reported in the label that correspond to $\mathrm{J} / \mathrm{J}=0.9$. The inset shows the comparision of the dendrite growth rates for the respective pore sizes reported in the main figure. 


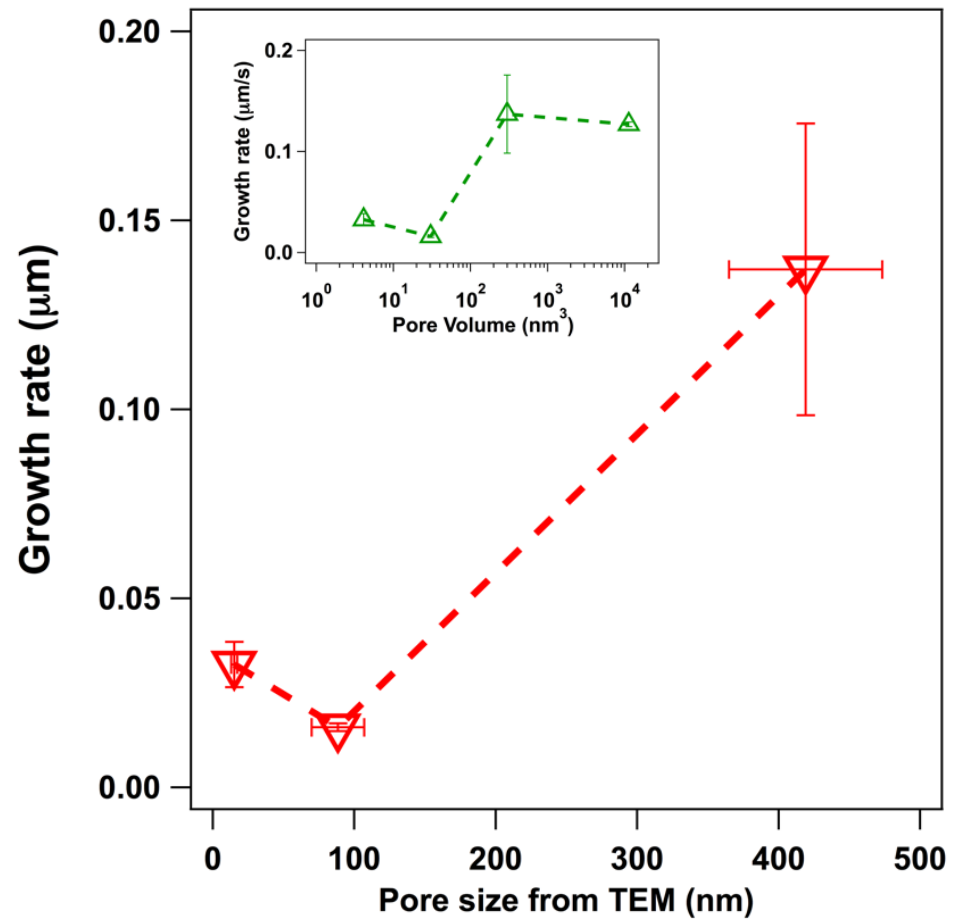

Supplementary Figure 6.12: Pore size dependence of dendrite growth at a current density of $8 \mathrm{~mA} / \mathrm{cm}^{2}$, where the pore size is obtained from the TEM analysis. The inset shows the growth rate as a function of pore volume obtained from the plateau modulus using the equation $(k T / G)$ 


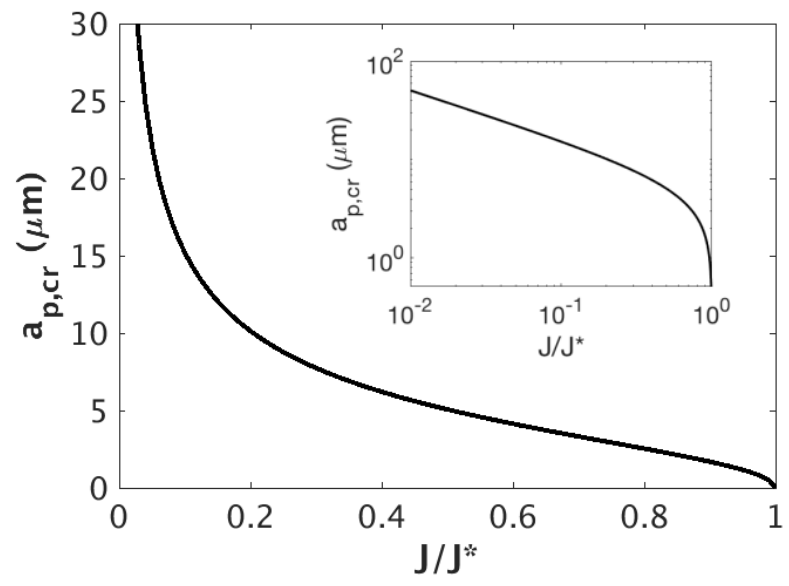

Supplementary Figure 6.13: Critical pore size, representative of crossover from positive to zero growth rate, at various normalized current density. The inset show the same graph in semi-logarithmic scale 
Supplementary Table 1: VFT parameters for fitting the dielectric relaxation times at different temperatures for the crosslinked and neat PPO.

\begin{tabular}{|c|c|c|c|}
\hline Sample & $\mathrm{A}(\mathrm{sec})$ & $\begin{array}{c}\mathrm{B} \\
(\mathrm{kJ} / \mathrm{mole})\end{array}$ & $\mathrm{T}_{\mathrm{o}}(\mathrm{K})$ \\
\hline Crosslinked PPO & $1.7 \times 10^{-9}$ & 20 & 155.4 \\
\hline Neat PPO & $1.6 \times 10^{-1}$ & 9.8 & 162.0 \\
\hline
\end{tabular}


Supplementary Table 6.2: Weight fraction of silica nanoparticles corresponding to the effective pore size obtained by the random packing fraction spherical particles

\begin{tabular}{|c|c|}
\hline $\begin{array}{c}\text { Effective Pore } \\
\text { size }\left[d_{p-p}\right](n m)\end{array}$ & $\begin{array}{c}\text { silica weight } \\
\text { fraction (\%) }\end{array}$ \\
\hline 20 & 6.3081 \\
\hline 100 & 0.1333 \\
\hline 500 & 0.0013 \\
\hline
\end{tabular}




\section{Chapter 7}

Soft Colloidal Glasses as Solid-state Electrolytes 


\subsection{Abstract}

Solid state electrolytes are regarded as an attractive alternative to liquid electrolytes in lithium batteries because of their intrinsic safety features and mechanical strength, however maintaining high bulk and interfacial ion fluxes in scalable electrolyte chemistries remains a significant challenge. In this work, we report on synthesis and electrochemical features of a class of solid state hybrid polymer electrolytes comprised of silica nanoparticles with grafted poly(ethylene oxide) chains. By regulating the salt content in the materials, we find that it is possible to drive microstructural changes, including nanoparticle arrangements, to achieve appreciable levels of room temperature ionic conductivity in a solid-state polymer composite. Additionally, we show that rationally designed salt additives can be used to create cathode-electrolyte interphases (CEI) that increase the oxidative stability of PEO-based electrolytes. In so doing, we report that solid-state lithium batteries comprised of a high-voltage nickel manganese cobalt oxide cathode, a metallic Li anode, and a solid state hybrid polymer electrolyte can be cycled stably with high levels of reversibility.

\subsection{Introduction}

Rechargeable batteries that utilize a metallic lithium anode simultaneously offer opportunities and challenges as reversible electrochemical storage systems. ${ }^{1-5}$ Lithium has the highest electronegativity and lowest atomic radius among other metals, however a major drawback is the propensity of the metal to form unstable, dendritic deposits during battery recharge, which produce premature battery failure by internal short-circuits or by voltage runaway when the deposited lithium reacts with electrolyte 
to form a thick, ion-retarding interphase. The ohmic heat generated by Li short circuits in a conventional liquid electrolyte poses a serious impediment to progress for at least three inter-related reasons. ${ }^{6-8}$ First a consequence of the flammability of the liquid electrolytes in current use is that release of such heat in a closed electrochemical cell would invariable end in fire, explosion, or both. Second, the relatively low melting point $\left(T_{\mathrm{m}}=180^{\circ} \mathrm{C}\right)$ of metallic lithium means that heat produced from a localized short can quickly destabilize the structural integrity of the electrode, causing catastrophic cell failure by thermal runaway. Finally, the intrinsic high reactivity with and exothermic reactions of metallic Li with commonly used fire-fighting reagents means that highly specialized procedures would be required to successfully intervene to stop a lithium metal battery fire.

Solid state electrolytes are attractive candidates for lithium metal cells both because of their non-flammable characteristics and the potential to eliminate leakage, meaning that cells in a wider range of form factors are possible. ${ }^{9-11}$ Additionally, a solid-state electrolyte can limit growth of Li dendrites and may limit transport of reactive species to a thin boundary region near the interface, localizing parasitic reactions between a $\mathrm{Li}$ anode and electrolyte components. The work by Li et al. ${ }^{12}$ provide the most compelling demonstration of how these features of a solid-state electrolyte can be used to advantage. Specifically, the authors show that a micro-lithium battery comprised of metallic lithium anode, a Nickel Manganese Cobalt Oxide (NCM) cathode, and a glassy Lithium Phosphorus Oxynitride (LiPON) solid electrode can be cycled stably for at least 10,000 cycles with minimal loss of capacity. Notwithstanding intense 
research by research teams world-wide, it has been a daunting challenge to achieve similar stability in larger scale versions of these cells. Myriad challenges ranging from the low bulk ionic conductivity of LiPON, its low mechanical toughness, and poor interfacial contact have been reported. ${ }^{13-20}$ Recently, Han et al. ${ }^{21}$ has demonstrated that some of these challenges can be overcome in solid-state batteries in which aluminacoated lithium is used in tandem with a $\mathrm{Li}_{7} \mathrm{La}_{2.75} \mathrm{Ca}_{0.25} \mathrm{Zr}_{1.75} \mathrm{Nb}_{0.25} \mathrm{O}_{12}$ (LLCZN) garnettype solid electrolyte, but these cells face other challenges associated with cost of the electrolyte, environmental stability of the electrolyte under conditions typically used for battery assembly, mechanical instability of the alumina coating layer, poor interfacial ion transport at phase boundaries between the solid-state electrolyte and intercalating cathodes, and the tendency of $\mathrm{Li}$ to form three-dimensional, dendritic deposits that appear to grow along the grain boundaries of the solid-state electrolyte.

Solid electrolytes based on amorphous or semi-crystalline polymers, most notably poly(ethylene oxide) (PEO), have been investigated for decades because of their flexible mechanics, straight forward manufacturability, and ability to transport lithium ions in both amorphous and crystalline forms. ${ }^{22,23}$ Perhaps, the greatest challenge in solid electrolytes common in both ceramics and polymers is the poor interfacial contact between the solid electrode and electrolyte and many recent articles have focused on developing novel strategies to ensure unrestricted electron and ion transport in such interfaces. ${ }^{24-26}$ Solid polymer electrolytes are problematic, however, because of their poor oxidative stability, strong coordination with $\mathrm{Li}$ and low molecular mobility, which make them unsuitable for either high-voltage or high-power 
cells capable of room temperature operation. Infusing inorganic fillers in the PEO electrolyte has attracted significant interest as a facile strategy for simultaneously increasing electrolyte modulus and fraction of high-conductivity amorphous phases in the polymer, potentially breaking the usual modulus-conductivity tradeoff., $71,27,28$ However, at high loading of fillers, new challenges associated with aggregation and phase separation of the polymer and particle phases obscure these benfits. ${ }^{7,29,30}$ In this work, we report what is to our knowledge the first example of a solid-state polymer electrolyte that offers the combination of oxidative stability, excellent mechanical properties, and high-enough ion mobility to enable stable operation of a lithium metal battery at $25^{\circ} \mathrm{C}$. Composed of short $\left(M_{\mathrm{w}}=5 \mathrm{KDa}\right) \mathrm{PEO}$ chains covalently grafted to $\mathrm{SiO}_{2}$ nanostructures, the electrolytes exhibit soft glassy behaviors, including existence of a yield stress that allows them to flow in response to an external load and to vitrify when the load is removed. We demonstrate the practical utility of these soft glassy electrolytes in electrochemical cells in which a metallic lithium anode is paired with a Nickel Cobalt Manganese Oxide (NCM) cathode. Further, it is shown that the oxidative instability of ether-based electrolytes at a high voltage cathode and morphological instability of Li during battery recharge can be simultaneously addressed in a simple solid-state electrolyte design.

\subsection{Results And Discussion}

\subsubsection{Synthesis and Chemical Analysis}

Figure 7.1 shows the schematic of the solid-state electrolyte design used in the study. Specifically, we synthesize silanized poly(ethylene oxide) by the reaction of amine- 


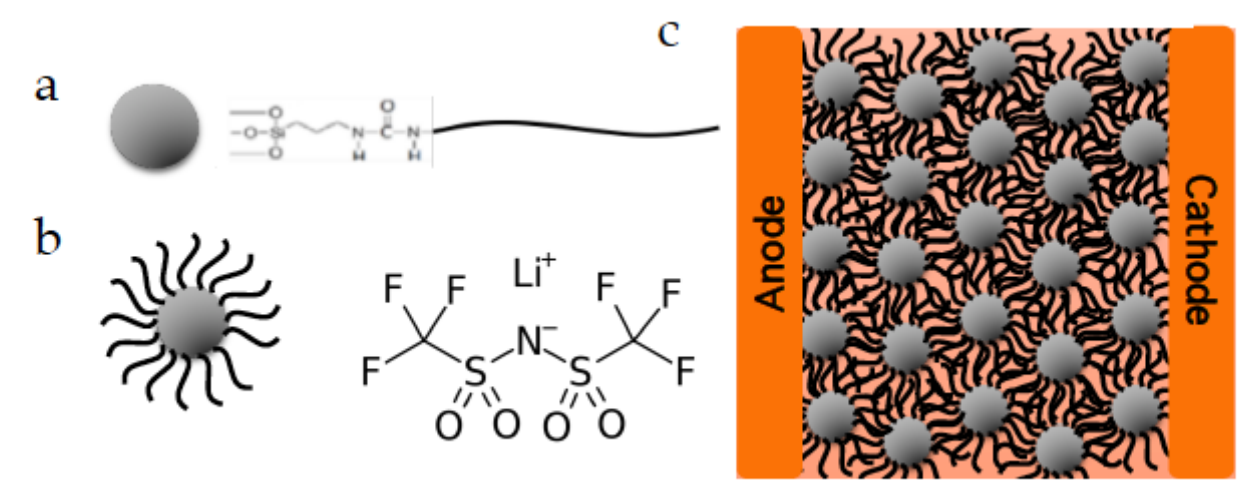

Figure 7.1: (a) Schematic of silica nanoparticle $(25 \mathrm{~nm})$ and silane- functionalized polyethylene oxide (5000Da) involved in the covalent grafting process; (b) Hairy nanoparticles blended with LiTFSI salt to form electrolytes; (c) Cartoon showing the soft glassy electrolyte sandwiched between two electrodes 
functionalized PEO (5KDa) with 3-(Triethoxysilyl)propyl isocyanate (in 1:1 molar basis) in anhydrous chloroform. ${ }^{31}$ Thereafter, silane-PEO is covalently grafted on the surface of silica nanoparticles (25nm) in aqueous media (Figure 7.1(a)). A rigorous washing process is carried out to expel the free polymer chains from the grafted nanoparticles. The presence of free chains in the composite is a concern because it can trigger similar adverse effects to those reported in plasticized solid polymer electrolytes, where free molecules migrate to the electrode-electrolyte interface to participate in parasitic reactions, similar to what is observed in liquid electrolytes. The self-suspended materials produced by this synthesis protocol are mixed with Bis(trifluoromethane) sulfonimide lithium salt (LiTFSI) at different ratios to provide a cation source in the composite (Figure 7.1(b)). The $\mathrm{SiO}_{2}$-PEO/LiTFSI composite forms the entire soft glassy electrolyte (SGE) composition (see Figure 7.1(c)). To understand the relationship between salt composition, physico-chemical, and transport properties of the SGE, we created $\mathrm{SiO}_{2}-\mathrm{PEO} / \mathrm{LiTFSI}$ electrolytes with different salt concentrations. In so doing it is possible to vary the ratio of $\mathrm{Li}+$ cation and ethylene oxide (EO) units in the composite from $r=0$ to $r=0.2$.

Figure 7.2 reports results from Fourier Transform- Infrared Spectroscopy (FTIR) measurements on the soft glassy electrolytes. The major differences in the FTIR spectra occur in the 'finger-print' region ranging from wavelength $900 \mathrm{~cm}^{-1}$ to $1500 \mathrm{~cm}^{-}$ ${ }^{1}$. Among the most obvious observations from these spectra is that absence of contamination associated with water absorption in the materials. ${ }^{32,33}$ It is also seen that there are several IR bands corresponding to similar vibrations in pure PEO $(r=0)$ and LiTFSI. The intensity of the $-\mathrm{CF}$ bond stretch at $1175 \mathrm{~cm}^{-1}$ can be utilized to 

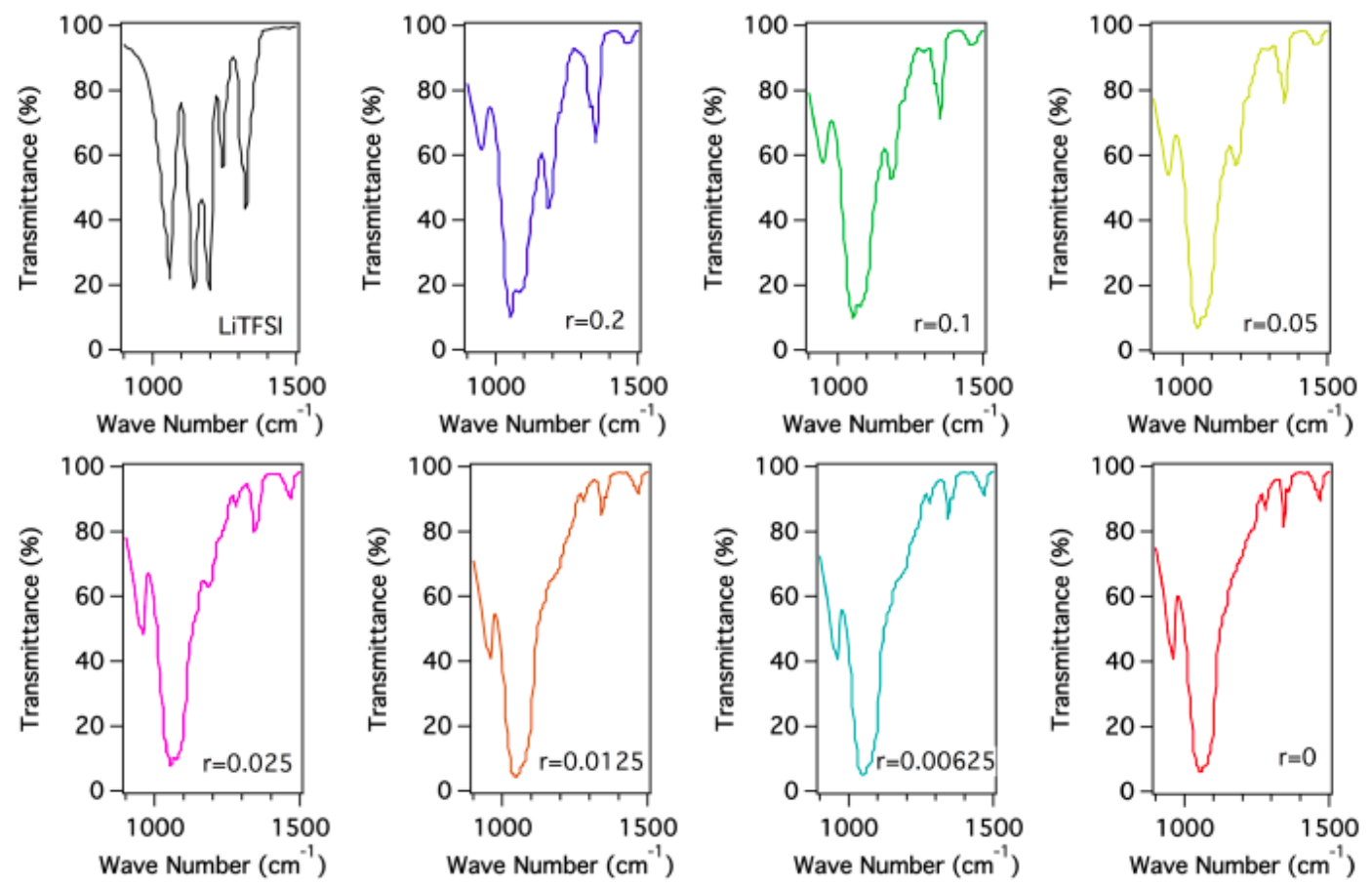

Figure 7.2: Transmitted infrared wave as a function of wavenumber obtained using FTIR. The different $r$ 's represent the ratio between lithium ions and ethylene oxide monomers in the glassy electrolyte 
understand molecular structuring in the salt in the SGE. At ratios, $r=0,0.00625$ and 0.0125 , the peak at $1175 \mathrm{~cm}^{-1}$ is absent. This observation is consistent with a model in which all $\mathrm{Li}^{+}$cations and TFSI ${ }^{-}$anions are dissociated and coordinated with PEO moieties in the composite. Further complexation of LiTFSI $(r=0.025$ and 0.05$)$ leads to the appearance of the $1175 \mathrm{~cm}^{-1}$ peak. At higher salt contents $(r=0.10$ and 0.20$)$ the intensity of the peak rises, implying that the LiTFSI forms aggregates in the composite with a low degree of dissociation, and consequently low fractions of mobile ions for charge transport in the electrolyte. These observations suggest that $r=0.025$ and 0.05 are close to the optimum salt concentrations for the studied SGE electrolytes as nearly complete salt dissociation is promoted by the particle tethered PEO chains.

\subsubsection{Calorimetry and Ion Transport}

The molecular structure of the SGE can be further analyzed using Differential Scanning Calorimetry (DSC). Figure 7.3(a) reports the gravimetric heat flow as a function of temperature for SGE with salt concentration ranging from $r=0$ to 0.2 . The sharp singlet peak at $\sim 54^{\circ} \mathrm{C}$ for the $\mathrm{r}=0$ sample is an indication of a lone crystallite structure in contrast to three melting peaks reported for free PEO polymer. ${ }^{31,32}$ It can be seen that for samples with LiTFSI salt, the thermogram still maintains a singular peak, however with differing intensities. Supplementary Figure 7.1 reports the melting temperatures $\left(\mathrm{T}_{\mathrm{m}}\right)$ for the different LiTFSI: EO ratios. Interestingly, it is seen that the $\mathrm{T}_{\mathrm{m}}$ is maintained very close to $\sim 54^{\circ} \mathrm{C}$ for $\mathrm{SPE}$ samples ranging from $\mathrm{r}=0$ to $\mathrm{r}=0.025$, although the intensity is seen to go down due to the decrease in the overall content of PEO moieties. The low degree of variation in the melting temperature in this range is 
a

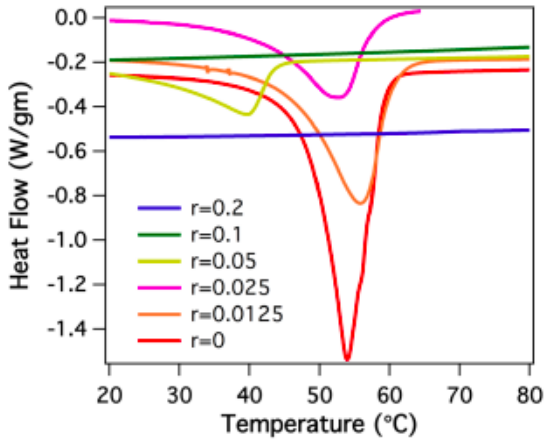

b

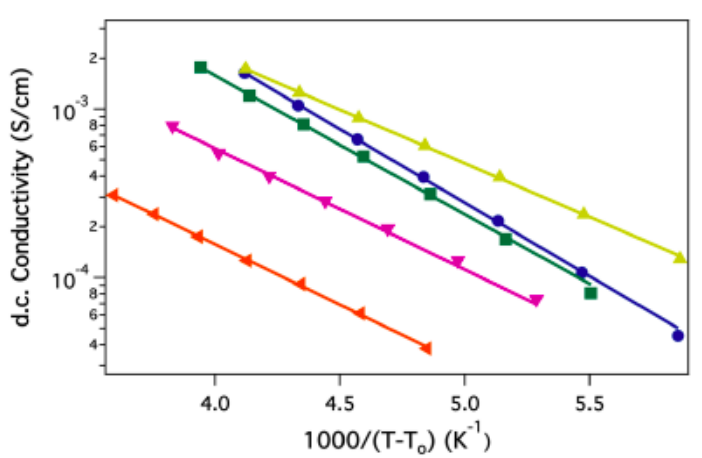

Figure 7.3: (a) Thermogram showing gravimetric heat flow as a function of temperature; (b) VFT fitted experimental data of conductivity in the temperature range above melting. 
thought to reflect the minimum influence of the salt in disrupting the crystallite structures of PEO. In other words, the interaction of LiTFSI with PEO in this range are ionic and effective in dissociating Li and TFSI ions in the salt, but appear to have no effect on the PEO crystallite size. At higher salt contents, there is a stiff drop in the $T_{m}$ at $\mathrm{r}=0.05$ to $\mathrm{T}_{\mathrm{m}}=\sim 40^{\circ} \mathrm{C}$, corresponding to this change, the crystallization peak in Figure 7.3(a) also significantly broadens in comparison to the lower or zero salt concentration. The decrease in $T_{m}$ provides evidence of molecular interaction between LiTFSI and PEO groups. At $\mathrm{r}=0.10$ and $\mathrm{r}=0.20$, no melting transition is observed, implying that interactions with the salt completely disrupt crystallization of PEG chains tethered to the $\mathrm{SiO}_{2}$ nanocores. Taken together, these observations imply that $\mathrm{r}$ $=0.05$ is a critical point in that it heralds a transition from less disruptive ionic to more disrupting molecular interactions between ions in the salt and tethered PEO chains. The molecular structure and ionic transport in an electric field can be further inferred using conductivity measurements of these composite materials. Supplementary Figure 7.2 reports the d.c. conductivity obtained using Dielectric Spectroscopy performed over a wide temperature range, plotted in Arrhenius form, for SGE ranging from $\mathrm{r}=$ 0.0125 to $r=0.2$. It is known that although PEO molecules can transport ions in amorphous as well as crystalline states, the transport timescales are considerably different. The conductivity values for $\mathrm{r}=0.0125,0.025$ and 0.05 are consistent with this understanding and reveal an abrupt change in slope at $48^{\circ} \mathrm{C}, 36^{\circ} \mathrm{C}$ and $24^{\circ} \mathrm{C}$, respectively. This observation is also in agreement with the DSC results, which reveal a crystallization transition in the materials. We isolated the temperature range from $48^{\circ} \mathrm{C}$ to $120^{\circ} \mathrm{C}$ for the measurements and fitted the measured conductivities with a 
Vogel-Fulcher-Tammann (VFT) model, $\sigma=\mathrm{A} \exp \left(-\mathrm{E}_{\mathrm{a}} / \mathrm{R}\left(\mathrm{T}-\mathrm{T}_{\mathrm{o}}\right)\right)$; where $\mathrm{A}$ is the prefactor, $E_{a}$ is the apparent activation energy for ion transport, $R$ is universal gas constant and $\mathrm{T}_{\mathrm{o}}$ is the shift temperature. That the VFT model provides a good fit to the data points in this range (see Figure 7.3(b)) indicates the absence of any thermal degradation of the material or temperature-induced abnormalities in ion transport. The fitting parameters are given in Supplementary Table 7.1. It is important to note that the previously observed temperature-induced jamming in the self-suspended hairy nanoparticles appears to have no noticeable effect on ionic motion. ${ }^{34-37}$ Figure 7.3(b) further shows that the ionic conductivity at $r=0.05$ is higher than the values measured at all other Li: EO ratios in the measured temperature range (see Supplementary Figure 7.3). The maxima in the conductivity in a material that is evidently still semicrystalline at low temperature provides support to our earlier suggestion that at this salt concentration the tethered PEO chains provide maximum dissociation of ion pairs in the LiTFSI salt. On this basis, one could further conclude that the salt concentration at lower ratios is insufficient to produce full complexation with all the available etheroxygens, while at higher than $r=0.05$, LiTFSI partially exist as undissociated and non-conducting ion-pairs. For this reason, we chose $r=0.05$ as the optimum electrolyte composition for the electrochemical studies discussed next.

\subsubsection{Structure Analysis and Rheology}

The bulk scale (nm- $\mu \mathrm{m})$ characteristics of SPEs are dominated by structural contributions from the $\mathrm{SiO}_{2}$ nanoparticle cores. For this reason we used small-angle Xray scattering and oscillatory shear rheology to analyze the electrolytes. 
a

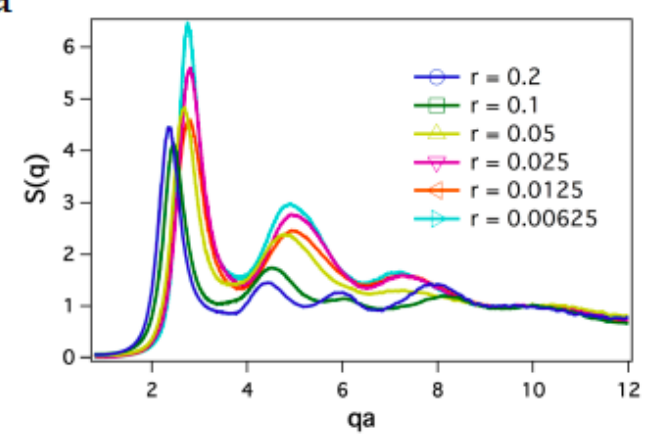

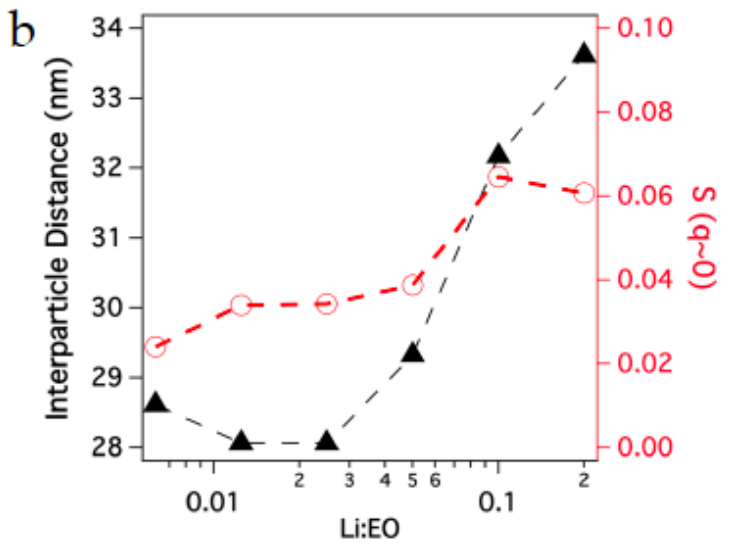

Figure 7.4: (a) Structure Factor obtained from SAXS analysis as a function of radius normalized wave vector; (b) Interparticle distance obtained from the first peak as well as value of $\mathrm{S}(\mathrm{q})$ as $\mathrm{q} \rightarrow 0$ for different salt compositions. 
Supplementary Figure 7.4 reports the scattered X-ray intensities plotted against the wave vector q, for measurements performed at $90^{\circ} \mathrm{C}$. Several features of the intensity profile can be used to understand the structure of these materials. At high q, the I(q) decay as the fourth power of the wave vector $\left(\mathrm{I}(\mathrm{q}) \sim \mathrm{q}^{-4}\right)$ with repeated oscillations, indicating that the particles are spherical in shape. Further at low q, the $\mathrm{I}(\mathrm{q})$ is independent of q, denoting the absence of long range density fluctuations and structure in the materials. ${ }^{38-40}$ Both characteristics are indicative of well-dispersed particles. Figure 7.4(a) reports the structure factor $(\mathrm{S}(\mathrm{q}))$ plotted as a function of wave vector normalized by the particle radius $\sim 12.5 \mathrm{~nm}$. Remarkably, in the limit as $\mathrm{q} \rightarrow 0, \mathrm{~S}(\mathrm{q})$ is seen to be significantly lower than previously obtained results for hard sphere suspensions. This behavior has been reported previously and reflects the effect of space-filling constrains on the tethered polymer chains which drive hyperuniformity in the materials, such that $\mathrm{S}(\mathrm{q}=0) \rightarrow 0 .{ }^{41,42}$ Figure $7.4(\mathrm{~b})$ reports $\mathrm{S}(\mathrm{q} \sim 0)$ for the different salt concentrations investigated. The results show there are no noticeable differences in $\mathrm{S}(0)$ until $\mathrm{r}=0.05$, however, upon increasing the salt concentration beyond this value, there is a jump in $S(0)$, reminiscence of long-range ordering. This finding lends support to our earlier inference that above the critical salt concentration LiTFSI is no longer associated with PEO chains and instead occupies space between the silica nanoparticles, reducing the strength of the space-filling constraint on grafted polymer chains. The location of the peaks in the S(q) (see Figure 7.4(a)) confirms this point. Specifically, the center-to-center distance between the silica nanoparticles can be estimated from the location of the first peak $S_{1}$, plotted in Figure 7.4(b). It is seen to rise steeply beyond $\mathrm{r}=0.05$ consistent with the existence of LiTFSI as undissociated 

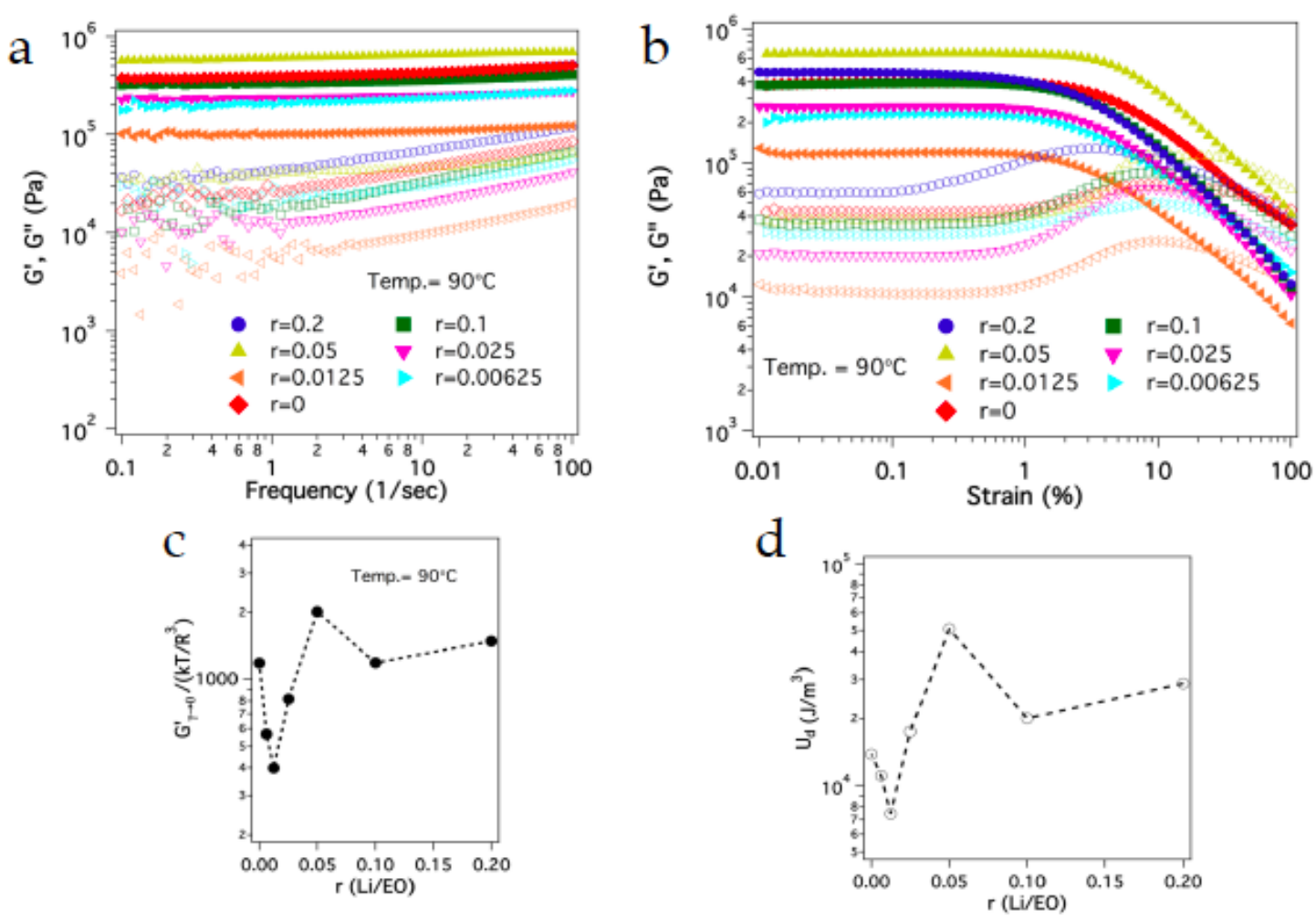

Figure 7.5: (a), (b) Frequency sweep and amplitude sweep measurements obtained from oscillatory shear measurements, respectively at $90^{\circ} \mathrm{C}$. The frequency sweep were done at strain $=0.1 \%$ and amplitude sweep at $\boldsymbol{\omega}=10 \%$; (c) Plateau modulus as strain $\rightarrow 0$ from different compositions of glassy electrolytes; (d) Dissipation energy obtained by calculating the area under the curve of G" maxima, obtained by lognormal fitting. 
salt clusters in the materials. It has been previously reported that the first peak of the structure factor $\left(\mathrm{S}_{1}\right)$ signifies the steric repulsions and the second peak $\left(\mathrm{S}_{2}\right)$ reflect the entropic attractions in these materials. ${ }^{31}$ Consistent with previous results using covalently grafted particles, the $S_{1}$ peak height is much larger than that of $S_{2}$, in contrast to their ionic counterparts; also signifying that the ionic linkages formed due to the salts do not significantly alter the macroscopic distribution of the nanoparticles. We performed oscillatory shear rheology on these materials to understand the relationships between their dynamics and bulk transport properties. Results from oscillatory shear measurements at a fixed shear strain $(\gamma=0.1 \%)$ and variable dynamic frequency $(\omega)$ and at a fixed frequency $\left(\omega=10 \mathrm{~s}^{-1}\right)$ and variable strain amplitude are reported in Figure 7.5(a) and 7.5(b), respectively. All measurements were performed at $90^{\circ} \mathrm{C}$. Interestingly, for all salt compositions, the storage modulus (G') dominates the loss modulus (G") in the low-strain, linear viscoelastic regime, indicating the materials possess solid-like, elastic consistency. Large Amplitude Oscillatory Shear (LAOS) measurements (Figure 5(b)) show that the materials are in fact soft glasses. ${ }^{43-}$ ${ }^{45}$ At low shear strain, G' $>>$ G' and nearly independent of $\omega$ (Figure 7.5a) and $\gamma$ (Figure 7.5b). In contrast at higher shear strain, G' decreases with increasing strain, while G" initially rises, then falls less rapidly than G'. As a result G" displays a local maximum, crosses G', and ultimately becomes larger than G' at high shear strains. This transition of the materials from solid-like (G' dominant) to liquid-like (G' dominant) consistencies at higher strains, along with the appearance of the G" maximum at an intermediate shear strain are all well-known traits of soft glasses. They are known to arise from arrested motion or caging of the $\mathrm{SiO}_{2}$ cores by the 
interdigitated tethered PEO molecules followed by strain-induced breakdown of the cages, yielding particles that slide past each other dissipating energy as a result of frictional contacts between the dislocated corona polymer chains..$^{31,40}$ Figure 7.5(c) reports the normalized elastic modulus obtained from the results in Figure 7.5(a) at different salt concentrations. Here $G^{\prime}$ is normalized by the Brownian Stress $\mathrm{kT} / \mathrm{R}^{3}$, such that values above unity imply that the stresses produced by caging are sufficient to prohibit uncorrelated, random motion of the cores. The results show that at all salt concentrations $<\mathrm{G}^{\mathrm{9}} /\left(\mathrm{kT} / \mathrm{R}^{3}\right)>$ is significantly larger than unity meaning that the particle motions are completely arrested by the interdigitated PEO corona. The PEO chains can therefore be thought of effective cross-links that lock the $\mathrm{SiO}_{2}$ cores in place to create a tortuous nanoporous medium in which ions must move in these electrolytes.

At low salt concentrations, results in Figure 7.5(c) show that addition of salt to the $\mathrm{SiO} 2$-PEO material causes $<\mathrm{G}^{\prime} /\left(\mathrm{kT} / \mathrm{R}^{3}\right)>$ to decrease. The decrease continues until $\mathrm{r}=$ 0.0125 , whereafter it begins to rise, reaching a maximum value at $r=0.05$. It is known that $\mathrm{Li}^{+}$cations are able coordinate with multiple EO moieties in an amorphous polymer, which would enhance the bridging effect produced by the interdigitated PEO chains. ${ }^{46,47}$ The saturation of the elastic modulus beyond $\mathrm{r}=0.05$ is consistent with our designation of $r=0.05$ as the critical salt concentration. The specific energy dissipated $\left(U_{d}\right)$ (shown in Figure 7.5(d)) during the cage breakage transition can be calculated from the area under the G' $(\gamma)$ curve, obtained by fitting the experimental results with a Normal Distribution function. The effect of salt concentration on $U_{d}$ tracks closely the $<\mathrm{G}^{\prime} /\left(\mathrm{kT} / \mathrm{R}^{3}\right)>$ data, indicating that the two effects originate from the 
a

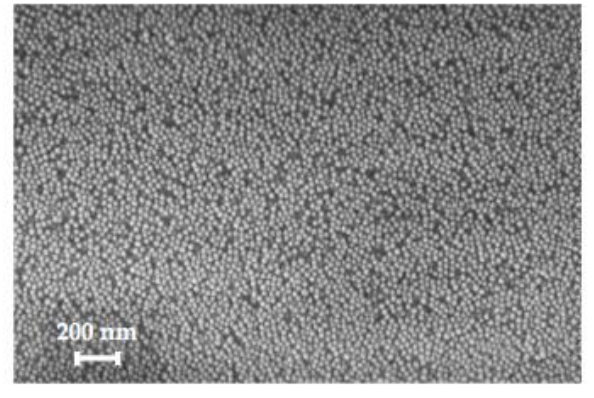

b

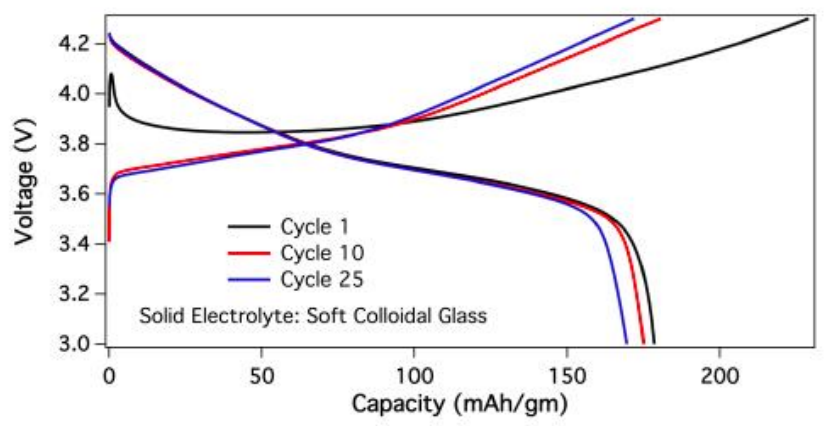

Figure 7.6: (a) SEM image of the surface of lithium metal electrode covered with multiple stacks of colloidal soft glassy electrolytes ; (b) Voltage profile for the Li||NCM cell at a current density of $0.20 \mathrm{~mA} / \mathrm{cm}^{2}$ for cycle 1,10 and 25 . 
same source and consistent with what one would expect from a cage breakage event arising from breakage of PEO cross-links.

To investigate electrochemical properties of SGE materials, we designed a lithium metal battery using lithium metal electrode and SGE with $r=0.05$ as the solid electrolyte. Figure 7.6(a) reports the Scanning Electron Microcopy (SEM) image of the surface of lithium metal laminated with the SGE. The particles are seen to be well dispersed without any visible aggregates. It is noteworthy than even after multiple layers, the particles are essentially randomly distributed in space, consistent with the idea that the materials can be conceptualized as nanoporous media, with pore size set by the inter-particle distance, which is of the order $4 \mathrm{~nm}$ for $\mathrm{r}=0.05$ (Figure 4(b)). On the basis of linear stability analysis ${ }^{48,49}$ and experiment ${ }^{11,50,51}$, we previously reported that electrolytes with such nanoporous morphology are effective in suppressing growth of dendrites during metal electrodeposition.

\subsubsection{Analysis of Electrochemical Performance}

As discussed earlier, the poor oxidative stability of PEO-based electrolytes has traditionally limited use of such electrolytes to batteries in which metallic lithium is paired with relatively low voltage $\left(<3.8 \mathrm{~V}\right.$ vs $\left.\mathrm{Li} / \mathrm{Li}^{+}\right)$cathode chemistries, including lithium titanate $\mathrm{LiTi}_{4} \mathrm{O}_{7}$ or lithium iron phosphate $\mathrm{LiFePO}_{4}$. This has in turn reduced practical interest in lithium batteries that utilize PEO-based polymers as solid-state electrolytes. We analyzed the voltage stability window of the soft glassy electrolyte using cyclic voltammetry using a lithium versus stainless steel electrochemical cell as shown in Supplementary Figure 7.5. It was observed that the oxidative potential for 
the $\mathrm{SGE}$ is $\sim 4.2 \mathrm{~V}$ vs. $\mathrm{Li} / \mathrm{Li}+$. This extended stability window can be asserted to the immobilization of the PEO groups by the surface grafting on silica nanoparticles. Similar improvement has been also reported in block-copolymer electrolytes based on PS-PEO with LiTFSI salt ${ }^{52}$. However, in addition to the physical approach, it is important to chemically inhibit the electrochemical oxidation of ether-oxide groups below $4.3 \mathrm{~V} \mathrm{vs} \mathrm{Li} / \mathrm{Li}+$ to enable stable cycling in a high voltage $\mathrm{Li} \| \mathrm{NCM}$ battery. Recently, we performed ab-initio calculations and inferred that a salt additive lithium bis(oxalate) borate ( $\mathrm{LiBOB}$ ) can be employed as a sacrificial agent in a liquid electrolyte to produce negatively charged ion clusters at the cathode electrolyte interphase (CEI) ${ }^{53} \mathrm{~A}$ negatively charged SEI is hypothesized to create a rectifying mechanism that restricts transport of oxidation products formed during electrochemical breakdown of PEO-based electrolytes. Here, we evaluate the effectiveness of this concept for enabling high voltage operation of a solid polymer electrolyte based on PEO. Specifically, we create a CEI on the surface of a NCM cathode (areal loading $=2 \mathrm{mAh} / \mathrm{cm}^{2}$ ) by first wetting the cathode with a LIBOBcontaining liquid electrolyte (0.4M LiBOB, 0.6M LiTFSI, 0.05M LiPF 6 - EC/DMC $)^{54}$ and use the wetted cathodes in electrochemical cells. $\mathrm{LiPF}_{6}$ is include in the formulation because it is thought to be important for preventing corrosion of the $\mathrm{Al}$ current-collector used for the cathode ${ }^{55}$, LiTFSI is the common ion carrier for transport at the CEI and in the bulk SGE phase.

Supplementary Figure 7.6 shows the impedance spectra of the Li $\| \mathrm{SGE} \mid$ liq. || $\mathrm{NCM}$ plotted in the for of a Nyquist plot at $30^{\circ} \mathrm{C}$. The experimental data was fitted to the equivalent circuit model shown in the inset of Figure 7.6, which comprises of a bulk 
resistor in series with two other resistors representing the anodic and cathodic interfaces parallel to constant phase elements representing the static charges in the debye layer. The bulk resistance is calculated to be $19 \Omega$, while the anodic and cathodic interfacial resistances are $127 \Omega$ and $435 \Omega$, respectively. It can be inferred from these resistances that the liquid electrolyte partially solvates the SGE at the anode side, thus offering low overall battery resistance for operation at room temperature at low to moderate currents. The ionic conductivity of the solvated SGE electrolyte can be calculated as $0.5 \mathrm{mS} / \mathrm{cm}$. Furthermore, we analyzed the impedance spectra of the battery after 100 cycles shown in the inset of Supplementary Figure 7.6. It can be seen that, while the bulk impedance $(20 \Omega)$ remains similar to the original value, the interfacial resistance drops from $127 \Omega$ to $40 \Omega$, which implies that formation of ionic pathways sue to wetting of the PEO chains by the liquid electrolyte. Although we operate the battery in this work at ambient conditions in the current work, it will be interesting in future to observe the physical behavior of the soft glassy electrolyte as well robustness of the interfacial layer when the operated at elevated temperature. However, it is important to note that the hairy nanoparticles maintain their viscoelastic nature at high temperatures and also the borate salts are thermally stable.

The CEI layer formed during cycling was analyzed using Fourier Transform Infrared Spectroscopy measurements. The IR spectra is shown in Supplementary Figure 7.7 after the battery was cycled 5 times and the relevant peaks are marked using dashed lines. The peaks at $1060 \mathrm{~cm}^{-1}$ represent O-B-O bond, while that at $1260 \mathrm{~cm}^{-1}$ and 1360 $\mathrm{cm}^{-1}$ indicate $\mathrm{C}-\mathrm{O}$ and B-O bonds. ${ }^{56}$ Thus, it can be argues that the CEI is dominated by carboxylic and boro-oxylate species. Figure 7.6(b) shows the voltage profiles for 
the $\mathrm{Li} \| \mathrm{NCM}$ cell operated at $0.20 \mathrm{~mA} / \mathrm{cm}^{2}$, cycled between $4.3 \mathrm{~V}$ to $3 \mathrm{~V}$. The first charging involves a long step associated with the formation of an interfacial phase. It is observed that the nominal discharge voltage is $\sim 3.7 \mathrm{~V}$ as expected for a NCM cathode in absence of any IR loss. The initial discharge capacity is obtained to be $\sim 178 \mathrm{mAh} / \mathrm{gm}$ and in 25 cycles the capacity retention is over $97 \%$. The coulombic efficiency of the first cycle is seen to be $\sim 78 \%$, thereafter it increases to more than 99\% in the course of cycling as seen in Supplementary Figure 7.8. The low C.E. in the initial cycle can be arise from the irreversible reactions to form the interfacial layer on the cathode. We further performed post-mortem analysis of the electrodes at different stages of cycling to fundamentally understand the cycling behavior. Supplementary Figure 7.9 displays the surface of lithium metal anode and the NCM cathode after 5 cycles. It can be seen that the lithium metal anode is covered with the multilayers of PEO-grafted silica nanoparticles similar to the anode surface before cycling shown in Figure 6(a). This supports many previous observations in nanocomposite electrolytes, where it is argued the hairy nanoparticles adsorb to the surface of the anode forming a mechanically stable artificial SEI layer. ${ }^{57,58}$ At the end of 100 cycles of stable charging and discharging the $\mathrm{Li} \| \mathrm{NCM}$ cell, it is seen from Supplementary Figure 7.10 that the surface of lithium metal anode is cracked with multiple wide openings, presumably due to the large volume expansion and contraction $(10 \mu \mathrm{m})$ during lithium plating and stripping. Unlike previous reports of battery cycling using liquid electrolytes, there is no sign of dendritic growth even after prolonged cycling due to the mechanical reinforcement by the soft glassy electrolytes. The SEM analysis of the NCM cathode 
after 5 and 100 cycles (as shown in Supplementary Figure 7.9 and 7.10 reveals that there is minimal disintegration of the cathode particles upon cycling.

\subsection{Conclusion}

In conclusion, we designed a glass-type solid polymer by covalent linkage between silica nanoparticles and polyethylene oxide polymer and blending with a lithium salt. Variation of the salt content in the solid electrolyte leads to number of interesting structural and transport properties. The molar ratio of 0.05 for $\mathrm{Li}+$ and ethylene oxide molecules was seen to be the optimum, such that above this value, lithium salt remain as aggregates, while at low content the number of ions inadequately complex with the tethered polymers. The amount of salt in the composite also leads to macroscopic changes in the modulus and nanoparticle arrangements. We further characterized the novel material in a lithium metal battery, where the colloidal particles were seen to arrange in a uniform multilayered fashion. As a first example of a safe, high voltage solid-state lithium metal battery, we demonstrated stable cycling of a Nickel Manganese Cobalt Oxide cathode after interfacial engineering with ionic additives. We believe, this work will open up a new avenue in the solid polymer electrolyte literature with ether-oxide based chemistries that often fail at high oxidation potentials.

\section{Acknowledgements}

The authors acknowledge support of the National Science Foundation, Division of Materials Research, through Award No. DMR-1609125. This work made use of the 
Cornell Center for Materials Research Shared Facilities, which are supported through the NSF MRSEC program (DMR-1719875). 


\section{REFERENCES}

(1) Cheng, X.; Zhang, R.; Zhao, C.; Wei, F.; Zhang, J.; Zhang, Q. A Review of Solid Electrolyte Interphases on Lithium Metal Anode. Adv. Sci. 2016, 3, 1-20.

(2) Tikekar, M. D.; Choudhury, S.; Tu, Z.; Archer, L. A. Design Principles for Electrolytes and Interfaces for Stable Lithium-Metal Batteries. Nat. Energy 2016, $1,16114$.

(3) Lin, D.; Liu, Y.; Cui, Y. Reviving the Lithium Metal Anode for High-Energy Batteries. Nat. Nanotechnol. 2017, 12, 194-206.

(4) Cheng, X.-B.; Zhang, R.; Zhao, C.-Z.; Zhang, Q. Toward Safe Lithium Metal Anode in Rechargeable Batteries: A Review. Chem. Rev. 2017, 117, 1040310473.

(5) Wei, S.; Choudhury, S.; Tu, Z.; Zhang, K.; Archer, L. A. Electrochemical Interphases for High-Energy Storage Using Reactive Metal Anodes. Acc. Chem. Res. 2018, 51, 80-88.

(6) Stalin, S.; Choudhury, S.; Zhang, K.; Archer, L. A. Multifunctional CrossLinked Polymeric Membranes for Safe, High-Performance Lithium Batteries.

Chem. Mater. 2018, 30, 2058-2066.

(7) Agrawal, A.; Choudhury, S.; Archer, L. A. A Highly Conductive, NonFlammable Polymer-Nanoparticle Hybrid Electrolyte. RSC Adv. 2015.

(8) Guo, Q.; Han, Y.; Wang, H.; Hong, X.; Zheng, C.; Liu, S.; Xie, K. Safer Lithium Metal Battery Based on Advanced Ionic Liquid Gel Polymer Nonflammable Electrolytes. RSC Adv. 2016, 6, 101638-101644. 
(9) Hu, J.; Wang, W.; Peng, H.; Guo, M.; Feng, Y.; Xue, Z.; Ye, Y.; Xie, X. Flexible Organic-Inorganic Hybrid Solid Electrolytes Formed via ThiolAcrylate Photopolymerization. Macromolecules 2017, 50, 1970-1980.

(10) Khurana, R.; Schaefer, J. L.; Archer, L. A.; Coates, G. W. Suppression of Lithium Dendrite Growth Using Cross-Linked Polyethylene/poly(ethylene Oxide) Electrolytes: A New Approach for Practical Lithium-Metal Polymer Batteries. J. Am. Chem. Soc. 2014, 136, 7395-7402.

(11) Choudhury, S.; Mangal, R.; Agrawal, A.; Archer, L. A. A Highly Reversible Room-Temperature Lithium Metal Battery Based on Crosslinked Hairy Nanoparticles. Nat. Commun. 2015, 6, 10101.

(12) Li, J.; Ma, C.; Chi, M.; Liang, C.; Dudney, N. J. Solid Electrolyte: The Key for High-Voltage Lithium Batteries. Adv. Energy Mater. 2015, 5, 1401408.

(13) Takada, K. Progress in Solid Electrolytes toward Realizing Solid-State Lithium Batteries. J. Power Sources 2018, 394, 74-85.

(14) Zheng, F.; Kotobuki, M.; Song, S.; Lai, M. O.; Lu, L. Review on Solid Electrolytes for All-Solid-State Lithium-Ion Batteries. J. Power Sources 2018, $389,198-213$.

(15) Put, B.; Vereecken, P.; Stesmans, A. On the Chemistry and Electrochemistry of LiPON Breakdown. J. Mater. Chem. A 2018, 6, 4848-4859.

(16) Le Van-Jodin, L.; Claudel, A.; Secouard, C.; Sabary, F.; Barnes, J.-P.; Martin, S. Role of the Chemical Composition and Structure on the Electrical Properties of a Solid State Electrolyte: Case of a Highly Conductive LiPON. Electrochim. Acta 2018, 259, 742-751. 
(17) Bai, L.; Xue, W.; Li, Y.; Liu, X.; Li, Y.; Sun, J. The Interfacial Behaviours of All-Solid-State Lithium Ion Batteries. Ceram. Int. 2018, 44, 7319-7328.

(18) Lei, F.; Shuya, W.; Siyuan, L.; Qi, L.; Yingying, L. Recent Progress of the Solid-State Electrolytes for High-Energy Metal-Based Batteries. Adv. Energy Mater. 2018, 8, 1702657.

(19) Yu, X.; Bates, J. B.; Jellison, G. E.; Hart, F. X. A Stable Thin-Film Lithium Electrolyte: Lithium Phosphorus Oxynitride. J. Electrochem. Soc. 1997, 144, $524-532$.

(20) Chih-Jung, C.; Tatsuhiro, M.; Anirudha, J.; Hung-Yu, L.; Nai-Hsuan, Y.; NaeLih, W.; Ho, C.; Shu-Fen, H.; Ru-Shi, L. Optimizing the Lithium Phosphorus Oxynitride Protective Layer Thickness on Low-Grade Composite Si-Based Anodes for Lithium-Ion Batteries. ChemistrySelect 2018, 3, 729-735.

(21) Han, X.; Gong, Y.; Fu, K. (Kelvin); He, X.; Hitz, G. T.; Dai, J.; Pearse, A.; Liu, B.; Wang, H.; Rubloff, G.; et al. Negating Interfacial Impedance in GarnetBased Solid-State Li Metal Batteries. Nat. Mater. 2016, 16, 572.

(22) Weidong, Z.; Zhengyuan, T.; Jiawei, Q.; Snehashis, C.; A., A. L.; Yingying, L. Design Principles of Functional Polymer Separators for High-Energy, MetalBased Batteries. Small 2018, 14, 1703001.

(23) Stephan, a. M. Review on Gel Polymer Electrolytes for Lithium Batteries. Eur. Polym. J. 2006, 42, 21-42.

(24) Zhang, W.; Zhuang, H. L.; Fan, L.; Gao, L.; Lu, Y. A “cation-Anion Regulation" Synergistic Anode Host for Dendrite-Free Lithium Metal Batteries. Sci. $A d v$. 2018, 4. 
(25) Cheng, X.-B.; Yan, C.; Zhang, X.-Q.; Liu, H.; Zhang, Q. Electronic and Ionic Channels in Working Interfaces of Lithium Metal Anodes. ACS Energy Lett. 2018, 3, 1564-1570.

Zhao, C.-Z.; Zhang, X.-Q.; Cheng, X.-B.; Zhang, R.; Xu, R.; Chen, P.-Y.; Peng, H.-J.; Huang, J.-Q.; Zhang, Q. An Anion-Immobilized Composite Electrolyte for Dendrite-Free Lithium Metal Anodes. Proc. Natl. Acad. Sci. 2017, 114, 11069 LP-11074.

(27) Baker, G. L.; Colsons, S. Composite Polymer Electrolytes Using Fumed Silica Fillers : Rheology and Ionic Conductivity. 1994, 2359-2363.

(28) Lu, Y.; Korf, K.; Kambe, Y.; Tu, Z.; Archer, L. a. Ionic-Liquid-Nanoparticle Hybrid Electrolytes: Applications in Lithium Metal Batteries. Angew. Chemie $\mathbf{2 0 1 4}, 126,498-502$.

(29) Stone, G. M.; Mullin, S. a.; Teran, a. a.; Hallinan, D. T.; Minor, a. M.; Hexemer, a.; Balsara, N. P. Resolution of the Modulus versus Adhesion Dilemma in Solid Polymer Electrolytes for Rechargeable Lithium Metal Batteries. J. Electrochem. Soc. 2012, 159, A222-A227.

(30) Bruce, P. Conductivity and Transference Number Measurements on Polymer Electrolytes. Solid State Ionics 1988, 28-30, 918-922.

(31) Choudhury, S.; Agrawal, A.; Kim, S. a; Archer, L. a. Self-Suspended Suspensions of Covalently Grafted Hairy Nanoparticles. Langmuir 2015, 31, $3222-3231$.

(32) Kim, S. A.; Archer, L. A. Hierarchical Structure in Semicrystalline Polymers Tethered to Nanospheres. Macromolecules 2014, 47, 687-694. 
(33) Wong, D. H. C.; Thelen, J. L.; Fu, Y.; Devaux, D.; Pandya, A. a; Battaglia, V. S.; Balsara, N. P.; DeSimone, J. M. Nonflammable Perfluoropolyether-Based Electrolytes for Lithium Batteries. Proc. Natl. Acad. Sci. U. S. A. 2014, 111, $3327-3331$.

(34) Agarwal, P.; Srivastava, S.; Archer, L. A. Thermal Jamming of a Colloidal Glass. Phys. Rev. Lett. 2011, 107, 268302.

(35) Agrawal, A.; Wenning, B. M.; Choudhury, S.; Archer, L. A. Interactions, Structure, and Dynamics of Polymer-Tethered Nanoparticle Blends. Langmuir 2016, 32, 8698-8708.

Agrawal, A.; Yu, H.-Y.; Sagar, A.; Choudhury, S.; Archer, L. A. Molecular Origins of Temperature Induced Jamming in Self-Suspended Hairy Nanoparticles. Macromolecules 2016.

(37) Agrawal, A.; Yu, H.-Y.; Srivastava, S.; Choudhury, S.; Narayanan, S.; Archer, L. Dynamics and Yielding of Binary Self-Suspended Nanoparticle Fluids. Soft Matter 2015, 11, 5224-5234.

(38) Yu, H.-Y.; Srivastava, S.; Archer, L. a; Koch, D. L. Structure Factor of Blends of Solvent-Free Nanoparticle-Organic Hybrid Materials: Density-Functional Theory and Small Angle X-Ray Scattering. Soft Matter 2014, 10, 9120-9135.

(39) Ashcroft, N. W.; Langreth, D. C. Structure of Binary Liquid Mixtures. I. Phys. Rev. 1967, 16, 685-692.

(40) Srivastava, S.; Choudhury, S.; Agrawal, A.; Archer, L. A. Self-Suspended Polymer Grafted Nanoparticles. Curr. Opin. Chem. Eng. 2017, 16, 92-101.

(41) Chremos, A.; Panagiotopoulos, A. Z.; Koch, D. L. Dynamics of Solvent-Free 
Grafted Nanoparticles. J. Chem. Phys. 2012, 136, 44902.

(42) Chremos, A.; Panagiotopoulos, A. Z.; Yu, H.-Y.; Koch, D. L. Structure of Solvent-Free Grafted Nanoparticles: Molecular Dynamics and DensityFunctional Theory. J. Chem. Phys. 2011, 135, 114901.

(43) Sollich, P. Rheological Constitutive Equation for a Model of Soft Glassy Materials. Phys. Rev. E 1998, 58, 738-759.

(44) Sollich, P.; Lequeux, F.; Hébraud, P.; Cates, M. Rheology of Soft Glassy Materials. Phys. Rev. Lett. 1997, 78, 2020-2023.

(45) Zaccarelli, E.; Mayer, C.; Asteriadi, a.; Likos, C.; Sciortino, F.; Roovers, J.; Iatrou, H.; Hadjichristidis, N.; Tartaglia, P.; Löwen, H.; et al. Tailoring the Flow of Soft Glasses by Soft Additives. Phys. Rev. Lett. 2005, 95, 268301.

(46) Nugent, J. L.; Moganty, S. S.; Archer, L. a. Nanoscale Organic Hybrid Electrolytes. Adv. Mater. 2010, 22, 3677-3680.

(47) Schaefer, J. L.; Moganty, S. S.; Yanga, D. a.; Archer, L. a. Nanoporous Hybrid Electrolytes. J. Mater. Chem. 2011, 21, 10094.

(48) Tikekar, M. D.; Archer, L. A.; Koch, D. L. Stabilizing Electrodeposition in Elastic Solid Electrolytes Containing Immobilized Anions. Sci. Adv. 2016, 2.

(49) Tikekar, M. D.; Archer, L. A.; Koch, D. L. Stability Analysis of Electrodeposition across a Structured Electrolyte with Immobilized Anions. $J$. Electrochem. Soc. 2014, 161, A847-A855.

(50) Tu, Z.; Zachman, M. J.; Choudhury, S.; Wei, S.; Ma, L.; Yang, Y.; Kourkoutis, L. F.; Archer, L. A. Nanoporous Hybrid Electrolytes for High-Energy Batteries Based on Reactive Metal Anodes. Adv. Energy Mater. 2017, 7, 1602367. 
(51) Tu, Z.; Nath, P.; Lu, Y.; Tikekar, M. D.; Archer, L. A. Nanostructured

Electrolytes for Stable Lithium Electrodeposition in Secondary Batteries. Acc.

Chem. Res. 2015, 48, 2947.

(52) Bouchet, R.; Maria, S.; Meziane, R.; Aboulaich, A.; Lienafa, L.; Bonnet, J.;

Phan, T. N. T.; Bertin, D.; Gigmes, D.; Devaux, D.; et al. Single-Ion BAB

Triblock Copolymers as Efficient Electrolytes for Lithium-Metal Batteries. Nat.

Mater. 2013, 12, 452-457.

(53) Choudhury, S.; Tu, Z.; Nijamudheen, A.; Zhao, Q.; Vu, D.; A., J. L. M.-C.;

Archer, L. A. Stabilizing Polymer Electrolytes in High-Voltage Lithium

Batteries. submitted.

(54) Zheng, J.; Engelhard, M. H.; Mei, D.; Jiao, S.; Polzin, B. J.; Zhang, J.-G.; Xu,

W. Electrolyte Additive Enabled Fast Charging and Stable Cycling Lithium

Metal Batteries. Nat. Energy 2017, 2, 17012.

(55) Zhang, S. S. A Review on Electrolyte Additives for Lithium-Ion Batteries. $J$.

Power Sources 2006, 162, 1379-1394.

(56) Beaucage, G. Small-Angle Scattering from Polymeric Mass Fractals of Arbitrary Mass-Fractal Dimension. J. Appl. Crystallogr. 1996, 29, 134-146.

(57) Choudhury, S.; Agrawal, A.; Wei, S.; Jeng, E.; Archer, L. A. Hybrid Hairy Nanoparticle Electrolytes Stabilizing Lithium Metal Batteries. Chem. Mater. 2016, 28, 2147-2157.

(58) Wei, S.; Xu, S.; Agrawral, A.; Choudhury, S.; Lu, Y.; Tu, Z.; Ma, L.; Archer, L. A. A Stable Room-Temperature Sodium-sulfur Battery. Nat. Commun. 2016, 7, 11722 . 


\section{APPENDIX}

Supplementary Information for Chapter 7

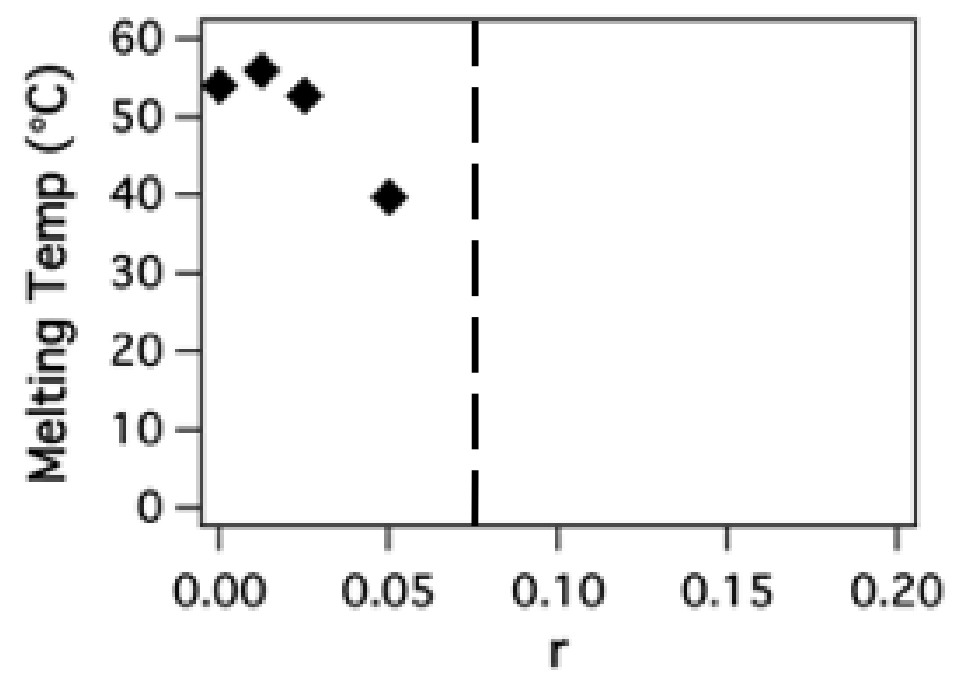

Supplementary Figure 7.1: Melting temperature obtained from DSC plotted with Li : EO ratio 


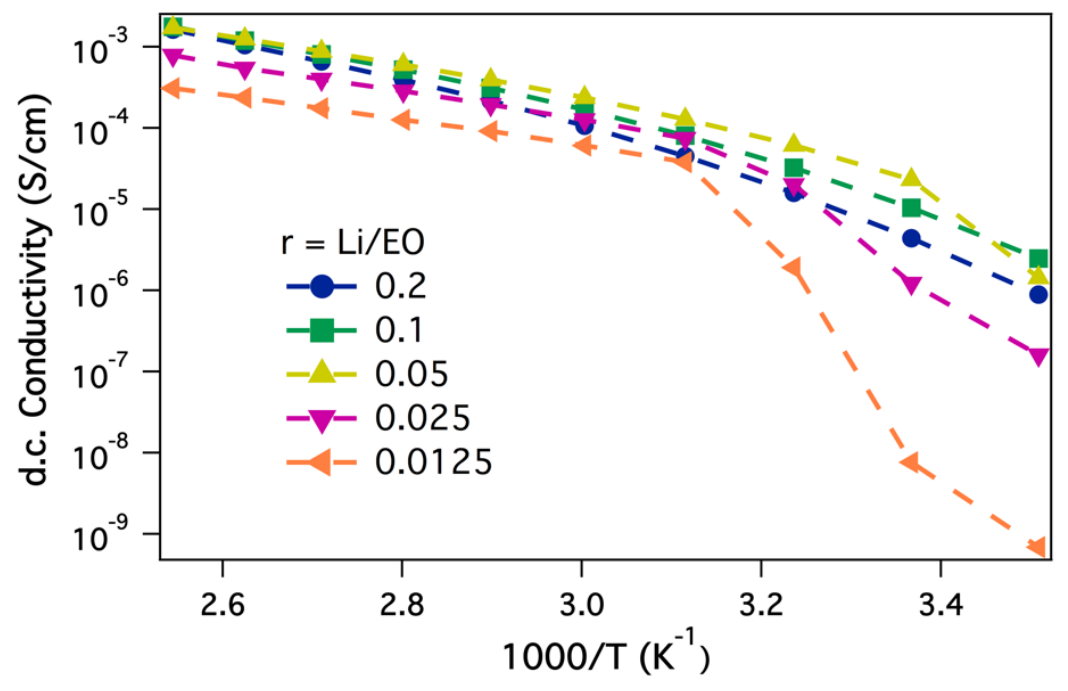

Supplementary Figure 7.2: d.c. conductivity plotted a function of Arrhenius temperature 


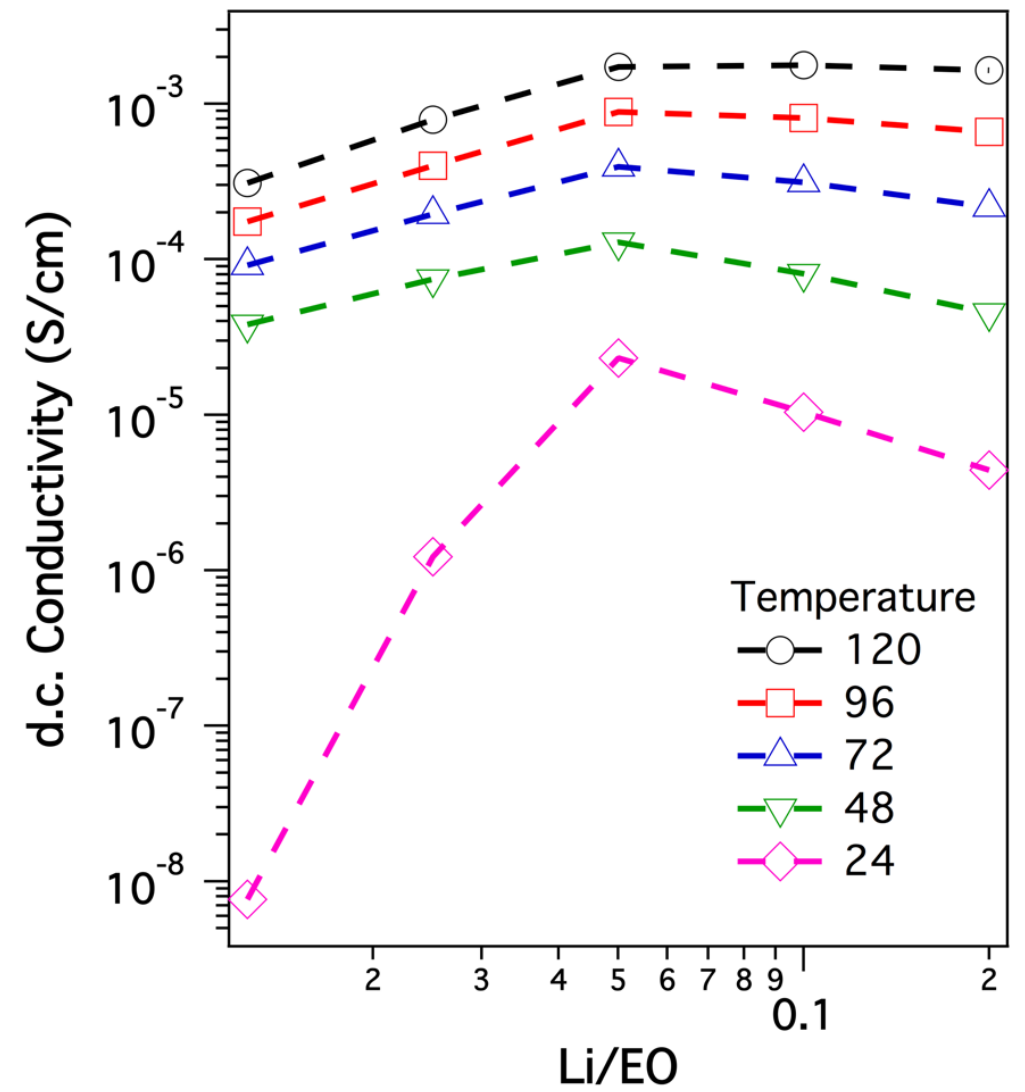

Supplementary Figure 7.3: Variation of $d . c$. conductivity with the Li/EO ratios at various temperatures 


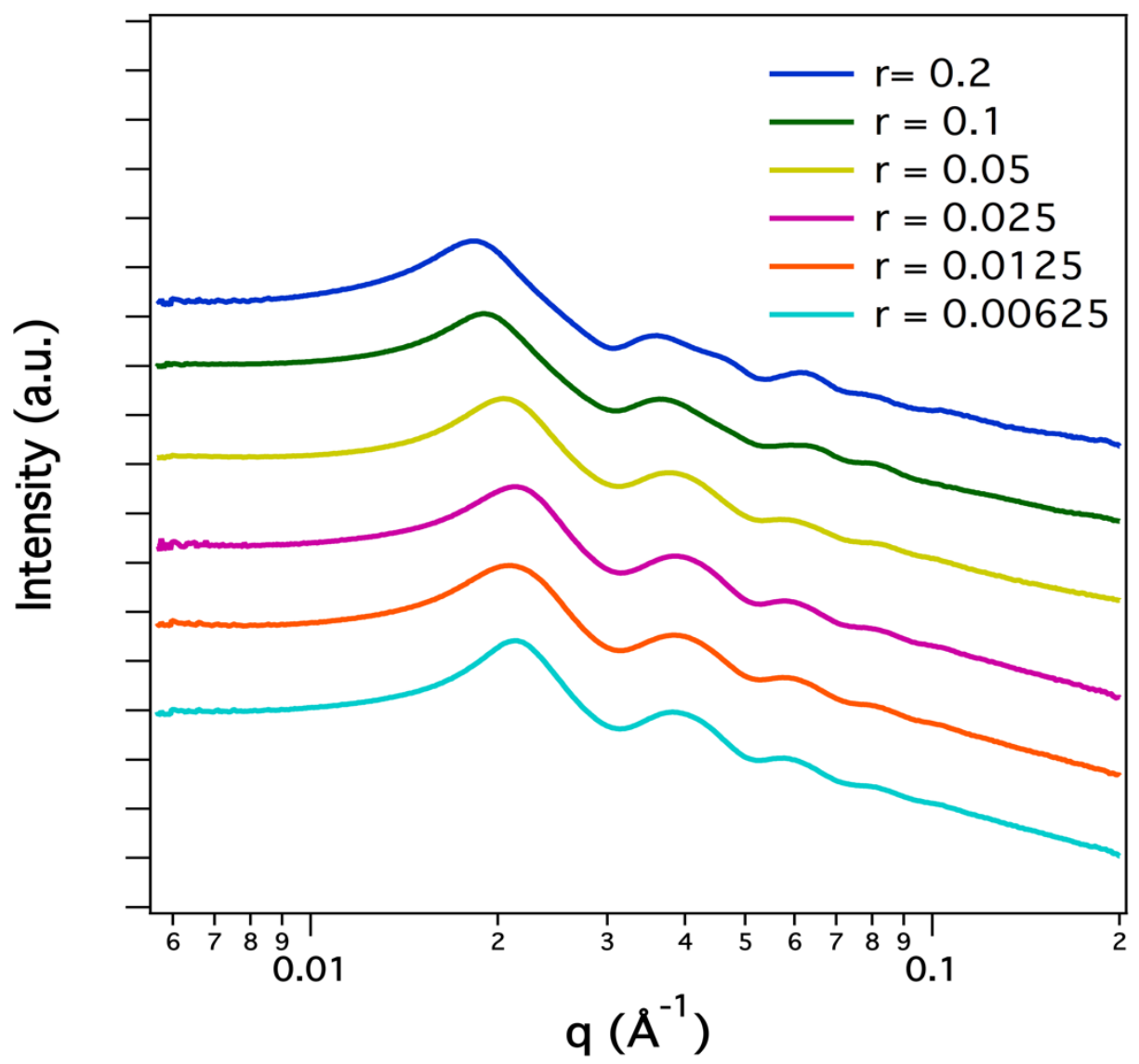

Supplementary Figure 7.4: Intensity of scattered $x$-ray as a function of wave vector for different glassy electrolytes with varying salt concentrations 


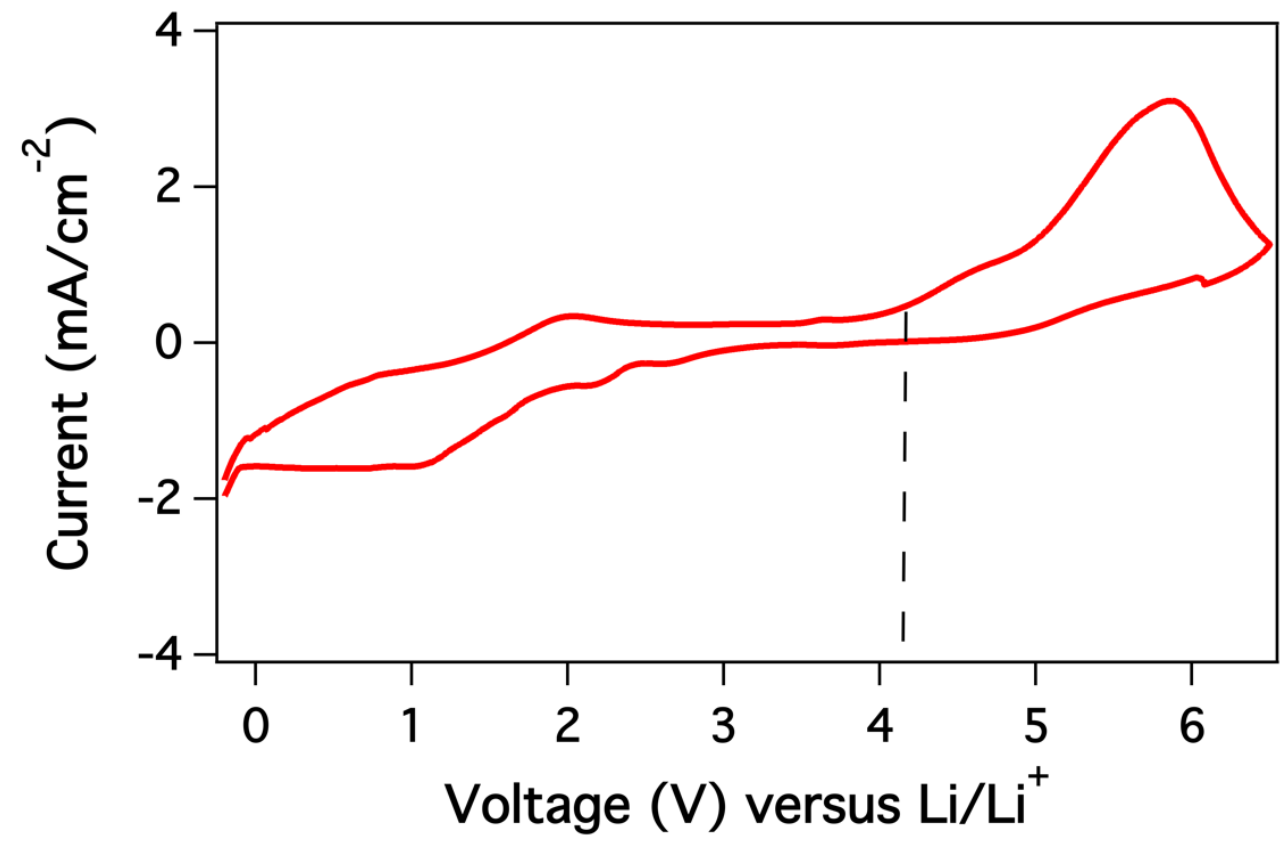

Supplementary Figure 7.5: Cyclic Voltammetry at $90^{\circ} \mathrm{C}$ for a coin cell comprising of lithium vs. stainless steel battery with the soft glassy electrolyte with $\mathrm{Li} / \mathrm{EO}$ ratio being 0.05 . The dotted line shows the oxidative breakdown potential at $\sim 4.2 \mathrm{~V}$ vs. $\mathrm{Li} / \mathrm{Li}^{+}$ 


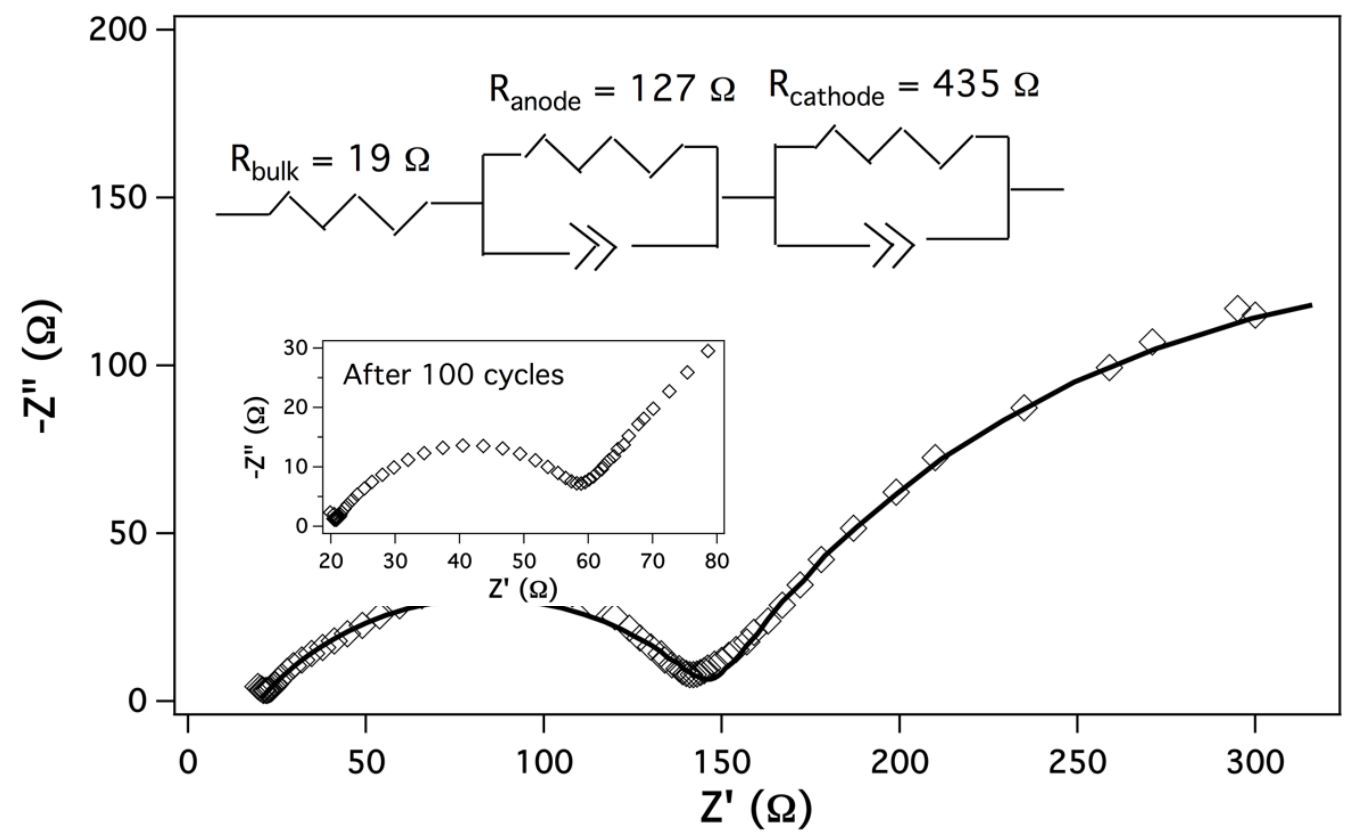

Supplementary Figure 7.6: Impedance spectra obtained at $30^{\circ} \mathrm{C}$ for a coin cell comprising of lithium vs. NCM battery with the soft glassy electrolyte with Li/EO ratio being 0.05 on the anode side along with a layer of liquid electrolyte on the cathode surface that comprises of $0.4 \mathrm{M} \mathrm{LiBOB}, 0.6 \mathrm{M} \mathrm{LiTFSI}$ and $0.05 \mathrm{M} \mathrm{LiPF}_{6}$ in EC/DMC. The inset shows the equivalent circuit model along with the corresponding resistances. The second inset shows the impedance profile of the same battery after 100 cycles 


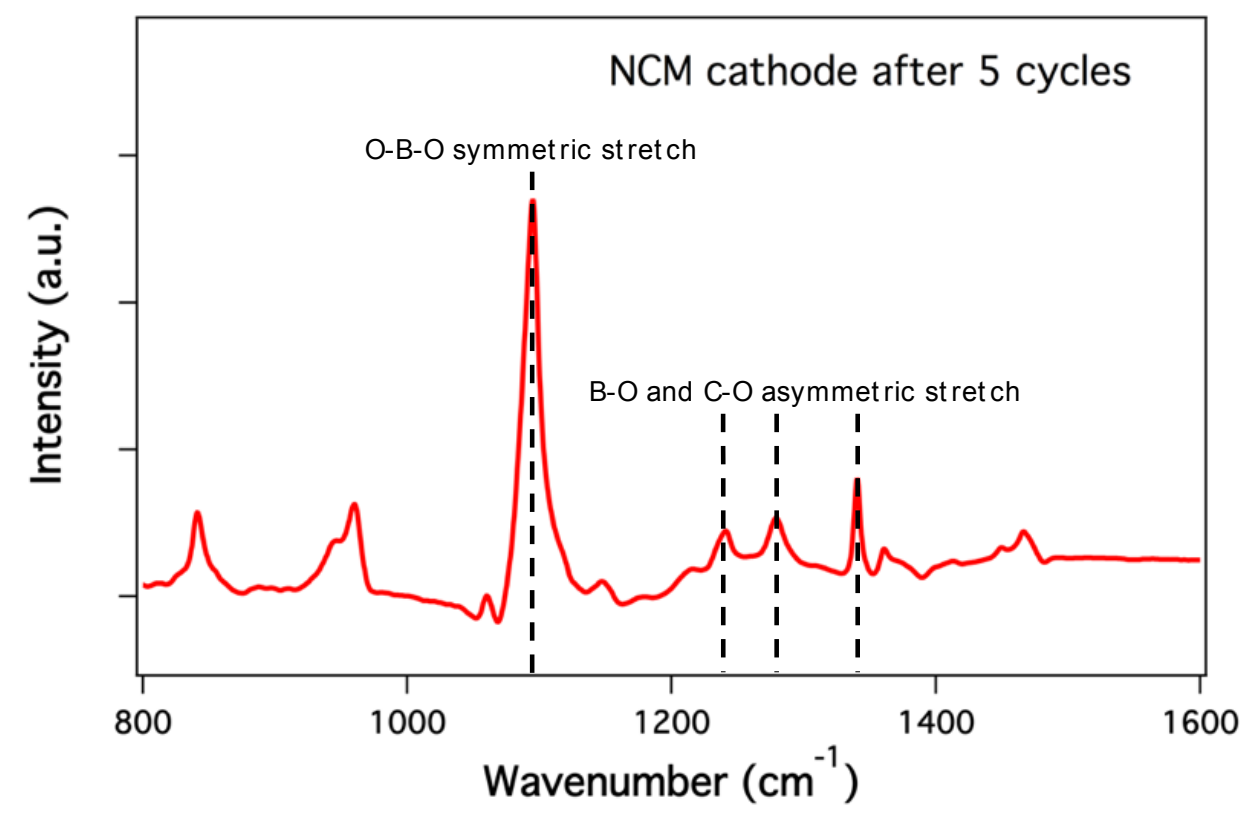

Supplementary Figure 7.7: FTIR analysis of the NCM cathode after cycling 5 times.

The dashed lines represent the location of the peaks for the carboxylic and borooxalate bonds signifying the chemistry of the cathode electrolyte interphase. 


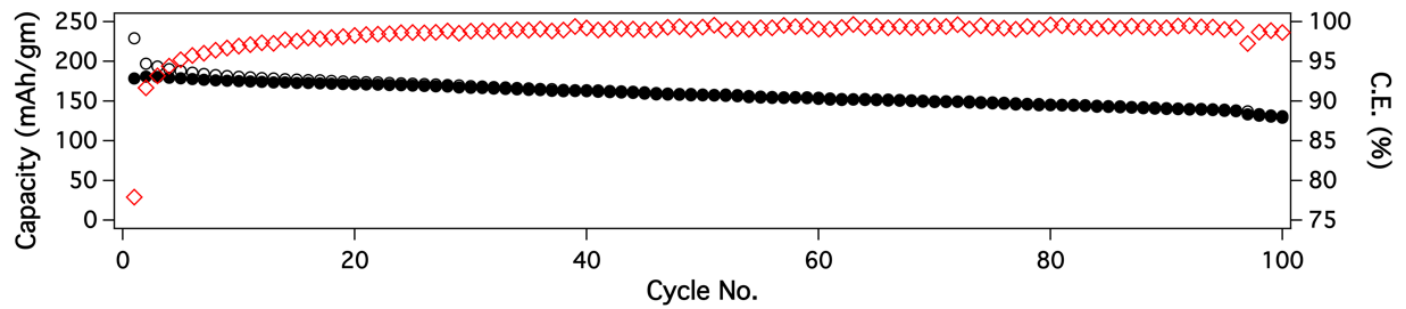

Supplementary Figure 7.8: Cycling performance of lithium vs. NCM battery with the soft glassy electrolyte with $\mathrm{Li} / \mathrm{EO}$ ratio being 0.05 on the anode side along with a layer of liquid electrolyte on the cathode surface that comprises of $0.4 \mathrm{M} \mathrm{LiBOB}, 0.6 \mathrm{M}$ LiTFSI and $0.05 \mathrm{M} \mathrm{LiPF}_{6}$ in EC/DMC. He inset shows the equivalent circuit model along with the corresponding resistances 

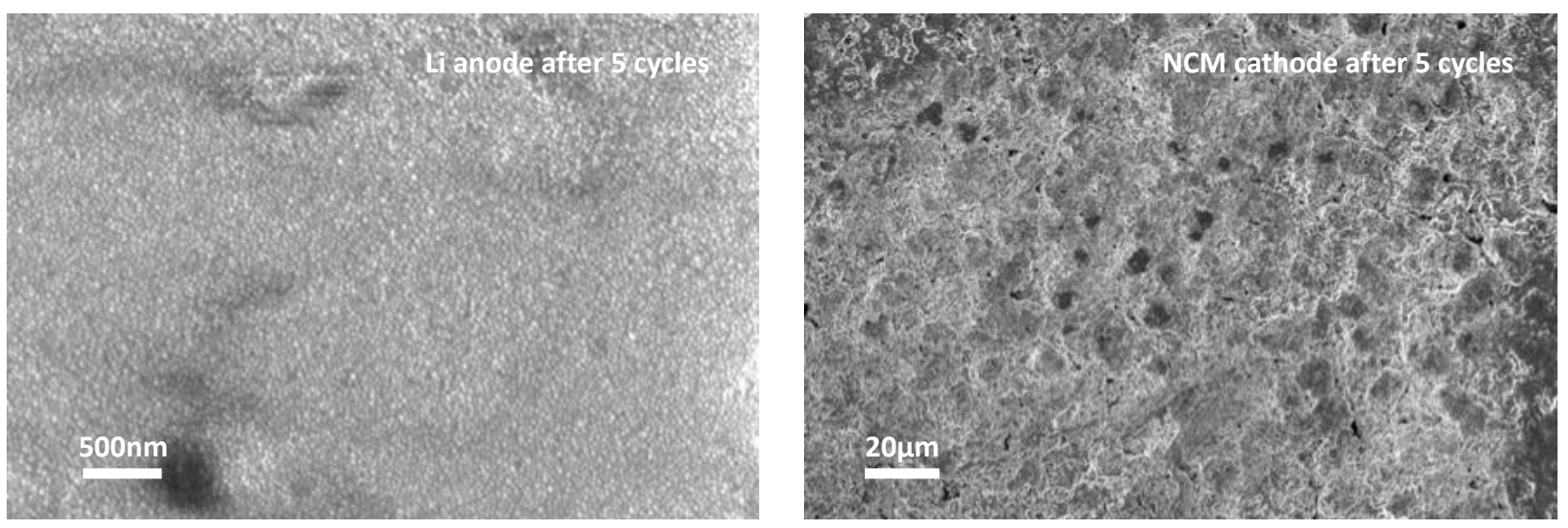

Supplementary Figure 7.9: SEM micrographs of the lithium metal anode and NCM cathode after 5 cycles. 

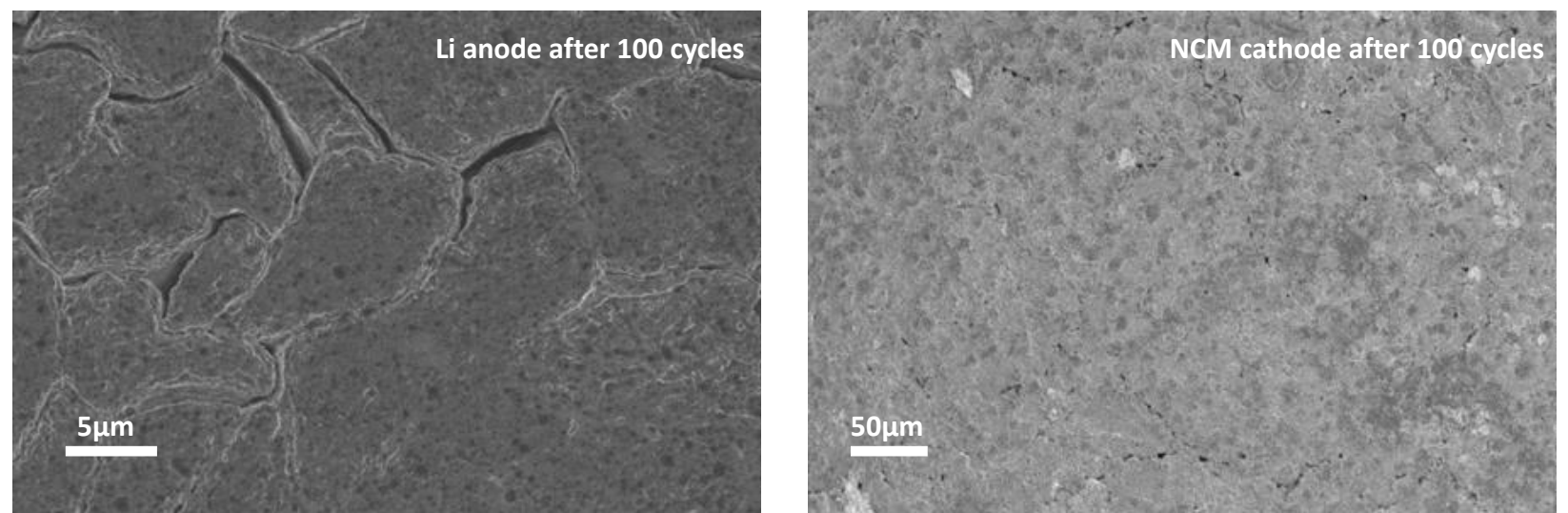

Supplementary Figure 7.10: SEM micrographs of the lithium metal anode and NCM cathode after 100 cycles. 
Supplementary Table 7.1: VFT parameters for different samples obtained $b$ fitting the experimental conductivity values using least squares method

\begin{tabular}{|c|c|c|c|}
\hline Sample & $\mathbf{A ~ ( S / c m ) ~}$ & $\mathbf{E}_{\mathbf{a}}(\mathbf{k J} / \mathbf{m o l e})$ & $\mathbf{T}(\mathbf{K})$ \\
\hline $\mathrm{r}=0.20$ & 6.38 & 16.7 & 150.2 \\
\hline $\mathrm{r}=0.10$ & 3.11 & 15.8 & 139.3 \\
\hline $\mathrm{r}=0.05$ & 0.73 & 12.2 & 150.4 \\
\hline $\mathrm{r}=0.025$ & 0.44 & 13.8 & 131.8 \\
\hline $\mathrm{r}=0.0125$ & 0.12 & 13.7 & 114.8 \\
\hline
\end{tabular}




\section{Materials and Methods:}

1. Synthesis of Self-Suspended Covalently grafted Hairy Nanoparticles:

Self-Suspended covalently grafted nanoparticles were prepared using a previously described method. Briefly, these nanoparticles are prepared using a two-step process, in which Polyethylene oxide was first functionalized with a silane group by reacting the isocyanate end in 3-(triethoxysilyl) propyl isocyanate (Sigma-Aldrich) with the amine end in amino-polyethylene oxide $(\mathrm{MW} \approx 5000 \mathrm{Da}, \mathrm{PDI} \approx 1.1$, purchased from Laysan Bio) in a stoichiometric ratio, creating a urethane bond in the process. The prepared silane functionalized polymer was condensed onto silica nanoparticles with diameter $25 \pm 2 \mathrm{~nm}$ by reaction with the hydroxyl groups on the surface of the particles ( TM-50, Sigma-Aldrich). Excess unreacted polymer chains were removed by repeated centrifugation in a choloroform-hexane mixture. The inorganic content of these hairy nanoparticles was analyzed using thermogravimetric analysis (TGA) on a TGA Q1000 (TA Instruments). The TGA for different samples revealed inorganic content of $51 \%$ corresponding to grafting density of approximately 1.47 chains $/ \mathrm{nm}^{2}$.

\section{Characterization:}

Glassy electrolytes were prepared by mixing the hairy nanoparticles with predetermined amount of LiTFSI salt (Sigma-Aldrich). The molecular structuring in the glassy electrolytes were studied using attenuated total reflectance-Fourier transform infrared spectroscopy (ATR-FTIR) on a Nicolet iS10 FTIR spectrometer (Thermo Fisher Scientific) equipped with a deuterated triglycine sulfate (DTGS) detector and a SMART iTR diamond ATR accessory. Melting transitions were then investigated 
using differential scanning calorimetry on a DSC Q2000 (TA Instruments) at a scan rate of $10^{\circ} \mathrm{C} / \mathrm{min}$. Morphology of the electrolytes at the electrode-electrolyte interface was studied using Scanning Electron Microscope (Zeiss Gemini 500) at the Cornell Centre for Materials Research (CCMR). Fourier transform infrared spectroscopy (ATR FTIR) was performed on a Nicolet iS10 FTIR spectrometer (Thermo Fisher Scientific) equipped with a deuterated triglycine sulfate (DTGS) detector and a SMART iTR diamond ATR accessory.

\section{Electrochemical Measurements}

2030 coin-type cells were assembled in a glovebox (MBraun Labmaster) with Nickel Cobalt Manganese Oxide (NCM) Cathode $\left(2 \mathrm{~mA} / \mathrm{cm}^{2}\right)$ as the cathode and lithium foil (Alfa Aesar) as the anode. The NCM cathode has $80 \%$ cative material (NCM 622), $10 \%$ binder (PTFE) and $10 \%$ carbon. The active material loading was $\sim 11 \mathrm{mg} / \mathrm{cm}^{2}$. The glassy electrolyte was sandwiched between the two electrodes, after wetting the cathode in a liquid electrolyte comprised of LiBOB (Oakwood Chemicals), LiTFSI (Sigma-Aldrich) and $\mathrm{LiPF}_{6}$ (Sigma-Aldrich) salts in an Ethylene Carbonate/Dimethyl Carbonate (Sigma Aldrich) mixture.

Ionic transport in the bulk and at the interface in this system was studied using conductivity and impedance measurements using a Novocontrol N40 broadband spectrometer fitted with a Quarto temperature control system. The samples were sandwiched between two gold-plated blocking electrodes. The cyclic voltametrry was done in lithium versus stainless steel configuration using a CHI600 potentiostat. The 
cycling characteristics of the cells were evaluated under galvanostatic conditions using Neware CT-3008 battery testers.

\section{Small-angle x-ray scattering measurements}

X-ray scattering (SAXS) measurements were performed on the solvent-less

SiO2-PEGME nanoparticles at sector 12-ID-B of Argonne National Laboratory, using a point collimated X-ray beam. The sample was smeared on a thermal cell, and the measurements were performed at different temperatures, all above melting point of PEG. The measured scattering intensity, I(q), depends on wave vector $\mathrm{q}$ and particle volume fraction $\varphi$ as

$$
\mathrm{I}(\mathrm{q}, \varphi)=\mathrm{P}(\mathrm{q}) \mathrm{S}(\mathrm{q}, \varphi)
$$

where $\mathrm{P}(\mathrm{q})$ and $\mathrm{S}(\mathrm{q}, \varphi)$ represent the particle form factor and the interparticle structure factor, respectively. Because in the limit of infinite dilution $S(q, \varphi \rightarrow 0) \approx 1$, the particle form factor can thus be obtained from the scattering intensities of dilute aqueous suspensions of particles. The structure factor can then be obtained by normalizing the scattering intensity with the form factor.

4. Rheology Measurements: Rheology Measurements. Oscillatory shear rheology measurements were performed at a temperature of $90^{\circ} \mathrm{C}$ using a Physica MCR 501 rheometer (Anton Paar at Cornell Energy Systems Institute (CESI)), outfitted with a cone and plate geometry ( $10 \mathrm{~mm}$ diameter, $2^{\circ}$ cone angle). To study the linear and nonlinear viscoelastic properties of the materials, variable strain amplitude 
measurements at a fixed angular frequency of $\omega=10 \mathrm{rad} / \mathrm{s}$ as well as variable frequency measurements at a fixed strain amplitude $\gamma=0.5 \%$, were employed. 


\section{Chapter 8}

Solid Polymer Interphases for Lithium Metal Batteries 


\subsection{Abstract}

Metal based batteries that comprise of a reactive metal anode like lithium, sodium or potassium are the future of energy storage devices because of their high volumetric and gravimetric energy density. However, these batteries fail by three distinct modes chemical instability due to internal reactions, morphological instability due to uneven electrodeposition and hydrodynamic instability due to convective flows at the vicinity of electrode-electrolyte interface. Both liquid based, and solid-state electrolytes have their individual advantages and disadvantages in mitigating these issues. Here, we show that solid-polymer interphases based on crosslinked polymer networks can essentially possess qualities from both of these worlds. We find that by tuning the thermodynamic interactions between the polymer network and oligomer diluents, one can control the bulk properties like ion transport and mass transfer rate. Thus, it is possible to design solid-like electrolyte-phases where the electroconvective flows can be inhibited, while maintaining high ionic conductivity. We further show that these polymer networks act as excellent interfacial layer for lithium metal electrode to inhibit dendrite growth and side reactions. On pairing with high voltage cathodes, the lithium metal battery at ambient conditions exhibit over 250 cycles of stable operation even at a high rate of $1 \mathrm{~mA} / \mathrm{cm}^{2}$.

\subsection{Introduction}

Powerful and long-lasting electrical energy storage devices are essential in modern times. One highly sought-after pathway to such devices is evolving today's ubiquitous lithium-ion batteries to so-called 'metal batteries' that replace the graphitic anode with 
an alkali metal block, including lithium, sodium and potassium. ${ }^{1,2}$ These batteries not only augment the anodic capacity by factors as high as ten times, but also enables use of higher-energy cathodes, including sulfur and oxygen $\cdot^{3-7}$ However, fundamental issues involving morphological, chemical, and hydrodynamic instabilities at the alkali metal anode must be addressed. First, rough electrodeposit structures grow on the metallic anode to eventually short-circuit the cell, inducing early battery failure and potential thermal runaway processes. ${ }^{8,9}$ Second, parasitic side-reactions between the electrolyte and the expanded anode promote electrolyte loss, ultimately lowering the cell efficiency over time. ${ }^{10-13}$ These effects are both exacerbated by the large volume change experienced by alkali metal electrodes during cycles of charge and discharge and by the tendency of associated interface strain to damage/destroy the passivating, but fragile interfacial material phases formed spontaneously on the electrode. A third mode of failure arises from unstable electro-convective flows in liquid electrolytes at current densities above the diffusion limit. This latter process is a consequence of ion depletion at the electrode interface and has been studied extensively in the context of electroplating processes. ${ }^{14}$

Conventional wisdom holds that solid-state electrolytes composed of mechanically strong and chemically inert materials may offer a unified strategy for mitigating all three sources of instability. The successes and failings of such all solid-state alkali metal batteries are beginning to emerge from fundamental ${ }^{9,15-17}$ and applicationfocused studies. ${ }^{18,11,19,12}$ Among the most important challenges that have emerged from such studies include: (i) the difficulty of finding materials that simultaneously offer sufficient mechanical rigidity to slow the growth kinetics of non-planar metal 
deposits and fast room-temperature bulk and interfacial ion transport — particularly when the alkali metal electrode is used in tandem with high-capacity intercalating cathodes such as $\mathrm{LiCoO}_{2}(\mathrm{LCO}), \mathrm{LiNiMnCoO}_{2}(\mathrm{NMC})$, and $\mathrm{LiNiCoAlO}_{2}(\mathrm{NCA})$; (ii) the inability for many of the best electrolyte candidates to reversibly deform and flex to accommodate volume change at the anode; and (iii) the complex, insulating interphases formed by solid-state electrolytes in contact with the reactive alkali metal electrode. Dense polyether-based networks with high crosslink densities have been reported in multiple recent studies ${ }^{18,20,21}$ to be effective in overcoming some of these challenges at low current densities. More recently, Wei, et al. ${ }^{22}$ reported that liquid electrolytes that incorporate high molar mass polymers to form molecular entanglements in the liquid and thereby impart viscoelasticity are effective in stabilizing deposition of metals at intermediate current densities, particularly at electrodes composed of softer alkali metals such as sodium. We report on the physical and electrochemical characteristics of thin, cross-linked polymer electrolyte interphases formed directly on the surface of Li metal anodes. We find that the interactions and composition of such interfaces can be easily tuned in the presence of a compatible liquid electrolyte host to create single-component, elastic membranes on the surface of a lithium metal anode. It is further shown that intrinsic chemical and physical features of the membrane design imparts high levels of reversibility to electrochemical processes at the anode, including elimination of hydrodynamic instability by indefinitely extending the diffusion limited ion migration regime. Deployment of the cross-linked polymer membranes in electrochemical cells composed of metallic Li anodes and commercial-grade Nickel Manganese Cobalt 
Oxide (NMC) cathodes reveals that the membranes facilitate high-columbic efficiency stable cell operations in cell configurations where the membranes function either as artificial solid electrolyte interfaces or as single component solid-state electrolytes. Direct visualization studies of Li electrodeposition reveal that the ability of the elastic interfaces formed in contact with the $\mathrm{Li}$ anode to promote compact deposition is an important source of the electrochemical stability and versatility of the materials.

\subsection{Results and Discussion}

Figure 8.1a shows a schematic of the synthesis scheme used to create the polymer membranes used in the present study. It is a facile, single-step polymerization process and does not involve incorporation of any undesired solvent. Specifically, poly(ethylene glycol) dimethacrylate (PEGDMA) of molecular weight $750 \mathrm{Da}$ is added to Bis(2-methoxyethyl) ether (diglyme) at varying volume fractions. In the presence of a suitable free-radical initiator, such as methyl benzoylformate (MBF), the DMA end groups form up to two linkages with other PEGDMA chains; rapidly crosslinking the material and trapping the mobile diglyme component in the pores. The ratio of lithium ions $\left(\mathrm{Li}^{+}\right)$to ethylene oxide (EO) was maintained at 0.10 via addition of a simple, low-cost lithium salt, Lithium Nitrate $\left(\mathrm{LiNO}_{3}\right)$. Cross-linking was achieved in the present work through photo polymerization via exposure of the interfaces to ultraviolet (UV) light $(320 \mathrm{~nm})$ irradiation. The result of the photopolymerization is a soft membrane, tightly bound to the underlying substrate. The degree of softness is dependent on the diglyme content in the overall mixture because only PEGDMA participates in cross-linking. Figure $8.1 \mathrm{~b}$ shows a membrane with $40 \%$ 
a.

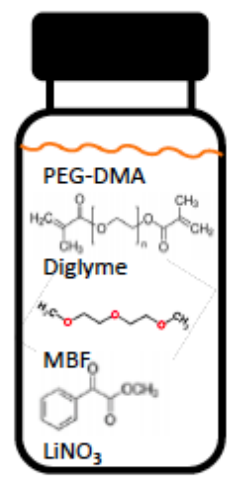

b.

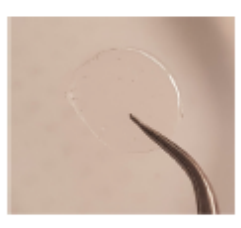

c.

$$
\text { UV LAMP }
$$

d.
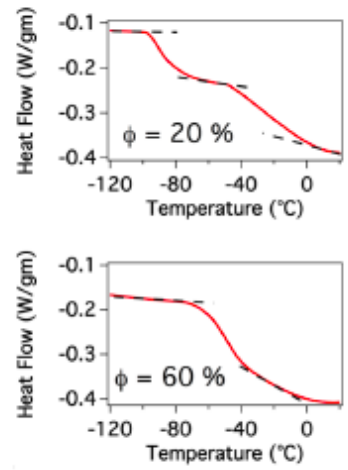

e.

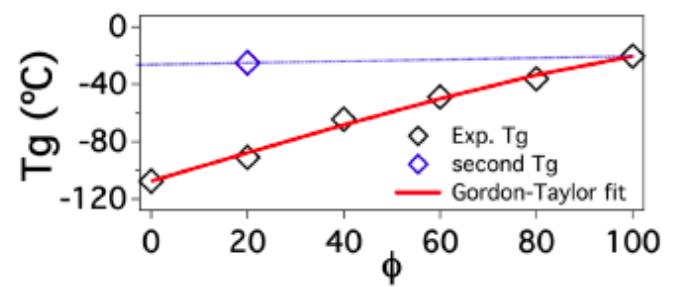

Figure 8.1: Physical Structure and Thermodynamic Characterizations: (a)

Chemical structures of the precursors involved in the synthesis of the polymer network;

(b) Photograph of the polymer network. (c) Schematic demonstrating the concept of insitu crosslinking on metal electrode; (d) Thermograms obtained from Differential Scanning Calorimetry for various fractions of PEGDMA $(\Phi)$ in the membranes. The dotted lines mark the step-change in the heat-flow; (e) Glass Transition temperature as a function of PEGDMA fraction in the membranes $(\Phi)$. The red line represent GordonTaylor fit 
PEGDMA content $(\phi)$ that is transparent and homogenous, exhibiting no observable aggregates or signs of crystallite formation. Further it can be seen in Supplementary Figure 8.1 that it has a rubbery texture and is able to recover fully even after macroscopic deformation. The curing process can be carried out on lithium or other substrates of lower surface energy (represented in the schematic of Figure 8.1c). This versatility is important as it allows the materials to be studied in detail either in the form of in-tact solid electrolyte interphases on $\mathrm{Li}$, as single-component solid-state electrolytes, or as free-standing films.

Evolution of the chemical bonding chemistry in the membranes with varying PEGDMA content in the precursor solution $(\phi)$ was studied via Fourier transform infrared spectroscopy (FTIR). The results illustrated in Supplementary Figure 8.2 show that the $C=O$ bond at $\sim 1,700 \mathrm{~cm}^{-1}$ associated with PEGDMA increases in intensity and shifts to lower wave number with increasing PEGDMA content. This shift is an indication of a reduction in the effective moment of inertia of the absorbers, which correlates with a reduction in the spacing between network points in the crosslinked material. Thus, increasing PEGDMA content results in higher crosslink density, which leads to membranes that are macroscopically more elastic and mechanically stronger.

Supplementary Figure 8.3 illustrates how such an in-situ cross-linked membrane might be used as artificial solid-electrolyte interphases (ASEI) to inhibit physical and chemical instabilities at an alkali metal electrode. Before assessing the electrochemical consequences of this ASEI design, we first consider the fundamental physical features of the materials and on that basis elucidate their versatility. Results from differential 
scanning calorimetry (DSC) of the of pure diglyme- $\mathrm{LiNO}_{3}(\phi=0 \%)$ as well as pure PEGDMA membrane-LiNO $3(\phi=100 \%)$ are shown in in Supplementary Figure 8.4, while the DSC thermograms for intermediate $\phi$ 's are provided in Figure 8.1d. In the specific ranges of temperatures displayed in these figures, a second-order phase transition is observed that is associated with the glass transition of the polymer membranes. This transition reflects the phase change from melt to a glassy state due to kinetic entrapment of the molecules due to free volume reduction. The glass transition temperature ( $\mathrm{Tg}$ ) observed for $\phi=0$, corresponding to pure diglyme- $\mathrm{LiNO}_{3}$ and $\phi=$ $100 \%$ (pure PEGDMA - $\mathrm{LiNO}_{3}$ ) are $-107.58^{\circ} \mathrm{C}$ and $-20.37^{\circ} \mathrm{C}$, respectively as shown in Supplementary Figure 8.4. Polymer blends with It is known that compatible polymeric mixtures show distinct Tg's intermediate of the respective materials, while that of a fully-mixed blend result in a single Tg that is dependent on the molecular dispersion and relative strength of intermolecular forces in the materials. The membrane with $20 \%$ PEGDMA content shows two distinct $\mathrm{Tg}$ values of $-91.01{ }^{\circ} \mathrm{C}$ and $-25^{\circ} \mathrm{C}$ (see Figure $8.1 \mathrm{~d}$ ), which can be asserted to two-phases that are rich in diglyme and PEGDMA, respectively. At this particular $\phi$, the membrane exists in two separate phases, which implies that the PEGDMA in the original mixture is not adequate to form a fully-connected network after crosslinking. This means that only a small fraction of the added diglyme interacts with the crosslinked phase, while the rest exists as a second phase that is essentially a free liquid.

At $\phi=40 \%$, the glass transition event occurs over a visibly broadened range of temperature, indicating that the PEGDMA network in the membrane is at that point at 
the limits where it exists as a one single component. At this composition, the separate Tg values begin to merge, indicating a chemically homogenous material such that the PEG chains are permeated evenly throughout the membrane and it inherits the mechanical toughness of a fully crosslinked network, while maintaining conductivity close to that of a neat (liquid) electrolyte. As the PEGDMA composition is increased further, the Tg values fully merge to a sharp transition (shown at $\phi=60 \%$ and above), indicating the formation of a single phase percolated network. At this point, the crosslinked networks exhibits characteristics of so-called solid-state electrolytes: mechanically resilient yet limited by low ionic conductivity. Figure 8.1e compares Tg values for the synthesized material at all PEGDMA content. The measured $\mathrm{Tg}$ values were fitted to the classical Gordon-Taylor relation: $\mathrm{Tg}=$ $\left(\mathrm{w}_{1} \mathrm{Tg}_{1}+\mathrm{K}_{\mathrm{o}} \mathrm{w}_{2} \mathrm{Tg}_{2}\right) /\left(\mathrm{w}_{1}+\mathrm{K}_{\mathrm{o}} \mathrm{w}_{2}\right)$, where $\mathrm{w}_{1}$ and $\mathrm{w}_{2}$ are weight fractions of diglyme- $\mathrm{LiNO}_{3}$ and PEGDMA-LiNO 3 , while $\mathrm{Tg}_{1}$ and $\mathrm{Tg}_{2}$ are the glass transition temperature of the same. $K_{o}$ here is 0.35 that is obtained from the least square fitting of the experimental values, which is indicative of the relative change in heat capacities compared to the pure components. The Gordon-Taylor relationships holds for noninteracting $(\chi=0)$ polymer mixtures. It was noted that the second glass transition temperature at $\phi=20 \%$ that doesn't follow the Gordon-Taylor relation was recorded to be close to the value at $\phi=100 \%$. Thus, it can be concluded that as the PEGDMA content in the overall mixture increases, the first phase (rich in PEG network) continue to grow and essentially engulf the diglyme molecules to form a dense and swollen percolated network of polymer chains. 

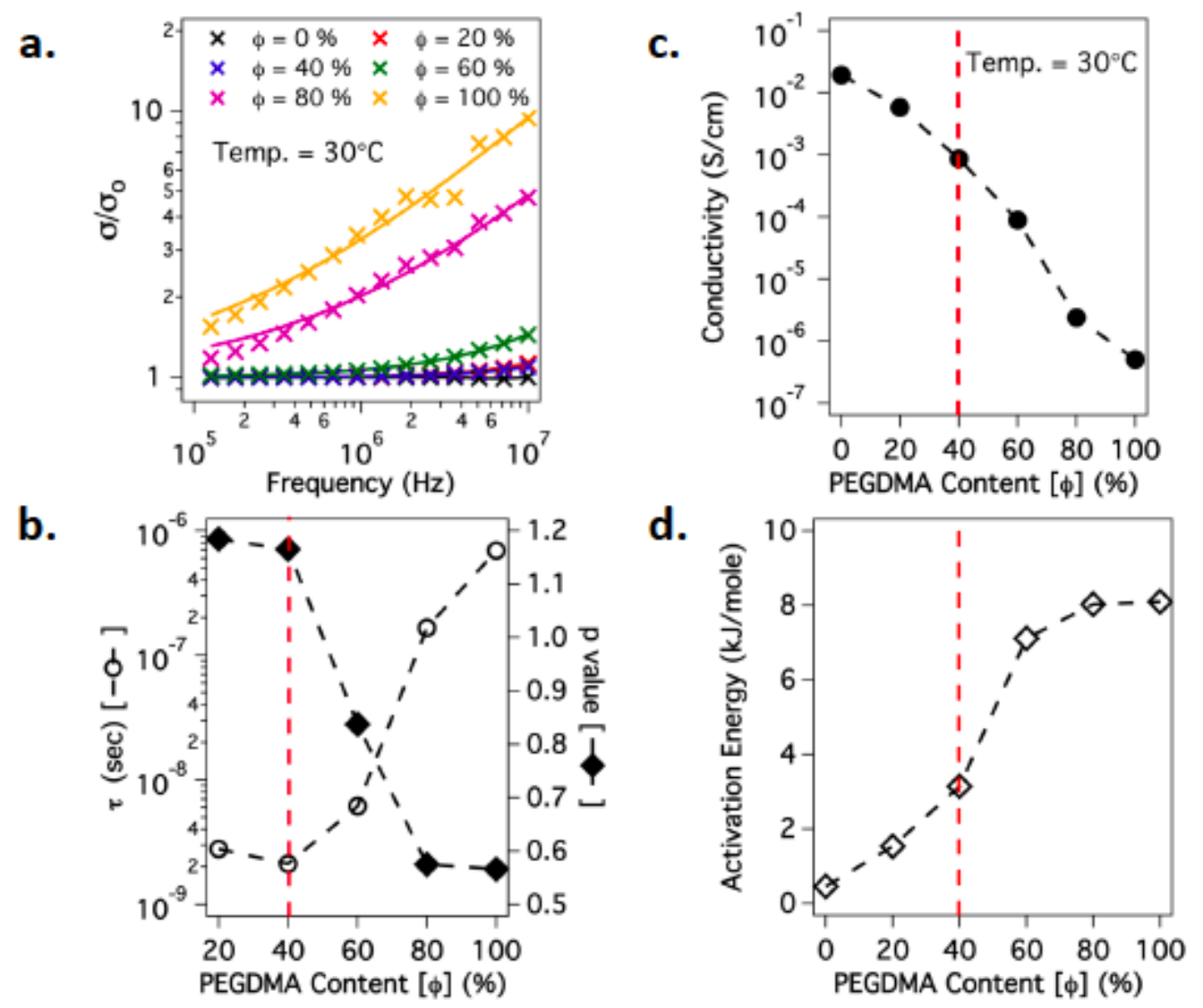

Figure 8.2: Structure dependent Ion Transport Properties: (a) Normalized ac Conductivity as a function of frequency. Measured values are shown as markers, while lines are the data fitted to Cramer's Equation. (b) Results from frequency dependent conductivity measurements. Left axis shows the ion transport relaxation time, and right axis describes ratio of back hop-rate over forward hop-rate obtained from the power-law model. (c) Conductivity as a function of PEGDMA content at fixed temperature. (d) Activation energy of ion transport as a function of PEGDMA content obtained by VFT model. 
Frequency dependent conductivity can reveal important information regarding the ion transport mechanisms as well as structural arrangements. Figure $8.2 \mathrm{a}$ reports the normalized real component of a.c. conductivity as a function of frequency for different PEGDMA content obtained using Dielectric Spectroscopy at $30^{\circ} \mathrm{C}$. The range of frequency values reported in Figure 8.2a only captures the high-to-mid values below which polarization effects at electrode-electrolyte interface start to dominate. It is seen that at lower frequencies the conductivity is independent of frequency denoting the bulk or d.c. conductivity $\left(\sigma_{0}\right)$. In the high frequency region, the conductivity values progressively rise beyond a critical frequency of $\omega_{c}$ due to correlated ionic motions and depend on the host media microstructure as well as temperature. Such behavior has also observed universally in several glassy and solid polymer electrolytes reported in the literature. The solid line fits in Figure 8.2a represent the jump-relaxation model proposed by Jonscher ${ }^{23}$ for thermally activated processes that was be mathematically reproduced by Cramer et al. ${ }^{24}$ as a power-law expression: $\sigma=\sigma_{\mathrm{o}}\left[1+(\tau \omega)^{\mathrm{p}}\right]$. Here $\tau$ is the timescale for dielectric relaxation that depends upon the coulombic interactions between the polymer chains and mobile ions. The power law exponent $\mathrm{p}$ denotes the ratio between the forward hop to backward hop relaxation time. The variation of $\tau$ and $\mathrm{p}$ with different PEGDMA content $(\phi)$ is represented in Figure 8.2b. It is seen that the ion hopping relaxation time $(\tau)$ increases rapidly beyond $\phi=40 \%$. This can be attributed to a transition of conduction mechanism from bulk motion of oligomers to ion hopping processes along the EO links in the percolated network. The evidence of this is the transition from a mixed diglyme-network to a single-phase solid electrolyte observed from the glass transition behavior at and beyond $\phi=40 \%$. Furthermore, the 
$\mathrm{p}$-value is seen to changes from $>1$ to less than unity at the same fraction. Previously in the literature, solid polymer electrolytes based on PEO have been reported to exhibit $\mathrm{p}<1$ due to the cationic nature of the ion transport where $\mathrm{Li}^{+}$ions hop through coordination with EO molecules, however, at elevated temperatures as well as for liquid electrolytes $\mathrm{p}$ is seen be higher than 1 . This argument similarly validates our proposed idea that all diglyme molecules are 'tightly' bound by the percolated network beyond $\phi=40 \%$.

Supplementary Figure 8.5 reports the d.c. conductivity obtained from the plateau region of the frequency dependent conductivity as a function of inverse temperature. The continuous lines here represent the Vogel-Fulcher-Tammann (VFT) model given by, $\sigma_{0}=A \exp \left(-E_{a} / R\left(T-T_{0}\right)\right)$, provides relationship between the ion transport rate and temperature. Here, A is a pre-factor related to the overall number of charge carrier, $E_{a}$ is the activation energy and $T_{o}$ denote the characteristic temperature below which the ion transport ceases to take place. The excellent fitting of the VFT model confirm the absence of any temperature induced chemical or morphological changes in the measured range. The comparison of the conductivity values at $30^{\circ} \mathrm{C}$ is plotted as a function of $\phi$ in Figure 8.2c. It is seen that the conductivity from $\phi=0$ to $40 \%$ only decreases by an order of magnitude and thereafter, as PEGDMA becomes the majority in mixture, the conductivity of the membrane quickly drops off by several orders of magnitude. Operation of cells created using the membrane of above $40 \%$ PEGDMA content as an electrolyte composite content would require increased operating temperatures similar to solid polymer electrolytes previously reported in the literature. ${ }^{20,25,26}$ Figure $8.2 \mathrm{~d}$ reports the activation energy obtained from the VFT 
a.

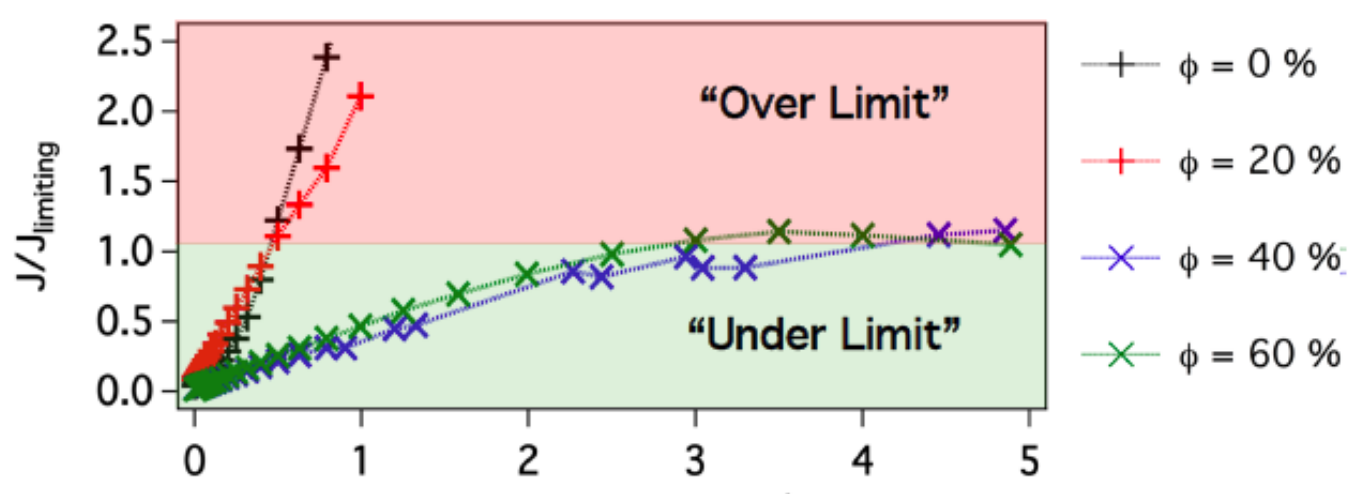

b.

Voltage (V) vs. $\mathrm{Li} \mathrm{Li}^{+}$

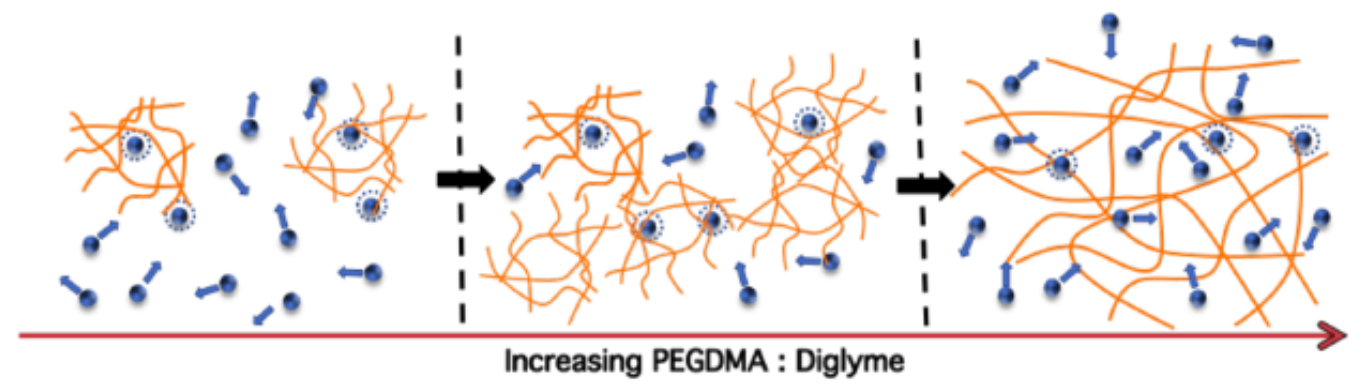

Figure 8.3: Hydrodynamic Stability during electrodeposition: (a) Normalized I-V curves for various membranes of different PEGDMA fractions $(\Phi)$. Here, the $y$-axis is normalized by the limiting current density of ion transport. (b) Cartoon showing the evolution of the crosslinked polymer architecture with increasing PEGDMA content. As $\Phi$ increases, free diglyme decreases in the network and PEGDMA becomes cross-linked in a singular percolated structure. 
fittings. It is evident that the activation energy for ion hopping increases sharply beyond $\phi=40 \%$. This implies that ion transport through the membrane occurs at more easily at lower PEGDMA content. Thus, the molecular interactions between the oligomers and polymer network ultimately regulates the bulk-scale ion transport processes.

In absence of forced convections, electrodeposition is a diffusion-limited process such that ion transport rate at every potential differences should be a function of ionic conductivity. However, at intermediate voltage differences, the ion transport rate should reach a maximum value, known as the limited current density, which can be calculated as $\mathrm{J}_{\mathrm{lim}}=\mathrm{zFDc} / \delta$, where $\mathrm{z}$ is the charge, $\mathrm{F}$ is Faraday constant, $\mathrm{c}$ is bulk concentration, $\mathrm{D}$ is ion diffusivity and $\delta$ is the diffusion layer thickness. At even higher voltages, the ion migration rate cannot exceed the diffusion rate, thus the electroneutrality fails to be maintained at the electrode-electrolyte interfaces resulting in creation of a space charge region. It has been experimentally observed as well as predicted from Nernst-Planck Theory that a large electric field near the electrodes generates a convective flow field driving the ions across the space charge region, thereby exceeding the limiting current to a 'over-limiting' value. Since, the current flow in these conditions occur not by the electroconvective flows rather than ion diffusion, it results in several unwanted events like electrolyte degradation and rampant dendritic growth on the electrodes. It can be safely argued that these instabilities are absent in all solid-state electrolytes but can occur in Newtonian fluids. Thus, such experiments are important with context of the present work to classify the crosslinked polymer networks. 
Supplementary Figure 8.6 reports current density as a function of voltage for our membranes of different PEGDMA content. The measurements were performed in symmetric ( $\mathrm{Li}|| \mathrm{Li})$ two-electrode cells. The voltage was scanned in stair-case progression from $0 \mathrm{~V}$ to $5 \mathrm{~V}$ vs. $\mathrm{Li} / \mathrm{Li}^{+}$, and the resultant steady-state current was recorded. In $\phi=0,20 \%$ PEGDMA content electrolytes, quick divergence of current can be observed, without any obvious plateau region in the current density as opposed to the higher PEGDMA contents. Figure 8.3a shows the data reported in Supplementary Figure 8.6 after normalization by the limiting current of ion transport. Jim for $\phi=0,20 \%$ were calculated from the theoretical expression using $\delta$ as the interelectrode spacing while, Jlim for the $\phi<40 \%$ was calculated from the observed plateau region. The data reports that for PEGDMA content $\phi<40 \%$ quickly rises to regions of transport instability, over the limiting current. Past the diffusion-limited current, electroconvection dominates ion transport. However, for $\phi \geq 40 \%$, the current through the electrochemical systems is limited at the critical current, signaling stable ion transport. As the PEGDMA polymer network architecture exist as a single percolating solid at higher (40-100\%) PEGDMA content, electroconvection is negated. A cartoon summarizing the observed effect is represented in Figure 8.3b. The architecture of the crosslinked PEGDMA chain network in the solid polymer interphase membrane is shown. With diglyme in excess, the physically wet membrane exhibits many free diglyme chains, and some interacting in a thinly populated PEGDMA supramolecules. As the PEGDMA content was incrementally increased, the percolated network exhibited more diglyme chains interactions. It is interesting that oligomer-polymer interactions not only regulate the thermodynamic behavior, but it is 
a.

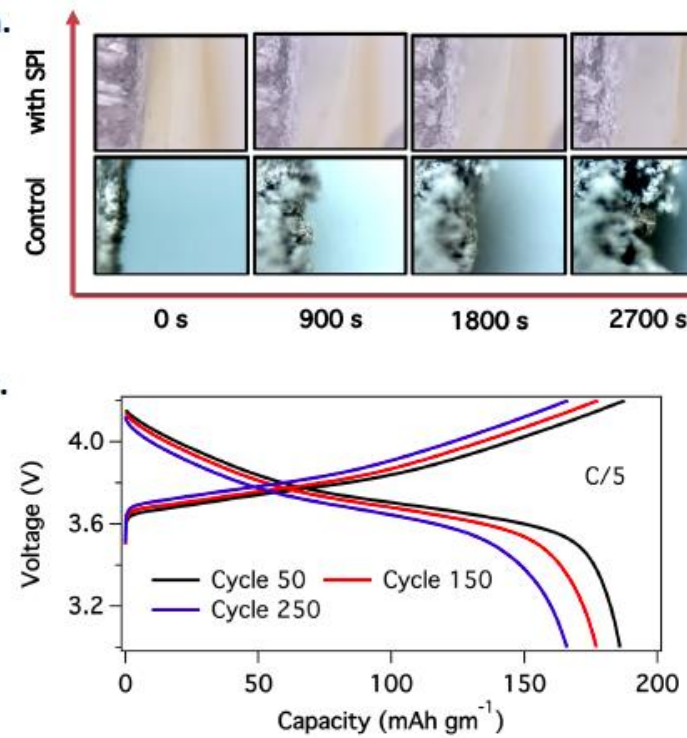

b.

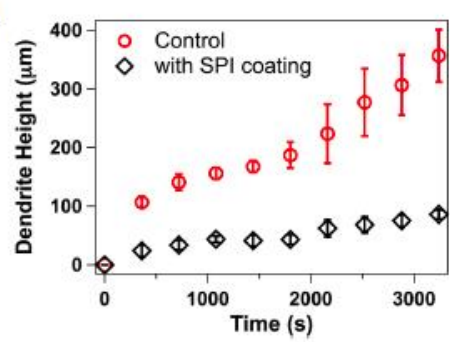

d.

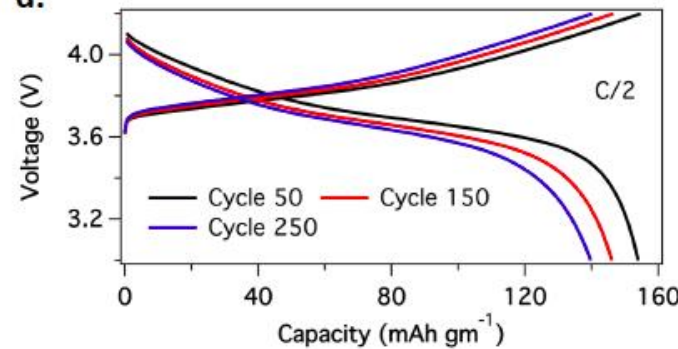

Figure 8.4: Morphological Stability and Electrochemical Performances: (a)

Snapshots from direct-visualization. The first row shows results lithium coated with the synthesized solid polymer interphase; the second row shows results with the uncoated lithium electrode. In both cases the electrolyte of $1 \mathrm{M} \mathrm{LiPF}_{6}$ in EC/DMC was used and current density was $4 \mathrm{~mA} / \mathrm{cm}^{2}$. (b) Average height of dendrite as a function of time. (c), (d) Voltage profiles for lithium vs. NCM batteries where the lithium anode is coated with the solid polymer interphase for C-rates of $\mathrm{C} / 5$ and $\mathrm{C} / 2$ respectively. The electrolyte used is $0.6 \mathrm{M} \mathrm{LiTFSI,} \mathrm{0.4M} \mathrm{LiBOB} 0.05 \mathrm{LiPF}_{6}$ in EC/DMC. 
also manifested in ion transport behavior and macro-scale hydrodynamics. Based on this, we can select PEGDMA content $\phi=40 \%$, as the optimum candidate for designing our Solid Polymer Interphase $(S P I)$ that simultaneously exhibit liquid and solid-like characteristics.

To characterize macroscopic morphological evolution during electrodeposition, an inhouse built visualization setup was utilized. The set-up contained dual-lithium metal rods as the electrodes coated with the $S P I$ and liquid electrolyte of $1 \mathrm{M}$ EC:DMC LiPF 6 in the interelectrode space, filling the center of the tube. Electrodeposition was visually recorded under an optical microscope using a current density of $4 \mathrm{~mA} \mathrm{~cm}^{-2}$. Electrodeposit morphology for both coated and uncoated electrodes was observed at regular intervals up to 1 hour, as shown in Figure 8.4a. For the control electrolyte, the electrodeposit morphology is "mossy" and rough as compared to the case with the solid polymer interphase. This effect is the result of three mechanisms. The physical barrier provided by the SPI provides the electrolyte with the mechanical modulus to resist uneven deposition. Further, the negation of electroconvection due to the permeating polymer network encourages stable ion transport towards the electrode surface. Lastly, at the electrode surface, the presence of the polymer composites prevents continuous side reactions with the carbonate electrolyte. Quantitative analysis of electrodeposit morphology was also reported. This was done by plotting the height of deposit at various times, shown in Figure $8.4 \mathrm{~b}$. In a linear regression of the data, the growth rate of the pristine lithium deposits with the control electrolyte was found to be $\sim 90 \mathrm{~nm} \mathrm{~s}^{-1}$, while that with the $S P I$ was found to be $\sim 20 \mathrm{~nm} \mathrm{~s}^{-1}$. Supplementary Figure 8.7 reports cell coulombic efficiency $(\mathrm{CE})$ as a function of cycle number. Results were 
obtained using an antisymmetric coin cell, with pristine Li electrode and stainless-steel counter electrode with and without the SPI coating. In the control case, the cell cycles stably at above $80 \% \mathrm{CE}$ for around 30 cycles, before the CE drops and the cell fails. In contrast, the coin cells fabricated with the $S P I$ coating exhibited $>80 \% \mathrm{CE}$ for at least 100 cycles. It is clear that the SPI can promote long term electrodeposition stability. Finally, we paired the SPI coated lithium metal anode with high voltage cathode of Nickel Manganese Cobalt Oxide (NMC). The electrolyte used is 0.6M LiTFSI, 0.4M LiBOB $0.05 \mathrm{LiPF}_{6}$ in $\mathrm{EC} / \mathrm{DMC}$ (1/1 by wt.), which has been reported to be stable in high potential operation. Figure $8.4 \mathrm{c}, 8.4 \mathrm{~d}$ report the voltage profiles at a rate of $\mathrm{C} / 5$ and $\mathrm{C} / 2$, respectively. The capacity and coulombic efficiencies as a function of cycle numbers are reported in Supplementary Figure 8.8a, 8.8b. Here, where 1C corresponds to a current density of $2 \mathrm{~mA} \mathrm{~cm}^{-2}$. It can be seen in both the C-rates, that the initial capacity is $\sim 100 \mathrm{mAh} \mathrm{gm}^{-1}$ that eventually increases to $\sim 185 \mathrm{mAh} \mathrm{gm}^{-1}$ and $\sim 155 \mathrm{mAh}$ $\mathrm{gm}^{-1}$ for $\mathrm{C} / 5$ and $\mathrm{C} / 2$ respectively. However, the coulombic efficiency is seen to be remain close to $100 \%$ throughout the battery operation, this indicates that initial rise in the capacity is due to the increasing interfacial conductivity as the liquid electrolyte wets the SPI. It can be seen at both C-rates that the discharge capacity retention even after 250 cycles of operation is more than $90 \%$.

In conclusion, we designed crosslinked solid polymer networks based on PEG chemistry for using as a solid polymer interphase on a lithium metal electrode. We systematically varied diglyme diluent in the polymer network to understand how thermodynamic interactions affect bulk properties. It was observed at high diglyme content, it partly interacts with the sporadic networks and the majority remains 
unbounded. Specifically, at 20\% PEGDMA two distinct Tg's were observed, followed by a fully interacting network at $40 \%$ and beyond. Thus at $40 \%$ PEGDMA content, the diglymes was loosely held by the network resulting in high ionic conductivity in contrast to higher content values. Also, at this critical content of PEGDMA, the solid electrolyte membrane did not manifest any electroconvective flows, thus possessing qualities of both solid and liquid electrolytes. Finally, we utilized the optimum crosslinked network as a solid interphase on lithium metal battery to show stable battery cycling due to inhibition of dendritic growth and electrode-electrolyte side reactions.

\subsection{Methods}

\subsubsection{Fabrication of crosslinked polymer network and coated lithium}

Poly(ethylene glycol) Dimethacrylate $(\mathrm{Mn}=750)$, Diglyme and Lithium Nitrate were purchased from Sigma Aldrich. All chemicals were thoroughly dried before usage. The PEGDMA and diglyme were mixed in different ratios as required, however the $\mathrm{LiNO}_{3}$ content was maintained at $L i: E O=0.1$ for all the samples. The mixture was thoroughly mixed to obtain a uniform solution. After addition of $4 \mathrm{wt} . \%$ of a photoinitiator methyl benzoylformate (MBF), the solution was casted on a desired substrate and exposed to UV light (VMR UVAC $115 \mathrm{~V} \sim 60 \mathrm{~Hz}$ 254/365 nm) for 20 minutes. After the reaction, the membranes were utilized as is for characterizations. The solid polymer interphase was formed using the same procedure, however the reaction was carried out on a flat piece of lithium metal anode in an Argon-filled glove-box. 


\subsubsection{Material Characterization}

The molecular structuring in the glassy electrolytes were studied using attenuated total reflectance-Fourier transform infrared spectroscopy (ATR-FTIR) on a Nicolet iS10 FTIR spectrometer (Thermo Fisher Scientific) equipped with a deuterated triglycine sulfate (DTGS) detector and a SMART iTR diamond ATR accessory. Melting transitions were then investigated using differential scanning calorimetry on a DSC Q2000 (TA Instruments) at a scan rate of $10^{\circ} \mathrm{C} / \mathrm{min}$.

\subsubsection{Electrochemical Characterization}

Ionic transport in the bulk and at the interface in this system was studied using conductivity and impedance measurements using a Novocontrol N40 broadband spectrometer fitted with a Quarto temperature control system. The samples were sandwiched between two gold-plated blocking electrodes. The I-V analysis were done using staircase voltammetry where each voltage steps comprise of 20 seconds using Maccor battery testers.

2030 coin-type cells were assembled in a glovebox (MBraun Labmaster) with Nickel Cobalt Manganese Oxide (NCM) Cathode $\left(2 \mathrm{~mA} / \mathrm{cm}^{2}\right)$ as the cathode and lithium foil (Alfa Aesar) as the anode. The solid-polymer coated lithium was paired with the NCM cathode, and the in a liquid electrolyte comprised of LiBOB (Oakwood Chemicals), LiTFSI (Sigma-Aldrich) and $\mathrm{LiPF}_{6}$ (Sigma-Aldrich) salts in an Ethylene Carbonate/Dimethyl Carbonate (Sigma Aldrich) mixture. 
The coulombic efficiency tests were performed in a cell configuration of lithium anode with or without the solid polymer coating paired against a stainless steel counter electrode. The electrolyte utilized was $1 \mathrm{M} \mathrm{EC:DMC} \mathrm{LiPF} 6$. In this measurement a fixed amount of lithium was plated onto the stainless steel electrode and stripped back, such that the ratio of stripped and plated lithium determined the coulombic efficiency for each cycle.

The direct visualization experiment was done using two lithium rod-type electrodes in a tube-like visualization cell. ${ }^{27}$ 


\section{REFERENCES}

1. Wei, S., Choudhury, S., Tu, Z., Zhang, K. \& Archer, L. A. Electrochemical Interphases for High-Energy Storage Using Reactive Metal Anodes. Acc. Chem. Res. 51, 80-88 (2018).

2. Cheng, X. et al. A Review of Solid Electrolyte Interphases on Lithium Metal Anode. Adv. Sci. 3, 1-20 (2016).

3. Lin, D., Liu, Y. \& Cui, Y. Reviving the lithium metal anode for high-energy batteries. Nat. Nanotechnol. 12, 194-206 (2017).

4. Cheng, X.-B., Zhang, R., Zhao, C.-Z. \& Zhang, Q. Toward Safe Lithium Metal Anode in Rechargeable Batteries: A Review. Chem. Rev. 117, 10403-10473 (2017).

5. Armand, M. \& Tarascon, J.-M. Building better batteries. Nature 451, 652-7 (2008).

6. Ma, L., Hendrickson, K. E., Wei, S. \& Archer, L. A. Nanomaterials: Science and applications in the lithium-sulfur battery. Nano Today 10, 315-338 (2015).

7. Aurbach, D., McCloskey, B. D., Nazar, L. F. \& Bruce, P. G. Advances in understanding mechanisms underpinning lithium-air batteries. Nat. Energy 1, 16128 (2016).

8. Choudhury, S. et al. Confining electrodeposition of metals in structured electrolytes. Proc. Natl. Acad. Sci. (2018).

9. Bai, P., Li, J., Brushett, F. R. \& Bazant, M. Z. Transition of lithium growth mechanisms in liquid electrolytes. Energy Environ. Sci. 9, 3221-3229 (2016). 
10. Zhang, S. S. A review on electrolyte additives for lithium-ion batteries. $J$. Power Sources 162, 1379-1394 (2006).

11. Suo, L. et al. Fluorine-donating electrolytes enable highly reversible 5-V-class Li metal batteries. Proc. Natl. Acad. Sci. (2018).

12. Choudhury, S. \& Archer, L. A. Lithium Fluoride Additives for Stable Cycling of Lithium Batteries at High Current Densities. Adv. Electron. Mater. 1-6 (2015). doi:10.1002/aelm.201500246

13. Lu, Y., Tu, Z. \& Archer, L. A. Stable lithium electrodeposition in liquid and nanoporous solid electrolytes. Nat. Mater. 13, 961-969 (2014).

14. Tikekar, M. D., Choudhury, S., Tu, Z. \& Archer, L. A. Design principles for electrolytes and interfaces for stable lithium-metal batteries. Nat. Energy 1, 16114 (2016).

15. Tikekar, M. D., Archer, L. A. \& Koch, D. L. Stabilizing electrodeposition in elastic solid electrolytes containing immobilized anions. Sci. Adv. 2, e1600320 (2016).

16. Liu, W., Lin, D., Pei, A. \& Cui, Y. Stabilizing Lithium Metal Anodes by Uniform Li-Ion Flux Distribution in Nanochannel Confinement. J. Am. Chem. Soc. 138, 15443-15450 (2016).

17. Zheng, G. et al. Interconnected hollow carbon nanospheres for stable lithium metal anodes. Nat. Nanotechnol. 9, 618-623 (2014).

18. Choudhury, S., Mangal, R., Agrawal, A. \& Archer, L. A. A highly reversible room-temperature lithium metal battery based on crosslinked hairy nanoparticles. Nat. Commun. 6, 10101 (2015). 
19. Kozen, A. C. et al. Next-Generation Lithium Metal Anode Engineering via Atomic Layer Deposition. ACS Nano 9, 5884-5892 (2015).

20. Khurana, R., Schaefer, J. L., Archer, L. A. \& Coates, G. W. Suppression of lithium dendrite growth using cross-linked polyethylene/poly(ethylene oxide) electrolytes: a new approach for practical lithium-metal polymer batteries. $J$. Am. Chem. Soc. 136, 7395-7402 (2014).

21. Bouchet, R. et al. Single-ion BAB triblock copolymers as efficient electrolytes for lithium-metal batteries. Nat. Mater. 12, 452-457 (2013).

22. Wei, S. et al. Stabilizing electrochemical interfaces in viscoelastic liquid electrolytes. Sci. $A d v$. 4, (2018).

23. Jonscher, A. K. The 'universal' dielectric response. Nature 267, 673-679 (1977).

24. Funke, K. \& Cramer, C. Conductivity spectroscopy. Curr. Opin. Solid State Mater. Sci. 2, 483-490 (1997).

25. Gurevitch, I. et al. Nanocomposites of Titanium Dioxide and PolystyrenePoly(ethylene oxide) Block Copolymer as Solid-State Electrolytes for Lithium Metal Batteries. J. Electrochem. Soc. 160, A1611-A1617 (2013).

26. Stone, G. M. et al. Resolution of the Modulus versus Adhesion Dilemma in Solid Polymer Electrolytes for Rechargeable Lithium Metal Batteries. J. Electrochem. Soc. 159, A222-A227 (2012).

27. Choudhury, S. et al. Electroless Formation of Hybrid Lithium Anodes for Fast Interfacial Ion Transport. Angew. Chemie Int. Ed. 56, 13070-13077 (2017). 


\section{APPENDIX}

Supplementary Information for Chapter 7

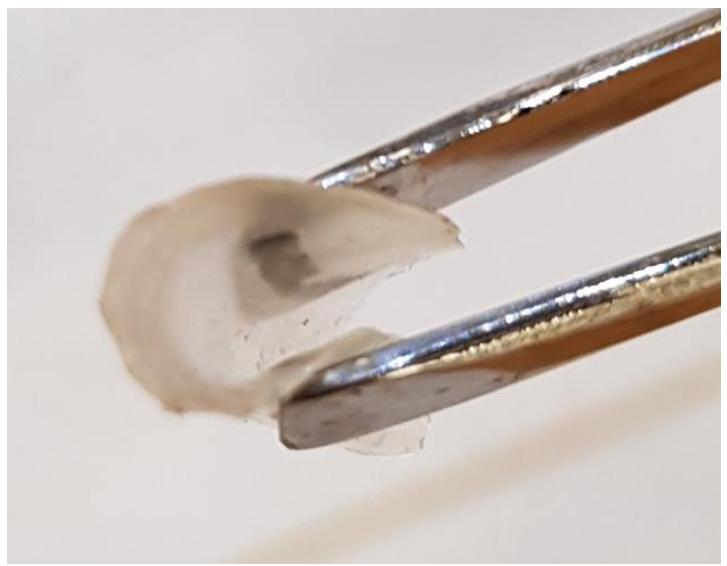

Supplementary Figure 8.1: Photograph of the flexible crosslinked membrane. 


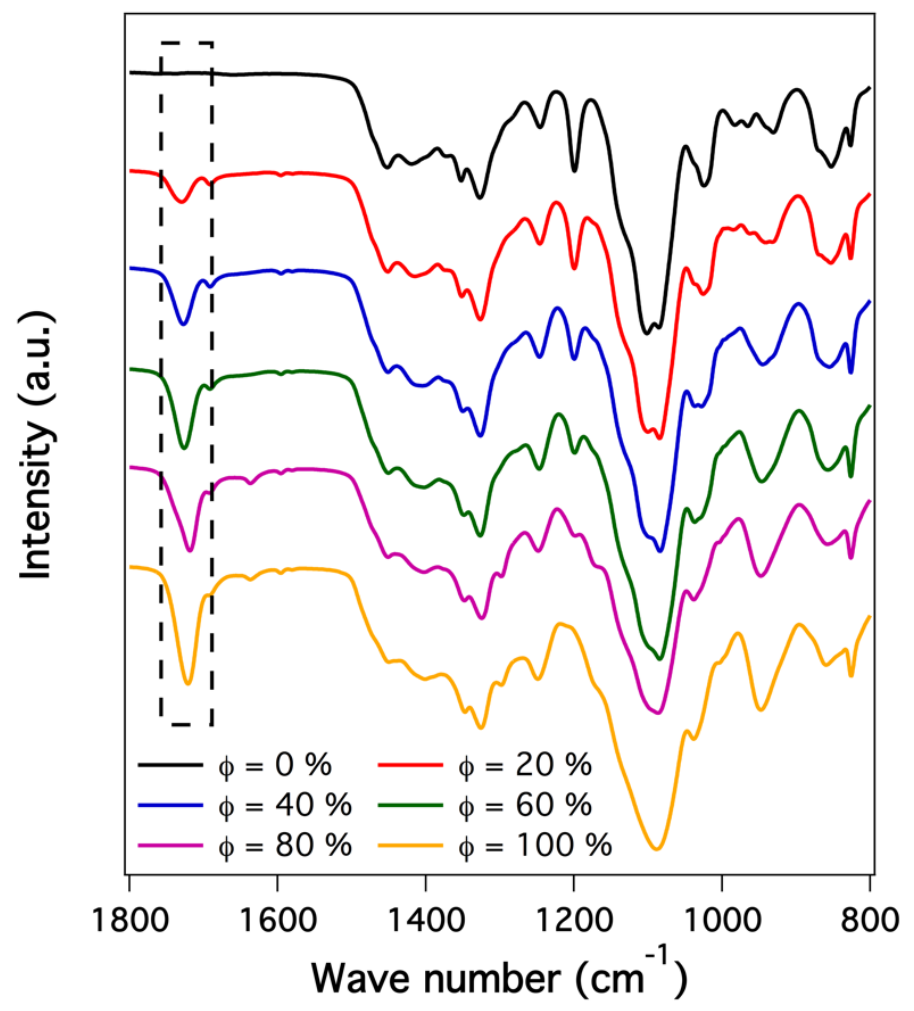

Supplementary Figure 8.2: FTIR analysis of the crosslinked membrane at various PEGDMA content that shows $\mathrm{C}=\mathrm{O}$ bond at $\left(1,700 \mathrm{~cm}^{-1}\right)$ shifting to lower intensity values as the volume percent PEGDMA is increased in solution. 

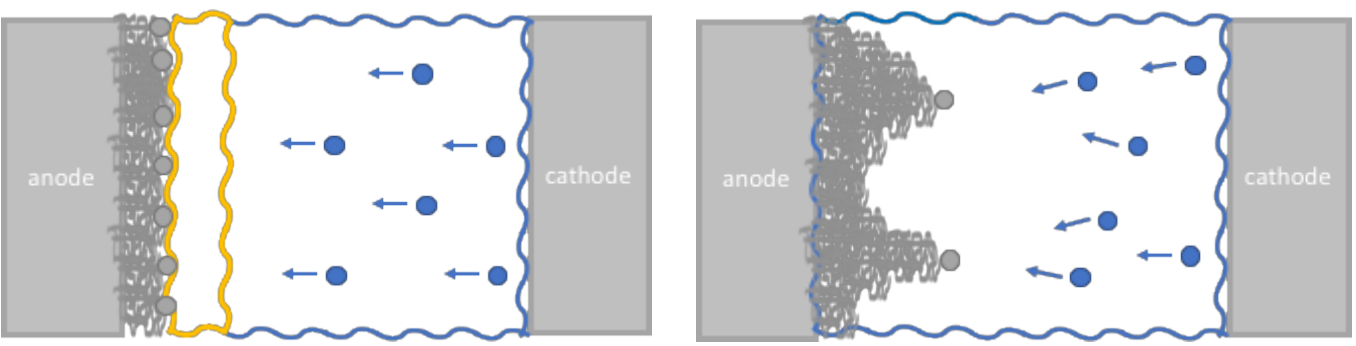

Supplementary Figure 8.3: Schematic demonstrating the concept of stabilization using a solid polymer interphase. 

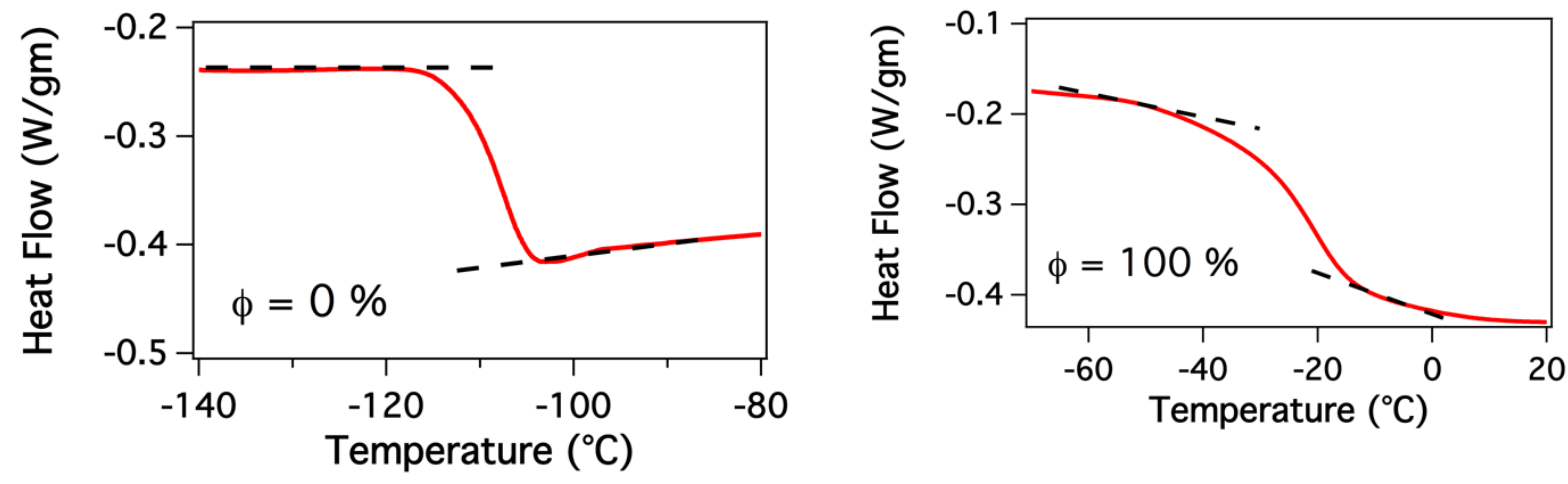

Supplementary Figure 8.4: Thermograms obtained from Differential Scanning Calorimetry for pure diglyme $(\Phi=0 \%)$ and pure PEGDMA network $(\Phi=100 \%)$. The dotted lines mark the step-change in the heat-flow 


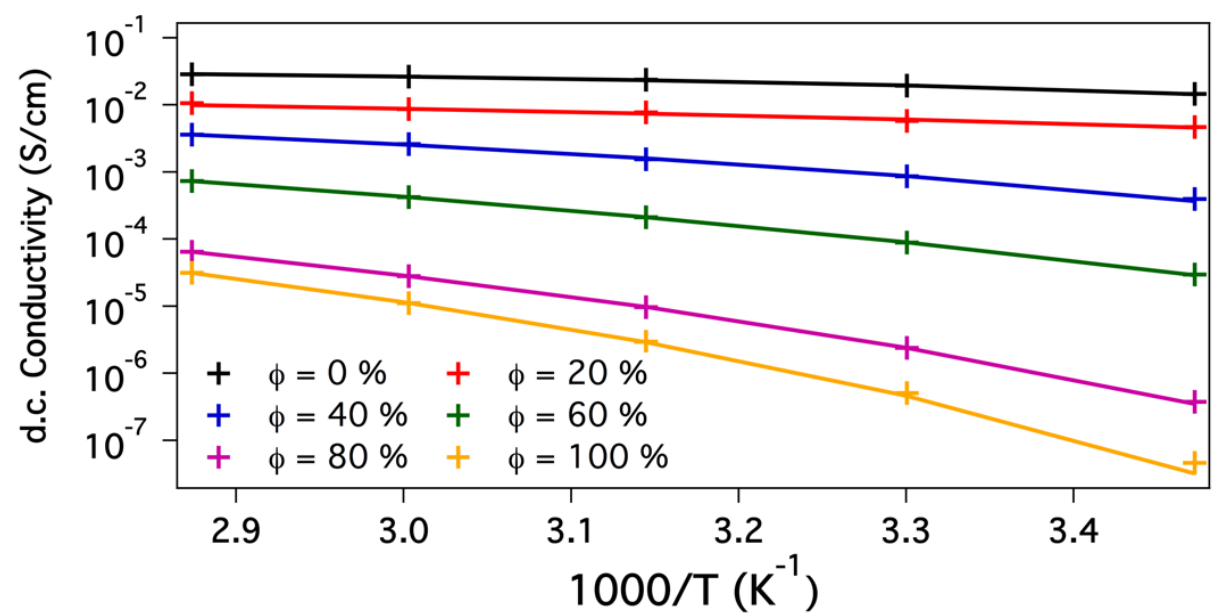

Supplementary Figure 8.5: d.c. conductivity as a function of inverse absolute

temperature. Measured values are shown as markers, and the data is fitted to Vogel Tamman Fulcher function. 


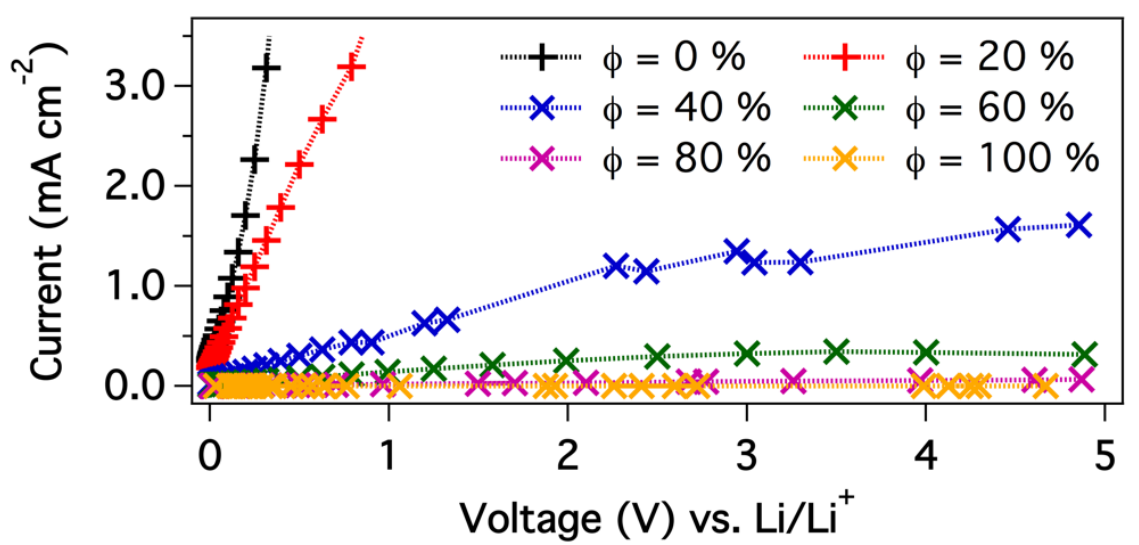

Supplementary Figure 8.6: Current as a function of voltage. Divergence in current was seen for $\Phi=0 \%$ and $\Phi=20 \%$, signaling the presence of electroconvection. For $\Phi=40 \%$ and beyond, the current reached a limiting value at higher voltages 


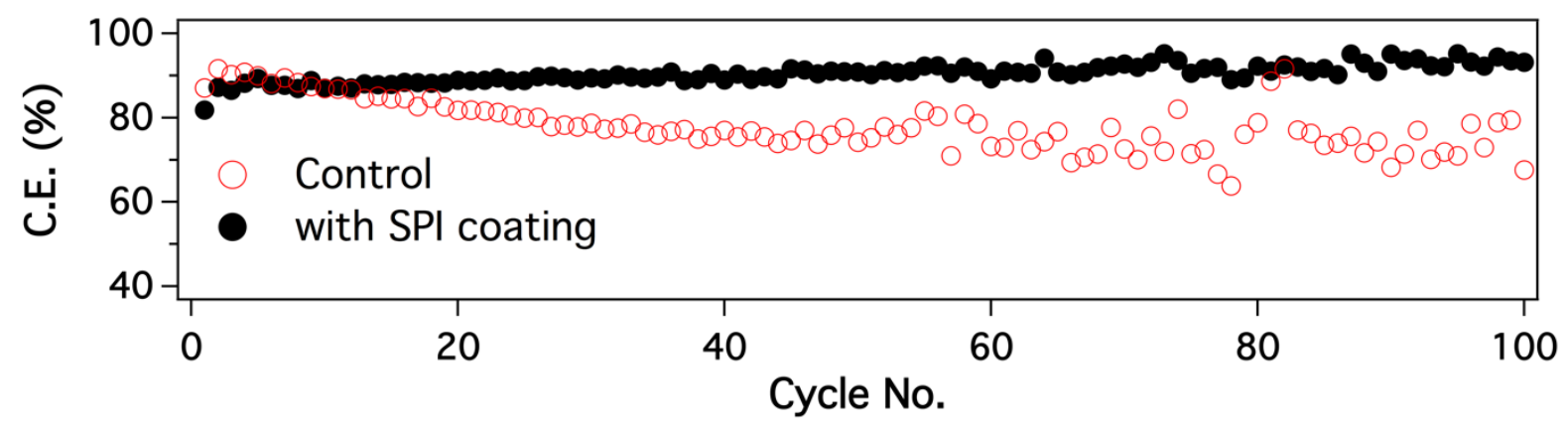

Supplementary Figure 8.7: Coulombic efficiency measurement in Li $\|$ stainless steel coin cell with and without the solid polymer interphase at a current density of $1 \mathrm{~mA}$ $\mathrm{cm}^{-2}$ and capacity of $1 \mathrm{mAh} \mathrm{cm}^{-2}$, using the $1 \mathrm{M} \mathrm{LiPF}_{6}$ in EC/DMC electrolyte. 


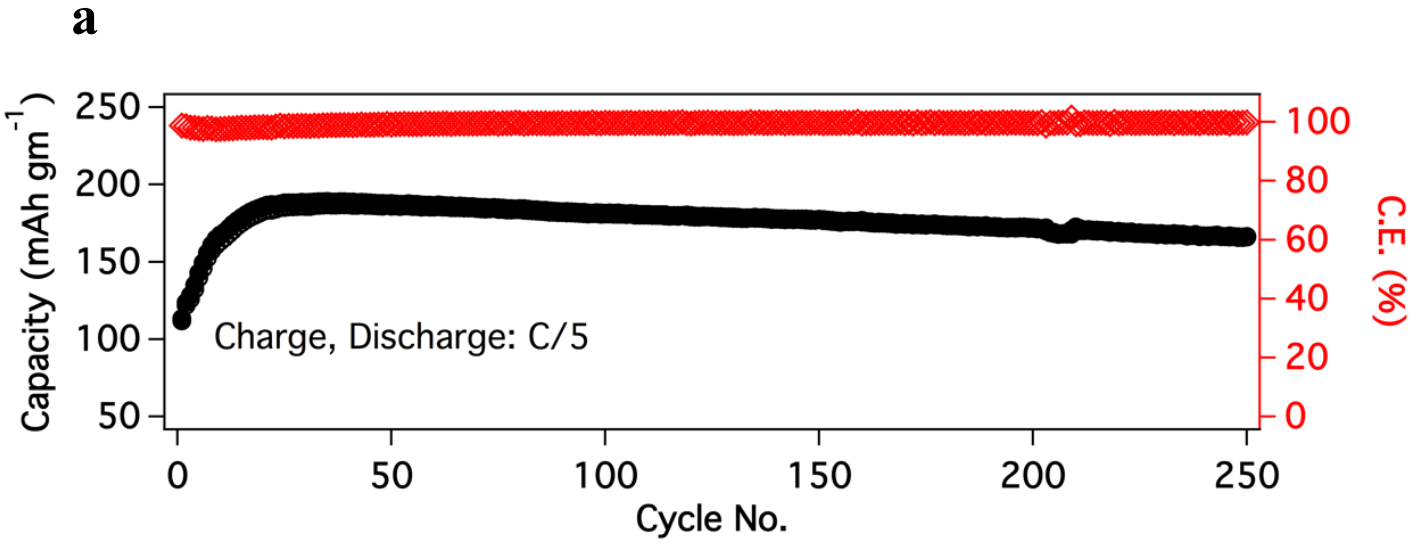

b

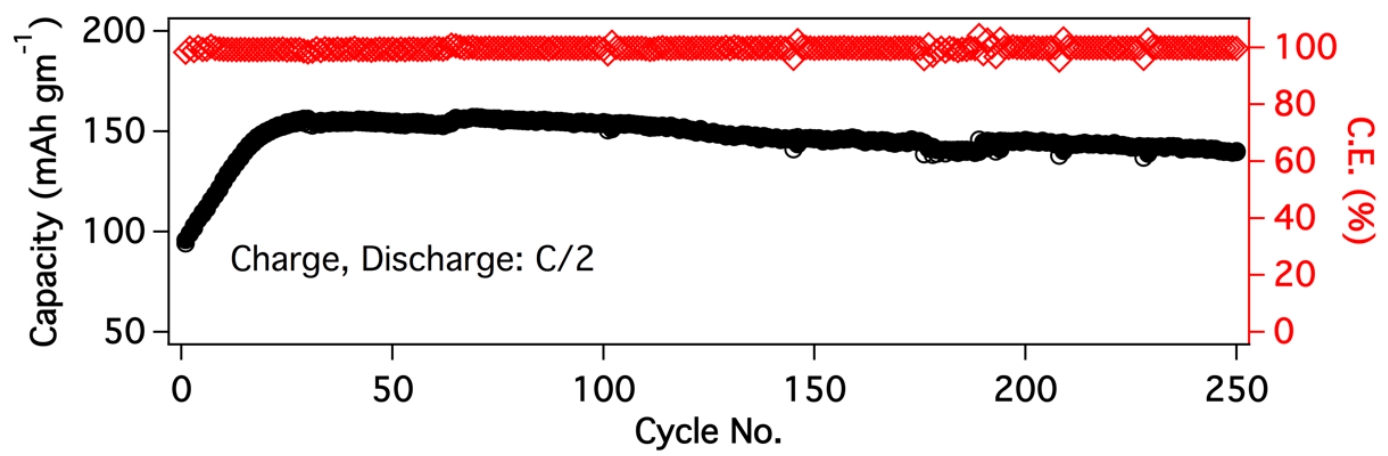

Supplementary Figure 8.8: Cycling performances for $\mathrm{Li} \| \mathrm{NMC}$ cell operated at a Crate of (a) $\mathrm{C} / 5$ and (b) $\mathrm{C} / 2$. Here the lithium metal electrode was coated with the solid polymer interphase that comprises of the polymer network and diglyme, with PEGDMA content of $40 \%$. The thickness of the polymer coating was $\sim 100 \mu \mathrm{m}$. The capacity of cathode is $2 \mathrm{mAh} / \mathrm{cm}^{2}$ and the electrolyte used here is $0.6 \mathrm{M}$ LiTFSI, $0.4 \mathrm{M}$ LiBOB, $0.05 \mathrm{LiPF}_{6}$ in EC/DMC (1:1 by wt.) 


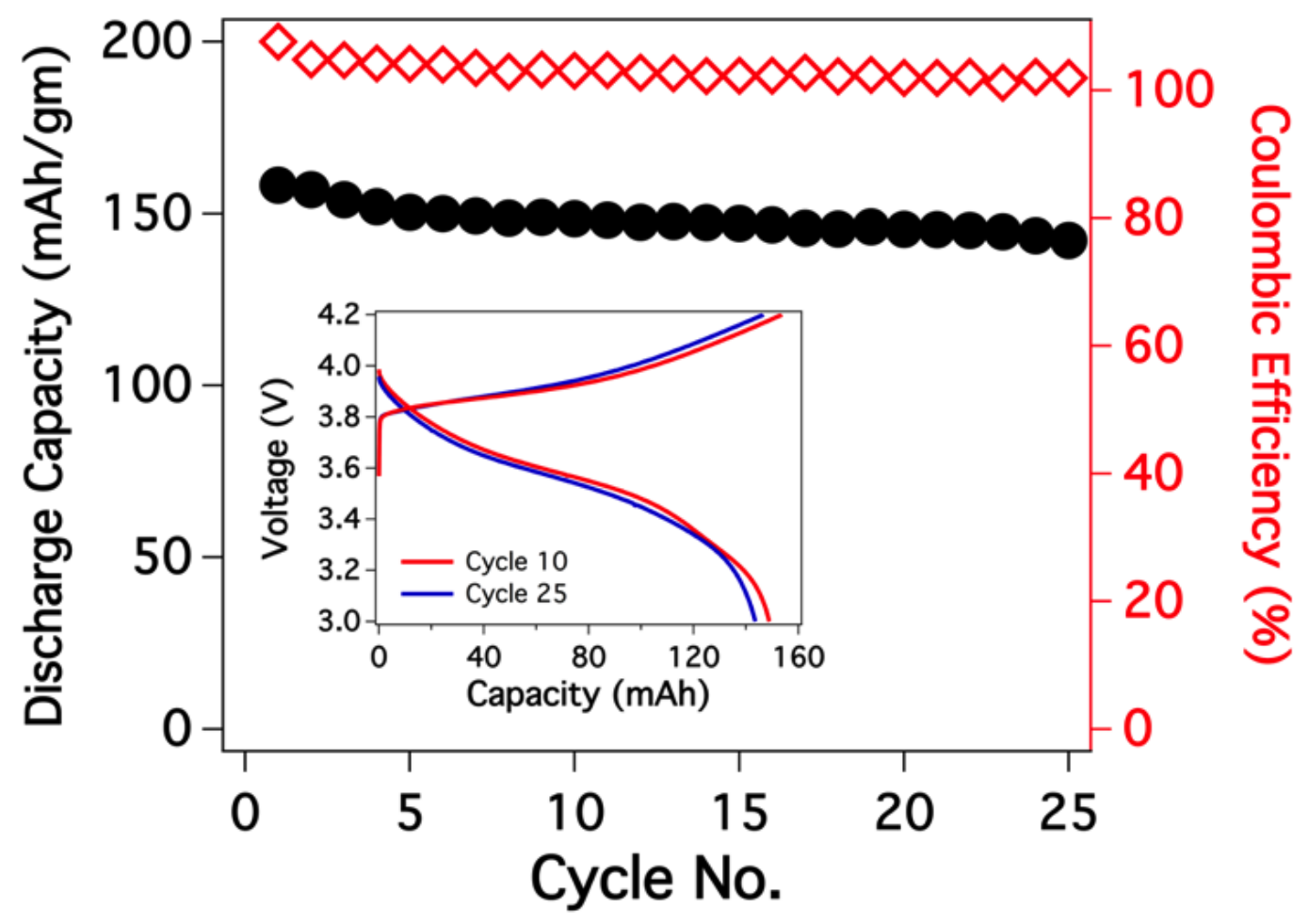

Supplementary Figure 8.9: Cycling performances for $\mathrm{Li} \| \mathrm{NMC}$ cell operated at a Crate of $\mathrm{C} / 5$. Here the electrolyte utilized was an all-solid state polymer electrolyte that comprises of the polymer network and diglyme, with PEGDMA content of $40 \%$ and with the salt $\mathrm{LiNO}_{3}(\mathrm{Li}: \mathrm{EO}=0.10)$ and $0.4 \mathrm{M} \mathrm{LiBOB}$ as additive. The thickness of the solid polymer electrolyte was $\sim 400 \mu \mathrm{m}$. The capacity of cathode is $2 \mathrm{mAh} / \mathrm{cm}^{2}$. The cathode surface was wetted by liquid electrolyte of diglyme- $\mathrm{LiNO}_{3}(\mathrm{Li}: \mathrm{EO}=0.1)$ and 0.4M LiBOB. 


\section{Chapter 9}

Stabilizing Polymer Electrolytes in High-Voltage Lithium Batteries 


\subsection{Abstract}

More than forty years after the first report of a rechargeable lithium battery, electrochemical cells that utilize metallic lithium anodes are again under active study for their potential to provide more energy dense storage in batteries. Electrolytes based on small-molecule ethers and their polymeric counterparts are known to form stable interfaces with alkali metal electrodes and for this reason are among the most promising choices for rechargeable lithium batteries. Uncontrolled anionic polymerization of the electrolyte at the low anode potentials and oxidative degradation at the working potentials of the most interesting cathode chemistries have led to a quite concession in the field that solid-state or flexible batteries based on polymer electrolytes can only be achieved in cells based on low- or moderate-voltage cathodes. Here we show that cationic chain transfer agents in an ether electrolyte provide a fundamental strategy for limiting polymer growth at the anode, enabling long term (at least 2000) cycles of high-efficiency operation of asymmetric lithium cells. Building on these ideas, we also report that cathode electrolyte interphases composed of anionic polymers and the superstructures they form spontaneously at high electrode potentials provide as fundamental a strategy for extending the high voltage stability of etherbased electrolytes to potentials well above conventionally accepted limits. Through computational chemistry, we discuss the mechanistic processes responsible for the extended high voltage stability and on this basis report Li $\mid$ NMC cells based on a simple diglyme electrolyte that offer unprecedented stability in extended galvanostatic cycling studies. 


\subsection{Introduction}

Small-molecule linear and cyclic ethers/glymes and their carbonate esters formed by reaction with carbon dioxide have emerged as the most important family of electrolytes for lithium batteries. These molecules are attractive for a variety of reasons, including their low viscosity and ability to coordinate with lithium ions, producing higher concentrations of mobile charge carriers than one would anticipate from classical theory, based on their dielectric constants alone. ${ }^{1-6}$ Macromolecular analogs, most notably polyethylene glycol dimethyl ether (PEGDME), have been reported to offer additional beneficial effects, including orders of magnitude higher mechanical modulus, low volatility and low flammability, making them attractive candidates for solid-state or flexible lithium batteries in a variety of form factors. ${ }^{7-11}$ A substantial body of work focused on charge carrier transport mechanisms in polyethers has shown that lithium ion mobility is coordinated with molecular motions and that charge carrier transport occurs predominantly in the amorphous phase of the materials where molecular mobility is highest. ${ }^{12-16} \mathrm{~A}$ less studied, but as important trait of ethers is the ease with which they can be electropolymerized at the reducing potentials at the lithium battery anode, as well as at the oxidizing potentials of the cathode. Almost nothing is known about how these processes can be regulated to produce self-limiting interphases and how fast ion transport at such interphases might be used to stabilize deposition at the Li anode.

Reduction of small-molecule ethers and carbonate esters at a lithium battery anode produces less mobile polymeric species by ring-opening and/or anionic 
chemistries. ${ }^{2,4,17-19}$ In favorable situations (e.g. at the graphitic carbon anode of stateof-the art lithium ion batteries) the reactions are self-limiting and produce a thin coating of a low-molar mass polymer-rich phase (interphase) at the electrode surface. This so-called solid-electrolyte interface (SEI) limits molecular access to the electrode surface and prevents continuous loss of electrolyte. The SEI is known to be crucial for stable, long-term battery operation, but almost nothing is known about how the tools of polymer chemistry can be used to harness it to achieve a similar electrochemical function at more unstable (chemical and morphological) alkali metal anodes. In cells that use lithium metal as anode, spontaneously formed interphases are in fact rarely self-limiting. Numerous studies have begun to appear that center on materials synthesis strategies for creation of specially designed self-limiting interfaces on such anodes using sacrificial, easily reduced species added to an electrolyte, ${ }^{20-23}$ or application of ion permeable coatings formed ex-situ. ${ }^{24-26}$

At the intercalating composite cathodes (e.g. NMC, LMO, LCO) of greatest contemporary interest for lithium cells, electrolyte-electrode interfaces are not restricted to planes. Designing self-limiting interphases able to reduce/prevent electrolyte oxidation is therefore far more complex. Because ethers are particularly vulnerable to oxidative attack, a concession is the field is that ether- and polyetherbased electrolytes cannot be used in practical electrochemical cells that employ highvoltage cathodes. ${ }^{27}$ As a consequence, solid-state ceramic electrolytes have emerged in recent years as the most promising candidates for all solid-state lithium batteries. 
Here, we consider the chemical processes responsible for uncontrolled interphase polymer chain growth at the anode and oxidative degradation of ethers at the cathode of a lithium cell and on that basis show that electrolytes based on ethers can be designed to overcome conventional limitations. We show in particular that inhibition of anionic polymerization of electrolytes based on chain transfer agents (CTAs) offer unusually high levels of interphase stability at a lithium metal anode. We further report that anionic species able to limit transport of polymer intermediates at the cathode are an integral component in designing self-limiting cathode electrolyte interfaces (CEIs) able to stabilize glymes at highly oxidizing electrode potentials. Taken together with recent work showing that polyethers in a variety of cross-linked configurations are able to inhibit rough, dendritic electrodeposition at a lithium metal anode during battery recharge, the results reported herein provide a path towards safe, cost-effective solid state and flexible batteries based on polymer electrolytes. ${ }^{7,28,29}$ The electrolyte used in the study is comprised of Bis(2-methoxyethyl) ether (diglyme) and Lithium Nitrate $\left(\mathrm{LiNO}_{3}\right)$ salt. The choice of $\mathrm{LiNO}_{3}$ is based on the fact that it is cheaper compared to other salts and it is known to form a stable interfacial layer on the anode.$^{30}$ Diglyme is chosen as the simplest oligo-ether that offers the combination of a high boiling point $\left(162^{\circ} \mathrm{C}\right)$ and appreciable ion transport rate at ambient temperature to be of interest as an electrolyte for the lithium metal battery. The chemical structure of the electrolyte, including the ease with which the molecule can be electropolymerized at the cathode or anode of an electrochemical cell is shared with all ether-based liquid and solid polymer electrolytes, which means that the interfacial polymerization, oxidative breakdown, and transport characteristics of diglyme at 
electrodes are to a reasonable approximation representative of a much broader class of polymer electrolyte candidates. The $\mathrm{LiNO}_{3}$ concentration in diglyme is systematically adjusted by varying the ratio, $r$, of $\mathrm{Li}^{+}$cations to ether oxygen (EO) molecules in the electrolyte.

\subsection{Results and Discussions}

Supplementary Figure 9.1a reports the effect of $r$ on the temperature-dependent electrolyte conductivity. The conductivity values at room temperature are seen exceed $1 \mathrm{mS} / \mathrm{cm}$ for all materials used in the study, but there are appreciable variations at subzero temperatures. It is clear from the results that diglyme- $\mathrm{LiNO}_{3}$ electrolytes with $r=$ 0.1 exhibit the highest conductivity compared across the range of measurement temperatures employed in the study. It is also notable that even at temperature of $30^{\circ} \mathrm{C}$, the conductivity of this electrolyte is $>1 \mathrm{mS} / \mathrm{cm}$, which makes it suitable for lowtemperature battery operation without any compromise in power density. The continuous lines in Supplementary Figure 9.1a shows that the Vogel-FulcherTammann (VFT) model, $\sigma=A \exp \left(-E_{a} / R\left(T-T_{o}\right)\right)$. Here, $\mathrm{E}_{\mathrm{a}}$ is related to the free volume required to move the ions and To is related to the glass transition temperature of the polymer (typically found to be in order of $\mathrm{T}_{\mathrm{g}}-50$ ). $E_{a}$, obtained from this analysis provides a measure of the facility with which ions move in an electrolyte plotted and are reported as a function of $r$ in Supplementary Figure 9.1b. $E_{a}$ is seen to increase monotonically with $r$, similar to the glass transition temperature ( $\left.T_{g}\right)$, also plotted in Supplementary Figure 9.1b. This result indicates that there are high levels of molecular association between the diglyme and the salt and is consistent with the idea 
that as the salt concentration is increased, diglyme molecules move in a more coupled manner. On the basis of these results, we utilize an electrolyte with $r=0.1$ for all subsequent studies.

Glyme or ether based electrolytes are known to undergo anionic polymerization at the surface of alkali metals, particularly at the highly reducing potentials at the anode. The resultant polymer-rich interphases are desirable because they passivate the electrode against parasitic chemical reactions with the electrolyte. Glymes are for this reason among the most preferred electrolytes for electrochemical cells in which alkali metals are to be used as anodes. ${ }^{1,19,30}$ Unfortunately, left unchecked, the polymers formed may grow to such high molecular weights that $\mathrm{Li}+$ transport to the electrode is severely retarded. Alkali metals are thought to initiate polymerization by cleaving a proton from the side-chain of a glyme molecule as shown in Figure 9.1a. The polymer chain grows by an addition process wherein the active anionic reactive center collides with another glyme molecule, extending the length of the chain. Because electrostatic interactions between active centers prevent collisions between growing chains and centers can be stabilized by Li ions in solution, the growth can in principle progress indefinitely to produce extremely large, poorly conductive polymer chains or until all available glyme molecules are integrated into the growing center. In either event, ion mobility in the electrolyte bulk falls and interfacial resistance rises, producing premature failure of the cell by voltage run-away. 

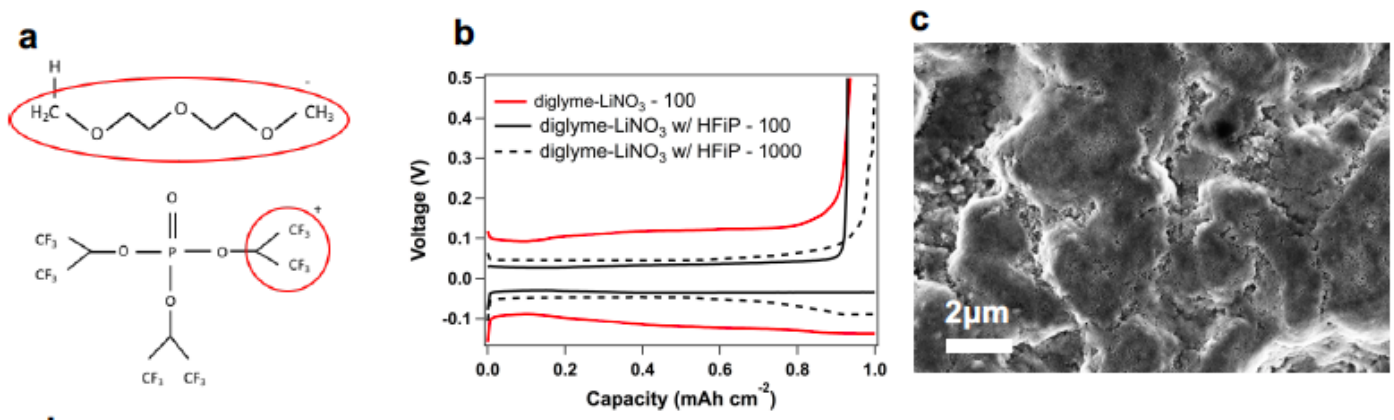

d

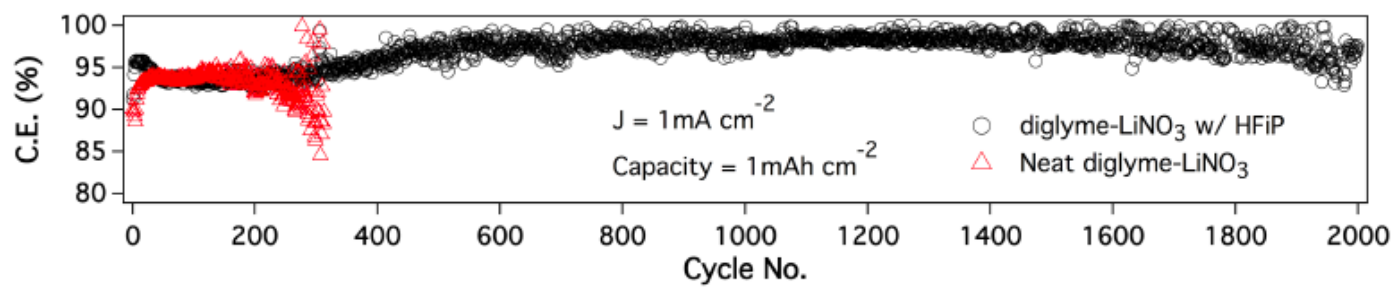

Figure 9.1: Enabling stable electrodeposition of lithium metal: (a) Schematic showing the possible cleavage sites for diglyme and HFiP molecules such that the uncontrolled polymerization of diglyme is quenched by the $\mathrm{CH}\left(\mathrm{CF}_{3}\right)_{2}{ }^{+}$radical; (b) Voltage profile for the electroplating and stripping of lithium metal at the same current density. The different numbers represent cycle no.; (c) Scanning electron microscopy image of stainless steel substrate after lithium deposition for 6 hours at the current density of $1 \mathrm{~mA} / \mathrm{cm}^{2}$; (d) Coulombic efficiency measurements in a $\mathrm{Li} \mid$ |stainless steel battery at a current density of $1 \mathrm{~mA} / \mathrm{cm}^{2}$ and capacity of $1 \mathrm{mAh} / \mathrm{cm}^{2}$. The black circles represent the diglyme-LiNO $\mathrm{L}_{3}$ electrolyte with the HFiP additive and red triangles are for neat electrolyte 
We hypothesize that an electrolyte that addresses this fundamental, termination-free characteristic of anionic addition polymerization could limit chain growth to produce self-limited SEI on a metallic electrode. To test this idea, we employ the molecule Tris(hexafluoro-iso-propyl)phosphate (HFiP) that is known to readily degenerate to form multiple $\mathrm{CH}\left(\mathrm{CF}_{3}\right)_{2}{ }^{+}$species per molecule ${ }^{31,32}$. The large number of electron withdrawing groups near the cationic fragments should enable rapid, and efficient quenching of anionic polymerization of glyme molecules by the chain transfer mechanism depicted in Figure 9.1a. As a proof of concept, we performed a simple analysis wherein lithium metal was dipped in diglyme- $\mathrm{LiNO}_{3}$ electrolyte with and without HFiP additive aged for a month. Supplementary Figure 9.2 shows the image vial bottles, where it is seen that the electrolyte without the CTA turns yellow due to uncontrolled polymerization of the diglyme molecules, while the lithium is blackened due to surface reactions. In comparison, the HFiP additive stabilizes not only the diglyme solution but also the lithium surface maintains its pristine form. Furthermore, surface of lithium from both vial bottles were analyzed using X-ray Photoelectron Spectroscopy (XPS) and reported in Supplementary Figure 9.3 \& 9.4. The F-1s XPS for the case with HFiP additive has a single peak at $688.9 \mathrm{eV}$ representing-CF3 bond, which is further confirmed from the C-1s XPS from the peak at $293.3 \mathrm{eV}$, while it is absent in the $\mathrm{C}-1 \mathrm{~s}$ for the lithium extracted from additive-free lithium. ${ }^{33}$ The absence of a metal-fluoride binding energy peak is a confirmation that the -CF3 groups do not decompose in the presence of the lithium metal electrode, ruling out an alternative stabilizing mechanism reported in our previous work. ${ }^{22,23}$ 
The effectiveness of the approach to create self-limited interphases on a $\mathrm{Li}$ anode was evaluated in an asymmetric electrochemical cell comprised of lithium metal and stainless-steel electrodes. By comparing the electric current generated when a specific amount of Li is stripped from the Li electrode and deposited onto the stainless-steel electrode, with the current required for the reverse process, the coulombic efficiency (CE) of the cell can be determined. Figure $9.1 \mathrm{~b}$ shows the voltage profile during a typical measurement in cells with and without the HFiP chain transfer agent. It can be seen that although for the $100^{\text {th }}$ cycle the CE values for the two electrolytes are the same, the overpotential for stripping and plating Li are vastly reduced by the chain transfer agent, consistent with expectations for the CTA's ability to terminate polymer chain growth. The consequence of these effects is quite clearly seen in Figure 9.1d and Supplementary Figure 9.5, which report the CE for electrolyte with and without the CTA, at current densities of $1 \mathrm{~mA} / \mathrm{cm}^{2}$ and $0.25 \mathrm{~mA} / \mathrm{cm}^{2}$, respectively with each half cycle comprising of 1 hour. This means that approximately $5 \mu \mathrm{m}$ and $1.25 \mu \mathrm{m}$ of the $450 \mu \mathrm{m} \mathrm{Li} \mathrm{electrode} \mathrm{is} \mathrm{stripped} \mathrm{and} \mathrm{plated} \mathrm{during} \mathrm{each} \mathrm{cycle,} \mathrm{respectively.} \mathrm{It} \mathrm{is}$ seen that the CE is maintained at a value $>98 \%$ for 2000 plate-strip cycles, even without efforts to optimize the composition of the CTA in the electrolyte or its efficiency in terminating addition polymerization! This level of stability has to our knowledge not been observed in a lithium metal cell using a liquid electrolyte. The benefits of the CTA are obvious when results for electrolytes with and without this species are compared (Figure 9.1d). It is observed that whereas the control diglyme$\mathrm{LiNO}_{3}$ electrolyte with/without the chain transfer agent have similar $\mathrm{CE}$ for the initial 200 cycles, upon longer-term cycling large fluctuations appear in the latter that are 
absent in the former. Similar behavior is observed at the lower current density of $0.25 \mathrm{~mA} / \mathrm{cm}^{2}$, however the fluctuations in CE are seen after 500 cycles. We further characterize the electrodeposition by SEM analysis of the stainless-steel electrode after electrodeposition of $6 \mathrm{mAh} / \mathrm{cm}^{2}$ (ca $30 \mu \mathrm{m}$ of Li) at $1 \mathrm{~mA} / \mathrm{cm}^{2}$ (Figure $9.1 \mathrm{c}$ ) and at $0.25 \mathrm{~mA} / \mathrm{cm}^{2}$ (Supplementary Figure 9.6), in the glyme electrolyte containing HFiP additive. It is seen that the deposits are compact at both these current densities; also the coverage of the smooth deposits span over several microns indicating the large scale uniformity.

The fluctuations in CE observed in the control electrolytes are associated with the sporadic electrical connections with electrically disconnected fragments of lithium ('orphaned lithium') formed during the electrodeposition process and are indicative of the irreversibility of the process. These findings are confirmed by postmortem analysis of the electrode surface after cycling the Li||stainless steel cell with and without HFiP additive at current density of $1 \mathrm{~mA} / \mathrm{cm}^{2}$ for 100 cycles, followed by depositing lithium of capacity $1 \mathrm{mAh} / \mathrm{cm}^{2}$ on the stainless steel electrode. The SEM images of the electrodeposited stainless steel reported in Supplementary Figure 9.7 indicate that in contrast to open, dendritic or needle-like deposits are observed in the control electrolyte, the CTA containing electrolyte resulted in compact structures. This difference underscores the consequence of faster diffusion of lithium ions and low charge transfer resistance for the anodic reaction: $\mathrm{Li}^{+}+e^{-} \rightarrow \mathrm{Li}$ at interphases where polymerization of the glyme is constrained. 
The continuous polymerization of the diglyme molecules without the HFiP chain transfer agent can lead to increased battery resistance over cycling which can be verified by impedance spectroscopy measurements of batteries. Supplementary Figure 9.8 reports the Nyquist plots of the cells containing the electrolyte of diglyme-LiNO 3 with and without the HFiP additive. The cells were comprised of lithium metal and stainless steel disk as electrodes and were cycled 100 times at $1 \mathrm{~mA} / \mathrm{cm}^{2}$ current density and $1 \mathrm{mAh} / \mathrm{cm}^{2}$ capacity with plating in the last step. After fitting the impedance spectra with the appropriate circuit model (shown in the inset of Supplementary Figure 9.8), it was seen that the interfacial resistance for the cell using control electrolyte was $77.5 \Omega$, while that of the CTA containing cell was $50.9 \Omega$. Thus, it can be argued that the chain transfer agent enables longer term stable cycling by preventing electrolyte degradation. We also analyzed the surface of lithium metal extracted from a $\mathrm{Li} \|$ stainless steel cell with the diglyme-LiNO $3-\mathrm{HFiP}$ electrolyte after 100 cycles using XPS (reported in Supplementary Figure 9.9). It was seen that the majority of the F-1s spectra comprised of the peak at $688.9 \mathrm{eV}$ corresponding to the $\mathrm{CF} 3$, it also shows presence of a peak at $684 \mathrm{eV}$ that can be ascertained to the presence of LiF species. Several previous works on electrode-electrolyte interfaces have demonstrated that LiF stabilizes electrodeposition of metallic lithium. ${ }^{23,34}$ The success of a CTA in limiting polymer growth under the reducing potentials at the $\mathrm{Li}$ anode lead us to hypothesize that an analogous approach might be used to enable all ether based electrolytes to be operated at higher potentials, where oxidative breakdown of the electrolytes is a well-known and longstanding barrier to ether-based electrolytes. Because the cathodes of greatest contemporary interest are porous 


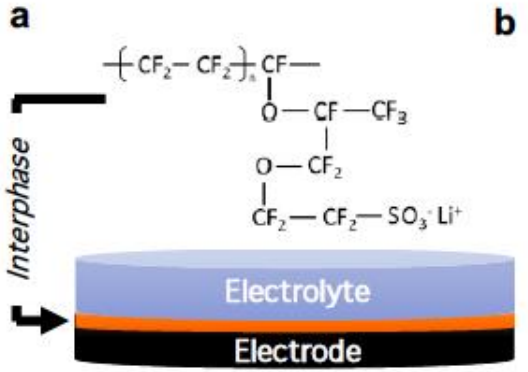

C

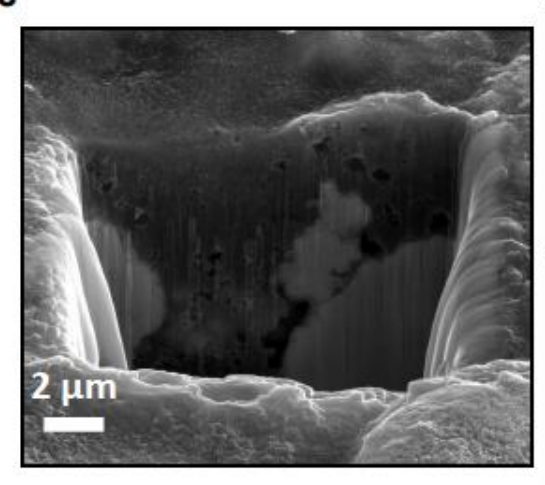

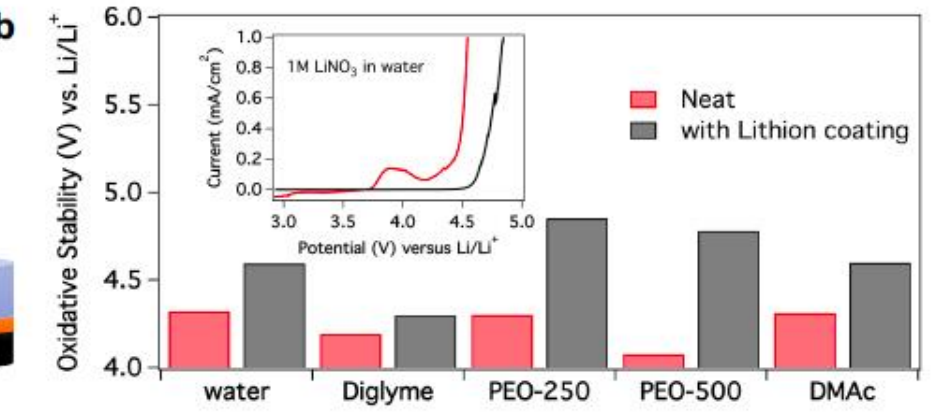

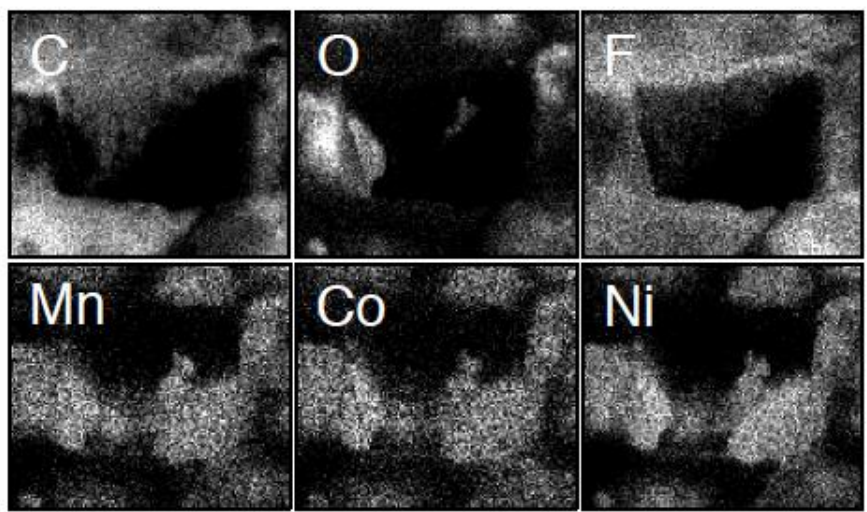

Figure 9.2: Designing a cathodic interface based on immobilized anions: (a) Schematic showing the structure of lithiated nafion (Lithion) utilized to form the artificial interface; (b) Bar chart compares the oxidative stability of different electrolytes with (black) and without (red) the Lithion coating on the electrode. The measurements were done in 3-electrode setup with $\mathrm{Ag} / \mathrm{AgCl}$ as reference and stainless steel as counter and working electrodes. The scan rate was $10 \mathrm{mV} / \mathrm{s}$. The electrolytes investigated are: $1 \mathrm{M} \mathrm{LiNO}_{3}$ in water, $r=0.1 \mathrm{LiNO}_{3}$ in diglyme, $r=0.05 \mathrm{LiNO}_{3}$ in PEO-250, $r=0.05 \mathrm{LiNO}_{3}$ in PEO-500 and $1 \mathrm{M} \mathrm{LiNO}_{3}$ in dimethylacetamide. The inset shows the linear san voltammetry for the $1 \mathrm{M} \mathrm{LiNO}_{3}$-water electrolyte. All the voltages are shifted with respect to $\mathrm{Li} / \mathrm{Li}^{+}$; (c) Cryo-SEM image of the cross-section for a Lithion coated Nickel Manganese Cobalt Oxide (NCM) electrode obtained by Focused Ion Beam milling. The images on the right represent the EDX mapping of different atoms present in the cross-section. 
materials and the active polymer centers once initiated can in principle react with electrolyte solvent able to diffuse from any other location in the cell, a localized strategy that limits active center diffusion away from the electrode-electrolyte interface and lowers solvent migration to the active center is evidently needed. Here, we chose to study interphases formed by the semi-crystalline anionic polymer electrolyte, Lithion (see Figure 9.2a). This choice is motivated by three primary considerations. First, we discovered that solutions of Lithion in aprotic carbonate ester solvents possess sufficiently low viscosity that the polymer can be transported by liquid carriers into the pores of preformed cathodes. Second, the immobilized anions on Lithion can be thought to provide a barrier to oxidation reactions of the negatively charged species and lewis bases in the electrolyte. We've previously explored electrokinetic attributes of this barrier and on that basis shown that the negative charge adopted by Lithion in solution provides an effective electrostatic shield that limits transport of negatively charged species at planar electrodes, yielding lithium transference numbers approaching unity in liquid electrolytes, ${ }^{35}$ Finally, the coexistence if hydrophobic and hydrophilic domains in Lithion means that at appropriate thicknesses it should be possible to retard molecular solvent transport, without compromising anion mobility.

To evaluate this concept, we performed linear scan voltammetry in a three-electrode cell using $\mathrm{Ag} / \mathrm{AgCl}$ as the reference electrode and Lithion coated stainless steel as the working and counter electrodes. A variety of liquid electrolytes were tested ranging from aqueous to oligomers and the electrochemical oxidation potential was compared to the case without the Lithion coating. The inset of Figure $9.2 \mathrm{~b}$ reports the oxidative 
window of $1 \mathrm{M} \mathrm{LiNO}_{3}$-water as electrolyte. It is seen that the Lithion coating elevates the degradation potential of water by $\sim 0.3 \mathrm{~V}$ even in a dilute concentration, in contrast to several recent reports involving high concentration (water-in-salt) aqueous electrolytes. This finding is important from the fundamental perspective of the working mechanism of supersaturated electrolytes, where it is argued that the solvent molecules bind with the ionic content to kinetically inhibit the electrochemical reactions at the electrode. Here, we demonstrate that enabling an immobilized layer of salt at the electrode surface can serve the same purpose of inhibiting electrolyte degradation at the interface. It is hypothesized that the immobilized ionic species strongly desolvate lithium ions in the interfacial region due to electrostatic interactions, thus the reactive solvent molecules are shielded away from high potentials. Furthermore, it is important to note that this methodology is not limited by solubility limit of salt in the bulk electrolyte and cost-effective from industrial pointof-view. We performed similar voltammetry analysis for non-aqueous electrolytes of dimethylacetamide, diglyme, PEG250 and PEG500 with $\mathrm{LiNO}_{3}$ salt and reported the results in Supplementary Figure 9.10. In all the reported electrolytes, an improvement in the oxidative potential was observed, as summarized in Figure 9.2b. The universality of this effect further supports our claim that we are able to isolate the interfacial degradation mechanism from the bulk electrolyte.

We believe that the generalized finding mentioned here, can be utilized to design stable interphases in different electrochemical systems (like $\mathrm{Li}-\mathrm{O}_{2}, \mathrm{Li} \| \mathrm{NMC}$ batteries) that operate at potentials beyond the thermodynamic stability limits of the electrolytes and at the same time do not have much room of electrolyte chemistry modifications. In 

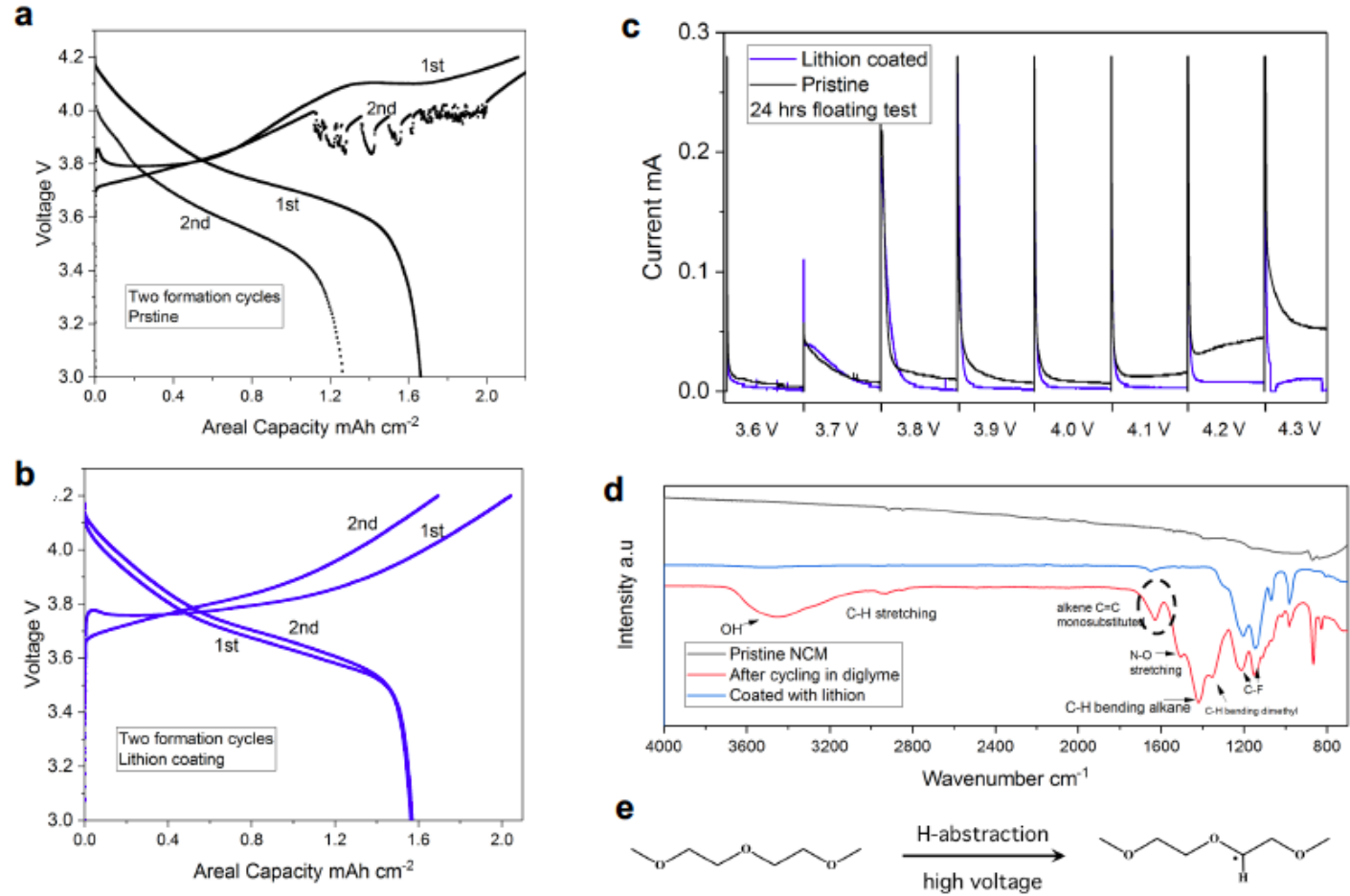

Figure 9.3: Immobilized anions on cathode prevent glyme oxidation: (a) Voltage profile of a lithium||NCM cell using the base electrolyte of diglyme$\mathrm{LiNO}_{3}-\mathrm{HFiP}$ at $\mathrm{C} / 10$ rate; (b) Voltage profile of $\mathrm{Li}|| \mathrm{NCM}$ cell using the same base electrolyte, however the cathode is coated with a layer of lithion, operated at $\mathrm{C} / 10$; (c) Floating point experiment in a $\mathrm{Li}|| \mathrm{NCM}$ cell, where the voltage is fixed at different values ranging from $3.6 \mathrm{~V}$ to $4.3 \mathrm{~V}$ for a period of 24 hours and the current response is measured. The black curves represent results for uncoated NCM and blue is for lithion-coated NCM; (d) Intensity profile obtained from Fourier Transform Infrared Spectroscopy (FTIR) for pristine (uncycled) NCM and NCM cathode extracted from a $\mathrm{Li} \| \mathrm{NCM}$ cell cycled twice at $\mathrm{C} / 10$ with and without the Lithion coating; (e) Schematic showing the proposed mechanism for the proton extraction from the diglyme molecule due to oxidation at high voltages 
this work, we demonstrate this studied electrochemical characteristics of a cell comprised of a lithium metal anode, NMC cathode and the base electrolyte (diglyme$\mathrm{LiNO}_{3}$-HFiP). Specifically, we use drop-cast method to form a coating on the NMC electrode disc. The thickness of the Lithion layer was analyzed using scanning electron microscopy at cryo temperatures shown in Supplementary Figure 9.11. It was observed that the Lithion drop-cast method yielded a thickness range of 90 to $30 \mu \mathrm{m}$, the edges being relatively thicker than the central regions. We further investigated the Lithion coating on the NMC active particles by a focused ion beam milling of the cross-section and EDX mapping of all the atoms as shown in Figure 9.2c and Supplementary Figure 9.12. It can be seen that the $\mathrm{C}$ and $\mathrm{F}$ atoms are highly populated around the individual metal atoms of $\mathrm{Ni}, \mathrm{Mn}$ and $\mathrm{Co}$, which implies that the Lithion layer not only laminates the surface but also conformally surrounds the NMC particles.

The baseline battery performance with the diglyme- $\mathrm{LiNO}_{3}-\mathrm{HFiP}$ electrolyte without the Lithion coating is demonstrated in Figure 9.3a, where it is seen that the voltage profile exhibits a prolonged charging step in the $1^{\text {st }}$ cycle and erratic fluctuations in the $2^{\text {nd }}$ cycle above $3.8 \mathrm{~V}$ vs. $\mathrm{Li} / \mathrm{Li}^{+}$. The discharge step does not show such fluctuations, however a high overpotential is observed in the $2^{\text {nd }}$ cycle indicative of high battery resistance due to the oxidative degradation of glyme electrolytes. These results can be compared with observations provided in Figure 9.3b where the the NMC electrode was coated with the mentioned Lithion layer. As seen from voltage profiles for the $1^{\text {st }}$ and $2^{\text {nd }}$ cycles in Figure 9.3b, the Li $\|$ NMC cells do not show the prolonged charging characteristics observed for the controls. Furthermore, the battery comprising of a thin 
metallic lithium $(50 \mu \mathrm{m})$ as anode shows stable cycling for over 100 cycles as seen in Supplementary Figure 9.13. In addition, we cycled the Lithion coated NMC cathodes in lithium metal batteries with varying charging potentials upto $4.4 \mathrm{~V}$, as shown in Supplementary Figure 9.14. Although previous works based on glyme electrolytes have demonstrated stable cycling with lithium iron phosphate cathode ${ }^{29}$, the facile coating technique is able to augment the stability limits for cycling a high voltage NMC cathode.

We further investigated the high voltage stabilization using electrochemical floating experiments. In this experiment, $\mathrm{Li} \| \mathrm{NMC}$ cells with and without the lithion coating are charged at voltages ranging from $3.6 \mathrm{~V}$ to $4.3 \mathrm{~V}$ in a step-wise ramp and the voltage maintained at a targeted value for a period of 24 hours, as shown in Figure 9.3c. The leakage current obtained at each voltage is recorded and can be used to directly assess the importance of electrochemical degradation of electrolytes in the fully charged state. The results show that the leak current is always higher for the control cells (ie. without the Lithion electrode treatment) than for those that utilize a lithion-coated NMC electrode. In addition, it is seen that the leakage current for the neat NMC cell start to exceed the modified NMC based cell at a faster rate beyond $4 \mathrm{~V}$, which is also consistent with the low coulombic efficiency in the Li||NMC half-cell cycling. Fourier Transform Infrared Spectroscopic analysis was used to provide deeper insight into the mechanism(s) through which the glyme molecules fail at high potentials and how Lithion coatings increase electrolyte stability in the cathode. The NMC cathodes after the formation cycles, with and without the Lithion layer were analyzed and the intensity profiles are plotted in Figure 9.3d. Although most of the chemical bonds are 
a<smiles>C=C1CC(OC(=O)C(CCC)CCCCC)CC1=O</smiles>

(a)

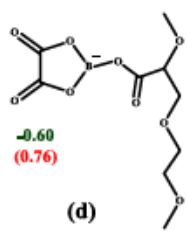

(d)

\section{C}

Calculated redox potentials calculated with respect to that of $\mathrm{Li} / \mathrm{Li}^{+}$couple for diglyme and its oligomers with BOB molecules.

\begin{tabular}{|c|c|c|}
\hline System & Ox/Red charge state & redox potential (eV) \\
\hline Diglyme & $0 /{ }^{-1}$ & 4.14 \\
\hline Dimer & $-1 / 0$ & -6.02 \\
\hline Trimer & $-1 / 0$ & -5.77 \\
\hline Tetramer & $-1 / 0$ & -6.07 \\
\hline
\end{tabular}

(f) b
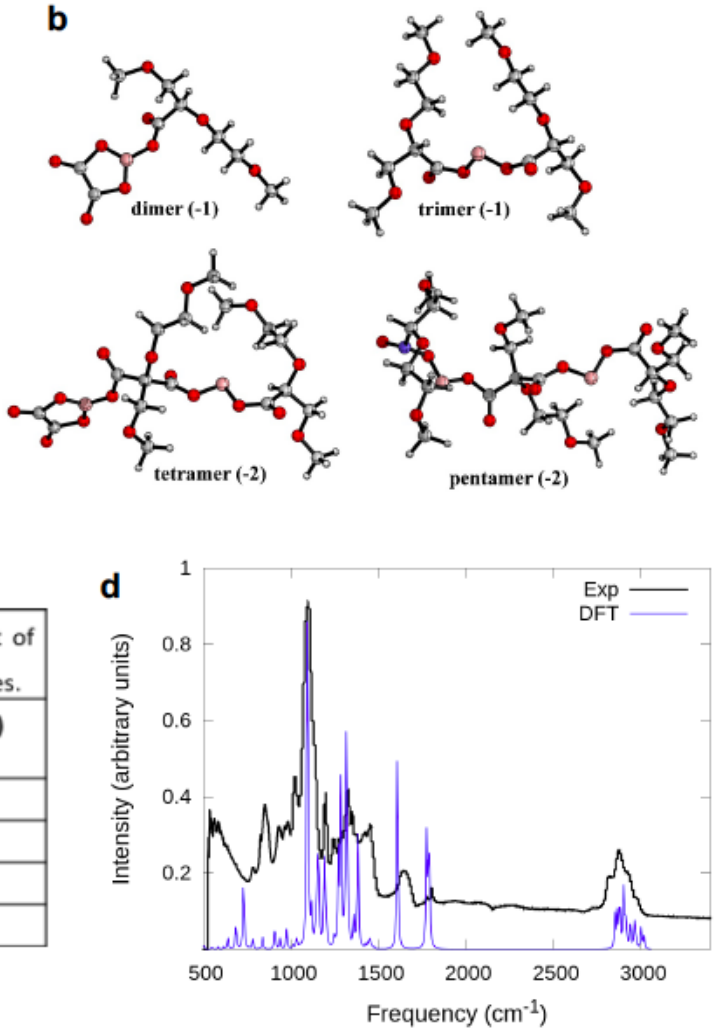

Figure 9.4: In-situ formation of anionic aggregates at cathode interface: (a) Structures of plausible coupling products of $\mathrm{BOB}^{2-}$ and diglyme. Calculated reaction free energies (in $\mathrm{eV}$ ) for the formation of anionic (green color) and neutral (red color) dimers are presented; (b) Optimized geometries for the dimer and higher order coupling products of $\mathrm{BOB}$ and diglyme. Respective charge is shown in the parenthesis; (c) Table showing calculated redox potentials for diglyme and its oligomers with BOB molecules. Oxidation/reduction potentials are calculated with respect to that of $\mathrm{Li} / \mathrm{Li}+$ couple. $\mathrm{A}$ positive or negative sign is used represent reduction and oxidation potentials, respectively; (d) Infrared (IR) spectra comparing the intensity profiles obtained from experiment and DFT calculations. The experimental profile was obtained from a NCM cathode harvested from a $\mathrm{Li}|| \mathrm{NCM}$ cell comprising of diglyme-LiNO 3 -HFiP electrolyte with $0.4 \mathrm{M} \mathrm{LiBOB}$ salt additive and the battery was cycled twice at $\mathrm{C} / 10$. 
present in both cases of with and without Lithion layer, the major difference is the occurrence of the peak at $1600 \mathrm{~cm}^{-1}$ without the Lithion coating that corresponds to the formation of unsaturated $\mathrm{C}=\mathrm{C}$ bonds due to the $\mathrm{H}$-abstraction from the diglyme molecules as shown in Figure 9.3e. The de-protonation of the ether oxide molecules during the high voltage charging can leads to self-polymerization and formation of side products on the electrode surface resulting in high overpotentials and battery fading. Similar, $\mathrm{C}=\mathrm{C}$ bond formation was also found in the electrolyte remains at the battery separator surface, shown in Supplementary Figure 9.15 that is absent in pristine diglyme solution.

The effectiveness of the Lithion cathode coatings suggests that other approaches that lead to in-situ formation of anionic polymer coatings throughout the cathode would be a more straightforward strategy for enabling ether-based electrolytes in lithium cells employing high voltage cathodes. To evaluate this concept, we use the lithium salt bis(oxalate)borate ( $\mathrm{LiBOB}$ ) salt as and electrolyte additive in the base electrolyte and, by means of hybrid density functional theory (DFT), computationally study the interphases the salt forms at various electrode potentials. The BOB anion is of interest because it has been reported in previous studies to readily form either an open, dianion by breaking a B-O bond, or can furnish dissociation products. ${ }^{36-38}$ The reactions of these intermediate species with diglyme would generate distinct coupling products. We calculated the reaction free energies for the formation of a series of neutral and anionic $\mathrm{O}-\mathrm{C}, \mathrm{C}-\mathrm{C}$, and $\mathrm{B}-\mathrm{C}$ coupling products from the diglyme and $\mathrm{BOB}$ dianion. These transformations proceed through the release of $\mathrm{CO}_{2}$ molecules. Unique coupling products considered here and the respective free energy changes are presented in 
Figure 9.4a. The calculations indicate that the formation of negatively charged species are thermodynamically more favorable than the respective neutral analogues. Among the anionic dimers, the $\mathrm{C}-\mathrm{C}$ coupling product (a, Figure 9.4a) formed by the release of a $\mathrm{CO}_{2}$ molecule is thermodynamically most favorable $(\Delta \mathrm{G}=-0.64 \mathrm{eV})$.

Starting from the negatively charged dimer, one could envision its subsequent reactions with diglyme and $\mathrm{BOB}^{2-}$, which will generate oligomers, polymers, or a supramolecular assembly at the electrode-electrolyte interface. We have calculated the reaction free energies for the step-wise generation of neutral or negatively charged dimer, trimer, tetramer, and pentamer from $\mathrm{BOB}^{2-}$ and diglyme (Figure 9.4b). These calculations reveal that the formation of neutral or negatively charged trimer and higher aggregates is thermodynamically unfavorable. The formation of anionic and neutral forms of trimer from the dimer is endothermic by $1-4 \mathrm{eV}$, whereas the generation of higher order coupling products is highly unfavorable $(\Delta \mathrm{G}>10 \mathrm{eV})$. At higher voltages, the trimers could still form, however it is very unlikely that further polymerization will occur. The higher oligomers with multiple charges may not be stable as they would readily dissociate to smaller charged dimers or trimers. We theoretically calculate the redox potentials of glyme molecule and its oligomers with BOB molecules, presented in Figure 9.4c using the computational methodology described in the Supplementary Information. It is seen that the glyme-BOB oligomers are charged and at the same time electrochemically stable even at high potentials. It can be argued that these initially generated oligomers would form a network at the cathode via strong non-covalent interactions; furnishing a charged supramolecular 

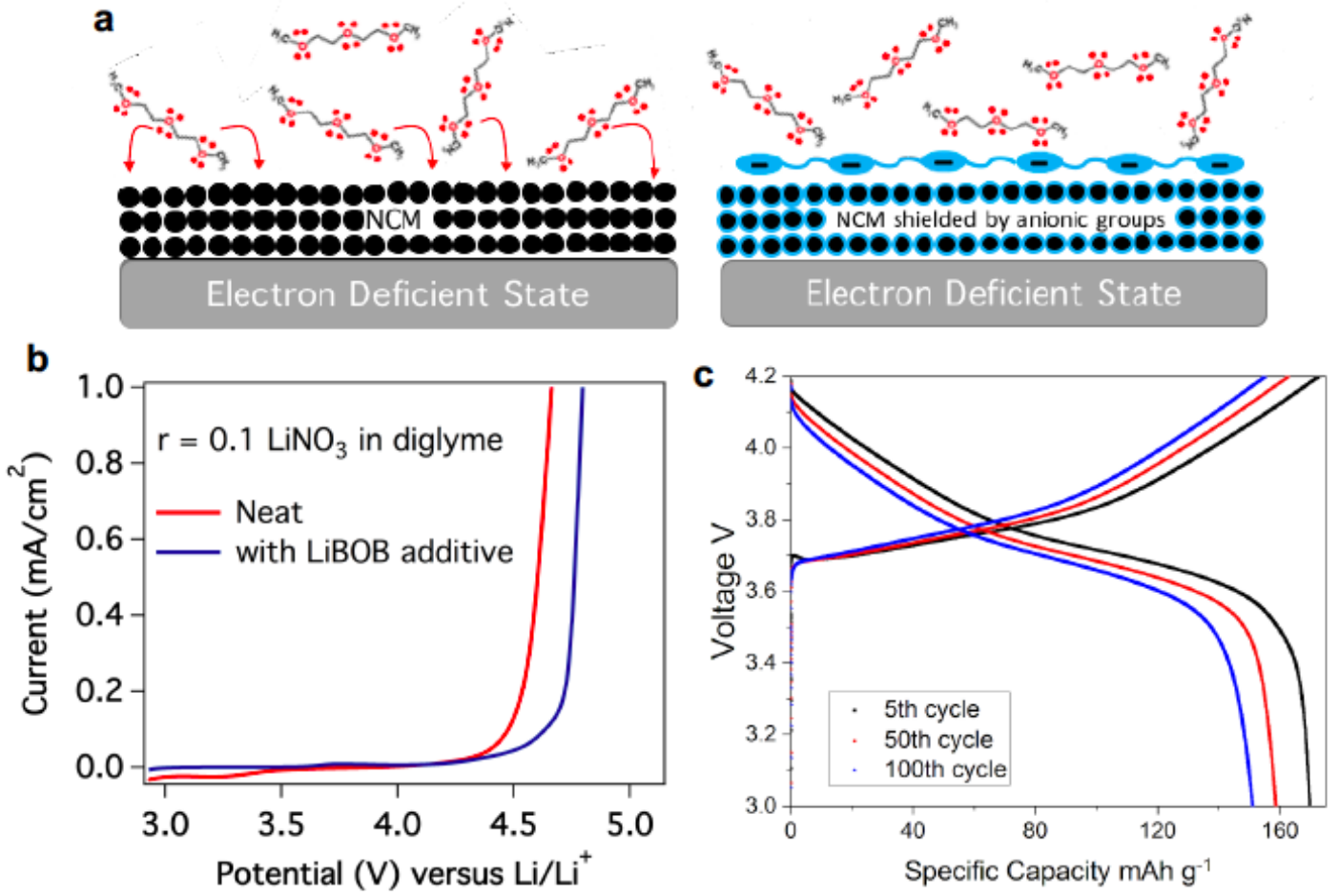

d

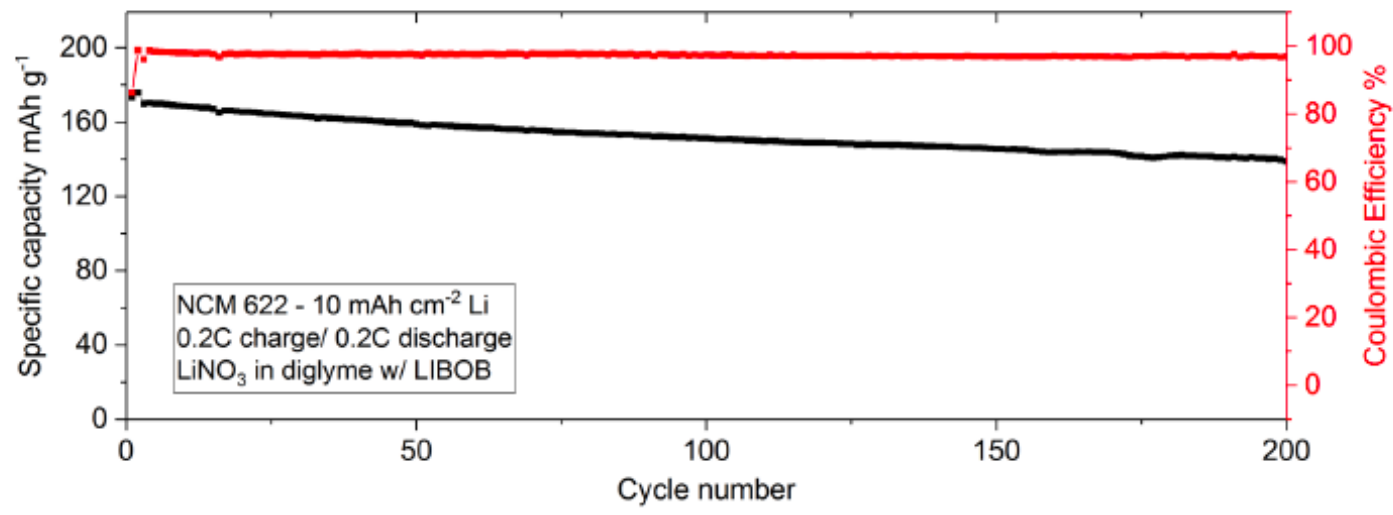

Figure 9.5: Enabling stable cycling of high voltage lithium battery: (a)

Schematic showing the proposed mechanism according to which the oxidation of glymes is inhibited by a layer of immobilized anions; (b) Poetential-current diagram obtained from linear scan voltammetry in a 3-electrode setup comprising of $\mathrm{Ag} / \mathrm{AgCl}$ reference electrode and stanless steel as working and counter-electrodes. The scan rate was $10 \mathrm{mV} / \mathrm{s}$. The electrolytes used here was diglyme- $\mathrm{LiNO}_{3}-\mathrm{HFiP}$, with (blue) and without (red) $0.4 \mathrm{M} \mathrm{LiBOB}$ salt additive. (c) Voltage profile for $5^{\text {th }}$, $50^{\text {th }}$ and $100^{\text {th }}$ cycles of Li $\mid$ NCM cycling using $0.4 \mathrm{M}$ LiBOB additive; (d) Discharge capacity retention and coulombic efficiency over 200 cycles in $\mathrm{Li} \| \mathrm{NCM}$ cell diglyme- $\mathrm{LiNO}_{3}-\mathrm{HFiP}$ electrolyte with $0.4 \mathrm{M}$ LiBOB additive. Here, the lithium used is $50 \mu \mathrm{m}$ thick, thus the anode to cathode capacity ratio is 5 . 
assembly (as shown in the schematic of Figure 9.5a. This might be the reason for the prevention of further oxidation of diglyme at the cathode.

To experimentally interrogate the cathode-electrolyte interphase (CEI) formed in the presence of a $\mathrm{LiBOB}$ salt, we cycled a $\mathrm{Li}|| \mathrm{NMC}$ battery with LiBOB additive in diglyme- $\mathrm{LiNO}_{3}-\mathrm{HFiP}$ (base) electrolyte twice at $\mathrm{C} / 10$ and extracted the NMC cathode for FTIR analysis and compared it with the computational IR-spectra of the predicted oligomeric species, as shown in Figure 9.4d. We find that there is a good agreement between the peak locations of the simulations and experiments. The differences in the relative intensities can be ascertained to the presence of additional species in the experiment as the electrolyte comprise of additional components (salt, additive, surface impurities) hat are not considered in the DFT calculations. The verification of our hypothesis that LiBOB additive in diglyme based electrolyte enhances the oxidation potential was done using 3-electrode linear scan voltammetry and electrochemical floating point test reported in Figure 9.5b and Supplementary Figure 9.16, respectively. It is seen that the degradation potential of diglyme electrolyte is enhanced by $\sim 0.3 \mathrm{~V}$ when $\mathrm{LiBOB}$ was used as additive (Figure 9.5b). Since, the measurement was done in a 3-electrode cell with stainless steel as working electrode, our argument that the LiBOB in-situ forms the CEI in the liquid-phase is further strengthened. The floating experiments with and without LiBOB additive was done using the same protocol as mentioned for the Lithion case, where we charged a Li||NMC battery at varying voltages for 24 hours each. The results (Supplementary Figure 9.16) show that the leak current for the cells containing LiBOB additive is lower than the control cells at all voltages. We also characterized the surface of 
lithium metal anode using XPS after two initial cycles of charge and discharge at $\mathrm{C} / 10$ rate (reported and discussed in Supplementary Figure 9.17).

Finally, we cycle a lithium metal battery with the NMC cathode and a thin lithium anode $(50 \mu \mathrm{m})$ such that the anode to cathode capacity ratio is $5: 1$ and using the base electrolyte (diglyme-LiNO $3-\mathrm{HFiP}$ ) and $\mathrm{LiBOB}$ as additive. The voltage profiles for the $5^{\text {th }}, 50^{\text {th }}$ and $100^{\text {th }}$ cycles are shown in Figure $9.5 \mathrm{c}$ and the cycle life in Figure $9.5 \mathrm{~d}$. It is seen that coulombic efficiency of the cells is high (>98\%) and that the discharge capacity is retained to more than $80 \%$ for at least 200 cycles at a rate of $\mathrm{C} / 5$. A similar performance is also observed at a relatively higher rate of discharge $(\mathrm{C} / 2)$ reported in Supplementary Figure 9.18. It is remarkable that by rational design of the cathodic interphases, we have been successful in enabling stable operation of glyme electrolytes in high voltage batteries for several hundreds of cycles, while they spontaneously fail without the interfacial modification. The concept of high voltage stabilization using anionic interfaces is not limited in oligomeric liquid electrolytes but also in other classes of polymer electrolytes including gels and crosslinked nanocomposites. We further designed gel electrolytes comprising of 1wt.\% PEG (100k) in diglyme electrolyte (image shown in Supplementary Figure 9.19) and operated Li||NMC cells with and without LiBOB salt additive at ambient conditions with a rate of C/5 as shown in Supplementary Figure 9.20. We find that unlike control liquid electrolyte, the gel electrolyte is able to show charge-discharge profiles even without LiBOB for first few cycles. However, beyond 20 cycles, there is sharp drop in the capacity followed by a noisy charge profile due to the breakdown of the gel electrolyte at high voltages similar to its liquid counterparts. The limited stabilization 
of the control gel electrolytes can be attributed to transport barrier of the viscous components preventing spontaneous breakdown in the initial cycles. In contrast, it is found that LiBOB additive in the gel electrolyte enables stable cycling and high capacity retention for at least 100 cycles. We also demonstrate the stable cycling behavior of a Li $\|$ NMC cell in Supplementary Figure 9.21 using a recently reported crosslinked nanoparticle membrane $\mathrm{e}^{39,40}$ as solid-electrolyte by infusing the base diglyme electrolyte with LiBOB additive.

In conclusion, we have shown that cationic chain-transfer agents can be used to terminate anionic polymerization of ether-/glyme-based electrolytes at a lithium metal electrode, producing self-limiting interfaces, high Coulombic efficiency, and extend the lifetime of the anode (to over 4000 hours) in asymmetric lithium||stainless steel cells. Building on these observations, we show that a longstanding barrier to deployment of glyme electrolytes can be removed using either ex- or in-situ generated interphases in the cathode that limit transport and reduce reactivity of active polymer centers by what we hypothesize to be an electrostatic shielding mechanism. Specifically, we show that a cathode electrolyte interphase (CEI) that hosts immobilized anions tethered to a polymeric backbone can act as a barrier for the oxidation reaction. Extending this concept to create an in-situ generated interphase composed of anionic polymer aggregates at the cathode result in significantly enhanced lifetime of a high voltage lithium metal battery. We believe, this work opens a new pathway for conventional, solid-state, and flexible lithium metal batteries based on ether and polyether-based electrolytes. 


\subsection{Methods}

\subsubsection{Computational details}

All structures are optimized in the gas-phase using wB97X-D ${ }^{41,42}$ functional and 6$311 \mathrm{G}(\mathrm{d}, \mathrm{p})^{43}$ basis sets implemented in the Gaussian suite of programs. ${ }^{44}$ Vibrational frequencies are calculated at the same level of theory to ensure that the optimized geometry represents a true minimum; i.e, no negative frequencies are found. Further, single point calculations are performed on these structures by employing a polarizable continuum model (PCM) to mimic the effects of diglyme. ${ }^{45} \mathrm{We}$ used a dielectric constant of 7.23 for diglyme. A value of $1.63 \mathrm{eV}$ is assumed for the electron solvation free energy. ${ }^{46}$

\subsubsection{Experimental details}

The detailed description of the synthesis procedure is provided in the Supplementary Information. 


\section{REFERENCES}

1. Qian, J. et al. High rate and stable cycling of lithium metal anode. Nat.

Commun. 6, 6362 (2015).

2. Cheng, X.-B., Zhang, R., Zhao, C.-Z. \& Zhang, Q. Toward Safe Lithium Metal Anode in Rechargeable Batteries: A Review. Chem. Rev. 117, 10403-10473 (2017).

3. Cheng, X. et al. A Review of Solid Electrolyte Interphases on Lithium Metal Anode. Adv. Sci. 3, 1-20 (2016).

4. Tikekar, M. D., Choudhury, S., Tu, Z. \& Archer, L. A. Design principles for electrolytes and interfaces for stable lithium-metal batteries. Nat. Energy 1, $16114(2016)$.

5. Wei, S., Choudhury, S., Tu, Z., Zhang, K. \& Archer, L. A. Electrochemical Interphases for High-Energy Storage Using Reactive Metal Anodes. Acc. Chem. Res. (2017). doi:10.1021/acs.accounts.7b00484

6. Lin, D., Liu, Y. \& Cui, Y. Reviving the lithium metal anode for high-energy batteries. Nat. Nanotechnol. 12, 194-206 (2017).

7. Long, L., Wang, S., Xiao, M. \& Meng, Y. Polymer electrolytes for lithium polymer batteries. J. Mater. Chem. A 4, 10038-10069 (2016).

8. Porcarelli, L., Gerbaldi, C., Bella, F. \& Nair, J. R. Super Soft All-Ethylene Oxide Polymer Electrolyte for Safe All-Solid Lithium Batteries. Sci. Rep. 6, 19892 (2016). 
9. Khurana, R., Schaefer, J. L., Archer, L. A. \& Coates, G. W. Suppression of lithium dendrite growth using cross-linked polyethylene/poly(ethylene oxide) electrolytes: a new approach for practical lithium-metal polymer batteries. $J$. Am. Chem. Soc. 136, 7395-7402 (2014).

10. Bouchet, R. et al. Single-ion BAB triblock copolymers as efficient electrolytes for lithium-metal batteries. Nat. Mater. 12, 452-457 (2013).

11. Agrawal, A., Choudhury, S. \& Archer, L. a. A highly conductive, nonflammable polymer-nanoparticle hybrid electrolyte. $R S C A d v . \mathbf{5 ,} 20800-20809$ (2015).

12. Srivastava, S., Schaefer, J. L., Yang, Z., Tu, Z. \& Archer, L. A. 25Th Anniversary Article: Polymer-Particle Composites: Phase Stability and Applications in Electrochemical Energy Storage. Adv. Mater. 26, 201-34 (2014).

13. Rojas, A. A. et al. Effect of Lithium-Ion Concentration on Morphology and Ion Transport in Single-Ion-Conducting Block Copolymer Electrolytes.

Macromolecules 48, 6589-6595 (2015).

14. Chintapalli, M. et al. Effect of Grain Size on the Ionic Conductivity of a Block Copolymer Electrolyte. Macromolecules 47, 5424-5431 (2014).

15. Schaefer, J. L., Yanga, D. A. \& Archer, L. A. High Lithium Transference Number Electrolytes via Creation of 3-Dimensional, Charged, Nanoporous Networks from Dense Functionalized Nanoparticle Composites. Chem. Mater. 25, 834-839 (2013).

16. Schaefer, J. L., Moganty, S. S., Yanga, D. a. \& Archer, L. a. Nanoporous hybrid 
electrolytes. J. Mater. Chem. 21, 10094 (2011).

17. (Youngman) Chusid, O., Ein Ely, E., Aurbach, D., Babai, M. \& Carmeli, Y. Electrochemical and spectroscopic studies of carbon electrodes in lithium battery electrolyte systems. J. Power Sources 43, 47-64 (1993).

18. Aurbach, D. Review of selected electrode-solution interactions which determine the performance of Li and Li ion batteries. J. Power Sources 89, 206-218 (2000).

19. Miao, R. et al. Novel dual-salts electrolyte solution for dendrite-free lithiummetal based rechargeable batteries with high cycle reversibility. J. Power Sources 271, 291-297 (2014).

20. Zhang, X., Cheng, X., Chen, X., Yan, C. \& Zhang, Q. Fluoroethylene Carbonate Additives to Render Uniform Li Deposits in Lithium Metal Batteries. Adv. Funct. Mater. 27, 1605989 (2017).

21. Choudhury, S. et al. Electroless Formation of Hybrid Lithium Anodes for Fast Interfacial Ion Transport. Angew. Chemie Int. Ed. 56, 13070-13077 (2017).

22. Choudhury, S. \& Archer, L. A. Lithium Fluoride Additives for Stable Cycling of Lithium Batteries at High Current Densities. Adv. Electron. Mater. 2, $1500246(2015)$.

23. Lu, Y., Tu, Z. \& Archer, L. A. Stable lithium electrodeposition in liquid and nanoporous solid electrolytes. Nat. Mater. 13, 961-969 (2014).

24. Zheng, G. et al. Interconnected hollow carbon nanospheres for stable lithium metal anodes. Nat. Nanotechnol. 9, 618-623 (2014).

25. Li, N.-W., Yin, Y.-X., Yang, C.-P. \& Guo, Y.-G. An Artificial Solid Electrolyte 
Interphase Layer for Stable Lithium Metal Anodes. Adv. Mater. 28, 1853-1858 (2016).

26. Tu, Z. et al. Fast ion transport at solid-solid interfaces in hybrid battery anodes. Nat. Energy (2018). doi:10.1038/s41560-018-0096-1

27. Suo, L. et al. Fluorine-donating electrolytes enable highly reversible 5-V-class Li metal batteries. Proc. Natl. Acad. Sci. (2018).

28. Stephan, a. M. Review on gel polymer electrolytes for lithium batteries. Eur. Polym. J. 42, 21-42 (2006).

29. Zhang, W. et al. Design Principles of Functional Polymer Separators for HighEnergy, Metal-Based Batteries. Small 1703001-n/a doi:10.1002/smll.201703001

30. Li, W. et al. The synergetic effect of lithium polysulfide and lithium nitrate to prevent lithium dendrite growth. Nat. Commun. 6, 7436 (2015).

31. Tan, S. et al. Tris(hexafluoro-iso-propyl)phosphate as an SEI-Forming Additive on Improving the Electrochemical Performance of the Li[Li0.2Mn0.56Ni0.16Co0.08]O2 Cathode Material. J. Electrochem. Soc. 160, A285-A292 (2013).

32. von Cresce, A. \& Xu, K. Electrolyte Additive in Support of 5 V Li Ion Chemistry. J. Electrochem. Soc. 158, A337-A342 (2011).

33. Verma, P., Maire, P. \& Novak, P. A review of the features and analyses of the solid electrolyte interphase in Li-ion batteries. Electrochim. Acta 55, 6332$6341(2010)$

34. Choudhury, S. \& Archer, L. A. Lithium Fluoride Additives for Stable Cycling 
of Lithium Batteries at High Current Densities. Adv. Electron. Mater. 1-6 (2015). doi:10.1002/aelm.201500246

35. Tu, Z. et al. Designing Artificial Solid-Electrolyte Interphases for Single-Ion and High-Efficiency Transport in Batteries. Joule (2017). doi:https://doi.org/10.1016/j.joule.2017.06.002

36. Amine, K. et al. Mechanism of capacity fade of $\mathrm{MCMB} / \mathrm{Li}_{1.1}\left[\mathrm{Ni}_{1 / 3} \mathrm{Mn}_{1 / 3} \mathrm{Co}_{1 / 3}\right]_{0.9} \mathrm{O}_{2}$ cell at elevated temperature and additives to improve its cycle life. J. Mater. Chem. 21, 17754-17759 (2011).

37. Xiao, A., Yang, L., Lucht, B. L., Kang, S.-H. \& Abraham, D. P. Examining the Solid Electrolyte Interphase on Binder-Free Graphite Electrodes. J.

Electrochem. Soc. 156, A318-A327 (2009).

38. Zhu, Y., Li, Y., Bettge, M. \& Abraham, D. P. Electrolyte additive combinations that enhance performance of high-capacity $\mathrm{Li}_{1.2} \mathrm{Ni}_{0.15} \mathrm{Mn}_{0.55} \mathrm{Co}_{0.1} \mathrm{O}_{2}-$ graphite cells. Electrochim. Acta 110, 191-199 (2013).

39. Choudhury, S., Mangal, R., Agrawal, A. \& Archer, L. A. A highly reversible room-temperature lithium metal battery based on crosslinked hairy nanoparticles. Nat. Commun. 6, 10101 (2015).

40. Choudhury, S. et al. Confining electrodeposition of metals in structured electrolytes. Proc. Natl. Acad. Sci. (2018).

41. Chai, J.-D. \& Head-Gordon, M. Long-range corrected hybrid density functionals with damped atom-atom dispersion corrections. Phys. Chem. Chem. Phys. 10, 6615-6620 (2008).

42. Chai, J.-D. \& Head-Gordon, M. Systematic optimization of long-range 
corrected hybrid density functionals. J. Chem. Phys. 128, 84106 (2008).

43. Hariharan, P. C. \& Pople, J. A. The influence of polarization functions on molecular orbital hydrogenation energies. Theor. Chim. Acta 28, 213-222 (1973).

44. M. J. Frisch, G. W. Trucks, H. B. Schlegel, G. E. Scuseria, M. A. Robb, J. R. Cheeseman, G. Scalmani, V. Barone, B. Mennucci, G. A. Petersson, H. Nakatsuji, M. Caricato, X. Li, H. P. Hratchian, A. F. Izmaylov, J. Bloino, G. Zheng, J. L. Sonnenberg, M. Had.

45. Tomasi, J., Mennucci, B. \& Cammi, R. Quantum Mechanical Continuum Solvation Models. Chem. Rev. 105, 2999-3094 (2005).

46. Zhan, C.-G. \& Dixon, D. A. The Nature and Absolute Hydration Free Energy of the Solvated Electron in Water. J. Phys. Chem. B 107, 4403-4417 (2003). 


\section{APPENDIX}

Supplementary Information for Chapter 9
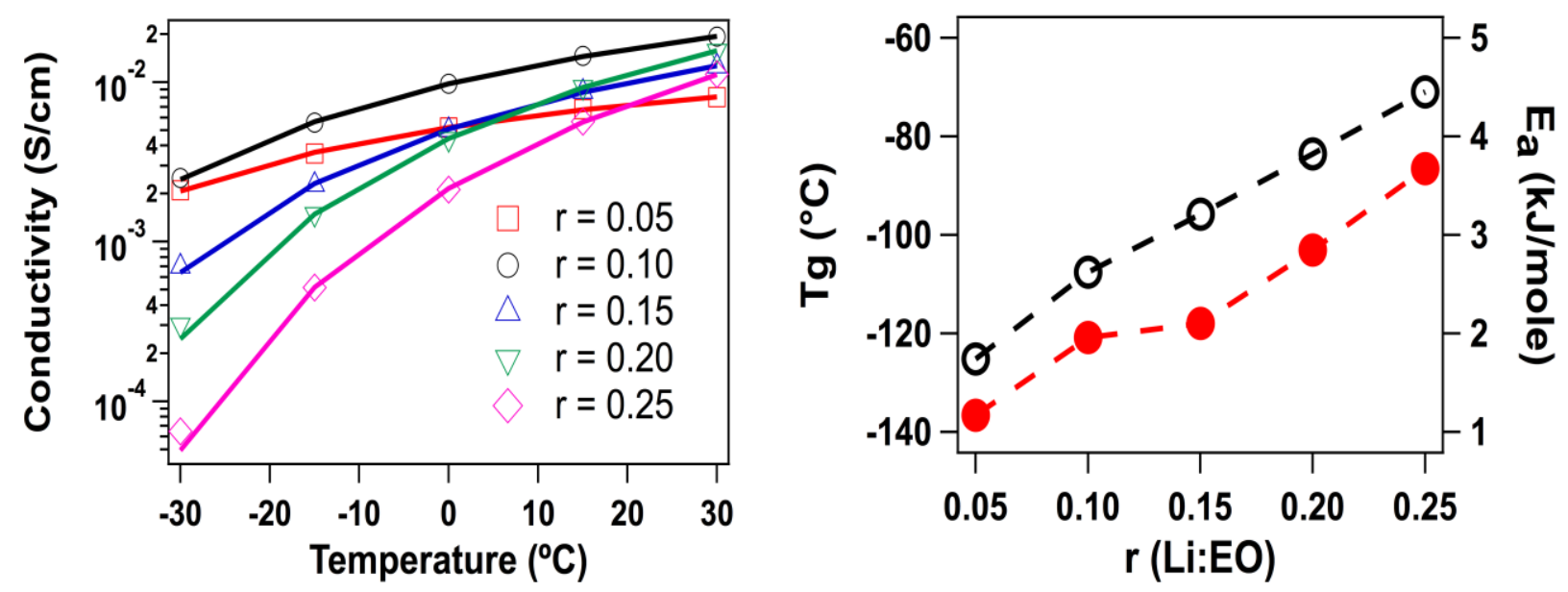

Supplementary Figure 9.1: (a) d.c. conductivity as a function of temperature for different Li:EO (r) ratios. The points represent experimental values and lines are the CFT fitting; (b) Variation in glass transition temperature obtained from Differential Scanning Calorimetry measurements in the left axis and Activation Energy obtained from the VFT fitting of conductivity data. 

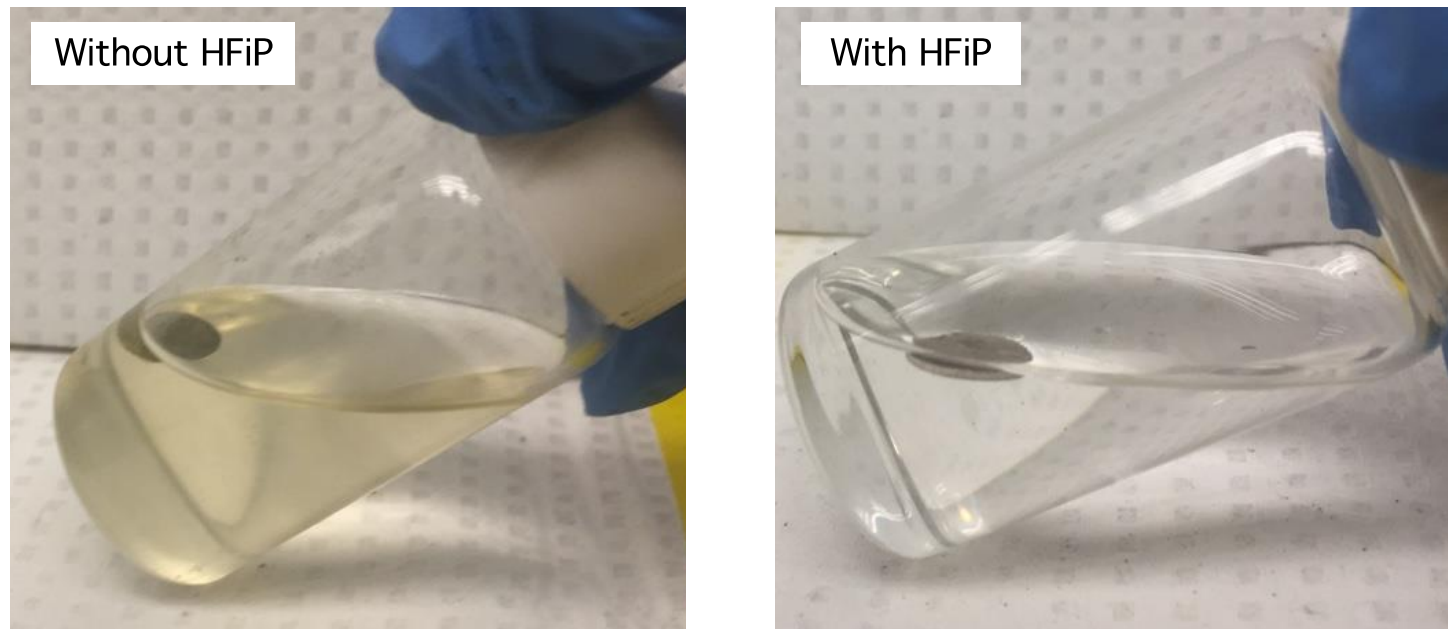

Supplementary Figure 9.2: A piece of lithium metal was added to the diglyme-LiNO electrolyte without 1wt.\% HFiP (left) and with 1wt.\% HFiP additive (right). The electrolyte solutions were aged for one month in a vial bottle. It is seen that the electrolyte without HFiP additive turns yellow and also the lithium surface becomes blackened, presumably, due to the polymerization of the glyme molecules 

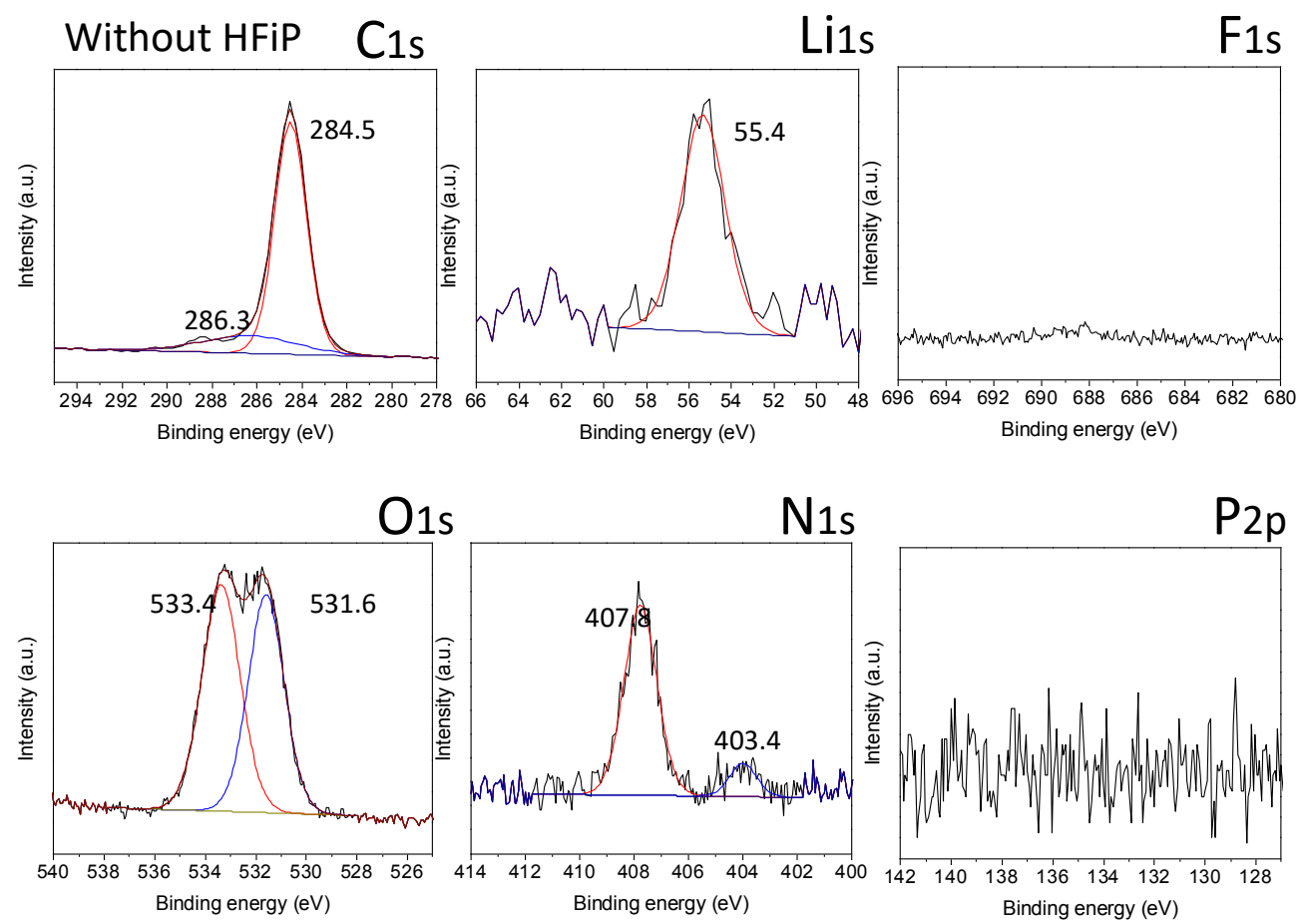

Supplementary Figure 9.3: Binding energies of different atoms on the surface of lithium obtained from X-ray Photoelectron Spectroscopy. The lithium metal was dipped in an electrolyte solution of diglyme- $\mathrm{LiNO}_{3}$ without any addition of HFIP 

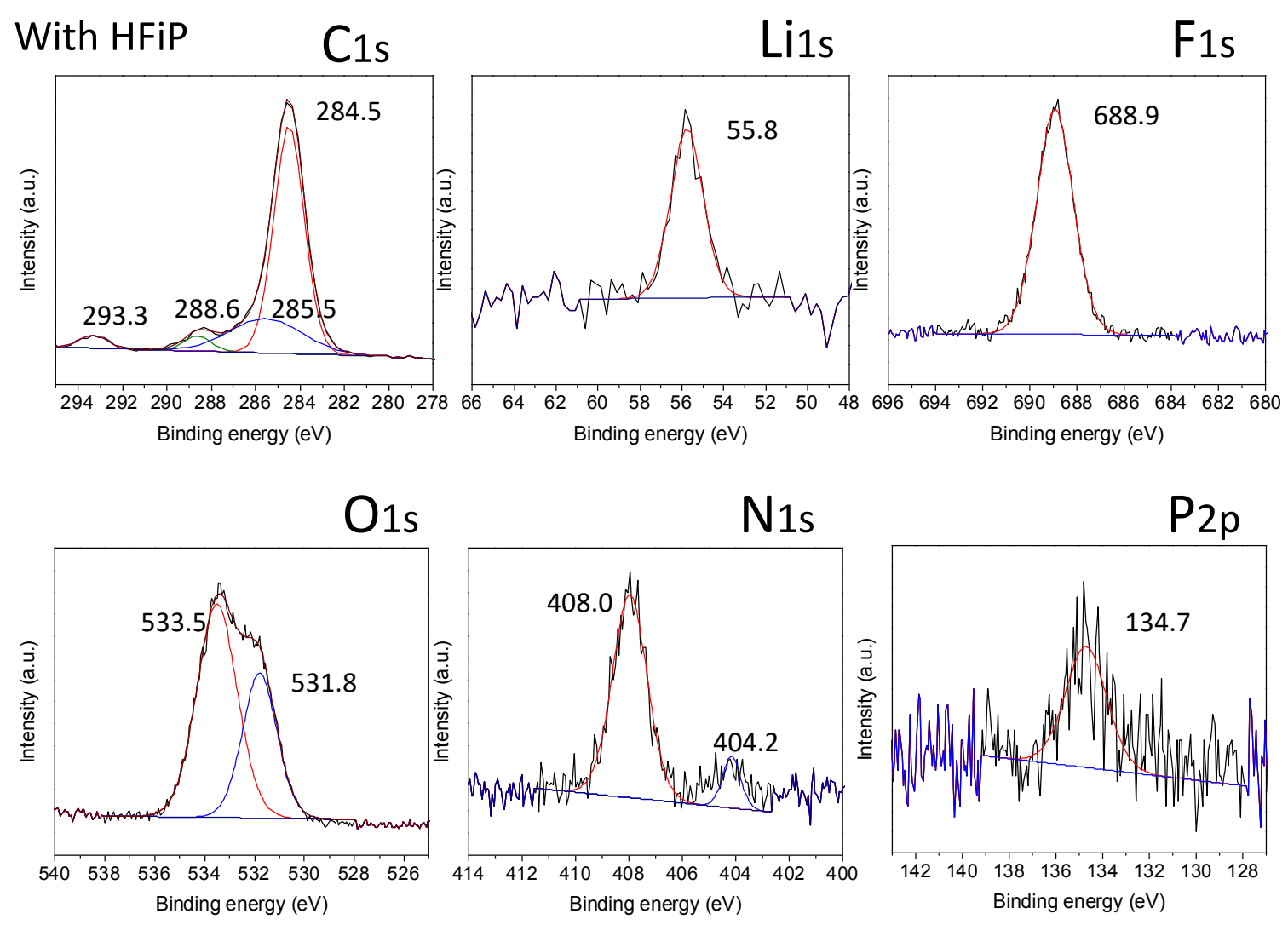

Supplementary Figure 9.4: Binding energies of different atoms on the surface of lithium obtained from X-ray Photoelectron Spectroscopy. The lithium metal was dipped in an electrolyte solution of diglyme- $\mathrm{LiNO}_{3}$ with $1 \mathrm{wt} . \%$ HFIP additive 


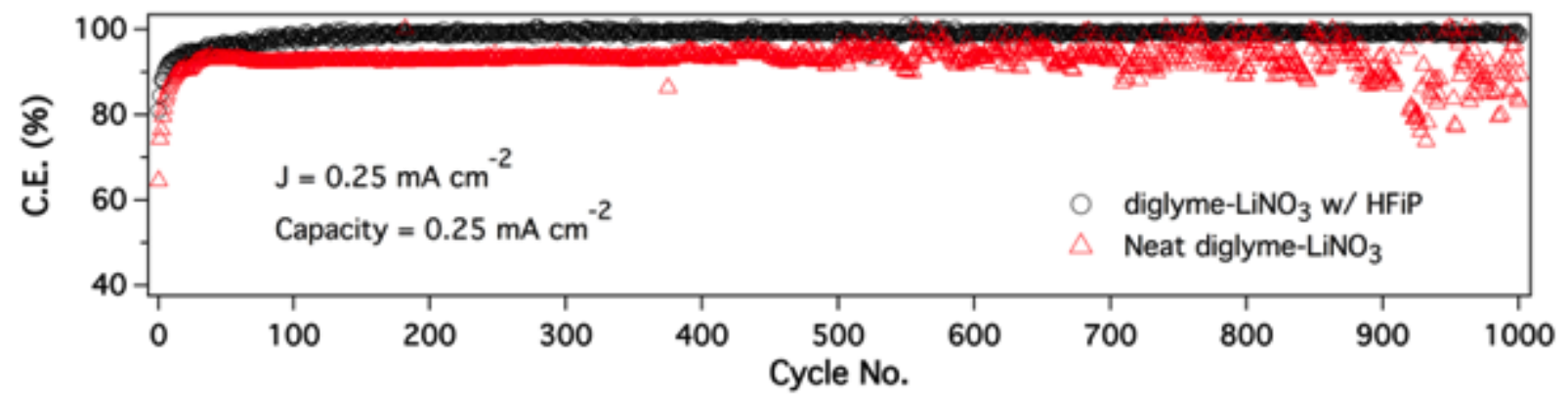

Supplementary Figure 9.5: Coulombic efficiency measurements in a Li||stainless steel battery at a current density of $0.25 \mathrm{~mA} / \mathrm{cm}^{2}$ and capacity of $0.25 \mathrm{mAh} / \mathrm{cm}^{2}$. The black circles represent the diglyme- $\mathrm{LiNO}_{3}$ electrolyte with the HFiP additive and red triangles are for neat electrolyte 

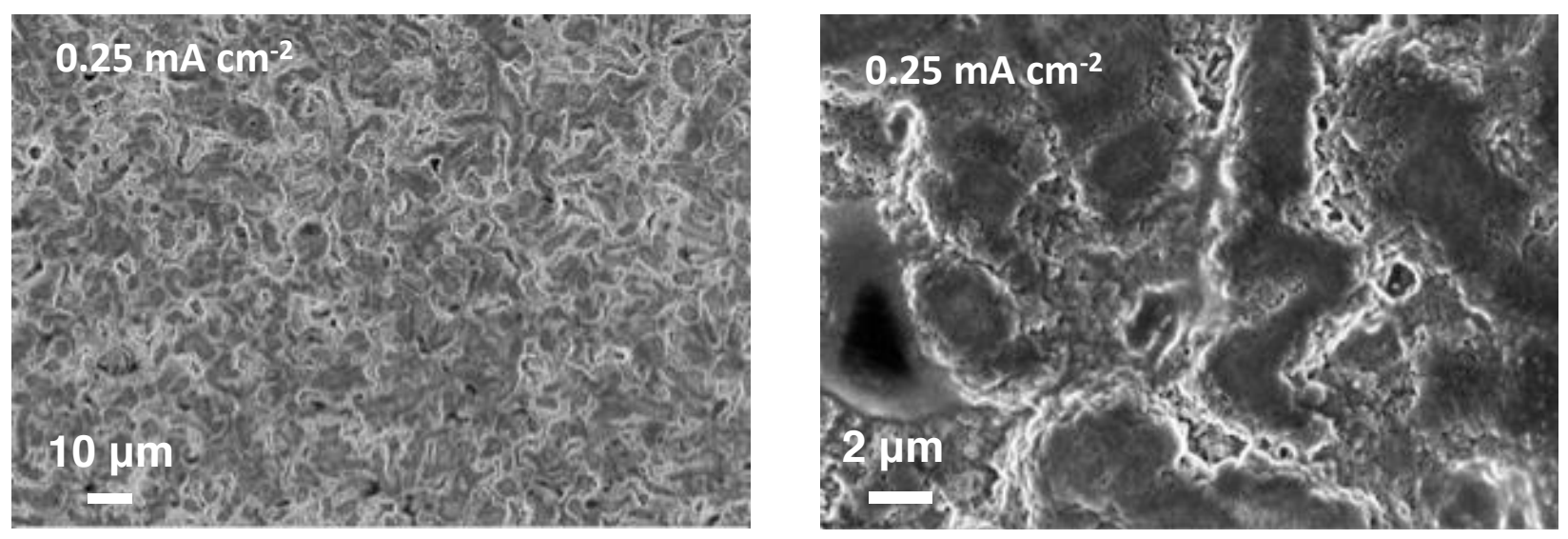

Supplementary Figure 9.6: Scanning electron microscopy image of stainless steel substrate after lithium deposition for 24 hours at the current density of $0.25 \mathrm{~mA} / \mathrm{cm}^{2}$, using the electrolyte diglyme- $\mathrm{LiNO}_{3}$ with 1 wt. $\%$ HFiP 


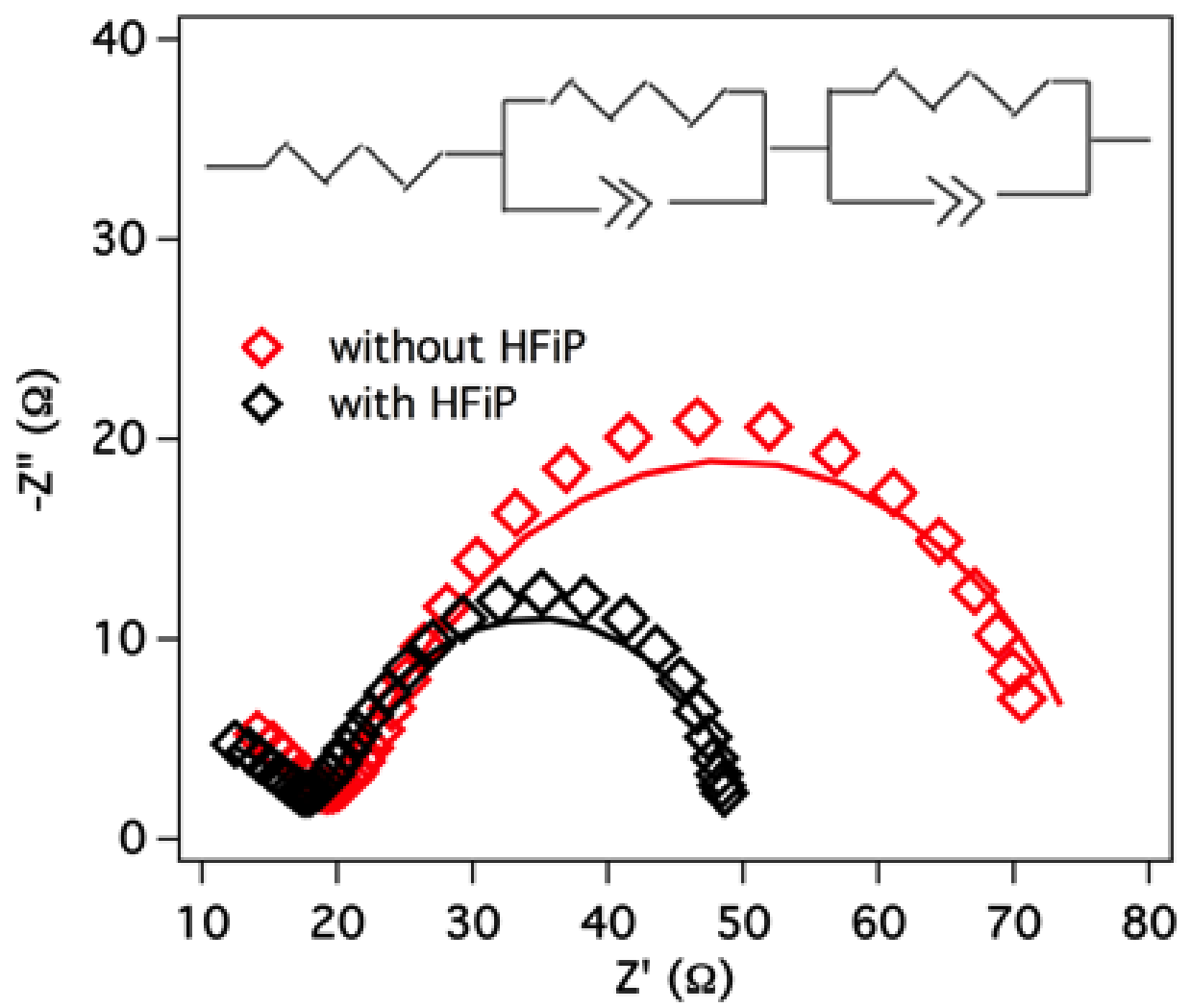

Supplementary Figure 9.7: Nyquist diagrams obtained from impedance spectroscopy for lithium vs. stainless steel cell that was cycled 100 times at a current density of $1 \mathrm{~mA} / \mathrm{cm}^{2}$ and capacity of $1 \mathrm{mAh} / \mathrm{cm}^{2}$ before depositing lithium onto the stainless steel electrode. The red symbols are for electrolyte of diglyme- $\mathrm{LiNO}_{3}$; while the black are for the same electrolyte with $1 \mathrm{wt} . \%$ HFiP additive. The inset shows the circuit model utilized to fit the data. 

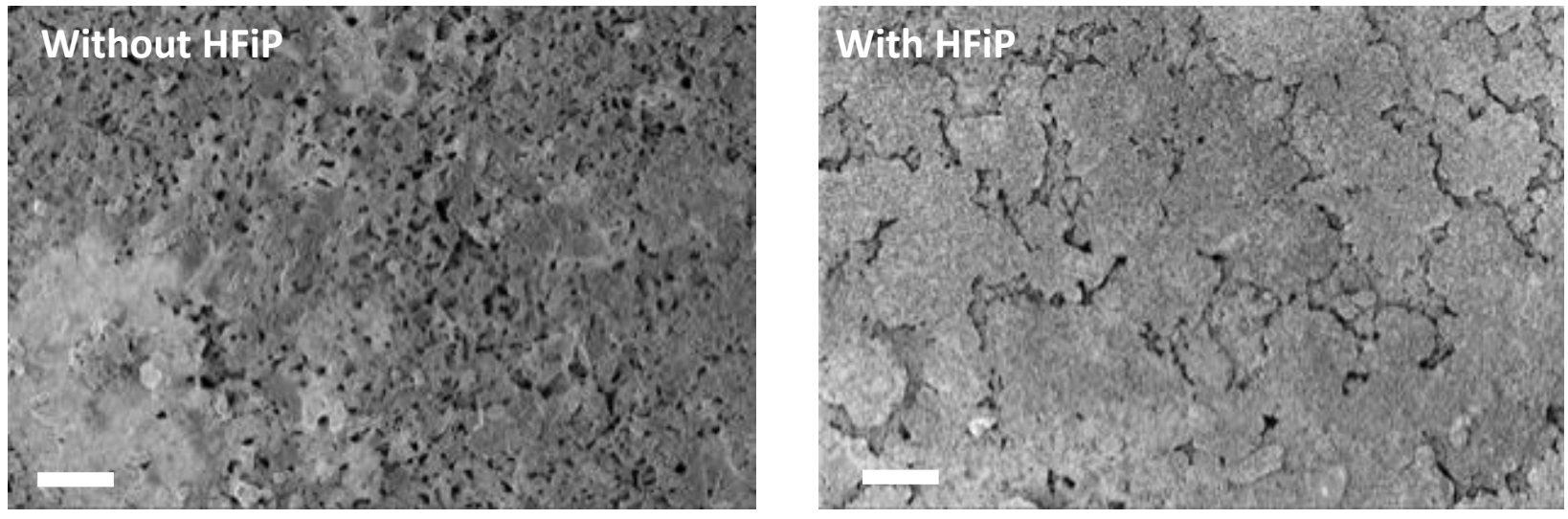

Supplementary Figure 9.8: Scanning electron microscopy image of stainless steel substrate after 100 cycles of plating and stripping lithium for 1 hour at the current density of 1 $\mathrm{mA} / \mathrm{cm}^{2}$. In the last step lithium metal was plated onto stainless steel electrode The left image is for the diglyme- $\mathrm{LiNO}_{3}$ electrolyte, without any additive and the right is for same electrolyte with 1wr.\% HFiP additive. The scale bar in both images represent $20 \mu \mathrm{m}$ 

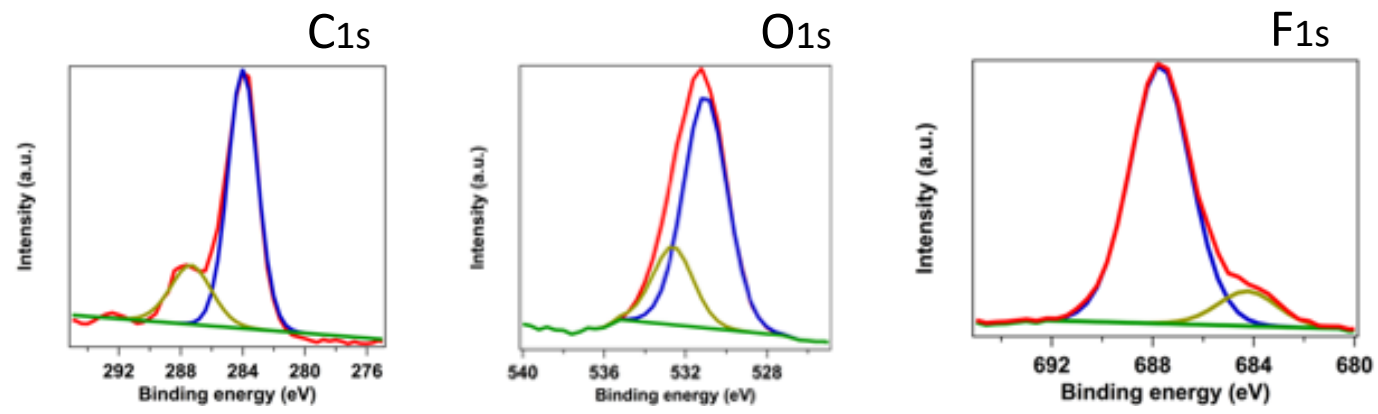

Supplementary Figure 9.9: Binding energies of different atoms obtained from X-ray Photoelectron Spectroscopy measurements for the lithium surface, extracted from a cell of after cycling 100 times at a current density of $1 \mathrm{~mA} / \mathrm{cm}^{2}$ and capacity of $1 \mathrm{mAh} / \mathrm{cm}^{2}$ in a cell with configuration of $\mathrm{Li}||$ stainless steel. electrolyte comprised of diglyme$\mathrm{LiNO}_{3}$ with 1 wt.\% HFiP additive. 

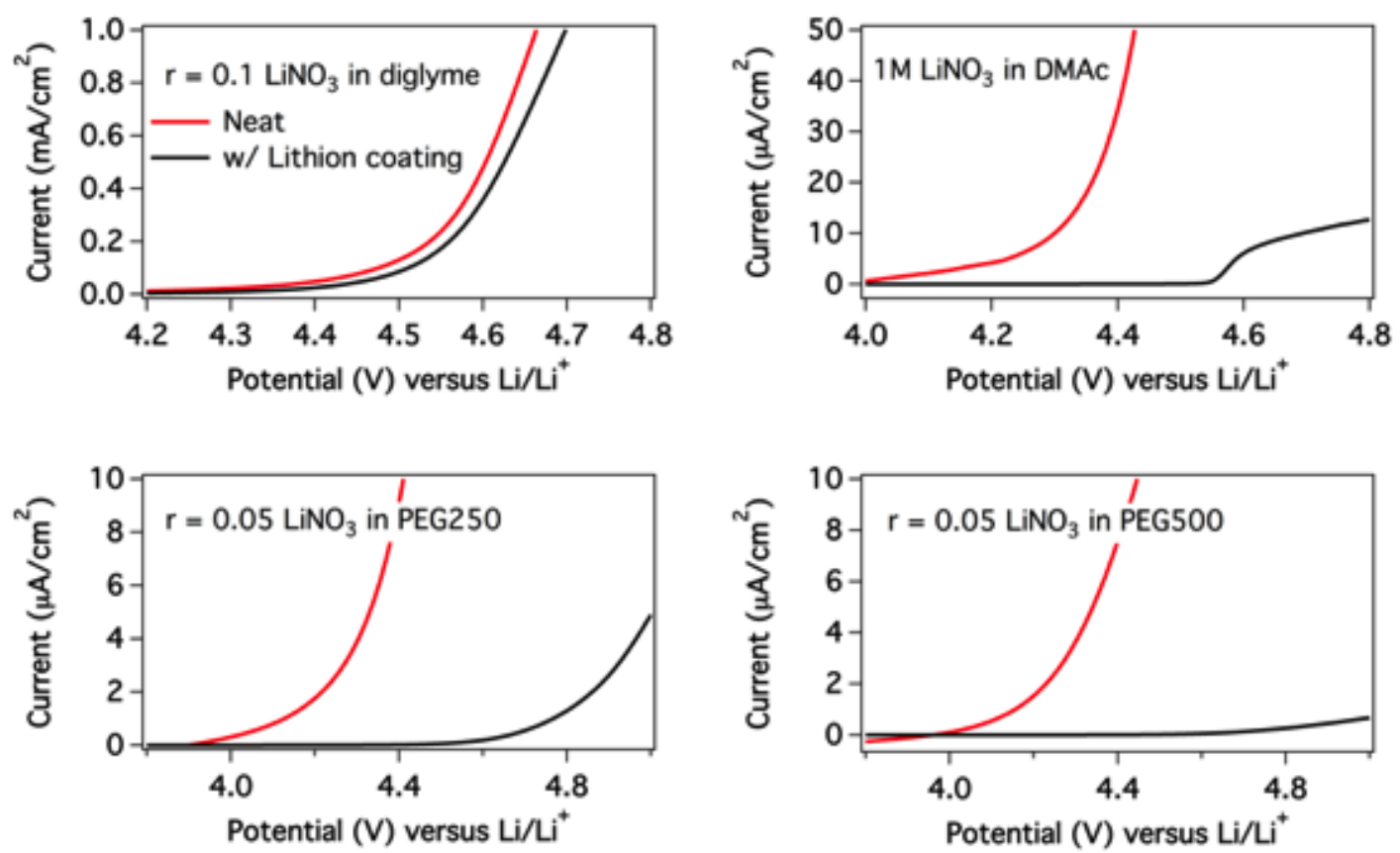

Supplementary Figure 9.10: Linear scan voltammetry in a three-electrode cell with $\mathrm{Ag} / \mathrm{AgCl}$ electrode as reference electrodes, while stainless steel used as both reference and counter electrodes. The scan rate utilized was $10 \mathrm{mV} / \mathrm{s}$ and the potentials were shifted with reference to $\mathrm{Li} / \mathrm{Li}^{+}$. The red curves represent cases where the stainless steel was coated with Lithion layer and black represent pristine stainless steel electrodes. 


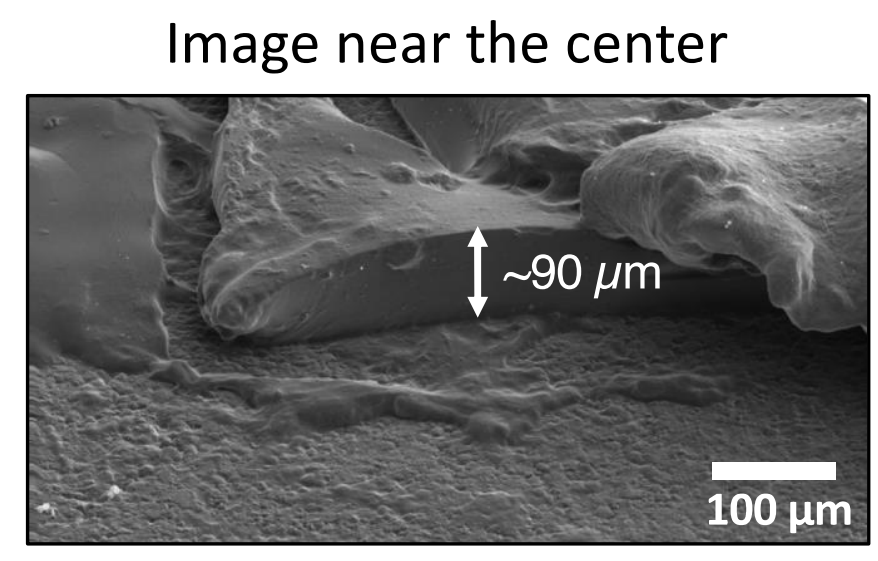

\section{Image near the edge}

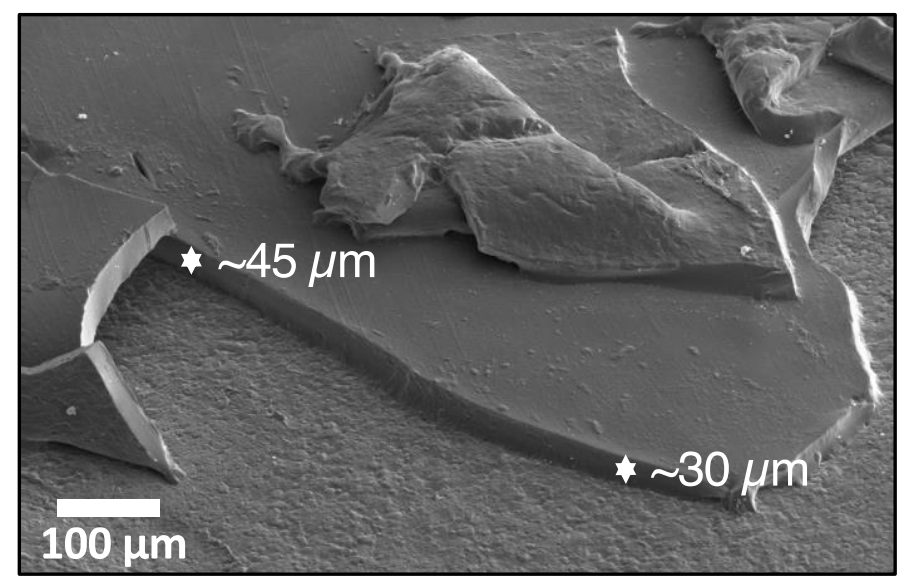

Supplementary Figure 9.11: SEM images of the lithion coated NCM surfaces at cryo-temperatures. A lithion layer was present on the NCM cathode (cracked during preparation). A thickness gradient was present, from $\sim 90 \mu \mathrm{m}$ thick near the center to $\sim 30 \mu \mathrm{m}$ near the edge. 

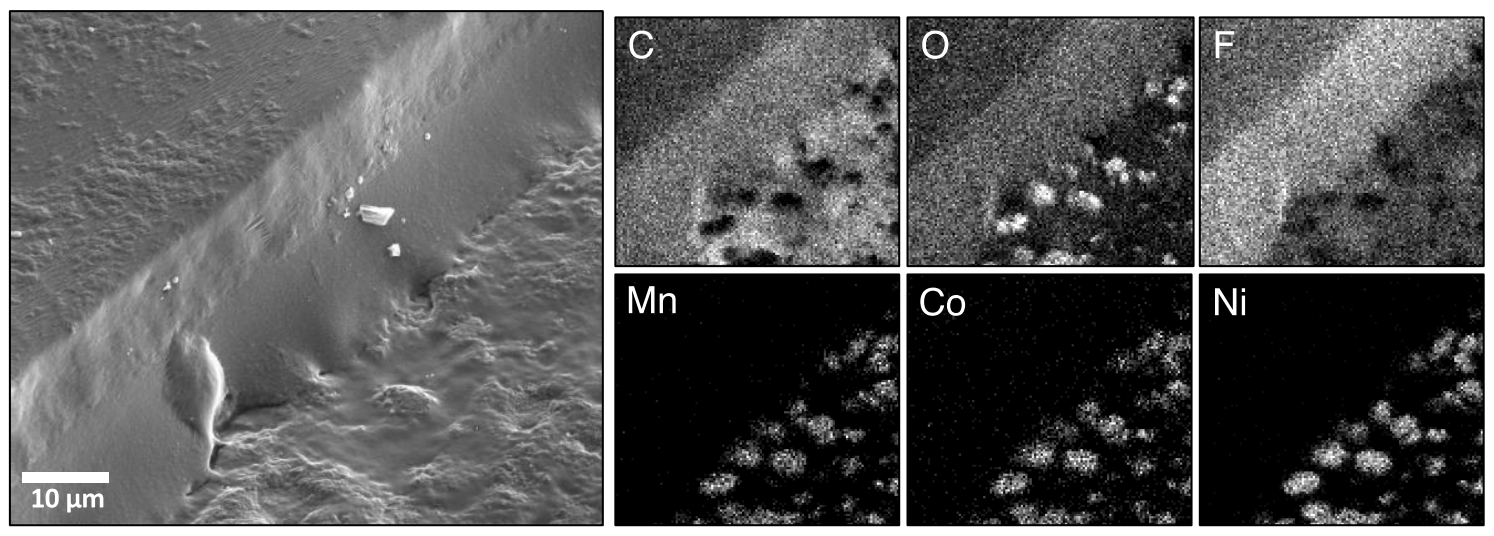

Supplementary Figure 9.12: EDX of the edge of the cracked lithion layer showed no nickel (or other metals) in the lithion. 


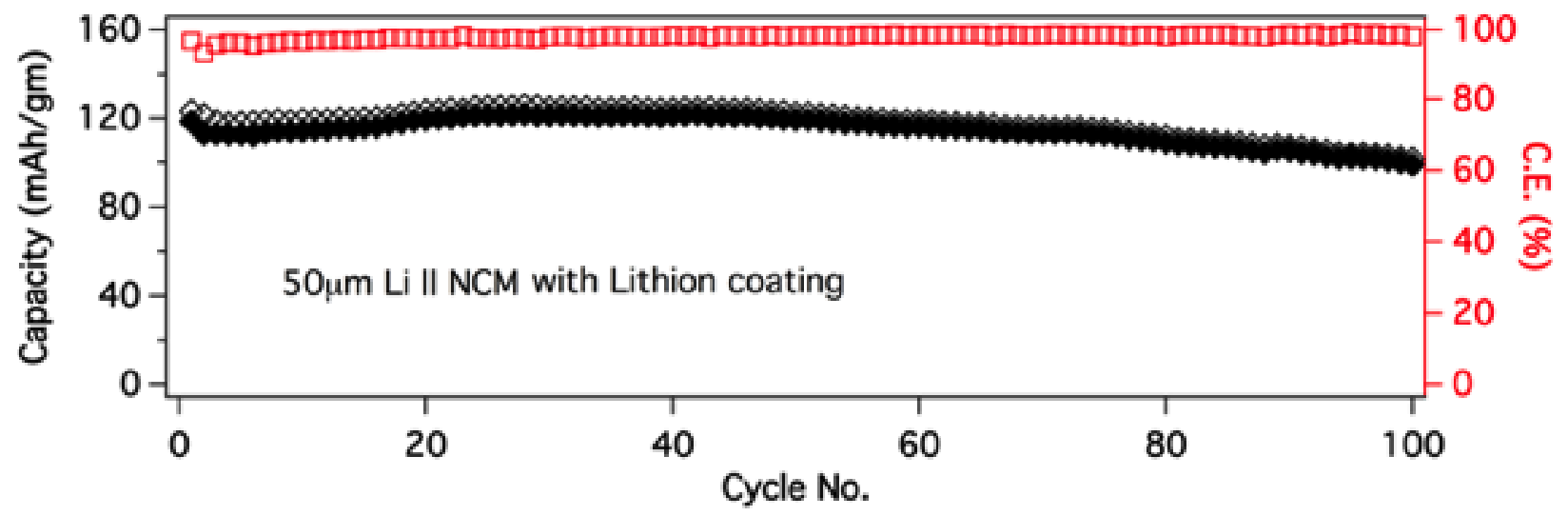

Supplementary Figure 9.13: Cycling of a thin lithium versus NCM battery, where the capacity of the lithium anode was $10 \mathrm{mAh} / \mathrm{cm}^{2}$ and that of cathode was $2 \mathrm{mAh} / \mathrm{cm}^{2}$. Here, the NCM cathode was coated with a layer of Lithion layer. The current density is $0.4 \mathrm{~mA} / \mathrm{cm}^{2}(\mathrm{C} / 5)$ 

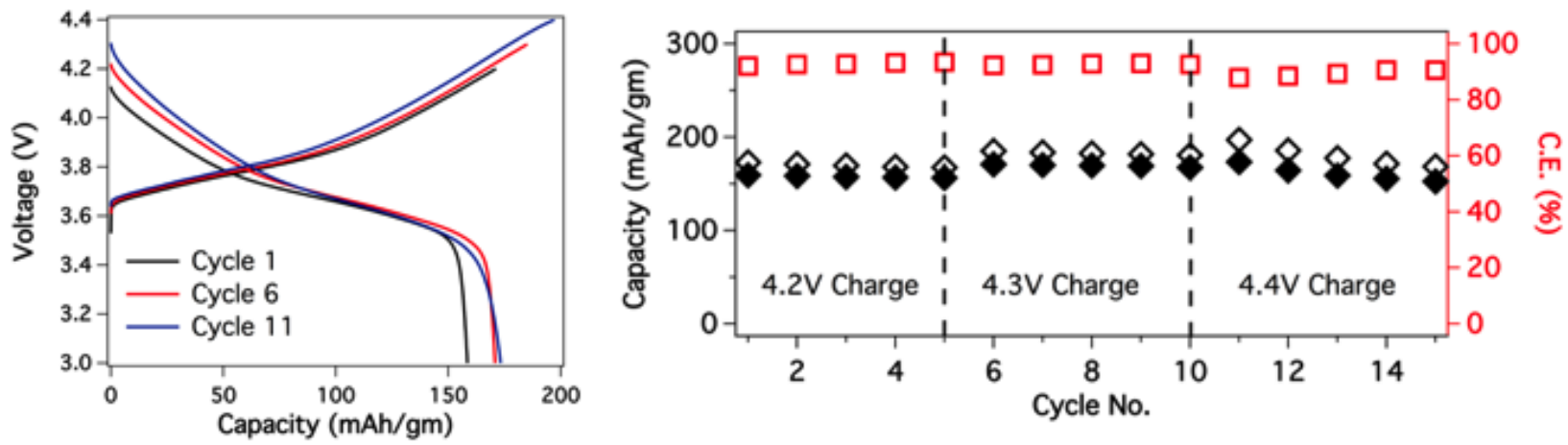

Supplementary Figure 9.14: Cycling of a thin lithium versus NCM battery, where the capacity of the lithium anode was $10 \mathrm{mAh} / \mathrm{cm}^{2}$ and that of cathode was $2 \mathrm{mAh} / \mathrm{cm}^{2}$. Here, the $\mathrm{NCM}$ cathode was coated with a layer of Lithion layer. The current density is $0.2 \mathrm{~mA} / \mathrm{cm}^{2}$ $(\mathrm{C} / 10)$ 


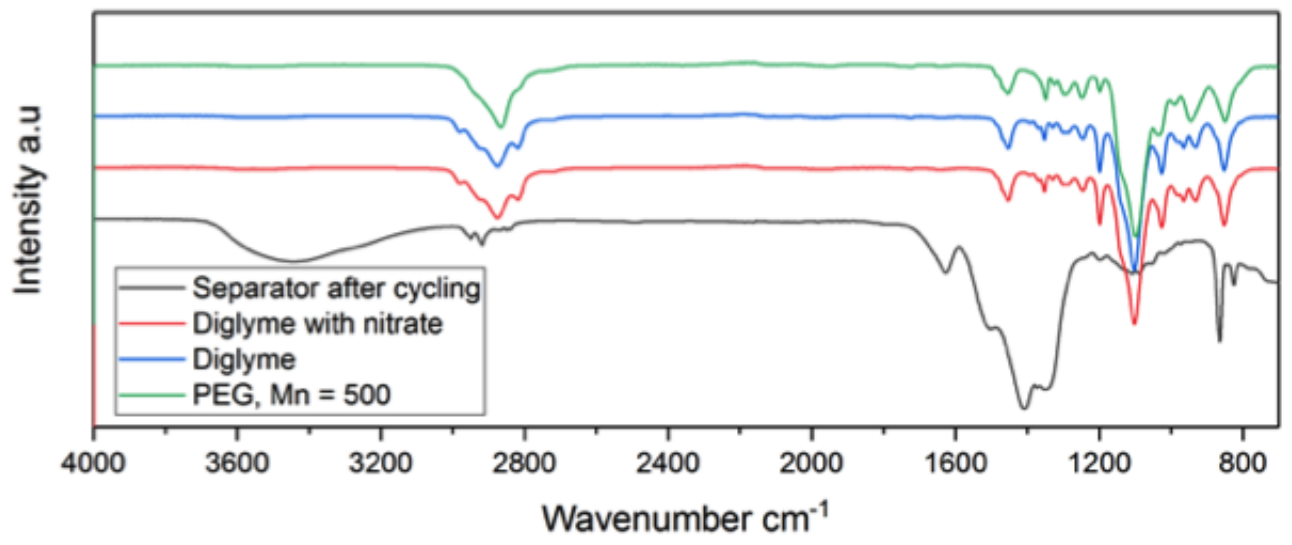

Supplementary Figure 9.15: FTIR Spectra for diglyme electrolyte infused separator extracted from a Li\|NCM cell without any Lithon coating, after it was cycled twice at $\mathrm{C} / 10$. It is compared with neat diglyme solvent, diglyme with $\mathrm{LiNO}_{3}$ and PEG500 liquid. 


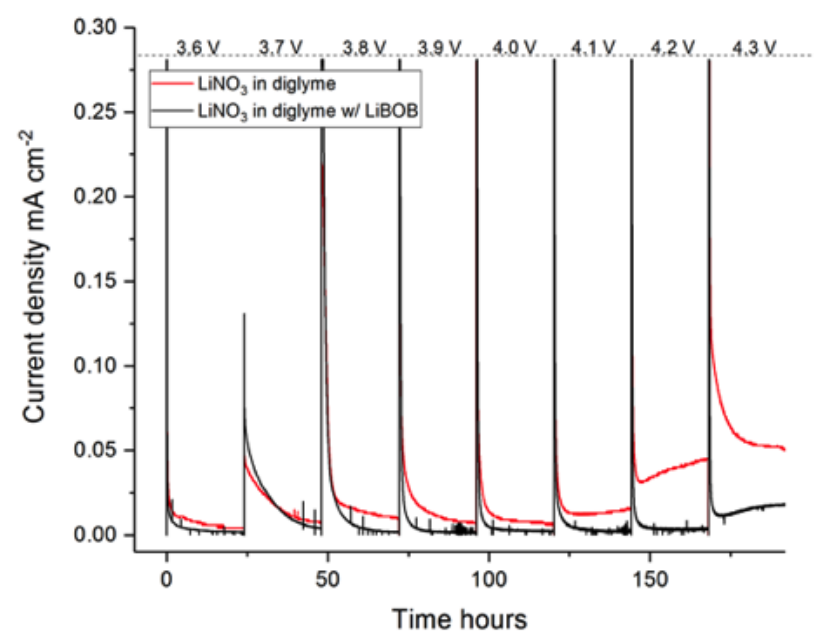

Supplementary Figure 9.16: Floating experiments using Li||NCM cell, where the cells are charged at different voltages for 24 hours each and the leak current is recorded to determine the side reactions at the operated voltages. The red line is for the diglyme- $\mathrm{LiNO}_{3}-\mathrm{HFiP}$ electrolyte, while the black line is result for the same electrolyte with $0.4 \mathrm{M} \mathrm{LiBOB}$ salt as additive 

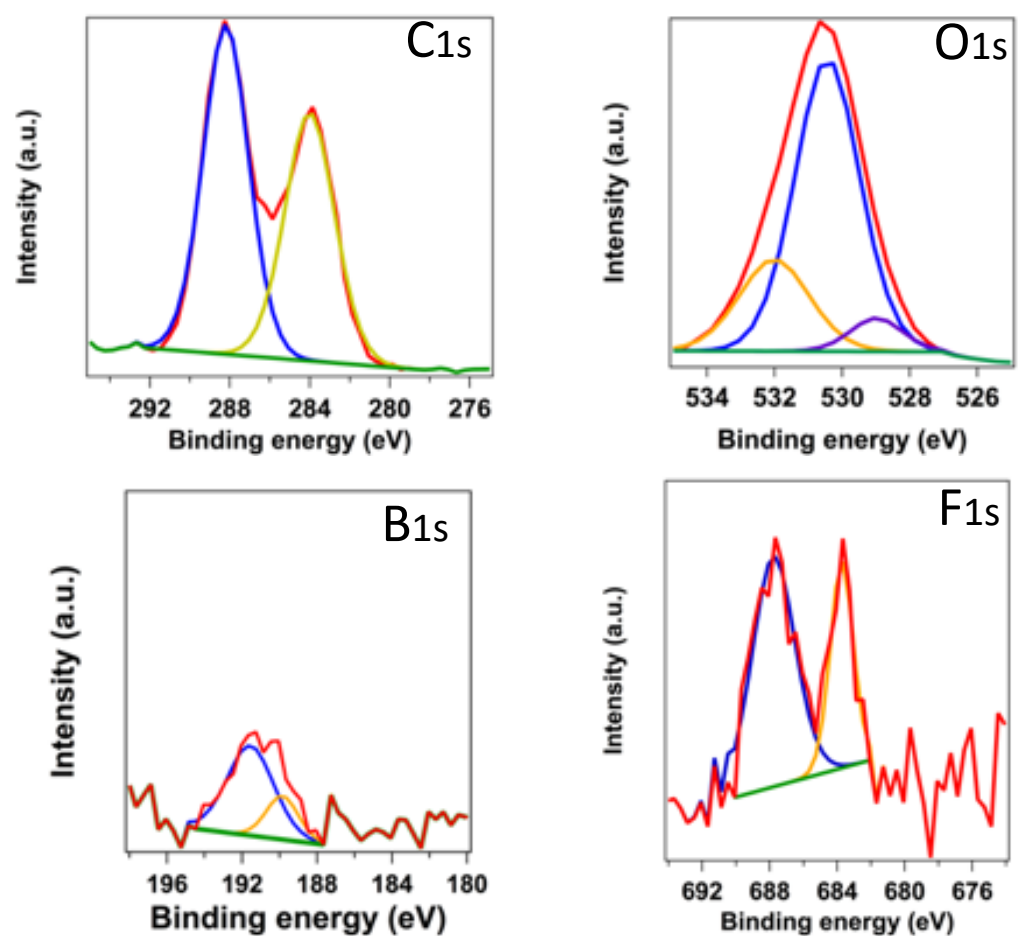

Supplementary Figure 9.17: XPS spectra from obtained from the surface of lithium metal anode harvested from a $\mathrm{Li} \| \mathrm{NCM}$ cell cycled twice at a $\mathrm{C} / 10$ rate using the electrolyte diglyme- $\mathrm{LiNO}_{3}$-HFiP with $0.4 \mathrm{M} \mathrm{LiBOB}$ additive. Here, the Fluorine spectra indicates that there is presence of both $\mathrm{LiF}$ and -CF3 content from the HFiP, while the LiBOB plays a role of forming boro-oxolate compounds in the anodic interfacial layer due to low potential reduction. 


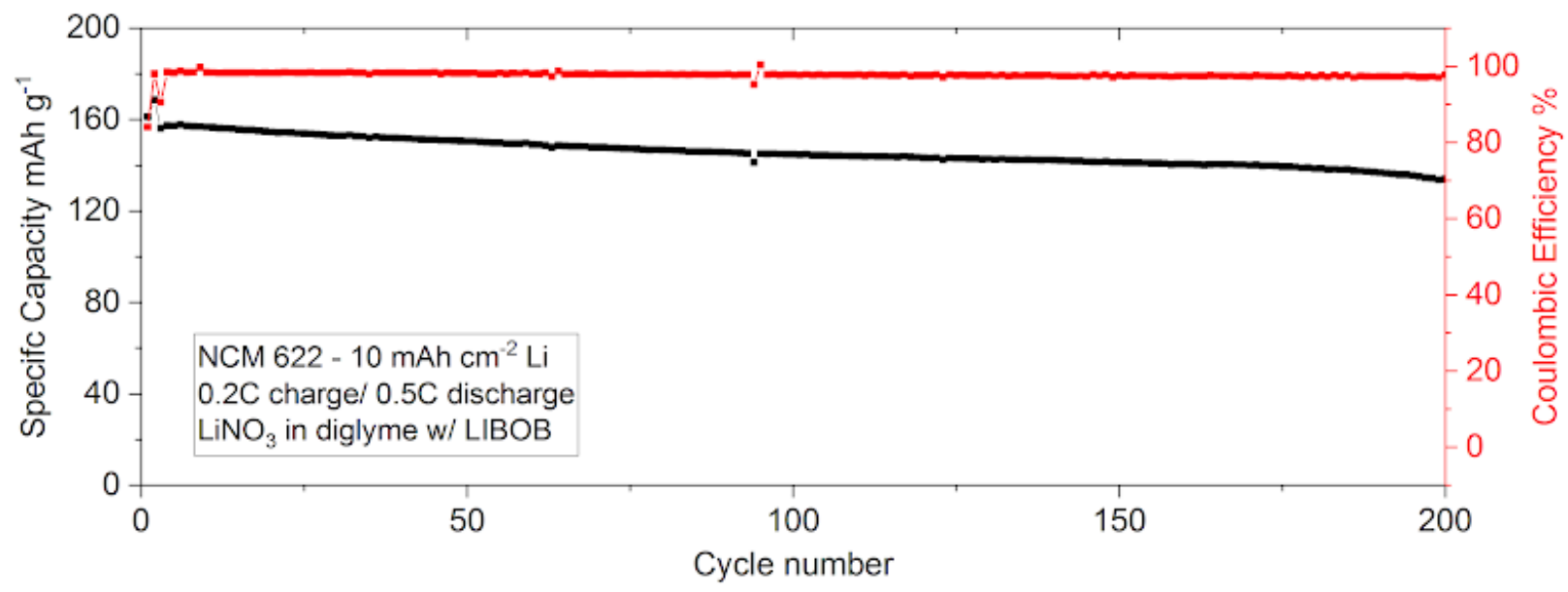

Supplementary Figure 9.18: $\mathrm{Li}|| \mathrm{NCM}$ cycling results using a rate of $0.2 \mathrm{C}$ charge and $0.5 \mathrm{C}$ discharge. The cathode loading is $2 \mathrm{mAh} / \mathrm{cm}^{2}$ and the lithium metal anode is $50 \mu \mathrm{m}$ thick that corresponds to $10 \mathrm{mAh} / \mathrm{cm}^{2}$ capacity. The electrolyte used here is diglyme$\mathrm{LiNO}_{3}$-HFiP with $0.4 \mathrm{M} \mathrm{LiBOB}$ salt additive. 


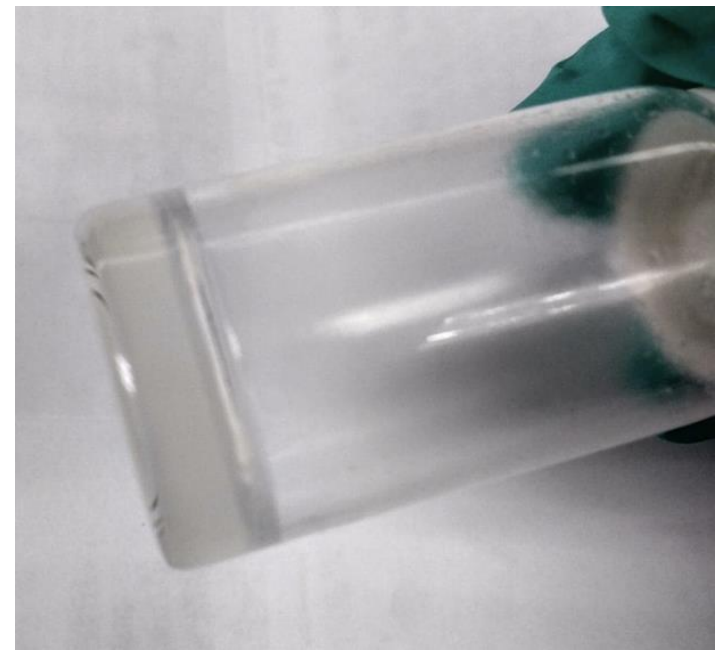

Supplementary Figure 9.19: Image showing the gel electrolyte used for cycling at room temperature. The composition is $1 \mathrm{wt} . \%$ 100k PEG in diglyme with $\mathrm{LiNO}_{3}$ and HFiP. 

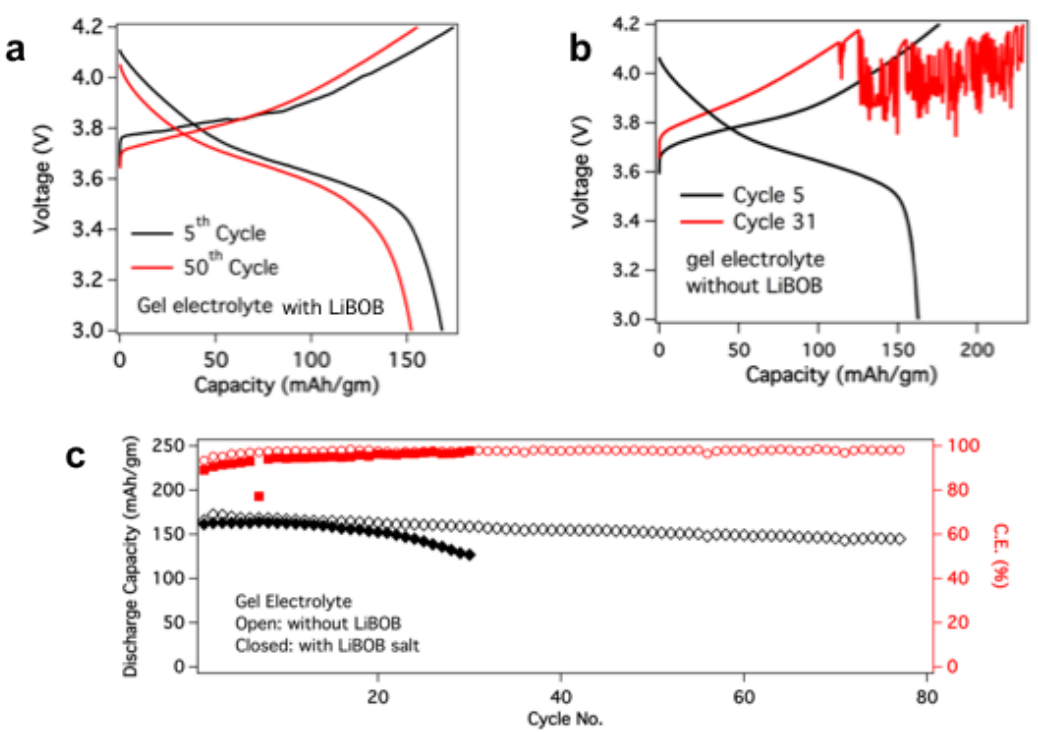

Supplementary Figure 9.20: Voltage profile of $\mathrm{Li} \| \mathrm{NCM}$ cell with a thin $(50 \mu \mathrm{m})$ lithium and $2 \mathrm{mAh} / \mathrm{cm}^{2}$ cathode cycled at $\mathrm{C} / 5$ rate using the gel electrolyte comprising of $1 \mathrm{wt} . \%$ PEG 100k in diglyme-LiNO 3 -HFiP, (a) with LiBOB salt additive and (b) without LiBOB. (c) Cycling performance of the gel electrolyte with (filled symbols) and without LiBOB salt (unfilled symbols). 


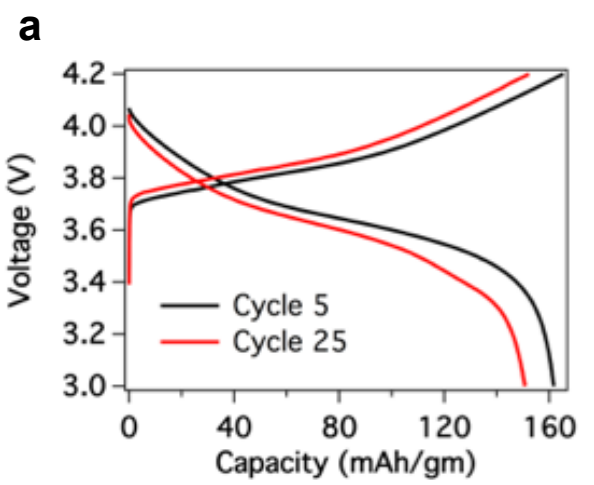

b

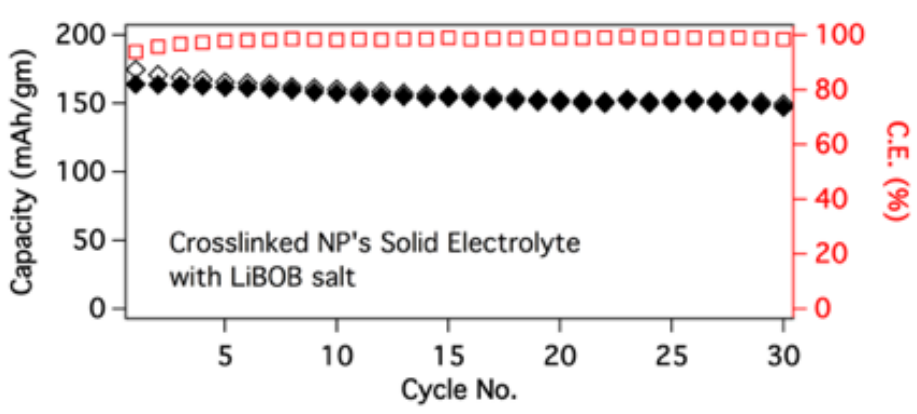

Supplementary Figure 9.21: (a) Voltage profile of Li||NCM cell with a thin $(50 \mu \mathrm{m})$ lithium and $2 \mathrm{mAh} / \mathrm{cm}^{2}$ cathode cycled at $\mathrm{C} / 10$ rate using crosslinked hairy nanoparticles soaked with the electrolyte diglyme- $\mathrm{LiNO}_{3}$-HFiP and LiBOB additive, (b) Cycling profile showing the coulombic efficiency and charge/discharge capacity 


\section{Methods}

Computational details:

Scheme used to calculate the redox potentials

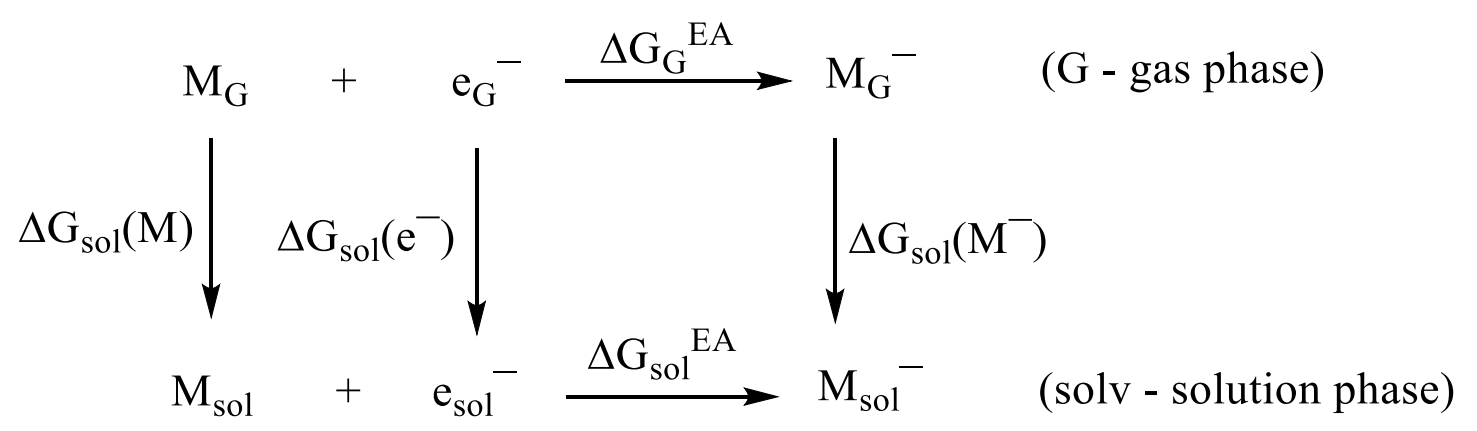

Scheme 1. Thermodynamic cycle used to calculate the oxidation/reduction potential of diglyme and its oligomers formed via reactions with BOB.

By using the thermodynamic cycle shown in scheme 1, Gibbs free energy change in solution phase $\left(\Delta G_{\text {sol }}^{E A}\right)$ during oxidation process could be estimated from eq. (1).

$\Delta G_{s o l}^{E A}=\Delta G_{G}^{E A}+\Delta G_{s o l}\left(M^{-}\right)-\Delta G_{s o l}(M)-\Delta G_{s o l}\left(e^{-}\right)$.

Then, the oxidation potential for a given molecule (M) is calculated as $E_{0}=-\Delta G_{\text {sol }}^{E A} / F$.

where $\mathrm{F}$ is the Faraday constant.

Practically, it is difficult to calculate the solvation free energy of an electron, $\overline{\Delta G_{\text {sol }}\left(e^{-}\right)}$ . Therefore, we have calculated the relative oxidation potential with respect to $\mathrm{Li} / \mathrm{Li}^{+}$ electrodes $(-3.04 \mathrm{eV})$ using eq. (3).

$E^{0}=-\left(\Delta G_{G}^{E A}+\Delta G_{\text {sol }}\left(M^{-}\right)-\Delta G_{\text {sol }}(M)\right) / F+3.04$. 


\section{Experimental Details}

\section{$\underline{\text { Materials }}$}

Lithium discs were obtained from MTI corporation. Diglyme, Lithium Nitrate were all purchased from Sigma Aldrich. Tris(hexafluoroisopropyl) phosphate was obtained from Synquest Laboratories. Celgard 3501 separator was obtained from Celgard Inc. Lithion solution (LITHion ${ }^{\mathrm{TM}}$ dispersion, $\sim 10 \mathrm{wt} \%$ in isoproponal) was purchased from Ion Power Inc. The Lithion is composed of a nafion-type perfluorinated polymer having the sulfonic acid groups $(\mathrm{EW} 1100)$ ion exchanged by lithium ions. Nickel Manganese Cobalt Oxide (NCM) cathodes were obtained from from Electrodes and More Co. All the chemicals were used as received in after rigorous drying in a $\sim 0 \mathrm{ppm}$ water level and $<0.1 \mathrm{ppm}$ oxygen glove box.

\section{Coating of NCM electrode with Lithion Solution}

NCM electrodes were punched out using a hole-punch of diameter 3/8". On a flat bench-top, the NCM cathodes were laid and $\sim 20 \mu 1$ of Lithion solution was dropped to evenly cover the entire surface. Thereafter the electrodes were dried in open air for 6 hours, followed by rigorous drying in a vacuum oven at a temperature of $60^{\circ} \mathrm{C}$ for 24 hours.

\section{Synthesis of gel and crosslinked nanoparticles electrolyte}

The gel electrolyte was prepared by dissolving 1wt.\% of PEG-100kDa (Sigma

Aldrich) in an electrolyte solution of diglyme- $\mathrm{LiNO}_{3}-\mathrm{HFiP}$ (with and without $0.4 \mathrm{M}$

LiBOB salt additive) and thereafter heating the solution to $60^{\circ} \mathrm{C}$ overnight. Thereafter 
the gel electrolyte was brought to room temperature before usage. It was used with a 3501 celgard separator for Lithium battery cycling.

The crosslinked solid electrolyte was prepared using the same procedure reported in our earlier work. ${ }^{1,2}$ After thoroughly drying, the membrane was soaked in the diglyme$\mathrm{LiNO}_{3}$-HFiP-LiBOB solution for a period of 2 days before using in the battery. No separator was used in these batteries.

\section{Dielectric Spectroscopy}

The ionic conductivities of the electrolytes were measured at room temperature the desired electrolytes between two gold-plated copper discs using a Novocontrol Broadband Dielectric spectrometer with a frequency range of $10^{-3}$ to $10^{6} \mathrm{~Hz}$. The electrolyte was sandwiched between the discs using a Teflon o-ring. The DC conductivities were obtained from the plateau of real part of the conductivity versus frequency curve. The Dielectric Spectroscopy instrument was calibrated initially using a $1 \mathrm{M} \mathrm{KCl}$ standard solution.

\section{Scanning electron microscopy}

Surface analysis of electrodeposited stainless-steel was done using SEM with the LEO155FESEM instrument. The sample was prepared by depositing $6 \mathrm{mAh} \mathrm{cm}^{-2}$ in battery comprising of lithium vs. stainless-steel comprising of diglyme- $\mathrm{LiNO}_{3}-\mathrm{HFiP}$ electrolyte and celgard separator. 
$\underline{X-\text { ray Photoelectron Spectroscopy }}$

XPS was conducted using Surface Science Instruments SSX-100 with operating pressure of $\sim 2 \times 10^{-9}$ torr. Monochromatic Al K- $\alpha$ X-rays (1486.6eV) with beam diameter of $1 \mathrm{~mm}$ were used. Photoelectrons were collected at an emission angle of $55^{\circ}$. A hemispherical analyzer determined electron kinetic energy, using pass energy of $150 \mathrm{~V}$ for wide survey scans and 50V for high-resolution scans. Samples were ion-etched using $4 \mathrm{kV}$ Ar ions, which were rastered over an area of $2.25 \times 4 \mathrm{~mm}$ with total ion beam current of $2 \mathrm{~mA}$, to remove adventitious carbon. Spectra were referenced to adventitious C $1 \mathrm{~s}$ at $284.5 \mathrm{eV}$. CasaXPS software was used for XPS data analysis with Shelby backgrounds. The lithium and NCM cathode samples were lightly washed in pure diglyme before XPS measurements. Also, the samples were transferred in an air-tight Argon filled puck from the glove box to the XPS chamber. Hence, there is minimal or no exposure to air.

\section{Floating-point Experiment}

Floating-point experiments were performed in a cell comprising of lithium vs. NCM using various electrolytes reported in the main text. The batteries were charged at constant current of $0.4 \mathrm{~mA} \mathrm{~cm}^{-2}$ upto different voltages from $3.6 \mathrm{~V}$ to $4.3 \mathrm{~V}$ and then held at a constant voltage for 24 hours and the values of the leak current at various voltages were measured.

\section{Fourier Transform Infrared Spectroscopy}


The NCM electrodes were harvested after constant voltage charge at $3.8 \mathrm{~V}$ for 24 hours in a battery comprising of lithium anode and NCM cathode using the electrolyte of diglyme-LiNO 3 -HFiP with and without $\mathrm{LiBOB}$ additive. After drying for 24 hours in the glove-box antechamber ATR-FTIR was used in the wavelength range of $800 \mathrm{~cm}^{-1}$ and $4000 \mathrm{~cm}^{-1}$.

\section{3-electrode Voltammetry}

3-electrode cell was prepared in in a vial-type cell that comprised of a $\mathrm{Ag} / \mathrm{AgCl}$ electrode (prior soaked in standard $1 \mathrm{M} \mathrm{KCl}$ brine) as the reference electrode and stainless steel disc $(2 \mathrm{~mm})$ as the working electrode at room temperature. The scan rate utilized was $10 \mathrm{mV} / \mathrm{s}$.

\section{Battery Performance}

2032 type Li||stainless-steel coin cells with and without HFiP additive in diglyme$\mathrm{LiNO}_{3}$ electrolyte were prepared inside an argon-filled glove box. The amount of electrolyte used for all battery testing was $60 \mu 1$. The cells were evaluated using galvanostatic cycling in a Neware CT-3008 battery tester. Coulombic Efficiency test was performed in Li $\|$ stainless steel cell with different current densities with one each cycle comprising of one hour. Half-cell test was performed in Li $\| \mathrm{NCM}$ at different $\mathrm{C}$ rates after initial two formation cycles of $\mathrm{C} / 10$. The cathode loading was $2 \mathrm{mAh} / \mathrm{cm}^{2}$ and all the $\mathrm{Li}|| \mathrm{NCM}$ experiments were performed using a thin lithium $(50 \mu \mathrm{m})$ as anode. Unless stated in the figure, the voltage ranges were chosen to $4.2 \mathrm{~V}$ to $3 \mathrm{~V}$. All the coin-cells were crimped to a pressure of $\sim 2500$ psi. Except for the results using the 
crosslinked nanoparticles electrolytes, all the battery comprised of a 3501 celgard separator. Unless stated otherwise, the LiNO3 content in the battery was $r=0.5$ (molar ratio between $\mathrm{EO}$ and $\mathrm{Li}$ ions). In all the battery measurements with the HFiP chaintransfer-agent, the amount added in the electrolyte was $1 \mathrm{wt} . \%$. In the measurements using LiBOB salt additive, the amount utilized was $0.4 \mathrm{M}$.

\section{References:}

1 S. Choudhury, R. Mangal, A. Agrawal and L. A. Archer, Nat. Commun., 2015, 6, 10101 .

2 S. Choudhury, D. Vu, A. Warren, M. D. Tikekar, Z. Tu and L. A. Archer, Proc. Natl. Acad. Sci., 2018. 
Chapter 10

Lithium Fluoride additives for stable cycling of Lithium Batteries at high current densities 


\subsection{Abstract}

Progress in advanced energy storage technologies, in particular rechargeable batteries, is limited by complex electrochemical and interfacial phenomena, which produce deposition instabilities on the most energetic anode materials. Uneven electrodeposition is a serious problem in almost all rechargeable batteries that use high-energy metals such as aluminum, lithium, sodium, or zinc as the anode because it leads to formation of dendritic structures that expose the device to a variety of failure modes, including catastrophic failure by internal short circuiting. We investigate the effect of lithium fluoride salt additives on electrodeposition of metals in batteries that use metallic lithium anodes. Through systematic electrochemical, spectroscopic, and microscopy studies, we find that these additives provide a robust strategy for improving both the lifetime and coulombic efficiency of a battery at both high and low current densities. We show that LiF simultaneously act to protect lithium metal anode surface and also improves interfacial Li-ion transport, thus enabling faster and flatter electrodeposition with longer cycle life. Finally, we demonstrate that a conventional electrolyte reinforced with as little as $0.5 \mathrm{w} \% \mathrm{LiF}$ salt can be used to enable $\mathrm{Li} / \mathrm{LiFePO} 4$ full cells that exhibit stable cycling for over 150 cycles of charge and discharge at high current density.

\subsection{Introduction}

Rechargeable batteries are key components in a growing list of technologies where portability and reliability are required. The commercial success of one particular family of rechargeable batteries, the Lithium-ion battery (LIB), has played a crucial 
role in the development of portable device technology that offer consistent performance, power, lifetime as well as enhanced safety. In a rechargeable Lithium metal battery (LMB), one replaces the inert carbon anode with metallic lithium and in so doing creates an energy storage platform with significantly improved energy density, portability, and power ${ }^{1-10}$. Two specific examples of such batteries, the Lisulfur and Li-air batteries, are currently under active investigation worldwide as potential platforms for increasing range, performance and lifetime costs in next generation electrified vehicle technologies, including electric cars ${ }^{11}$. It is understood, however, that even pairing a metallic lithium anode with any of the currently used LIB cathode materials (e.g. $\mathrm{Li} / \mathrm{LiFePO}_{4} ; \mathrm{Li} / \mathrm{LiCoO}_{2}, \mathrm{Li} / \mathrm{LiNiCoO}$, etc), provide more straightforward opportunities for engineering LMBs with energy-storage and performance characteristics that are superior to today's work-horse LIB technologies.

A key barrier to progress in development of rechargeable batteries in any of these configurations is the complex electrochemistry of deposition at metallic surfaces in a liquid electrolyte. In course of successive charge-discharge processes in LMBs, uneven plating of the anode can be caused by electroconvection and other deposition instabilities leading to premature cell failure ${ }^{4,8}$. In the most extreme cases, the uneven electrodeposition on the anode results in the formation and growth of dendritic structures that ultimately bridge the inter-electrode space and short-circuit the cell $1^{4,12,13}$. However, the most common mode of cell failure occurs by loss of the active electrode material by various interrelated electrochemical and interfacial processes. For example, the ohmic energy in and fragility of a growing dendrite can cause it to break before it spans the inter-electrode space. This produces regions of electrically 
disconnected or dead lithium that is no longer able to exchange electrons with the electrode mass and contributes to a low columbic efficiency and shortened lifetime of a battery. An even more challenging failure mode results from the loss of lithium metal and electrolyte due to side reactions at the roughened electrode-electrolyte interface. Indeed while such reactions always occur when liquids are in conformal contact with reactive metals, such as $\mathrm{Li}$, during each cycle of deposition the roughened metal surface exposes new $\mathrm{Li}$ metal to the electrolyte that leads to continuous formation of new so called SEI layer that ultimately depletes the electrolyte and causes formation of 'mossy' Lithium. ${ }^{13}$ All of these situations are exacerbated by the common use of flammable organic liquids as electrolyte solvents in rechargeable batteries to improve ionic conductivity of the electrolyte, which now add the threat of fire or even explosion to the list of potential failure modes $^{4,14,15}$.

Over the years, several efforts have been made to eliminate the possibility of dendrite-induced short-circuits in batteries by designing high modulus electrolytes through which a growing metal dendrite cannot penetrate ${ }^{16-18}$. These efforts have largely met with, at most, limited success because of the fundamental difficulty in designing materials that are simultaneously mechanically strong-enough to stop dendrites, but in which fast ionic transport needed to sustain battery performance can be achieved at moderate temperatures. A notable exception is the work of Tu et. al., ${ }^{19}$ which shows that a $\mathrm{Al}_{2} \mathrm{O}_{3}$ ceramic separator with uniform, nanometer-sized pores that hosts a liquid electrolyte in its pores is able to perform both functions. However, as none of these approaches address the root cause of the electrodeposition instabilities that trigger dendrite formation, more elegant solutions in the form of SEI additives 
have been sought to stop dendrites at the nucleation stage. There are generally two approaches that have been investigated in the previous literature: 1) direct addition, wherein specific chemical agents are used as electrolyte additives to promote stable SEI formation but do not take part in the bulk ion transfer. Hydrofluoric Acid ${ }^{20}$, Vinylene Carbonate ${ }^{21-23}$, Lithium bis(oxalato)borate ${ }^{24,25}$, Lithium Nitrate $^{26}$, and Organic Sultones ${ }^{27,28}$ have all been reported to function in this way. While each of these additives have been shown to improve the cell stability to an extent, wider use of all are challenged by the associated decrease in ionic conductivity and gradual loss of efficacy due to decomposition ${ }^{26} .2$ ) indirect formation of stabilizing layers. This approach involves the formation of a stable layer by internal reactions of two or more components added to the electrolyte. A recent example by Miao et. al. ${ }^{29}$ showed that a binary mixture of LiTFSI and LiFSI can significantly improve the cyclability of a LMB due to the formation of a LiF layer by degradation of these salts on the surface of the lithium metal anode. Also, recently Qian et. al..$^{30}$ showed similar improvement in performance by use of LiFSI in excess concentration, which may also be rationalized as producing $\mathrm{LiF}$ at the electrolyte/electrode interface as seen using XPS, and predicted previously by ab initio calculations that show the tendency of LiFSI to reduce to $\mathrm{LiF}^{31}$.

\subsection{Experimental Section:}

\subsubsection{Materials}

Lithium foil was bought from Alfa Aesar. 1M EC:DMC- LiPF, High purity Lithium Fluoride were obtained from and opened as received in an Argon filled glove box. All 
coin cell parts and $\mathrm{LiFePO}_{4}$ sheets $\left(\sim 2 \mathrm{mAh} / \mathrm{cm}^{2}\right)$ were bought from MTI Corporation. Polypropylene based separator with commercial name Celgard 3501 was obtained from Celgard LLC.

\subsubsection{Methods}

For the preparation of modified electrolyte system, $30 \mathrm{ml}$ of $1 \mathrm{M} \mathrm{EC:} \mathrm{DMC} \mathrm{LiPF}_{6}$ and $0.5 \%$ (by wt.) of $\mathrm{LiF}$ were added to it. This particular additive approximately equals to the $30 \%$ molar of LiF previously used as reinforced electrolyte system used by $\mathrm{Lu}$ et. al. ${ }^{12}$ This mixture of electrolyte-additive was continuously stirred overnight to ensure proper mixing owing to the fact that $\mathrm{LiF}$ is partially insoluble in this electrolyte. Coin cells of different types were prepared in 2032 type configurations. For symmetric cells, both electrodes comprised of Lithium foil, whereas for coulombic efficiency test, thoroughly cleaned stainless steel plate was taken as one of the electrodes. For making half-cells, $\mathrm{LiFePO}_{4}$ sheets were punched, kept in vacuum for 12 hours and weight of the electrodes were weighted individually, before using as cathode. For all kinds of configurations (in exception of those made for impedance spectroscopy measurements), the batteries were allowed to rest for a period of 7 days before any electrochemical analysis.

\subsubsection{Electrochemical Characterization}

Impedance Spectroscopy measurements were done using a Solatron frequency analyzer. The measurements were done in a symmetric cell configuration at a frequency range of $10^{-3} \mathrm{~Hz}$ to $10^{7} \mathrm{~Hz}$. All galvanostatic measurements were done using 
a Newar CT-3008 battery tester. Coulombic Efficiency tests were done in a LiStainless Steel configuration, where the batteries were initially charged and discharged between $0.5 \mathrm{~V}$ to $0 \mathrm{~V}$ for 10 cycles at $0.01 \mathrm{~mA} / \mathrm{cm}^{2}$ to enable a stable SEI formation. Next, they were discharged at different current densities with a time control and then charged until the voltage was $0.5 \mathrm{~V}$. The coulomic efficiency was calculated from ratio of charge to discharge capacity. Strip-Plate measurements were done symmetric cells with time-controlled charge and discharge cycles. The half-cells comprising of Lithium anode and $\mathrm{LiFePO}_{4}$ cathode were cycled in the voltage window of $3.8 \mathrm{~V}$ to 2.5V. The SEM analysis of the electrode surface was done using a LEO155FESEM instrument after the disassembling of Lithium symmetric cells, which were subjected to polarization at different current densities.

\subsection{Results}

Ion transfer through SEI layer has been an extensive topic of research, recent findings from Joint Density Functional Theoretical (JDFT) studies by the Arias and co-workers underscore the importance of SEI additives such as LiF in controlling surface diffusion of metal ions during electrodeposition. ${ }^{32,33}$ Figure 10.1 is a schematic of the mechanism through which an enhanced Li-ion surface mobility would enable stable electrodeposition. The JDFT analysis ${ }^{33}$ in fact, shows that the barrier energy for surface diffusion of $\mathrm{Li}$ ions over a surface of $\mathrm{LiF}$ is lower by $0.09 \mathrm{eV}(=3.5 \mathrm{kT})$, compared to that of Lithium Carbonate, which is the most abundant component in a SEI layer. This means that the rate of transport of Li-ions on a $\mathrm{LiF}$ surface is more than 30 times larger than $\mathrm{LiCO}_{3}$ substrate. It is proposed that this enhanced surface 

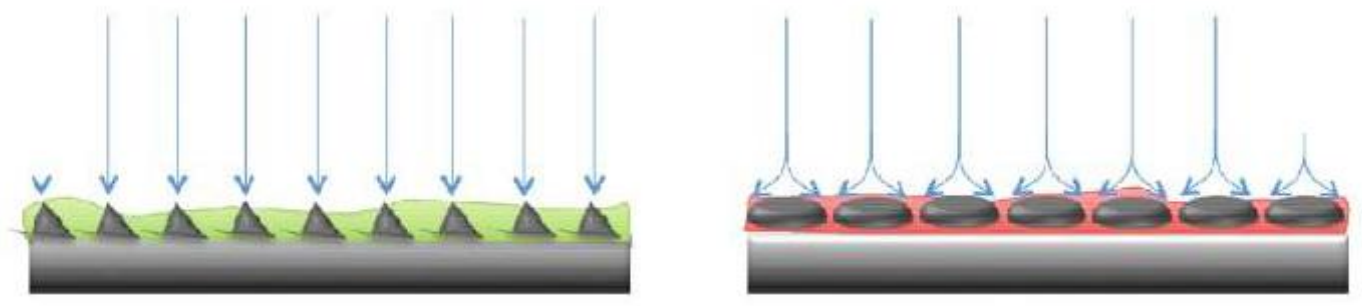

$\checkmark$ Li+ Diffusion near surface a Li Deposits L LiF rich SEl layer Usual SEl layer

Figure 10.1. Pictorial representation of proposed mechanism: Lithium diffusion near the surface of electrodes represented by blue arrows. LiF rich SEI is shown in red, while usual SEI is indicated by green color. Due to lower lateral diffusion barrier on LiF crystals, the Li + ions form smooth electrodeposits, while in usual SEI needle-like lithium plating is expected. 
diffusion is likely to promote smooth lithium plating in contrast to the more typical rough, dendritic electroplating. Additionally, the JDFT calculations by Ozhabes et. al. ${ }^{33}$ show that the surface energy of a LiF surface is more than three times that of $\mathrm{LiCO}_{3}$ and close to eight times of $\mathrm{LiOH}$. This means that a lithium metal surface with a coating of $\mathrm{LiF}$ is much more resistant to roughening than one with a similar coating of $\mathrm{LiCO}_{3}$ or $\mathrm{LiOH}$.

In a departure from the previous "sacrificial" methods and motivated by the JDFT calculations, Lu et al. ${ }^{12}$ reported a potentially simpler approach based on partially reinforcing the liquid electrolyte with halide salt additives that can deposit on the anode surface. This method was reported to produce significant improvements in the short circuit time of LMBs cycled at moderate to low current densities. However, the study did not investigate the effect of these additives at current densities typical for electrochemical transport in batteries and also did not evaluate the effect of $\mathrm{LiF}$ on cell failure modes associated with interfacial reactions and degradation of the electrolyte at the lithium metal anode. Additionally, the study by Lu et al. considered electrolytes primarily based on propylene carbonate (PC) as solvent. This choice is a limitation because $\mathrm{PC}$ is a poor solvent for $\mathrm{LiF}$ and is typically not used as the electrolyte solvent in lithium batteries because of its reactivity towards carbon. Finally, in an effort to demonstrate the effectiveness of the LiF salt additives, the study by $\mathrm{Lu}$ et al. interrogated cells using a so-called membrane-less configuration in which O-ring shaped separators are employed, which is again not a widely practiced approach.

Herein we report on the deposition of metallic lithium at low and high current densities in LMBs containing conventional carbonate electrolytes, EC:DMC-LiPF, 
reinforced with halide additives. In an attempt to advance fundamental understanding of how and why LiF salt additives work to impede dendrite proliferation and electrochemical degradation under typical cell running conditions, and to evaluate the broader relevance of salt additives to LMBs, the present work systematically removes all of the aforementioned constraints in the study by Lu et al. In so doing, we find that electrolytes containing as little as $0.5 \%$ by wt. $\mathrm{LiF}$ in a 1 molar electrolyte solution simultaneously offer remarkable dendrite suppression abilities and enhanced electrolyte stability during cell cycling. The results reported in the present work therefore expand significantly upon current knowledge and define a path towards LMBs with lifetime and safety characteristics compatible with requirements for commercially important cells.

Various recent works have considered the role of electrolyte chemistry on coulombic efficiency of Li-metal electrodes. The electrolyte blend DOL:DME-LiTSFI has received significant attention because it is known to exhibit good stability in the presence of metallic lithium and high coulombic efficiency due to its ability to form a stable SEI layer, particularly in the presence of $\mathrm{LiNO}_{3}{ }^{13}$. In a departure from this

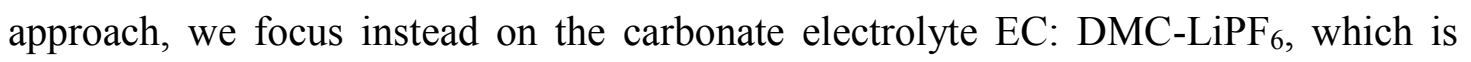
more commonly used in a high voltage Lithium ion battery, owing to its high vaporization temperature, cost and compatibility with high voltage cathode materials. In order to monitor the stability of the SEI layer in a LMB, symmetric cells were constructed using neat and $\mathrm{LiF}$ reinforced electrolytes, and impedance spectroscopy measurements were performed at different time intervals to characterize the interfacial resistance in the cells. Figure 10.2(a) shows the Nyquist plots for the corresponding 
(a)

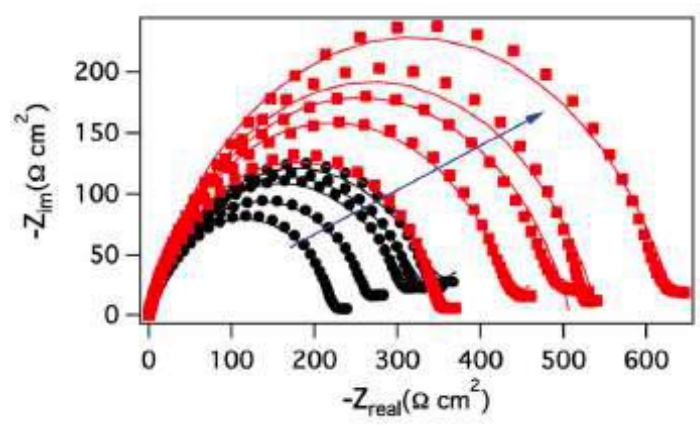

(b)

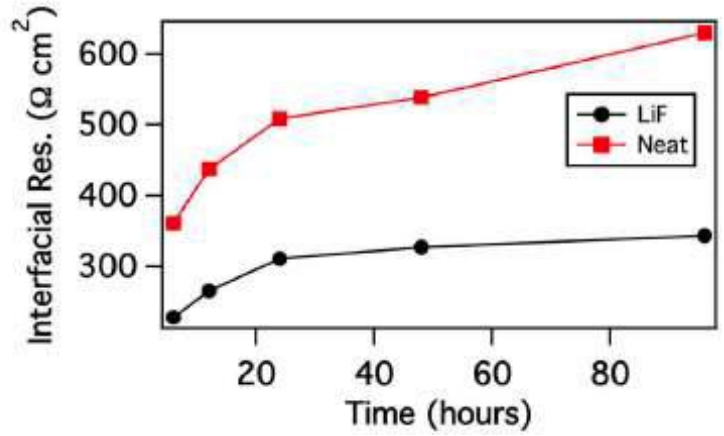

Figure 10.2. Impedance spectroscopy as a function of time: a) Nyquist plots obtained from impedance spectrocopy for symmetric cells having neat electrolyte (shown in red) and modifi ed electrolyte with LiF additive (shown in black). The blue arrow shows the direction of increasing time from 6 to $96 \mathrm{~h}$. b) Interfacial resistance for neat and LiF-based batteries shown as a function of time. It is obtained by fi tting to an equivalent circuit model and approximately it corresponds to the width of the semicircle in the Nyquist diagram. 
impedance spectra. The interfacial resistance obtained by fitting the plots to the equivalent circuit depicted in Supplementary Figure 10.1 is plotted against time in Figure 10.2(b). At initial times, the interfacial resistance for batteries containing the neat (no LiF) electrolytes is over $350 \Omega \mathrm{cm}^{2}$, consistent with the formation of a thick carbonate rich SEI. In contrast, electrolytes containing LiF based electrolyte demonstrated a much lower values, close to $200 \Omega \mathrm{cm}^{2}$. Furthermore, the interfacial resistance is shown to increase with time for the neat electrolyte system, which is a clear trait of an unstable SEI formation that leads to side reaction between the bare Lithium and electrolyte creating an insulating interface. On comparison, cells having LiF based electrolytes show slight increase in the interfacial resistance in initial time, there after it becomes constant. LiF salt being partially insoluble in the used electrolytes forms a thin coating over the surface of Lithium, thus stabilizing the SEI layer and preventing any side reaction.

The coulombic efficiency for batteries consisting of electrolytes with/without LiF additive was examined in a two-electrode setup consisting of metallic lithium and stainless steel (SS) electrodes. Since a symmetric cell has virtually infinite lithium source, the Lithium reserve in the electrode can compensate for any Li loss in forming the SEI or in side reactions with the electrolyte during cell operation. Thus, it is not possible to quantify the effects of many of the failure modes outlined in the introduction in a symmetric cell. However, in the asymmetric Li-SS cells used in the present study, the Lithium loss in SEI formation or by side reactions can be quantified by the difference between the amount of Lithium plated and successively stripped. Recent reports by Cui and co-workers ${ }^{13}$ have shown $\sim 99 \%$ efficiency in a composite 
electrolyte of DOL: DME-LiTFSI $+\mathrm{Li}_{2} \mathrm{~S}_{6}+\mathrm{LiNO}_{3}$, however with carbonate based electrolyte such as PC, the coulombic efficiency from similar tests ${ }^{34}$ have been reported to rarely exceed $\sim 77 \%$, which is attributed to the relative poor SEI forming characteristics of the carbonate solvents in comparison to DOL:DME. Figure 10.3 (a), (b) reports the coulombic efficiencies of cells with and without LiF additive at rates ranging from $0.25 \mathrm{~mA} / \mathrm{cm}^{2}$ and $0.50 \mathrm{~mA} / \mathrm{cm}^{2}$ respectively, with $1.00 \mathrm{mAh} / \mathrm{cm}^{2}$ of Lithium cycled in each run. It is seen that at $0.25 \mathrm{~mA} / \mathrm{cm}^{2}$, the coulombic efficiency for the neat electrolyte is close to $80 \%$ in agreement with reported values in literature ${ }^{34}$, while for cells with LiF additive, the coulombic efficiency exceeds 90\%. In addition, it is observed that for at least 120 cycles, the values remain essentially constant for cells containing the LIF salt-reinforced electrolyte. In contrast for the control batteries that do not include $\mathrm{LiF}$ in the electrolyte, the coulombic efficiency is observed to begin fluctuating beyond 50 cycles. Also, at current density of $0.50 \mathrm{~mA} / \mathrm{cm}^{2}$, the LiFreinforced electrolytes are seen to exhibit coulombic efficiency close to $90 \%$, while their unreinforced counterpart show poor performance. The respective voltage profile as a function of areal capacity is shown in Supplementary Figure 10.2. It is clear that the $\mathrm{LiF}$ additive is enabling a stable SEI formation and flat electrodeposition, hence improving the coulombic efficiency by at least $10 \%$ compared to convention battery. It is remarkable that addition of just $0.5 \%$ by weight of LiF additive can significantly improve the lifetime and performance of a battery without the need of any other modification of the conventional electrolyte.

In order to more carefully investigate the aforementioned characteristics of Lithium deposition onto the electrode, scanning electron microscopy was performed 
(a)

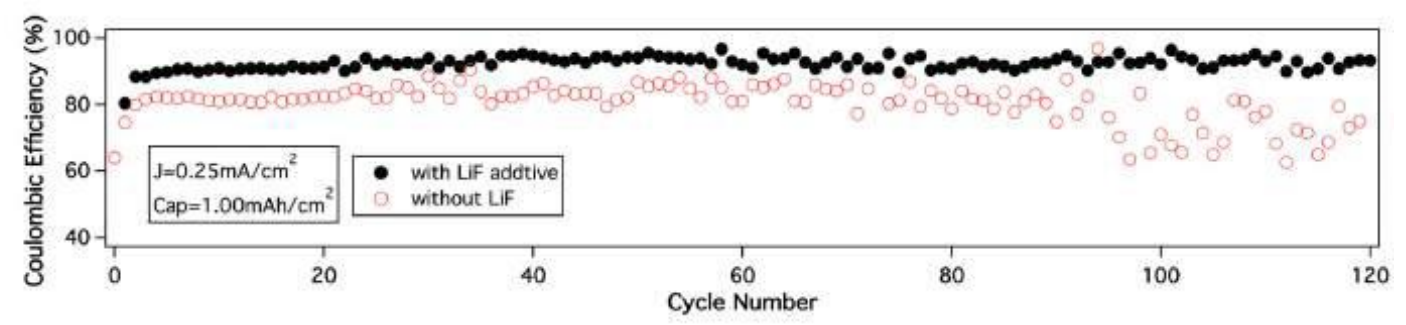

(b)

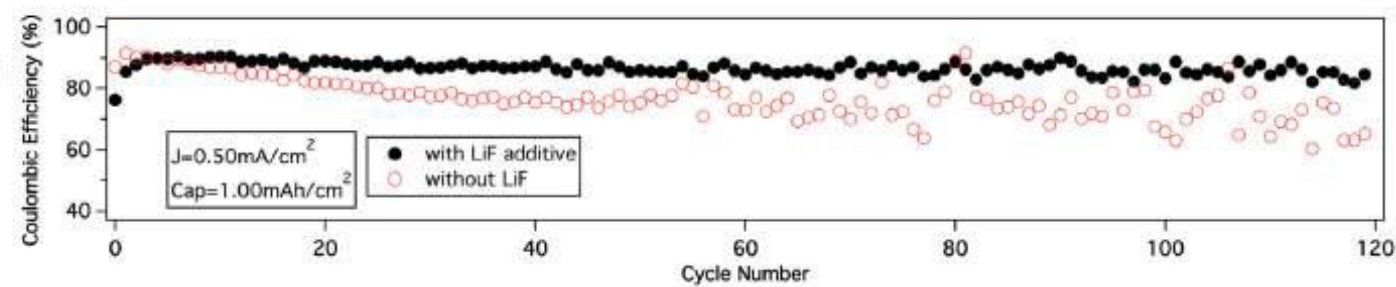

Figure 10.3. Coulombic efficiency test of lithium cells: Batteries with neat electrolyte (shown in red) and with $0.5 \% \mathrm{LiF}$ additive (shown in black) were cycled at different current densities a) at current density of $0.25 \mathrm{~mA} \mathrm{~cm}{ }^{-2}$ with capacity of $1 \mathrm{mAh} \mathrm{cm}^{-2}$; b) at current density of $0.25 \mathrm{~mA} \mathrm{~cm}^{-2}$ with capacity of $1 \mathrm{mAh} \mathrm{cm}^{-2}$. 
on the Lithium metal surface after Li deposition as shown in Figure 10.4. For these experiments, symmetric cells were constructed and a fixed amount of Lithium $\left(4 \mathrm{mAh} / \mathrm{cm}^{2}\right)$ was passed from one electrode to the other at current densities of $2 \mathrm{~mA} / \mathrm{cm}^{2}$ and $4 \mathrm{~mA} / \mathrm{cm}^{2}$. It is seen that the Lithium deposits obtained using the neat electrolyte are uneven and needle-like, while for the LiF-reinforced electrolytes; the Li deposition is significantly flatter. The SEM images of the Lithium anodes after deposition at $2 \mathrm{~mA} / \mathrm{cm}^{2}$ were analyzed to determine the size of the deposits as given in the Supplementary Figure 10.4. It is seen that the electrode with neat electrolyte had dendrites in mostly conical or cylindrical shape with mean length of $\sim 20 \mu \mathrm{m}$; while for the electrolyte with LiF additive, the deposits were mostly spherulites with a mean diameter of $\sim 11.5 \mu \mathrm{m}$. A mechanism that explains these large effects in terms of enhanced lateral mobility of Li-ions on a LiF surface is provided in Figure 10.1. The surface morphology of the Lithium electrode in the presence of LiF electrolytes is qualitatively consistent with expectations based on this concept.

Further, to evaluate the hypothesis that LiF-reinforced electrolytes yield LMBs with higher resistance to dendrite formation and thus more resistant to failure by dendriteinduced short circuits, 'strip-plate' measurements were carried out in symmetric Lithium cells. In these experiments the $\mathrm{Li} / \mathrm{Li}$ cells are subjected to alternating periods of charge and discharge at a range of current densities. The experiments were deliberately performed under relatively harsh conditions, such that $4 \mathrm{mAh} / \mathrm{cm}^{2}$ of Lithium was deposited in each charge and discharge cycles, which is at least twice as high as the areal capacity of commercially available cathode sheets. Figure 10.5 shows the voltage profiles as a function of time for batteries with and without LiF additive at 

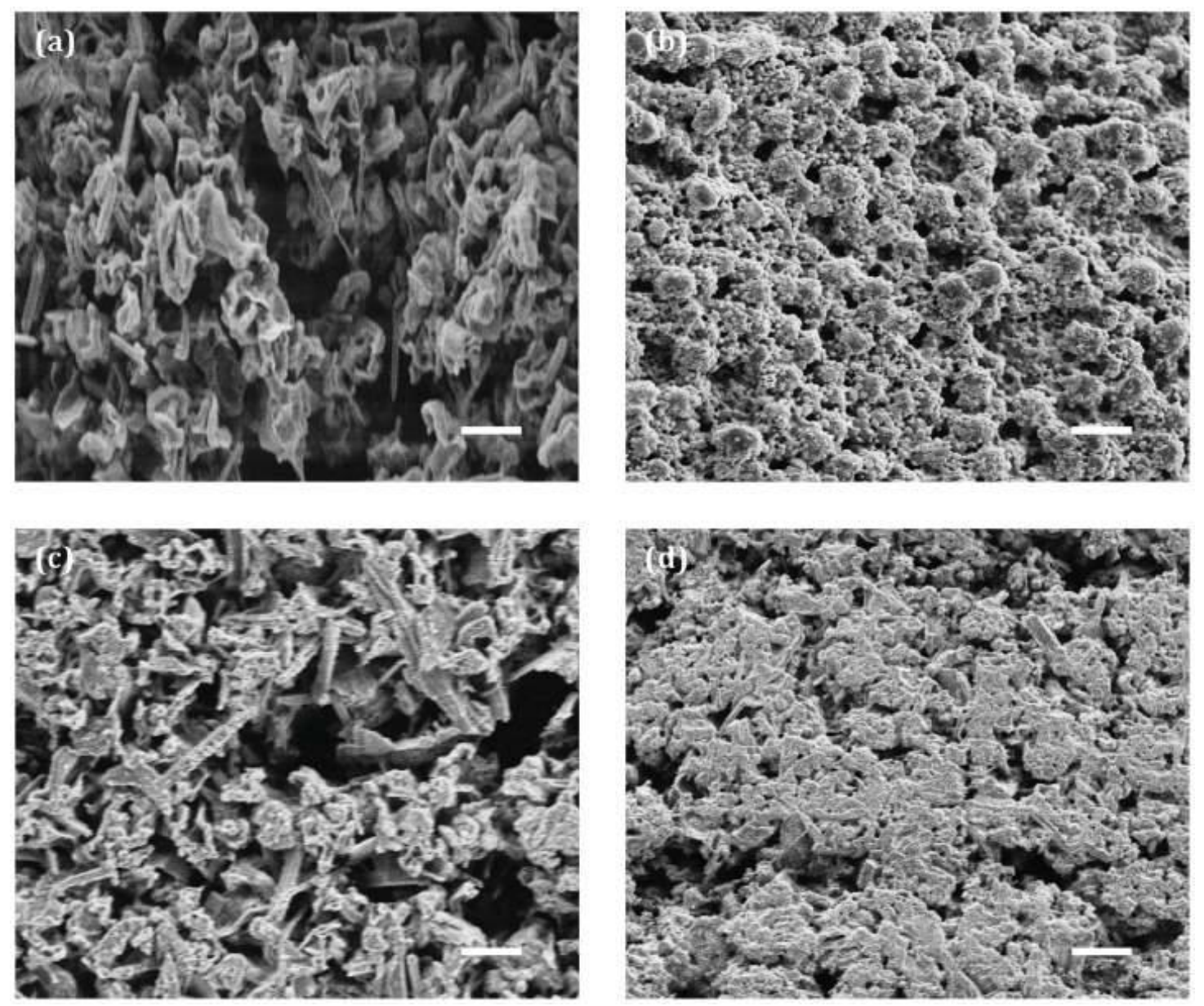

Figure 10.4. Surface morphology of lithium anode: Lithium symmetric cells were polarized at different conditions before disassembling them for SEM analysis of the surface features a) without $\mathrm{LiF}$ at $2 \mathrm{~mA} \mathrm{~cm}^{-2}$ for $2 \mathrm{~h} \mathrm{~b}$ ) with $0.5 \% \mathrm{LiF}$ at $2 \mathrm{~mA} \mathrm{~cm}^{-2}$ for $2 \mathrm{~h}$; c) without $\mathrm{LiF}$ at $4 \mathrm{~mA} \mathrm{~cm}^{-2}$ for $1 \mathrm{~h} \mathrm{~d}$ ) with $0.5 \% \mathrm{LiF}$ at $4 \mathrm{~mA} \mathrm{~cm}^{-2}$ for $1 \mathrm{~h}$. All scale bars: $20 \mu \mathrm{m}$. 
current densities of 1,2 and $4 \mathrm{~mA} / \mathrm{cm}^{2}$. It is seen that at the highest current density of $4 \mathrm{~mA} / \mathrm{cm}^{2}$, the cells that do not contain $\mathrm{LiF}$ additives in the electrolyte short circuit within 80 hours of the start of the test. In contrast, those in which LiF is used show lifetimes in excess of 140 hours. The cell lifetime is also seen to double at a current density of $2 \mathrm{~mA} / \mathrm{cm}^{2}$ upon addition of $0.5 \mathrm{wt} \% \mathrm{LiF}$ to the electrolyte; at a current density of $1 \mathrm{~mA} / \mathrm{cm}^{2}$, the neat electrolyte-based battery fails within 900 hours, while its counterpart continues to cycle to more than 1700 hours without any sign of short circuit. Thus, it is evident that $\mathrm{LiF}$ additive can not only prolong short circuit time at high current density, it can also prevent short circuit at low current densities, making Lithium metal batteries viable for practical applications. Another important conclusion that can be drawn from the Figure 10.5 is the fact that the voltage profiles for neat electrolytes show gradual increment with time, which is an indication of electrolyte degradation by side reactions. This is attributed to the unstable SEI formation in usual Lithium batteries as mentioned earlier, while, LiF based batteries show significant improvement in the stability of voltage profiles.

The practical viability of $\mathrm{LiF}$ as an electrolyte for LMBs was analyzed in the simplest LMBs in which a metallic lithium anode is paired with a $\mathrm{LiFePO}_{4}$ cathode as shown in Figure 10.6. Figure 10.6(a), (b) reports the voltage profiles obtained from galvanostatic measurements for different cycle numbers for Li|EC:DMC$\mathrm{LiPF}_{6} \mid \mathrm{LiFePO}_{4}$ and $\mathrm{Li} \mid \mathrm{EC}: \mathrm{DMC}_{-} \mathrm{LiPF}_{6}-0.5 \% \mathrm{LiF}^{-} \mathrm{LiFePO}_{4}$, respectively; while, Figure 10.3(c) shows the cyclability of these cells. The active material loading of the cathode used in this experiment of $\sim 2 \mathrm{mAh} / \mathrm{cm}^{2}$ is higher than most previous studies and the current density used in experiment is $\sim 0.50 \mathrm{~mA} / \mathrm{cm}^{2}$, corresponds to a C-rate of $\mathrm{C} / 4$. 
(a)

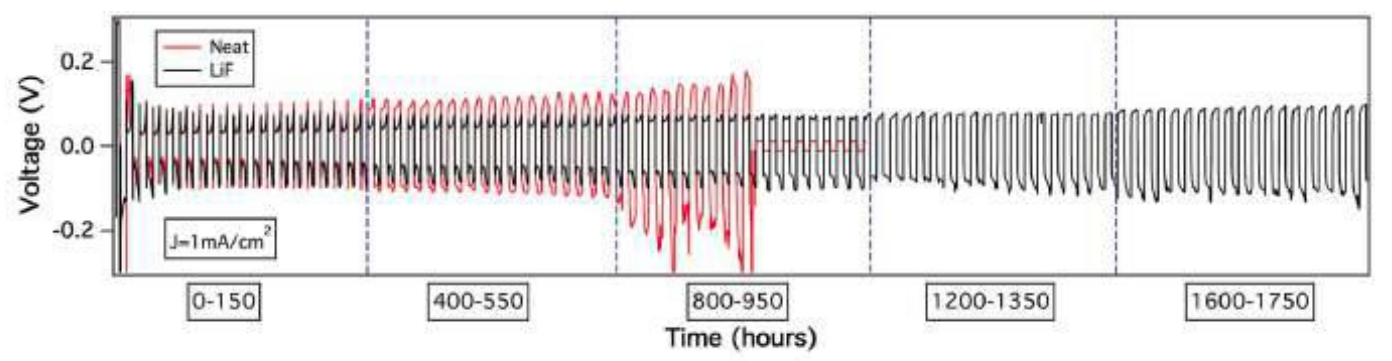

(b)

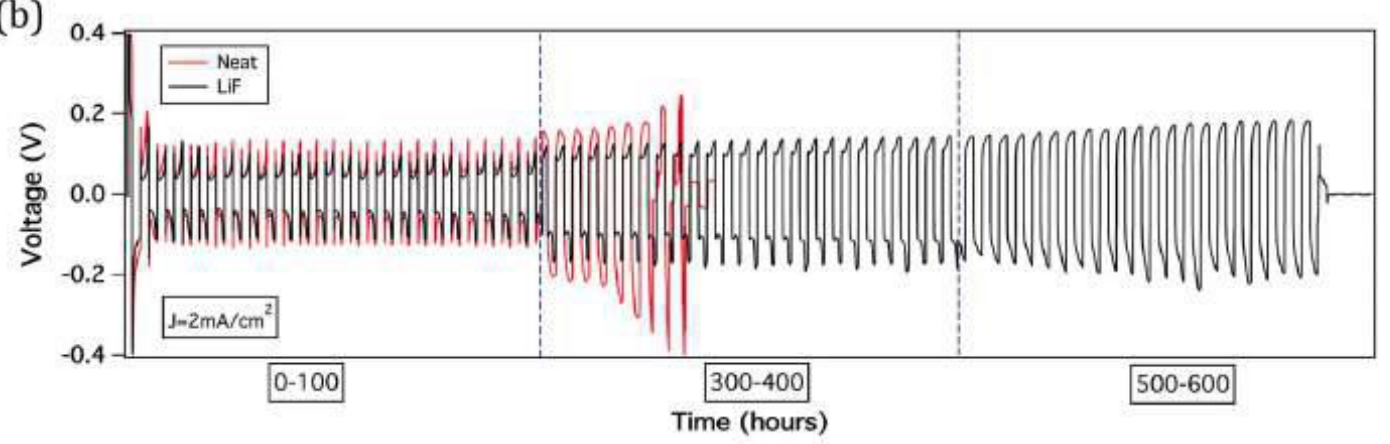

(c)

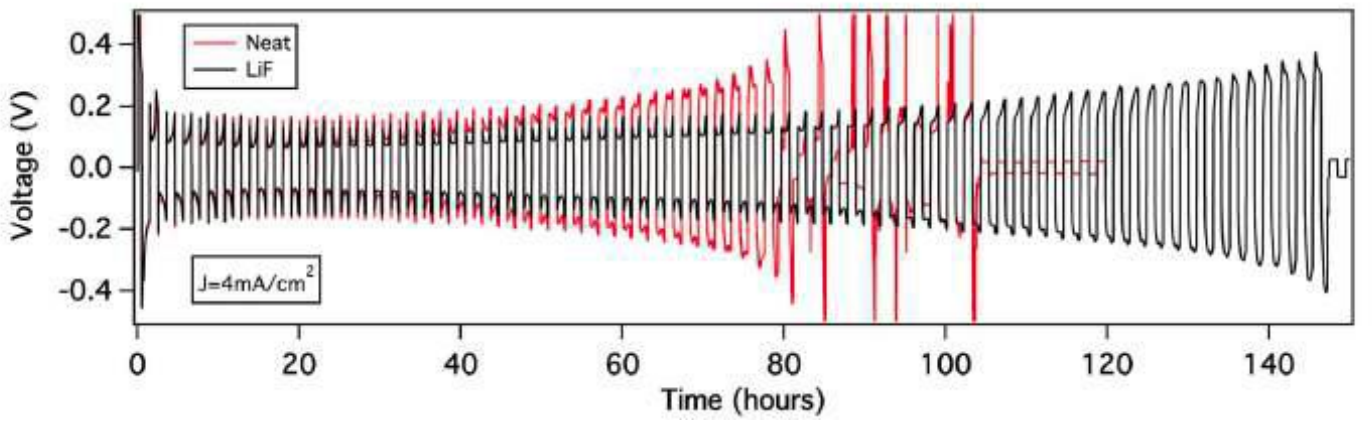

Figure 10.5. Strip-plate tests: Symmetric lithium cells were charged and discharged cycles with neat electrolyte (shown in red) and with $0.5 \% \mathrm{LiF}$ additive (shown in black) at different conditions: a) at current density of $1 \mathrm{~mA} \mathrm{~cm}^{-2}$ with capacity of 4 $\left.\mathrm{mAh} \mathrm{cm}{ }^{-2} ; \mathbf{b}\right)$ at current density of $2 \mathrm{~mA} \mathrm{~cm}^{-2}$ with capacity of $4 \mathrm{mAh} \mathrm{cm}^{-2}$; c) at current density of $4 \mathrm{~mA} \mathrm{~cm}^{-2}$ with capacity of $4 \mathrm{mAh} \mathrm{cm}^{-2}$. 
Both types of cells show coulombic efficiency of $\sim 99 \%$, which is to an extent intuitive; there is a reservoir of virtually infinite Lithium at the anode. In the initial cycle, both the batteries are observed to have a discharge capacity of $120 \mathrm{mAh} / \mathrm{gm}$. In successive cycles, this value increases to about $130 \mathrm{mAh} / \mathrm{gm}$ as the battery reaches

steady state. However, the discharge capacity for the neat electrolyte systems shows a gradual decrement as a result of side reactions in the electrolyte and uneven electrodeposition. Beyond 100 cycles, it is seen that the neat electrolyte system becomes significantly unstable and ultimately fails. The LiF based battery show good performance for at least 150 cycles with a near constant discharge capacity, which is certainly a remarkable result considering that the battery operates at room temperature and at a relatively high current density. Results for batteries operating at even higher current density of $1 \mathrm{~mA} / \mathrm{cm}^{2}$ are shown in Figure S3 of supplementary information, where a similar behavior is observed for the two types of batteries.

\subsection{Conclusion}

In summary, we have demonstrated that addition of $0.5 \%$ by weight of LiF to a conventional electrolyte can significantly improve the stability and reversibility of a battery. The rationality behind this observation is attributed to the recent JDFT calculations $^{33}$ that predicted high surface diffusivity of $\mathrm{Li}$ ions over a layer of $\mathrm{LiF}$ crystals as well as its higher surface stability over other SEI components. The batteries with $\mathrm{LiF}$ additive show low interfacial resistance and more than $90 \%$ columbic efficiency owing to better protection of Lithium meta compared to usual electrolytes. In addition, it enables suppression of dendrite growth by facilitating smooth 
(a)
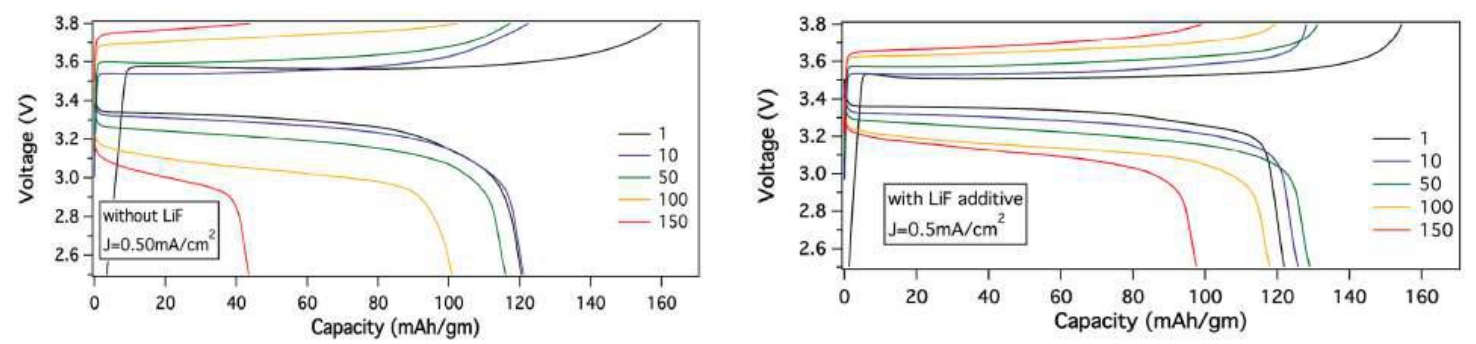

(c)

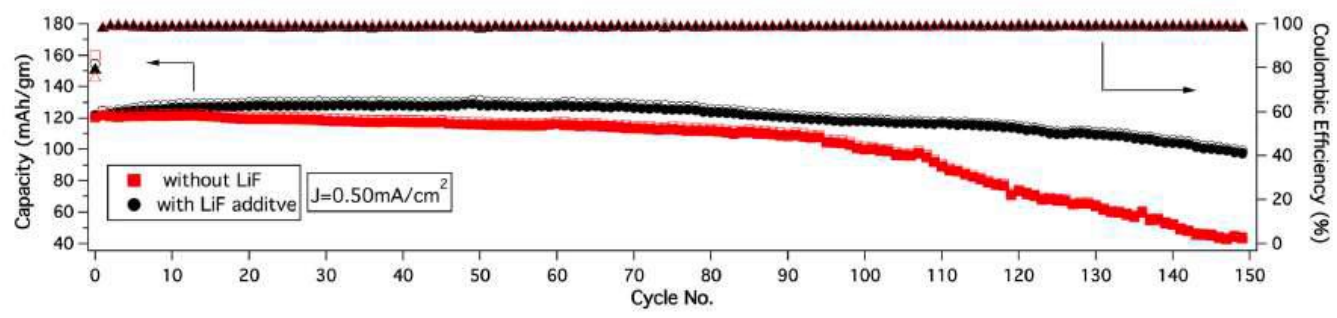

Figure 10.6. Half-cell tests: Lithium metal batteries comprising of lithium anode and LiFePO 4 were charged and discharged with neat electrolyte and with $0.5 \% \mathrm{LiF}$ additive: a) voltage profi le at current density of $0.5 \mathrm{~mA} \mathrm{~cm}^{-2}$ for neat electrolyte; b) voltage profile at current density of $0.5 \mathrm{~mA} \mathrm{~cm}{ }^{-2}$ with $\mathrm{LiF}$ additive; c) Cycling performance of these batteries up to 150 cycles. 
electrodeposition, thus increasing the lifetime of cells to hundreds of hours. The LiF additive is also successful in improving the discharge capacity and cyclability of a $\mathrm{LiFePO}_{4}$ half-cell for at least 150 cycles while a usual battery fails within 100 cycles. All these features of LiF additive, in addition to the convenience of usage make it a viable option for practical applications.

\section{Acknowledgements}

The material reported in this paper is based on work supported as part of the Energy Materials Center at Cornell, an Energy Frontier Research Center funded by the U.S. Department of Energy, Office of Science, Office of Basic Energy Sciences under Award Number DESC0001086. Financial support from the National Science Foundation, Partnerships for Innovation (Grant\#IIP-1237622) is also gratefully acknowledged. Electron microscopy, X-ray diffractometry and X-ray spectroscopy facilities available through the Cornell Center for Materials Research (CCMR) were used for this work (NSF Grant DMR-1120296). 


\section{REFERENCES}

(1) Dunn, B.; Kamath, H.; Tarascon, J.-M. Electrical Energy Storage for the Grid: A Battery of Choices. Science 2011, 334, 928-935.

(2) Dresselhaus, M. S.; Thomas, I. L. Alternative Energy Technologies. Nature 2001, 414, 332-337.

(3) Armand, M.; Tarascon, J.-M. Building Better Batteries. Nature 2008, 451, 652657.

(4) Tarascon, J. M.; Armand, M. Issues and Challenges Facing Rechargeable Lithium Batteries. Nature 2001, 414, 359-367.

(5) Aricò, A. S.; Bruce, P.; Scrosati, B.; Tarascon, J.; Schalkwijk, W. V. A. N.; Picardie, U. De; Verne, J.; Umr-, C. Nanostructured Materials for Advanced Energy Conversion and Storage Devices. 2005, 4.

(6) Yang, P.; Tarascon, J.-M. Towards Systems Materials Engineering. Nat. Mater. 2012, 11, 560-563.

(7) Kang, B.; Ceder, G. Battery Materials for Ultrafast Charging and Discharging. Nature 2009, 458, 190-193.

(8) Goodenough, J. B.; Kim, Y. Challenges for Rechargeable Li Batteries †. Chem. Mater. 2010, 22, 587-603. 
(9) Ji, L.; Lin, Z.; Alcoutlabi, M.; Zhang, X. Recent Developments in Nanostructured Anode Materials for Rechargeable Lithium-Ion Batteries. Energy Environ. Sci. 2011, 4, 2682.

(10) Whittingham, M. S. Lithium Batteries and Cathode Materials. Chem. Rev. 2004, $104,4271-4301$.

(11) Bruce, P. G.; Freunberger, S. a; Hardwick, L. J.; Tarascon, J.-M. Li-O2 and LiS Batteries with High Energy Storage. Nat. Mater. 2012, 11, 19-29.

(12) Lu, Y.; Tu, Z.; Archer, L. A. Stable Lithium Electrodeposition in Liquid and Nanoporous Solid Electrolytes. Nat. Mater. 2014, 13, 961-969.

(13) Zheng, G.; Lee, S. W.; Liang, Z.; Lee, H.-W.; Yan, K.; Yao, H.; Wang, H.; Li, W.; Chu, S.; Cui, Y. Interconnected Hollow Carbon Nanospheres for Stable Lithium Metal Anodes. Nat. Nanotechnol. 2014, 9, 618-623.

(14) Agrawal, A.; Choudhury, S.; Archer, L. A. A Highly Conductive, NonFlammable Polymer-nanoparticle Hybrid Electrolyte. RSC Adv. 2015, 5, 20800-20809.

(15) Kashiwagi, T.; Du, F.; Douglas, J. F.; Winey, K. I.; Harris, R. H.; Shields, J. R. Nanoparticle Networks Reduce the Flammability of Polymer Nanocomposites. Nat. Mater. 2005, 4, 928-933. 
(16) Monroe, C.; Newman, J. The Effect of Interfacial Deformation on Electrodeposition Kinetics. J. Electrochem. Soc. 2004, 151, A880.

(17) Monroe, C.; Newman, J. The Impact of Elastic Deformation on Deposition Kinetics at Lithium/Polymer Interfaces. J. Electrochem. Soc. 2005, 152, A396.

(18) Hallinan, D. T.; Mullin, S. a.; Stone, G. M.; Balsara, N. P. Lithium Metal Stability in Batteries with Block Copolymer Electrolytes. J. Electrochem. Soc. 2013, 160, A464-A470.

(19) Tu, Z.; Kambe, Y.; Lu, Y.; Archer, L. A. Nanoporous Polymer-Ceramic Composite Electrolytes for Lithium Metal Batteries. Adv. Energy Mater. 2014, $4, \mathrm{n} / \mathrm{a}-\mathrm{n} / \mathrm{a}$.

(20) Kanamura, K. Electrochemical Deposition of Very Smooth Lithium Using Nonaqueous Electrolytes Containing HF. J. Electrochem. Soc. 1996, 143, $2187-$ 2197.

(21) Aurbach, D.; Gamolsky, K.; Markovsky, B.; Gofer, Y.; Schmidt, M.; Heider, U. On the Use of Vinylene Carbonate (VC) as an Additive to Electrolyte Solutions for Li-Ion Batteries. Electrochim. Acta 2002, 47, 1423-1439.

(22) Chen, L.; Wang, K.; Xie, X.; Xie, J. Effect of Vinylene Carbonate (VC) as Electrolyte Additive on Electrochemical Performance of Si Film Anode for Lithium Ion Batteries. J. Power Sources 2007, 174, 538-543. 
(23) Guo, J.; Wen, Z.; Wu, M.; Jin, J.; Liu, Y. Vinylene carbonate-LiNO3: A Hybrid Additive in Carbonic Ester Electrolytes for SEI Modification on Li Metal Anode. Electrochem. commun. 2015, 51, 59-63.

(24) Xu, K.; Zhang, S.; Jow, T. R. LiBOB as Additive in LiPF[sub 6]-Based Lithium Ion Electrolytes. Electrochem. Solid-State Lett. 2005, 8, A365.

(25) Pieczonka, N. P. W.; Yang, L.; Balogh, M. P.; Powell, B. R.; Chemelewski, K.; Manthiram, A.; Krachkovskiy, S. a.; Goward, G. R.; Liu, M.; Kim, J. H. Impact of Lithium Bis(oxalate)borate Electrolyte Additive on the Performance of HighVoltage Spinel/graphite Li-Ion Batteries. J. Phys. Chem. C 2013, 117, 2260322612.

(26) Zhang, S. S. A Review on Electrolyte Additives for Lithium-Ion Batteries. $J$. Power Sources 2006, 162, 1379-1394.

(27) Pires, J.; Timperman, L.; Castets, A.; Santos-pena, J.; Dumont, E.; Levasseur, S.; Dedryvere, R.; Tessier, C.; Anouti, M. Role of Propane Sultone as Additive to Improve the Performance of Lithium-Rich Cathode Material at High Potential. RSC Adv. 2015.

(28) Li, B.; Xu, M.; Li, T.; Li, W.; Hu, S. Prop-1-Ene-1,3-Sultone as SEI Formation Additive in Propylene Carbonate-Based Electrolyte for Lithium Ion Batteries. Electrochem. commun. 2012, 17, 92-95. 
(29) Miao, R.; Yang, J.; Feng, X.; Jia, H.; Wang, J.; Nuli, Y. Novel Dual-Salts Electrolyte Solution for Dendrite-Free Lithium-Metal Based Rechargeable Batteries with High Cycle Reversibility. J. Power Sources 2014, 271, 291-297.

(30) Qian, J.; Henderson, W. a; Xu, W.; Bhattacharya, P.; Engelhard, M.; Borodin, O.; Zhang, J.-G. High Rate and Stable Cycling of Lithium Metal Anode. Nat. Commun. 2015, 6, 6362.

(31) Philippe, B.; Gorgoi, M.; Edstro, K. Improved Performances of Nanosilicon Electrodes Using the Salt LiFSI : A Photoelectron Spectroscopy Study. J. Am. Chem. Soc. 2013, 135, 9829-9842.

(32) Gunceler, D.; Letchworth-Weaver, K.; Sundararaman, R.; Schwarz, K. a; Arias, T. a. The Importance of Nonlinear Fluid Response in Joint Density-Functional Theory Studies of Battery Systems. Model. Simul. Mater. Sci. Eng. 2013, 21, 074005 .

(33) Ozhabes, Y.; Gunceler, D.; Arias, T. a. Stability and Surface Diffusion at Lithium-Electrolyte Interphases with Connections to Dendrite Suppression. arXiv 2015, 1504.05799, 1-7.

(34) Ding, F.; Xu, W.; Graff, G. L.; Zhang, J.; Sushko, M. L.; Chen, X.; Shao, Y.; Engelhard, M. H.; Nie, Z.; Xiao, J.; et al. Dendrite-Free Lithium Deposition via Self-Healing Electrostatic Shield Mechanism. J. Am. Chem. Soc. 2013, 135, $4450-4456$. 


\section{APPENDIX}

Supplementary Information for Chapter 10

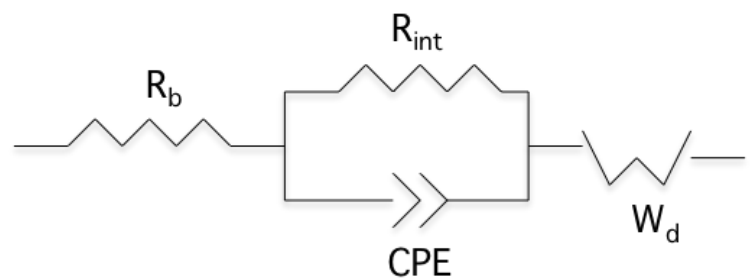

Supplementary Figure 10.1: Equivalent circuit: The impedance spectroscopy results are fitted with equivalent circuit model comprising of a bulk impedance, interfacial resistance and a solid-state diffusion element 

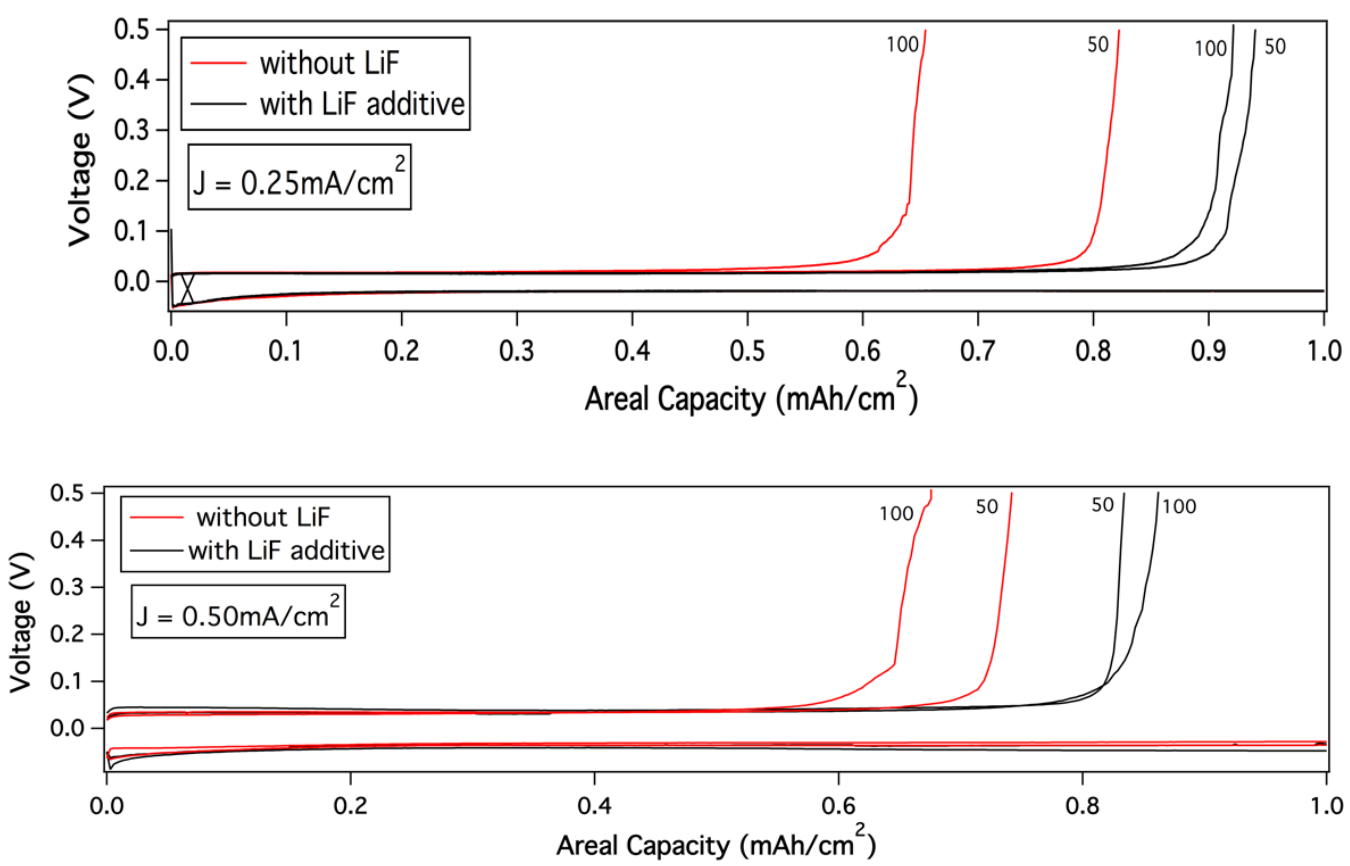

Supplementary Figure 10.2: Coulombic Efficiency Test: Voltage profiles for batteries with Li-Stainless Steel configuration comprising of neat electrolyte (shown in red) and with $0.5 \% \mathrm{LiF}$ additive (shown in black) at different conditions: (a) at current density of $0.25 \mathrm{~mA} / \mathrm{cm}^{2}$ for $1 \mathrm{mAh} / \mathrm{cm}^{2}$ capacity; (b) at current density of $0.50 \mathrm{~mA} / \mathrm{cm}^{2}$ for $1 \mathrm{mAh} / \mathrm{cm}^{2}$ capacity 

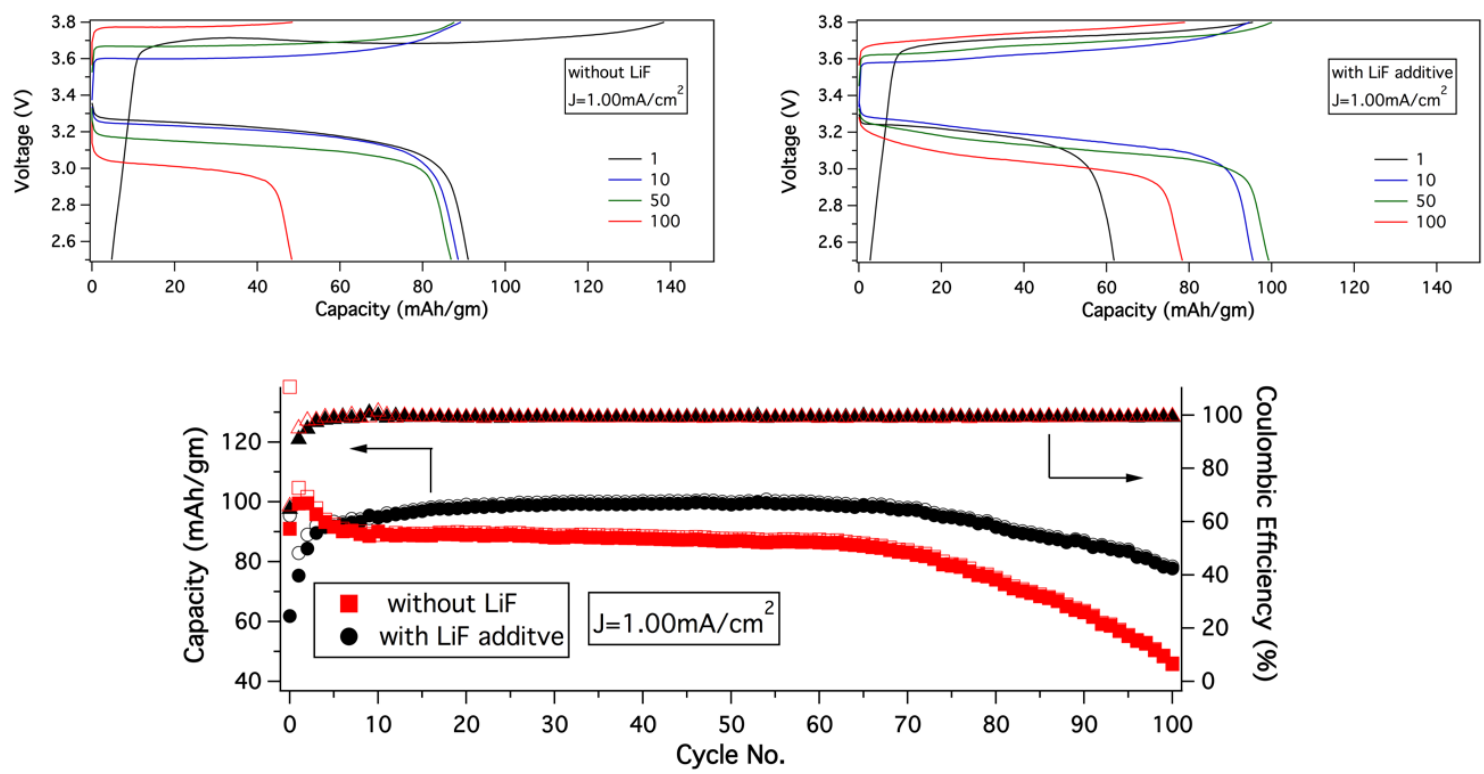

Supplementary Figure 10.3: Half-cell Test: Performance for batteries with Li$\mathrm{LiFePO}_{4}$ configuration comprising of neat electrolyte and $0.5 \% \mathrm{LiF}$ additive: (a) voltage profiles of batteries with neat electrolytes at current density of $1.00 \mathrm{~mA} / \mathrm{cm}^{2}$ for $1 \mathrm{mAh} / \mathrm{cm}^{2}$ capacity; (b) voltage profiles of batteries with LiF additive at current density of $1.00 \mathrm{~mA} / \mathrm{cm}^{2}$; (c) Cyclability of these batteries for 100 cycles 

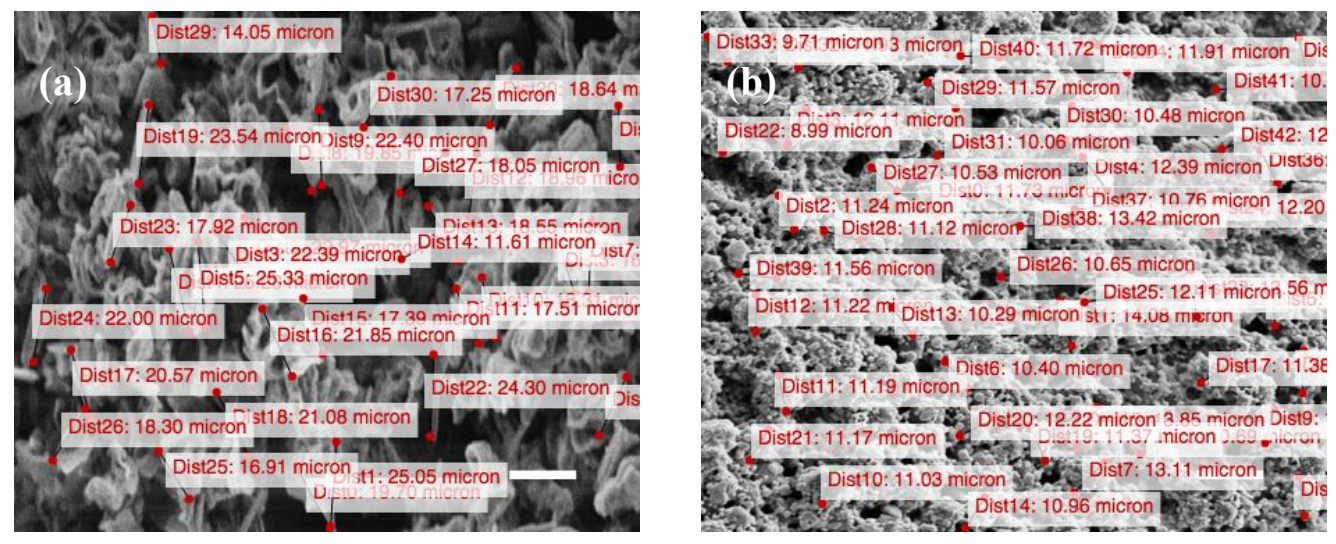

\section{Supplementary Figure 10.4: Post-mortem analysis of electrodeposited anode:}

Anode surfaces electrodeposited at $2 \mathrm{~mA} / \mathrm{cm}^{2}$, without and with $\mathrm{LiF}$ additive (see Figure $4 a, b)$ are analyzed to obtain the morphology of dendrites. (a) With neat electrolytes, the deposits are mostly conical or cylindrical with a mean length of $\sim 20 \mu \mathrm{m}$; (b) with LiF additive, the deposits are mostly spherulites with a mean diameter of $\sim 11.5 \mu \mathrm{m}$ 


\section{Chapter 11}

Designing Solid-liquid Interphases for Sodium Batteries 


\subsection{Abstract}

Secondary batteries based on earth-abundant sodium metal anodes are desirable for both grid-level, stationary storage and for portable electrical energy storage. Roomtemperature sodium metal batteries are impractical today because morphological instability during battery recharge leads to dendritic electrodeposition. Chemical instability of liquid electrolytes in contact with metallic sodium also leads to premature cell failure by depleting the electrolyte and electrode via parasitic reactions. Here we show by means of Joint Density-Functional Theoretical analysis that the surface diffusion barrier for ion transport across a metal/liquid interface is a sensitive function of the chemistry of solid-electrolyte interphase. In particular, we find that a sodium bromide interphase presents an exceptionally low energy barrier to ion transport, comparable to that of metallic magnesium, which can be recharged in liquid electrolytes without forming dendrites. We evaluate this prediction by means of electrochemical measurements and direct visualization studies. These experiments reveal an approximately three-fold reduction in activation energy for ion transport across the sodium bromide interphase. By means of direct visualization of sodium electrodeposition at planar interfaces and by electrochemical analysis we further show that the reduction in transport barrier at a sodium-bromine-liquid electrolyte interphase yields large improvements in stability of sodium deposition in liquid electrolytes.

\subsection{Introduction}

Rechargeable batteries based on lithium and sodium metal anodes are of interest for high-energy storage solutions in portable and stationary applications ${ }^{1,2}$. Although 
sodium-based batteries pre-date those based on lithium ${ }^{3}$, Li has received more recent attention for a variety of reasons, including its greater electronegativity, higher specific energy, low atomic radius ${ }^{4,5}$, and the commercial success of related Li-ion battery technology. The greater natural abundance of sodium and its availability in regions all over the world provide significant cost advantages over Li that have within the last decade helped re-ignite interest in Na-based batteries. ${ }^{6-8}$ Metallic sodium has other attractive features as a battery anode, including its relatively high electronegativity and low atomic weight, which combine to give the $\mathrm{Na}$ anode a specific capacity $\left(1166 \mathrm{mAh} \mathrm{gm}^{-1}\right)$ that is competitive with $\mathrm{Li}\left(3860 \mathrm{mAh} \mathrm{gm}^{-1}\right)$ in many applications. ${ }^{6}$ Additionally, recent studies have shown that rechargeable batteries that pair a $\mathrm{Na}$ anode with highly energetic $\mathrm{O}_{2}$-based cathodes are intrinsically more stable during discharge than their $\mathrm{Li}$ analogs because the species generated electrochemically in the cathode, the metal superoxide, is more stable when the anode is $\mathrm{Na}$, as opposed to $\mathrm{Li}^{9-10}$.

As with rechargeable batteries comprising Li metal anodes, the Achilles heel of the rechargeable sodium battery is the anode's susceptibility to failure during the charging process. Specifically, during battery recharge $\mathrm{Na}$ ions deposit in rough, low density and uneven patches on the negative electrode, even at current densities below the limiting current where classical instabilities such as electroconvection that drive rough, dendritic deposition are expected to be unimportant. ${ }^{11,12}$ Instead, dendrites on $\mathrm{Na}$ (and Li) arise from inhomogeneities in the resistance of the solid-electrolyte interphase (SEI), formed spontaneously on the anode surface when in contact with an electrolyte. The resultant concentration of electric field lines on faster growing regions 
of the interface drives the morphological instability loosely termed dendritites. ${ }^{12,13} \mathrm{At}$ later stages, uncontrolled dendritic deposition leads to metallic structures able to bridge the inter-electrode space, ultimately short-circuiting the cell. Short-circuits lead to two catastrophic failure mechanisms: (i) Thermal runaway which drives chemical reactions in the electrolyte, ending the cell life by fire, explosion or both ${ }^{12,14-16}$; and (ii) Melting and breakage of the dendrites, which electrically disconnects the material from the electrode mass ${ }^{4,17}$, causing rapid or gradual reduction in the storage capacity of the anode. Unlike $\mathrm{Li}$, where dendrite-induced short circuits are considered the dominant failure mode, chemical reaction between the electrolyte and metal anode are regarded as the most important mechanism of cell failure for batteries based on a $\mathrm{Na}$ anode. $\mathrm{Na}$ also has a lower melting point than $\mathrm{Li}$, which makes batteries based on $\mathrm{Na}$ more prone than their Li counterparts to failure by thermal runaway and/or dendrite breakage ${ }^{6,18,19}$.

Few studies have addressed the challenges associated with stabilizing a $\mathrm{Na}$ anode. ${ }^{18}$ In contrast, several approaches have been reported for preventing/retarding Li dendrite proliferation in Li metal batteries ${ }^{11,12}$. Some of the approaches include using high modulus electrolyte or nanoporous/tortuous separator ${ }^{14,20-22}$, modifying the ion transport in electrolytes by using single ion conductors and ionic liquids ${ }^{23-27}$, or forming a stable electrode-electrolyte interface to suppress the nucleation of dendrites $^{4,13,28-30}$. In addition to preventing dendrite induced short circuits, the last approach may impede unwanted parasitic reactions between the electrode and electrolyte that lead to formation of insulating products and loss of electrochemically active material, causing decay in the battery capacity with increasing charge-discharge 
cycles $^{12}$. A common approach for the formation of artificial SEI on the metal involves use of special electrolyte additives such as vinylene carbonate ${ }^{31,32}$, fluoroethylene carbonate $^{33}$, dioxane $\mathrm{e}^{34}$, sultones ${ }^{30,35}$, or functional ionic liquids ${ }^{7}$ which can electropolymerize on the surface of electrode to form an elastic coating that protects the metal surface and accommodate volume changes in the electrode during charge and discharge. There are also recent reports of protecting the electrode interface by direct formation of a barrier layer by deliberate reaction between electrodes and reactive species in electrolytes ${ }^{36-38}$. Various indirect methods have also been reported for stabilizing a Li anode during battery recharge. These include use of a functional nanoparticles ${ }^{6,11,23-24}$, and mixtures of salts (e.g. LiTFSI-LiFSI) ${ }^{39}$, use of concentrated electrolytes (e.g. 5M LiFSI in DME) ${ }^{40}$, or use of polysulfides and $\mathrm{LiNO}_{3}{ }^{29}$ as electrolyte additives. A common feature of these methods is that they produce lithium fluoride $(\mathrm{LiF})$ in the SEI. In recent studies ${ }^{13,22}$, direct incorporation of $\mathrm{LiF}$ as an additive in liquid electrolytes was reported to yield dramatic enhancements to battery lifetime in Li metal cells at both high an low current densities. Cui and co-workers ${ }^{18}$ were among the first to show that application of this concept to Na metal batteries, through electrolyte additives that generate sodium fluoride $(\mathrm{NaF})$, leads to markedly higher coulombic efficiencies (as high as $99 \%$ ) in $\mathrm{Na} \| \mathrm{Cu}$ cells.

With the specific aim of developing rational strategies for stabilizing the anode of $\mathrm{Na}$ batteries during cell recharge, we herein investigate how the chemistry of the SEI alters ion transport at Na- and Li-electrolyte interfaces by means of Joint DensityFunctional Theory (JDFT) calculations and experiment. Our focus on interfacial transport derives from the observation that magnesium metal anodes, which do not 
form uneven deposits under charging at currents below the limiting current ${ }^{41}$, present the lowest barriers for interfacial metal ion transport ${ }^{42}$. Remarkably, we find that a $\mathrm{Na}$ metal anode protected by $\mathrm{NaBr}$ presents a barrier of only $\sim 0.02 \mathrm{eV}$ per atom (i.e. comparable to $\mathrm{Mg}$ metal) for interfacial ion transport. By means of direct visualization studies and electrochemical analysis, we investigate the stability imparted to the $\mathrm{Na}$ electrode by a $\mathrm{NaBr}$ protective coating.

\subsection{Methods}

\subsubsection{Materials}

Sodium cubes, Bromo-propane, Chloro-propane, Iodo-propane, Propylene Carbonate, Ethylene Carbonate, Diglyme, Dimethoxyethane, Sodium Hexafluorophosphate, Magnesium(II) Bis(trifluoromethanesulfonyl)imide, were all purchased from Sigma Aldrich. Celgard 3501 separator was obtained from Celgard Inc. Glass fiber separator was bought from Whatman Inc. All the chemicals were used as received in after rigorous drying in a $\sim 0 \mathrm{ppm}$ water level and $<5 \mathrm{ppm}$ oxygen glove box; in order to make sure the sodium metal is not oxidized.

\subsubsection{Sodium Bromide and other halide coating formation}

Sodium-cube pieces were taken out of mineral oil and cleaned with kimwipes. Then with a sharp knife, thin slices of sodium pieces were cut before punching with $1 / 4$ th inch punch. For the coating of solid electrolyte interface, $15 \mu 1$ of Bromo-propane was added to the sodium electrode before drying in vacuum ante-chamber for five minutes. It is known that the reaction is instantaneous due to high reactivity of sodium metal. 
Further the by-product obtained by reaction- hexane is believed to vaporize rapidly in vacuum owing to its low boiling point characteristics. Coating with $\mathrm{NaCl}$ and $\mathrm{NaI}$ was done in exact same procedure, however, with Chloro-propane and Iodo-propane respectively.

\subsubsection{Physical characterization}

XPS was conducted using Surface Science Instruments SSX-100 with operating pressure of $\sim 2 \times 10^{-9}$ torr. Monochromatic Al K- $\alpha$ X-rays $(1486.6 \mathrm{eV})$ with beam diameter of $1 \mathrm{~mm}$ were used. Photoelectrons were collected at an emission angle of $55^{\circ}$. A hemispherical analyzer determined electron kinetic energy, using pass energy of $150 \mathrm{~V}$ for wide survey scans and 50V for high-resolution scans. Samples were ionetched using $4 \mathrm{kV}$ Ar ions, which were rastered over an area of $2.25 \times 4 \mathrm{~mm}$ with total ion beam current of $2 \mathrm{~mA}$, to remove adventitious carbon. Depth profile was obtained was obtained by ion etching at $2 \mathrm{kV}, 22 \mu \mathrm{A}$ over $2 * 3 \mathrm{~mm}$, which yielded an atch rate of approximately $5 \mathrm{~nm} \mathrm{~min}{ }^{-1}$. The etching was done for 395 minutes. Spectra were referenced to adventitious $\mathrm{C}$ 1s at $284.5 \mathrm{eV}$. CasaXPS software was used for XPS data analysis with Shelby backgrounds. Br $3 d$ was assigned to double peaks $\left(3 \mathrm{~d}_{5 / 2}\right.$ and $3 \mathrm{~d}_{3 / 2}$ ) for each bond with $1.05 \mathrm{eV}$ separation. Residual SD was maintained close to 1.0 for the calculated fits. Samples were exposed to air only during the short transfer time to the XPS chamber (less than 5 seconds).

XRD was carried out on a Scintag Theta-Theta X-ray diffractometer using $\mathrm{Cu} \mathrm{K}_{\alpha}$

radiation at $\lambda=1.5406 \AA$. All samples were covered with Kapton tape to ensure that 
the sodium metal is not oxidized in air. For the sodium metal samples after cycling, the symmetric cell of $\mathrm{NaBr}$ coated sodium was charged-and-discharged five times at a current density of $0.5 \mathrm{~mA} \mathrm{~cm} \mathrm{~cm}^{-2}$.

\subsubsection{Electrochemical characterization}

The impedance spectroscopy measurement ionic conductivity of electrolytes was measured as a function of temperature using a Novocontrol N40 Broadband Dielectric instrument. Symmetric cells were prepared using two sodium pristine metal pieces using the electrolyte $1 \mathrm{M} \mathrm{NaPF}_{6} \mathrm{EC} / \mathrm{PC}$ with a glass fiber separator. For understanding the impedance of halide based interfacial layer, both sodium pieces were coated with respective halides using the same method (as described for $\mathrm{NaBr}$-coating section) before performing the experiment. For the symmetric cell with $\mathrm{Mg}$ electrodes, the electrolyte $0.3 \mathrm{M} \mathrm{Mg}(\mathrm{TFSI})_{2}$ in $\mathrm{DME} /$ diglyme was used with a glass fiber separator. The measurements were done in a frequency range from $10^{-3}$ to $10^{7} \mathrm{~Hz}$.

\subsubsection{Scanning Electron Microscopy}

Postmortem characterization of the sodium metal electrodes was done to understand the morphology of sodium metal deposition and also failure mechanisms involved in short circuits. For this reason, the cells with symmetric sodium cells with or without $\mathrm{NaBr}$ layer was charged at a current density of $1 \mathrm{~mA} \mathrm{~cm} \mathrm{~cm}^{-2}$ for 2 hours before disassembling the cell inside the glovebox. The charged sodium metal pieces were washed with the electrolyte-solvent and were transferred carefully to the microscopy 
facility minimizing the exposure of sodium metal to atmosphere. The SEM analysis was done using the LEO155FESEM instrument.

\subsubsection{Focused Ion Beam/Scanning Electron Microscopy}

An FEI Strata 400 Focused Ion Beam (FIB) was used for the FIB/SEM experiments. The FIB is fitted with a Quorum PP3010T Cryo-FIB/SEM Preparation System that enables cryogenic experiments. For room temperature experiments, the sample was removed from an inert environment and loaded onto an aluminum stub. The stub was subsequently attached to a shuttle and placed in a loadlock that pumps down to vacuum before inserting into the FIB. For cryogenic experiments, the sample was removed from an inert environment, attached to a stub, and immediately plunged into slush nitrogen. This shortened exposure time and minimized any reactions with air or moisture. The sample was then transferred into the FIB in a transfer device at liquid nitrogen temperature and subsequently maintained at $-165^{\circ} \mathrm{C}$ in the cryo-FIB.

\subsubsection{In situ visualization studies}

The visualization experiment was carried out for understanding the in-operando observation of electrodeposition in sodium metal batteries. In all experiments the electrolyte- $1 \mathrm{M} \mathrm{NaPF}_{6}-\mathrm{EC} / \mathrm{PC}$ was used. For control experiments pristine sodium was used as both electrodes, while for understanding the role of stable SEI, $\mathrm{NaBr}$ coated sodium pieces were used. The sodium metal pieces were attached to current collectors and fixed in an air-tight cuvette-chamber as shown in Supplementary Figure 3. In these experiment, it is made sure that the electrodes are fully facing each other and 
then one electrode is continuously charged with a current density of $1 \mathrm{~mA} / \mathrm{cm}^{2}$. The electrode, being charged was monitored over time and images of sodium deposition at different intervals were captured from an optical microscope.

\subsubsection{Cell lifetime and failure studies}

Symmetric 2032 type $\mathrm{Na} \mid \mathrm{Na}$ coin cells with and without $\mathrm{NaBr}$ coating containing liquid electrolyte of $1 \mathrm{M} \mathrm{NaPF}_{6} \mathrm{EC} / \mathrm{PC}(1: 1 \mathrm{v} / \mathrm{v})$ inside an argon-filled glove box. The cells were evaluated using galvanostatic (strip-plate) cycling using a Neware CT-3008 battery tester. In the 'strip-plate' experiments, the batteries were repeatedly charged and discharged with each half-cycle 0.5 hours long. Failure was deduced from irregularities in the voltage profile as well as excessive increment in the overpotential indicating excessive formation of electrolyte by-products completely insulating the electrodes.

\subsubsection{Sulfur-PAN cathode cycling}

Galvanostatic measurements with the Sulfur-PAN composite cathode was done in a 2032-type coin cell comprising a sodium metal anode with and without the $\mathrm{NaBr}$ coating. Celgard 3501 polypropylene membranes were used as the separator. $40 \mu \mathrm{L}$ $1 \mathrm{M} \mathrm{NaClO}_{4}$ in a mixture ethylene carbonate (EC) and Propylene Carbonate (PC) (v:v $=1: 1)$ was used as the electrolyte. The cathode consisted of $70 \mathrm{wt} \%$ of the active material, $15 \mathrm{wt} \%$ of carbon black (Super-P Li from TIMCAL) as a conductivity aid, and $15 \mathrm{wt} \%$ of polymer binder (PVDF, polyvinylidene fluoride, Aldrich). A carboncoated aluminum foil used for current collector. The mass loading of the cathode was 
$\sim 0.85 \mathrm{mg}$ SPAN $\mathrm{cm}^{-2}$. Detailed synthesis of the PAN-Sulfur composite can be found in recent paper by Wei et al. ${ }^{52}$ Cell assembly was carried out in an argon-filled glovebox. The measurements were done in Neware CT-3008 battery tester.

\subsection{Results}

\subsubsection{Joint Density-Functional Theory (JDFT) study of SEI}

It has been argued that the concentration of electric field lines at protrusions on the electrode surface leads to non-uniform ion distribution and deposition rate, which serves to seed dendrites ${ }^{43}$. We have previously proposed through density-functional simulations that enhanced surface diffusion at the electrode-electrolyte interface could serve as a counter mechanism by smoothing protrusions on the surface and thus prevent formation of dendrites ${ }^{44-46}$.

To understand these effects in the context of the sodium anode, we simulated sodium adatoms on the surface of different passivated sodium electrodes using a similar methodology as described in detail in our previous work focused on density-functional calculations of transport barriers for halogenated SEI salt layers in lithium-metal batteries $^{44}$. The surface diffusion barrier is affected by the presence of the liquid electrolyte at the interface and its calculation is thus non-trivial. Regular densityfunctional theory can provide the total energy of a given configuration (or snapshot) at fixed atomic positions, but to accurately compute the free energy of a solid-liquid interface one must also sample the configuration space of the liquid. While this can be done by molecular-dynamics methods (example: $\mathrm{QM} / \mathrm{MM}$ ), such calculations are

computationally demanding, and to-date haven't been reported for the systems of 
interest. Joint density-functional theory ${ }^{45}$, which works with thermodynamic averages of the fluid variables, provides an economical alternative to molecular dynamics and provides direct access to free energies without the need for sampling.

We performed all electronic structure calculations with $\mathrm{JDFTx}^{46}$, an open-source implementation of joint density-functional theory. To account for the effect of the electrolyte, we used the nonlinear polarizable continuum model ${ }^{45}$ generalized to nonaqueous solvents ${ }^{47}$, which is taken to be acetonitrile in the present work. All calculations employ a plane-wave basis with a cutoff of 20 Hartrees, and we use ultrasoft pseudopotentials from GBRV library ${ }^{48}$.

Results for these calculations are reported in Figure 11.1. The binding energy of a $\mathrm{Na}$ adatom depends on where it binds onto the Na-halide surface (as shown in Figure 1a). For the smallest halide, F, the minimum energy position for the sodium adatom is directly on top of the fluoride-ion, we refer this site as the "anion site". Again, for F the saddle point on the diffusion path is in the middle of two neighboring anion sites, we call this middle point the "in-between site". Traversing down the periodic table, it is observed that with increasing anion size, the binding energy of the in-between-site becomes relatively closer to the anion-site, and eventually the saddle point becomes the minimum. This transition happens with $\mathrm{NaBr}$ and results in the lowest diffusion barrier for interfacial ion transport.

Comparing the surface diffusion barriers of $\mathrm{NaBr}$ with other sodium halide salts and lithium salts as well as pure elements, lithium, sodium and magnesium (Figure 11.1b) places our finding in perspective with recent theory-supported strategies for suppressing dendritic deposition at metal electrodes. It is notable that the diffusion 

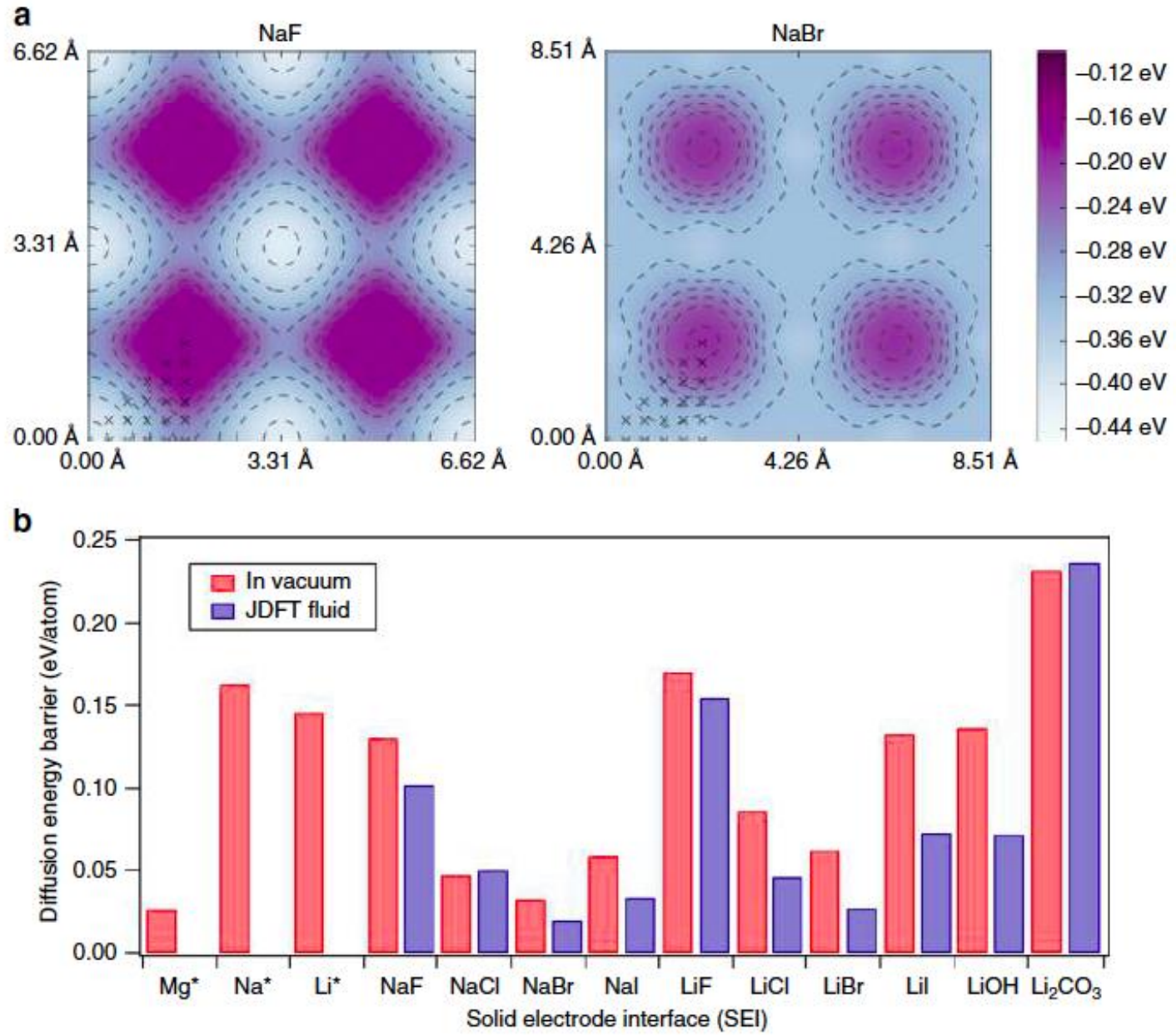

Figure 11.1 Surface diffusion barriers calculated using joint density functional theory. a) Surface binding energy vs. binding site for $\mathrm{NaF}$ (left) and $\mathrm{NaBr}$ (right) obtained from JDFT analysis of adatom diffusion. The entire contour plot is generated by symmetry using the data points indicated by cross symbols. b) Diffusion energy barriers computed for $\mathrm{Mg}, \mathrm{Na}$, and Li adatoms on surfaces with the chemistries noted. The red bars denote surface in contact with vacuum and blue bars indicate the same in presence of acetonitrile. The * symbol marks the data points obtained from ref. 42 
barrier for $\mathrm{NaBr}$ adatoms is substantially lower than for $\mathrm{NaF}$, and even in a liquid electrolyte is comparable to those computed for $\mathrm{Mg}$ in vacuum. Based on earlier reports that $\mathrm{LiF}$ coatings on $\mathrm{Li}$ metal dramatically stabilize electrodeposition of $\mathrm{Li}^{13,22}$, and that $\mathrm{NaF}$ coatings on $\mathrm{Na}$ has a similar large-stabilizing effect on $\mathrm{Na}$ deposition ${ }^{18}$, we hypothesize that a $\mathrm{Na}$ anode protected by a coating of $\mathrm{NaBr}$ would be particularly attractive for room-temperature sodium batteries employing liquid electrolytes.

\subsubsection{Formulation and stability a NaBr-based SEI layer on sodium metal}

To evaluate the JDFT prediction we first developed a method for uniformly coating $\mathrm{NaBr}$ on a $\mathrm{Na}$ metal electrode. Unlike previous experiments, where the source of halide salts in SEI layer of anode is degradation of active materials ${ }^{18,39,40}$ or precipitation of a poorly soluble electrolyte salt additive ${ }^{13,22}$, we here employ a wellknown chemical reaction to create a layer of $\mathrm{NaBr}$ at the interface. Specifically, we carried out a reaction of the sodium metal anode with bromopropane to undergo Wurtz reaction as illustrated in the Figure 11.2a. This reaction is widely used for production of symmetric alkanes, with the side product being a sodium halide. In the present case, along with $\mathrm{NaBr}$, hexane is formed, which is removed by evaporation. X-ray diffraction (XRD) analysis (Figure 11.2b) for pristine and treated sodium metal confirms that crystalline $\mathrm{NaBr}$ is formed on the surface of the $\mathrm{Na}$ electrode surface. The morphology of the $\mathrm{NaBr}$ layer was interrogated using survey scanning electron microscopy (SEM) for different exposure times of sodium metal piece with 1bromopropane. This exposure time corresponds to the time the sodium metal piece was dipped into the 1-bromopropane liquid (which we define that as the nominal 

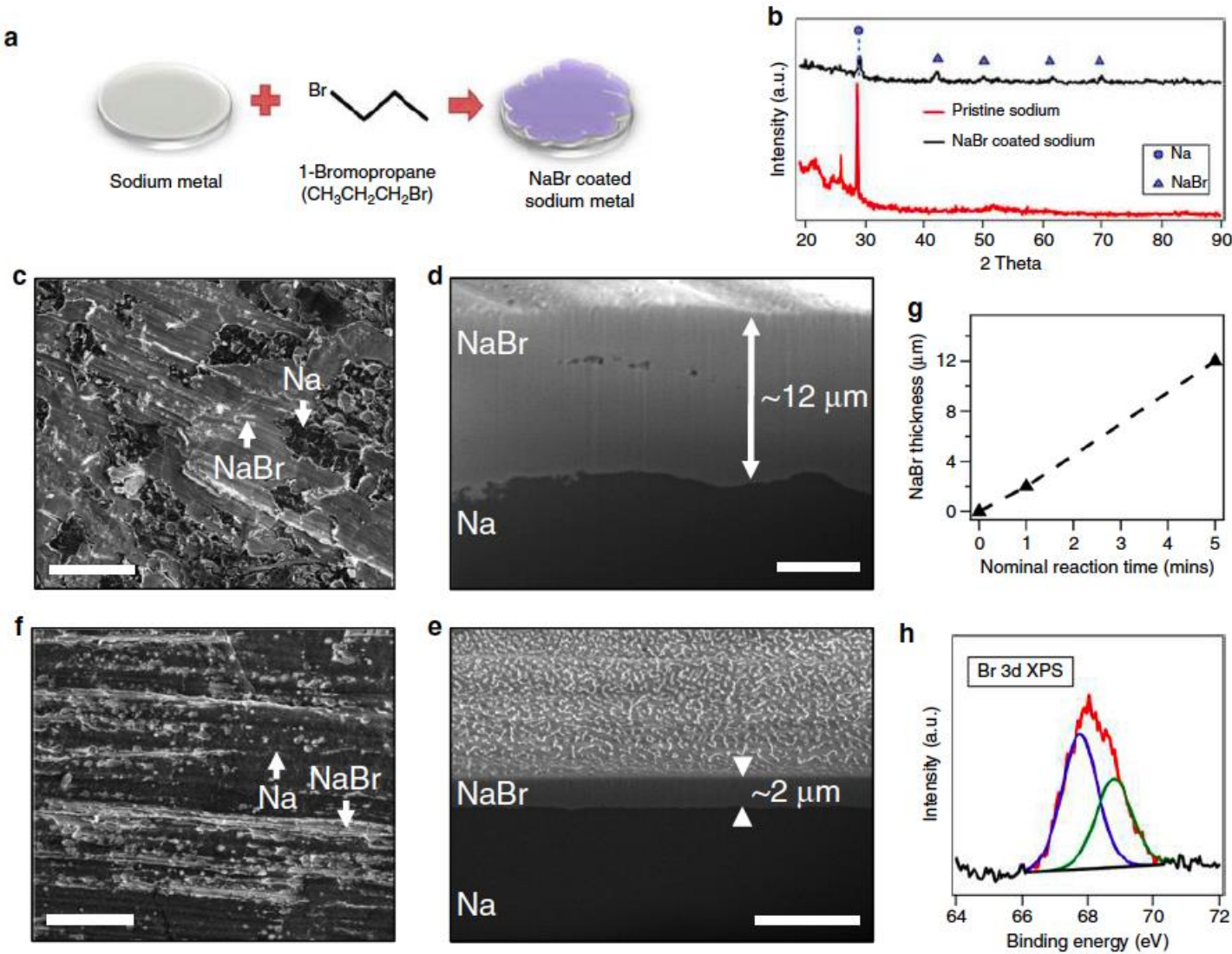

Figure 11.2 Formulation and characterization of sodium bromide layer. a) Schematic showing the procedure used to coat $\mathrm{Na}$ with $\mathrm{NaBr}$. b) XRD analysis of pristine and $\mathrm{NaBr}$-coated sodium showing that $\mathrm{NaBr}$ exists in crystalline form in the coatings on $\mathrm{Na}$. c) Cryo-SEM image of $\sim 12 \mu \mathrm{m}$ thick $\mathrm{NaBr}$ coating on sodium metal surface. Light regions are $\mathrm{NaBr}$ coating and dark regions are sodium metal, as confirmed by EDX; scale bar, $200 \mu \mathrm{m}$. d) Cryo-SEM image of a cross section through the $\mathrm{NaBr}$ coating obtained by focused ion beam milling under cryogenic conditions. Cross-sectional SEM imaging was used for layer thickness analysis, and the layer composition was confirmed by EDX mapping; scale bar, $5 \mu \mathrm{m}$. e, f) Complementary images and scale bars as $(\mathrm{d}, \mathrm{c})$, respectively, but for a $\mathrm{Na}$ substrate exposed to 1bromopropane for $1 \mathrm{~min}$. A thinner $(2 \mu \mathrm{m}) \mathrm{NaBr}$ coating is formed in this case. $\mathbf{g}$ ) Thickness of the NaBr-based SEI layer on sodium metal at various nominal reaction times. h) X-ray photoelectron spectrum centered on the $\mathrm{Br} 3 \mathrm{~d}$ bands confirms the existence of metallic bromide bond 
reaction time, though the actual time of reaction is difficult to quantify). Figures $11.2 \mathrm{c}$ and $11.2 \mathrm{f}$ show the SEM images of the sample surface for exposure times of $5 \mathrm{~min}$ and 1 min, respectively. Figure $11.2 \mathrm{c}$ clearly shows large regions of a dense, smooth deposit of $\mathrm{NaBr}$ on $\mathrm{Na}$ that is interspersed with smaller, less well-coated regions as confirmed by EDX. In contrast, fewer well-coated regions are seen in Figure 11.2f. More in-depth information about the Na electrode coatings was obtained using cryofocused ion beam-scanning electron microscopy (cryo-FIB-SEM) and the results are shown in Figures 11.2d and 11.2e after 5 and 1 mins of treatment of the electrodes with 1-bromopropane, respectively. The thicknesses and depth-dependent composition of the coating layers were determined by SEM imaging and energy dispersive X-ray (EDX) mapping (Supplementary Figure 11.1a) of cross sections produced by FIB milling. The EDX element mapping confirms that the top layer is predominantly $\mathrm{Br}$, while the bottom comprises of essentially pure $\mathrm{Na}$ metal. The thickness of the $\mathrm{NaBr}$ layer is plotted as a function of nominal reaction time in Figure 11.2g. For further studies, $\mathrm{NaBr}$ coated sodium samples with nominal reaction times between 1 to 5 mins were utilized. X-ray photoelectron spectroscopy (XPS) of the $\mathrm{Br} 3 d$ peaks was used to more carefully analyze the bromine containing compounds in contact with the sodium metal surface. A high-resolution scan was performed after $45 \mathrm{~s}$ sputtering to remove any oxide layer that may form when transferring samples to the XPS chamber. As shown in Figure 11.2h, two deconvoluted peaks for $\mathrm{Br} 3 d 5 / 2$ and $3 / 2$ at $68.8 \mathrm{eV}$ and $67.7 \mathrm{eV}$ respectively are observed, which correspond to the predominant presence of metallic-bromide bonds on the sodium surface ${ }^{49-51}$. 
The effectiveness of the $\mathrm{NaBr}$ coating in protecting sodium can be most easily evaluated by comparing SEM images of the pristine (Figure 11.3a) and $\mathrm{NaBr}$ protected (Figure 11.3b) Na electrodes following brief air exposure during transfer to FIB/SEM chamber at room temperature. The former is seen to be covered with a porous oxide layer, which is entirely absent from the $\mathrm{NaBr}$-coated $\mathrm{Na}$. The oxide layer on the pristine sodium surface was $\sim 5 \mu \mathrm{m}$ thick, as seen from the cross-sectional image in Supplementary Figure $11.1 \mathrm{~b}$. The stability of the $\mathrm{NaBr}$ interphase layer during electrochemical cycling was characterized by post-mortem analysis of the $\mathrm{Na}$ anode after five cycles of charge and discharge in a symmetric cell at a fixed current density of $0.5 \mathrm{~mA} \mathrm{~cm}^{-2}$. Figure $11.3 \mathrm{c}$ shows that the $\mathrm{NaBr}$ crystal structure is retained, confirming that the anode-protection mechanism is sustained. Results from SEM analysis of the cycled anodes are reported in Figure 11.3d. The surface morphology is seen to remain relatively flat and compact. XPS analysis for the $\mathrm{Br} 3 d$ peaks (inset of Figure 11.3e) was further performed on the sodium sample with $\mathrm{NaBr}$ coating after cycling. The depth profile was obtained by etching the surface at $2 \mathrm{kV}, 2 \mu \mathrm{A}$ over an area of $2 \mathrm{~mm} \times 3 \mathrm{~mm}$, at a rate of $5 \mathrm{~nm} \mathrm{~min}^{-1}$ for $395 \mathrm{mins}$. It is seen from Figure 3e that the $\operatorname{Br} 3 d$ atomic content decreases for the first 167 mins, followed by a steady state $\mathrm{Br} 3 d$ atomic content; this indicates that at least $2 \mu \mathrm{m}$ thick $\mathrm{NaBr}$ layer is retained even after cycling. The surface atomic composition of the cycled sample is deduced from both EDX mapping and XPS analysis (prior to etching) as seen in Supplementary Figure 11.2. In both cases, the $\mathrm{Br}$ element is seen to co-exist with other elements (Phosphorus, Fluorine, Carbon, Oxygen), typical for degradation of the EC/PC NaPF 6 electrolyte. It is also seen that with the exception of carbon, the atomic compositions 


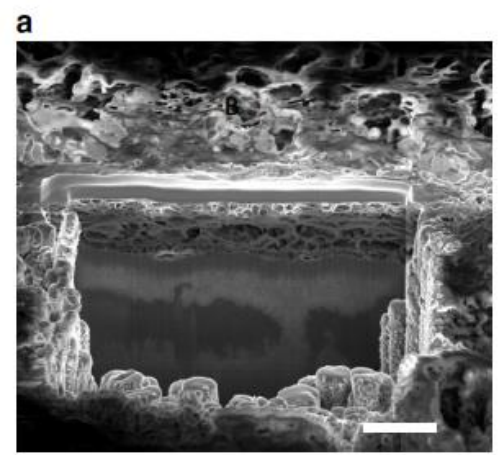

C

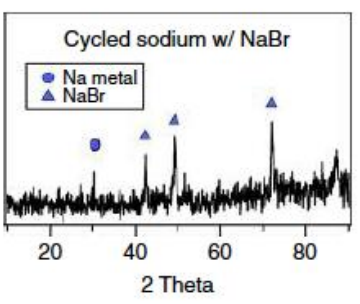

d b
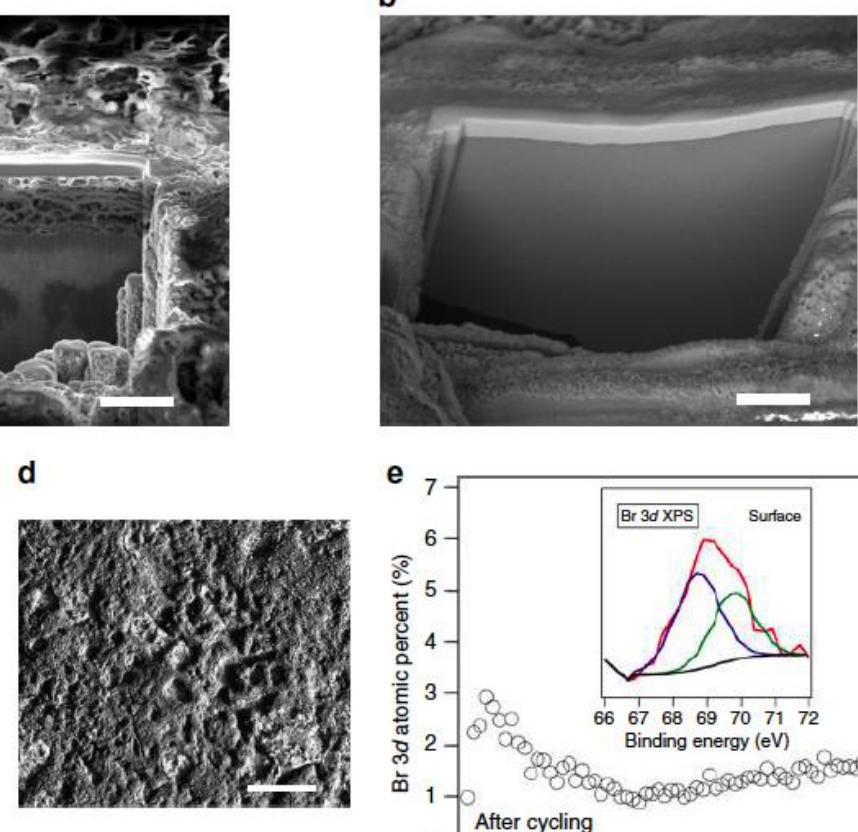

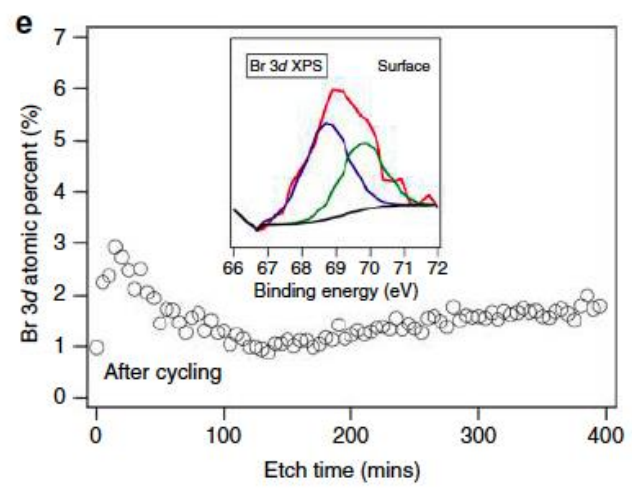

Figure 11.3 Air sensitivity and stability of sodium bromide interphase. a Milled region on pristine sodium metal obtained using focused ion beam milling at room temperature. The porous layer indicates severe oxidation of sodium during sample transfer; scale bar, $5 \mu \mathrm{m}$. b Same as a but with $\mathrm{NaBr}$ coating. In both $(\mathrm{a}, \mathrm{b})$ the thin top solid layer is a platinum protective coating, deposited inside the FIB prior to milling. c XRD showing intensity of $\mathrm{Na}$ metal and $\mathrm{NaBr}$ peaks for sodium anode with $\mathrm{NaBr}$ coating and after cycling at $0.5 \mathrm{~mA} \mathrm{~cm}-2$ for five times in a symmetric sodium cell. $\mathbf{d}$ SEM image of NaBr-coated sodium metal anode after cycling; scale bar, $200 \mu \mathrm{m}$. e Depth profiling of cycled anode obtained by ion etching and XPS measurements. The atomic content of $\mathrm{Br} 3 \mathrm{~d}$ is shown as a function of etch time. The etching rate is $\sim 5 \mathrm{~nm} / \mathrm{min}$. The inset shows the Br $3 \mathrm{~d}$ XPS result for the cycled sodium anode surface (before etching) 
deduced from the two techniques are comparable.

Quantitative assessment of interfacial transport of $\mathrm{Na}$ ions in sodium halide coatings was made using impedance spectroscopy. These experiments were performed using symmetric sodium cells with/without halide salt coatings on $\mathrm{Na}$ and, for comparison, symmetric magnesium cells. Figures $11.4 \mathrm{a}$ and $11.4 \mathrm{~b}$ reports Nyquist plots at different temperatures for pristine sodium and $\mathrm{NaBr}$ coated sodium metal symmetric cells, respectively. By fitting the Nyquist plots to an equivalent circuit model (Supplementary Figure 11.3) it is possible to deduce from the data the bulk resistance $\left(\mathrm{R}_{\mathrm{b}}\right)$, representing ion transport in the electrolyte, and two interfacial resistances $\left(\mathrm{R}_{\mathrm{int}}\right.$ and $\mathrm{R}_{\text {int2 }}$ ) representing ion transport through the passivating layer on $\mathrm{Na}$ as well as electronic transport. The temperature dependence of the interfacial ion conductivity can be used to extract information about how the halide coating alters the energy barrier for transport. The reciprocal of bulk impedance and net interfacial resistance are plotted with temperature in Arrhenius form as in Figure 11.4c. The temperaturedependent analogs of these plots for $\mathrm{NaCl}, \mathrm{NaI}$ coated sodium as well as that of $\mathrm{Mg}$ are provided in the Supplementary Figure 11.4. The lines through the data in Figure $11.4 \mathrm{c}$ are fits obtained using the Vogel-Fulcher-Tammann (VFT) formula ${ }^{1}, \sigma=A$ $\exp \left(-E_{a} / R\left(T-T_{o}\right)\right)$, commonly used for modeling ion transport in liquid electrolytes. Here $A$ is the prefactor, $E_{a}$ is the apparent activation energy for ion transport, $R$ is the universal gas constant and $T_{o}$ is the reference temperature. The respective VFT coefficients for all materials used in the study are tabulated in Supplementary Table 11.1. It is seen that the bulk impedance for sodium cells utilizing pristine and halide 
coated sodium are similar, indicating that such coatings have at best a minimal effect on ion transport in the liquid electrolyte. In contrast, the interfacial conductivity and its temperature dependence are seen to be very sensitive to the chemistry of the SEI. Figure $11.4 \mathrm{c}$ for example shows that whereas $1 / \mathrm{R}_{\text {int }}$ for pristine $\mathrm{Na}$ is higher than for the NaBr-coated material, it decreases more rapidly with temperature. This latter behavior can be captured in terms of the apparent activation energy $E_{a}$ for interfacial ion transport, which is reported in Figure 11.4d for various pristine and halide-coated sodium electrodes, as well as for $\mathrm{Mg}$. It is observed that $E_{a}$ for the pristine sodium $\left(\sim 0.175 \mathrm{eV}\right.$ atom $\left.^{-1}\right)$ is higher by a factor of around 3 than the corresponding $\mathrm{NaBr}-$ or $\mathrm{NaCl}$-coated metal. This means that at any temperature transport of $\mathrm{Na}$ ions is 20 times or faster in a SEI composed of $\mathrm{NaBr}$ or $\mathrm{NaCl}$, in comparison to the SEI formed spontaneously at the pristine $\mathrm{Na}$ electrode. These experiments also reveal that the apparent interfacial activation energy for the $\mathrm{Mg}$-symmetric cell $\left(\sim 0.02 \mathrm{eV}\right.$ atom $\left.^{-1}\right)$ is around 10-times lower than for pristine $\mathrm{Na}$, although the value of interfacial resistance for Mg is two orders of magnitude higher. As illustrated in Supplementary Figure 11.5, these conclusions are broadly insensitive to the model (VFT or Arrhenius) used to extract $E_{a}$ from the temperature-dependent electrochemical impedance measurements.

A lower $E_{a}$ for a halide-rich SEI on Na means that at any current density deposition of the ions at the $\mathrm{Na}$ interface is less restricted. This result is consistent with the earlier prediction based on our JDFT analysis and is interpreted here to mean that a low diffusion energy barrier for halide adatom transport contributes to the low interfacial 
a

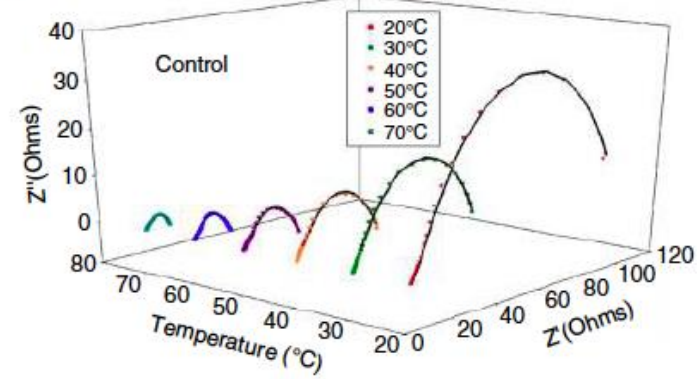

C

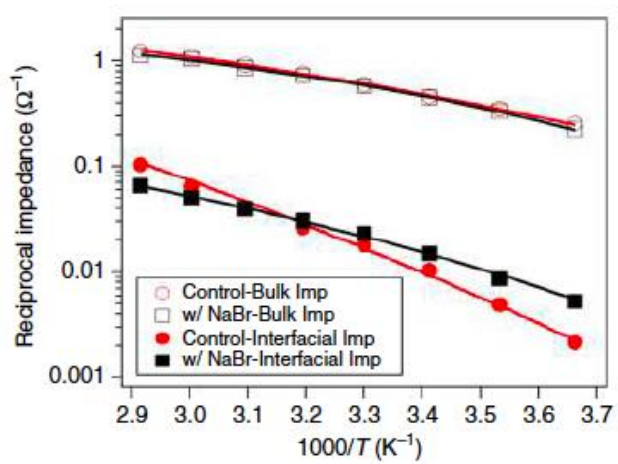

b

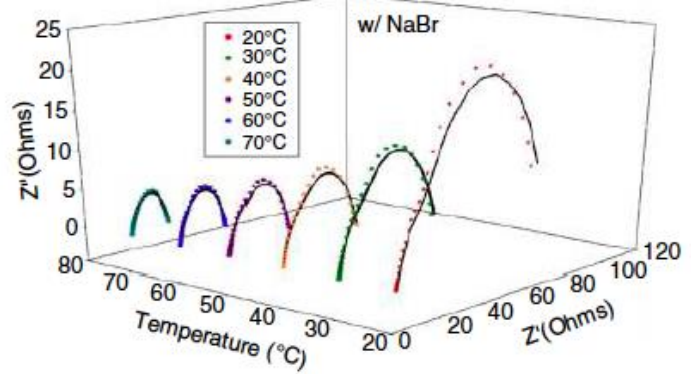

d

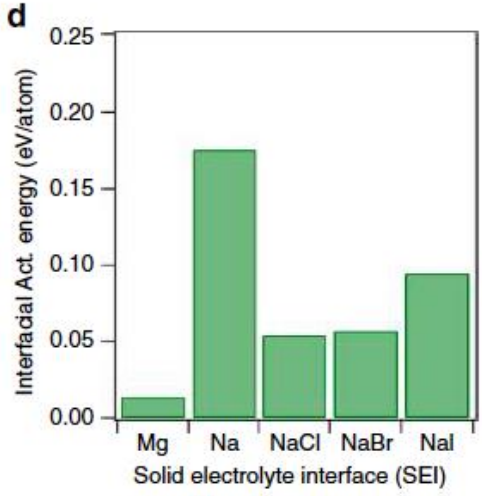

Figure 11.4 Electrochemical impedance spectroscopy analysis temperature-dependent Nyquist plots for a symmetric sodium cell with (a) pristine sodium and (b) $\mathrm{NaBr}$ coated sodium. $\mathbf{c}$ Reciprocal bulk and interface impedance as a function of reciprocal temperature; the lines are VFT model fits. $\mathbf{d}$ The apparent interfacial activation energy obtained from VFT fits of the temperature-dependent reciprocal resistance for various interphase chemistries. Here, $\mathrm{Na}$ and $\mathrm{Mg}$ symbolize results from symmetric cell studies with pristine sodium and magnesium electrodes, while $\mathrm{NaCl}, \mathrm{NaBr}, \mathrm{NaI}$ are for the corresponding salt-coated $\mathrm{Na}$ electrodes 
activation energy measured here. Additionally, the fact that we experimentally capture both the trends with Na-halide salts and the dramatically lower $E_{a}$ values for $\mathrm{Mg}$ electrolyte interphases predicted by the JDFT analysis implies that the diffusion barrier to adatom transport dominates the overall energy barrier to ion transport at the solid-electrolyte interphase. Comparison of the $E_{a}$ values in bulk electrolyte and at interphases composed of halide salts (see Supplementary Table 11.1) indicates that there is only a modest change in the barrier for ion movement as ions leave the bulk electrolyte and cross the electrolyte-electrode interface during deposition, which would be expected to favor more stable deposition. This inference is confirmed by the fact that the difference in energy barrier is lowest for Mg cells, which do not form dendrites.

\subsubsection{Electrodeposition of sodium metal with $\mathrm{NaBr}$ coated anode}

It is known that sodium metal is more reactive than lithium ${ }^{6}$, thus in contact with a liquid electrolyte it is expected to fail more easily by the first of the three mechanisms discussed in the introduction. Protecting the $\mathrm{Na}$ surface with a coating like $\mathrm{NaBr}$ that does not increase the barrier for $\mathrm{Na}$ ion transport at the interface should therefore inhibit failure by this mechanism. Additionally, since rough deposition is triggered by formation of an inhomogeneous SEI, cell failure by dendrite-induced short circuits should also be lower. To evaluate these statements, we directly visualize the surface of the $\mathrm{Na}$ anode with/without $\mathrm{NaBr}$ coatings during electrodeposition and quantify the growth of surface roughness as a function of time. A symmetric cuvette-type optical cell (see Supplementary Figure 11.6) was used for this component of the study. In a

typical experiment, the cell is polarized with a fixed current density of $1 \mathrm{~mA} \mathrm{~cm}{ }^{-2}$ and 
the morphology of the electrode-surface viewed in an up-right Olympus optical microscope outfitted with $6.10 \mathrm{~mm}$ Extra Long Working Distance (EWLD) 10X objectives. Videos of the visualization-experiment are provided (with a 300X speed). Figure 11.5a reports images obtained from these measurements at discrete time points separated by a fixed 10-minute interval. It is observed that a pristine $\mathrm{Na}$ electrode is prone to form moss-like dendritic structures. In comparison, the $\mathrm{NaBr}$ coated sodium metal is seen to electrodeposit without the formation any dendritic structure; however, it is seen that the overall volume (thickness) of the electrode is increasing over time due to the deposition under the $\mathrm{NaBr}$ coating. Figure $11.5 \mathrm{~b}$ plots the tip length of the dendrites over time. It is seen that for the pristine $\mathrm{Na}$ electrode, dendrites begin to grow immediately upon imposition of the current and grow throughout the electrode surface. The number density and reactivity of dendrites can be estimated from the brightness of the respective images. Reaction of $\mathrm{Na}$ with electrolyte is known to cause the metal to lose its shiny appearance. This, along with the much greater number density of dendritic structures is the source of the darker appearance of the images obtained using the pristine $\mathrm{Na}$ electrode. Figure $11.5 \mathrm{c}$ reports the comparative Lightness $\left(\mathrm{L}^{*}\right)$ of different spots on the sodium metal for both cases. Here, Lightness $\left(\mathrm{L}^{*}\right)$ is defined as the relative brightness of a spot, such that a white spot would correspond $\mathrm{L}^{*}$ of 100 and that of a black spot would be zero. $\mathrm{L}^{*}$ of pristine sodium drops down close to zero within 1 min of electrodeposition, while that of sodium with $\mathrm{NaBr}$ maintains $\mathrm{L}^{*}>90$ for entire time of measurement. It is observed that the reduction in brightness (or synonymously formation of undesired by-products) is synchronous to the growth of dendrite-like structure. It can be hypothesized that the 
a

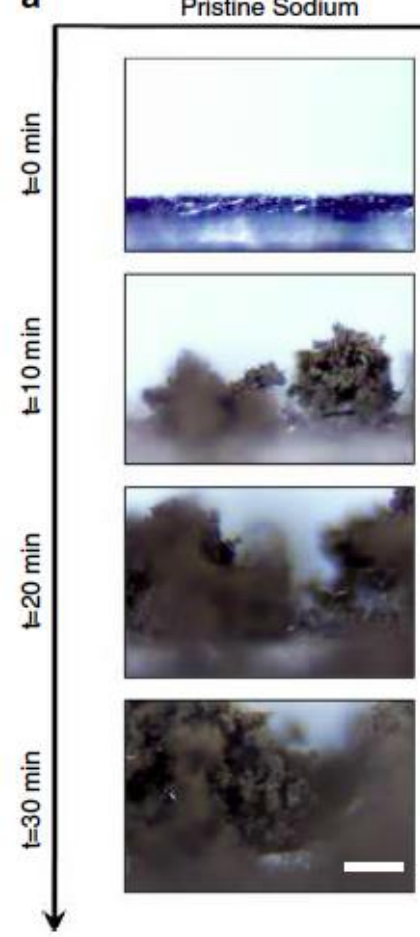

Sodium w/ $\mathrm{NaBr}$
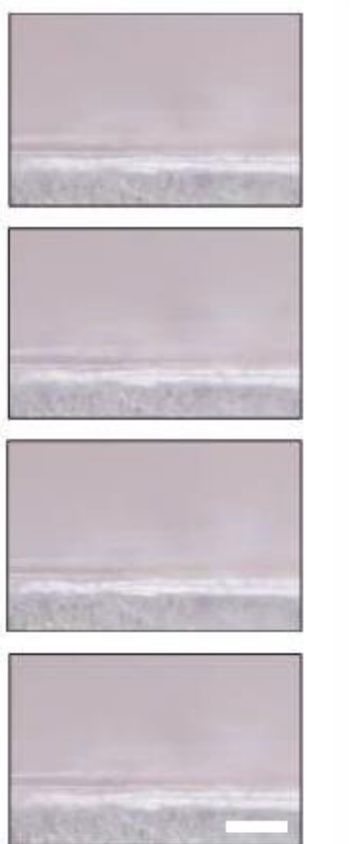

b

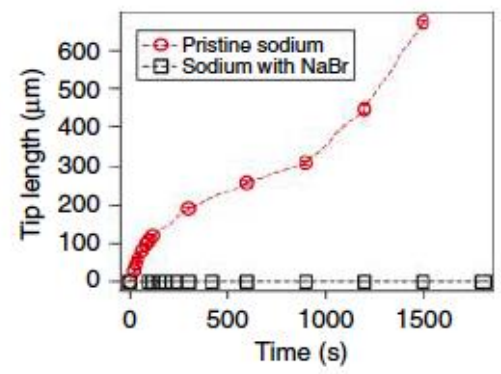

C

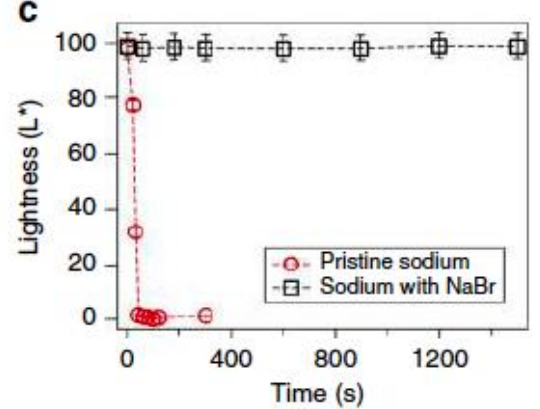

Figure 11.5 Visualization of sodium electrodeposition. a Snapshots from video microscopy of the pristine $\mathrm{Na}$ - (left column) and $\mathrm{NaBr}$-coated $\mathrm{Na}$ - (right column) electrolyte interface at $0,10,20$ and $30 \mathrm{~min}$ (top to bottom) after onset of $\mathrm{Na}$ deposition at $\mathrm{J}=1 \mathrm{~mA} \mathrm{~cm}-2$; scale bar, $100 \mu \mathrm{m}$. b Na dendrite tip length as a function of time. c Relative brightness $\left(\mathrm{L}^{*}\right)$ of electrolyte near the electrode-electrolyte interface as a function of time. This variable captures the obvious darkening of the $\mathrm{Na}$ deposits in the pristine case and the complete absence of this effect for the $\mathrm{NaBr}$ coated electrodes. In parts $(b, c)$, the error bars represent the standard deviation of measurements taken at different points in the same image 
distribution of the insulating products is heterogeneous, which leads to a nonuniformity of local current density on the electrode surface that ultimately causes the formation of rough and needle-like structures. These dendrites further increment the local current densities due to their sharp edges causing a cascade of instabilities.

Figure 11.6 reports the electrochemical performance of sodium-metal cells in different configurations. To simulate the performance of a working $\mathrm{Na}$ metal battery, a symmetric Na coin cell was cycled galvanostatically at various fixed current densities of $0.25 \mathrm{~mA} \mathrm{~cm}^{-2}, 0.5 \mathrm{~mA} \mathrm{~cm}{ }^{-2}$ and $1.0 \mathrm{~mA} \mathrm{~cm}^{-2}$ and the voltage profile is reported as a function of time in Figure 11.6a, b and c respectively. For all the different current density values, the voltage profiles are flat. The voltage hysteresis, of the sodium metal plating and stripping, represented by the mid-voltage value of charge-anddischarge, is plotted in Figure 11.6d. On comparing results for pristine and $\mathrm{NaBr}$ coated $\mathrm{Na}$ electrodes (in Figure 11.6b, d), it is seen that for cell with pristine $\mathrm{Na}$, the voltage diverges to $>0.5 \mathrm{~V}$ after 30 hours of cycling, meaning that the effective interfacial resistance is $>15 \mathrm{k}^{\prime} \Omega$. In contrast, the cell comprising $\mathrm{NaBr}$-coated $\mathrm{Na}$ is stable for at least 250 hours with minimal rise in cell voltage and hence effective resistance. The divergence in cell resistance is most likely a result of formation of a thick and insulating SEI layer; such an observation with pristine sodium anodes complements the result obtained from visualization experiments explained in Figure 11.5. However, it is remarkable that with the $\mathrm{NaBr}$ coating the overpotential is nearly constant for 250 hours, indicating that the $\mathrm{NaBr}$ coating produces nearly a 10 -fold improvement in the cell lifetime. 
Post-mortem analysis of polarized sodium anodes was done using SEM after continuously electrodepositing at the rate of $1 \mathrm{~mA} \mathrm{~cm} \mathrm{~cm}^{-2}$ for 2 hours. Figure $11.6 \mathrm{e}$ shows the morphology of the sodium metal with and without $\mathrm{NaBr}$ coating. It is seen that the pristine $\mathrm{Na}$ electrode develops a non-uniform surface with few protruding sharp structures compared to a relatively smooth surface for the $\mathrm{NaBr}$-coated electrode. It is important to note that in contrast to visualization experiments the general electrodeposition is more uniform, which can be attributed to the stabilizing effect of compression forces exerted by the separator on $\mathrm{Na}$ electrodes in the coin cells.

Finally, the effectiveness of the $\mathrm{NaBr}$-coated $\mathrm{Na}$ metal was evaluated in a full $\mathrm{Na} \| \mathrm{S}$ electrochemical cell, comprising a sulfur-polyacrylonitrile composite (SPAN) cathode. In this cathode, molecular sulfur is covalently trapped in a PAN framework, which has been reported to completely eliminate polysulfide dissolution and shuttling effects with carbonate based solvents in lithium-sulfur cells ${ }^{52}$. However, unlike their $\mathrm{Li}$ counterparts, the anodes in Na||SPAN cells have been reported to develop "black mossy dendrites" within a few cycles, which results in an unstable voltage profile during the cell recharge and low coulombic efficiency ${ }^{53}$. This effect is apparent in the inset to Figure 11.6g for the Na||SPAN cells based on pristine Na anodes. The lower coulombic efficiency measured in Na||SPAN cells employing pristine $\mathrm{Na}$ as anode is also observed from Figure 11.6f. Because the reactivity of sodium with electrolytes increases with voltage, we conclude that $\mathrm{Na}|| \mathrm{SPAN}$ cell configuration provides a more 

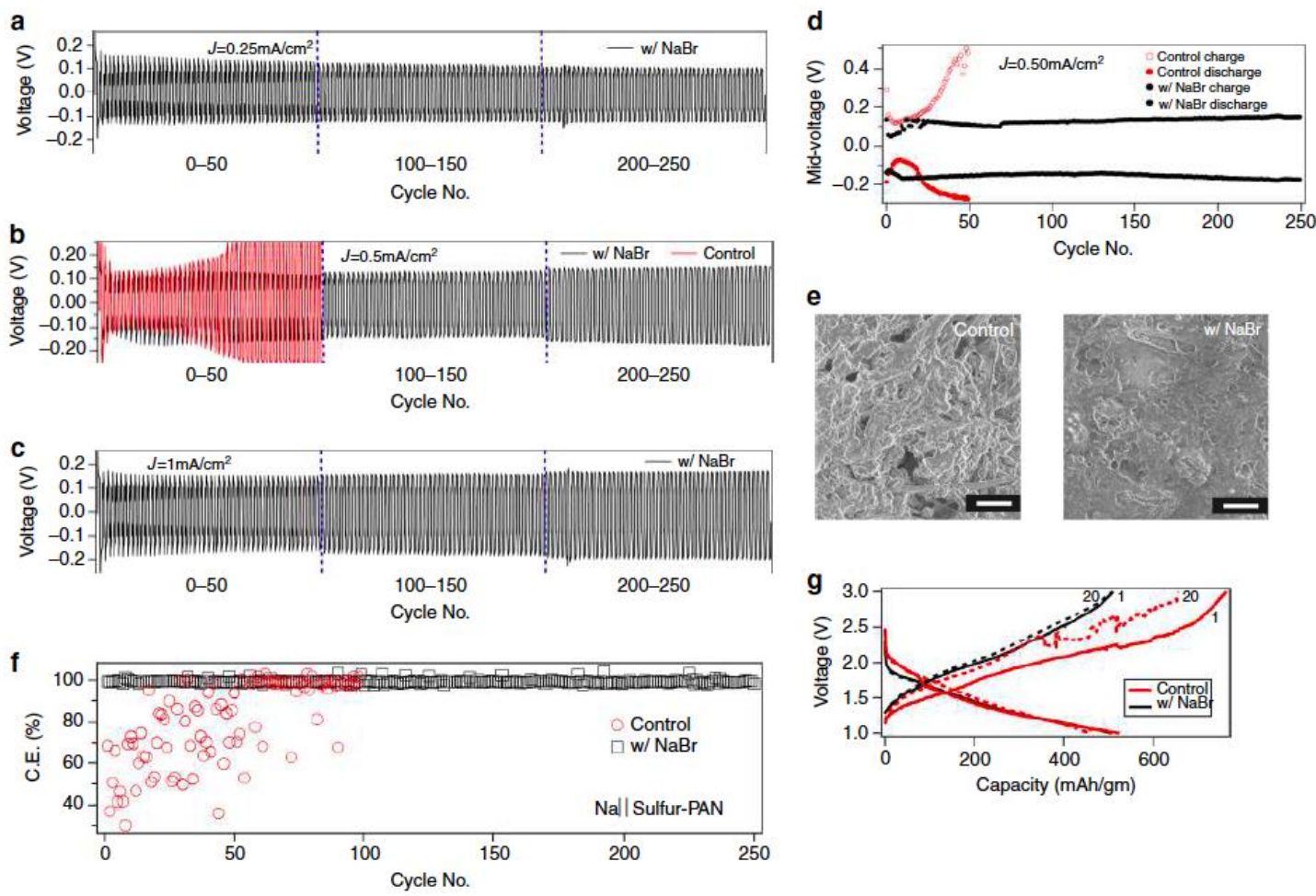

Figure 11.6 Galvanostatic cycling performance of Na anodes. Voltage profile obtained by consecutively charging and discharging a symmetric sodium cell at current densities of $\mathbf{a} 0.25 \mathrm{~mA} \mathrm{~cm}{ }^{-2}, \mathbf{b ~} 0.5 \mathrm{~mA} \mathrm{~cm}^{-2}, \mathbf{c} 1 \mathrm{mAcm}^{-2}$. In each experiment, one complete cycle was $1 \mathrm{~h}$ long, with each charge and discharge time is half-an-hour. The profiles in black represent sodium metals with $\mathrm{NaBr}$ coating and red stands for pristine sodium (control). d The voltage hysteresis represented by the mid-voltage values of charge and discharge is plotted as a function of cycle no. for $\mathrm{NaBr}$ coated and control sodium cells corresponding to (b). e Morphology of sodium metal electrode obtained from post-mortem SEM analysis. The cells were charged at a rate of $1 \mathrm{~mA} \mathrm{~cm}-2$ for $2 \mathrm{~h}$ before being taken apart for the post-mortem analysis. The left image are results for pristine $\mathrm{Na}$, while the image to the right is for $\mathrm{NaBr}$-coated $\mathrm{Na}$; for both images: scale bar, $5 \mu \mathrm{m}$. f Coulombic efficiency of a $\mathrm{Na}||$ Sulfur-PAN composite half-cell as a function of cycle number. $\mathbf{g}$ Voltage profile of $\mathrm{Na}||$ SulfurPAN during charging and discharging at the 1st and 20th cycle numbers. The red lines/symbols represent control, while black shows the result for $\mathrm{NaBr}$-coated sodium 
rigorous assessment of electrode reactivity and stability, in comparison to the $\mathrm{Na}|| \mathrm{Cu}$ type cells ${ }^{18}$ used in previous work. Here we observe that coating $\mathrm{Na}$ with $\mathrm{NaBr}$ protects the metal and in a liquid electrolyte, and in the presence of a conversion cathode, yields columbic efficiency $>99 \%$ for at least 250 cycles with minimal fade in discharge capacity as plotted in Supplementary Figure 11.7a. Also, the SEM image of the $\mathrm{Na}$ anode (with $\mathrm{NaBr}$ protection) is shown in Supplementary Figure 11.7b along with the EDX mapping of elements. No dendritic features are seen even after the longterm cycling, and in addition the sulfur species are absent, indicating immunity of the anode from side reactions. This observation can be attributed to the near complete protection of the metal from parasitic reactions, without compromising ion transport across the SEI. Thus, we conclude that consistent with the JDFT calculations a NaBrcoated $\mathrm{Na}$ anode opens the possibility of stable, room-temperature rechargeable sodium metal batteries able to operate using liquid electrolytes.

\subsection{Discussion}

We used Density-Functional Theory calculations to analyze the surface diffusion barriers for $\mathrm{Na}$ and $\mathrm{Li}$ adatom transport on various salts. It was observed that the usually formed SEI components on $\mathrm{Li}$, including $\mathrm{LiOH}$ and $\mathrm{Li}_{2} \mathrm{CO}_{3}$, have very high activation energy barriers, which is thought to increase the propensity of the metal to form needle-like, dendrite nucleates. In contrast, energy barriers for adatom diffusion on metal halide salts, including $\mathrm{LiF}, \mathrm{NaF}, \mathrm{NaBr}$, are low, with the energy barrier for diffusion on $\mathrm{NaBr}$ as low as that of Magnesium, which is known to form spherical nucleates on charging. We evaluate these predictions using $\mathrm{Na}$ electrodes on which an 
artificial SEI composed of pure $\mathrm{NaBr}$ is used to protect the metal. By means of XRD, EDAX, XPS, and cryo-FIB-SEM measurements, we confirm that $\mathrm{NaBr}$ coatings with thicknesses ranging from $2 \mu \mathrm{m}-12 \mu \mathrm{m}$ are achieved on Na. Further, impedance spectroscopy measurements at different temperatures show that $\mathrm{NaBr}$ coated sodium anodes exhibit at least three times lesser interfacial ion-transport activation energy compared to pristine sodium. In-situ visualization was performed to contrast the electrodeposition-stability with and without $\mathrm{NaBr}$ layer on sodium anodes. It showed that the $\mathrm{NaBr}$ coating not only restricts dendritic formation, but also prevents unwanted side-reactions between the electrode and electrolyte. This observation is in line with the charge-discharge measurements in symmetric cells as well as in coulombic measurement tests in Na||SPAN type half-cells. Thus, we think that this rational analysis of SEI layers in reactive metal-batteries, as well as the methodology of incorporating the desired component in the SEI, can provide a new outlook towards low-cost and long lasting secondary batteries.

\section{Acknowledgements}

This work was supported by the Department of Energy, Advanced Research Projects Agency - Energy (ARPA-E) through award \#DE-AR0000750. The work made use of electrochemical characterization facilities in the KAUST-CU Center for Energy and Sustainability, supported by the King Abdullah University of Science and Technology (KAUST) through Award \# KUS-C1-018-02. Electron microscopy facilities at the Cornell Center for Materials Research (CCMR), an NSF-supported MRSEC through

Grant DMR-1120296, were also used for the study. M.J.Z. and L.F.K. acknowledge support by the NSF (DMR-1654596) 


\section{REFERENCES}

1. Armand, M. \& Tarascon, J.-M. Building better batteries. Nature 451, 652-657 (2008).

2. Tarascon, J. M. \& Armand, M. Issues and challenges facing rechargeable lithium batteries. Nature 414, 359-367 (2001).

3. Hueso, K. B., Armand, M. \& Rojo, T. High temperature sodium batteries: status, challenges and future trends. Energy Environ. Sci. 6, 734-749 (2013).

4. Zheng, G. et al. Interconnected hollow carbon nanospheres for stable lithium metal anodes. Nat. Nanotechnol. 9, 618-623 (2014).

5. Whittingham, M. S. Lithium Batteries and Cathode Materials. Chem. Rev. 104, 4271-4301 (2004).

6. Wei, S. et al. A stable room-temperature sodium-sulfur battery. Nat. Commun. 7, $11722(2016)$

7. Wei, S. et al. Highly stable sodium batteries enabled by functional ionic polymer membranes, Adv. Mater. 29, 1605512 (2017).

8. Cohn, A. P., Muralidharan, N., Carter, R., Share, K., \& Pint, C. L. Anode-Free Sodium Battery through in Situ Plating of Sodium Metal. Nano Letters. 17, 1296-1301 (2017).

9. Choudhury, S. et al. Designer interphases for lithium-oxygen electrochemical cell. Sci. Adv. 3, (2017).

10. Yadegari, H., Sun, Q. \& Sun, X. Sodium-Oxygen Batteries: A Comparative Review from Chemical and Electrochemical Fundamentals to Future Perspective. Adv. Mater. 28, 7065-7093 (2016). 
11. Tu, Z., Nath, P., Lu, Y., Tikekar, M. D. \& Archer, L. A. Nanostructured Electrolytes for Stable Lithium Electrodeposition in Secondary Batteries. Acc. of Chem. Res. 48, 2947-2956 (2015).

12. Tikekar, M. D., Choudhury, S., Tu, Z. \& Archer, L. A. Design principles for electrolytes and interfaces for stable lithium-metal batteries. Nat. Energy 1, 16114 (2016).

13. Choudhury, S. \& Archer, L. A. Lithium Fluoride Additives for Stable Cycling of Lithium Batteries at High Current Densities. Adv. Electron. Mater. 2, $1500246(2015)$

14. Choudhury, S., Mangal, R., Agrawal, A. \& Archer, L. A. A highly reversible room-temperature lithium metal battery based on crosslinked hairy nanoparticles. Nat. Commun. 6, 10101 (2015)

15. Agrawal, A., Choudhury, S. \& Archer, L. A. A highly conductive, nonflammable polymer-nanoparticle hybrid electrolyte. $R S C A d v . \mathbf{5}, 20800-20809$ (2015).

16. Wong, D. H. C. et al. Nonflammable perfluoropolyether-based electrolytes for lithium batteries. Proc. Natl. Acad. Sci. U. S. A. 111, 3327-3331 (2014).

17. Wu, H. et al. Stable Li-ion battery anodes by in-situ polymerization of conducting hydrogel to conformally coat silicon nanoparticles. Nat. Commun. 4, 1943 (2013).

18. Seh, Z. W., Sun, J., Sun, Y. \& Cui, Y. A Highly Reversible Room-Temperature Sodium Metal Anode. ACS Cent. Sci. 1, 449-455 (2015)

19. Pan, H., Hu, Y.-S. \& Chen, L. Room-temperature stationary sodium-ion 
batteries for large-scale electric energy storage. Energy Environ. Sci. 6, 2338 (2013).

20. Tu, Z. et al. Nanoporous hybrid electrolytes for high energy batteries based on reactive metal anodes. Adv. Energy Mater. 7, 1602367 (2017).

21. Hallinan, D. T., Mullin, S. A., Stone, G. M. \& Balsara, N. P. Lithium Metal Stability in Batteries with Block Copolymer Electrolytes. J. Electrochem. Soc. 160, A464-A470 (2013).

22. Lu, Y., Tu, Z. \& Archer, L. A. Stable lithium electrodeposition in liquid and nanoporous solid electrolytes. Nat. Mater. 13, 961-969 (2014).

23. Lu, Y., Das, S. K., Moganty, S. S. \& Archer, L. A. Ionic liquid-nanoparticle hybrid electrolytes and their application in secondary lithium-metal batteries. Adv. Mater. 24, 4430-4435 (2012).

24. Schaefer, J. L., Yanga, D. A. \& Archer, L. A. High Lithium Transference Number Electrolytes via Creation of 3-Dimensional, Charged, Nanoporous Networks from Dense Functionalized Nanoparticle Composites. Chem. Mater. 25, 834-839 (2013).

25. Bouchet, R. et al. efficient electrolytes for lithium-metal batteries. Nat. Mater. 12, 452-457 (2013).

26. Feng, S. et al. Single lithium-ion conducting polymer electrolytes based on poly[(4-styrenesulfonyl)(trifluoromethanesulfonyl)imide] anions. Electrochim. Acta 93, 254-263 (2013).

27. Bertasi, F. et al. Single-Ion-Conducting Nanocomposite Polymer Electrolytes for Lithium Batteries Based on Lithiated-Fluorinated-Iron Oxide and 
Poly(ethylene glycol) 400. Electrochim. Acta 175, 113-123 (2015)

28. Guo, J., Wen, Z., Wu, M., Jin, J. \& Liu, Y. Vinylene carbonate- $-\mathrm{LiNO}_{3}$ : A hybrid additive in carbonic ester electrolytes for SEI modification on Li metal anode. Electrochem. Commun. 51, 59-63 (2015).

29. Li, W. et al. The synergetic effect of lithium polysulfide and lithium nitrate to prevent lithium dendrite growth. Nat. Commun. 6, 7436 (2015).

30. Pires, J. et al. Role of propane sultone as additive to improve the performance of lithium-rich cathode material at high potential. $R S C A d v .5,42088-42094$ (2015)

31. Aurbach, D. et al. On the use of vinylene carbonate (VC) as an additive to electrolyte solutions for Li-ion batteries. Electrochim. Acta 47, 1423-1439 (2002).

32. Chen, L., Wang, K., Xie, X. \& Xie, J. Effect of vinylene carbonate (VC) as electrolyte additive on electrochemical performance of Si film anode for lithium ion batteries. J. Power Sources 174, 538-543 (2007).

33. Etacheri, V. et al. Effect of fluoroethylene carbonate (FEC) on the performance and surface chemistry of Si-nanowire li-ion battery anodes. Langmuir 28, 965976 (2012).

34. Miao, R. et al. A new ether-based electrolyte for dendrite-free lithium-metal based rechargeable batteries. Sci. Rep. 6, 21771 (2016).

35. Li, B., Xu, M., Li, T., Li, W. \& Hu, S. Prop-1-ene-1,3-sultone as SEI formation additive in propylene carbonate-based electrolyte for lithium ion batteries.

Electrochem. Commun. 17, 92-95 (2012). 
36. Choudhury, S. et al. Designer interphases for the lithium-oxygen electrochemical cell. Sci. Adv. 3, 1-12 (2017).

37. Li, N., Yin, Y., Yang, C. \& Guo, Y. An Artificial Solid Electrolyte Interphase Layer for Stable Lithium Metal Anodes. Adv. Mater. 28, 1853-1858 (2016).

38. Ye, H. et al. Nano Energy Synergism of Al-containing solid electrolyte interphase layer and Al-based colloidal particles for stable lithium anode. Nano Energy 36, 411-417 (2017).

39. Miao, R. et al. Novel dual-salts electrolyte solution for dendrite-free lithiummetal based rechargeable batteries with high cycle reversibility. J. Power Sources 271, 291-297 (2014).

40. Qian, J. et al. High rate and stable cycling of lithium metal anode. Nat. Commun. 6, 6362 (2015).

41. Ha, S. et al. Magnesium (II) Bis(trifluoromethane sulfonyl) Imide-Based Electrolytes with Wide Electrochemical Windows for Rechargeable Magnesium Batteries. ACS Appl. Mater. Interfaces 6, 4063-4073 (2014).

42. Jäckle, M. \& Groß, A. Microscopic properties of lithium, sodium, and magnesium battery anode materials related to possible dendrite growth. $J$. Chem. Phys. 141, (2014).

43. Chazalviel, J.-N. Electrochemical aspects of the generation of rampified metallic electrodeposits. Phys. Rev. A 42, 7355-7367 (1990).

44. Ozhabes, Y., Gunceler, D. \& Arias, T. A. Stability and surface diffusion at lithium-electrolyte interphases with connections to dendrite suppression. arXiv 1504.05799, 1-7 (2015). 
45. Gunceler, D., Letchworth-Weaver, K., Sundararaman, R., Schwarz, K. a \& Arias, T. a. The importance of nonlinear fluid response in joint densityfunctional theory studies of battery systems. Model. Simul. Mater. Sci. Eng. 21, 74005 (2013).

46. Gunceler, Deniz, et al. Nonlinear solvation models: Dendrite suppression on lithium surfaces. 16th International Workshop on Computation Physics and Materials Science: Total Energy and Force Methods. 10, (2013).

47. Gunceler, D. \& Arias, T. A. Universal iso-density polarizable continuum model for molecular solvents. arXiv 1403.6465, 1-11 (2014).

48. Garrity, K. F., Bennett, J. W., Rabe, K. M. \& Vanderbilt, D. Pseudopotentials for high-throughput DFT calculations. Comput. Mater. Sci. 81, 446-452 (2014).

49. Zangmeister, D. Z., Turner, A. T. \& Pemberton, E. P. Segregation of $\mathrm{NaBr} / \mathrm{NaCl}$ crystals grown from aqueous solutions: Implications for sea salt surface chemistry. Geophys. Res. Lett. 28, 995-998 (2001).

50. Wang, X. et al. A Chelation Strategy for In-situ Constructing Surface Oxygen Vacancy on $\{001\}$ Facets Exposed BiOBr Nanosheets. Sci. Rep. 6, 24918 (2016).

51. NIST X-ray Photoelectron Spectroscopy Database, Version 4.1. National Institute of Standards Technology, Gaithersburg (2012).

52. Wei, S., et al. Metal-Sulfur Battery Cathodes Based on PAN-Sulfur Composites. J. Am. Chem. Soc. 137, 12143-12152 (2015).

53. Wang, J. et al. Room temperature $\mathrm{Na} / \mathrm{S}$ batteries with sulfur composite cathode materials. Electrochem. Commun. 9, 31-34 (2007). 


\section{APPENDIX}

Supplementary Information for Chapter 11

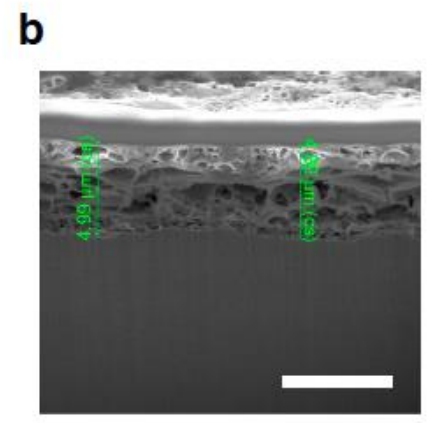

Supplementary Figure 11.1: Cross-sectional images of $\mathrm{NaBr}$ coated sodium and pristine sodium (a) EDX mapping of cross sections of sodium anodes with $\mathrm{NaBr}$ surface layers after reacting for $1 \mathrm{~min}$ (left) and $5 \mathrm{mins}$ (right). The cross sections were obtained by cryo-focused ion beam milling; scale bar, $3 \mu \mathrm{m}$. (b) Room temperature FIB-SEM cross-sectional imaging of pristine sodium shows a $5 \mu \mathrm{m}$ thick oxidation layer on the surface; scale bar, $5 \mu \mathrm{m}$. 


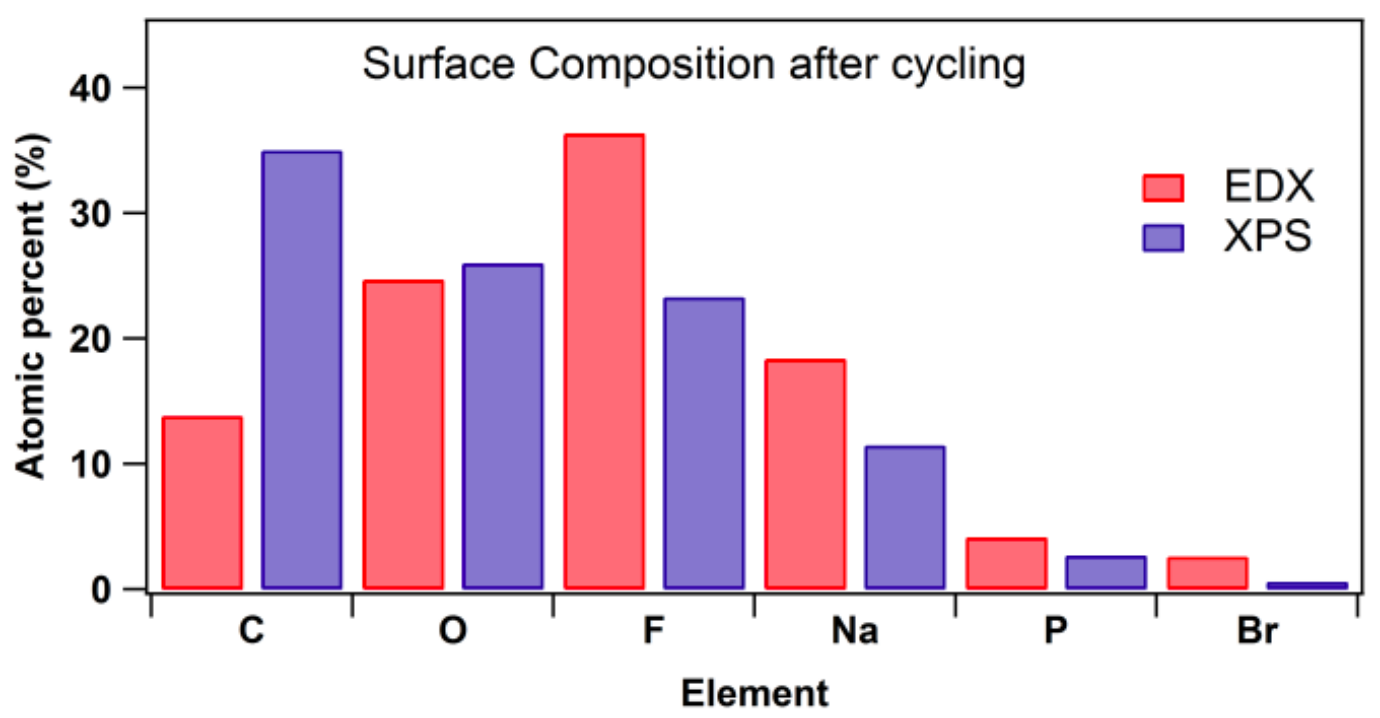

Supplementary Figure 11.2: Surface composition of cycled sodium metal with $\mathrm{NaBr}$ coating obtained by two separate methods of EDX and XPS. 


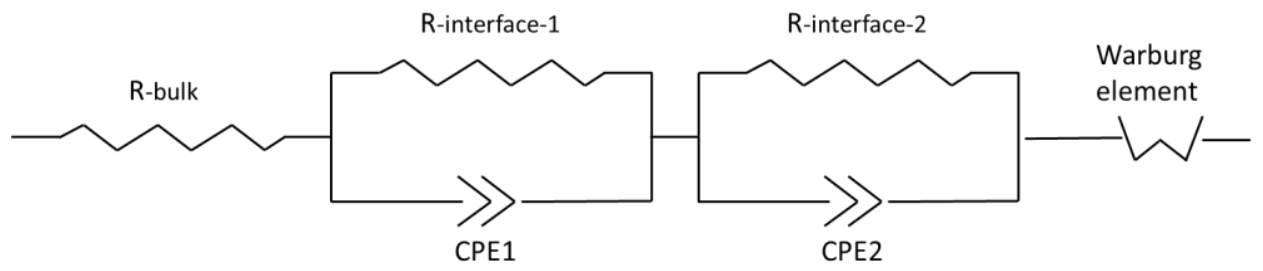

Supplementary Figure 11.3: Equivalent circuit model for fitting Nyquist plots of impedance measurements. Here, R-bulk represents the ion transport in bulk electrolyte. R-interface1 represents interfacial resistance associated with the passivation layer between electrode and electrolyte. R-interfaces denotes the electronic transport in the interface. CPE1, CPE2 represent the constant phase elements. Warburg element stands for solid-state diffusion contribution 
a

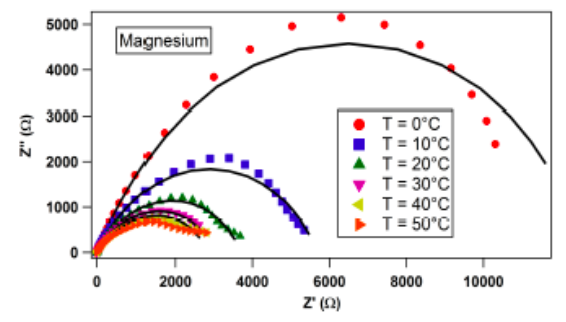

C

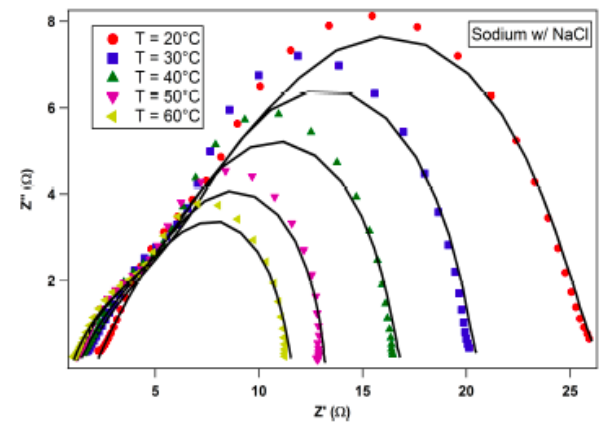

e

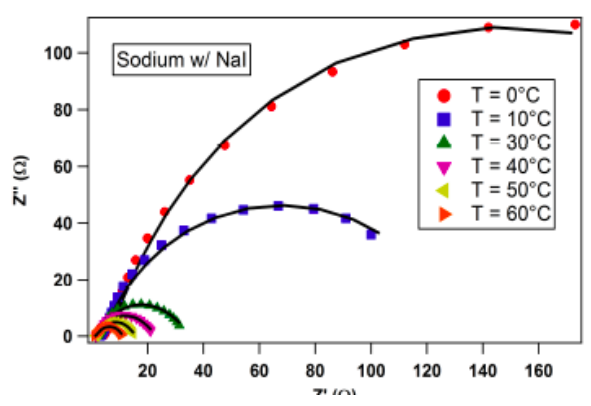

D

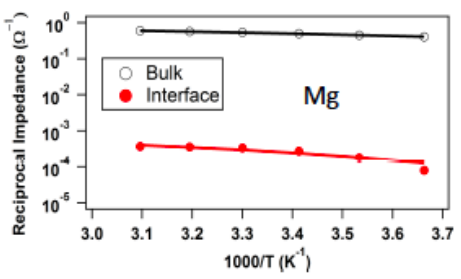

d

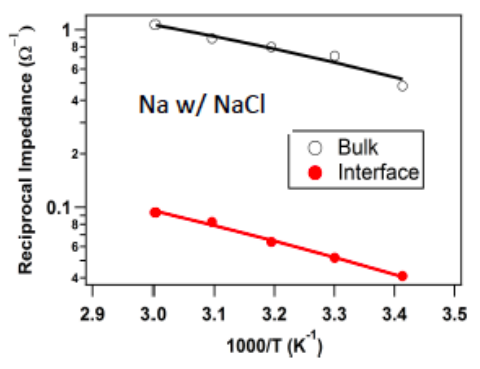

$\mathbf{f}$

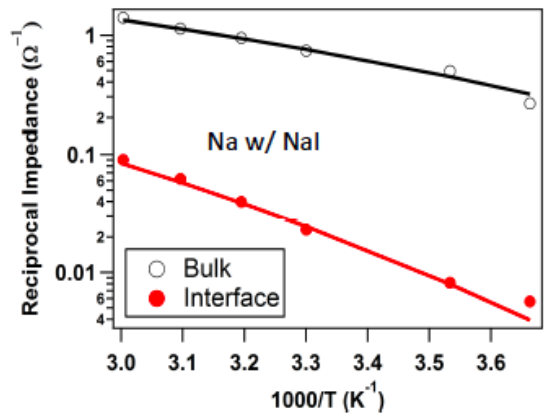

Supplementary Figure 11.4: Impedance Spectroscopy for different anodes Nyquist Plots at various temperatures for (a) $\mathrm{Mg}$, (c) $\mathrm{Na}$ with $\mathrm{NaCl}$, (e) $\mathrm{Na}$ with $\mathrm{NaI}$. Figures (b), (d) and (f) represent the temperature dependence of the reciprocal impedances (both bulk and interface) is plotted as a function of Arrhenius Temperature, the lines represent corresponding VFT fits. The labels represent the type of electrode/interface used for the experiment 

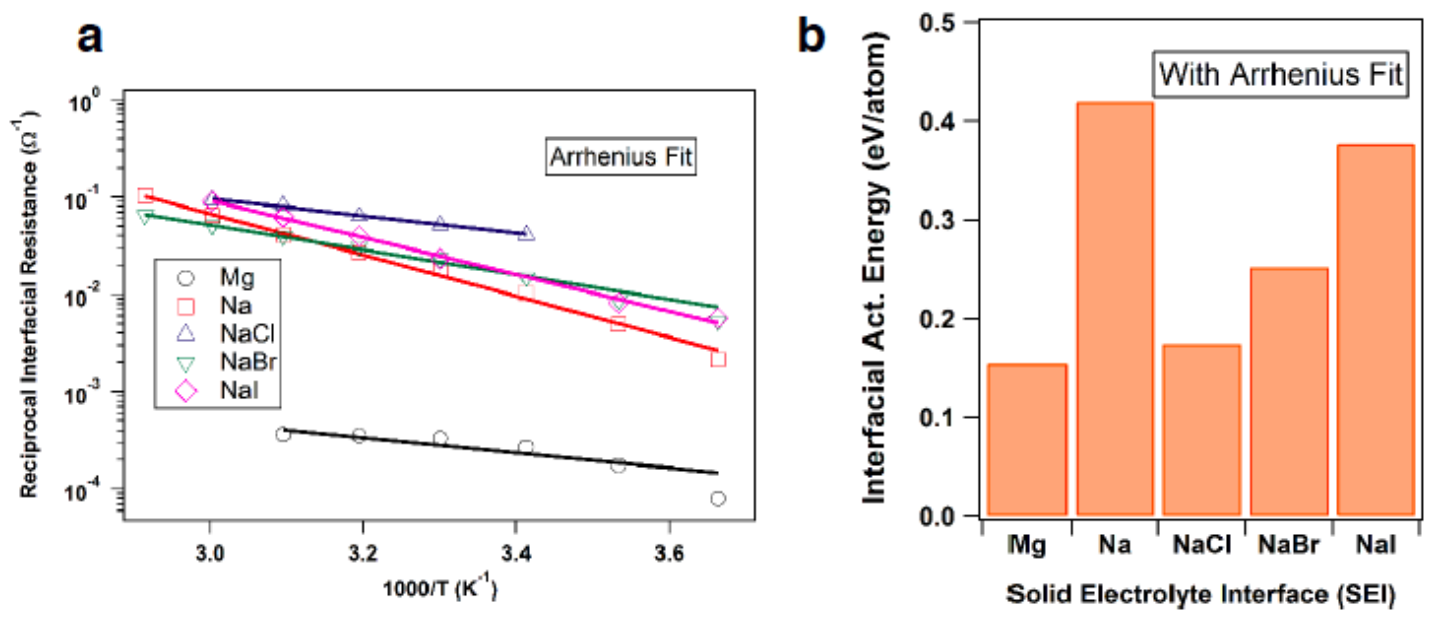

Supplementary Figure 11.5: Activation energy obtained by Arrhenius Analysis (a) Reciprocal of interfacial resistance plotted as a function of inverse temperature. The fits represent prediction from Arrhenius equation; (b) The activation energy is plotted for different interface or metal electrodes, obtained by Arrhenius fitting. $\mathrm{Mg}, \mathrm{Na}$ represents cells with magnesium and sodium electrodes respectively without any modification, while $\mathrm{NaCl}, \mathrm{NaBr}, \mathrm{NaI}$ represent data for respective halide coated sodium metal. 


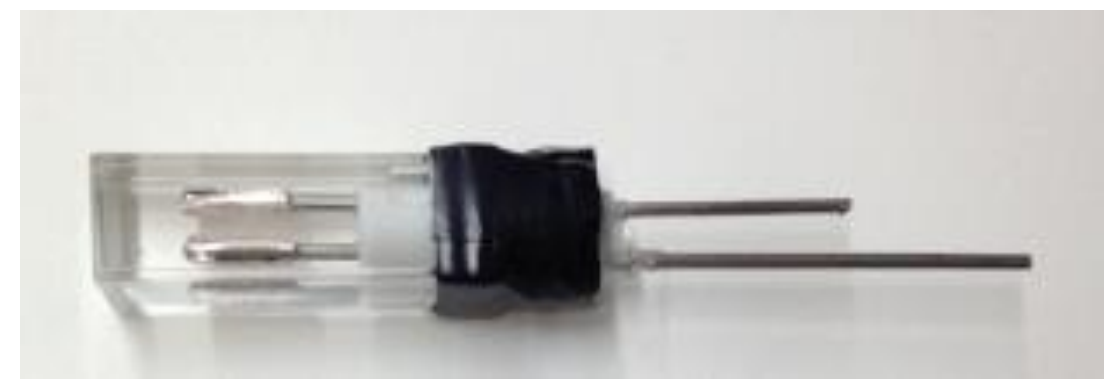

Supplementary Figure 6: Electrochemical setup for in-situ visualization of sodium electrodeposition consisting of an airtight cuvette and two rods serving as current collectors for attaching the sodium electrodes. The cap is well sealed with a black tape to ensure that there is no leakage of electrolyte or air-contamination. 

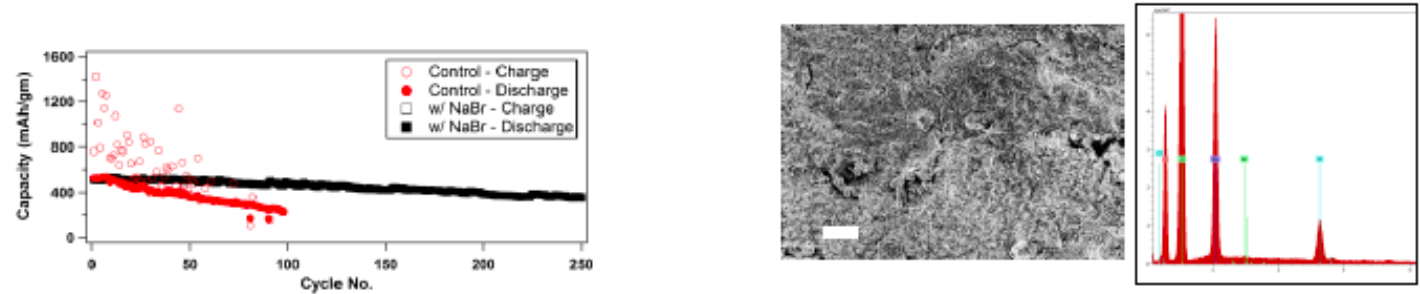

Supplementary Figure 7: Performance and characterization of Na||SPAN cell (a) Charge and discharge capacity as a function of cycle no. for pristine sodium-based half-cell and $\mathrm{NaBr}$ coated sodium-based half-cell comprising of the SPAN cathode. (b) SEM image of sodium metal after cycling in $\mathrm{Na}|| \mathrm{SPAN}$ cell with $\mathrm{NaBr}$ coating anode; scale bar, $100 \mu \mathrm{m}$. The adjacent image shows the EDX mapping of the elements of $\mathrm{Na}$ anode 
Supplementary Table 11.1: Parameters of VFT Analysis Bulk impedance represents the ion transport in the electrolyte media; while interfacial impedance indicates the ion transport across electrode-electrolyte interface. The temperature dependent data is fitted to VFT model.

\begin{tabular}{|c|c|c|c|c|c|c|}
\hline $\begin{array}{c}\text { Type of } \\
\text { Impedance }\end{array}$ & Component & $\begin{array}{l}\text { Prefactor } \\
\text { (A) }\left(\Omega^{-1}\right)\end{array}$ & $\begin{array}{l}\text { Activation } \\
\text { Energy (Ea) } \\
(\mathrm{kJ} \mathrm{mole-1)}\end{array}$ & $\begin{array}{l}\text { Activation } \\
\text { Energy (Ea) } \\
\left(\mathrm{eV} \text { atom }^{-1}\right)\end{array}$ & $\begin{array}{l}\text { Reference } \\
\text { Temp. } \\
\text { (To) (K) }\end{array}$ & $\begin{array}{l}\text { Goodness } \\
\text { of fit }\left(\chi^{2}\right)\end{array}$ \\
\hline \multirow{5}{*}{ Bulk } & $\mathrm{Mg}$ & 1.38 & 1.095 & 0.011 & 164.88 & $6.9 \times 10^{-5}$ \\
\hline & Sodium & 14.86 & 3.61 & 0.037 & 167.25 & $1.2 \times 10^{-3}$ \\
\hline & $\mathrm{NaCl}$ & 7.64 & 2.51 & 0.026 & 180 & $6.4 \times 10^{-3}$ \\
\hline & $\mathrm{NaBr}$ & 6.73 & 2.14 & 0.022 & 197.82 & $1.9 \times 10^{-3}$ \\
\hline & $\mathrm{NaI}$ & 20.30 & 3.91 & 0.041 & 160 & $2.1 \times 10^{-2}$ \\
\hline \multirow{5}{*}{ Interface } & $\mathrm{Mg}$ & 0.0016 & 1.32 & 0.014 & 211 & $6.1 \times 10^{-9}$ \\
\hline & Sodium & 755.81 & 16.94 & 0.18 & 112.84 & $1.4 \times 10^{-4}$ \\
\hline & $\mathrm{NaCl}$ & 2.44 & 5.20 & 0.054 & 140.43 & $1.4 \times 10^{-5}$ \\
\hline & $\mathrm{NaBr}$ & 2.70 & 5.48 & 0.057 & 166.40 & $5.4 \times 10^{-6}$ \\
\hline & $\mathrm{NaI}$ & 37.42 & 9.14 & 0.095 & 152.99 & $2.0 \times 10^{-5}$ \\
\hline
\end{tabular}


Chapter 12

Electroless Formation of Hybrid Lithium Anodes for High Interfacial Ion

Transport 


\subsection{Abstract}

Rechargeable batteries based on metallic anodes provide promising platforms for fundamental and applications-focused studies of chemical and physical kinetics of liquids at solid interfaces. It is known that the intrinsic chemical instability of the metals to undergo parasitic side reactions with liquid electrolytes presents a difficult challenge for long-term stability. Approaches that allow facile creation of uniform coatings on these metals to prevent physical contact with liquid electrolytes, while enabling high rate ion-transport, are essential to address the anode failure issues in these batteries. Here, we report a simple electroless ion-exchange chemistry for creating uniform and functional coatings of the metal Indium on lithium. By means of Joint- Density Functional theory and interfacial characterization experiments, we show that these coatings provide multiple stabilization mechanisms, including exceptionally low surface diffusion barriers for lithium ion transport and high chemical resistance to liquid electrolytes. Indium coatings undergo reversible alloying reactions with lithium ions, enabling the design of high-capacity hybrid In-Li anodes that utilize both alloying and plating chemistries for charge storage. By means of direct visualization, we further show that the coatings enable remarkably compact and uniform electrodeposition. The resultant $\mathrm{In}-\mathrm{Li}$ anodes are shown to exhibit minimal capacity fade in extended galvanostatic cycling when paired with commercial-grade cathodes.

\subsection{Introduction}

Secondary batteries comprising of metallic anodes (e.g. Li, Na, Al) have drawn significant recent attention due to their promise for enabling large (as high as ten-fold) 
increase in the anodic capacity, in comparison to state-of-art lithium-ion anode chemistry. ${ }^{[1,2]}$ A major barrier to the successful implementation of such anodes is the uneven electrodeposition of the metal in the charging process, which leads to formation of rough, so-called dendritic structures. These structures are diffusionlimited and as such under extended battery operation can grow to fill the interelectrode space, short-circuiting the battery (often accompanied with fire and /or cell explosion). ${ }^{[3-5]}$ Previously, using continuum modeling, Tikekar et al. ${ }^{[6,7]}$ proposed that dendrite induced short-circuits can be prevented using electrolytes with moderate mechanical modulus (10 MPa) if a fraction of the anions are tethered to prevent cell polarization at high current densities and to maintain electrolyte conductivity in the region near the anode. There is also a large body of experimental work showing that dendrite-growth can be regulated using solid state electrolytes ${ }^{[8]}$, nanoporous polymers or ceramics that host liquids in their pores; ${ }^{[9-11]}$ cross-linked polymers ${ }^{[12,13]}$; as well as with high transference number gel-like electrolytes ${ }^{[14-17]}$. Although researchers are now able to address the safety concerns by variety of means, but this progress has difficult challenges associated with uncontrolled parasitic reactions between liquid electrolytes and a reactive metal electrode. ${ }^{[18-19]}$

In rechargeable batteries that employ metallic anodes, particularly those based on Li and $\mathrm{Na}$, the electrolyte would ideally react with the metal surface to form a stable and self-limiting passivation layer termed the solid-electrolyte interphase (SEI). Ion and mass transport through this poorly understood interfacial layer are crucial for the longterm stability of any rechargeable battery that utilizes a reactive metal anode. Continuous expansion and contraction of the electrode during cycles of battery charge 
and discharge, unfortunately degrades the SEI, exposing the native metal to the electrolyte, thus forming further reaction products ${ }^{[3,4]}$. The parasitic reactions can be checked by minimizing the exposed surface to the electrolyte, which can be attained by either implementing an artificial protection film on the electrode ${ }^{[17,20]}$ or through physical means that enable smooth (flat) electrodeposition using appropriate salt chemistries $^{[21,22]}$. Zheng et al. ${ }^{[23]}$ for example utilized interconnected hollow carbon nanospheres for fabricating a protective film able to shield a Li metal anode from the electrolyte media resulting in high columbic efficiencies. Similar methodologies based on ex-situ coating processes such as atomic layer deposition of metal oxides, most notably alumina ${ }^{[24]}$ on $\mathrm{Li}$ and electrospun polymer fibers ${ }^{[25]}$ have also been reported to protect Li from reacting with liquid electrolytes. Further, there are several recent studies based on in-situ generation of specialized interface materials using electrolyte additives including liquids, such as Fluoroethylene carbonate (FEC $)^{[26]}$ and salts like $\mathrm{LiFSI}^{[27]}, \mathrm{LiPF}_{6}{ }^{[28]}, \mathrm{LiF}^{[29]}$ and $\mathrm{CsF} \cdot{ }^{[21]}$

The successes of these methods in simultaneously suppressing dendritic deposition and parasitic side reactions all appear to hinge upon formation of a SEI enriched with species such as $\mathrm{LiF}$ that facilitate fast $\mathrm{Li}$-ion diffusion at the electrolyte-metal interface. It also underscores the importance of fundamentally based strategies able to control the dendrite nucleation processes at reactive metal/liquid electrolyte interfaces. Previous work has shown that low energy barriers for surface diffusion i.e. high predicted diffusion rates correlate well with long short-circuit times in experiment i.e. low dendrite formation rate, for lithium ${ }^{[20,30]}$ as well as sodium batteries ${ }^{[31]}$. Low diffusion barriers also correlate well with low surface energies, ${ }^{[30,32-33]}$ presumably 
because this results in weaker binding of electrodeposited atoms overall, with small energy differences between the weakly and tightly-bound spots, and therefore a low barrier for diffusion. These approaches therefore all appear to take advantage of processes that compete with growth of high-curvature regions on the electrode surface. Here, we report a new approach to diffusion barrier minimization that exploits the effects of the solvent (and electrolyte) at the interface. The key idea is to utilize strong interactions of the solvent with the electrodeposited atom to weaken its binding to the electrode surface and flatten the energy landscape for atom motion in the plane. Aprotic solvents used in battery electrolytes will interact most strongly with charged species. Stable charging of the surface atom should be possible under these conditions by employing a difference in electropositivity between the deposited atom and the electrode. We illustrate these ideas using indium metal coatings on lithium metal anodes by an in-situ electroless plating technique. The high electropositivity of lithium relative to indium is expected to result in (partially) positively charged lithium atoms on the In surfaces.

\subsection{Results}

In order to evaluate the energetic landscapes at the electrode-electrolyte interphase, we perform density-functional theory calculations of lithium atom diffusion on the surface of indium, both in vacuum and in an electrolyte environment described using a

previously established continuum solvation methodology ${ }^{[33,34]}$. The two most stable surfaces of indium, (011) and (001), are very close in surface energy, so we examine lithium ion diffusion on both these surfaces (Table ST1 and Figure 12.1). In vacuum, 
we find a large difference between the diffusion barriers on the two surfaces. The highest-density $\operatorname{In}(011)$ surface has a small diffusion barrier of $0.04 \mathrm{eV}$ (less than $2 k_{B} T$ ) between adjacent hollow sites, whereas the $\operatorname{In}(001)$ surface has a much larger barrier of $0.30 \mathrm{eV}\left(\sim 11 k_{B} T\right)$, also in a path connecting adjacent hollow sites over a bridge site. Supplementary Table 12.1 compares the predicted surface energies of the low-index surfaces of indium in vacuum as well as solution, with previous calculations for other metal electrodes. Importantly, the surface energies of indium are comparable to the other metals (larger than $\mathrm{Na}$, but smaller than $\mathrm{Li}$ and $\mathrm{Mg}$ ) and are not significantly affected by the solvent.

The solvent dramatically alters the energy landscape for Li diffusion on both these surfaces. For the (011) surface, the minimum energy path is qualitatively similar (connecting adjacent hollow sites), but the barrier reduces to $0.015 \mathrm{eV}\left(<k_{B} T\right)$. For the (001) surface, the change is much more dramatic: the stable binding sites switch from the hollow to the atop sites because the latter are more accessible by the solvent and hence stabilized further. The barrier drops to $0.013 \mathrm{eV}\left(<k_{B} T\right)$ for this surface as well. Lowdin charge analysis suggests a charge ranging from +0.3 to +0.5 for the Li atom on the solvated In surfaces; while the exact value of atomic charges is not particularly meaningful, this does agree with the qualitative picture of solvent stabilization in this case. In summary, our JDFT calculations reveal a dramatic reduction in the diffusion barriers for Li on solvated In surfaces due to solvent stabilization. For the two most stable surfaces of In, the barriers are less than $k_{B} T \sim 0.026 \mathrm{eV}$ at room temperature, and smaller than those reported for other electrode or coating materials ${ }^{[32]}$. Significantly, as seen from the bar chart in Figure 12.1e, the diffusion barrier is much lower than the 


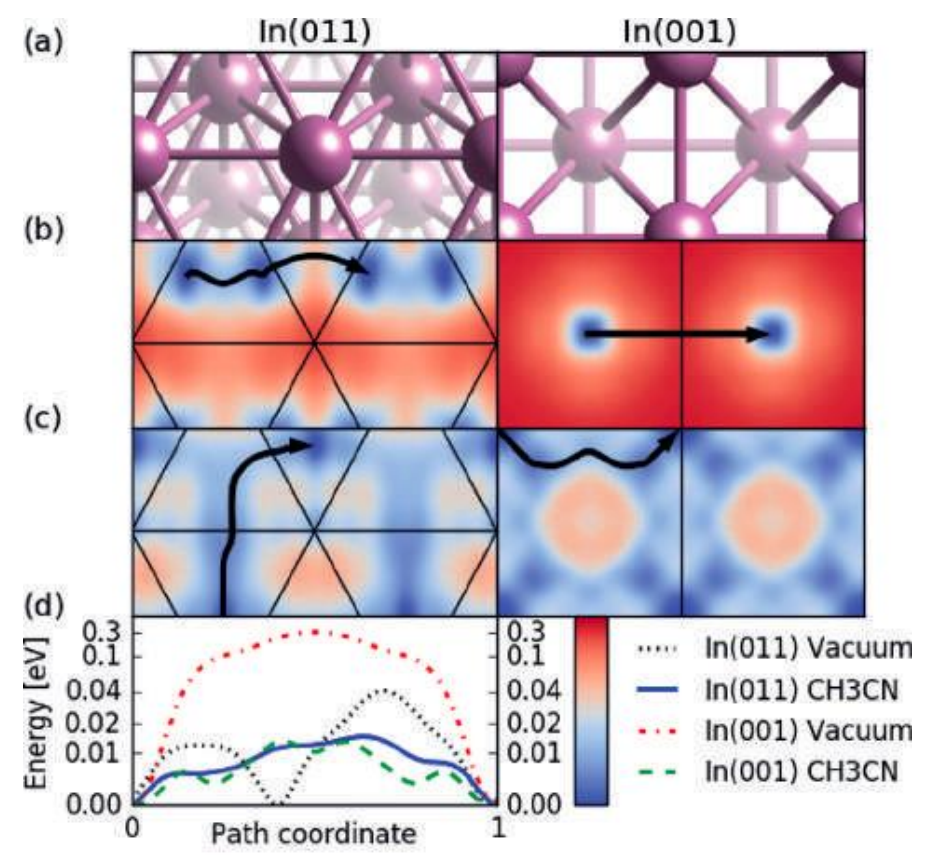

(e)

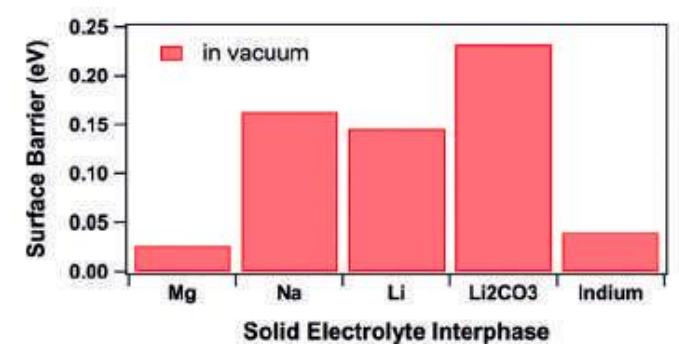

\section{Figure 12.1: Joint-Density Functional Theory for ion transport on Indium} surface: (a) Surface structures, and energy landscapes of Li diffusion in (b) vacuum and (c) acetonitrile solvent on the $\operatorname{In}(011)$ surface (left panels) and the $\operatorname{In}(001)$ surface (right panels). The arrows annotate the minimum energy paths, and (d) compares the energy along the path for all four cases from (b) and (c). Note that the energy axis and color scale are mapped nonlinearly (as $y=E /\left(E+k_{B} T\right)$ ) to show features on the $k_{B} T$ scale while capturing the larger energy range of the data. Solvation substantially reduces the energy barriers for $\mathrm{Li}$ diffusion on $\operatorname{In}(011)$ as well as $\operatorname{In}(001)$; (e) Comparison of surface diffusion barrier of Indium with other interphases reported before, including $\mathrm{Mg}, \mathrm{Na}, \mathrm{Li}, \mathrm{Li}_{2} \mathrm{CO}_{3}$ 
commonly generated SEI component lithium carbonate, but is comparable to that of Magnesium, which is known to electrodeposit at moderate currents in the diffusioncontrolled regime, without dendrite formation.

We choose to focus on Indium for this first study because in addition to its low surface diffusion barrier of indium metal, an additional advantage is its property of alloying with lithium. Commonly used intercalating materials like graphite, tin and silicon are known to form carbonates and oxides by side reactions. Here, we utilize Indium metal coating, which is relatively inert and non-reactive with most electrolyte components. Figure 12.2a shows the schematic of the proposed lithium metal protection technique using Indium metal. In this method, $\operatorname{In}(T F S I)_{3}$ salt is used in the electrolyte as additive to form the electroless coating by reduction reaction given by: $3 \mathrm{Li}+\operatorname{In}(\mathrm{TFSI})_{3} \rightarrow$ 3 LiTFSI + In. The predicted field lines of Li ions indicate the enhanced surface diffusion, followed by alloying in the Indium buffer layer before electrodeposition. To demonstrate the electroless plating method, lithium metal anodes were dipped in a solution of $12 \mathrm{mM} \operatorname{In}(T F S I)_{3}$ in EC:DMC (1:1) and allowed to incubate for 6 hours, followed by washing and drying under vacuum. Figure $12.2 \mathrm{~b}$ shows the treated lithium surface using scanning electron microscopy (SEM). The chemical composition was mapped using Energy Dispersive X-ray Spectroscopy (EDX) shown in Figure 12.2c. It is seen that Indium is evenly plated on the lithium surface. Further, X-Ray Diffraction (XRD) was utilized to analyze the surface of Indium coated lithium metal as shown in Figure 12.2d. The presence of In, In-Li alloy peaks in addition to lithium XRD-peak confirm the Indium metal formation by the reaction of $\operatorname{In}(T F S I)_{3}$ with 
lithium. We later show that it is possible to create a self-healing version of these coatings by adding $12 \mathrm{mM} \operatorname{In}(T F S I)_{3}$ as a co-salt in a standard battery electrolyte.

Figure 12.2e shows the X-ray Photon Spectroscopy (XPS) results for the lithium electrode after conditioning at a low current density of $0.1 \mathrm{~mA} \mathrm{~cm}{ }^{-2}$ for 10 cycles in a symmetric cell with the elctrolyte $1 \mathrm{M} \mathrm{LiPF}_{6} \mathrm{EC} / \mathrm{DMC}+12 \mathrm{mM} \operatorname{In}(T F S I)_{3}$. Figure 12.2f report the analogous results when the electrodes are cycled at 10 times at $1 \mathrm{~mA}$ $\mathrm{cm}^{-2}$. The In $3 \mathrm{~d}$ spectra shows two fully split peaks of $3 \mathrm{~d}_{3 / 2}$ and $3 \mathrm{~d}_{5 / 2}$ at $352 \mathrm{eV}$ and $344 \mathrm{eV}$ respectively for both before and after cycling at high current densities. ${ }^{[35]}$ The presence of metallic Indium peaks even after cycling indicate that the coating doesn't break down during the expansion and contraction of lithium electrode during charge and discharge, respectively. Typical peaks of $55 \mathrm{eV}$ in $\mathrm{Li} 1 \mathrm{~s}$ representing organometallic compounds are further seen. The C1s spectra peaks $(284.8,285.5,286$ and $288.5 \mathrm{eV}$ ), confirm the presence of $\mathrm{ROCO}_{2} \mathrm{Li}$ and polycarbonates before and after

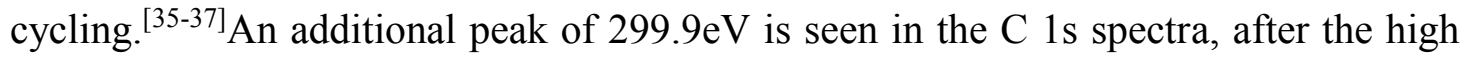
current density cycling of the cell, this represent additional formation of carbonates. ${ }^{[37]}$ The peaks of 533.5 and $531 \mathrm{eV}$ in case of $\mathrm{O} 1 \mathrm{~s}$ spectra, also confirm the presence of carbonates and $\mathrm{ROCO}_{2} \mathrm{Li}$ species. ${ }^{[38]}$ In case of $\mathrm{F} 1$ spectra, $685 \mathrm{eV}$ peak indicates LiF, while that at $688-689 \mathrm{eV}$ represent organo-fluorides (C-F bond), with a shift in the energy for $\mathrm{CF}_{2}$ and $\mathrm{CF}_{3}$ as seen in the spectra before cycling. The $\mathrm{LiF}$, here is predicted to be generated by the interaction between the anion species of $\mathrm{TFSI}^{-1}$ and $\mathrm{PF}_{6}^{-1}$. As LiF generation is often associated with degradation of $\mathrm{PF}_{6}^{-1}$, its absence confirms the ability of Indium layer in preventing side reactions. Overall, it can be concluded that Indium metal forms a conformal coating on the lithium anode along 
(a)

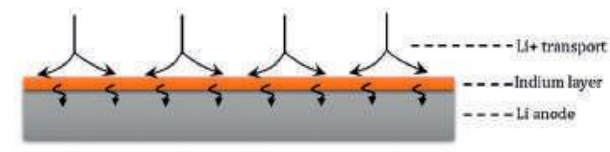

(d)

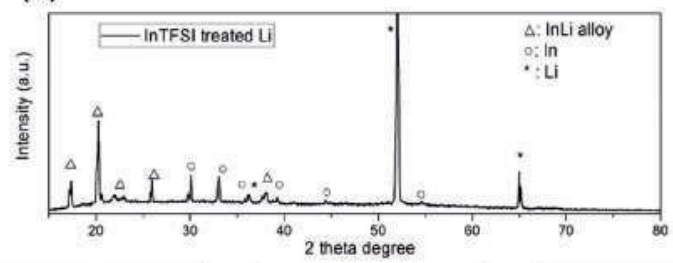

(e)

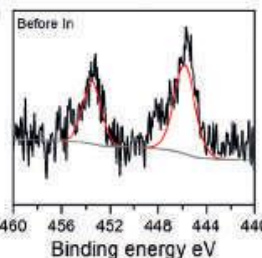

(f)

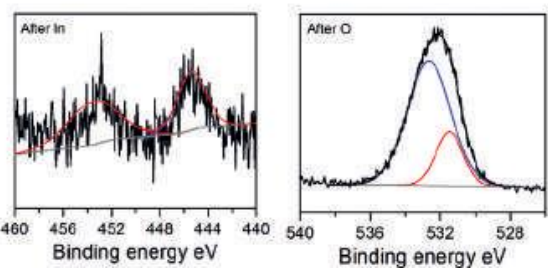

(b)

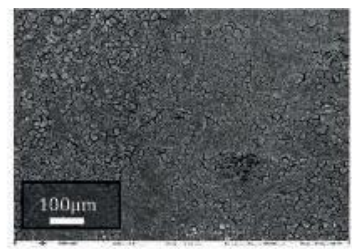

(c)
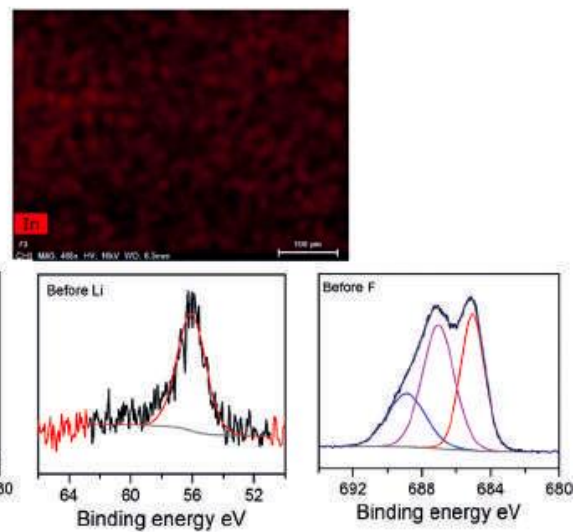
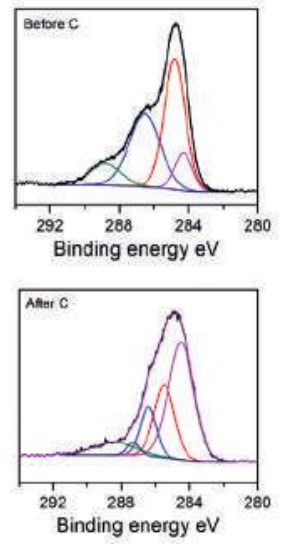

Binding energy $\mathrm{eV}$

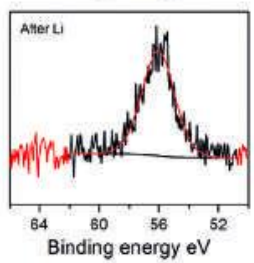

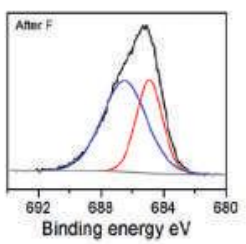

Figure 12.2: Surface Characterization of Indium metal coated lithium anode: (a)

Schematic of predicted $\mathrm{Li}^{+}$ion transport near the interface and through Indium layer on lithium. (b) SEM image of lithium metal after treatment with $\operatorname{In}(T F S I)_{3}$ solution. (c) EDX mapping of Indium atom of lithium after surface- treatment. (d) XRD showing presence of Indium metal and In-Li alloy phases on the surface of lithium. Here * indicated Li, o is Indium and $\Delta$ stands for In-Li alloy. (e) Energy spectra obtained by XPS measurement of lithium electrode surface after cycling at low current density of $0.1 \mathrm{~mA} \mathrm{~cm} \mathrm{~cm}^{-2}$. (f) Energy spectra of lithium anode after cycling at high current density of $1 \mathrm{~mA} \mathrm{~cm}^{-2}$ for 10 cycles 
with other carbonate species. Further it is confirmed that the chemical composition of this coating is unperturbed even after long term cycling. The approach therefore provides a facile method for creating In-Li electrodes that take advantage of the electrochemical attributes of both metals.

We performed impedance spectroscopy measurements for control electrolyte comprising of $1 \mathrm{M} \mathrm{LiPF}_{6} \mathrm{EC} / \mathrm{DMC}$ and electrolyte reinforced with $12 \mathrm{mM} \operatorname{In}(\mathrm{TFSI})_{3}$. Figure 12.3a shows the Nyquist diagram obtained at $30^{\circ} \mathrm{C}$, the lines are fits obtained using the equivalent circuit diagram shown in Supplementary Figure 12.1. It is seen that the interfacial resistance of the cell using the control electrolyte is essentially unchanged relative to the one where which $\operatorname{In}(\mathrm{TFSI})_{3}$ was introduced. However, after five cycles at a low current density $\left(0.1 \mathrm{~mA} \mathrm{~cm}^{-2}\right)$, the interfacial resistance of the latter cells is seen to reduce significantly, by about 10 times. The high interfacial resistance initially observed is consistent with the blockage of direct electrolyte access to the $\mathrm{Li}$ electrode. The fact that the cell without initial conditioning has the same interfacial resistance as control cell is also unsurprising because it is known that lithium metals form a native coating of oxides and carbonates even with slightest exposure of organic solvent and oxygen (even inside glove-box). The low current density conditioning, thus appears to have the effect of cleaning off the surface for in-situ reaction fresh $\mathrm{Li}$ with Indium salts. Further the interfacial conductance or reciprocal of interfacial resistance is plotted in Figure 12.3b for all three cases and fitted by Arrhenius rule, $R_{\text {int }}{ }^{-1}=A \exp (-B / T)$ and the values of activation energy $B$, are tabulated in Supplementary Table 12.2. The results show that the interfacial activation energy for a $\mathrm{Li}$ anode in presence of Indium salts without conditioning $(0.39 \mathrm{eV} /$ atom $)$ is similar to 
that of pristine lithium $(0.43 \mathrm{eV} /$ atom $)$ in the same baseline electrolyte. After generation of the Indium coating on anode, however, the value drops to $0.29 \mathrm{eV} /$ atom, which is a substantial decrease in comparison to the thermal energy $k \mathrm{~T}$. As previously reported $^{[31]}$, the lower interfacial activation energy reflects the reduced diffusion barrier for surface transport of $\mathrm{Li}$ ad atoms on the In surface.

Figure $12.3 \mathrm{c}$ reports results the cyclic voltammetry analysis in a two-electrode cell comprised of Li foil as reference as well as counter electrode and Indium metal $(100 \mu \mathrm{m})$ as the working electrode using $1 \mathrm{M} \mathrm{LiPF}_{6} \mathrm{EC} / \mathrm{DMC}$ electrolyte. On backward scanning, a positive current peak of $-4 \mathrm{~mA} / \mathrm{cm}^{2}$ appears at $\sim 0.5 \mathrm{~V}$ (vs. $\mathrm{Li} / \mathrm{Li}+$ ), indicative of lithium alloying in Indium metal; while on reverse scanning, a deintercalation peak appears at $\sim 1.2 \mathrm{~V}$ (vs. $\mathrm{Li} / \mathrm{Li}+$ ). A similar experiment was conducted in a Lithium versus stainless steel configuration using $12 \mathrm{mM} \operatorname{In}(\mathrm{TFSI})_{3}$ salt additive and the results is plotted in Figure 12.3d. Current peaks at $0.5 \mathrm{~V}$ and $1.2 \mathrm{~V}$ (vs. $\mathrm{Li} / \mathrm{Li}+$ ) are also observed, however with lower magnitude. These peaks are associated with the lithium alloying and de-alloying from the in-situ formed Indium layer on stainless steel. During battery operation, this layer is hypothesized to act as buffer passage for consequent plating on underlying Li layer. Further, it is seen that the peak heights do not change significantly over 5 cycles, indicating good stability of Indium layer during the charge-discharge processes. In comparison, on cycling the thick Indium electrode there is significant shift in the current peaks as well as it becomes noisy (see Supplementary Figure 12.3). A simplistic calculation was done to calculate the thickness of the in-situ formed In layer based on the measured capacity of the Indium foil $(100 \mu \mathrm{m})$ and It was found to be $1.25 \mu \mathrm{m}$ thick. This layer is evidently much 
(a)

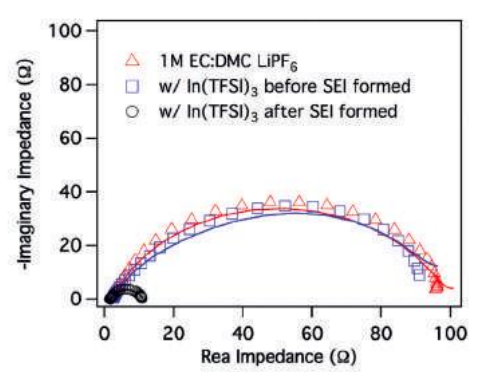

(c)

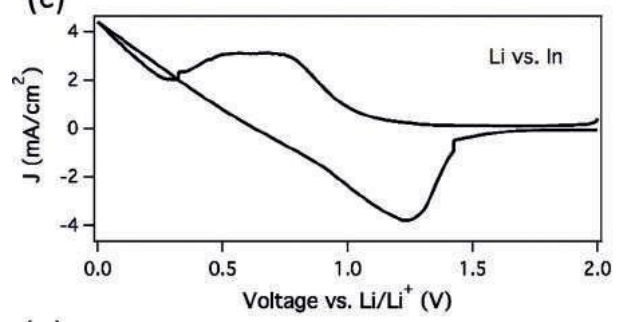

(e)

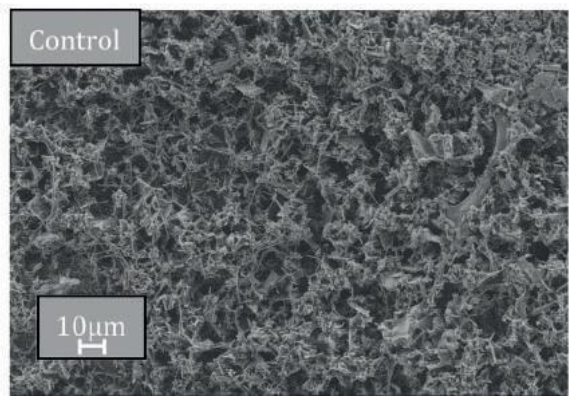

(b)

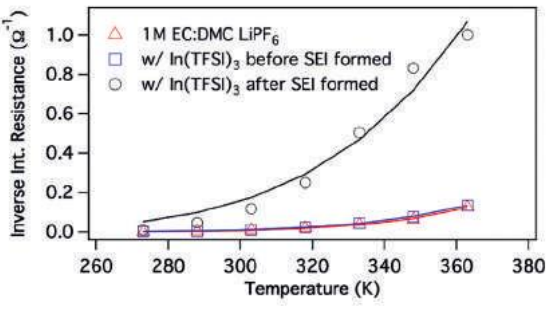

(d)

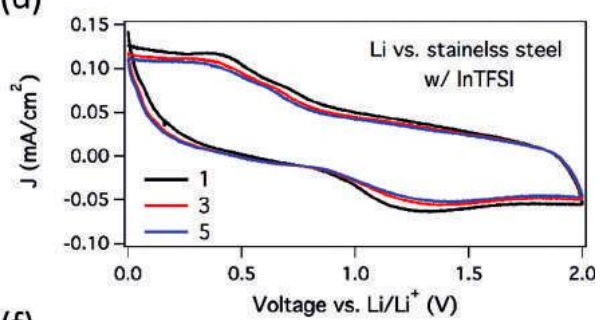

(f)

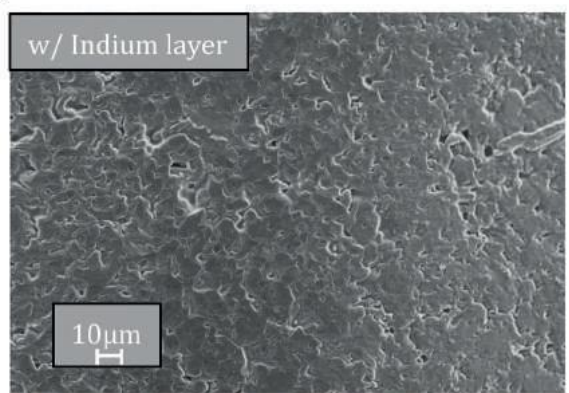

Figure 12.3: Electrochemical and Morphological Analysis of interfacial ion

transport: (a) Nyquist plots for control electrolyte as well as $\operatorname{In}(T F S I)_{3}$ added electrolytes before and after condition at low current density obtained by Impedance Spectroscopy measurements. b) Reciprocal of interfacial Impedance obtained by circuit model fitting of impedance curves. The solid lines represent Arrhenius plot for interfacial impedances. (c) Cyclic voltammetry for Li vs. Indium type cell performed at the rate of $1 \mathrm{mV} / \mathrm{sec}$. (d) Cyclic voltammetry of Li vs stainless steel battery with $\operatorname{In}(T F S I)_{3}$ added electrolyte. The scan rate is $1 \mathrm{mV} / \mathrm{sec}$. Morphology of lithium deposition on stainless steel at the rate of $0.5 \mathrm{~mA} / \mathrm{cm}^{2}$ for 6 hours with (e) Control electrolyte and (f) electrolyte with addition of $\operatorname{In}(T F S I)_{3}$. Images obtained using scanning electron microscopy. 
thicker than spontaneously formed SEI coatings on reactive metals such as $\mathrm{Li}$, which leads us to describe the resultant anodes as In-Li hybrid anodes because of their ability to utilize both reversible alloying and electroplating of $\mathrm{Li}$ ions for energy storage.

The morphology of Li deposits, with and without Indium coating was obtained using scanning electron microscopy (SEM) after plating stainless steel electrode at the rate $0.5 \mathrm{~mA} / \mathrm{cm}^{2}$ for 6hours. It is seen that for the control electrolyte, lithium deposition is rough and needle-like, while with Indium protection it is smooth and uniform. Since, the lithium deposits with control electrolyte is more perforated, there is a higher degree of contact between the electrolyte and electrode resulting in high side-reaction sites, also the lithium spikes can cause rupture in the SEI layer. Also, it is important to note that the interconnected and flat deposits achieved with In protection testifies to the low energy barrier for $\mathrm{Li}$ ion diffusion on Indium surface, similar to previously observed Magnesium electrodeposition. ${ }^{[39]}$

It is hypothesized that the macroscopic morphological evolution during electrodeposition are dependent on the interfacial ion mobility and initial nucleation process. We performed direct-visualization experiments under optical microscope to monitor the electrodeposition on longer time scales. Specifically, an in-house built visualization setup was utilized (as shown in Supplementary Figure 12.4) comprised of a lithium rod as one electrode and a stainless disc as the counter-electrode, with electrolyte filled in the center tube. Using a current density of $8 \mathrm{~mA} \mathrm{~cm}{ }^{-2}$, morphological changes of the electrode was recorded at equal time intervals upto $15 \mathrm{mins}\left(2 \mathrm{mAh} \mathrm{cm}^{-2}\right)$, as shown in Figure 12.4a. It can be seen that for the control electrolyte (1M LiTFSI-PC), deposition morphology is uneven and rough compared to 
the smooth and compact deposits with the Indium coating. Further, in comparison to the electrodeposition with Indium salt additives, the lithium deposits with the PC based neat electrolyte is seen to be darker in color, this is an indication of instantaneous side reactions because of an unstable SEI. ${ }^{[3]}$ The quantitative comparison of the morphological changes was done by plotting the height of deposit at different times as shown in Figure 12.4b. Using a linear fit, the growth rate of pristine lithium deposits were found to be $\sim 90 \mathrm{~nm} / \mathrm{sec}$, while that of In-Li electrode was $\sim 12 \mathrm{~nm} / \mathrm{sec}$. Thus, it is evident the Indium layer results in improved long-term electrodeposition stability.

Figure $4 \mathrm{c}$ shows the voltage profile of a galvanostatic charge-discharge experiment for a symmetric lithium cell, where the cell was charged and discharged for 1 hour at a current density of $1 \mathrm{~mA} / \mathrm{cm}^{2}$. The control electrolyte is $1 \mathrm{M}$ LiTFSI in PC, while in the other case, $12 \mathrm{mM} \operatorname{In}(T F S I)_{3}$ salt was added. It can be seen that the within $\sim 30$ cycles the overpotential of the control cell increase upto over twice its initial value. This can be attributed to the formation of an insulating SEI layer by continuous side reactions between $\mathrm{Li}$ and the electrolyte, which hinder ion transport at the interface. In contrast, cells based on the In-Li anodes continue to cycle with lower overpotentials for over 200 hours. A similar experiment was done using the base electrolyte comprised of commercial grade 1M LiPF6 in EC: DMC (1:1 by vol.), and the cells were cycled at a current density of $1 \mathrm{~mA} / \mathrm{cm}^{2}$ for the same charge and discharge time of 1 hour (Figure 12.4d). It is seen that after 120 hours of cycling, there is a sudden drop in the voltage, following rise in overpotential, which is indicative of internal short-circuit; in contrast long-term stability is attained with Indium coating. 
(a)

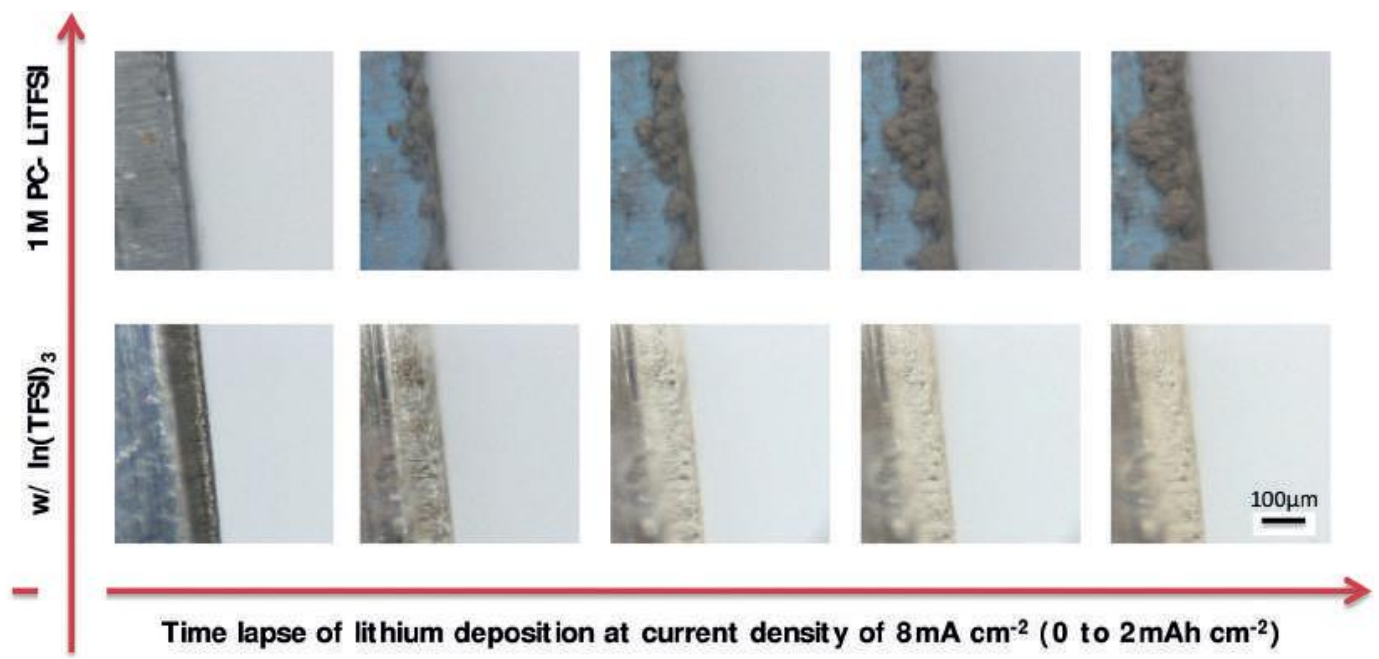

(b)

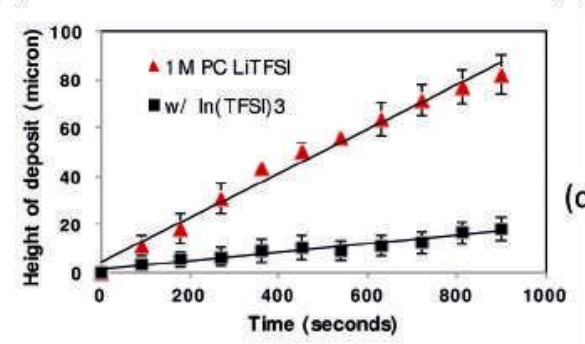

(c)

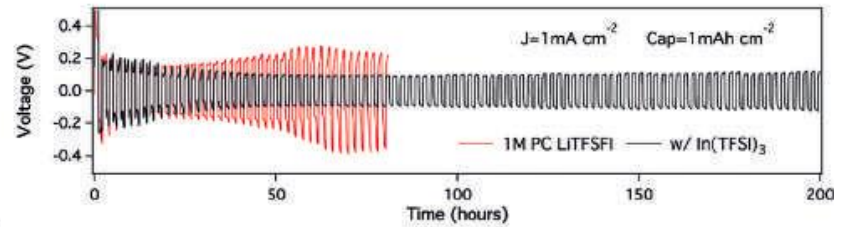

(d)

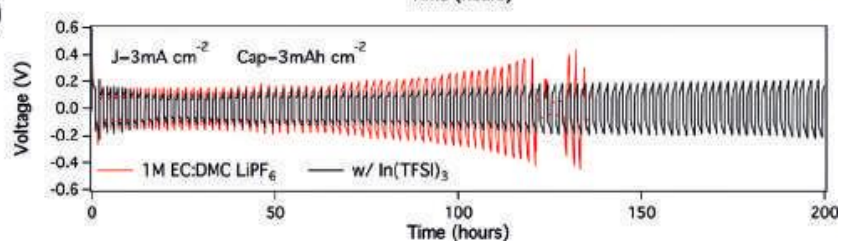

Figure 12.4: Inhibition of dendrite growth during Electrodeposition process (a)

Snapshots of stainless steel electrode during electrodeposition of lithium, obtained by direct-visualization under optical microscope. The first row represents results with the electrolyte $1 \mathrm{M}$ LiTFSi in PC; while in the second row $12 \mathrm{mM} \operatorname{In}(T F S I)_{3}$ was added. The electrochemical cells were operated at a current density of $8 \mathrm{~mA} \mathrm{~cm}^{-2}$. The scale bar is for all the images. (b) Height of individual dendrite as a function of time for control and $\operatorname{In}(\text { TFSI })_{3}$ added electrolyte, obtained by visualization experiments (c) Voltage profile for symmetric lithium cell at current density of $1 \mathrm{~mA} / \mathrm{cm}^{2}$ with each half-cycle is 1 hour. The red curve represents control cell with the electrolyte $1 \mathrm{M}$ LiTFSI in PC, while the black is with $12 \mathrm{mM} \operatorname{In}(\text { TFSI })_{3}$ added in the same electrolyte. (d) Voltage profile of symmetric cells cycled at $3 \mathrm{~mA} / \mathrm{cm}^{2}$ with each half-cycle is 1 hour. Here the control electrolyte, shown in red, is $1 \mathrm{M} \mathrm{LiPF} 6 \mathrm{EC}$ : DMC and the black lines represent cell with the same electrolyte and $12 \mathrm{mM} \operatorname{In}(T F S I)_{3}$ additive. 
Figure 12.5a reports the coulombic efficiency (CE) measurements in cells comprising of Li and stainless steel electrodes. In this experiment, lithium is deposited onto the stainless steel at a current density of $1 \mathrm{~mA} / \mathrm{cm}^{2}$ for 1 hour and then stripped back at the same current density until the voltage rises to $0.5 \mathrm{~V}$ and the $\mathrm{CE}$ is defined as ratio of stripping and plating time. The base electrolyte (control) used here comprises of $1 \mathrm{M}$ $\mathrm{LiPF}_{6}$ in $\mathrm{EC}$ : DMC with addition of 10\%(vol.) fluoroethylene carbonate (FEC), which is known to stabilize the electrode interphase by generating lithium fluoride, ${ }^{[26]}$ while in the other cell the base electrolyte was reinforced with $12 \mathrm{mM} \operatorname{In}(T F S I)_{3}$. It can be seen in Figure 5a that the initial CE for both electrolytes is $>95 \%$, however for the control cell the CE begins to fluctuate and reverts to $<70 \%$ at higher cycle numbers (see Figure 12.5b), in contrast cells based on the In-Li electrodes maintain high CE values ( $>95 \%)$ for over 150 cycles. The fluctuations in the $\mathrm{CE}$ can be shown to arise from sporadic electrical connections of broken pieces of lithium from previous cycles (so called 'orphaned lithium') in the cell. The addition of $\operatorname{In}(T F S I)_{3}$ salt is hypothesized to result in formation of an Indium layer not only on the Li anode, but also on the bare stainless steel electrode, thus preventing side reactions when fresh $\mathrm{Li}$ is deposited on either electrode. On this basis it is argued that the protection technique should be applicable for stabilizing lithium metal anodes in presence of reactive solvents like dimethylacetamide or dimethyl sulfoxide, which are commonly utilized in lithium-oxygen batteries ${ }^{[20,40]}$.

The effectiveness of protection mechanism was finally evaluated in full-cells comprising of Li ||LTO with a very high mass loading cathode $\left(24 \mathrm{mg} / \mathrm{cm}^{2}\right.$ or $3 \mathrm{mAh} / \mathrm{cm}^{2}$ ) at a rate of $1 \mathrm{C}$. Figure $12.5 \mathrm{c}$ and $12.5 \mathrm{~d}$ report the capacity, coulombic 
(a)

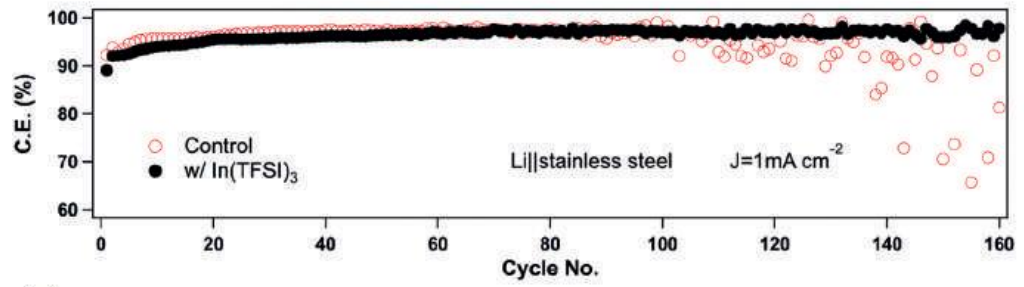

(c)

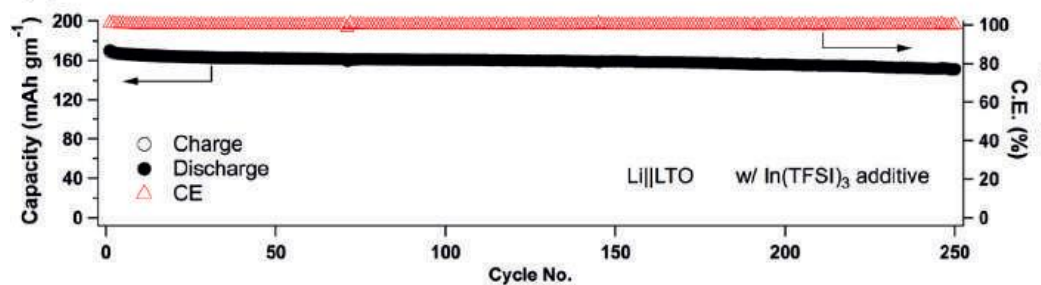

(e)

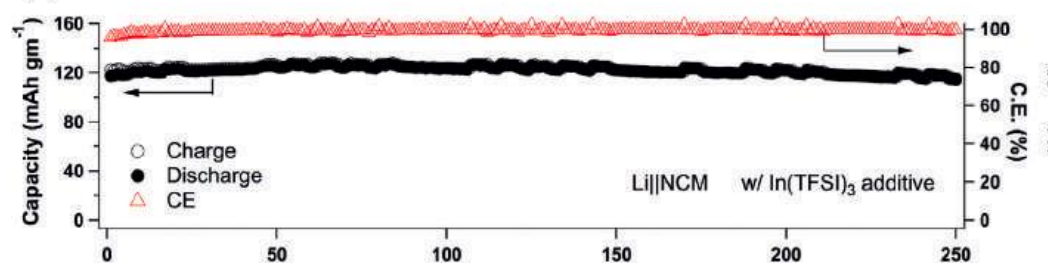

Figure 12.5: Long-term galvanostatic cycling performance: (a) Coulombic efficiency measurement in Li||stainless steel configuration at a current density of $1 \mathrm{~mA} / \mathrm{cm}^{2}$ and capacity of $1 \mathrm{mAh} / \mathrm{cm}^{2}$. The red marker is control electrolyte of $1 \mathrm{M}$ $\mathrm{LiPF}_{6}$ in EC: DMC and red is with $12 \mathrm{mM} \operatorname{In}(\text { TFSI })_{3}$ additive. In both the types of electrolyte 10\% (by vol.) of fluoroethylene carbonate (FEC) has been added. (b) Voltage profile of the Li||stainless steel cells for the $150^{\text {th }}$ cycle. (c) Half-cell cycling with Lithium Titanate cathode using the electrolyte of $1 \mathrm{M} \mathrm{EC/DMC} \mathrm{LiPF}_{6}$ with the additives $12 \mathrm{mM} \operatorname{In}(T F S I)_{3}$ and $10 \%$ (by vol.) FEC. The areal capacity is $3 \mathrm{mAh} / \mathrm{cm}^{2}$ and the C-rate is 1C. (d) Voltage profiles for the Li||LTO cell for cycle numbers 1, 100 and 250. (e) Cycling with Lithium Nickel Cobalt Manganese Oxide cathode using the same electrolyte. The areal capacity is $2 \mathrm{mAh} / \mathrm{cm}^{2}$. The charging cycling rate is $\mathrm{C} / 2$ and the discharging rate is $1 \mathrm{C}$. (f) Voltage profiles for the $\mathrm{Li} \| \mathrm{NCM}$ for $1^{\text {st }}, 100^{\text {th }}$ and $250^{\text {th }}$.

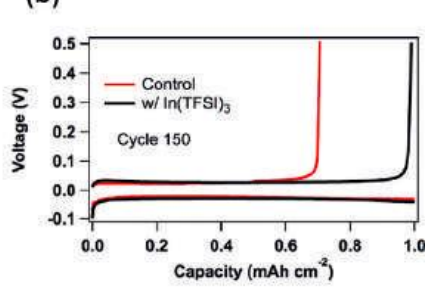

(d)

(f)

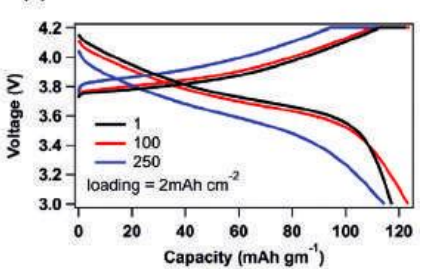


efficiency and voltage profiles of these cells. It is seen that the cell shows over $85 \%$ capacity retention and high coulombic efficiency for at least 250 cycles. Further, we utilized the Indium protection method with high voltage Nickel Manganese Cobalt Oxide (NCM) cathode using the same electrolyte $(1 \mathrm{M} \mathrm{LiPF} 6 \mathrm{EC}$ : DMC $(10 \% \mathrm{FEC}$, $\left.\left.12 \mathrm{mM} \operatorname{In}(\text { TFSI })_{3}\right)\right)$ as shown in Figure 12.5e and 12.5f. The cell was charged at a fixed rate of $0.5 \mathrm{C}$ and discharged at a $1 \mathrm{C}$. Since, $\mathrm{TFSI}^{-}$anion is unstable at high voltages, a very long charge capacity is observed for the initial cycle, shown in Supplementary Figure 12.5. Similar to cells based on LTO cathodes, the coulombic efficiency of the cell remains high ( $>98 \%$ ) for at least 250 cycles, while there is close to $95 \%$ capacity retention. We believe that the long cycle life of the hybrid In-Li anode (paired with a high voltage cathode) is an important development in comparison to recently reported protected anodes $^{[41,42]}$ and it can serve as a suitable, high-energy replacement for the graphitic anode in currently used Li-ion batteries.

\subsection{Conclusion}

In conclusion, using JDT calculations we show that Li ions are very loosely bound to the surface, thus enabling faster relaxation and migration to form more uniform electrodeposits. Experimentally, we demonstrated this idea by an electroless plating method to form a protective Indium layer on lithium metal anode. Using characterization tools like electron microscopy and $\mathrm{x}$-ray spectroscopy, it was shown that the layer is uniform and is stable even after battery cycling. Further, it was found the interfacial resistance is significantly reduced due to presence of the coating, which also acts as a buffer layer, where lithium ions alloys and diffuses before depositing 
onto the underneath lithium electrode. As a result of the enhanced interfacial ion transport mechanism, electrodeposition at long time-scales was visually observed to be compact and uniform. As a result of the stable electrodeposition using the electroless Indium coating, lithium metal batteries with high loading cathodes of LTO and NCM could be stably cycled for over 250 cycles with close to $90 \%$ capacity retention.

\section{Acknowledgements}

We are grateful to the Advanced Research Projects Agency- Energy (ARPA-E) through award number 1002-2265, DEFOA- 001002 for supporting this study. The study also made use of the electrochemical characterization facilities of the KAUSTCU Center for Energy and Sustainability, which is supported by the King Abdullah University of Science and Technology (KAUST) through award number KUS-C1018- 02. Electron microscopy facilities at the Cornell Center for Materials Research (CCMR), an NSF-supported MRSEC through Grant DMR-1120296, were also used for the study. 


\section{REFERENCES}

[1] M. Armand, J. -M. Tarascon. Nature 2008, 451, 652-657.

[2] J. -M. Tarascon, M. Armand. Nature 2001, 414, 359-67.

[3] M. D. Tikekar, S. Choudhury, Z. Tu, L. A. Archer. Nat. Energy 2016, 1, 16114.

[4] X. Cheng, R. Zhang, C. Zhao, F. Wei, J. Zhang, Q. Zhang, Adv. Sci. 2016, 3, 120.

[5] Z. Tu, P. Nath, Y. Lu, M. D. Tikekar, L. A. Archer, Acc. Chem. Res. 2015, 48, 2947-2956.

[6] M. D. Tikekar, L. A. Archer, D. L. Koch, J. Electrochem. Soc. 2014, 161, A847-A855.

[7] M. D. Tikekar, L. A. Archer, D. L. Koch, Sci. Adv. 2016, 2.

[8] J. C. Bachman, S. Muy, A. Grimaud, H. Chang, N. Pour, S. F. Lux, O. Paschos, F. Maglia, S. Lupart, P. Lamp, et al., Chem. Rev. 2016, 116, 140-162.

[9] Z. Tu, Y. Kambe, Y. Lu, L. A. Archer, Adv. Energy Mater. 2014, 4, 1300654.

[10] S. -O. Tung, S. Ho, M. Yang, R. Zhang, N. A Kotov, Nat. Commun. 2015, 6, 6152. 
[11] Z. Tu, M. J. Zachman, S. Choudhury, S. Wei, L. Ma, Y. Yang, L. F. Kourkoutis, L. A. Archer, Adv. Energy Mater. 2017, 7, 1602367.

[12] S. Choudhury, R. Mangal, A. Agrawal, L. A. Archer, Nat. Commun. 2015, 6, 10101.

[13] R. Khurana, J. L. Schaefer, L. A Archer, G. W. Coates, J. Am. Chem. Soc. 2014, 136, 7395-7402.

[14] X. B. Cheng, T. Z. Hou, R. Zhang, H. J. Peng, C. Z. Zhao, J. Q. Huang, Q. Zhang, Adv. Mater. 2016, 28, 2888-2895.

[15] Y. Lu, M. Tikekar, R. Mohanty, K. Hendrickson, L. Ma, L. A. Archer, $A d v$. Energy Mater. 2015, 5, 1402073.

[16] J. L. Schaefer, D. A. Yanga, L. A. Archer, Chem. Mater. 2013, 25, 834-839.

[17] H. Wu, G. Yu, L. Pan, N. Liu, M. T. McDowell, Z. Bao, Y. Cui, Nat. Commun. 2013, 4, 1943.

[18] D. Aurbach, E. Zinigrad, Y. Cohen, H. Teller, Solid State Ionics 2002, 148, 405-416.

[19] D. Lin, Y. Liu, Y. Cui, Nat. Nanotechnol. 2017, 12, 194-206.

[20] S. Choudhury, C. T. Wan, W. I. Al Sadat, Z. Tu, S. Lau, M. J. Zachman, L. F. Kourkoutis, L. A. Archer, Sci. Adv. 2017, 3. 
[21] F. Ding, W. Xu, G. L. Graff, J. Zhang, M. L. Sushko, X. Chen, Y. Shao, M. H. Engelhard, Z. Nie, J. Xiao, et al., J. Am. Chem. Soc. 2013, 135, 4450-4456.

[22] S. Choudhury, L. A. Archer, Adv. Electron. Mater. 2015, 2, 1500246.

[23] G. Zheng, S. W. Lee, Z. Liang, H.-W. Lee, K. Yan, H. Yao, H. Wang, W. Li, S. Chu, Y. Cui, Nat. Nanotechnol. 2014, 9, 618-623.

[24] A. C. Kozen, C. Lin, A. J. Pearse, M. a Schroeder, X. Han, L. Hu, S. Lee, G. W. Rubloff, M. Noked, ACS Nano 2015, 9, 5884-5892.

[25] Z. Liang, G. Zheng, C. Liu, N. Liu, W. Li, K. Yan, H. Yao, P.-C. Hsu, S. Chu, Y. Cui, Nano Lett. 2015, 15, 2910-2916.

[26] X. Zhang, X. Cheng, X. Chen, C. Yan, Q. Zhang, Adv. Funct. Mater. 2017, 27, 1605989.

[27] J. Qian, W. a Henderson, W. Xu, P. Bhattacharya, M. Engelhard, O. Borodin, J.-G. Zhang, Nat. Commun. 2015, 6, 6362.

[28] Z. W. Seh, J. Sun, Y. Sun, Y. Cui, ACS Cent. Sci. 2015, 1, 449-455.

[29] Y. Lu, Z. Tu, L. A. Archer, Nat. Mater. 2014, 13, 961-969.

[30] Y. Ozhabes, D. Gunceler, T. A. Arias, arXiv 2015, 1504.05799, 1-7.

[31] S. Choudhury, S. Wei, Y. Ozhabes, D. Gunceler, et al. Nat. Commun. 2017, doi:10.1038/s41467-017-00742-X 
[32] M. Jäckle, A. Groß, J. Chem. Phys. 2014, 141, 174710.

[33] D. Gunceler, K. A. Schwarz, K. L. R. Sundararaman, T. A. Arias, 16th Int. Work. Comput. Phys. Mater. Sci. Total Energy Force Methods 2013.

[34] D. Gunceler, K. Letchworth-Weaver, R. Sundararaman, K. A. Schwarz, T. A. Arias, Model. Simul. Mater. Sci. Eng. 2013, 21, 074005.

[35] NIST X-ray Photoelectron Spectroscopy Database, Version 4.1 (National Institute of Standards and Technology, Gaithersburg, 2012)

[36] D. Aurbach, B. Markovsky, a. Shechter, Y. Ein-Eli, H. Cohen, J. Electrochem. Soc. 1996, 143, 3809-3820.

[37] H. Ota, Y. Sakata, X. Wang, J. Sasahara, E. Yasukawa, J. Electrochem. Soc. 2004, 151, A437-A446.

[38] P. Verma, P. Maire, P. Novak, Electrochim. Acta 2010, 55, 6332-6341.

[39] S. Ha, Y. Lee, S. W. Woo, B. Koo, J. Kim, J. Cho, K. T. Lee, N. Choi, ACS Appl. Mater. Interfaces 2014, 6, 4063-4073.

[40] T. Zhang, K. Liao, P. He, H. Zhou, Energy Environ. Sci. 2015, 9, 1024-1030.

[41] N.-W. Li, Y.-X. Yin, C.-P. Yang, Y.-G. Guo, Adv. Mater. 2016, 28, $1853-$ 1858. 
[42] X.-B. Cheng, C. Yan, X. Chen, C. Guan, J.-Q. Huang, H.-J. Peng, R. Zhang, S.T. Yang, Q. Zhang, Chem 2017, 2, 258-270. 


\section{APPENDIX \\ Supplementary Information for Chapter 12}

Supplementary Table 12.1: Comparison of predicted surface energies and $\mathrm{Li}$ diffusion barriers for various low-index surfaces of indium in vacuum and acetonitrile solvent (this work) against those for the most stable surfaces of lithium, sodium and magnesium ${ }^{1}$. While surface energies and vacuum diffusion barriers are comparable to previous cases, dramatic reduction in diffusion barriers for Li on indium surfaces in solution suggests reduced dendrite formation on indium electrodes.

\begin{tabular}{|c|c|c|c|c|c|}
\hline & \multirow[t]{2}{*}{ Surface } & \multicolumn{2}{|c|}{ Surface energy $\left[\mathrm{J} / \mathrm{m}^{2}\right]$} & \multicolumn{2}{|c|}{$\begin{array}{c}\text { Li diffusion barrier } \\
{[\mathrm{eV}]}\end{array}$} \\
\hline & & Vacuum & $\mathrm{CH}_{3} \mathrm{CN}$ & Vacuum & CH3CN \\
\hline \multirow{4}{*}{$\begin{array}{l}\text { This } \\
\text { work }\end{array}$} & $\operatorname{In}(011)$ & 0.25 & 0.25 & 0.04 & 0.015 \\
\hline & $\operatorname{In}(001)$ & 0.26 & 0.26 & 0.30 & 0.013 \\
\hline & $\operatorname{In}(110)$ & 0.29 & 0.29 & - & - \\
\hline & $\operatorname{In}(100)$ & 0.31 & 0.30 & - & - \\
\hline \multirow{3}{*}{ Ref. 1} & $\operatorname{Li}(001)$ & 0.46 & - & 0.14 & - \\
\hline & $\mathrm{Na}(001)$ & 0.22 & - & 0.16 & - \\
\hline & $\operatorname{Mg}(0001)$ & 0.52 & - & 0.02 & - \\
\hline
\end{tabular}


Supplementary Table 12.2: Values of Arrhenius fitting parameters for inverse interfacial resistance

\begin{tabular}{|c|c|c|}
\hline Type of cell & $\begin{array}{c}\text { Prefactor (A) } \\
\left({ }^{-1}\right)\end{array}$ & $\begin{array}{c}\text { Activation } \\
\text { Energy (Ea) } \\
(\mathrm{eV} / \text { atom) }\end{array}$ \\
\hline Control & 123660.9 & 0.43 \\
\hline $\begin{array}{c}\text { w/ In salt (before } \\
\text { conditioning) }\end{array}$ & 36997.2 & 0.39 \\
\hline $\begin{array}{c}\text { w/ In salt (after } \\
\text { conditioning) }\end{array}$ & 9979.9 & 0.29 \\
\hline
\end{tabular}




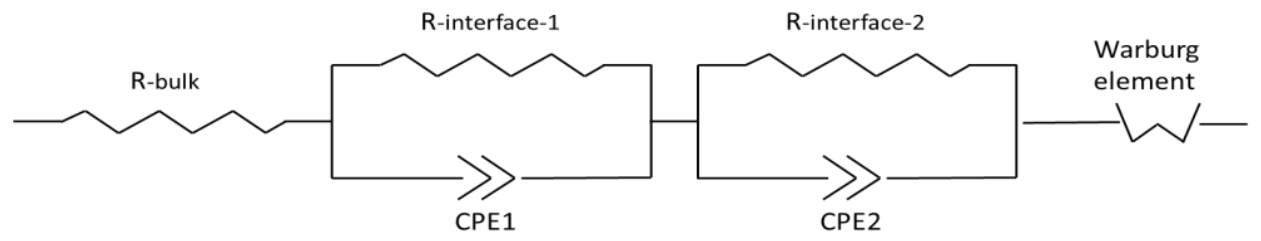

Supplementary Figure 12.1: Equivalent circuit model for fitting Nyquist plots of impedance measurements. R-bulk represent bulk electrolyte. R-interface ${ }_{1}$ represent interfacial resistance associated with the passivation layer and R-interface 2 denotes the charge transfer resistance. CPE1, CPE2 represent the constant phase elements. Warburg element is the solid-state diffusion contribution 
(a)

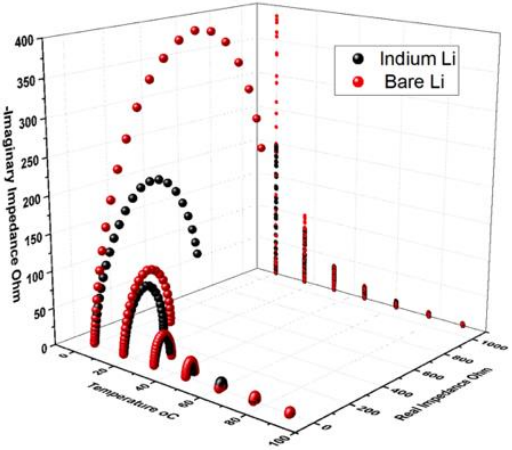

(b)

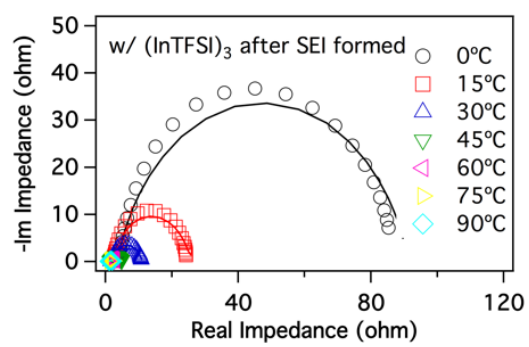

Supplementary Figure 12.2: (a) Nyquist plots as a function of temperature for control electrolyte and $\operatorname{In}(T F S I)_{3}$ added electrolyte before conditioning obtained by Impedance Spectroscopy measurements; (b) Nyquist diagram for $\operatorname{In}(T F S I)_{3}$ added electrolyte, after cycling at low current densities 


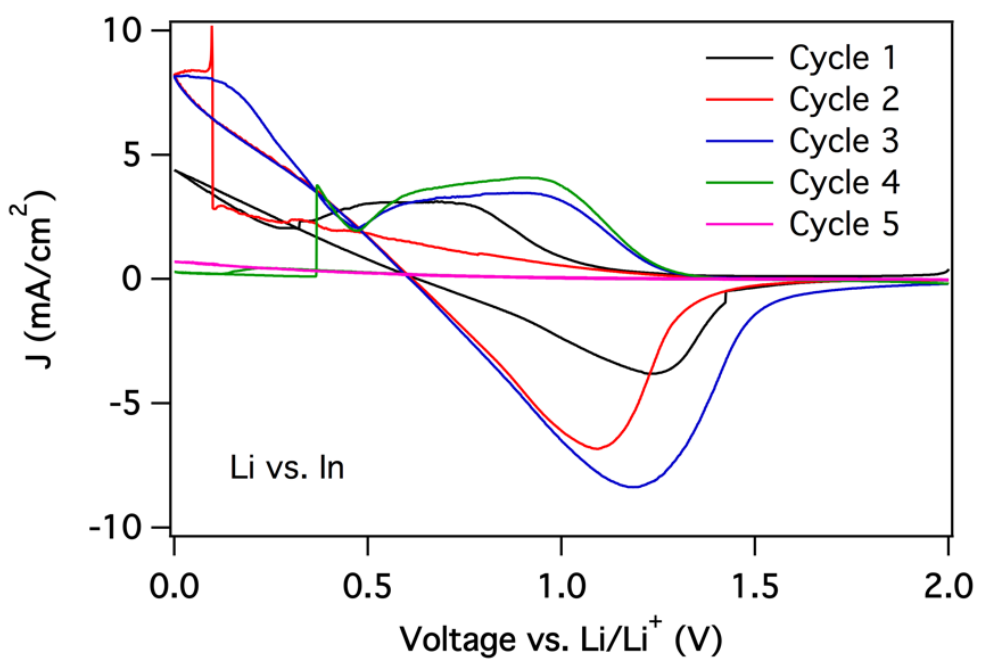

Supplementary Figure 12.3: Cyclic voltammetry result of Indium electrode $(100 \mu \mathrm{m})$ vs. Lithium electrode for five cycles. It is seen that the cycling result is 'noisy' and there is significant shift in the current peaks. 


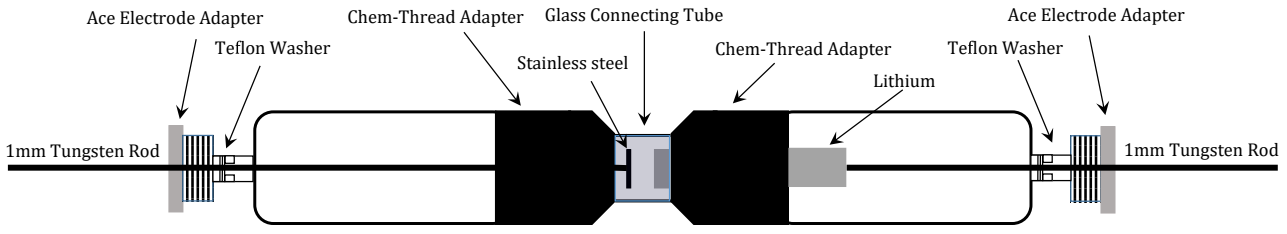

Supplementary Figure 12.4: In-house built electrochemical setup for visualization experiments involving electrodeposition of lithium metal onto stainless steel electrode. 


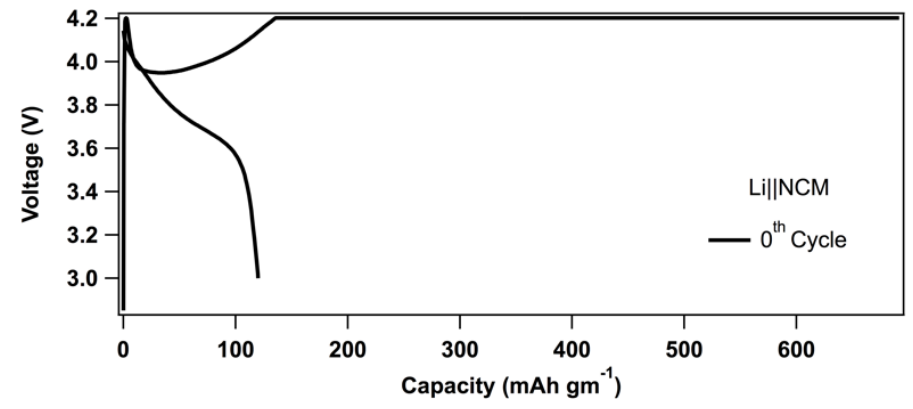

Supplementary Figure 12.5: Initial cycle of Li||NCM cell using the electrolyte 1M $\mathrm{LiPF}_{6}$ in EC: DMC with additives $10 \% \mathrm{FEC}$ and $12 \mathrm{mM} \operatorname{In}(T F S I)_{3}$. The long charging curve is due to the formation of SEI by breakdown of TFSI anions. 


\section{Methods}

\section{Computational details}

We perform density-functional theory calculations using the open-source JDFTx software $^{2}$, employing a plane-wave basis with ultrasoft pseudopotentials ${ }^{3}$ at the recommended kinetic energy cutoffs of 20 and 100 Hartrees for wavefunctions and charge densities respectively. We use the PBE generalized-gradient approximation to the exchange-correlation functional ${ }^{3}$, and the Nonlinear PCM model ${ }^{4}$ to describe solvation in acetonitrile ${ }^{5}$ along with $1 \mathrm{M}$ non-adsorbing electrolyte. (Electrolytes in continuum solvation models are non-adsorbing by definition). We use k-point grids that correspond to an effective supercell size of at least $30 \mathrm{~A}$ in each periodic direction, and Fermi smearing with a width of 0.01 Hartrees.

For the body-centered tetragonal lattice of bulk Indium, we obtain lattice constants $a=$ 3.27 A and $c=4.96 \mathrm{~A}$, in excellent agreement with experiment (errors $+0.5 \%$ and $+0.4 \%$ respectively). For the surfaces, we use inversion-symmetric 5 layer slab models with a vacuum/solvent gap of $15 \mathrm{~A}$, along with truncated Coulomb potentials to exactly eliminate interactions between periodic images along the slab normal ${ }^{6}$. For diffusion calculations, we use $3 \times 3$ supercells along the surface directions, and map the energy landscape of an Li atom over a symmetry-irreducible wedge of one-unit cell. Specifically, we use (the irreducible wedges of) an 8x8 uniform grid of Li positions for the (001) surface and a $6 \times 6$ grid for (011). All ionic positions are optimized selfconsistently, except for the central layer held at bulk geometry for surface 
calculations, and planar coordinates of $\mathrm{Li}$ constrained for mapping the energy landscape.

\section{Experimental details}

\section{$\underline{\text { Materials }}$}

Lithium discs were obtained from MTI corporation. Indium foil (0.1micron), Indium(III) tris(trifluoromethanesulfonimide), Ethylene Carbonate, Diethylene carbonate, Propylene Carbonate, Lithium Hexafluorophosphate, Lithium bis(trifluoromethanesulfonimide) were all purchased from Sigma Aldrich. Fluoroethylene carbonate was obtained from Alfa Aesar. Celgard 3501 separator was obtained from Celgard Inc. Lithium Titanate was obtained from NEI Corporations. Nickel Cobalt Manganese Oxide cathode was bought from Electrodes and More Co. All the chemicals were used as received in after rigorous drying in a $\sim 0 \mathrm{ppm}$ water level and $<5$ ppm oxygen glove box.

\section{$\underline{\text { Scanning electron microscopy and EDX }}$}

Surface analysis of Indium coated lithium samples was done using SEM and EDX techniques using the LEO155FESEM instrument. The samples were prepared by 6 hours treating of lithium disc in a solution of $12 \mathrm{mM} \operatorname{In}(\mathrm{TFSI})_{3}$ in EC/DMC solvent,

followed by 2 days drying in glove-box antechamber. Morphology of electrodeposition was studied by a post-mortem analysis of a $\mathrm{Li}||$ stainless steel battery using the electrolyte $1 \mathrm{M} \mathrm{LiPF} 6 \mathrm{EC} / \mathrm{DMC}$ with a celgard separator. For the case of 
Indium coating, $12 \mathrm{mM} \operatorname{In}(\mathrm{TFSI})_{3}$ was added in the control electrolyte. The battery was discharged at a rate of $0.5 \mathrm{~mA} / \mathrm{cm}^{2}$ for 6hours.

\section{$\underline{X-R a y \text { Diffraction }}$}

XRD was carried out on a Scintag Theta-Theta X-ray diffractometer using Cu K- $\alpha$ radiation at $\lambda=1.5406 \AA$. Samples were quickly transferred to the XRD chamber with minimal exposure to air. For XRD same Indium coated lithium samples were used as for SEM characterizations.

\section{X-ray Photoelectron Spectroscopy}

XPS was conducted using Surface Science Instruments SSX-100 with operating pressure of $\sim 2 \times 10^{-9}$ torr. Monochromatic Al K- $\alpha$ x-rays $(1486.6 \mathrm{eV})$ with beam diameter of $1 \mathrm{~mm}$ were used. Photoelectrons were collected at an emission angle of $55^{\circ}$. A hemispherical analyzer determined electron kinetic energy, using pass energy of $150 \mathrm{~V}$ for wide survey scans and 50V for high-resolution scans. Samples were ionetched using $4 \mathrm{kV}$ Ar ions, which were rastered over an area of $2.25 \times 4 \mathrm{~mm}$ with total ion beam current of $2 \mathrm{~mA}$, to remove adventitious carbon. Spectra were referenced to adventitious C 1s at $284.5 \mathrm{eV}$. CasaXPS software was used for XPS data analysis with Shelby backgrounds. Samples were exposed to air only during the short transfer time to the XPS chamber (less than 10 seconds).

\section{Impedance Spectroscopy}


The impedance spectroscopy measurement was done using a Novocontrol N40 Broadband Dielectric instrument. Symmetric cells were prepared using two lithium discs using the electrolyte $1 \mathrm{M} \mathrm{LiPF} 6$ EC/DMC with a celgard separator. The Indium based cells were prepared by addition of $12 \mathrm{mM} \mathrm{In(TFSI)})_{3}$ as the co-salt in the control electrolyte. The measurements were done in a frequency range from $10^{-3}$ to $10^{7} \mathrm{~Hz}$.

\section{Cyclic voltammetry}

Cyclic voltammetry was performed in two-electrode setup. In one of the test the cell comprised of lithium as reference and counter-electrode, and Indium foil as working electrode; while in another experiment stainless steel was used as the working electrode. The electrolyte used in the former case was $1 \mathrm{M} \mathrm{LiPF} 6 \mathrm{EC} / \mathrm{DMC}$, while in the later the same electrolyte was used with $12 \mathrm{mM} \operatorname{In}(\mathrm{TFSI})_{3}$. In both cases the separator used was celgard and the scanning rate was $1 \mathrm{mV} / \mathrm{sec}$ operated between 0 to 2V (vs. Li/Li $\left.{ }^{+}\right)$.

\section{Direct Visualization experiments}

The visualization experiment was carried out for understanding the electrodeposition process with and without Indium coatings. The electrolyte utilized was $1 \mathrm{M} \mathrm{LiPF}$ - EC: DMC and the same electrolyte with $12 \mathrm{mM} \operatorname{In}(\mathrm{TFSI})_{3}$ additive. The setup consists of a glass chamber with a lithium rod on one side and stainless steel disc on the other, connected using tungsten rods. The glass-tube was tightly closed and sealed with Teflon film to ensure complete inert environment inside the cell. In these experiment, the stainless steel electrode was continuously charged with a current density of 
$8 \mathrm{~mA} / \mathrm{cm}^{2}$. The electrode, being charged was monitored over time and images of lithium deposition at different intervals were captured from an optical microscope.

\section{Battery Performance}

2032 type $\mathrm{Li} \| \mathrm{Li}$ coin cells with and without $12 \mathrm{mM} \operatorname{In}(\mathrm{TFSI})_{3}$ were prepared inside an argon-filled glove box. The amount of electrolyte used for all battery testing was $60 \mu 1$. The cells were evaluated using galvanostatic (strip-plate) cycling in a Neware CT3008 battery tester. The batteries were repeatedly charged and discharged with each half-cycle 1 hour long. Coulombic Efficiency test was performed in Li ||stainless steel cell with a current density higher current density of $1 \mathrm{~mA} / \mathrm{cm}^{2}$ and capacity $1 \mathrm{mAh} / \mathrm{cm}^{2}$, $10 \%$ (vol.) fluoroethylene carbonate was used in additive in both control and Indium based electrolytes. Half-cell test was performed in Lithium versus Lithium Titanate cell at a C-rate of $1 \mathrm{C}$. The cathode loading was $3 \mathrm{mAh} / \mathrm{cm}^{2}$ and the voltage range was between $1 \mathrm{~V}$ to $3 \mathrm{~V}$. For cycling NCM cells with $2 \mathrm{mAh} \mathrm{cm}^{-2}$, the voltage range was chosen to be $4.2 \mathrm{~V}$ to $3 \mathrm{~V}$. A constant voltage step was applied at the end of the charge cycle at 4.2 , until the current reduced to $10 \%$ of the current used in galvanostatic charging process. The charging was done at $\mathrm{C} / 2$ rate and discharge at $1 \mathrm{C}$. 


\section{REFERENCES}

1. Jäckle, M. \& Groß, A. Microscopic properties of lithium, sodium, and magnesium battery anode materials related to possible dendrite growth. $J$. Chem. Phys. 141, (2014).

2. R. Sundararaman, D. Gunceler, K. Letchworth-Weaver, K. S. and T. A. A. JDFTx code. http://jdftx.sourceforge.net (2012).

3. Garrity, K. F., Bennett, J. W., Rabe, K. M. \& Vanderbilt, D. Pseudopotentials for high-throughput DFT calculations. Comput. Mater. Sci. 81, 446-452 (2014).

4. Gunceler, D., Letchworth-Weaver, K., Sundararaman, R., Schwarz, K. A. \& Arias, T. A. The importance of nonlinear fluid response in joint densityfunctional theory studies of battery systems. Model. Simul. Mater. Sci. Eng. 21, 74005 (2013).

5. Gunceler, D. \& Arias, T. A. Towards a generalized iso-density continuum model for molecular solvents in plane-wave DFT. Model. Simul. Mater. Sci. Eng. 25, (2017).

6. Sundararaman, R. \& Arias, T. A. Regularization of the Coulomb singularity in exact exchange by Wigner-Seitz truncated interactions: Towards chemical accuracy in nontrivial systems. Phys. Rev. B 87, 165122 (2013). 
Chapter 13

Designer interphases for the lithium-oxygen electrochemical cell 


\subsection{Abstract}

An electrochemical cell based on the reversible oxygen reduction reaction (ORR): $2 \mathrm{Li}^{+}+2 \mathrm{e}^{-}+\mathrm{O}_{2} \leftrightarrow \mathrm{Li}_{2} \mathrm{O}_{2}$, provides among the most energy dense platform for portable electrical energy storage. Such Lithium-Oxygen $\left(\mathrm{Li}^{-} \mathrm{O}_{2}\right)$ cells offer theoretical specific energies competitive with fossil fuels and have long been considered an important storage technology for enabling electrified transportation. Multiple, fundamental challenges with the cathode, anode, and electrolyte have limited practical interest in $\mathrm{Li}-\mathrm{O}_{2}$ cells because these fundamental problems lead to practical shortcomings, including poor rechargeability, high overpotentials, and specific energies well below theoretical expectations. We create and study in-situ formation of solid-electrolyte interphases (SEIs) based on bromide ionomers tethered to the $\mathrm{Li}$ anode that take advantage of three powerful, fundamental processes for overcoming the most stubborn of these challenges. Formed in-situ, the ionomer SEIs are specifically shown to exhibit three attributes required for stable $\mathrm{Li}-\mathrm{O}_{2}$ cell operation. First, they protect the $\mathrm{Li}$ anode against parasitic reactions and also stabilize Li electrodeposition during cell recharge. Second, bromine species liberated during the anchoring reaction function as a redox mediator for the recharge reaction at the cathode, reducing the charge overpotential. Finally, the ionomer SEI form an exceptionally stable interphase with $\mathrm{Li}$, which is shown to protect the metal in high Gutmann donor number liquid electrolytes. Such electrolytes have been reported to exhibit rare stability against nucleophilic attack by $\mathrm{Li}_{2} \mathrm{O}_{2}$ and other cathode reaction intermediates but are known for their reactivity with Li metal anodes. We conclude 
that rationally designed SEIs able to regulate transport of matter and ions at the electrolyte/anode interface provide a highly promising materials platform for addressing the major barriers to practical $\mathrm{Li}-\mathrm{O}_{2}$ storage technology.

\subsection{Introduction}

The rechargeable lithium-oxygen $\left(\mathrm{Li}-\mathrm{O}_{2}\right)$ electrochemical cell is peerless among energy storage technologies for its high theoretical specific energy $(3500 \mathrm{Wh} / \mathrm{kg})$, which far exceeds that of current state-of-the-art Li-ion battery technology. ${ }^{1-4} \mathrm{Li}-\mathrm{O}_{2}$ cells are under intense study for applications in electrified transportation because they are viewed as the gateway to Li-Air storage technology able to offer competitive specific storage capacities to fossil fuels. A $\mathrm{Li}-\mathrm{O}_{2}$ cell consists of a $\mathrm{Li}$ metal anode, an electrolyte that conducts $\mathrm{Li}^{+}$ions, and uses $\mathrm{O}_{2}$ gas hosted in a porous carbon or metal support as the active material in the positive electrode (cathode). Ideally, the cell operates on the principle that $\mathrm{Li}_{2} \mathrm{O}_{2}$ is reversibly formed and decomposed in the cathode, with the net electrochemical reaction of $2\left(\mathrm{Li}^{+}+\mathrm{e}^{-}\right)+\mathrm{O}_{2} \leftarrow \rightarrow \mathrm{Li}_{2} \mathrm{O}_{2}$ at an equilibrium potential of $2.96 \mathrm{~V}$ vs. $\mathrm{Li} / \mathrm{Li}^{+}$. In practice, however, the physicochemical processes in $\mathrm{Li}-\mathrm{O}_{2}$ cells rarely, if ever, live up to these ideals. The insoluble electrically insulating $\mathrm{Li}_{2} \mathrm{O}_{2}$ is, for example, difficult to oxidize, which leads to high charging overpotential (charge voltage $\sim 4.3 \mathrm{~V}$ ) on the oxygen evolution reaction (OER) and limits the cell efficiency to $\sim 60-70 \%{ }^{5-7}$. Additionally, decomposition reactions between the electrolyte and reactive oxygen species at the positive electrode and lithium metal at the negative electrode form undesirable products that further limit cell life and efficiency ${ }^{8,9}$. Finally, insoluble $\mathrm{Li}_{2} \mathrm{O}_{2}$ produced upon discharge 
accumulates in the cathode, eventually clogging pores in the cathode support, which simultaneously limits the $\mathrm{Li}_{-} \mathrm{O}_{2}$ cell discharge capacity and compromise rechargeability. ${ }^{10,11}$ A grand challenge in the field concerns the development of materials and cell running protocols that are able to overcome all of these limitations without compromising the favorable attributes of the $\mathrm{Li}-\mathrm{O}_{2}$ cell.

Several approaches have been proposed for overcoming each of the challenges with the $\mathrm{Li}-\mathrm{O}_{2}$ cell, but a frustrating observation is that promising solutions to one problem often come at the expense of others or in some cases create new problems ${ }^{1,4}$. For example, significant theoretical and experimental efforts to lower the overpotential of the OER have resulted in the exploration of soluble redox mediators as electrolyte additives. Redox mediators are first oxidized electrochemically at a lower potential than $\mathrm{Li}_{2} \mathrm{O}_{2}$; the oxidized form of a soluble redox mediator can therefore diffuse to and oxidize otherwise electrochemically inaccessible $\mathrm{Li}_{2} \mathrm{O}_{2}$ deposited on the cathode surface, regenerating the mediator and allowing the $\mathrm{Li}-\mathrm{O}_{2}$ battery to be recharged at a lower overpotential. Since their introduction by Bruce and co-workers ${ }^{12}$, multiple successful demonstrations of this concept has been reported using Tetrathiafulvalene $(\mathrm{TTF})^{12}, \quad\left(2,2,6,6\right.$-tetramethylpiperidin-1-yl) oxidanyl $(\mathrm{TEMPO})^{13}$, nitroxides $^{13,14}$, Lithium iodide $(\mathrm{LiI})^{15-17}$, tris[4-(diethylamino) phenyl] amine (TDPA) $)^{18}$, iron phthalocyanine $(\mathrm{FePc})^{19}$, and $\mathrm{LiBr}^{20}$ as electrolyte additives. A drawback of this approach is that, with few exceptions, ${ }^{13,21}$ the redox mediator is free to diffuse throughout the cell and is reduced by Li metal in a parasitic process that depletes both the anode and redox mediator. Likewise, efforts to improve the stability of electrolytes 
in the presence of the highly nucleophilic $\mathrm{O}_{2}$. species produced at the cathode and the Li metal anode ${ }^{22,23}$ have produced mixed results.

It is now known that electrolytes based on ethers, carbonates, ketones, and esters are all broken down at the cathode of a $\mathrm{Li}-\mathrm{O}_{2}$ cell by the highly nucleophilic $\mathrm{Li}_{2} \mathrm{O}_{2}$ discharge product. At the anode, no liquid electrolyte presently exists that can survive long-term contact with metallic $\mathrm{Li}$ and few form a stable solid electrolyte interphase (SEI) with $\mathrm{Li}^{24}$. Results from electrochemical mass spectrometry studies have shown that straight-chain alkyl amides N,N-dimethylformamide (DMF) and $\mathrm{N}, \mathrm{N}-$ dimethylacetamide (DMA) are unique among electrolyte solvents for their stability against nucleophilic attack at the $\mathrm{Li}-\mathrm{O}_{2}$ cathode ${ }^{25,26}$. Burke et. al. ${ }^{27}$ reported that the high donor number solvents (like, DMSO in their case) induces a solution mediated reaction pathway at the cathode by stabilizing $\mathrm{LiO}_{2}$ intermediates and the anion $\mathrm{NO}_{3}{ }^{-}$, which leads to higher cell discharge capacity. ${ }^{27-31} \mathrm{~A}$ perhaps obvious drawback is that these high donor number electrolytes undergo continuous chemical reaction with the $\mathrm{Li}$ anode, degrading the anode and electrolyte. $\mathrm{LiNO}_{3}$ salt additives have been investigated for its ability to form stable coatings on Li metal in certain electrolytes, which passivate the metal against attack even by electrolytes containing oxidizing sulfur species ${ }^{32-35}$. In an important study, Walker et al ${ }^{36}$ showed that electrolytes that combine the beneficial effects of $\mathrm{LiNO}_{3}$ and $\mathrm{N}, \mathrm{N}$-dimethylacetamide do in fact enable longer term cycling of $\mathrm{Li}^{-\mathrm{O}_{2}}$ cells, underscoring the synergistic benefits of a high donor number electrolyte and anode protection in the $\mathrm{Li}-\mathrm{O}_{2}$ cell.

An unprotected Li metal anode can fail by other, more catastrophic processes than those precipitated by uncontrolled chemical reaction with a liquid electrolyte ${ }^{24,37,38}$. 
Electrodeposition of lithium metal during battery recharge is known to be physically unstable towards formation of rough/dendritic structures on the anode that ultimately grow to short-circuit the cell. The ohmic heat generated by this process can trigger thermal run-away of the cell in organic liquid electrolytes leading to cell failure by fire and/or explosions ${ }^{39-41}$. Furthermore, because rough electrodeposition increases the surface area of $\mathrm{Li}$ in contact with liquid electrolytes, physical instability of the $\mathrm{Li}$ anode exacerbates chemical instability at the anode/electrolyte interface. Three recent reviews provides a comprehensive assessment of the strengths and shortcomings of practiced strategies for stabilizing rechargeable lithium batteries against failure by dendrite-induced short-circuits. ${ }^{24,42,43}$ An important conclusion is that because Li deposition is fundamentally unstable, fundamentally-based approaches that take advantage of multiple physical processes are likely to be the most successful in guaranteeing long-term stability of rechargeable batteries that use metallic lithium as anode.

Herein, we report on the stability of $\mathrm{Li}_{-} \mathrm{O}_{2}$ cells employing liquid electrolytes containing an ionomer salt additive that spontaneously forms a multifunctional solidelectrolyte interphase (SEI) at the anode. The additive and in-situ-formed-SEI it forms are deliberately designed to take advantage of three, fundamentally-based mechanisms for stabilizing electrochemical processes at the anode and cathode of the $\mathrm{Li}_{2} \mathrm{O}_{2}$ cell. First, consistent with predictions from recent continuum ${ }^{44,45}$ and density functional analyses of lithium deposition ${ }^{46}$, we report that ionomer electrolyte additives able to ensure low diffusion barriers and high cation fluxes in the SEI at the anode are highly effective in stabilizing deposition of Li. We demonstrate the success of these additives 
by means of electrochemical analysis and post-mortem imaging. Second, we show that if the ionomer additives are designed to form thin conformal coatings at the Li surface, it is possible to passivate the anode surface against chemical attack by high donor number $(\mathrm{DN}=27.8)$ liquid electrolytes capable of stabilizing oxide intermediates on the cathode. Finally, we report that the same material that stabilizes Li deposition on the anode also functions as an effective redox mediator that lowers the overpotential for the OER reaction at the $\mathrm{Li}-\mathrm{O}_{2}$ cathode.

\subsection{Results and Discussion}

\subsubsection{Understanding the anode protection mechanism}

\subsubsection{Characterization of the anode}

The electrolyte ionomer salt additive (2-bromo-ethanesulfonate lithium salt) investigated in the present study is illustrated in Figure 13.1(a). The material is chosen because of its ability to react with Lithium to simultaneously anchor lithiumethanesulfonate at the anode/electrolyte interface and to generate partially soluble lithium bromide $(\mathrm{LiBr})$ in the electrolyte. The specific ionomer chemistry selected for the study is motivated by four fundamental considerations. First, recent continuum theoretical analysis ${ }^{44,45}$ and experiment ${ }^{47-49}$, indicate tethering anions such as sulfonates at the anode/electrolyte interface lowers the potential at the interface during Li deposition and in so doing stabilizes the deposition. Second, Joint Density Functional (JDFT) calculations ${ }^{46}$, show that the energy barrier $E_{a}$ for $\mathrm{Li}^{+}$diffusion at a $\mathrm{Li}$ anode coated with $\mathrm{LiBr}$ salt $\left(E_{\mathrm{a}, \mathrm{LiBr}} \approx 0.03 \mathrm{eV}\right)$ is much lower, by a factor of around 8, compared to $\mathrm{Li}_{2} \mathrm{CO}_{3}\left(E_{\mathrm{a}, \mathrm{Li} 2 \mathrm{CO}} \approx 0.24 \mathrm{eV}\right)$, which forms naturally when aprotic 
solvents react with Li. This means that under isothermal conditions, stable deposition of $\mathrm{Li}$ in given electrolyte can occur at deposition rates more than three orders of magnitude higher on a $\mathrm{LiBr}$ coated $\mathrm{Li}$ anode than on an anode with a spontaneously formed $\mathrm{Li}_{2} \mathrm{CO}_{3}$-rich SEI. Third, the short hydrocarbon stem that connects the tethered sulfonate groups to Li should allow a dense hydrocarbon brush to form at the interface to protect the Li electrode from chemical attack by a high $\mathrm{DN}$ electrolyte required for stability at the cathode. Finally, soluble $\mathrm{LiBr}$ undergoes electrochemical oxidation and reduction in an appropriate potential window to function as a soluble redox mediator. Cryo-focused ion beam (cryo-FIB) was used to characterize the morphology and thickness of the ionomer-enriched electrode-electrolyte interface with the liquidelectrolyte intact but cryo-immobilized. In this technique, a symmetric Lithium cell (with ionomer-based electrolyte) was opened manually and the sample was snapfrozen by immediately plunging it into slush nitrogen to preserve the electrolyte and to avoid air exposure. The sample was then transferred under vacuum into an FEI Strata 400 FIB fitted with a Quorum PP3010T Cryo-FIB/SEM Preparation System and maintained at $-165^{\circ} \mathrm{C}$ for the duration of the experiment. To produce a cross section of the interface, the focused gallium ion beam was used to mill through the frozen electrolyte and into the electrode. This interface was then examined by scanning electron microscopy (SEM) and energy dispersive X-ray spectroscopy (EDX) directly in the cryo-FIB. SEM images revealed an interfacial layer up to approximately $25 \mathrm{~nm}$ thick in most areas. An example of the observed layer is shown in Figure 13.1(b). EDX could not confirm an increased bromine concentration in this layer, owing to the similar atomic composition of the reactant and product (in this case bulk electrolyte 
and interface). Therefore, it is important to perform x-ray analysis of washed electrode surface (i.e, excluding bulk liquid) to understand chemical compositions of the interface.

The proposed reaction mechanism was evaluated by means of EDX and highresolution XPS analytical measurements. The XPS measurements are performed using monochromatic Al K- $\alpha$-x-rays $(1489.6 \mathrm{eV})$ with a beam diameter of $1 \mathrm{~mm}$ and the results originate from a surface layer on the electrodes approximately $15-25 \mathrm{~nm}$ thick. Supplementary Figure 13.1 reports the 2-D EDX results on a lithium anode that was thoroughly washed after cycling. Sulfur and bromine signals are clear everywhere on the surface of the materials as expected. XPS analysis was performed using postmortem measurements on lithium anodes harvested from $\mathrm{Li}-\mathrm{O}_{2}$ cells subjected to different running conditions. High resolution scans for anodes retrieved after cycling or after a single discharge with the ionomer additive in $1 \mathrm{M} \mathrm{LiNO}_{3}-\mathrm{DMA}$ electrolyte are reported in Figure 13.1(c, d, e). The corresponding results without the ionomer are shown in Supplementary Figure 13.2. In Figure 13.1(c), it is apparent that after the first discharge a $\mathrm{Li} 1 \mathrm{~s}$ peak at $55.2 \mathrm{eV}$ is observed on anodes with/without the ionomer present in the electrolyte. The peak may be attributed to the presence of $\mathrm{LiOH}, \mathrm{Li}_{2} \mathrm{O}_{2}$ and $\mathrm{Li}_{2} \mathrm{CO}_{3}{ }^{50-57}$. A more prominent $\mathrm{Li} 1 \mathrm{~s}$ peak is observed at $53.8 \mathrm{eV}$, accounting for about $85 \%$ of lithium, only in spectra of anodes cycled in the presence of the ionomer additive. This peak is indicative of the formation of a different SEI in electrolytes containing the ionomer; Li 1s peaks with comparable binding energy are reported for organometallics containing Li-C bonds $(54.2 \mathrm{eV})^{58,59}$. This observation is consistent with the hypothesis that the ionomer reacts at the $\mathrm{Li}$ anode surface to form a lithium- 
ethanesulfonate-rich SEI at the interface. Also, the fact that this binding energy is observed in the cycled anodes, confirms that the SEI layer is stable and present even after repeated insertion and extraction of lithium ions into the underlying electrode.

Further evidence that the ionomer additive forms a stable SEI on Li can be deduced from the $\mathrm{O} 1 \mathrm{~s}$ (Figure 13.1(d)) and $\mathrm{Br} 3 \mathrm{~d}$ (Figure 13.1(e)) high resolution scans. The $\mathrm{O}$ 1s peak at $532.2 \mathrm{eV}$ comprises approximately $18 \%$ of the oxygen signal in cells without the ionomer additive, whether the anodes originate from cells that were subjected to a single discharge or were cycled. The $532.2 \mathrm{eV}$ peak has been previously reported to originate from sulfonates ${ }^{64}$ which, accounts for respectively $27 \%$ and $38 \%$ of the oxygen signal when the anode is discharged once or cycled in the presence of the ionomer additive. The corresponding sulfur atomic contribution for the same materials can be computed from the wide survey scans (Supplementary Table 13.1) to be about $2 \%$ for the once discharged anode and about twice as high for the cycled anodes. The high-resolution scans of $\mathrm{Br} 3 \mathrm{~d}$ reveal the formation of a single bond ( $\mathrm{a}$ $3 \mathrm{~d}_{5 / 2}$ and $3 \mathrm{~d}_{3 / 2}$ doublet) with a $\mathrm{Br} 3 \mathrm{~d}_{5 / 2}$ peak at $68.5 \mathrm{eV}$ when the anode is discharged once in the presence of the ionomer. We attribute this peak to the formation of $\mathrm{Br}-\mathrm{Li}$ bond, which has been previously reported to occur at a binding energies between 68.8 and $69.5^{53,60}$. The same peak persists when the anode is cycled in the presence of the ionomer however with a contribution of only around $15 \%$. The reduced $\mathrm{Li}-\mathrm{Br}$ species in the anodes of cycled cells is an indication of LiBr being solvated by the DMA electrolyte that can further participate in the redox mediation of oxygen cathode recharging. In fact, a more prominent $\mathrm{Br} 3 \mathrm{~d}$ peak at $67.0 \mathrm{eV}$ is observed only for the cycled anodes that is likely to originate from $\mathrm{Br}-\mathrm{C}$ bonds (binding energies between 
66.7 and $\left.71.0 \mathrm{eV}^{60-65}\right)$ in the SEI originating from untethered ionomer. The untethered ionomer in the electrolyte can help in the regeneration of the SEI layer in repeated cycling. Our results based on XPS analysis thus shows that the ionomer added electrolyte forms a SEI layer of lithium-ethanesulfonate and $\mathrm{LiBr}$, in accordance to the proposed reaction mechanism.

The effectiveness of ionomer-based SEI on $\mathrm{Li}$ was analyzed using Impedance Spectroscopy measurements on symmetric lithium cells. The results are shown in Figure 13.1(f) with Nyquist-type plots at progressive time periods for control cells and those that contain $10 \%$ (wt.) of ionomer additive. The Nyquist plots for $5 \%$ ionomer added cells are shown in Supplementary Figure 13.3. The experimental data points are fitted with the circuit model illustrated in Supplementary Figure 13.4 to deduce the bulk and interfacial resistances (Figure 13.1(g)) as a function of time for the control electrolyte as well as with $10 \%$ and $5 \%$ (by wt.) ionomer additive. It is seen that the bulk resistance for all cells remain essentially constant for approximately 20 hours, beyond which the bulk resistance of the control diverges (the increase is much larger as see Supplementary Figure 13.5 for the results for the control cells after 48 and 56 hours). The time-dependent interfacial impedance provides an even more sensitive indicator of the stability of the anode-electrolyte interphase in a high donor number solvent. It is seen that the initial interfacial resistances for control and ionomer-SEI stabilized Li electrodes are approximately equal $(\sim 50 \Omega)$. However, there is an exponential rise in the interfacial resistance of the control cell over time consistent with rapid reaction between Li and DMA. It is important to note that this reaction is observed even though $\mathrm{LiNO}_{3}$ is present at large concentration in the electrolyte. These 

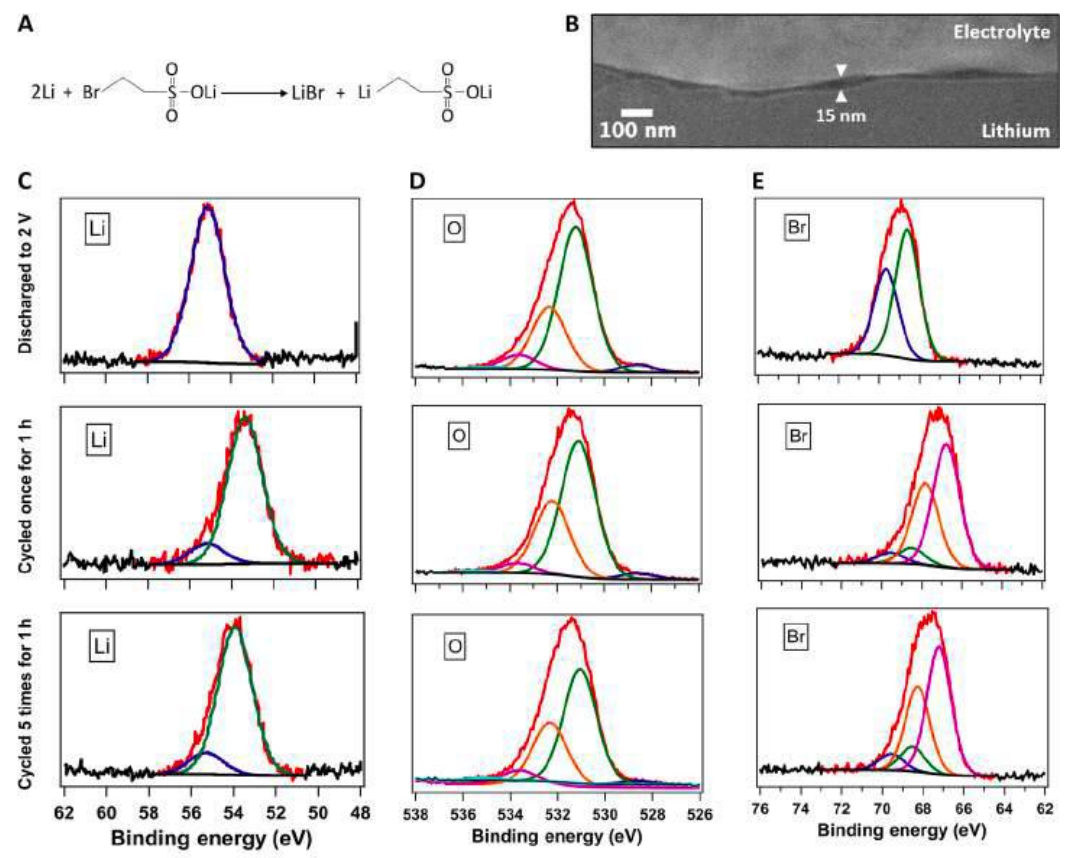

F
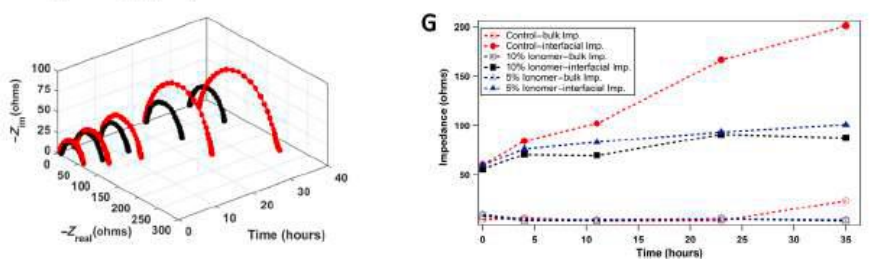

Figure 13.1. Artificial SEI concept and experimental verification of its proposed operating mechanism. (A) Schematic for the reaction of lithium 2bromoethanesulfonate with lithium metal forming $\mathrm{LiBr}$ and lithium-based organometallic. (B) SEM image of the interfacial layer between an intact electrolyte and a lithium electrode, revealed in a cross section produced by cryo-FIB milling. (C) Lithium 1s peak obtained from XPS of the lithium metal anode of a Li-O2 battery with the electrolyte ionomer [10\% (by weight)] in 1 M LiNO3-DMA. (D) Oxygen 1s peak of the lithium anode. (E) Bromine 3d peak of the lithium anode. In (C) to (E), the first row shows the postmortem analysis after discharging until $2 \mathrm{~V}$, the second row shows the result after cycling once with each half-cycle 5 hours long, and the third row shows the result after cycling five times with each half-cycle 1 hour long. (F) Threedimensional diagram of Nyquist plots obtained by impedance measurements at different intervals of time using symmetric lithium cells, in which -Zim is the imaginary component of the impedance and -Zreal is the real component of the impedance. (G) Comparison of interfacial and bulk impedance values for ionomerbased and control electrolytes as a function of time. In $(\mathrm{F})$ and $(\mathrm{G})$, the red symbols denote results with the control electrolyte (1 M LiNO3-DMA), whereas the black and blue symbols represent batteries with 10 and 5\% (by weight) ionomer additive, respectively, with the same electrolyte. 
results therefore challenge the view that $\mathrm{LiNO}_{3}$ provides an effective means of passivating Li metal anodes against reactive liquid electrolytes. In contrast, the results in Figure 13.1 $(\mathrm{g})$ show that the interfacial resistance remains constant (see also Supplementary Figure 13.4) when the ionomer-based SEI is present. It is seen that the stabilization with $10 \%$ ionomer additive is marginally better than $5 \%$ ionomer. Together these findings demonstrate that a SEI based on bromide ionomers has a large stabilizing effect on Li anodes in DMA-based electrolyte solvents.

\subsubsection{Lithium-electrolyte stability}

Figure 13.2(a, b) report on the quality of lithium ion deposition on stainless steel substrates mediated by control and ionomer-containing $1 \mathrm{MLiNO}_{3}-\mathrm{DMA}$ electrolytes. For these experiments, cells were assembled with lithium as anode and stainless steel as a virtual cathode. Lithium of capacity $10 \mathrm{mAh} / \mathrm{cm}^{2}$ was deposited at a rate of $1 \mathrm{~mA} / \mathrm{cm}^{2}$ onto stainless steel after which the cell was rested for a period of 10 hours and the voltage monitored over time. Figure 13.2(b) shows that in case of a control electrolyte $\mathrm{Li}$ deposition takes place at a higher voltage compared to the ionomercontaining electrolyte. Also, it can be observed that after the rest period, the voltage measured in the control cells immediately rises to approximately 0.5 volts. Such a high open circuit potential after Li deposition is a reflection of the complete decomposition of Li deposits on stainless steel due to corrosion by the electrolyte. It is again worth noting that despite using the $\mathrm{Li}$-passivating salt $\mathrm{LiNO}_{3}$ at high concentrations in the electrolyte, the freshly deposited lithium reacts completely with the electrolyte solvent. Figure 13.2(b) also reports the corresponding voltage profiles observed in 
rested cells containing the ionomer as an electrolyte additive. It is seen that the cell voltage remains close to 0 volts $\left(\right.$ vs. $\left.\mathrm{Li} / \mathrm{Li}^{+}\right)$, i.e. near the open circuit potential of a symmetric lithium cell, which means that the Li electrode is chemically stable in the reactive DMA electrolyte solvent.

To further examine the morphology of Li deposits, post-mortem analysis was performed, wherein the surface features of the electrodes were visualized under a scanning electron microscope (SEM). Figure 13.2(a) shows the SEM image of the surface of stainless steel in the control and ionomer-based electrolytes. For the control, there are few patches of $\mathrm{Li}$ observed and large sections of bare stainless steel are clearly visible. In contrast, in electrolytes containing the ionomer, the stainless-steel surface is covered with a thick layer of lithium. It is also seen that Li electrodeposits formed in the latter electrolytes are evenly sized and spherical in shape, even at a relatively high current density of $1 \mathrm{~mA} / \mathrm{cm}^{2}$. This observation is consistent with previous reports of more compact electrodeposition of $\mathrm{Li}$ in electrolytes with halidesalt enriched SEIs and single ion conducting features ${ }^{24,42}$.

To fundamentally understand the basis of these observations, electrochemical stability of the electrolytes was characterized by means of linear scan voltammetry in the range -0.2 to $5 \mathrm{~V}$ vs. $\mathrm{Li} / \mathrm{Li}^{+}$, at a fixed scan rate of $1 \mathrm{mV} / \mathrm{s}$. Figure $13.2(\mathrm{c})$ shows current as a function of voltage in a two-electrode setup of Li||stainless steel. It is seen that for the control (indicated by red curve), the current diverges at a value around $4 \mathrm{~V}$ vs. $\mathrm{Li} / \mathrm{Li}^{+}$, while for electrolytes containing ionomer additives the current diverges at a higher voltage, around $4.3 \mathrm{~V} \mathrm{vs} \mathrm{Li} / \mathrm{Li}^{+}$. This improved stability is consistent with previous reports of electrolyte composites with tethered anions ${ }^{49}$, wherein anions fixed at/near 
the electrode surface limit access to and chemical reaction of anions in an electrolyte with the negative electrode. Another important feature of the results can be seen at a potential close to 0 volts vs. $\mathrm{Li} / \mathrm{Li}^{+}$. The significant current peak apparent at approx. $0.2 \mathrm{~V}$ vs. $\mathrm{Li} / \mathrm{Li}^{+}$for both control and ionomer-containing electrolytes is a characteristic of lithium plating onto stainless steel. However, as the voltage is progressively increased, the corresponding Li stripping peak is not seen in the control cell but is readily apparent in cells with ionomer-containing electrolyte. This behavior is indicative of the complete consumption of Lithium deposits on stainless steel in the control cells and is consistent with previous results of SEM.

Figure 13.2(d) and (e) report results from so-called galvanostatic "plating-stripping" experiments. These experiments are used to evaluate the stability of $\mathrm{Li}$ electrodeposition and to assess the propensity of the material to electrodeposit as rough, dendritic structures. In contrast to previous studies ${ }^{38}$, where thick $(\sim 0.75 \mathrm{~mm})$ Li foil is used on both electrodes employed in pate-strip protocols, we performed these experiments using asymmetric $\mathrm{Li} / \mathrm{Li}$ cells comprised of one thick $\mathrm{Li}$ and one $\mathrm{Li}$-lean $\left(10 \mathrm{mAh} / \mathrm{cm}^{2}\right.$ of Li deposited on stainless steel at $\left.1 \mathrm{~mA} / \mathrm{cm}^{2}\right)$ electrode. The stability of the $\mathrm{Li}$ deposition reaction is normally assessed using three criteria: 1) The overpotential of lithium deposition. It can be seen from Figure 13.2(e) that at a fixed current density $\left(0.05 \mathrm{~mA} / \mathrm{cm}^{2}\right)$, the voltage response for cells with ionomer-based SEI is low (approx $6 \mathrm{mV}$ ), while the corresponding value for the control is much higher (approx $150 \mathrm{mV}$ ). This difference is indicative of formation of insulating products on the surface of the Li electrodes. 2) Steep decrease of the cell voltage to zero with continuous charge-discharge. This is an indication of short-circuiting of the cell when 

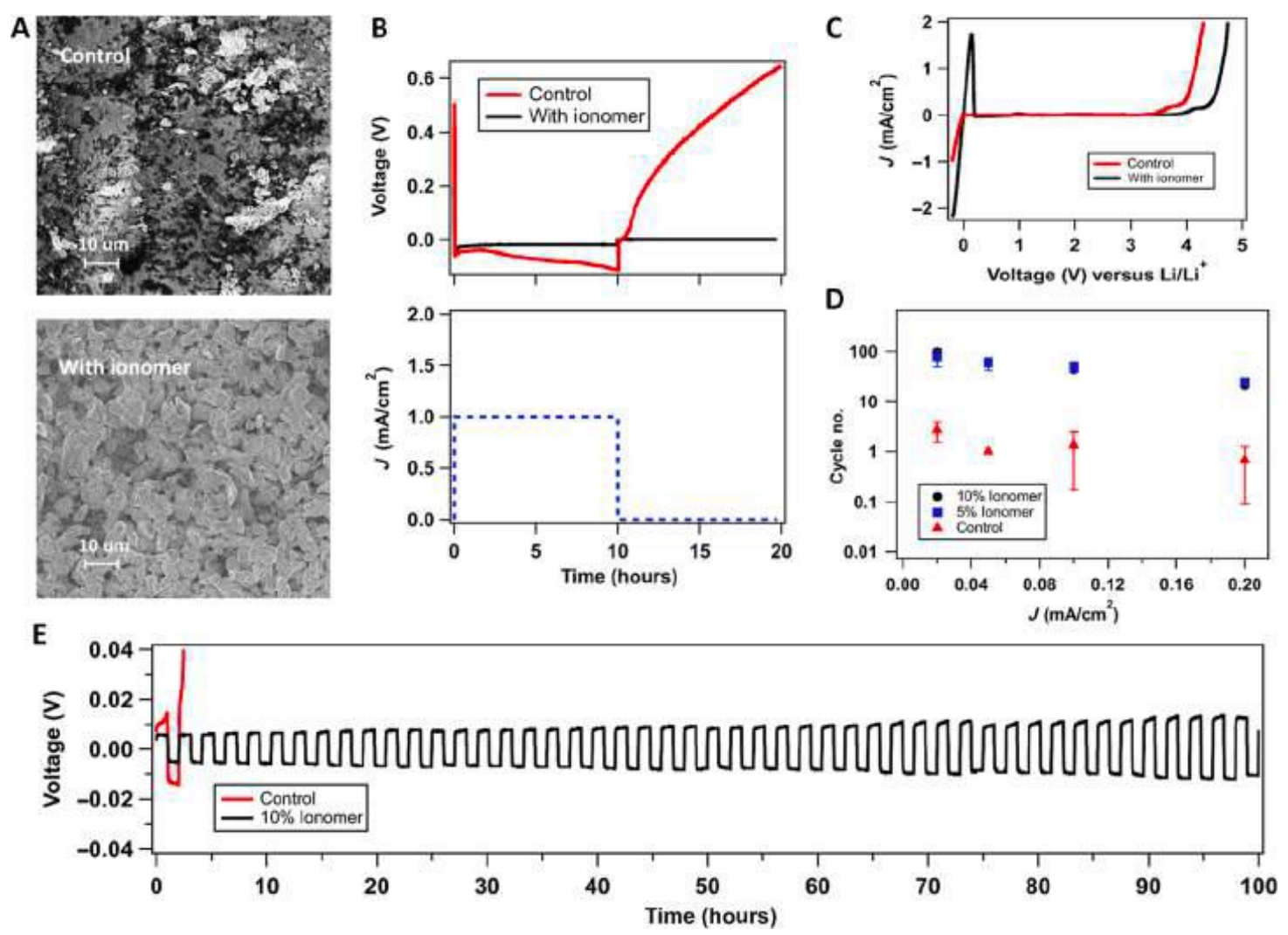

Figure 13.2. Stabilizing the lithium-electrolyte interface. (A) SEM images of stainless steel (SS) electrode after depositing lithium $\left(10 \mathrm{mAh} / \mathrm{cm}^{2}\right)$ in a $\mathrm{Li} \| \mathrm{SS}$ cell with and without the ionomer additive using the same electrolyte of $1 \mathrm{M} \mathrm{LiNO3-}$ DMA. (B) Voltage profile of the Li\||SS cell plotted over time. In this experiment, Li+ ions were deposited onto the stainless-steel side at a current density of $1 \mathrm{~mA} / \mathrm{cm}^{2}$ for 10 hours, after which the cell was kept at rest for an additional 10 hours, as shown in the current-versus-time curve. In the voltage-versus-time graph, the red line represents the profile of the control electrolyte ( $\left.1 \mathrm{M} \mathrm{LiNO}_{3}-\mathrm{DMA}\right)$, whereas the black line is for the same electrolyte enriched with $10 \%$ (by weight) ionomer additive. The dashed blue line in the current-versus-time graph is the applied current for both cases. (C) Linear scan voltammetry showing current as a function of voltage versus $\mathrm{Li} / \mathrm{Li}+$, with $\mathrm{Li}$ as both working and reference electrode and SS being the counter electrode. (D) In a $\mathrm{Li} \| \mathrm{SS}$ cell, lithium with $10-\mathrm{mAh} / \mathrm{cm}^{2}$ capacity is deposited onto SS, and the battery was charged and discharged consecutively at various current densities. The cycle number associated with the divergence of voltage is plotted against the respective current densities. (E) Voltage profile for the strip-and-plate experiment under the abovementioned condition using a current density of $0.05 \mathrm{~mA} / \mathrm{cm}^{2}$. In all figures, red indicates the control electrolyte ( $1 \mathrm{M} \mathrm{LiNO}_{3}$-DMA) and black represents the addition of $10 \%$ (by weight) ionomer additive, whereas blue denotes $5 \%$ (by weight) addition. 
dendritic lithium formed at one or both electrodes bridges the two electrodes. It is apparent that this phenomenon is not observed in either the control or for the ionomerSEI stabilized electrodes. 3) A steady increase of the voltage over extended cycles of charge and discharge. This observation is indicative of an unstable SEI that grows continuously, eventually consuming the Li deposited on the stainless-steel substrate. As seen in Supplementary Figure 13.6, after only two cycles at both current densities studied, the control cell fails after a steep rise in voltage. This is quite different from what is observed for cells in which Li is stabilized by an ionomer SEI, which are stable for over 150 cycles. Figure 13.2(d) reports the number of cycles at which the cell voltage diverges as a function of current density $(J)$. The ionomer-based SEI are seen to improve cell life time at a fixed current density by nearly two orders of magnitude. These results underscore the effectiveness of the ionomer-based SEI in stabilizing electrodeposition of $\mathrm{Li}$ in amide-based electrolytes, which were previously thought to be unfeasible for lithium metal batteries due to their high reactivity with and ready decomposition by Li.

\subsubsection{Anode protection mechanism}

We hypothesize that the stability of $\mathrm{Li}$ anode in DMA originates from two fundamental sources: (i) accumulation of $\mathrm{LiBr}$ salt at the $\mathrm{Li} /$ electrolyte interface, which facilitates Li-ion transport to the Li electrode during charging; and (ii) the existence of tethered sulfonate anions at the interface, which lowers the electric field at the electrode. Previous Joint-Density Functional Theoretical (JDFT) analysis

revealed that the presence of lithium halides in the SEI of Li-metal anode lowers the 
activation energy barrier by an order of magnitude or more for lateral Li diffusion at a Li/electrolyte interface, thereby increasing the tendency of $\mathrm{Li}$ to form smooth deposits. ${ }^{46}$ Comparing the surface diffusion barriers for various constituents of a typical SEI layer, Arias et al. ${ }^{46}$ found that $\mathrm{Li}_{2} \mathrm{CO}_{3}$, a common SEI constituent in carbonate electrolytes, has an energy barrier of $0.23 \mathrm{eV}$, while the barrier for a SEI composed of $\mathrm{LiF}$ is $0.17 \mathrm{eV}$. This difference has been argued previously to explain the much greater tendency of $\mathrm{Li}$ to form flat, compact deposits during battery recharge as revealed by experiments in which weakly soluble LiF salts are enriched in the SEI by precipitating out of liquid electrolytes. ${ }^{37}$ Interestingly, the JDFT analysis shows that the activation energy barrier for $\mathrm{Li}$-ion diffusion at a $\mathrm{LiBr} / \mathrm{Li}$ interface is much lower $(0.062 \mathrm{eV})$ and comparable to that of Magnesium ${ }^{46,66}$, which is in known in the literature to electrodeposit without formation of dendrites ${ }^{67}$. Thus, the $\mathrm{LiBr}$ created during the formation of the SEI should provide an even more powerful (than LiF) stabilizing effect on Li deposition.

In addition to the presence of $\mathrm{LiBr}$, the SEI created by the ionomer contains bound anionic groups in form of Lithium-ethanesulfonate $\left(\mathrm{Li}_{-}-\mathrm{CH}_{2} \mathrm{CH}_{2}-\mathrm{SO}_{3}^{-}\right)$. Thus, the electrolyte consists of a combination of free and tethered anion. In the past, researchers have realized the importance of single ion conducting electrolytes ${ }^{42,68}$, as they prevent the formation of ion concentration regions within a cell, leading to stable ion transport even at high charge rate. Interestingly, recent linear stability analysis of electrodeposition by Tikekar et al..$^{24,44,45}$ showed that the stability of an electrolyte can be significantly enhanced by immobilizing only a small fraction $(10 \%)$ of the anions. The design of our electrolyte comprising of a fraction of anions near the anodic 
surface, with $\mathrm{LiNO}_{3}$ as the free salt, is explicitly motivated by this theoretical framework. Thus, a modified SEI based on bromide ionomers tethered to the Li anode provides a powerful combination of processes that stabilize the anode against unstable electrodeposition.

\subsubsection{Understanding the cathode stabilization mechanism}

\subsubsection{Characterizing cathode products}

Figure 13.3a shows a representative voltage profile for the galvanostatic discharge and charge for a $\mathrm{Li}_{-} \mathrm{O}_{2}$ cell with $1 \mathrm{M} \mathrm{LiNO}_{3}$ in an ionomer-enriched DMA electrolyte. Cutoff voltages of $2.2 \mathrm{~V}$ and $4.3 \mathrm{~V}$, respectively, were used for the discharge and charge cycles and both processes were performed at a fixed current density of 31.25 $\mu \mathrm{A} / \mathrm{cm}^{2}$. Post-mortem SEM analysis was employed to study the evolution of discharge

products on the cathode at three stages of discharge (D1, D2, D3) and two stages of charge $(\mathrm{C} 1, \mathrm{C} 2)$. The SEM images show that the reversible formation and decomposition of an insoluble solid product on the cathode. Complementary XRD analysis (Figure 13.3b) shows that the cathode product is exclusively $\mathrm{Li}_{2} \mathrm{O}_{2}$ (and no other products such as $\mathrm{LiOH}$ ) are observed. The SEM analysis shows that $\mathrm{Li}_{2} \mathrm{O}_{2}$ particles grow increasingly larger as the discharge progresses and nucleation sites for growth are filled, and the full discharge capacity of the cell is reached. Analysis of the particle sizes on discharge (see Supplementary Figure 13.7) reveals that at low current densities (e.g. $\left.15 \mu \mathrm{A} / \mathrm{cm}^{2}\right)$ large $\mathrm{Li}_{2} \mathrm{O}_{2}$ particles $(1 \mu \mathrm{m}$ and higher) are formed. Comparing these results to those reported by Lau et al. ${ }^{10}$ for $\mathrm{Li}-\mathrm{O} 2$ cells discharged in a $1 \mathrm{M}$ LiTF in TEGDME (a low donor no. solvent), the $\mathrm{Li}_{2} \mathrm{O}_{2}$ particles formed in DMA 
A
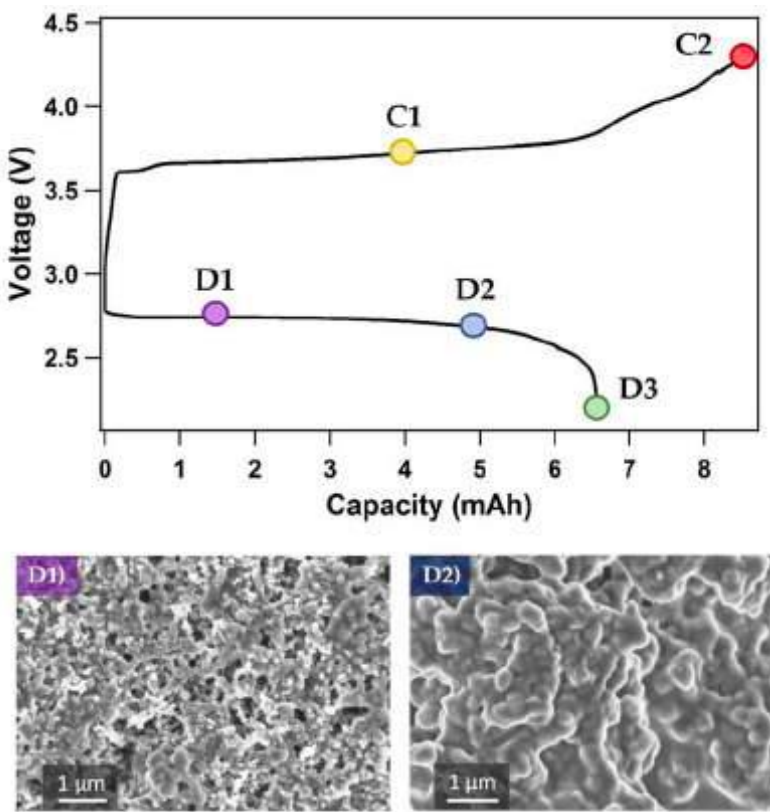

B

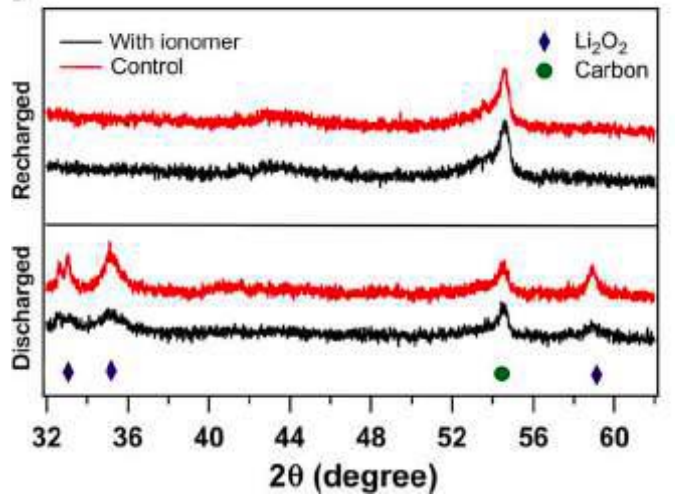

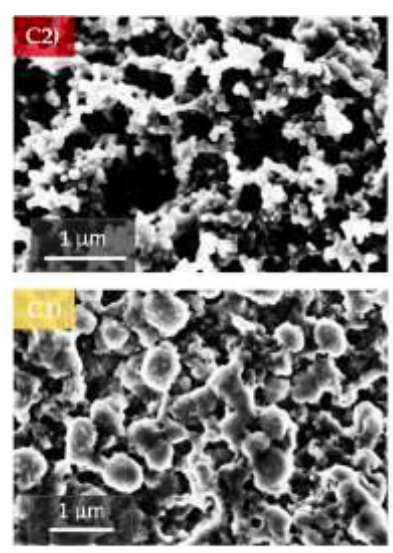

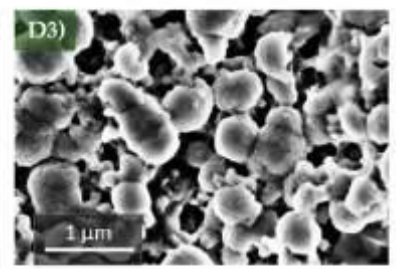

C

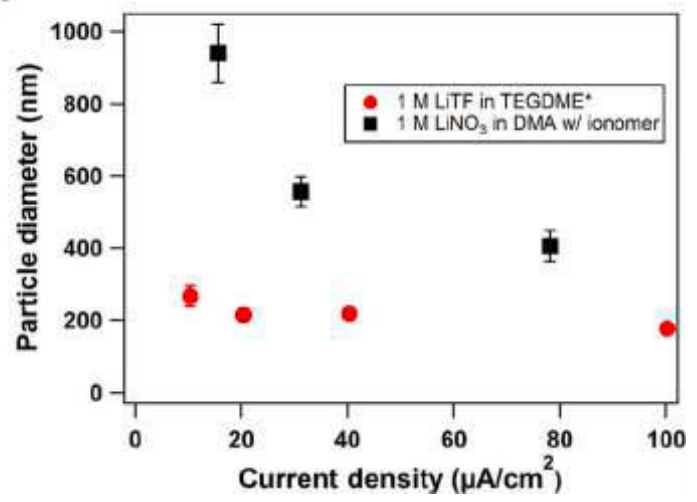

Figure 13.3. Characterization and electrochemical analysis of oxygen cathode. (A) Full charge-discharge cycle of a $\mathrm{Li} \| \mathrm{O}_{2}$ cell using ionomer-enriched $1 \mathrm{MLiNO}_{3}$ DMA electrolyte operated at a current density of $31.25 \mathrm{~mA} / \mathrm{cm}^{2}$. The different points on the voltage profile indicate various stages at which the same-type cells were stopped for ex situ analysis. The images below the voltage profile show the surface of a carbon cathode at the D1, D2, and D3 discharge phases. The size of the $\mathrm{Li}_{2} \mathrm{O}_{2}$ is seen to be increasing over the course of discharge. $\mathrm{C} 1$ and $\mathrm{C} 2$ show the stages of recharge; it is seen in $\mathrm{C} 1$ that the cathode is absent of $\mathrm{Li}_{2} \mathrm{O}_{2}$ particles. (B) XRD analysis showing various characteristic peaks for a fully discharged and a recharged $\mathrm{Li} \| \mathrm{O}_{2}$ battery. Here, diamonds denote $\mathrm{Li}_{2} \mathrm{O}_{2}$ peak and circles represent carbon. The red lines refer to the control electrolyte (1M LiNO3-DMA), whereas black lines show the result for the same electrolyte with the ionomer additive. (C) The diameter of $\mathrm{Li}_{2} \mathrm{O}_{2}$ particles obtained by fully discharging a $\mathrm{Li} \| \mathrm{O}_{2}$ cell is plotted as a function of current density. Here, black indicates the electrolyte (1MLiNO $3-\mathrm{DMA})$ with the ionomer additive, whereas red represents data from Lau and Archer's paper that used the electrolyte $1 \mathrm{MLiTF}$ in TEGDME. *From Lau and Archer (10). 
are at least four-times larger (see Figure 13.3(c)). These findings are consistent with expectation for the high donor number of DMA, which solvates $\mathrm{Li}^{+}$cations and enables a solution mediated mechanism, circumventing capacity limitations from the passivation layer formed at the cathode, which enables deep discharge ${ }^{31}$. At higher current densities, the particle size at the voltage cutoff decreases drastically, consistent with the idea that kinetic diffusion limitations ${ }^{27}$ set the maximum particle size. Upon charge, the SEM images (R1, R2) show a cathode that closely resembles that of the pristine electrode prior to discharge. Redox mediation from lithium 2bromoethanesulfonate is thought to aid in the electrochemical decomposition of the large, insulating $\mathrm{Li}_{2} \mathrm{O}_{2}$ particles formed on the cathode. Support for this hypothesis comes from the effectiveness of the recharge process as well as from the flat charge profile observed until the full capacity of the discharge is reached; the voltage ultimately begins to rise because of the set voltage limit of $4.3 \mathrm{~V}$. Thus, Figure 13.3 shows that a $\mathrm{Li}-\mathrm{O}_{2}$ cell with $1 \mathrm{M} \mathrm{LiNO}_{3}$ in DMA with an ionomer-based SEI on $\mathrm{Li}$ is able to reach a high capacity through $\mathrm{LiO}_{2}$ disproportionation; can fully utilize the formed $\mathrm{Li}_{2} \mathrm{O}_{2}$ during the recharge; and cycles with features indicative of the presence of a redox mediator.

\subsubsection{Cycling Performance}

To evaluate the hypothesis that a high donor number electrolyte solvent and redox mediator provide significant synergistic benefits for $\mathrm{Li}-\mathrm{O}_{2}$ cells, we compare the voltage profiles for fully discharged cells without and with these attributes (see Figure 13.4(a)). It is seen that the discharge capacity of $\mathrm{Li}_{2}-\mathrm{O}_{2}$ cells with a $1 \mathrm{M} \mathrm{LiNO}_{3} \mathrm{DMA}+$ 
Ionomer electrolyte is noticeably higher $(\sim 6.5 \mathrm{mAh})$ than with a conventional $1 \mathrm{M}$ LiTFSI- diglyme $(\sim 5.1 \mathrm{mAh})$ with same cathode loading. This finding is consistent with the observation of large-sized lithium peroxide structures owing to the solutionmediated nucleation of peroxides. Comparison of the charge cycle shows that with the diglyme electrolyte, the voltage diverges to $>4.2 \mathrm{~V}$ in $\sim 3.5 \mathrm{mAh}$ capacity, which is believed to be an indication of $\mathrm{Li}_{2} \mathrm{CO}_{3}$ formation and its effect on the charging process; whereas with ionomer based electrolyte, the voltage diverges at $\sim 6.5 \mathrm{mAh}$ (same as discharge). Figure 13.4(b) shows the cyclic voltammetry experiment for a lithium-oxygen cell in a two-electrode setup with lithium as both reference and counter electrode. The measurements were performed between $1.9 \mathrm{~V}$ to $4.5 \mathrm{~V}$ (vs. $\mathrm{Li} / \mathrm{Li}^{+}$) at a scan rate of $1 \mathrm{mV} / \mathrm{s}$ and normalized current is plotted against voltage. The current peaks for the ionomer based electrolyte are an order of magnitude higher than the control electrolyte. Thus, it can be inferred that there is higher electrochemical activity with owing to higher stability of the electrolyte and redox mediation due to presence of $\mathrm{LiBr}$. The peak seen at $\sim 3.5 \mathrm{~V}$ can be attributed to $\mathrm{Br}_{3}{ }^{-} \mathrm{Br}^{-}$redox couple. The inset shows 3 cycles with ionomer added electrolytes, where there is slight shift of the current peaks to lower values.

Discharge and charge profiles for cells having the electrolyte $1 \mathrm{M} \mathrm{LiNO}$-DMA with and without ionomer with a capacity cutoff of $3000 \mathrm{mAh} / \mathrm{gm}$ and current density of $0.04 \mathrm{~mA} / \mathrm{cm}^{2}$ is displayed in figure $13.4(\mathrm{c})$. It is seen that both discharge and charge voltage curves tend to diverge to lower and higher values respectively. Further it can be seen from the inset of Figure 13.4(c) that the voltage profile becomes extremely noisy in the fifth cycle of the control electrolyte, while that with ionomer additive is 
A

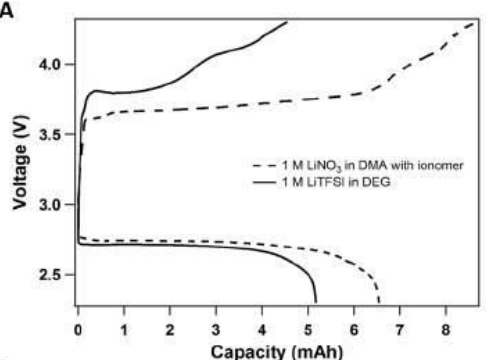

B
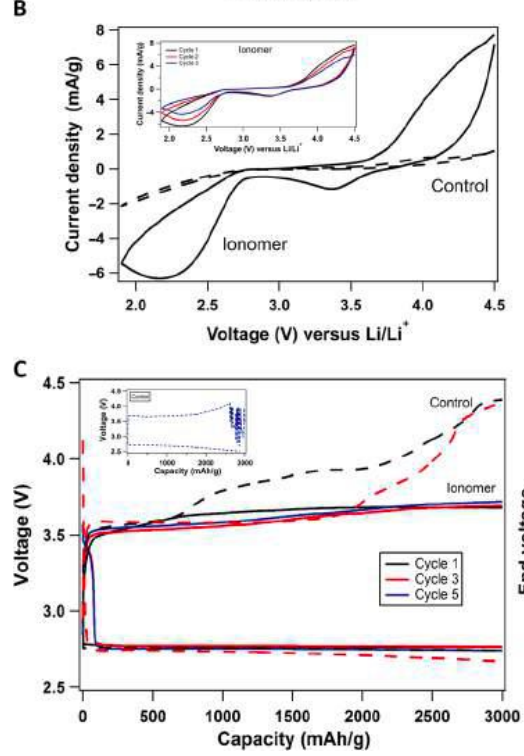
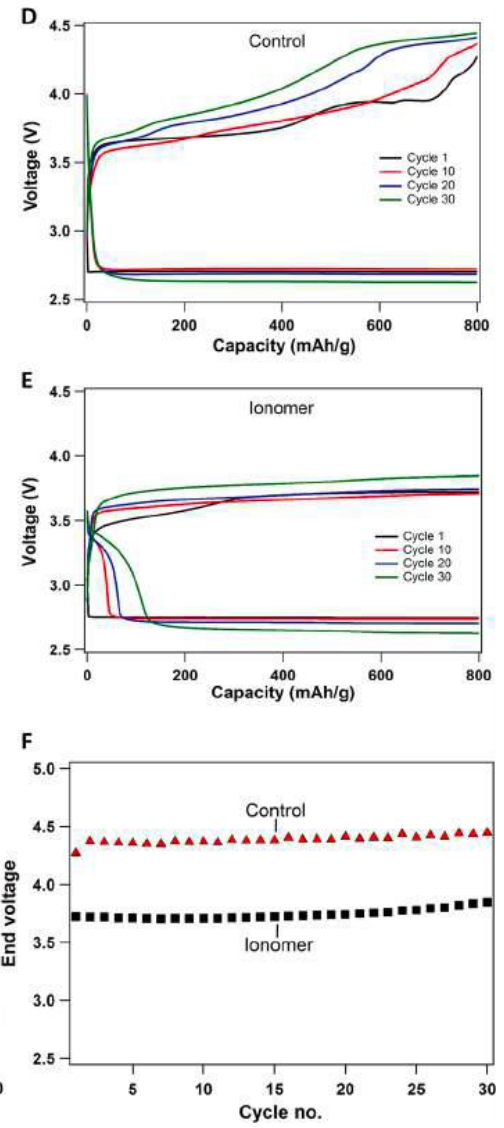

Figure 13.4. Galvanostatic cycling performance of lithium-oxygen electrochemical cell. (A) Voltage profile for batteries fully discharged and recharged with $1 \mathrm{M} \mathrm{LiNO}_{3}$ - DMA + ionomer electrolyte (shown with a solid black line) and a low-donor number electrolyte, 1 M LiTFSI-diglyme (shown with a dashed black line), at a current density of $31.25 \mathrm{~mA} / \mathrm{cm}^{2}$. (B) Comparison of cycling voltammetry results for the control electrolyte (1 $\mathrm{M} \mathrm{LiNO}_{3}$-DMA; shown with dashed lines) and the same electrolyte with the ionomer additive (shown with solid lines). The inset shows three cycles of cyclic voltammetry for the ionomer case. (C) Voltage profile of the $\mathrm{Li} \| \mathrm{O}_{2}$ battery with a cutoff capacity of $3000 \mathrm{mAh} / \mathrm{g}$ and a current density of $0.04 \mathrm{~mA} / \mathrm{cm}^{2}$. The solid lines indicate ionomer-based electrolytes, whereas the control is shown with dashed lines. The inset shows the noisy profile of the fifth cycle with the control electrolyte. (D) Voltage profile with a capacity cutoff of $800 \mathrm{mAh} / \mathrm{g}$ and a current density of $0.08 \mathrm{~mA} / \mathrm{cm}^{2}$ for a $\mathrm{Li} \| \mathrm{O}_{2}$ cell using the control electrolyte $\left(1 \mathrm{M} \mathrm{LiNO}_{3}-\right.$ DMA). (E) Voltage-versus-capacity curve with the same cutoff of $800 \mathrm{mAh} / \mathrm{g}$ using the ionomer additive in the electrolyte. (F) End voltage of charging cycle for the control and the ionomer-added electrolyte is plotted as function of cycle number. 
stable. This instability without ionomer can be attributed to the degradation of the electrolyte by reaction with the unprotected lithium metal. One major benefit of cells cycled with ionomer is reduced overpotential during charge relative to that of the control cell, thus increasing cycling efficiency. This is studied in a Li-O ${ }^{2}$ battery with a lower capacity cutoff of $800 \mathrm{mAh} / \mathrm{gm}$ at a current density of $0.08 \mathrm{~mA} / \mathrm{cm}^{2}$ for control (Figure 13.4(d)) and ionomer added electrolyte (Figure 13.4(e)). As demonstrated in Figure 13.4(e), the highest voltage on charge for cells with ionomer is approximately $3.7 \mathrm{~V}$, close to the $\mathrm{Br}^{-} / \mathrm{Br}_{3}{ }^{-}$redox reaction at $3.48 \mathrm{~V}^{1}$. Control cells with solely $1 \mathrm{M}$ $\mathrm{LiNO}_{3}$ in DMA reach voltages of around $4.45 \mathrm{~V}$ as seen in Figure 13.4(d). This suggests similar action to a redox mediator, in which $\mathrm{Li}_{2} \mathrm{O}_{2}$ is oxidized by $\mathrm{Br}_{3}{ }^{-}$to reform $\mathrm{Br}^{-}$in a cycle that lowers charge overpotential. The discharge and charge profiles remain similar over 30 cycles for cells with additive, while the charge profile in untreated cells increases more drastically. The distinct gentle slope of the initial portion of the discharge profile in cells with ionomer can be attributed to the presence of bromine species in the system. Figure 13.4(f) compared the end voltage of recharge with and without the ionomer additive. The $\sim 1 \mathrm{~V}$ improvement in the round-trip efficiency not only saves loss of input energy, but also ensures long life cycling by preventing electrolyte decomposition ${ }^{4}$.

\subsubsection{Cathode stabilization mechanism}

At the cathode surface, $\mathrm{LiBr}$ is thought to participate in the redox mediation that promotes the OER reaction. In this process, the $\mathrm{Li}_{2} \mathrm{O}_{2}$ can be co-reduced with $\mathrm{Br}^{-}$to form $\mathrm{O}_{2}$ and $\mathrm{Br}_{3}^{-}$. The potential for $\mathrm{Br}^{-} \rightarrow \mathrm{Br}_{3}^{-}$is known to be $3.48 \mathrm{~V}$, thus the charging 
of a $\mathrm{Li}-\mathrm{O}_{2}$ cell can be limited to this voltage. DMA's ability to dissolve peroxides also aids in the effective electrolyte-side redox mediation. Support for the uniqueness of these ideas come from recent experiments which demonstrate the efficacy of LiI and $\mathrm{LiBr}$ as redox mediators in $\mathrm{Li}_{2} \mathrm{O}_{2}$ cells based on glymes ${ }^{20}$. In the absence of water in the electrolyte, LiI was reported to produce a gradual rise in the discharge voltage due to formation of iodine and similar products. $\mathrm{LiBr}$ was found to be ineffective in maintaining a steady charge voltage. In electrolytes with high water contents and LiI, $\mathrm{LiOH}$ has been shown to be the primary discharge product, which has been reported to be thermodynamically impossible to undergo OER. Our results therefore clearly show that protecting the $\mathrm{Li}$ anode in a $1 \mathrm{M} \mathrm{LiNO}_{3}$-DMA electrolyte with a SEI based on bromide ionomer overcome fundamental limitations of the anode, cathode, and electrolyte in previously studied systems and enables stable cycling of these cells.

\subsection{Conclusions}

In summary, we demonstrate that addition of lithium 2-bromoethanesulfonate (ionomer) to $1 \mathrm{M} \mathrm{LiNO}_{3}$ in $\mathrm{DMA}$ electrolytes produces a $\mathrm{SEI}$ at lithium surface that stabilizes the anode in $\mathrm{Li}_{-} \mathrm{O}_{2}$ cells by at least two powerful processes. Compared to control, cells with the ionomer $\mathrm{SEI}, \mathrm{Li}_{2} \mathrm{O}_{2}$ cells based on lithium 2bromoethanesulfonate exhibit flatter, more stable charge profiles and are able to withstand deeper cycling. Furthermore, we show that electrochemical charge discharge processes in the cells coincide with formation and decomposition of large $\mathrm{Li}_{2} \mathrm{O}_{2}$ particles as the principal OER product in the cathode. Analysis by linear scan voltammetry and 'plate-strip' cycling analysis of the Li anode show that a SEI based 
on lithium 2-bromoethanesulfonate ionomer on the anode provides chemical stability to Li against attack by DMA, as well as physical stability against rough, dendritic electrodeposition. Although we expect the "perfect" electrolyte for $\mathrm{Li}^{-\mathrm{O}_{2}}$ cells significant additional work, by addressing fundamental issues that limit performance of the anode and cathode, we predict that multifunctional SEIs of the sort discussed in this study will emerge as critical to further progress. 


\section{REFERENCES}

1. Bruce, P. G., Freunberger, S. a, Hardwick, L. J. \& Tarascon, J.-M. Li-O2 and Li-S batteries with high energy storage. Nat. Mater. 11, 19-29 (2012).

2. Luntz, A. C. \& McCloskey, B. D. Nonaqueous Li-air batteries: A status report. Chem. Rev. 114, 11721-11750 (2014).

3. Girishkumar, G., McCloskey, B., Luntz, A. C., Swanson, S. \& Wilcke, W. Lithium-air battery: Promise and challenges. J. Phys. Chem. Lett. 1, 2193-2203 (2010).

4. Aurbach, D., McCloskey, B. D., Nazar, L. F. \& Bruce, P. G. Advances in understanding mechanisms underpinning lithium-air batteries. Nat. Energy 1, $16128(2016)$.

5. Lu, Y.-C., Gasteiger, H. a., Parent, M. C., Chiloyan, V. \& Shao-Horn, Y. The Influence of Catalysts on Discharge and Charge Voltages of Rechargeable LiOxygen Batteries. Electrochem. Solid-State Lett. 13, A69 (2010).

6. Mccloskey, B. D. et al. Combining accurate $\mathrm{O} 2$ and Li2O2 assays to separate discharge and charge stability limitations in nonaqueous Li-O 2 Batteries. $J$. Phys. Chem. Lett. 4, 2989-2993 (2013).

7. McCloskey, B. D. et al. Limitations in Rechargeability of Li-O2Batteries and Possible Origins. J. Phys. Chem. Lett. 3, 3043-3047 (2012).

8. McCloskey, B. D. et al. Twin problems of interfacial carbonate formation in nonaqueous Li-O2 batteries. J. Phys. Chem. Lett. 3, 997-1001 (2012).

9. Ottakam Thotiyl, M. M. et al. A stable cathode for the aprotic Li-O2 battery. Nat. Mater. 12, 1050-6 (2013). 
10. Lau, S. \& Archer, L. A. Nucleation and Growth of Lithium Peroxide in the Li$\mathrm{O}<$ inf $>2<$ inf $>$ Battery. Nano Lett. 15, 5995-6002 (2015).

11. Højberg, J. et al. An electrochemical impedance spectroscopy investigation of the overpotentials in Li-O2 batteries. ACS Appl. Mater. Interfaces 7, 4039-4047 (2015).

12. Chen, Y., Freunberger, S. a, Peng, Z., Fontaine, O. \& Bruce, P. G. Charging a Li-O2 battery using a redox mediator. Nat. Chem. 5, 489-94 (2013).

13. Lee, D. J., Lee, H., Kim, Y. J., Park, J. K. \& Kim, H. T. Sustainable Redox Mediation for Lithium-Oxygen Batteries by a Composite Protective Layer on the Lithium-Metal Anode. Adv. Mater. 28, 857-863 (2016).

14. Bergner, B. J. et al. Understanding the Fundamentals of Redox Mediators in LiO2 Batteries: A Case Study on Nitroxides. Phys. Chem. Chem. Phys. 17, 31769-31779 (2015).

15. Liu, T. et al. Cycling Li-O2 batteries via $\mathrm{LiOH}$ formation and decomposition. Science (80-. ). 350, 3-6 (2015).

16. Kwak, W.-J. et al. Understanding the behavior of Li-oxygen cells containing LiI. J. Mater. Chem. A 3, 8855-8864 (2015).

17. Lim, H. D. et al. Superior rechargeability and efficiency of lithium-oxygen batteries: Hierarchical air electrode architecture combined with a soluble catalyst. Angew. Chemie - Int. Ed. 53, 3926-3931 (2014).

18. Kundu, D., Black, R., Adams, B. \& Nazar, L. F. A Highly Active Low Voltage Redox Mediator for Enhanced Rechargeability of Lithium-Oxygen Batteries. ACS Cent. Sci. 1, 510-515 (2015). 
19. Sun, D. et al. A Solution-Phase Bifunctional Catalyst for Lithium - Oxygen Batteries. J. Am. Chem. Soc. 136, 8941-8946 (2014).

20. Hirshberg, D. et al. Li-O 2 cells with $\mathrm{LiBr}$ as an Electrolyte and Redox Mediator. Energy Environ. Sci. 9, 2334-2345 (2016).

21. Zhang, T., Liao, K., He, P. \& Zhou, H. A self-defense redox mediator for efficient lithium-O2 batteries. Energy Environ. Sci. 9, 1024-1030 (2015).

22. Veith, G. M., Nanda, J., Delmau, L. H. \& Dudney, N. J. In fl uence of Lithium Salts on the Discharge Chemistry of Li - Air Cells. J. Phys. Chem. Lett. 3, $1242-1247$ (2012).

23. Black, R. et al. Screening for superoxide reactivity in Li-O2 batteries: effect on Li2O2/LiOH crystallization. J. Am. Chem. Soc. 134, 2902-5 (2012).

24. Tikekar, M. D., Choudhury, S., Tu, Z. \& Archer, L. A. Design principles for electrolytes and interfaces for stable lithium-metal batteries. Nat. Energy 1, 16114 (2016).

25. Freunberger, S. A. et al. Reactions in the rechargeable lithium-O2 battery with alkyl carbonate electrolytes. J. Am. Chem. Soc. 133, 8040-8047 (2011).

26. Bryantsev, V. S. et al. The Identification of Stable Solvents for Nonaqueous Rechargeable Li-Air Batteries. J. Electrochem. Soc. 160, A160-A171 (2012).

27. Burke, C. M., Pande, V., Khetan, A., Viswanathan, V. \& Mccloskey, B. D. Enhancing electrochemical intermediate solvation through electrolyte anion selection to increase nonaqueous Li - O 2 battery capacity. Proc. Natl. Acad. Sci. 2, 201505728 (2015).

28. Johnson, L. et al. The role of $\mathrm{LiO} 2$ solubility in $\mathrm{O} 2$ reduction in aprotic solvents 
and its consequences for Li-O2 batteries. Nat. Chem. 6, 1091-9 (2014).

29. Khetan, A., Luntz, A. \& Viswanathan, V. Trade-offs in capacity and rechargeability in nonaqueous Li-O2 batteries: Solution-driven growth versus nucleophilic stability. J. Phys. Chem. Lett. 6, 1254-1259 (2015).

30. Knudsen, K. B., Vegge, T., McCloskey, B. D. \& Hjelm, J. SI - An Electrochemical Impedance Spectroscopy Study on the Effects of the Surfaceand Solution-Based Mechanisms in Li-O 2 Cells. J. Electrochem. Soc. 163, A2065-A2071 (2016).

31. Aetukuri, N. B. et al. Solvating additives drive solution-mediated electrochemistry and enhance toroid growth in non-aqueous $\mathrm{Li}-\mathrm{O} 2$ batteries. Nat. Chem. 7, 50-56 (2015).

32. Rosenman, A. et al. The Effect of Interactions and Reduction Products of LiNO3, the Anti-Shuttle Agent, in Li-S Battery Systems. J. Electrochem. Soc. 162, A470-A473 (2015).

33. Zhang, S. S. Role of LiNO3 in rechargeable lithium/sulfur battery. Electrochim. Acta 70, 344-348 (2012).

34. Liang, X. et al. Improved cycling performances of lithium sulfur batteries with LiNO 3-modified electrolyte. J. Power Sources 196, 9839-9843 (2011).

35. Aurbach, D. et al. On the Surface Chemical Aspects of Very High Energy Density, Rechargeable Li-Sulfur Batteries. J. Electrochem. Soc. 156, A694A702 (2009).

36. Walker, W. et al. A Rechargeable Li-O-2 Battery Using a Lithium Nitrate/N,NDimethylacetamide Electrolyte. J. Am. Chem. Soc. 135, 2076-2079 (2013). 
37. Choudhury, S. \& Archer, L. A. Lithium Fluoride Additives for Stable Cycling of Lithium Batteries at High Current Densities. Adv. Electron. Mater. 1-6 (2015). doi:10.1002/aelm.201500246

38. Choudhury, S., Mangal, R., Agrawal, A. \& Archer, L. A. A highly reversible room-temperature lithium metal battery based on crosslinked hairy nanoparticles. Nat. Commun. 1-9 (2015). doi:10.1038/ncomms10101

39. Agrawal, A., Choudhury, S. \& Archer, L. a. A highly conductive, nonflammable polymer-nanoparticle hybrid electrolyte. $R S C A d v . \mathbf{5 ,}$ 20800-20809 (2015).

40. Tarascon, J. M. \& Armand, M. Issues and challenges facing rechargeable lithium batteries. Nature 414, 359-67 (2001).

41. Kashiwagi, T. et al. Nanoparticle networks reduce the flammability of polymer nanocomposites. Nat. Mater. 4, 928-33 (2005).

42. Tu, Z., Nath, P., Lu, Y., Tikekar, M. D. \& Archer, L. a. Nanostructured Electrolytes for Stable Lithium Electrodeposition in Secondary Batteries. Acc. Chem. Res. acs.accounts.5b00427 (2015). doi:10.1021/acs.accounts.5b00427

43. Cheng, X. et al. A Review of Solid Electrolyte Interphases on Lithium Metal Anode. Adv. Sci. 3, 1-20 (2016).

44. Tikekar, M. D., Archer, L. a. \& Koch, D. L. Stability Analysis of Electrodeposition across a Structured Electrolyte with Immobilized Anions. $J$. Electrochem. Soc. 161, A847-A855 (2014).

45. Tikekar, M. D., Archer, L. A. \& Koch, D. L. Stabilizing electrodeposition in elastic solid electrolytes containing immobilized anions. Sci. $A d v$. 2:e1600320, 
(2016).

46. Ozhabes, Y., Gunceler, D. \& Arias, T. a. Stability and surface diffusion at lithium-electrolyte interphases with connections to dendrite suppression. arXiv 1504.05799, 1-7 (2015).

47. Lu, Y. et al. Stable Cycling of Lithium Metal Batteries Using High Transference Number Electrolytes. Adv. Energy Mater. 5, n/a-n/a (2015).

48. Schaefer, J. L., Yanga, D. a. \& Archer, L. a. High Lithium Transference Number Electrolytes via Creation of 3-Dimensional, Charged, Nanoporous Networks from Dense Functionalized Nanoparticle Composites. Chem. Mater. 25, 834-839 (2013).

49. Bouchet, R. et al. efficient electrolytes for lithium-metal batteries. Nat. Mater. 12, 452-457 (2013).

50. Yao, K. P. C. et al. Thermal Stability of Li2O2 and Li2O for Li-Air Batteries: In Situ XRD and XPS Studies. J. Electrochem. Soc. 160, A824-A831 (2013).

51. Lu, Y.-C. et al. In situ ambient pressure X-ray photoelectron spectroscopy studies of lithium-oxygen redox reactions. Sci. Rep. 2, 715 (2012).

52. Kundu, D., Black, R., Berg, E. J. \& Nazar, L. F. A highly active nanostructured metallic oxide cathode for aprotic $\mathrm{Li}-\mathrm{O}_{2}$ batteries. Energy Environ. Sci. 8, $1292-1298$ (2015).

53. Moulder, J., Stickle, W., Sobol, P. \& Bomben, K. Handbook of X-ray Photoelectron Spectroscopy. PerkinElmer Corp. Eden Prairie 1992 (1992).

54. Zhang, Z. et al. Increased stability toward oxygen reduction products for lithium-air batteries with oligoether-functionalized silane electrolytes. J. Phys. 
Chem. C 115, 25535-25542 (2011).

55. Lu, J. et al. Magnetism in lithium-oxygen discharge product. ChemSusChem 6, 1196-1202 (2013).

56. Basile, A., Bhatt, A. I. \& O’Mullane, A. P. Stabilizing lithium metal using ionic liquids for long-lived batteries. Nat. Commun. 7, 1-11 (2016).

57. Hausbrand, R. et al. Fundamental degradation mechanisms of layered oxide Liion battery cathode materials: Methodology, insights and novel approaches. Mater. Sci. Eng. B Solid-State Mater. Adv. Technol. 192, 3-25 (2015).

58. Xiong, S., Xie, K., Diao, Y. \& Hong, X. Properties of surface film on lithium anode with LiNO 3 as lithium salt in electrolyte solution for lithium-sulfur batteries. Electrochim. Acta 83, 78-86 (2012).

59. Wu, Y., Fang, S. \& Jiang, Y. Effects of nitrogen on the carbon anode of a lithium secondary battery. Sci. York 120, 117-123 (1999).

60. NIST X-ray Photoelectron Spectroscopy Database, Version 4.1. Natl. Inst. Stand. Technol. Gaithersbg. 1, 2012 (2012).

61. Ferrighi, L. et al. Control of the intermolecular coupling of dibromotetracene on $\mathrm{Cu}(110)$ by the sequential activation of C-Br and C-H bonds. Chem. - A Eur. J. 21, 5826-5834 (2015).

62. Basagni, A. et al. On-surface photo-dissociation of $\mathrm{C}-\mathrm{Br}$ bonds: towards room temperature Ullmann coupling. Chem. Commun. 51, 12593-12596 (2015).

63. Gutzler, R. et al. Ullmann-type coupling of brominated tetrathienoanthracene on copper and silver. Nanoscale 6, 2660-8 (2014).

64. Desai, S. M., Solanky, S. S., Mandale, A. B., Rathore, K. \& Singh, R. P. 
Controlled grafting of $\mathrm{N}$-isoproply acrylamide brushes onto self-standing isotactic polypropylene thin films: Surface initiated atom transfer radical polymerization. Polymer (Guildf). 44, 7645-7649 (2003).

65. Di Giovannantonio, M. et al. Insight into organometallic intermediate and its evolution to covalent bonding in surface-confined ullmann polymerization. $A C S$ Nano 7, 8190-8198 (2013).

66. Jäckle, M. \& Groß, A. Microscopic properties of lithium, sodium, and magnesium battery anode materials related to possible dendrite growth. $J$. Chem. Phys. 141, (2014).

67. Ha, S. et al. Magnesium ( II ) Bis ( tri fl uoromethane sulfonyl ) Imide-Based Electrolytes with Wide Electrochemical Windows for Rechargeable Magnesium Batteries. ACS Appl. Mater. Interfaces 6, 4063-4073 (2014).

68. Feng, S. et al. Single lithium-ion conducting polymer electrolytes based on poly[(4-styrenesulfonyl)(trifluoromethanesulfonyl)imide] anions. Electrochim. Acta 93, 254-263 (2013). 


\section{APPENDIX}

Supplementary Information for Chapter 13
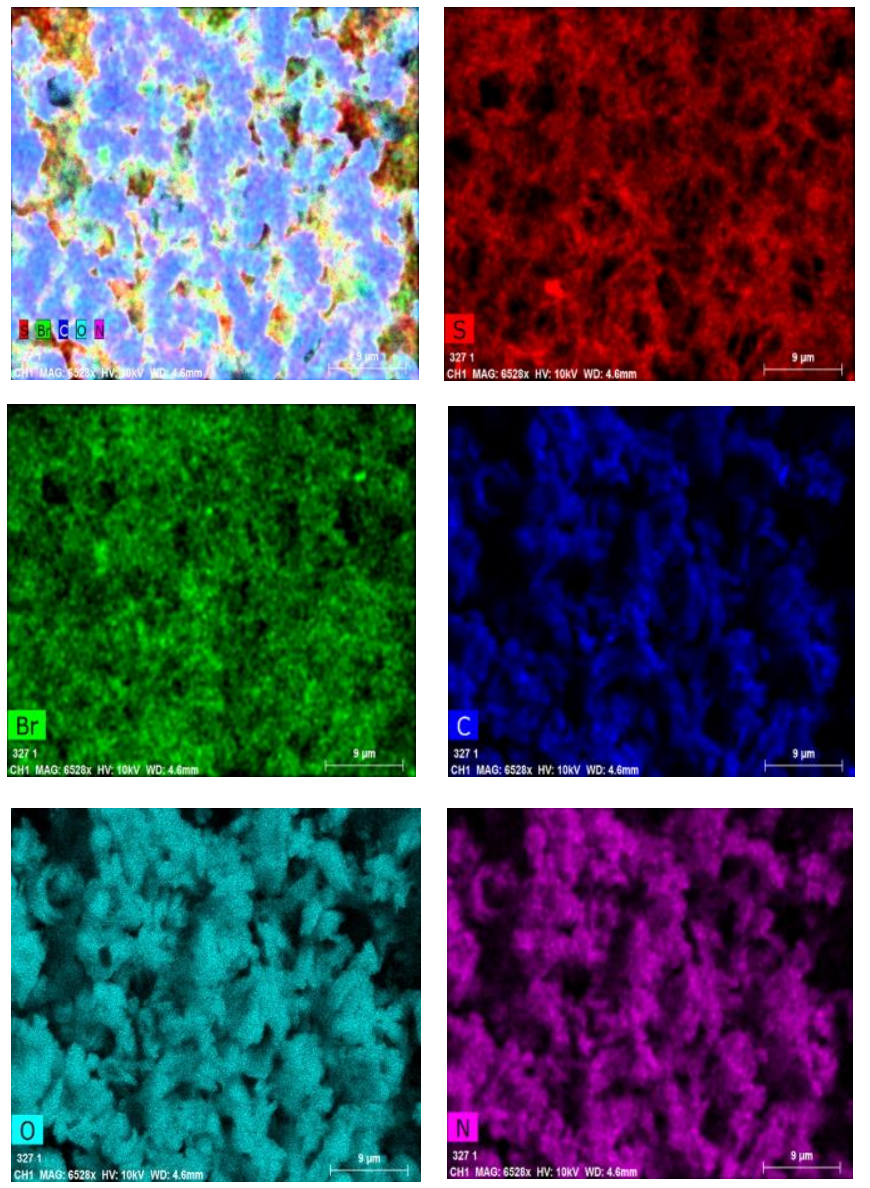

Supplementary Figure 13.1: 2D EDAX mapping of lithium-deposited stainless-steel substrate with $1 \mathrm{M} \mathrm{LiNO}_{3}$-DMAc electrolyte and $10 \%$ ionomer additive. The atoms taken into consideration are Sulfur, Bromine, Carbon, Oxygen and Nitrogen 

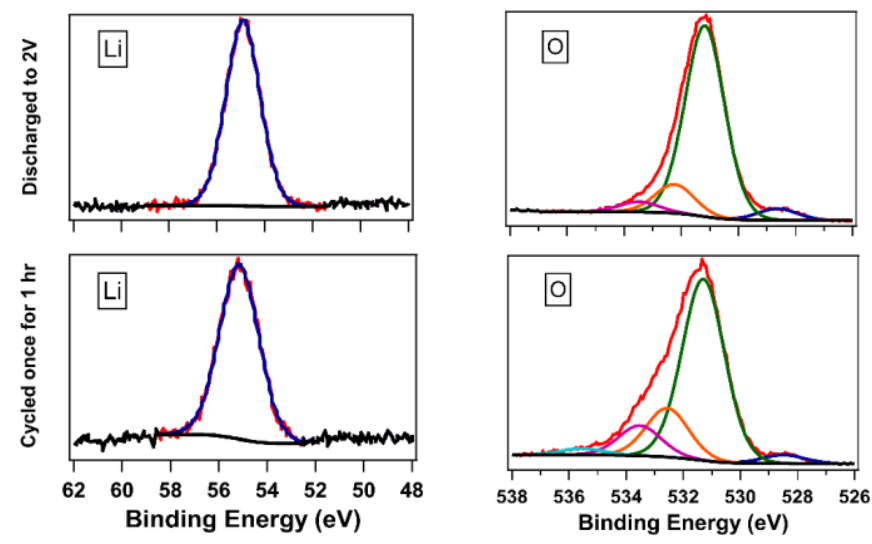

Supplementary Figure 13.2: XPS results showing the binding energy of Li and $\mathrm{O}$ atom with control electrolyte of $1 \mathrm{M} \mathrm{LiNO}_{3}$-DMAc electrolyte. The first row shows results when the battery is discharged to $2 \mathrm{~V}$, the second row shows results when the $\mathrm{Li}-\mathrm{O}_{2}$ battery is cycled once for 1hour. 


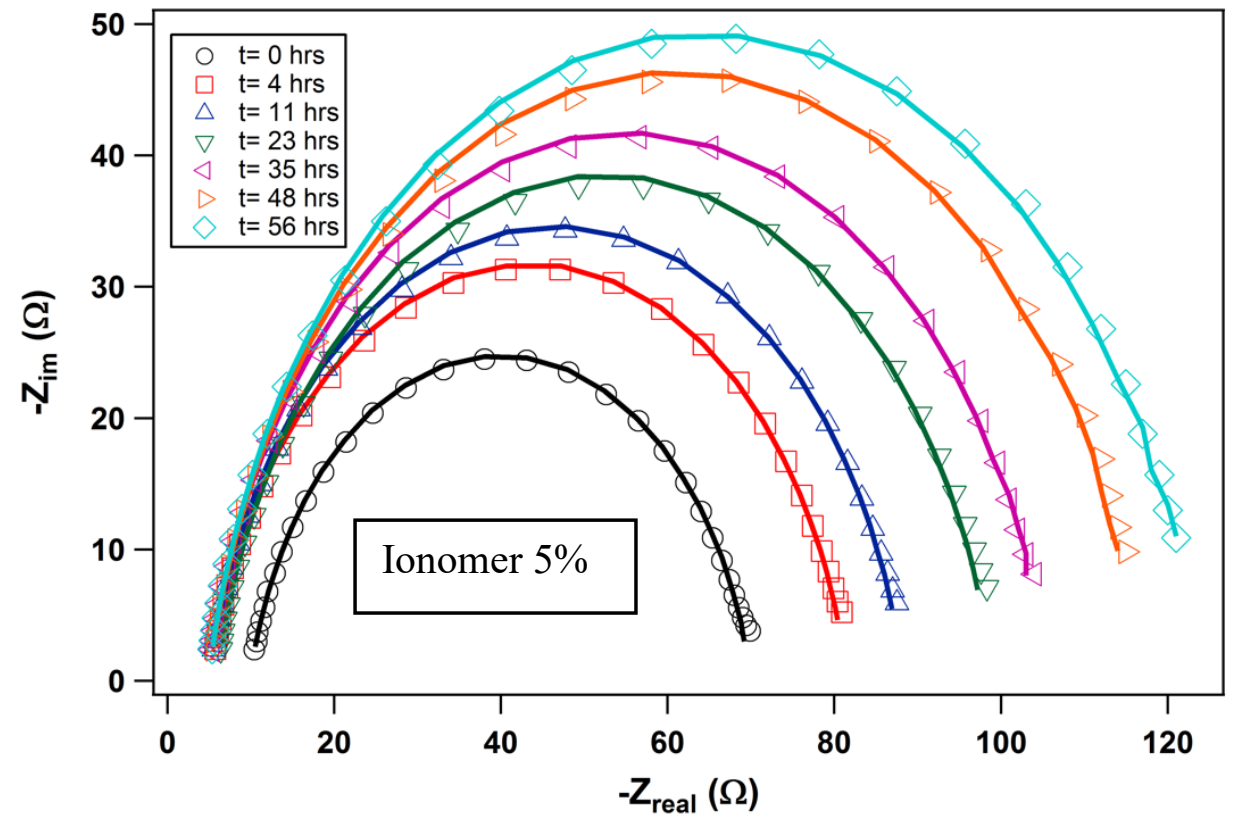

Supplementary Figure 13.3: Nyquist plots of $1 \mathrm{M} \mathrm{LiNO}_{3}$-DMAc enriched with 5\% (by wt.) of ionomer additive, showing impedance for different storage time of the battery 


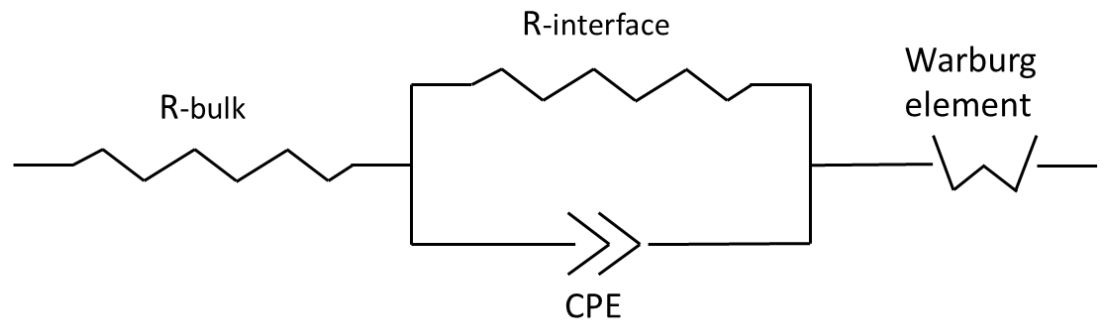

Supplementary Figure 13.4: Equivalent circuit model to fit the Nyquist plot obtained from impedance spectroscopy measurement comprising of bulk resistance, interfacial resistance parallel to a constant phase element and a solid-state diffusion element 

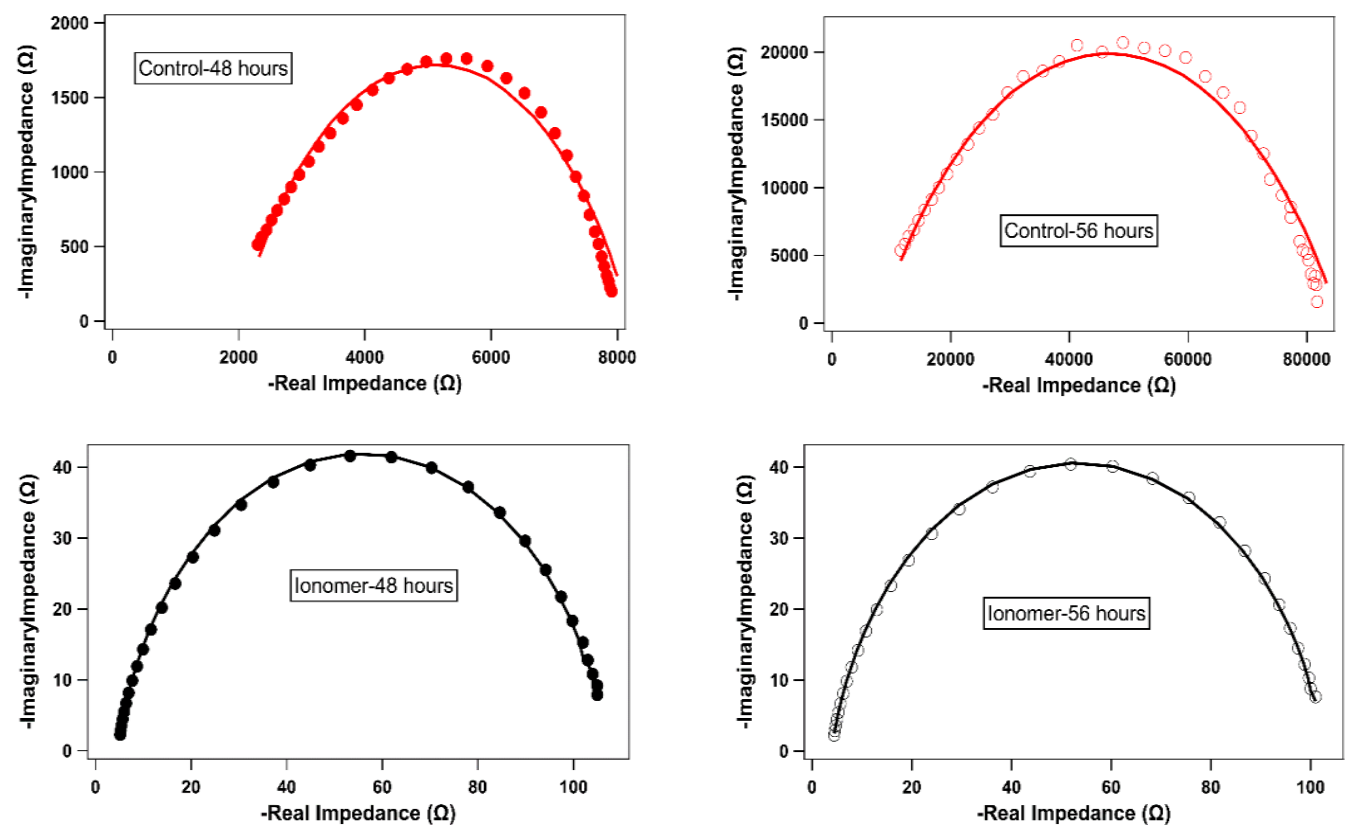

Supplementary Figure 13.5: Nyquist plots showing experimental as well as circuit-model fitted results of impedance measurements with symmetric cells for control electrolyte and ionomer added batteries at after $48 \mathrm{hrs}$ and $56 \mathrm{hrs}$ of storage. The red plot represents control and black shows data for ionomer added electrolyte. 


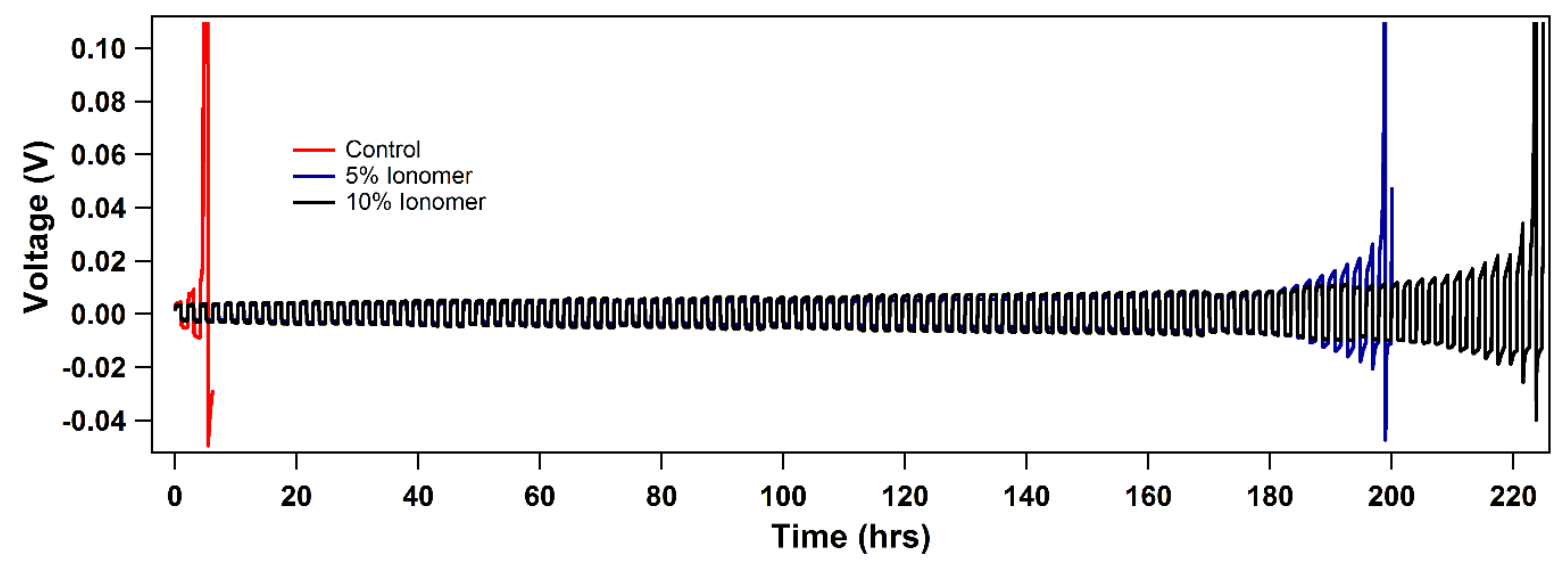

Supplementary Figure 13.6: Stripping and plating of Li vs. SS cell after depositing $10 \mathrm{mAh} / \mathrm{cm}^{2}$ of lithium onto Stainless Steel. It is seen that for all cells the voltage diverges for all cells however at different point of times. 

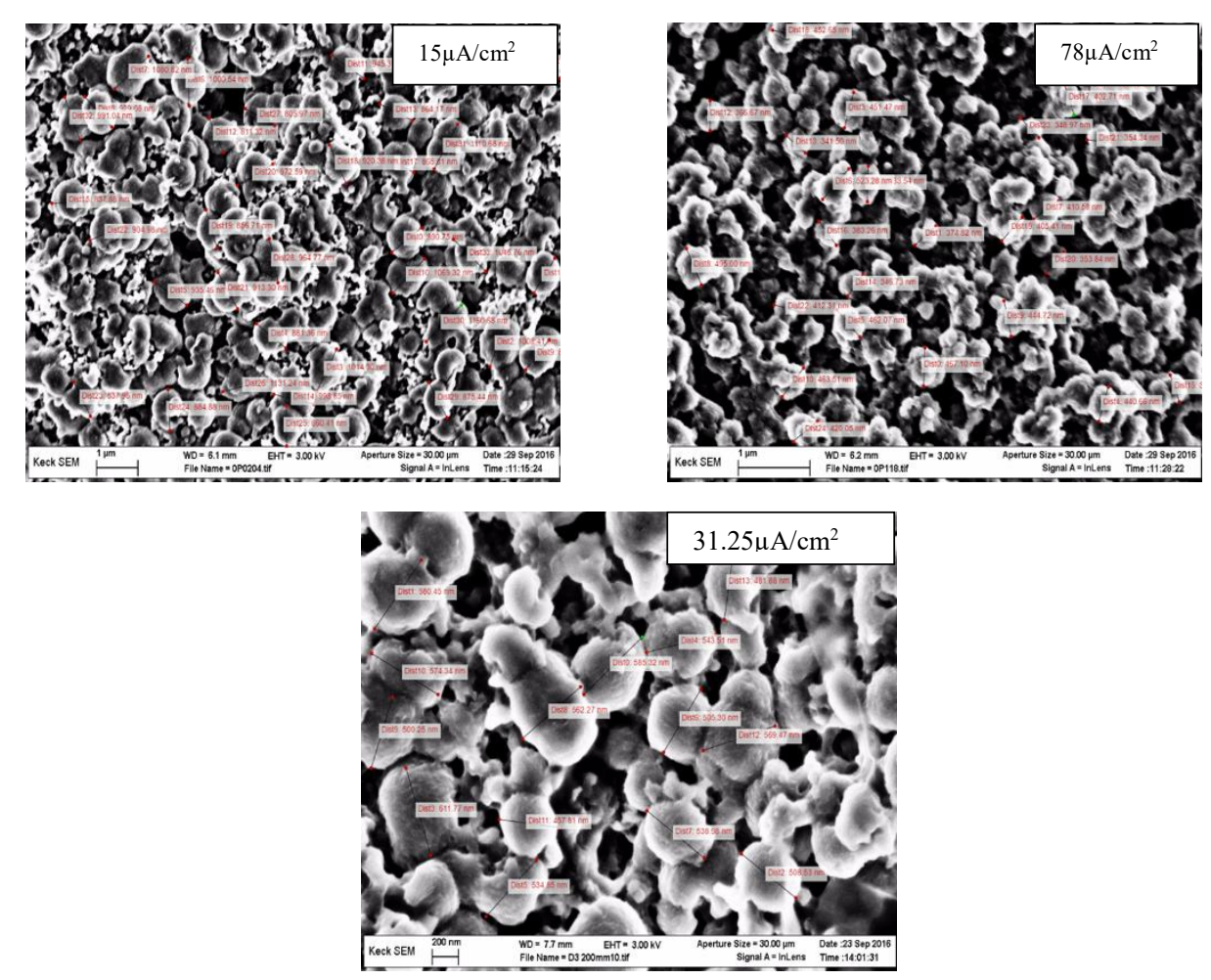

Supplementary Figure 13.7: Size analysis of lithium peroxide particles after discharging a $\mathrm{Li}-\mathrm{O}_{2}$ cell with $1 \mathrm{M} \mathrm{LiNO}_{3}-\mathrm{DMAc}$ electrolyte and ionomer additive at different current densities as indicated in the box 
Supplementary Table 13.1: Atomic percentage of detected elements on lithium anodes. Samples (1) without ionomer discharged to $2 \mathrm{~V}$, (2) without ionomer discharged and recharged for one hour, (3) with ionomer discharged to $2 \mathrm{~V}$, (4) with ionomer discharged and recharged for one hour and (5) with ionomer cycled five times for one hour each.

\begin{tabular}{|l|l|l|l|l|l|}
\hline \multirow{2}{*}{ Component } & \multicolumn{4}{|l|}{ Anode } & \multicolumn{4}{|l|}{} & \multicolumn{2}{l|}{$\mid$} & \\
\cline { 2 - 6 } & 1 & 2 & 3 & 4 & 5 \\
\hline O 1s & 44.93 & 38.42 & 42.35 & 40.11 & 40.99 \\
\hline C 1s & 10.52 & 15.23 & 12.88 & 16.4 & 16.42 \\
\hline N 1s & 2.02 & 3.61 & 2.61 & 2.84 & 2.92 \\
\hline F 1s & 1.12 & 5.12 & 1.25 & 1.73 & 2.35 \\
\hline Br 3p & & & 1.01 & 1.74 & 1.71 \\
\hline S 2p & & & 2.11 & 3.80 & 4.09 \\
\hline Li 1s & 41.42 & 37.62 & 37.8 & 33.38 & 31.52 \\
\hline
\end{tabular}




\section{Experimental}

\section{$\mathrm{Li}-\mathrm{O}_{2}$ battery methods and materials:}

\section{Cathode preparation}

A cathode slurry was prepared by mixing $180 \mathrm{mg}$ of Super P carbon (TIMCAL), 20

mg of polyvinylidene fluoride (PVDF; Aldrich), and $2000 \mathrm{mg}$ of N-Methyl-2pyrrolidone (NMP; Aldrich) in a ball mill at $50 \mathrm{~Hz}$ for 1 hour. Toray TGP-H-030 carbon paper was coated with an $80 \mu \mathrm{m}$ thick layer of carbon slurry using a doctor blade. The resulting coated carbon paper was dried at $100{ }^{\circ} \mathrm{C}$ overnight under vacuum and transferred into an argon filled glovebox $\left(\mathrm{O}_{2}<0.2 \mathrm{ppm}, \mathrm{H}_{2} \mathrm{O}<1.0 \mathrm{ppm}\right.$; Innovative Technology) without exposure to air. 5/8-inch diameter disks were punched and weighed from the carbon paper to yield individual carbon cathodes. The weight of the active carbon layer (not including the carbon paper) averaged $1.0 \mathrm{mg} \pm$ $0.1 \mathrm{mg}$.

\section{Electrolyte preparation}

$\mathrm{LiNO}_{3}$ and LiTFSI were heated under vacuum overnight at $100{ }^{\circ} \mathrm{C}$ to remove all traces of water and transferred directly into the glovebox. N,N-dimethylacetamide (DMA; Aldrich) and bis(2-methoxyethyl) ether (diglyme; Aldrich) solvents were dried over 3 $\AA ̊$ molecular sieves (Aldrich). Lithium 2-bromoethanesulfonate was obtained through ion exchange with sodium 2-bromoethanesulfonate (Alrdich). 


\section{Coin cell assembly}

First, a 1/2-inch $(12.7 \mathrm{~mm}$ ) diameter hole was punched in the top (cathode) side of each CR2032 case. Then, a stainless steel wire cloth disk, 3/4-inch $(19 \mathrm{~mm})$ disk diameter, 0.0055-inch $(0.140 \mathrm{~mm})$ wire diameter from McMaster-Carr was added, followed by a cathode disk, 3/4 inch diameter separator (either Whatman GF/D glass fiber or Celgard 3501), $100 \mu \mathrm{L}$ of desired electrolyte, $1 / 2$ inch diameter lithium metal, $15.5 \mathrm{~mm}$ diameter stainless steel spacer disk, stainless steel wave spring (MTI Corporation), and anode cap of the CR2032 case. The assembly was crimped to a pressure of $14 \mathrm{MPa}$ with a hydraulic coin cell crimple (BT Innovations).

\section{Testing environment}

Cells were tested at a regulated pure $\mathrm{O}_{2}$ environment of $1.3 \mathrm{~atm}$, and allowed to equilibrate for 6 hours prior to electrochemical testing. Galvanostatic measurements were conducted using a Newar CT-3008 battery tester.

\section{Cyclic Voltammetry}

The cyclic voltammetry test was done in a two-electrode setup of Li\|air cathode. The batteries were cycled between $1.9 \mathrm{~V}$ to $4.5 \mathrm{~V}$ at a scan rate of $1 \mathrm{mV} / \mathrm{sec}$ several times.

\section{Anode stability methods and materials:}

Impedance Spectroscopy

Cells in the symmetric configuration were assembled in an Ar glovebox. Measurements were done using a Solatron frequency analyzer at a frequency range of 
$10^{-3}$ to $10^{7} \mathrm{~Hz}$. The data was fitted into Nyquist-type plots using the equivalent circuit shown in Supplementary Figure 10.2 with the software zsimpwin. Impedance was conducted at room temperature at various time intervals.

\section{Linear scan voltammetry}

Linear scan voltammetry was done in a $\mathrm{Li} \| \mathrm{SS}$ cell. The batteries were first swept to $0.2 \mathrm{~V}$ vs $\mathrm{Li} / \mathrm{Li}^{+}$, then they were swept in reverse direction until the voltage diverges.

\section{Lithium vs. stainless steel cycling}

For cycling tests, Lithium vs. stainless steel cells were prepared and were cycled at $0.01 \mathrm{~mA} / \mathrm{cm}^{2}$ between 0 to $0.5 \mathrm{~V}$ ten times in order to form a stable SEI layer. Then different tests were done as given in the manuscript.

\section{Characterization Techniques:}

Scanning Electron Microscopy and EDAX

Discharged cells were disassembled inside the glovebox, and the cathodes were removed and transported to the scanning electron microscope (Zeiss LEO 1550 Field Emission SEM) within an airtight container. The cathodes were loaded onto the stage in the presence of a nitrogen stream. Images were taken with a single pass after focusing on a nearby region. EDAX measurements were done by taking multiple counts on a small section of sample. 


\section{X-Ray Diffraction}

Cathodes were mounted on a glass microscope slide inside an argon-filled glovebox and coated with paraffin oil to protect them from air during the x-ray diffraction (XRD) measurements. Measurements were done on a Scintag Theta-Theta X-ray diffractometer using $\mathrm{Cu}$ K- $\alpha$ radiation at $\lambda=1.5406 \AA$ and fitted with a 2 -dimensional detector. Frames were captured with an exposure time of 10 minutes, after which they were integrated along $\chi$ (the polar angle orthogonal to $2 \theta$ to yield an intensity vs $2 \theta$ plot.

\section{X-Ray Photoelectron Spectroscopy}

XPS was conducted Surface Science Instruments SSX-100 with operating pressure of $\sim 2 \times 10^{-9}$ torr. Monochromatic Al K- $\alpha$ x-rays $(1486.6 \mathrm{eV})$ with beam diameter of $1 \mathrm{~mm}$ were used. Photoelectrons were collected at an emission angle of $55^{\circ}$. A hemispherical analyzer determined electron kinetic energy, using a pass energy of $150 \mathrm{~V}$ for wide survey scans and 50V for high-resolution scans. Samples were ion-etched using 4kV Ar ions, which were rastered over an area of $2.25 \times 4 \mathrm{~mm}$ with total ion beam current of $2 \mathrm{~mA}$, to remove adventitious carbon. Spectra were referenced to adventitious $\mathrm{C} 1 \mathrm{~s}$ at $284.5 \mathrm{eV}$. CasaXPS software was used for XPS data analysis with Shelby backgrounds. Li 1s and $\mathrm{O} 1 \mathrm{~s}$ were assigned to single peaks for each bond, whereas $\mathrm{Br}$ $3 \mathrm{~d}$ was assigned to double peaks $\left(3 \mathrm{~d}_{5 / 2}\right.$ and $\left.3 \mathrm{~d}_{3 / 2}\right)$ for each bond with $1.05 \mathrm{eV}$ separation. Residual SD was maintained close to 1.0 for the calculated fits. Samples

were exposed to air only during the short transfer time to the XPS chamber (less than 5 seconds). 


\section{Bibliography}

1. Stabilizing Electrodeposition of Metals by Confinement in Structured Electrolytes

S. Choudhury*, D. Vu*, A. Warren, M. Tikekar, Z. Tu, L. A. Archer. Proceedings of the National Academy of Sciences, 115 (26) 6620-6625 (2018)

2. Fast Ion Transport at Solid-Solid Interfaces in hybrid battery anodes. Z. Tu*, S. Choudhury*, M. J. Zachman, S. Wei, K. Zhang, L. F. Kourkoutis and L. A. Archer. Nature Energy (2018) (DOI:10.1038/s41560-018-0096-1)

3. Designer Interphases for the Lithium-Oxygen Electrochemical Cell. S. Choudhury*, C. T. Wan*, W. I. Al Sadat, Z. Tu, S. Lau, M. J. Zachman, L. F. Kourkoutis and L. A. Archer. Science Advances, E1602809 (2017)

4. Designing Solid-liquid Interphases for Sodium Batteries S. Choudhury*, S. Wei*, Y. Ozhabes, D. Gunceler, M. J. Zachman, Z. Tu, J. H. Shin, P. Nath, A. Agrawal, L. F. Kourkoutis, T. A. Arias and L. A. Archer. Nature Communications (2017)

5. Electroless Formation of Hybrid Lithium Anodes for Fast Interfacial Ion Transport 
S. Choudhury*, Z. Tu*, K. Fawole, S. Stalin, D. Gunceler, R. Sundararaman and L. A. Archer.

6. Highly stable sodium batteries enabled by functional ionic polymer membranes.

S. Wei*, S. Choudhury*, J. Xu, P. Nath, Z. Tu, and L. A. Archer. Advanced Materials, 29, 1605512 (2017)

7. Designing artificial solid-electrolyte interphases for single-ion, high-efficiency transport in batteries

Z. Tu*, S. Choudhury*, M. J. Zachman, S. Wei, K. Zhang, L. F. Kourkoutis, L. A. Archer. Joule-Cell Press, 1, 1-13 (2017)

8. Lithium Fluoride Additives for Stable Cycling of Lithium Batteries at High Current Densities.

S. Choudhury and L. A. Archer. Advanced Electronic Materials, 2, 1500246 (2016)

9. Hybrid Hairy Nanoparticles Stabilize Lithium Metal Batteries.

S. Choudhury*, A. Agrawal*, S. Wei, E. Jeng and L. A. Archer. Chemistry of Materials, 28 (7), 2147-2157 (2016) 
10. A Highly Reversible Room Temperature Lithium Metal Battery based on Cross-linked Hairy Nanoparticles.

S. Choudhury, R. Mangal, A. Agrawal and L. A. Archer. Nature

Communications, 6: 10101 (2015)

11. Self-suspended Suspensions of Covalently Grafted Hairy Nanoparticles.

S. Choudhury*, A. Agrawal*, S A Kim, and L. A. Archer. Langmuir, 31 (10) $3222-3231(2015)$

12. A Highly Conductive, Non-flammable Polymer-nanoparticle Hybrid Electrolyte.

A. Agrawal*, S. Choudhury*, and L. A. Archer. RSC Advances, 5, 20800$20809(2015)$

13. Electrochemical Interphases for High-Energy Storage Using Reactive Metals Anodes

S. Wei, S. Choudhury, Z. Tu, K. Zhang and L. A. Archer. Accounts of Chemical Research, 51 (1), 80-88 (2018)

14. Multifunctional Cross-Linked Polymeric Membranes for Safe, HighPerformance Lithium Batteries

S. Stalin, S. Choudhury, K. Zhang and L. A. Archer. Chemistry of Materials, 30 (6), 2058-2066 (2018) 
15. Self-suspended Polymer Grafted Nanoparticles.

S. Srivastava, S. Choudhury, A. Agrawal. Current Opinion in Chemical Engineering, 16, 92-101 (2017)

16. Design Principles for Electrolytes and Interfaces for Stable Lithium-metal Batteries.

M. D. Tikekar, S. Choudhury, Z. Tu, and L. A. Archer. Nature Energy, $1: 16114(2016)$

17. Nanoporous Hybrid Electrolytes for High Energy Batteries Based on Reactive Metal Anodes.

Z. Tu, M. J. Zachman, S. Choudhury, S. Wei, L. Ma, Y. Yang, L. F. Kourkoutis, L. A. Archer. Advanced Energy Materials, 1602367 (2017)

18. Multifunctional Separator Coatings for High-Performance Lithium-Sulfur Batteries

M. S. Kim, L. Ma, S. Choudhury, L. A. Archer. Advanced Materials Interfaces, 3 (22) (2016)

18. Fabricating Multifunctional Nanoparticle Membranes by a Fast Layer-byLayer Langmuir-Blodgett Process: Application in Lithium-Sulfur Batteries 
M. S. Kim, L. Ma, S. Choudhury, S. S. Moganty, S. Wei, L. A. Archer. Journal of Materials Chemistry A, 4, 14709-14719 (2016)

19. Interactions, Structure, and Dynamics of Polymer-Tethered Nanoparticle Blends

A. Agrawal, B. M. Wenning, S. Choudhury. Langmuir 32 (34), 8698-8708 (2016)

20. Multiscale Dynamics of polymers in Particle-Rich Nanocomposites R. Mangal, Y. H. Wen, S. Choudhury, L. A. Archer. Macromolecules 49 (14), $5202-5212(2016)$

21. Electronic and Chemical Properties of Germanene: The Crucial Role of Buckling.

A. Nijamudheen, R. Bhattacharjee, S. Choudhury, and A. Datta. Journal Physical Chemistry C, 119 (7), pp 3802-3809 (2015)

22. Dynamics of nanoparticles in entangled polymer solutions P. Nath, R. Mangal, F. Kohle, S. Choudhury, S. Narayanan, U. Wiesner, L. A. Archer. Langmuir, 34 (1), 241-249 (2018)

23. Design principles of functional polymer separators for high-energy metalbased batteries 
W. Zhang, Z. Tu, J. Qian, S. Choudhury, Y. Lu and L. A. Archer. Small (2018) (DOI: $10.1002 / \mathrm{smll} .201703001)$

24. A Stable Room Temperature Sodium-sulfur Battery.

S. Wei, S. Xu, A. Agrawral, S. Choudhury, Y. Lu, Z. Tu, L. Ma, L. A. Archer. Nature Communications, 7: 117222 (2016)

25. Molecular Origins of Temperature-Induced jamming in Self-Suspended Hairy Nanoparticles

A. Agrawal, H.Y. Yu, A. Sagar, S. Choudhury, L. A. Archer. Macromolecules, 49 (22), 8738-8747 (2016)

26. Dynamics and yielding of binary self-suspended Nanoparticle Fluids.

A. Agrawal, H. Y. Hsiu, S. Srivastava, S. Choudhury, S. Narayanan and L. A. Archer. Soft Matter, 11, 5224-5234 (2015)

27. Building Organic/Inorganic Hybrid Interphases for Fast Interfacial Transport in Rechargeable Metal Batteries.

Q. Zhao, Z. Tu, S. Wei, K. Zhang, S. Choudhury, X. Liu, L. A. Archer, Angewandte Chemie International Ed., 57, 992 (2018)

29. Stabilizing Polymer Electrolytes in High-Voltage Lithium Batteries 
S. Choudhury*, Z. Tu*, A. Nijamudheen, M. J. Zachman, S. Stalin, Y. Deng, Q. Zhao, D. Vu, L. F. Kourkoutis, J. Mendoza-Cortes, L. A. Archer. (Under Review)

30. Soft Colloidal Glasses as Solid-State Electrolytes S. Choudhury, S. Stalin, Y. Deng, L. A. Archer. (Under Review)

31. Cryo-STEM mapping of solid-liquid interfaces and dendrites in Li-metal batteries

M. J. Zachman, Z. Tu, S. Choudhury, S. Stalin, L. A. Archer, L. F. Kourkoutis. Nature (In press)

32. Stabilizing protic and aprotic liquid electrolytes at high-bandgap oxide interphases

Z. Tu1, M. J. Zachman, S. Choudhury, K. A. Khan, Q. Zhao, L. F. Kourkoutis, L. A. Archer. Chemistry of Materials (Accepted) 